\title{
Stress Parameter Distribution on an Earthquake Fault Based on a Stochastic Modeling Approach
}

\author{
Submitted by \\ Karen Assatourians

\begin{abstract}
A thesis submitted to
The Faculty of Graduate Studies and Research

In partial fulfillment of the requirements for the degree of

Doctor of Philosophy
\end{abstract}

Ottawa-Carleton Geoscience Center

And Department of Farth Sciences

Carleton University

Ottawa. Ontario

Winter 2008

(c) 2008 Karen Assatourians 


$\begin{array}{ll}\begin{array}{l}\text { Library and } \\ \text { Archives Canada }\end{array} & \begin{array}{l}\text { Bibliothèque et } \\ \text { Archives Canada }\end{array} \\ \begin{array}{l}\text { Published Heritage } \\ \text { Branch }\end{array} & \begin{array}{l}\text { Direction du } \\ \text { Patrimoine de l'édition }\end{array} \\ \begin{array}{l}\text { 395 Wellington Street } \\ \text { Ottawa ON K1A 0N4 }\end{array} & \begin{array}{l}\text { 395, rue Wellington } \\ \text { Ottawa ON K1A ON4 }\end{array} \\ \text { Canada } & \begin{array}{l}\text { Canada } \\ \end{array}\end{array}$

Yourfile Votre référence

ISBN: 978-0-494-36775-9

Ourfile Notre référence

ISBN: 978-0-494-36775-9

NOTICE:

The author has granted a nonexclusive license allowing Library and Archives Canada to reproduce, publish, archive, preserve, conserve, communicate to the public by telecommunication or on the Internet, loan, distribute and sell theses worldwide, for commercial or noncommercial purposes, in microform, paper, electronic and/or any other formats.

The author retains copyright ownership and moral rights in this thesis. Neither the thesis nor substantial extracts from it may be printed or otherwise reproduced without the author's permission.
AVIS:

L'auteur a accordé une licence non exclusive permettant à la Bibliothèque et Archives Canada de reproduire, publier, archiver, sauvegarder, conserver, transmettre au public par télécommunication ou par l'Internet, prêter, distribuer et vendre des thèses partout dans le monde, à des fins commerciales ou autres, sur support microforme, papier, électronique et/ou autres formats.

L'auteur conserve la propriété du droit d'auteur et des droits moraux qui protège cette thèse. $\mathrm{Ni}$ la thèse ni des extraits substantiels de celle-ci ne doivent être imprimés ou autrement reproduits sans son autorisation.
In compliance with the Canadian

Privacy Act some supporting forms may have been removed from this thesis.

While these forms may be included in the document page count, their removal does not represent any loss of content from the thesis.
Conformément à la loi canadienne sur la protection de la vie privée, quelques formulaires secondaires ont été enlevés de cette thèse.

Bien que ces formulaires aient inclus dans la pagination, il n'y aura aucun contenu manquant.

\section{Canadä}




\section{Abstract}

This work compares the strengths and limitations of three variations of stochastic modeling of ground motions of earthquakes. The considered models all do a reasonable job of reproducing salient ground-motion measures in the time and frequency domains overall, though finite-source method of Motazedian and Atkinson (2005) is superior to other models over the range of magnitude, distance and frequencies that are of practical interest in engineering seismology. However, this stochastic finite-source technique is flawed in terms of its ability to predict directivity effects at lower frequencies.

The modeling technique of Motazedian and Atkinson (2005) is modified to simulate the effects of variable stress parameter on the fault. The spectrum of each sub-source is multiplied by a correction spectrum that leaves the low frequency portion of the spectrum intact and multiplies the high-frequency end of the spectrum by a constant proportional to $2 / 3$ power of the stress parameter of each sub-source.

The variable-stress implementation of stochastic finite-fault modeling is coupled with an inversion algorithm that effectively inverts observed response spectral data to derive the stress parameter distribution on the fault surface. This inversion tool applies the Levenberg-Marquardt method to minimize differences of average $(\log )$ response spectra ordinates at high frequencies between observations and simulations at all stations. Application of this inversion tool to observed ground-motion data from well-recorded earthquakes could be used to deduce the distribution of stress parameter across the fault plane. This is the first such application of stochastic-modeling techniques to determine the distribution of source strength on the fault.

Experiments for studying the effects of fault dip angle, number of simulation iterations used to define the model ground motions at each station, initial guess of the stress parameter distribution, and station distribution show that the tool works well if there is a sufficient number of observations about the fault plane. Also, this tool is capable of resolving the relative stress parameters of multiple asperities on a fault plane. 
Application of the inversion tool to the data of four well-recorded California earthquakes shows that the derived stress parameter distributions are in agreement with slip distribution results by other researchers. 


\section{Acknowledgments}

To a casual observer, a doctoral dissertation may appear to be a solitary work. However, to complete a project of this magnitude requires a network of support, and I am indebted to many people. First and foremost, I would like to send my utmost gratitude to my supervisor, Dr. Gail M. Atkinson, for her guidance, support, encouragement, and perspective. Also I would like to thank my co-supervisor, Dr. Dariush Motazedian, for useful discussions and comments about stochastic finite fault technique and related topics. I gained better understanding about seismological, statistical, and optimization aspects of the study from discussions with Dr. Ali Fatehi, Dr. David Boore, Grigor Karimian, Nader Fanai, and Kasgin Khaheshi.

I would like to thank my parents, whose love and guidance are with me in whatever I

pursue. Most importantly, I wish to thank my loving and supportive wife. Ani, who provides unending inspiration.

I should thank many individuals, friends and colleagues who have not been mentioned here personally in making this educational process a success.

This study is financially supported by Carleton University and an NSERC (Natural Sciences and Engineering Research Council of Canada) grant to G. Atkinson. 


\section{Table of Contents}

Title Page $\quad$ i

Abstract

Acknowledgements $\quad$ iv

Table of Contents $\quad$ v

List of Tables $\quad x$

List of Figures $\quad$ xii

List of Appendices $\quad x x i$

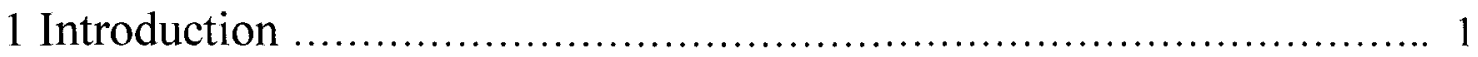

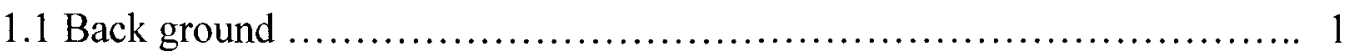

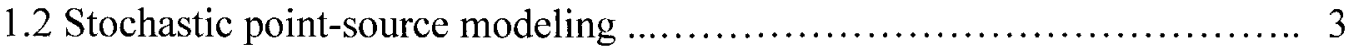

1.3 Stochastic finite-source modeling (FINSIM) $\ldots \ldots \ldots \ldots \ldots \ldots \ldots \ldots \ldots$

1.4 Stochastic finite-source modeling (EXSIM) ........................ 10

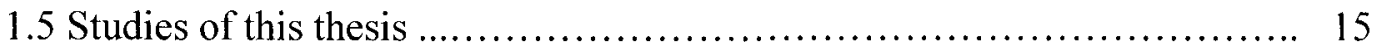

2 Stochastic Forward Modeling Experiments .................................. 17

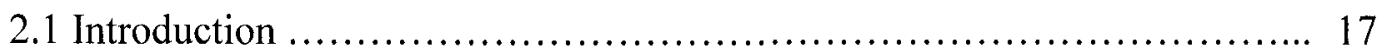

2.2 Directivity from EXSIM simulations ............................... 18

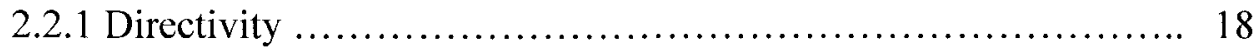

2.2.2 Measurement quantity ................................ 19

2.2.3 Simulation parameters and results $\ldots \ldots \ldots \ldots \ldots \ldots \ldots \ldots \ldots \ldots, 19$

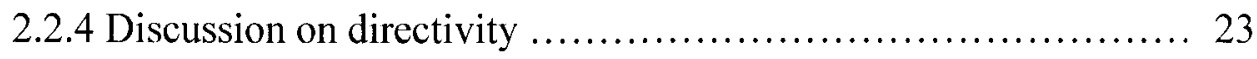

2.3 Sub-source size independence of EXSIM simulations................... 25

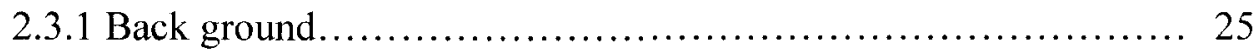


2.3.2 Simulation parameters and results

2.3.3 Discussion of sub-source size independence ................ 32

2.4 Spectral comparisons of different simulation algorithms .............. 35

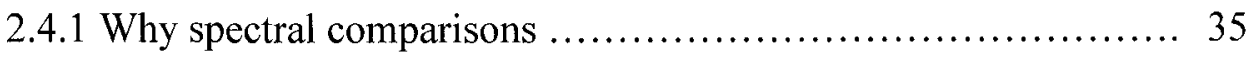

2.4.2 Comparing Fourier spectra of SMSIM (point-source) and EXSIM (extended source) ............................... 35

2.4.3 Comparing PSAs of SMSIM and EXSIM ................... 50

2.4.4 Comparing EXSIM with and without dynamic corner frequency . 53

2.5 Comparing SMSIM, FINSIM, and EXSIM against data ............... 57

2.5.1 Purpose ...................................................... 57

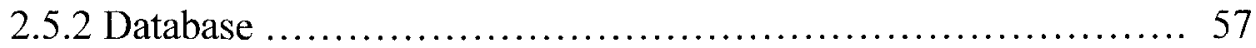

2.5.3 Reference magnitudes and distances ..................... 58

2.5.4 Correction of recorded PSAs to reference level ................ 60

2.5.5 SMSIM, FINSIM, and EXSIM simulations ................ 61

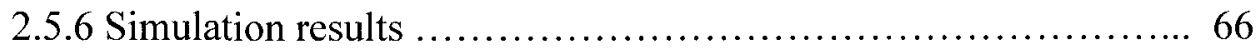

2.5.7 Error measure ........................................ 66

2.5.8 Error measurement results .............................. 67

2.5.9 Discussion and conclusion on three program comparisons ....... 70

3 Extended Applications of Stochastic Finite Fault Modeling .............. 89

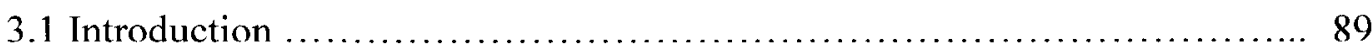

3.2 Implementation of variable rupture velocity in EXSIM ................ 89

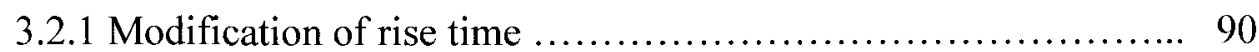

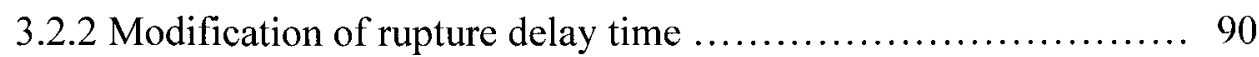


3.2.3 Rupture velocity variability experiments

3.3 Implementation of variable stress parameter in EXSIM ................... 105

3.3.1 Assumptions and formulations of variable stress parameter concept ................................................. 105

3.3.2 Stress parameter variability experiments ..................... 107

3.3.3 Stress parameter variability versus slip variability ............. 108

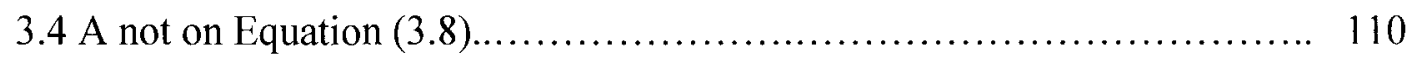

4 Inversion Program Development and Performance ......................... 116

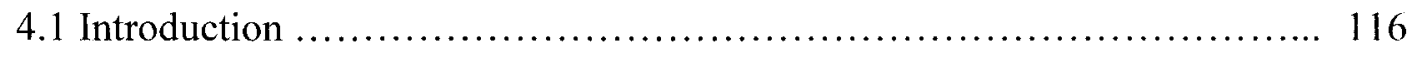

4.2 Development of inversion tool for stress parameter distribution............ 118

4.2.1 Error measurements to evaluate model performance ............. 119

4.2.2 Formulation of inversion tool ............................. 121

4.2.3 Functional form of objective function and Jacobian matrix

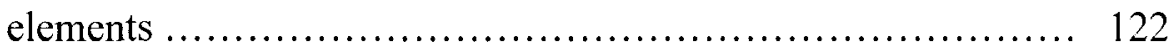

4.2.4 Inversion procedure ....................................... 124

4.3 Model performance studies.......................................... 125

4.3.1 Effect of EXSIM iterations and fault dip angle ................. 126

4.3.2 Multiple asperities .......................................... 127

4.3.3 Effect of initial guess .................................. 142

4.3.4 Effect of station distribution and asperity location .............. 150

4.3.5 A note on resolution........................................... 158

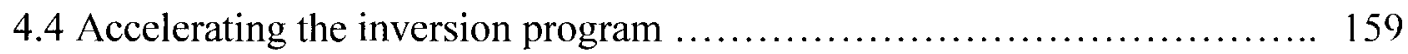

4.4.1 "Contrast amplifier" program ................................ 159 
4.4.2 Application and performance of "Contrast amplifier" program ... 160

4.5 Application of Method to the Parkfield Earthquake.................. 169

4.5.1 Data used and site corrections ............................ 169

4.5.2 Inversion of Recorded Parkfield Data ..................... 171

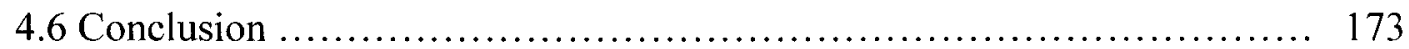

5 Implementation of Inversion Tool for Three Events ................... 182

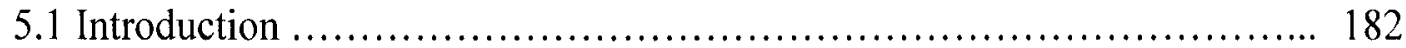

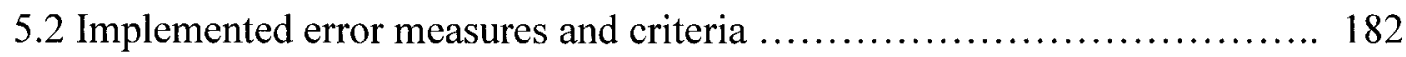

5.3 Data preparation and modeling parameters $\ldots \ldots \ldots \ldots \ldots \ldots \ldots \ldots \ldots \ldots . \ldots \ldots$

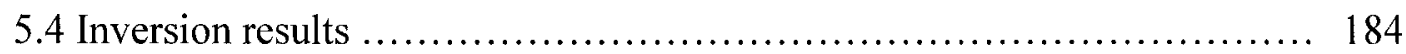

5.4.1 M7.1, 1989 Loma Prieta earthquake ..................... 184

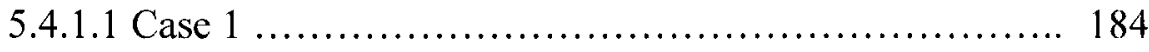

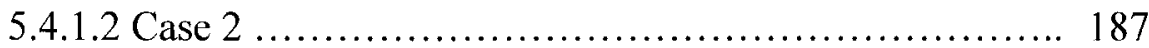

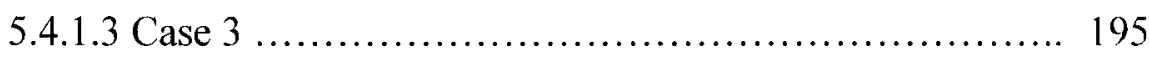

5.4.1.4 Loma Prieta earthquake slip model from other studies .. 196

5.4.2 M7.3, 1992 Landers earthquake ........................ 197

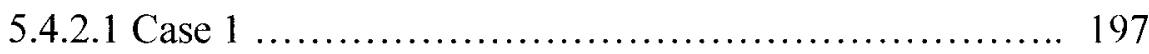

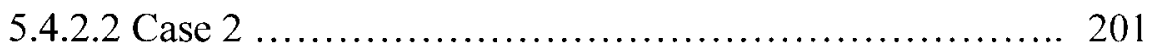

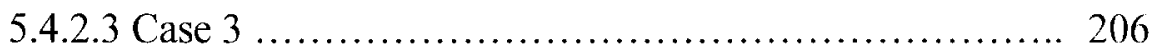

5.4.2.4 Landers earthquake slip model from other studies ...... 207

5.4.3 M6.7, 1994 Northridge earthquake ........................ 208

5.4.3.1 Case 1 ........................................ 208

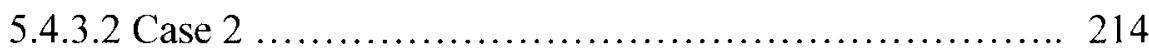

viii 
5.4.3.4 Northridge earthquake slip model from other studies ... 223

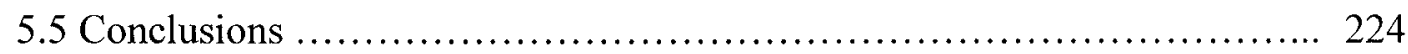

6 Summary and Conclusions ........................................... 229

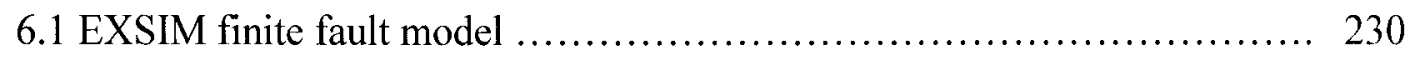

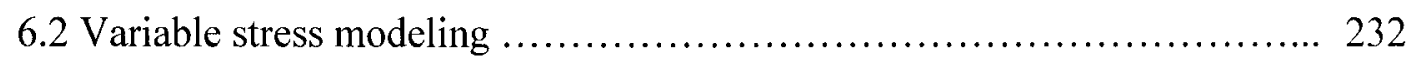

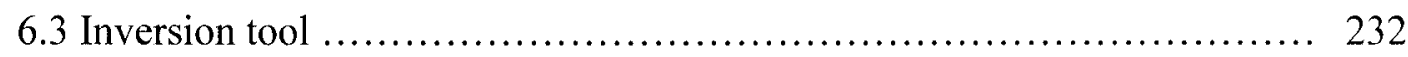

6.4 Variable stress modeling of California earthquakes .................... 234

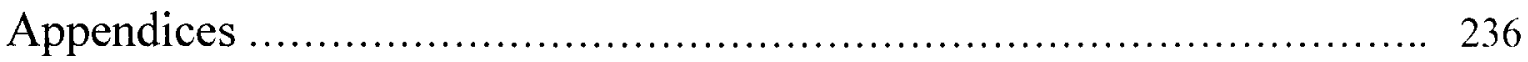

A Residual study of SMSIM, FINSIM, and EXSIM............... 236

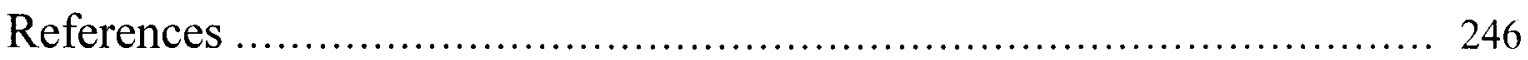




\section{List of Tables}

2.1 First set of simulation parameters for directivity study $\ldots \ldots \ldots \ldots \ldots \ldots \ldots \ldots \ldots \ldots \ldots \ldots$

2.2 Second set of simulation parameters for directivity study .................... 29

2.3 Third set of simulation parameters for directivity study ..................... 29

2.4 Simulation parameters for sub-source size independence study $\ldots \ldots \ldots \ldots \ldots \ldots \ldots . \ldots 33$

2.5 Simulation parameters for SMSIM and EXSIM Fourier spectra comparisons ..... 39

2.6 Information of extracted subset of NGA database used in SMSIM, FINSIM, and

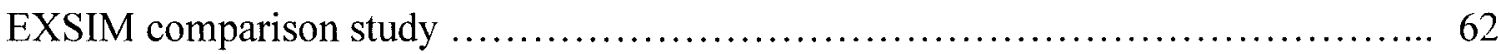

2.7 Common parameter values used in the three simulation approaches of SMSIM, FINSIM, and EXSIM comparison study

2.8 Reference magnitude-distance combinations of performed simulations in SMSIM, FINSIM, and EXSIM comparison study

2.9 Information of records and used earthquakes in Magnitude-distance ranges along with PSA differences between records and simulations averaged over $0.1 \mathrm{~Hz}-10 \mathrm{~Hz} \ldots .77$

2.10 Information of records and used earthquakes in Magnitude-distance ranges along with PSA differences between records and simulations at $0.33 \mathrm{~Hz}, 1 \mathrm{~Hz}$, and $3.3 \mathrm{~Hz} \ldots .79$

2.11 Results of simulation methods' error measure ( $\varepsilon$-value) distribution similarity tests performed in different frequencies and different sub-sets of $\varepsilon$-values

2.12 Preference table for choosing appropriate simulation technique among SMSIM, FINSIM, and EXSIM for considered scenario and frequency range

3.1 Common simulation parameters for 441 sites for studying the effect of variable

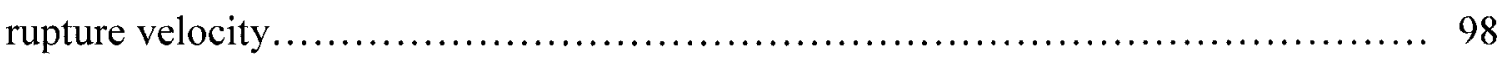

3.2 The correlation coefficients of PSA distribution maps at different frequencies in variable rupture velocity study 
3.3 The correlation coefficients of PSA distribution maps at different frequencies in variable stress parameter distribution study

4.1 Simulation parameters for the derivation of $D_{r}$ values for a range of distances and stress parameters of a sub-source

4.2 Simulation parameters for model performance studies

4.3 The correlation coefficients of inversion results with target distributions for different fault dip angles

4.4 List of stations used for M6, 2004 Parkfield earthquake study 176

5.1 Simulation parameters of Loma Prieta, Landers, and Northridge earthquakes 188

A.1 Preference table for choosing appropriate simulation technique among SMSIM, FINSIM, and EXSIM for considered scenario and frequency range based on residuals 245 


\section{List of Figures}

1.1 Steps of development of synthetic accelerogram using stochastic point source modeling

1.2 Subdivision of a fault into sub-sources and summing effects of all sub-sources at recording site in time domain

2.1 Earthquake fault subdivision to sub-sources implemented in three simulations for directivity study

2.2 Log of geometrical averages of PSA ordinates $\left(D_{r}\right)$ as a function of direction for $0.45 \mathrm{~Hz}-10 \mathrm{~Hz}$ and $2.5 \mathrm{~Hz}-25 \mathrm{~Hz}$ frequency ranges

2.3 Duration of simulated time series as a function of azimuth

2.4 Log of PSA ordinates as a function of direction at frequencies: $25 \mathrm{~Hz}, 10 \mathrm{~Hz}, 5 \mathrm{~Hz}$, $2 \mathrm{~Hz}, 1 \mathrm{~Hz}, 0.5 \mathrm{~Hz}, 0.2 \mathrm{~Hz}$, and $0.1 \mathrm{~Hz}$ for an $\mathbf{M} 6$ earthquake

2.5 Log of PSA ordinates as a function of direction at frequencies: $25 \mathrm{~Hz}, 5 \mathrm{~Hz}, 1 \mathrm{~Hz}$, and $0.2 \mathrm{~Hz}$ for an $\mathbf{M} 6$ earthquake with no distance dependent sub-source duration ...... 30

2.6 Log of PSA ordinates as a function of direction at frequencies: $25 \mathrm{~Hz}, 5 \mathrm{~Hz}, 1 \mathrm{~Hz}$, and $0.2 \mathrm{~Hz}$ for an $\mathbf{M} 7$ earthquake with no distance dependent sub-source duration

2.7 Surface trace of vertical fault for $\mathbf{M} 7.0$ earthquake and location of 500 sites at 15 $\mathrm{km}$ distance from fault

2.8 Averages and standard deviations of Fourier acceleration spectra of 500 simulations

2.9 Averages of Fourier acceleration spectra of 500 simulations for a site located at $300 \mathrm{~km}$ distance from a fault with no sub-divisions at magnitudes M5, M6, and M7 ... 40 2.10 Averages of Fourier acceleration spectra of 500 simulations for a site located at $300 \mathrm{~km}$ distance from a fault with 30 sub-divisions at magnitudes $\mathbf{M} 5, \mathbf{M} 6$, and $\mathbf{M} 7 \ldots 41$ 
2.11 Average of Fourier acceleration spectra of 500 simulations for sites located at $\mathrm{R}_{\mathrm{JB}}=15 \mathrm{~km}$ and $\Delta \sigma=50$ bars at magnitudes $\mathbf{M} 5, \mathbf{M} 6, \mathbf{M} 7$, and $\mathbf{M} 8$

2.12 Average of Fourier acceleration spectra (FAS) of 500 simulations for sites located at $\mathrm{R}_{\mathrm{JB}}=15 \mathrm{~km}$ and $\Delta \sigma=120$ bars at magnitudes $\mathbf{M} 5, \mathbf{M} 6, \mathbf{M} 7$, and $\mathbf{M} 8$

2.13 Average of Fourier acceleration spectra (FAS) of 500 simulations for sites located at $\mathrm{R}_{\mathrm{JB}}=300 \mathrm{~km}$ and $\Delta \sigma=50$ bars at magnitudes $\mathbf{M} 5, \mathbf{M} 6, \mathbf{M} 7$, and $\mathbf{M} 8$

2.14 Average of Fourier acceleration spectra (FAS) of 500 simulations for sites located at $\mathrm{R}_{\mathrm{IB}}=300 \mathrm{~km}$ and $\Delta \sigma=120$ bars at magnitudes $\mathbf{M} 5, \mathbf{M} 6, \mathbf{M} 7$, and $\mathbf{M} 8$

2.15 Average of pseudo spectral accelerations (FAS) of 500 simulations for sites located at $\mathrm{R}_{\mathrm{JB}}=15 \mathrm{~km}$ and $\Delta \sigma=120$ bars at magnitudes $\mathbf{M} 5, \mathbf{M} 6, \mathbf{M} 7$, and $\mathbf{M} 8$

2.16 Comparisons of Averages of FSAs of 500 simulations at magnitudes M5, M6, M7, and M8 when dynamic corner frequency is and is not implemented

2.17 Magnitude-distance distribution of events used to evaluate simulation performance

2.18 Distribution of hypocentral and fault upper corner depths of 26 events

2.19 Average of corrected PSAs of M6.2, 1999 ChiChi earthquake aftershock in distances $\mathrm{R}_{\mathrm{IB}}=1 \mathrm{~km}, \mathrm{R}_{\mathrm{JB}}=10 \mathrm{~km}, \mathrm{R}_{\mathrm{JB}}=30 \mathrm{~km}$, and $\mathrm{R}_{\mathrm{JB}}=200 \mathrm{~km}$

2.20 Average of corrected PSAs of M6.6, 1994 Northridge earthquake in distances $\mathrm{R}_{\mathrm{JB}}=1 \mathrm{~km}, \mathrm{R}_{\mathrm{JB}}=10 \mathrm{~km}, \mathrm{R}_{\mathrm{JB}}=30 \mathrm{~km}$, and $\mathrm{R}_{\mathrm{JB}}=200 \mathrm{~km}$

2.21 Average of corrected PSAs of M7.0, 1989 Loma Prieta earthquake in distances $\mathrm{R}_{\mathrm{JB}}=1 \mathrm{~km}, \mathrm{R}_{\mathrm{JB}}=10 \mathrm{~km}, \mathrm{R}_{\mathrm{JB}}=30 \mathrm{~km}$, and $\mathrm{R}_{\mathrm{JB}}=200 \mathrm{~km}$

2.22 Average of corrected PSAs of M7.2, 1992 Landers earthquake in distances $\mathrm{R}_{\mathrm{JB}}=1 \mathrm{~km}, \mathrm{R}_{\mathrm{JB}}=10 \mathrm{~km}, \mathrm{R}_{\mathrm{IB}}=30 \mathrm{~km}$, and $\mathrm{R}_{\mathrm{IB}}=200 \mathrm{~km}$ 74

2.23 Average of corrected PSAs of M7.6, 1999 ChiChi earthquake in distances $\mathrm{R}_{\mathrm{JB}}=1 \mathrm{~km}, \mathrm{R}_{\mathrm{JB}}=10 \mathrm{~km}, \mathrm{R}_{\mathrm{JB}}=30 \mathrm{~km}$, and $\mathrm{R}_{\mathrm{JB}}=200 \mathrm{~km}$ 75 
2.24 Average of corrected PSAs of M8.0, 2002 Denali earthquake in distances $\mathrm{R}_{\mathrm{IB}}=1 \mathrm{~km}, \mathrm{R}_{\mathrm{IB}}=30 \mathrm{~km}$, and $\mathrm{R}_{\mathrm{JB}}=200 \mathrm{~km}$

2.25 Scatter plot of Error " $\varepsilon$-values" as a function of "Number of records in reference distance" for SMSIM, FINSIM, and EXSIM

2.26 Comparisons of averages of sub-sets of " $\varepsilon$-values" derived from three different simulation methods in $1 \mathrm{~Hz}-10 \mathrm{~Hz}$ frequency range

2.27 Comparisons of averages of sub-sets of " $\varepsilon$-values" derived from three different simulation methods at $0.333 \mathrm{~Hz}$ frequency

2.28 Comparisons of averages of sub-sets of " $\varepsilon$-values" derived from three different simulation methods at $1 \mathrm{~Hz}$ frequency

2.29 Comparisons of averages of sub-sets of " $\varepsilon$-values" derived from three different simulation methods at $3.333 \mathrm{~Hz}$ frequency

3.1 Earthquake fault subdivisions into sub-sources illustrating the parameters and geometry for calculating the delay time

3.2 Distribution of simulation sites around the trace of the fault

3.3 Map of changes of $\log P S A$ values (E-values) at $25 \mathrm{~Hz}$, calculated for variable rupture velocity study

3.4 Map of changes of average $\log P S A$ values (E-values) in the $2.5 \mathrm{~Hz}$ to $20 \mathrm{~Hz}$, calculated for variable rupture velocity study

3.5 Map of changes of average $\log P S A$ values (E-values) in the $0.45 \mathrm{~Hz}$ to $10 \mathrm{~Hz}$, calculated for variable rupture velocity study

3.6 Map of significant changes of $\log P S A$ values ( $E$-values) at $25 \mathrm{~Hz}$, calculated for variable rupture velocity study

3.7 Map of significant changes of average $\log P S A$ values ( $E$-values) in the $2.5 \mathrm{~Hz}$ to $20 \mathrm{~Hz}$, calculated for variable rupture velocity study 
3.8 Map of significant changes of average $\log P S A$ values ( $E$-values) in the $0.45 \mathrm{~Hz}$ to $10 \mathrm{~Hz}$, calculated for variable rupture velocity study

3.9 Map of $\log P S A$ values ( $D_{r}$ values) at $25 \mathrm{~Hz}$, calculated for the study of variable stress parameter effect.

3.10 The map of average $\log P S A$ values ( $D_{r}$ values) in the $2.5 \mathrm{~Hz}$ to $20 \mathrm{~Hz}$, calculated for the study of variable stress parameter effect.

3.11 The map of average $\log P S A$ values ( $D_{r}$ values) in the $0.45 \mathrm{~Hz}$ to $10 \mathrm{~Hz}$, calculated for the study of variable stress parameter effect

3.12 The distribution of average $\log P S A \mathrm{~s}$ in $0.45 \mathrm{~Hz}$ to $10 \mathrm{~Hz}$ frequency range from EXSIM simulations, comparison of variable slip and variable stress effects

3.13 The empirical cumulative distribution function of average $\log P S A \mathrm{~s}$

4.1 $D_{r}$ values of simulations at constant distances from a sub-source for varying stress parameters

4.2 $D_{r}$ values of simulations for constant stress parameters at varying distances from a sub-source

4.3 Distribution of hypothetical stations around the fault for performance studies

4.4 The succession of inversion program solutions for $83^{\circ}$ fault dip angle and 10 EXSIM trials

4.5 The succession of inversion program solutions for $5^{\circ}$ fault dip angle and 10 EXSIM trials

4.6 The succession of inversion program solutions for $25^{\circ}$ fault dip angle and 10 EXSIM trials

4.7 The succession of inversion program solutions for $45^{\circ}$ fault dip angle and 10 EXSIM trials

4.8 Evolution of error $\Xi$ by inversion iteration number for four fault dip angles 
4.9 Evolution of error $\Xi$ by inversion iteration number for fault dip and iteration \# ... 138

4.10 The succession of solutions for the derivation of location and size of asperities ... 139

4.11 The succession of solutions for the derivation of location of close asperities .... 140

4.12 The succession of solutions for the derivation of location and size of multi-size

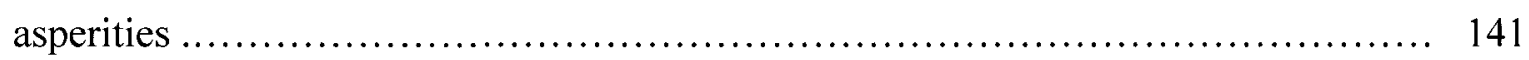

4.13 Target distribution, uniform initial guess, and solution after 5 iterations ......... 144

4.14 Target distribution, accurate initial guess, and solution after 5 iterations ........ 145

4.15 Target distribution, uniform initial guess, and solution after 5 iterations ......... 146

4.16 Target distribution, inaccurate initial guess, and solution after 5 iterations ...... 147

4.17 Target distribution, very poor initial guess, and solution after 2 iterations ....... 148

4.18 Target distribution, poor initial guess, and solution after 35 iterations ......... 149

4.19 Distribution of actual stations around the Parkfield fault ................... 152

4.20 Target stress parameter distributions and corresponding inversion results when forward modeling and inversions are performed for the actual stations ............... 153

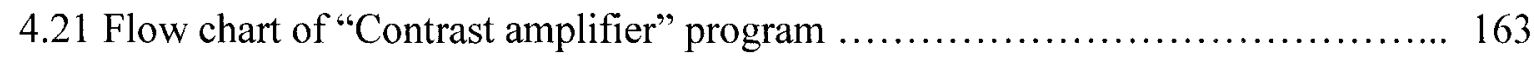

4.22 Target distribution, inversion program output after 1 iteration, and CA program output after processing inversion program output for $5^{\circ}$ fault dip angle ............ 164

4.23 Target distribution, inversion program output after 1 iteration, and CA program output after processing inversion program output for $45^{\circ}$ fault dip angle ............ 165

4.24 The evolution of the correlation coefficient of target distribution with inversion program output and with CA program output for $45^{\circ}$ fault dip angle $\ldots \ldots \ldots \ldots \ldots \ldots . \ldots 7$

4.25 Evolution of error $\Xi$ with successive iterations in the application of ordinary inversion and in the application of CA program .............................. 168

4.26 Error $\Xi$ for different values of pulsing area and uniform stress parameter $\ldots \ldots \ldots \quad 178$ 
4.27 Residuals of simulations at each station $\varepsilon_{\mathrm{j}}$ around the fault, for PSA at $5 \mathrm{~Hz} \ldots \ldots \quad 178$

4.28 Average error $\Xi_{i}$ at all stations as a function of frequency, for uniform stress parameter distribution and preferred stress parameter distribution

4.29 Initial guess, stress parameter distributions derived for M6, 2004 Parkfield earthquake from the fault surface sub-division into 30 and 36 sub-sources

4.30 Slip distribution on the Parkfield fault surface derived from Liu et al., 2006 .....

5.1 Distribution of stations and fault location used for Loma Prieta earthquake study in the case 1

5.2 Derived stress parameter distribution on the Loma Prieta fault in the case 1

5.3 Evolution of average absolute error $(\Xi)$ and average error $(\Phi)$ with successive inversion iteration in the case 1 of Loma Prieta study

5.4 Average absolute error $\left(\Xi_{i}\right)$ as a function of frequency in the case 1 of Loma Prieta inversion study

5.5 Spatial distribution of error improvement criterion $\Psi_{\mathrm{j}}$ from initial guess to final solution in the case 1 of Loma Prieta study

5.6 Distribution of stations and fault location used for Loma Prieta earthquake study in the cases 2 and 3

5.7 Derived stress parameter distribution on the Loma Prieta fault in the case 2

5.8 Evolution of average absolute error $(\Xi)$ and average error $(\Phi)$ with successive inversion iteration in the case 2 of Loma Prieta study

5.9 Average absolute error $\left(\Xi_{i}\right)$ as a function of frequency in the case 2 of Loma Prieta inversion study

5.10 Spatial distribution of error improvement criterion $\Psi_{\mathrm{j}}$ from initial guess to final solution in the case 1 of Loma Prieta study 198

5.11 Derived stress parameter distribution on the Loma Prieta fault in the case 3 198 
5.12 Evolution of average absolute error $(\Xi)$ and average error (D) with successive inversion iteration in the case 3 of Loma Prieta study

5.13 Average absolute error $\left(\Xi_{i}\right)$ as a function of frequency in the case 3 of Loma Prieta inversion study.

5.14 Spatial distribution of error improvement criterion $\Psi_{j}$ from initial guess to final solution in the case 3 of Loma Prieta study 200

5.15 Slip distribution of Loma Prieta earthquake by Steidl et al. (1991)

5.16 Distribution of stations and fault location used for Landers earthquake study in the case 1

5.17 Derived stress parameter distribution on the Landers fault in the case 1 203

5.18 Evolution of average absolute error $(\Xi)$ and average error $(\Phi)$ with successive inversion iteration in the case 1 of Landers study 204

5.19 Average absolute error $\left(\Xi_{\mathrm{i}}\right)$ as a function of frequency in the case 1 of Landers inversion study 204

5.20 Spatial distribution of error improvement criterion $\Psi_{j}$ from initial guess to final solution in the case 1 of Landers study 205

5.21 Distribution of stations and fault location used for Landers earthquake study in the cases 2 and 3 205

5.22 Derived stress parameter distribution on the Landers fault in the case 2 209

5.23 Evolution of average absolute error $(\Xi)$ and average error $(\Phi)$ with successive inversion iteration in the case 2 of Landers study

5.24 Average absolute error $\left(\Xi_{i}\right)$ as a function of frequency in the case 2 of Landers inversion study....

5.25 Spatial distribution of error improvement criterion $\Psi_{\mathrm{j}}$ from initial guess to final solution in the case 1 of Landers study 
5.26 Derived stress parameter distribution on the Landers fault in the case 3

5.27 Evolution of average absolute error $(\Xi)$ and average error $(\Phi)$ with successive inversion iteration in the case 3 of Landers study

5.28 Average absolute error $\left(\Xi_{\mathrm{i}}\right)$ as a function of frequency in the case 3 of Landers inversion study

212

5.29 Spatial distribution of error improvement criterion $\Psi_{\mathbf{j}}$ from initial guess to final solution in the case 3 of Landers study

5.30 Slip distribution of Loma Prieta earthquake by Cohee and Beroza, (1994).

5.31 Distribution of stations and fault location used for Northridge earthquake study in the case 1

5.32 Derived stress parameter distribution on the Northridge fault in the case 1

5.33 Evolution of average absolute error $(\Xi)$ and average error $(\Phi)$ with successive inversion iteration in the case 1 of Northridge study

5.34 Average absolute error $\left(\Xi_{\mathrm{i}}\right)$ as a function of frequency in the case 1 of Northridge inversion study

5.35 Spatial distribution of error improvement criterion $\Psi_{\mathrm{j}}$ from initial guess to final solution in the case 1 of Northridge study

5.36 Distribution of stations and fault location used for Northridge earthquake study in the cases 2 and 3

5.37 Derived stress parameter distribution on the Northridge fault in the case 2

5.38 Evolution of average absolute error $(\Xi)$ and average error $(\Phi)$ with successive inversion iteration in the case 2 of Northridge study

5.39 Average absolute error $\left(\Xi_{\mathrm{i}}\right)$ as a function of frequency in the case 2 of Northridge inversion study 
5.40 Spatial distribution of error improvement criterion $\Psi_{\mathrm{j}}$ from initial guess to final solution in the case 1 of Northridge study

5.41 Derived stress parameter distribution on the Northridge fault in the case 3

5.42 Evolution of average absolute error $(\Xi)$ and average error $(\Phi)$ with successive inversion iteration in the case 3 of Northridge study

5.43 Average absolute error $\left(\Xi_{i}\right)$ as a function of frequency in the case 3 of Northridge inversion study....

5.44 Spatial distribution of error improvement criterion $\Psi_{j}$ from initial guess to final solution in the case 3 of Northridge study 227

5.45 Slip distribution of Northridge earthquake by Wald et al., (1996)

A.1 Average residuals of Log PSAs of M7.0, 1989 Lomaprieta earthquake simulations in $\mathrm{R}_{\mathrm{JB}}=1 \mathrm{~km}, \mathrm{R}_{\mathrm{JB}}=10 \mathrm{~km}, \mathrm{R}_{\mathrm{JB}}=30 \mathrm{~km}$, and $\mathrm{R}_{\mathrm{JB}}=200 \mathrm{~km}$.

A.2 Average residuals of Log PSAs of M7.2, 1992 Landers earthquake simulations in $R_{I B}=1 \mathrm{~km}, R_{I B}=10 \mathrm{~km}, R_{I B}=30 \mathrm{~km}$, and $R_{I B}=200 \mathrm{~km}$.

A.3 Average residuals of Log PSAs of M6.6, 1994 Northridge earthquake simulations in $\mathrm{R}_{\mathrm{JB}}=1 \mathrm{~km}, \mathrm{R}_{\mathrm{JB}}=10 \mathrm{~km}, \mathrm{R}_{\mathrm{JB}}=30 \mathrm{~km}$, and $\mathrm{R}_{\mathrm{JB}}=200 \mathrm{~km}$.

A.4 Average residuals of $\log$ PSAs at $\mathrm{R}_{\mathrm{JB}}=1 \mathrm{~km}$ as a function of magnitude in four frequencies $\mathrm{f}=0.3 \mathrm{~Hz}, \mathrm{f}=1 \mathrm{~Hz}, \mathrm{f}=3 \mathrm{~Hz}$, and $\mathrm{f}=1 \mathrm{~Hz}-10 \mathrm{~Hz}$

A.5 Average residuals of Log PSAs at $\mathrm{R}_{\mathrm{JB}}=10 \mathrm{~km}$ as a function of magnitude in four frequencies $\mathrm{f}=0.3 \mathrm{~Hz}, \mathrm{f}=1 \mathrm{~Hz}, \mathrm{f}=3 \mathrm{~Hz}$, and $\mathrm{f}=1 \mathrm{~Hz}-10 \mathrm{~Hz}$

A.6 Average residuals of Log PSAs at $\mathrm{R}_{\mathrm{JB}}=30 \mathrm{~km}$ as a function of magnitude in four frequencies $\mathrm{f}=0.3 \mathrm{~Hz}, \mathrm{f}=1 \mathrm{~Hz}, \mathrm{f}=3 \mathrm{~Hz}$, and $\mathrm{f}=1 \mathrm{~Hz}-10 \mathrm{~Hz}$

A.7 Average residuals of $\log$ PSAs at $\mathrm{R}_{\mathrm{JB}}=200 \mathrm{~km}$ as a function of magnitude in four frequencies $\mathrm{f}=0.3 \mathrm{~Hz}, \mathrm{f}=1 \mathrm{~Hz}, \mathrm{f}=3 \mathrm{~Hz}$, and $\mathrm{f}=1 \mathrm{~Hz}-10 \mathrm{~Hz}$ 


\section{List of Appendices}

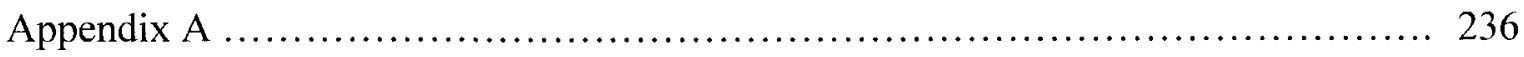




\section{Chapter 1: Introduction}

\subsection{Back ground}

Occurrences of earthquakes have serious impacts on human life. They may cause loss of life, economic losses and damage to vital infrastructure. Mitigating earthquake damage requires a good knowledge of earthquake strong ground motion characteristics. One successful and widely used approach for seismologists to gain insight into the characteristics of earthquakes is a technique called waveform modeling, in which synthetic seismograms are generated and compared to actual recorded seismograms. Records are generated as a combined effect of the source, path, and site effects, and thus waveform modeling offers insight into these processes.

There have been two successful classes of methods in investigating observed seismograms. One is based on a deterministic model and the other based on a stochastic model. The deterministic approach assigns exact models to the source, path, and site processes of the earthquake and tries to model the details as exactly as possible, while the stochastic approach uses a simplified representation of source, path and site effects, in the context of a random noise model, and does not aim to reproduce the details. The quick and easy stochastic approach may find solutions for difficult problems much sooner than a deterministic approach alone (Aki, 1989), as the deterministic approach is more complex and detailed. Since high-frequency signals have short wavelengths, these waves are sensitive to small size heterogeneities. As the earth is more heterogeneous in small scale than large scale, it is expected to see more stochastic behavior in high-frequency seismic signals than low-frequency ones. So there has been a long tradition of generating synthetic high-frequency seismograms by manipulation of a combination of stochastic noise and theoretical assumptions (e.g. Saragoni and IIart (1974)). Likewise, the simulation of long-period signals has tended to focus on deterministic methods.

Hanks and McGuire (1981) performed an important study that led to the popularization of the stochastic modeling approach. By studying a large number of time histories, they showed that accelerograms can be modeled as band-limited white Gaussian noise within 
the S-wave arrival window, with an underlying amplitude spectrum as specified by a simple seismological model of source and propagation processes. The S-window is of most interest for earthquake engineers since it usually contains the highest amplitudes. Their observations launched one of the most useful tools in the study of observed ground motions, stochastic point source modeling, which has been implemented in numerous studies (Boore, 2003, 1983; Boore and Atkinson, 1987; Toro and McGuire, 1987; Ou and Herrmann, 1990; Boore et al., 1992; Atkinson and Boore, 1995). The theory behind stochastic point source modeling is described in detail by Boore $(2003,1983)$ and will be reviewed in a later section.

The effects of a large finite source, including rupture propagation, directivity, and source receiver geometry, can profoundly influence the amplitudes, frequency content, and duration of ground motion (Beresnev and Atkinson, 1997). A common approach for modeling these effects is to subdivide the fault into smaller parts, each of which is then treated as a point source (after Hartzell, 1978). Then the ground motion at an observation point is obtained by summing the contributions over all sub-sources. By treating subsources as stochastic point sources, a stochastic finite fault model is developed (Schneider et al., 1993). There are two approaches for modeling path effects in stochastic finite fault techniques. One approach implements synthetic path effects and the other implements empirical path effects. Zeng et al., (1994) developed a composite $\omega^{2}$ source model and combined it with a version of synthetic Green's function (Zeng and Anderson, 1995) to simulate strong ground motion of earthquakes from complex and finite sources. This approach is successfully implemented for the derivation of the slip model of a number of major earthquakes (Su et al., 1994; Zeng and Anderson, 1996; Finite-sourcerupture model database, http://www.seismo.ethz.ch/srcmod/Homepage.html). Another approach combines a stochastic finite source model with empirical path effects to model ground motion. One of the studies that implemented this approach was Beresnev and Atkinson (1997), who used an $\omega^{2}$ stochastic point source to describe the source effect of subsources along a propagating finite-fault; an empirical attenuation and duration model was used to describe the path effects. Beresnev and Atkinson (1998) developed a computer 
program called "FINSIM" (Finite fault simulation) that follows their (1997) modeling method. This program was later modified by Motazedian and Atkinson (2005) to include the dynamic corner frequency concept and other conceptual improvements in finite fault modeling and is called "EXSIM".

The aims of this thesis are to evaluate stochastic methods, exploring their strengths and limitations, develop improvements to "EXSIM", and to develop an inversion tool for the derivation of stress drop distribution along a fault plane based on stochastic finite-fault modeling. I concentrate on the EXSIM modeling approach of Motazedian and Atkinson (2005) as a convenient starting point for the investigations, and a means of focusing the scope. The following subsections will provide an overview of selected stochastic waveform modeling methods.

\subsection{Stochastic point source modeling}

The stochastic point source model assumes that the source is concentrated at a point, and that the acceleration time series generated at a site carry both deterministic and random aspects of ground motion shaking. The deterministic aspects are specified by the average Fourier spectrum, typically as a function of magnitude and distance. The stochastic aspects are treated by modeling the motions as noise with the specified underlying spectrum. The point-source assumption is reasonable when source to site distance is much larger than source dimensions (Boore, 2003 and 1983, Boore and Atkinson, 1987; Atkinson and Boore, 1995 and 1997; Atkinson and Silva, 1997 and 2000). Figure (1.1) illustrates the stochastic point-source technique. This method multiplies a normalized spectrum of a Gaussian noise by the theoretical spectrum of ground motion resulting from a point source, and converts back the results to the time domain.

Boore (2003) synthesized the method of stochastic point source modeling and presented the procedure of time series generation. He developed a computer program called "SMSIM" (1996, 2005) for simulating strong ground motion using the stochastic point source method. The steps of this method are reviewed in the following: 
a) Generate a white Gaussian noise signal. The length of the signal will be equal to the duration of the source, which is a function of magnitude, plus the duration attributable to path effects:

$$
T_{w}=T+T_{d}
$$

where $T_{w}$ is total duration of signal, $T$ is duration of source, and $T_{d}$ is path-effect duration. The average source duration is equal to half of the fault rupture length divided by the rupture velocity (Beresnev and Atkinson, 1997). Since there are relations between rupture length and earthquake magnitude (Wells and Coppersmith, 1994), the $T$ term is a function of size of earthquake. The effect of path on duration, $T_{d}$, results from back/multiple scattering of the waves that originally left the source in different directions and are received at the recording site; it is a function of distance (e.g., Atkinson, 1995; Boore, 1996 and 2005)

b) Window the signal by multiplying it by a window function. Following Boore (2005) the windowing function is a tapered boxcar (rectangle) or an exponential (Saragoni and Hart, 1974). Tapering smooths the ends of the signal to avoid unwanted computational errors in the Fourier transform of boxcar-windowed signals. The duration of the boxcar is equal to $(1+\alpha) \times T_{w}$, where $\alpha$ is the tapering ratio. A Saragoni-Hart (1974) window is an appropriate average envelope shape for earthquake signals; its equation is:

$$
w\left(t ; t_{\eta}\right)=a\left(t / t_{\eta}\right)^{b} \exp \left(-c t / t_{\eta}\right)
$$

where $w\left(t ; t_{\eta}\right)$ is the window function:

$$
b=-(\varepsilon \ln \eta) /[1+\varepsilon(\ln \varepsilon-1)] ; \quad c=b / \varepsilon ; \quad a=(\exp (1) / \varepsilon)^{h} ; \quad t_{\eta}=2 T_{w} ;
$$

$\varepsilon$ is fraction of $t / t_{\eta}$ when $w\left(t ; t_{\eta}\right)$ reaches to its maximum, which is equal to unity, and $\eta$ is the value of $w\left(t=t_{\eta} ; t_{\eta}\right)$. The recommended values are: $\varepsilon=0.2$ and $\eta=0.05$.

c) Calculate the Fourier transform of the windowed noise.

d) Normalize the result so that the average amplitude spectrum equals unity. 
e) Calculate the theoretical (deterministic) point source spectrum. The total point source spectrum is calculated by the following equation:

$$
Y\left(M_{0}, R, f\right)=E\left(M_{0}, f\right) P(R, f) G(f)
$$

where $Y\left(M_{0}, R, f\right)$ is total point source spectrum observed at recording site, $E\left(M_{0}, f\right)$ is the source spectrum at unit distance, $P(R, f)$ is the path effect, $G(f)$ is the site response operator, $M_{0}$ is seismic moment (Aki, 1967), $R$ is distance from the source to site, and $f$ is frequency. The general form of the source spectrum is:

$$
E\left(M_{0}, f\right)=C M_{0} S(f)
$$

where $C$ is a constant and $S(f)$ is the source spectral shape. The constant $C$ relates a set of physical properties of the medium and geometry of wave propagation and it is derived from equation (1.6):

$$
C=\left\langle R_{\Theta \Phi}\right\rangle V F /\left(4 \pi \rho_{s} \beta_{s}^{3} R_{0}\right)
$$

where $\left\langle R_{\Theta \Phi}\right\rangle$ is average radiation pattern of $\mathrm{S}$ waves and is equal to 0.55 if the geometrical mean is calculated over whole focal-sphere (Boore and Boatwright, 1984), $V$ is average partitioning of $\mathrm{S}$ wave onto one horizontal component, and is equal to $(1 / 2)^{1 / 2}, F$ is the free surface effect $(=2), \rho_{\mathrm{s}}$ and $\beta_{\mathrm{s}}$ are density and shear wave velocity of waves of medium surrounding the source, and $R_{\ell}$ is the unit distance.

Hanks and Kanamori (1979) introduced the following equation for relating seismic moment, $M_{\ell}$, to moment magnitude, $\mathbf{M}$, which can be used for replacement in Equation (1.5):

$$
\boldsymbol{M}=\frac{2}{3} \log _{10} M_{0}-10.7
$$

There are many models available for source spectral shape $S(f)$ (e.g., Brune, 1970 and 1971; Boatwright and Choy, 1992; Atkinson and Boore, 1995; Frankel et al., 1996; Haddon, 1996; Atkinson and Silva, 2000). Most of these models intend to mimic some aspects of spectral forms of finite sources. The most widely used source model is Brune's point source model (or $\omega^{2}$ model) with the following functional form: 


$$
S(f)=\frac{4 \pi^{2} f^{2}}{1+\frac{f^{2}}{f_{c}^{2}}}
$$

where $S(f)$ is acceleration spectral shape, and $f_{c}$ is corner frequency of the spectrum, which may be calculated from the following equation (Boore, 2003, 1983):

$$
f_{c}=4.9 \times 10^{6} \beta_{s}\left(\frac{\Delta \sigma}{M_{0}}\right)^{1 / 3}
$$

where $\Delta \sigma$ is the stress parameter in bars, $M_{0}$ is in dyne $\mathrm{cm}$ and $\beta_{\mathrm{s}}$ is in $\mathrm{km} / \mathrm{s}$. For a specified magnitude $M_{0}$ can be calculated using Equation (1.7). The set of Equations (1.5) to (1.9) provide the relations necessary for the calculation of source spectrum, $E\left(M_{0, f}\right)$, in Equation (1.4). The Brune model assigns a circular surface to the fracture and measures distances from that surface, assuming the distance is much larger than the fracture radius; in addition it only considers shear deformation propagation. Implementation of Brune's model as the specified source term allows the point source model to synthesize just the "S" component of the waveform. Note that Brune's simplistic model assumes that an instantaneous drop in shear stress across the fault causes shear deformation, that propagates from the source, which is treated as a point. The value of the stress drop controls the corner frequency of the omega-squared spectrum, and hence the highfrequency level. In this thesis, the modeled stress drop parameter is that of Brune model, and specifically that inferred by the high-frequency spectral level. We refer to this as the "stress parameter" or "stress drop"; it is roughly equivalent to the dynamic shear stress drop on the fault surface.

The path operator, $P(R, f)$, includes the effects of both geometrical spreading and inelastic attenuation. The general functional form of the path spectrum following Boore (2003) is:

$$
P(R, f)=Z(R) \exp \left[-\pi R f / \beta_{s} Q(f)\right]
$$

where $Z(R)$ is the geometrical spreading term, $R$ is distance from source to site, and $Q(f)$ is the quality factor, which is an inverse measure of inelastic attenuation of the area. Generally the geometrical spreading term is modeled by the following equation. 


$$
Z(R)= \begin{cases}\frac{R_{0}}{R} & R \leq R_{1} \\ Z\left(R_{1}\right)\left(\frac{R_{1}}{R}\right)^{p_{1}} & R_{1} \leq R \leq R_{2} \\ \mathrm{M} & \\ Z\left(R_{n}\right)\left(\frac{R_{n}}{R}\right)^{p_{n}} & R_{n} \leq R\end{cases}
$$

where $R_{i}$ S are distances from source to site where the behavior of geometrical spreading changes and takes different exponent, $p_{i}$.

The site operator $G(f)$ includes effects of site amplification due to decrease of impedance by getting closer to the earth's surface (Boore and Joyner, 1997), and the near-surface high-frequency de-amplification effect (Hanks, 1982; Anderson and Hough, 1984). Accordingly the site response spectrum may be presented as the product of both amplification and de-amplification effects:

$$
G(f)=A(f) D(f)
$$

where $A(f)$ is the site amplification and $D(f)$ is high frequency de-amplification. The amplification term, $A(f)$ is available for different site types as tables in some publications (Boore and Joyner, 1997) or it can be calculated if the wave velocity structure of the site is available (e.g., quarter wavelength approach). The high frequency de-amplification component has been formulated in functional forms whose constants $\left(\kappa\right.$ and $f_{\max }$ ) are available from studies for an area or obtainable from processing of records available for earthquakes of an area. Either of the following equations can be used:

$$
D(f)=\exp (-\pi \kappa f) \quad \text { or } \quad D(f)=\frac{1}{\sqrt{1+\frac{f^{8}}{f_{\max }^{8}}}}
$$

where $\kappa$ and $f_{\max }$ are parameters of the filter and needed to be known or calculated for an area.

f) Combine the results of the Equations (1.4) to (1.13) with the normalized noise spectrum by multiplying the two spectra. 
g) Calculate the inverse Fourier transform of the results and get the simulated accelerogram.

The above steps are shown in Figure (1.1) schematically. Synthetic accelerograms generated with this method are on average reasonable representations of actual motions that would occur with the parameters implemented in the synthetics. To obtain an average of ground-shaking level at different vibration periods at a site (assuming source, path and site parameters are known), multiple simulations using this method are generally performed and the average ground-shaking level is calculated. Boore (1996) developed a program package called "SMSIM" that performs stochastic point source modeling as explained here (Boore, 2005 is the latest version of the program).

\subsection{Stochastic finite source modeling (FINSIM)}

The stochastic point source modeling assumption that the source is concentrated at a point ignores finite fault aspects like faulting geometry, the effects of distributed rupture, and rupture inhomogeneity. This is a poor assumption for large events at close distances, and may result in poor prediction of the spectral shape of accelerograms, ground-shaking levels in the vicinity of the faults, and the directional dependence of amplitudes (directivity). In an attempt to overcome this kind of problem, Hartzell (1978) subdivided the fault surface of an earthquake to a grid of sub-sources and assigned to each of them an aftershock of that earthquake as a source (treated as an EGF, or Empirical Green's Function). He summed the contributions to the total motion of sub-sources at the recording site, considering proper delay of sub-sources due to rupture propagation, in order to synthesize the record of the main shock. The EGF method automatically takes into account the effect of path and site, as they are embedded in the EGF. This method has been implemented in many studies (Irikura, 1983; Irikura, 1992; Irikura and Katsuhiro, 1994; Bour and Cara, 1997).

Following the idea of discretization of the earthquake fault surface and summation of effects of each sub-source at a recording point, as shown on Figure 1.2, many researchers 
implemented this approach in their studies, but with different methods of determination of source and path spectra ( $E$ and $P$ in Equation (1.4)) for the sub-sources (Irikura, 1983; Somerville et al., 1991; Hartzell and Langer, 1993; Yu et al., 1995; Schneider et al., 1993).

To model a finite fault, Beresnev and Atkinson (1997) discretized the fault and applied $\omega^{2}$ stochastic point sources to each of the sub-source activations $(Y(t))$. The properly delayed and reactivated sub-sources' effects are added in the time domain to generate the finite fault model:

$$
A(t)=\sum_{i=1}^{N} \sum_{j=1}^{n(i)} Y_{i}\left(t+\Delta t_{i}+T_{j}\right)
$$

where $A(t)$ is the total seismic signal at site, $Y,(t)$ is the signal of $\mathrm{i}^{\text {th }}$ sub-source activation, $N$ is total number of sub-sources, $n(i)$ is the number of activations of the $\mathrm{i}^{\text {th }}$ sub-source, depending on the value of slip on the sub-source, $\Delta t_{i}$ is the delay time of the sub-source, and $T_{j}$ is a random time delay between the $\mathrm{j}-1$ and $\mathrm{j}^{\text {th }}$ rise time. The $T_{j}$ component is added for further randomness. In their model the sub-source containing the hypocenter triggers first and begins the rupture propagation. A sub-source is triggered when the rupture front reaches its center. The delay time of a sub-source is the time required for the rupture to get to the element plus the travel time of the waves to cover the distance between the subsource and the site. Since each $Y(t)$ is a stochastic point source (like the one discussed before), the method is called stochastic finite fault modeling. Beresnev and Atkinson (1998) developed a computer program that performs stocharic finite fault modeling using this approach and called it "FINSIM".

Beresnev and Atkinson (1997) chose Brune's model as the underlying source spectral shape $(S(f))$. They showed that $Y(f)$, the spectrum of $Y(t)$, after algebraic simplifications is derived from Equation (1.15).

$$
Y(f)=C\left(\Delta \sigma M_{0}\right)^{1 / 2}\left(\frac{y z \beta}{\pi}\right)^{2} \Delta l^{-1 / 2}
$$


where $C$ is calculated using Equation (1.6), $\Delta \sigma$ is the stress drop of faulting, $y$ is the ratio of fault rupture velocity and shear wave velocity, $z$ is called the high frequency strength factor, which is proportional to maximum slip velocity, $\Delta l$ is sub-source size, and the other parameters are as explained before.

Beresnev and Atkinson $(1997,1998,2001)$ reported two problems associated with this modeling method; i) the strength factor is not a well-constrained quantity; and ii) simulations will be a function $\Delta l$ or sub-source size dependent. They solved these problems by calibrating the method against available data. They recommended $z=1.68$ for the high frequency strength factor if the $\omega^{2}$ model is implemented. For slow and fast slipping faults the " $z$ " value can be multiplied by a factor in the range of 0.5 to 2 . They also recommended a sub-source size in the range of $5 \mathrm{~km}$ to $15 \mathrm{~km}$ for the optimum performance of the method. Beresnev and Atkinson (2001) found an empirical equation that relates the optimal sub-source size to moment magnitude:

$$
\log (\Delta l)=0.4 M-2.0
$$

where $M$ is the moment magnitude and $\Delta l$ is the sub-source size. Their study showed that $\Delta l$ found by Equation (1.16) is in good agreement with the size of asperities of real events.

The FINSIM simulations performed in studies of this thesis implemented $z=1.68$ and Equation (1.16) for quantification of parameters.

\subsection{Stochastic finite source modeling (EXSIM)}

The stochastic point source modeling assumptions of Beresnev and Atkinson (1997 and 1998) were modified to introduce the concept of "dynamic corner frequency" (Motazedian, 2002; Motazedian and Atkinson, 2005) to overcome the difficulties associated with ambiguity of the strength factor value, and the dependence of FINSIM results on sub-source sizes. Motazedian and Atkinson (2005) developed a new computer 
program for stochastic finite fault modeling based on their dynamic-corner-frequency approach and called it "EXSIM".

EXSIM first subdivides an earthquake fault surface to a grid of sub-sources, and assigns to each of them a stochastic point source, as discussed in section (1.2).

In EXSIM each sub-source is activated once with an appropriate delay time of $\Delta t_{i}$ (Figure (1.2)). In each activation a stochastic point source waveform with an underlying $\omega^{2}$ source spectrum is generated. Properly normalized and delayed sub-source contributions are summed in the time domain as:

$$
A(t)=\sum_{i=1}^{N} H_{i} \times Y_{i}\left(t+\Delta t_{i}+T_{i}\right)
$$

where $A(t)$ is the total seismic signal at site, $H_{i}$ is a normalization factor for the $\mathrm{i}^{\text {th }}$ sub-that aims to conserve energy, $Y_{(}(t)$ is the signal of $\mathrm{i}^{\text {th }}$ sub-source activation, $N$ is the total number of sub-sources, $\Delta t_{i}$ is delay time of the sub-source, and $T_{i}$ is a fraction of rise time. Slip distribution is modeled by scaling the moment of the sub-sources:

$$
M_{0 i}=\frac{M_{0} \times u_{i}}{\sum_{i=1}^{N} u_{i}}
$$

where $M_{0}$ and $M_{0_{i}}$ are moments of source and ith sub-source respectively, and $u_{i}$ is the slip of the $\mathrm{i}^{\text {th }}$ sub-source. $M_{0 i}$ from Equation (1.18) replaces $M_{0}$ in the Equation (1.5) for synthetics of the $\mathrm{i}^{\text {th }}$ sub-source in EXSIM. 
a) Gaussian noise and window function

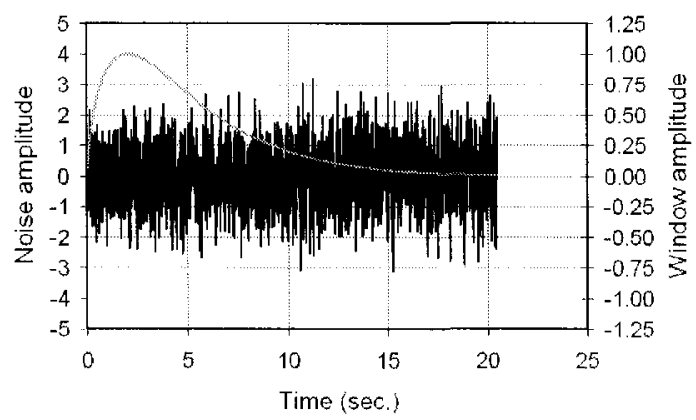

c) Calculate Fourier spectrum

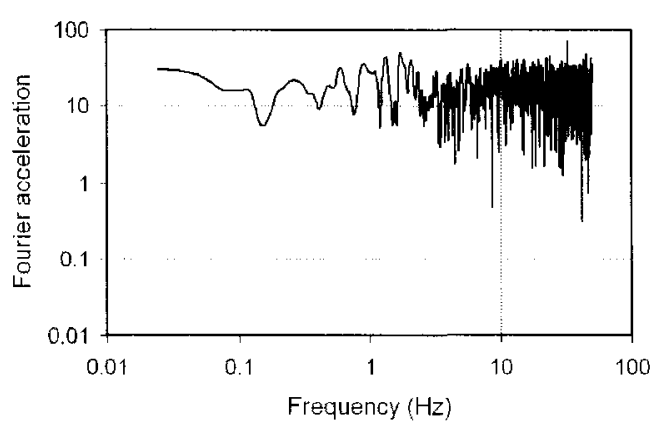

e) Calculate model, f) Combine results

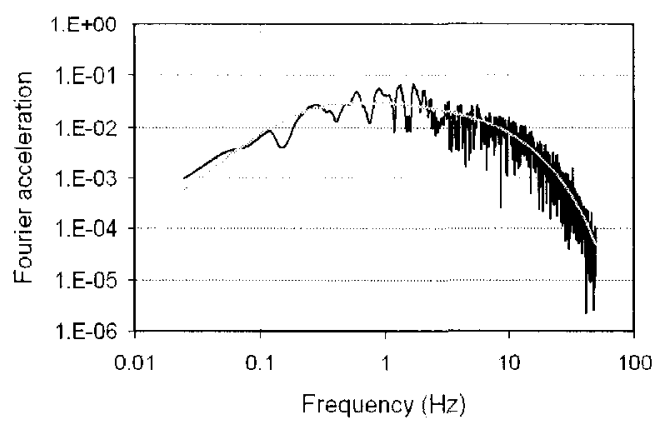

b) Multiply noise by window

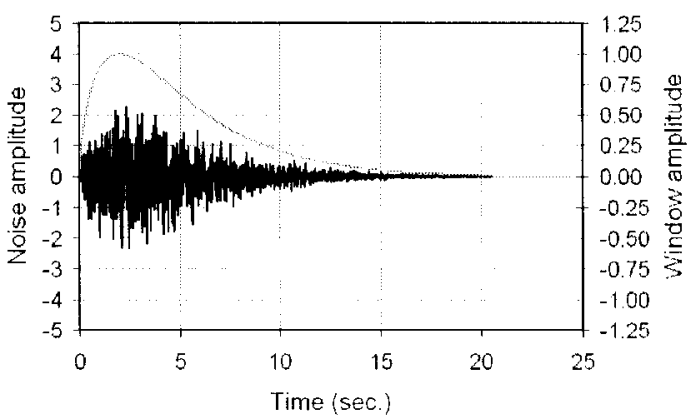

d) Normalize spectrum to 1

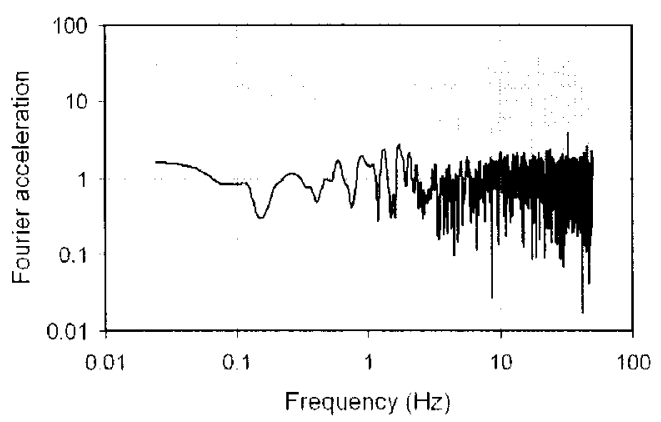

g) Calculate inverse Fourier

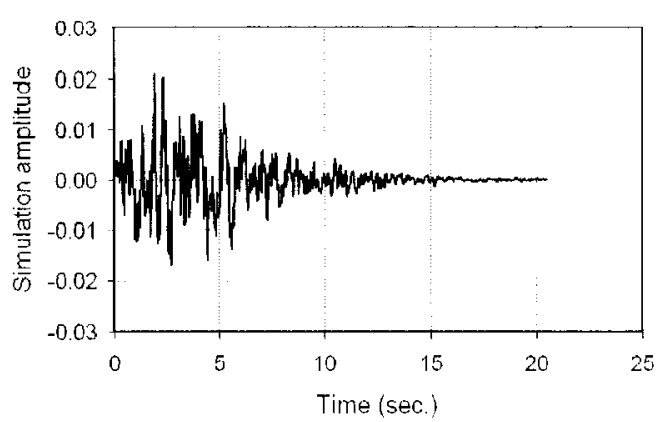

Figure (1.1) Steps of development of synthetic accelerogram using stochastic point source modeling 

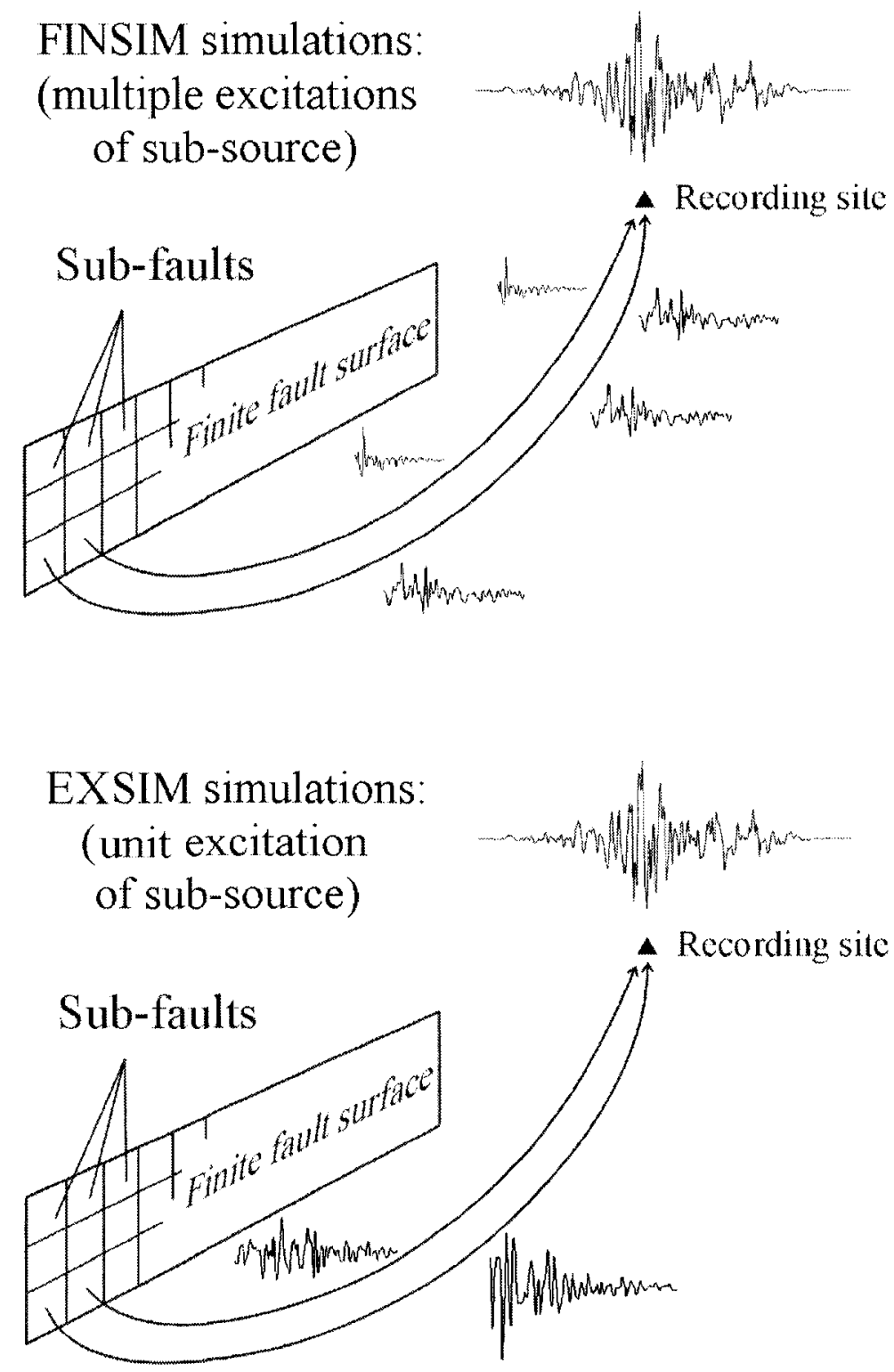

Figure (1.2) Subdivision of a fault into sub-sources and summing effects of all subsources at recording site in time domain. Top: FINSIM simulations where each subsource may radiate multiple (proportional to slip) almost similar waveforms; Bottom: EXSIM simulations where sub-sources radiate one scaled waveform (proportional to slip). 
Motazedian (2002) and Motazedian and Atkinson (2005) introduced the "dynamic corner frequency" concept to solve two difficulties associated with FINSIM. This concept assigns a variable (with location) corner frequency to the sub-sources so that corner frequencies of newly activated elements get smaller as the rupture progresses thus resulting in a frequency content that shifts lower as the rupture grows. This process continues until the active ruptured surface gets to a predefined limiting fraction of the fault surface, say $50 \%$ of its total surface, after which the corner frequency of newly activated elements remain constant:

$$
f_{c i}=4.9 \times 10^{6} \beta\left(\frac{N \times \Delta \sigma}{N_{R} \times M_{0}}\right)^{1 / 3}
$$

where $f_{c i}$ is corner frequency of $\mathrm{i}^{\text {th }}$ sub-source, $\beta$ is shear wave velocity, $\Delta \sigma$ is the average stress drop, $M_{0}$ is moment of earthquake, $N$ is total number of sub-sources, and $N_{R}$ is total number of active sub-sources at the time of $i^{\text {th }}$ sub-source activation. Equation $(1.19)$ replaces Equation (1.9) for synthetics of the $i^{\text {th }}$ sub-source.

Calculation of $H_{i}$ is necessary to complete Equation (1.17). Motazedian and Atkinson (2005) assumed that high frequency energies radiated from all sub-sources are equal and their sum equals the total high frequency energy of the earthquake. They calculated high frequency energy by adding squares of Fourier velocity spectra ordinates. With these assumptions they obtain the following equation for calculation of $H_{i}$ :

$$
H_{i}=\frac{M_{0}}{M_{0 i}} \sqrt{\frac{\sum_{j}\left(\frac{f_{0}^{2} f_{j}}{f_{0}^{2}+f_{j}^{2}}\right)^{2}}{N \sum_{j}\left(\frac{f_{0 i}^{2} f_{j}}{f_{0 i}^{2}+f_{j}^{2}}\right)^{2}}}
$$

where $M_{0}$ and $M_{0 i}$ are moments of the fault and the $\mathrm{i}^{\text {th }}$ sub-source respectively, $f_{0}$ and $f_{\phi_{i}}$ are corner frequencies of the whole fault and the $i^{\text {th }}$ sub-source respectively, $N$ is the number of sub-sources, and $f_{j}$ is the $\mathrm{j}^{\text {th }}$ frequency ordinate; summation is performed over these frequencies. 
The result of Equation (1.19) is to allow sub-sources to generate a range of low frequency to high frequency spectra, such that the final waveform of the simulation will contain sufficient low-frequency energy even if very small sub-sources sizes are chosen. Scaling simulated time series before summation by Equation (1.20) forces the method to generate a constant amount of energy no matter how many sub-sources are contributed in the summation. In other words, Equations (1.19) and (1.20) on average make the spectral shape and spectral level of the resultant accelerograms independent of sub-source size.

\subsection{Studies of this thesis}

The purpose of this thesis is to extend our knowledge and range of applicability of stochastic finite fault modeling by analyzing the output of the EXSIM approach in different circumstances, comparing its performance with other similar approaches, and improving it by adding more capabilities. In particular, I use the model to develop an inversion tool for derivation of stress parameter distribution on the fault surface, in which I combine the enhanced EXSIM model with an inversion algorithm; I test the performance of the inversion tool using synthetic and real data.

In this thesis, three stochastic methods are implemented. I use "SMSIM" (Boore, 1996 and 2005) for stochastic point source modeling, "FINSIM" for stochastic finite fault modeling with a static corner frequency (Beresnev and Atkinson, 1998), and "EXSIM" for stochastic finite fault modeling with a dynamic corner frequency (Motazedian and Atkinson, 2005).

Chapter two describes results of EXSIM forward modeling and its comparisons with two other modeling methods. This includes a study of sub-source size independence at short distances, the directivity effect in EXSIM, and Fourier and response spectra comparisons of EXSIM and SMSIM. Comparisons of SMSIM/FINSIM/EXSIM simulations against recorded time series from the PEER-NGA (Pacific Earthquake Engineering Research Center - Next Generation Attenuation Models, http://peer.berkeley.edu/nga/index.html) 
database are used to draw conclusions about the ranges of application of the three methods.

Chapter three presents new modifications to EXSIM to increase its range of applications. One modification is the introduction of a variable rupture velocity, while the other is the introduction of a variable stress parameter on the fault surface. The chapter explores sensitivity of the spatial distribution of ground motion parameters with changes of rupture velocity and stress parameter distribution on the fault surface.

Chapter four introduces the incorporation of the modified EXSIM with an analytical inversion tool. The purpose of this development is to make a tool to deduce stress parameter distribution on the fault surface from the observations of response spectra of records. Results of EXSIM forward modeling, with known initial conditions, are input to the newly developed inversion tool to test its performance and sensitivity to some parameters including: fault dip angle, asperity distributions, initial guess of stress parameter distribution, the station distribution, and EXSIM averaging of response spectra. Finally the method is applied to the M6.0, 2004 Parkfield earthquake and a stress parameter distribution model is derived, which is then consistent with EXSIM.

Chapter five demonstrates implementation of the stress parameter derivation tool, developed in the previous chapter, to the case of three major Californian earthquakes. The purpose of this chapter is to explore the conditions under which the method works reasonably well for inverting real data, and to demonstrate its performance also when the conditions are not ideal. The results of this chapter put an empirical constraint on the number of sub-divisions of the fault for acceptable results.

Chapter six draws together and summarizes the most important results and conclusions. 


\section{Chapter 2: Stochastic forward modeling experiments}

\subsection{Introduction}

This chapter explores three stochastic modeling methods - SMSIM (point-source stochastic), FINSIM (finite-fault stochastic), and EXSIM (finite-fault stochastic). The main motivation to use a finite-fault method (as opposed to point-source) is to include finite fault effects such as rupture propagation, directivity, and source-receiver geometry. Directivity refers to the azimuthal dependence of ground motion characteristics on the rupture propagation direction (e.g., Benioff, 1955; Ben-Menahem, 1961; Somerville et al., 1997). Directivity effects predicted by EXSIM are part of studies in this chapter.

As discussed in chapter one, stochastic finite fault modeling discretizes an earthquake fault into sub-sources and assigns a stochastic point source (Boore 1983; 2003) to each sub-source; the fracture expands from the hypocenter, triggering each element when the fracture front gets to the center of that sub-source. A critical element is the scaling and shaping of the sub-source spectrum, $E\left(M_{0 i}, f\right)$. In the "FINISIM" approach, Beresnev and Atkinson (1997 and 1998) used empirical constraints to determine sub-source properties. In "EXSIM" Motazedian and Atkinson (2005) introduced the concept of dynamic corner frequency, and used a conservation of energy constraint. This chapter explores the behavior of these methods and their applicability, when averaged over many events.

The origin and evolution of the alternative stochastic simulation methods, as discussed earlier, suggests the possibility of a large degree of similarity between the methods' outputs. This similarity should be pronounced if the limiting conditions push one method to be approximately equivalent to the other (For example, at large distances an extended fault may appear as a point-source, hence point-source and finite-source modeling results should be similar at large distances). To gain insight into how point source and finite source methods behave, Fourier acceleration spectra and response spectra of SMSIM and EXSIM simulations are compared at short and long distances, in low and high stress-drop regimes. The results of these comparisons are presented in a subsection of this chapter. 
The differences in predictions between the three simulation methods suggest that they are not behaving in the same way in different magnitude and distance ranges, so comparing the simulation results from these methods with real data can suggest magnitude and distance ranges in which one method on average performs the best. I base such data comparisons on the PEER-NGA database (http://peer.berkeley.edu/nga/index.html). The PEER-NGA (Pacific Earthquake Engineering Research Center - Next Generation Attenuation) database is a reference database for development of ground motion prediction equations; it consists of a database of corrected strong ground motion records along with corresponding causative earthquakes' and time series/spectral parameters. 'The results of this study cover the last subsection of this chapter.

This chapter covers those studies that directly implemented SMSIM, FINSIM, and EXSIM for forward modeling; no modifications were applied to the modeling methods. First, some modeling experiments are performed to explore the behavior of EXSIM (our preferred modeling tool). Then, some comparisons between methods are made. Finally, the methods are evaluated against data.

\subsection{Directivity from EXSIM simulations}

\subsubsection{Directivity}

The radiated seismic pulse varies in duration as a function of azimuth from the rupture direction, due to the effects of rupture propagation along a finite rupture length. In simple terms, we "see" a shortened pulse if the rupture propagates towards us, versus an extended pulse if it is rupturing away from us. Because the area of the pulse must be the same at all azimuths (to conserve seismic moment), the amplitude of the pulse varies inversely with its duration. This characteristic of finite sources is mentioned in classical texts as "directivity" (e.g., Lay and Wallace, 1995; Stein and Wysession, 2003). 


\subsubsection{Measurement quantity}

One simulation by EXSIM generates one acceleration time series, for a specified site, given source, path, and site information, for one random trial. The program configuration file has a parameter that sets the numbers trials. The output of an EXSIM run includes a sample simulated time series, an average pseudo spectral acceleration or PSA (Chopra, 2006; Trifunac, 2006), and an average Fourier spectrum of time series. The averaging is performed for the specified number of trials, as fixed in the configuration file.

The measured quantity for this study to explore predicted directivity is based on the PSA ordinates for each trace:

$$
D_{r}=\frac{1}{N_{f 2}-N_{f 1}+1} \sum_{N=N_{f 1}}^{N_{f 2}} \log [P S A(N)]
$$

where $D_{r}$ is the defined directivity measure quantity, $N_{f l}$ and $N_{f 2}$ are ordinate numbers of frequencies $f 1$ and $f 2$ respectively, and $P S A(N)$ is $N^{t h}$ ordinate of PSA. If $D_{r}$ is required for just one frequency then $N_{f l}=N_{f 2}$, and no averaging over frequency is performed.

\subsubsection{Simulation parameters and results}

Simulations are performed for magnitude $\mathbf{M} 6$ and $\mathbf{M} 7$ earthquakes. For magnitude $\mathbf{M} 6$ two scenarios are considered. For the first set of simulations the parameters of the M6, 2004 Parkfield earthquake are applied; for simplicity of presentation the fault is rotated to the east-west direction so that the rupture propagates from east to west. The discretization of the fault to sub-sources is shown in Figure (2.1) and simulation parameters are given in Table (2.1). The fracture starts at the shaded sub-source and propagates mainly to the west. Simulations are performed for a circular array of sites at radii of $100 \mathrm{~km}, 200 \mathrm{~km}$, and $300 \mathrm{~km}$ around the center of the fault. The sites are located on $2^{\circ}$ intervals on a circular array. The $D_{r}$ values of 30 simulation PSAs per site are calculated in $2.5 \mathrm{~Hz}-20 \mathrm{~Hz}$ and $0.45 \mathrm{~Hz}-10 \mathrm{~Hz}$ frequency ranges. Figures (2.2.A) and (2.2.B) show how average log PSA amplitudes change with orientation in $2.5 \mathrm{~Hz}-20 \mathrm{~Hz}$ and $0.45 \mathrm{~Hz}-10 \mathrm{~Hz}$ frequency 
ranges respectively (the graphs show directional $D$, values plus a constant of " 2 " to avoid difficulties of plotting in the polar coordinate system). These graphs demonstrate the ability of the simulation approach in predicting directivity effect.

Figure (2.3.A) shows the azimuthal dependence of the simulated traces' duration for three distances, where the processed duration, including zero padding, is shown. Figure (2.3.B) shows the same dependence for trace duration, which is the signal duration in the trace, without zero padding. The shortening of signal duration is responsible for the predicted increase of spectral acceleration levels.

Somerville et al., (1997) presented a study on directivity effect based on data of 21 earthquakes (mainly California events) in the range of magnitudes M6.0 to M7.5; they demonstrated that directivity is effective on PSA ordinates with periods $0.6 \mathrm{~s}$ and more (less than $1.7 \mathrm{~Hz}$ ) and gets stronger with increasing period. To see if EXSIM is capable of reproducing this observation I calculated log averages of PSA for 30 simulation trials per site, for $25 \mathrm{~Hz}, 10 \mathrm{~Hz}, 5 \mathrm{~Hz}, 2 \mathrm{~Hz}, 1 \mathrm{~Hz}, 0.5 \mathrm{~Hz}, 0.2 \mathrm{~Hz}$, and $0.1 \mathrm{~Hz}$ frequencies. As shown in Figure (2.4), in EXSIM the predicted directivity effect increases with frequency, to a maximum rate around $5 \mathrm{~Hz}$. To demonstrate that this is not sensitive to the choice of simulation parameters, two other sets of simulations are performed with different parameter values. The sub-source duration and fault length are the parameters that can affect the duration of the simulated signal and are thus important in predicting directivity. Accordingly the second set of simulations is performed for an M6.0 earthquake with the parameters of Table (2.2). These values are the same as those of Table (2.1) except that the "sub-source duration" is set to be fixed and equal to the sub-source rise time. The log PSA values averaged over 30 trials for this case are shown for $25 \mathrm{~Hz}, 5 \mathrm{~Hz}, 1 \mathrm{~Hz}$, and $0.2 \mathrm{~Hz}$ in Figure (2.5). Again, we see that EXSIM predicts strong directivity effects at high frequencies and weak effects at low frequencies, despite our expectations based on the Somerville et al., (1997) observations. This test demonstrates that the dependence of sub-source duration on distance is not responsible for this contradiction of our expectations. 


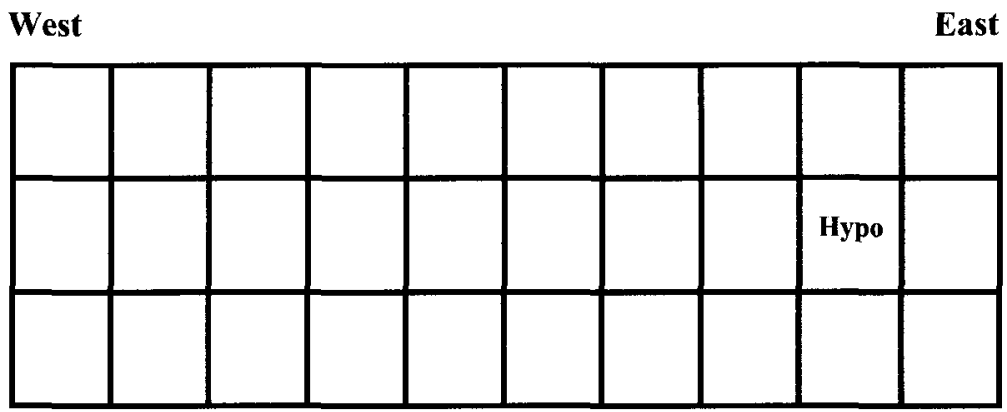

Figure (2.1) earthquake fault subdivision to sub-sources implemented in three simulations for directivity study. Fracture starts at hypocenter (shaded cell) and propagates towards the west

Table (2.1) First set of simulation parameters for directivity study:

M6, 2004 Parkfield earthquake

\begin{tabular}{cc}
\hline Parameter & Parameter value \\
\hline Fault orientation & Strike $90^{\circ}$, Dip $83^{\circ}$ \\
Depth of top & $0 \mathrm{~km}$ \\
Fault dimensions & Length $40 \mathrm{~km}$, Width $13 \mathrm{~km}$ \\
Number of sub-faults & Along length 10, Along width 3 \\
FFT points, sample interval & $8192,0.02 \mathrm{~s}$ \\
Shear wave velocity, Density & $3.5 \mathrm{~km} / \mathrm{s}, 2.8 \mathrm{~g} / \mathrm{cm}^{3}$ \\
Rupture velocity & $0.8 \times$ shear wave velocity \\
$Q(f)$ & $180 f^{0.45}$ \\
Moment magnitude & 6.0 \\
Average stress parameter & 50 bars \\
Kappa & 0.035 \\
Geometrical attenuation & If $\mathrm{R}<40, \mathrm{R}^{-1}$, else $\mathrm{R}^{-0.5}$ \\
Sub-source duration & $\propto 0.05 \times$ distance $(\mathrm{sec})$. \\
Windowing function & Saragoni-Hart \\
Amplification function & Boore and Joyner $(1997)$ for $\mathrm{V}_{\mathrm{s} 30}=620 \mathrm{~m} / \mathrm{s}$ \\
Damping & $5 \%$ of critical damping \\
Number of EXSIM trials & 30 (per site) \\
\hline
\end{tabular}


Directional Log Geometrical Mean of PSAs for $0.45 \mathrm{~Hz}-10 \mathrm{~Hz}$ at
Different Distances from Center of a Fault Oriented E-W

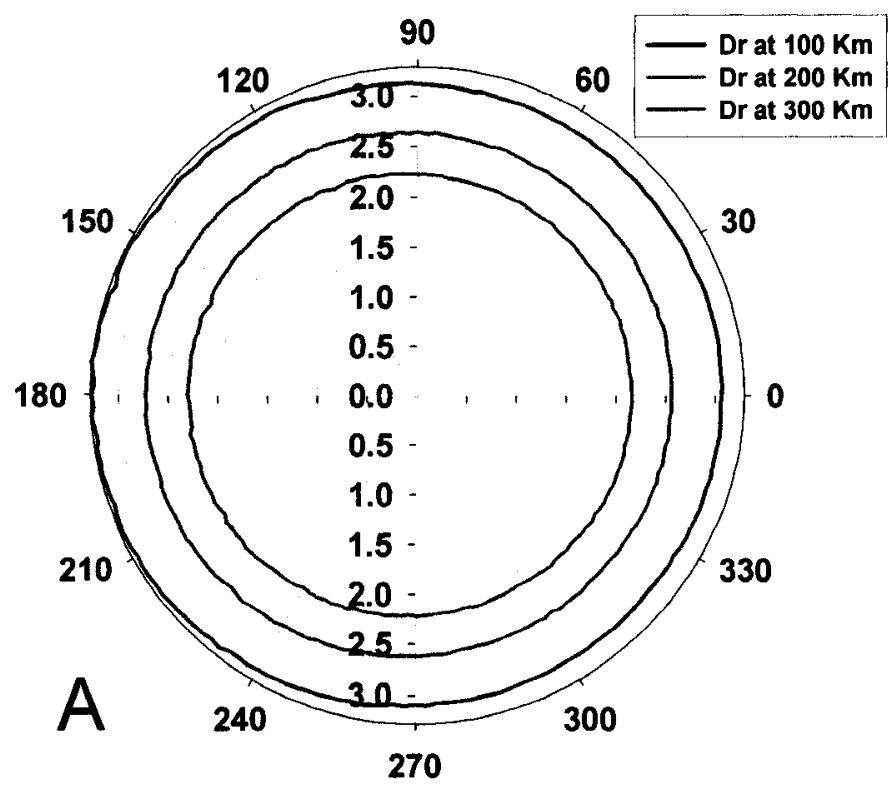

\section{Directional Log Geometrical Mean of PSAs for $2.5 \mathrm{~Hz}-20 \mathrm{~Hz}$ at Different Distances from Center of a Fault Oriented E-W}

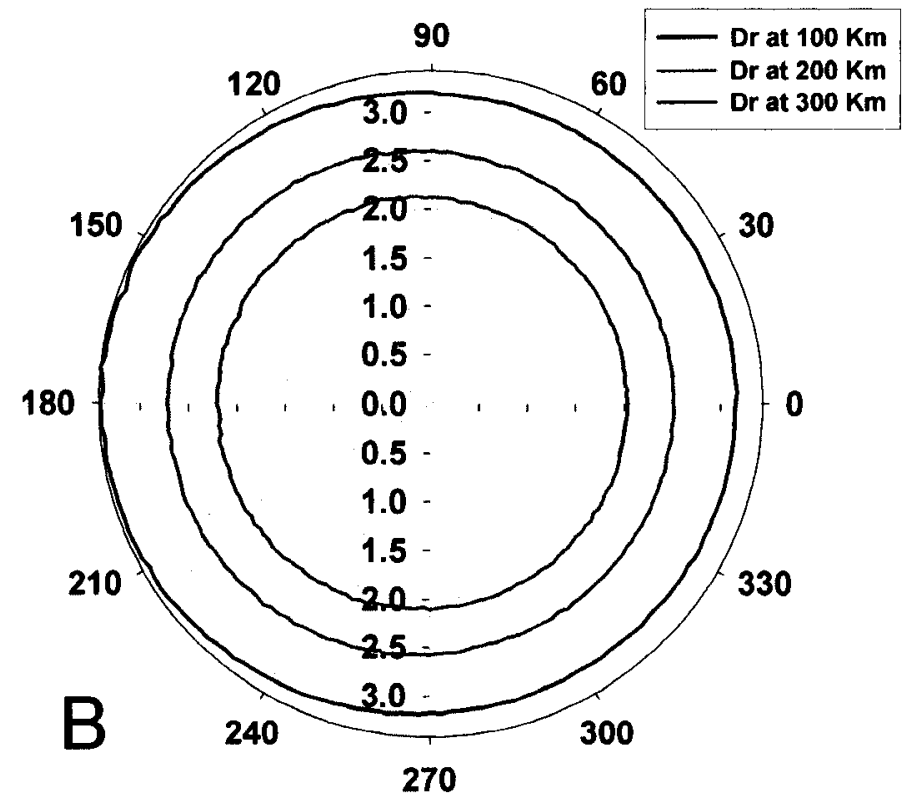

Figure (2.2) Log of geometrical averages of PSA ordinates $\left(D_{r}\right)$ as a function of direction for geometry of Figure (2.1) and Table (2.1) derived for $100 \mathrm{Km}, 200 \mathrm{Km}$, and $300 \mathrm{Km}$ distances from center of fault as predicted by EXSIM for:(A) for $0.45 \mathrm{~Hz}-10 \mathrm{~Hz}$; (B) for $2.5 \mathrm{~Hz}-20 \mathrm{~Hz}$. 
The third set of simulations is for a M7.0 earthquake with the parameters of Table (2.3). For this set "sub-source duration" is set to be fixed and equal to the sub-source rise time, the event is assigned to a $50 \mathrm{~km} \times 15 \mathrm{~km}$ fault surface, and the remaining parameters are those of Table (1.1). The PSA predictions are shown for $25 \mathrm{~Hz}, 5 \mathrm{~Hz}, 1 \mathrm{~Hz}$, and $0.2 \mathrm{~Hz}$ in Figure (2.6). This supports the conclusions reached from Figures (2.4) and (2.5). This experiment demonstrates that source magnitude and dimensions are not responsible for the directivity effect with frequency in the simulations.

\subsubsection{Discussion on directivity}

The reason why EXSIM can predict some level of directivity effect is that the signal at the recording site, which is the sum of signals of sub-sources at that point, shows variable duration depending on the azimuth of the site with respect to a point on the fault and the rupture propagation orientation. Figure (2.3) shows the azimuthal dependence of the simulated trace duration for three distances. This effect is similar to the pulse duration variation with azimuth effect that Stein and Wysession (2003) use for explaining directivity. Considering that the signals arriving at the observation point carry energy, and knowing that the rule of conservation of energy holds, concentration of the energy in a short time window will result in overall higher amplitudes, hence higher average log PSAs.

Let us look at energy from the random vibration point of view. Since EXSIM adds scaled time series of sub-faults with appropriate time delays, the sub-source signals are concentrated and added in smaller time intervals, towards the west, as compared to other orientations (Figures (2.3)). Newland (2005) gives the following equation for the superposition of many uncorrelated random signals:

$$
E\left[y^{2}\right]=\sum_{r=1}^{N} \int_{-\infty}^{+\infty}\left|H_{r}(\omega)\right|^{2} S_{x_{r}}(\omega) d \omega
$$


where $E\left[y^{2}\right]$ is the average power of the signal, $r$ is the number of sub-sources, $\left|H_{r}((1))\right|$ is the $\mathrm{r}^{\text {th }}$ sub-source's transfer function amplitude, $S_{x r}$ is power spectral density function of the $r^{\text {th }}$ sub-source signal, and $\omega$ is angular frequency (notations are those of Newland (2005)). The above equation represents the conservation of energy law in the case of random signals. So this equation will work if the signals added together at the observation point are random and uncorrelated. By reducing the frequency, the number of cycles in time the window reduces, and low-frequency components will not have time to show their random nature, so the above equation will not work for low frequencies. On the contrary, high frequency components of sub-source traces will show their random nature, because many cycles will fall in the time window. This explains why EXSIM predicts considerable directivity effect at high frequencies, $f \geq 2 \mathrm{~Hz}$, and weak directivity effect at low frequencies. It is a consequence of modeling the signal as a stochastic process and an inherent limitation of the stochastic approach.

The difference between arrival times of waves from two ends of a unilateral fracturing fault at a point is the apparent fracture time or pulse duration. According to Stein and Wysession (2003), if the periods of the arrival signals are much larger than the apparent fracture time, then the wavelength of waves will be too large to see the finiteness of the source. On the contrary if the periods of the arriving signals are much smaller than the apparent rupture time, then the wavelengths will be small enough to capture finiteness of the source. For example, a $10 \mathrm{~km}$ fault would be considered as a finite source for waves with period of $1 \mathrm{~s}$ while it would be considered as a point source for a surface wave with period of $50 \mathrm{~s}$. With the simulation parameters applied here, for a $40 \mathrm{~km}$ fault, $0.35 \mathrm{~Hz}$ waves will have periods equal to apparent fracture duration along the east-west direction (maximum directivity direction). My experiments don't show much sensitivity of EXSIM to directivity at such frequencies. Considering that the stochastic methods lack the presence of deterministic low frequency components, putting them in the finite source context will not improve them in a way to predict large directivity effects at low frequencies. To solve this problem, Motazedian and Atkinson (2005) improved EXSIM by allowing the specification of an analytical pulse shape of near-fault ground motion in 
their program (after the approach of Mavroeidis and Papageorgiou (2003)). It is done by convolving the end product of the stochastic finite fault simulation with a near-fault ground motion analytical signal. The ultimate result is the presence of a directivity pulse as well as considerable low frequency components in the spectrum of the EXSIM simulations. Thus in order to model directivity properly in stochastic finite fault models, an additional step is required.

\subsection{Sub-source size independence of EXSIM simulations}

\subsubsection{Background}

Motazedian and Atkinson's (2005) version of stochastic finite fault modeling, EXSIM, was developed to cope the difficulties associated with dependence of the spectrum level and spectral content distribution with sub-source size that characterized previous stochastic finite fault modeling approaches. In their paper, Motazedian and Atkinson (2005) performed simulations to demonstrate the independence of results from subsource size at a large distance from an M7.0 earthquake-generating fault. This subsection examines the independence of EXSIM simulation results from sub-source sizes for short distances from the fault. 


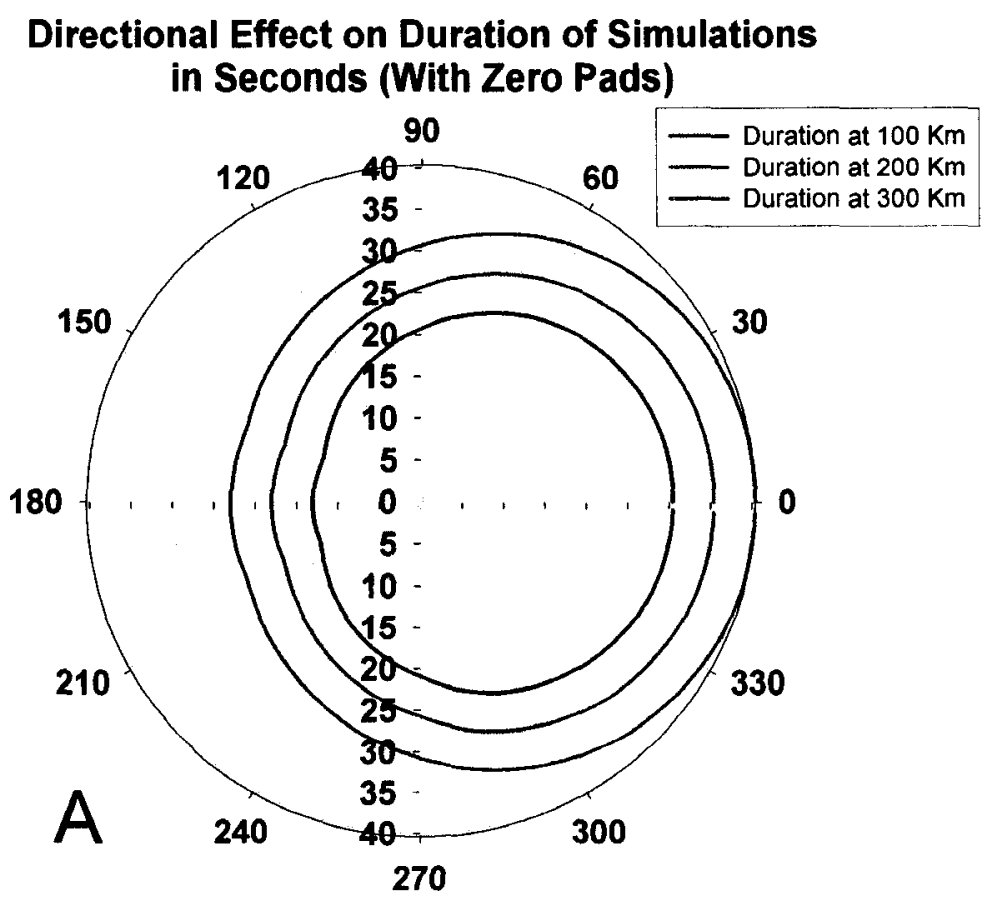

\section{Directional Effect on Duration of Simulations in Seconds (Pad Zeros Removed)}

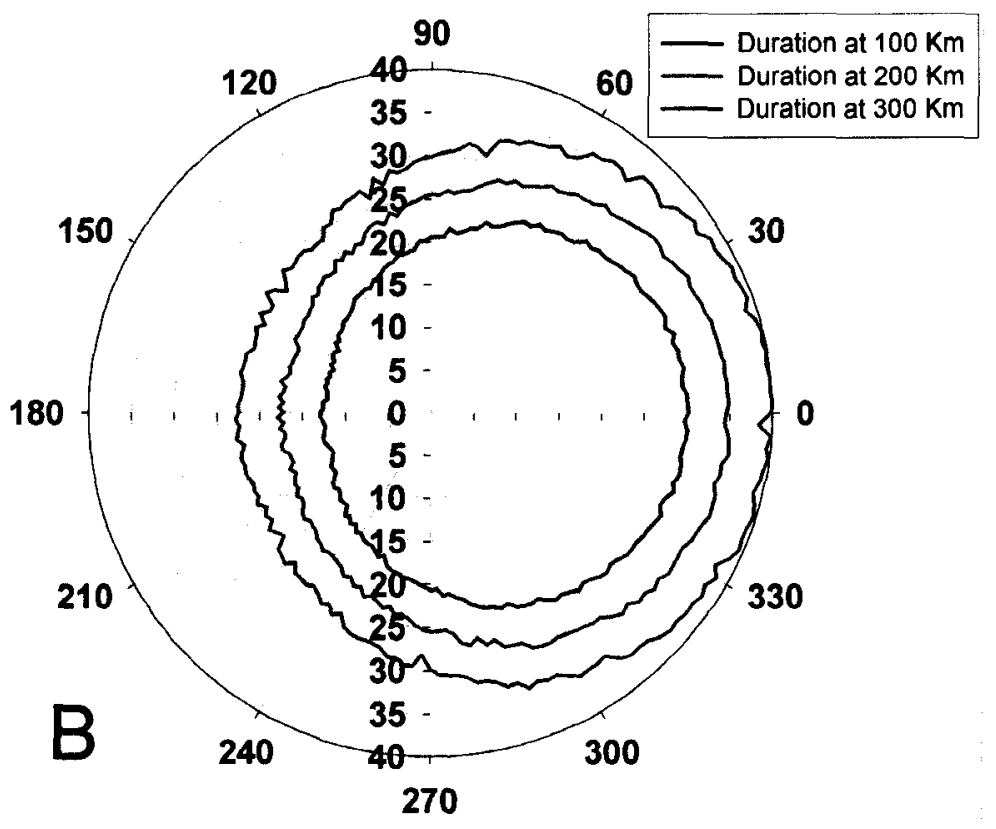

Figure (2.3) Duration of simulated time series as a function of azimuth (in seconds) for $100 \mathrm{Km}, 200 \mathrm{Km}$, and $300 \mathrm{Km}$ distances calculated by EXSIM for geometry of Figure (2.1) and Table (2.1): (A) total simulated trace duration, including zero-padding for processing; (B) actual trace duration, with zero pads removed. 
Directional Effect on Log PSAs of $25 \mathrm{~Hz}$ in Different Distances from Center of a Fault in E-W Direction

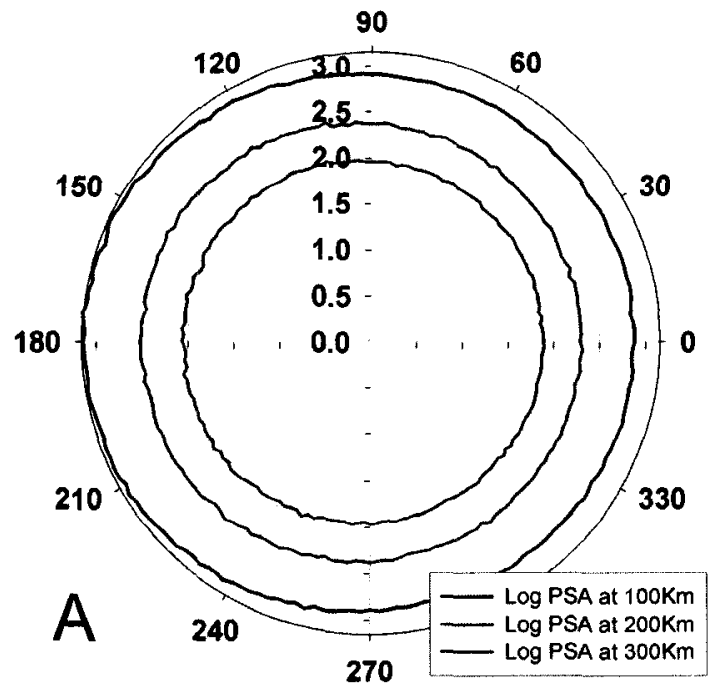

Directional Effect on Log PSAs of $5 \mathrm{~Hz}$ in Different Distances from Center of a Fault in E-W Direction

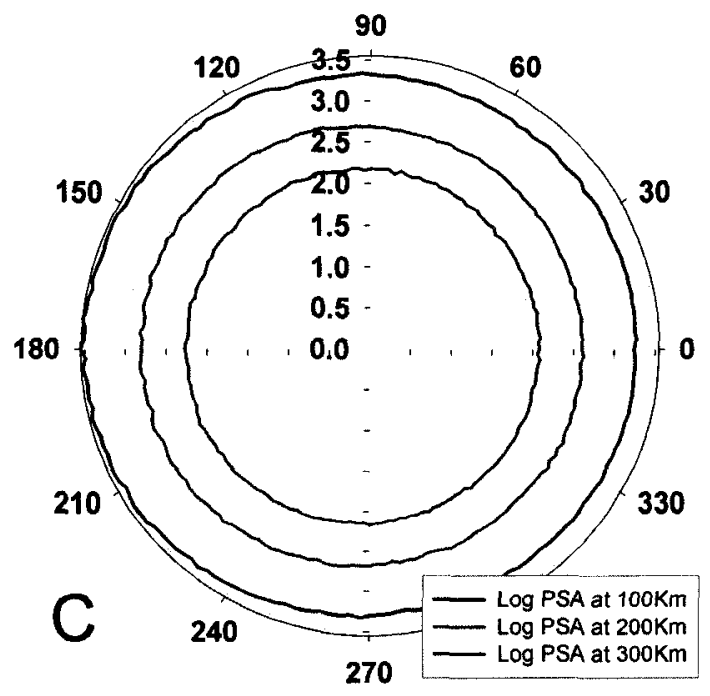

Directional Effect on Log PSAs of $10 \mathrm{~Hz}$ in Different Distances from Center of a Fault in E-W Direction

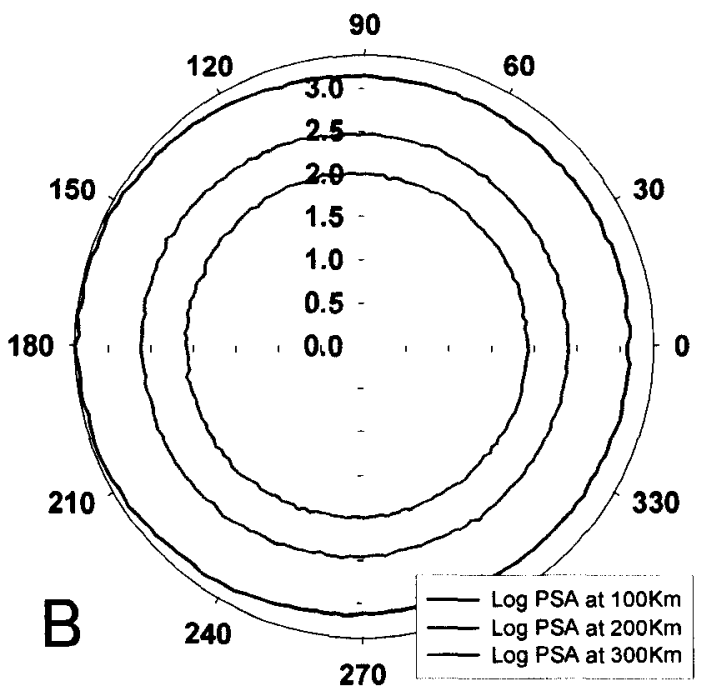

Directional Effect on Log PSAs of $2 \mathrm{~Hz}$ in Different Distances from Center of a Fault in E-W Direction

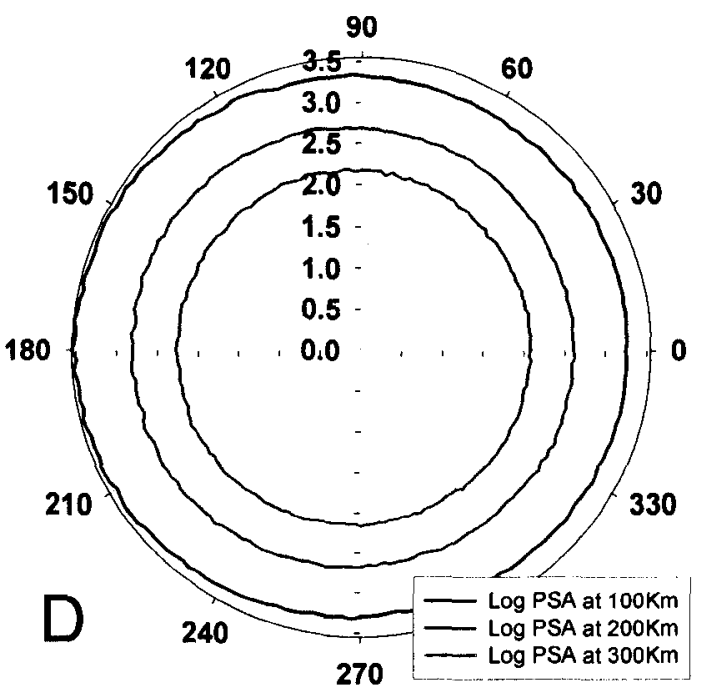

Figure (2.4) Log of PSA ordinates as a function of direction for geometry of Figure (2.1) and Table (2.1) derived for $100 \mathrm{Km}, 200 \mathrm{Km}$, and $300 \mathrm{Km}$ distances from center of fault as predicted by EXSIM for:(A) for $25 \mathrm{~Hz}$; (B) for $10 \mathrm{~Hz}$; (C) for $5 \mathrm{~Hz}$; and (D) for $2 \mathrm{~Hz}$. 

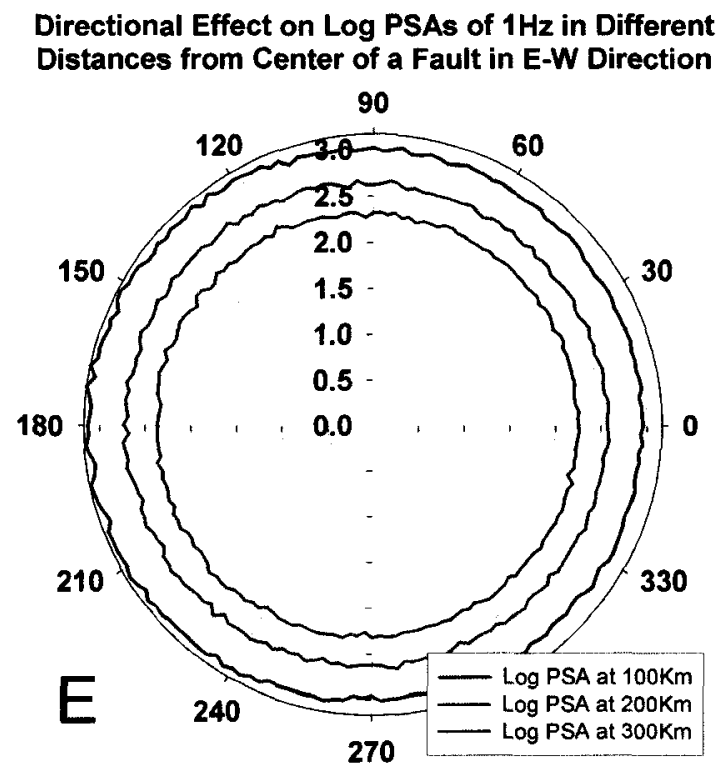

Directional Effect on Log PSAs of $0.2 \mathrm{~Hz}$ in Different Distances from Center of a Fault in E-W Direction

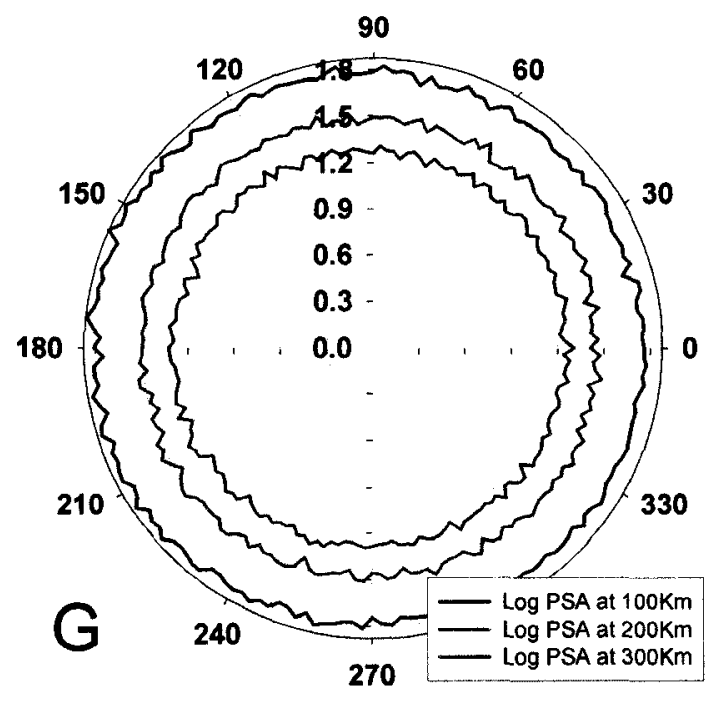

Directional Effect on Log PSAs of $0.5 \mathrm{~Hz}$ in Different Distances from Center of a Fault in E-W Direction

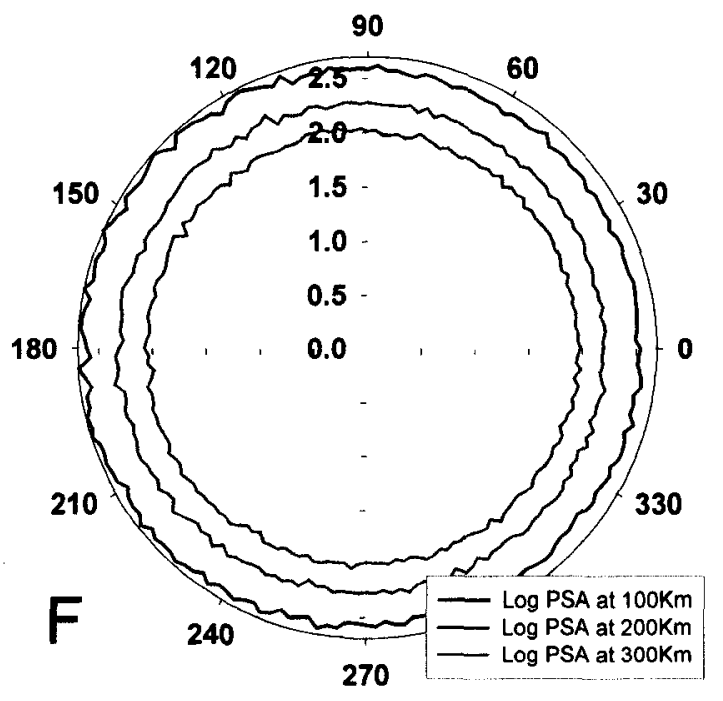

Directional Effect on Log PSAs of $0.1 \mathrm{~Hz}$ in Different Distances from Center of a Fault in E-W Direction

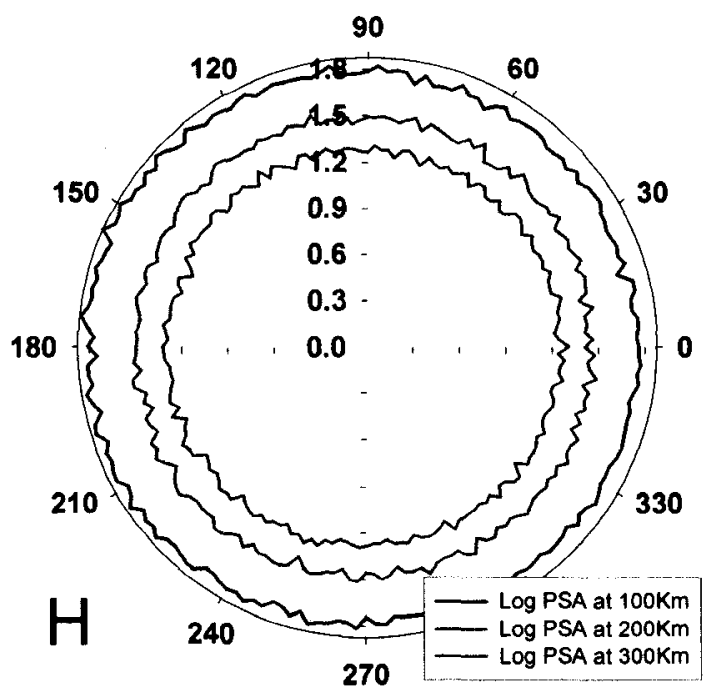

Figure (2.4) Log of PSA ordinates as a function of direction for geometry of Figure (2.1) and Table (2.1) derived for $100 \mathrm{Km}, 200 \mathrm{Km}$, and $300 \mathrm{Km}$ distances from center of fault as predicted by EXSIM for:(E) for $1 \mathrm{~Hz}$; (F) for $0.5 \mathrm{~Hz}$; $(\mathrm{G})$ for $0.2 \mathrm{~Hz}$; and $(\mathrm{H})$ for $0.1 \mathrm{~Hz}$. 
Table (2.2) Second set of simulation parameters for directivity study

\begin{tabular}{cc}
\hline Parameter & Parameter value \\
\hline Fault orientation & Strike $90^{\circ}$, Dip $83^{\circ}$ \\
Depth of top & $0 \mathrm{~km}$ \\
Fault dimensions & Length $40 \mathrm{~km}$, Width $13 \mathrm{~km}$ \\
Number of sub-faults & Along length 10, Along width 3 \\
FFT points, sample interval & $8192,0.02 \mathrm{~s}$ \\
Shear wave velocity, Density & $3.5 \mathrm{~km} / \mathrm{s}, 2.8 \mathrm{~g} / \mathrm{cm}^{3}$ \\
Rupture velocity & $0.8 \times$ shear wave velocity \\
$Q(f)$ & $180 f^{0.45}$ \\
Moment magnitude & 6.0 \\
Average stress parameter & 50 bars \\
Kappa & 0.035 \\
Geometrical attenuation & If R<40, $\mathrm{R}^{-1}$, else $\mathrm{R}^{-0.5}$ \\
Sub-source duration & Sub-source rise time \\
Windowing function & Saragoni-Hart \\
Amplification function & Boore and Joyner $(1997)$ for $\mathrm{V}_{\mathrm{s} .30}=620 \mathrm{~m} / \mathrm{s}$ \\
Damping & $5 \%$ of critical damping \\
Number of EXSIM iterations & 30 (per site) \\
\hline
\end{tabular}

Table (2.3) Third set of simulation parameters for directivity study

\begin{tabular}{cc}
\hline Parameter & Parameter value \\
\hline Fault orientation & Strike $90^{\circ}$, Dip $83^{\circ}$ \\
Depth of top & $0 \mathrm{~km}$ \\
Fault dimensions & Length $50 \mathrm{~km}$, Width $15 \mathrm{~km}$ \\
Number of sub-faults & Along length 10, Along width 3 \\
FFT points, sample interval & $8192,0.02 \mathrm{~s}$ \\
Shear wave velocity, Density & $3.5 \mathrm{~km} / \mathrm{s}, 2.8 \mathrm{~g} / \mathrm{cm}^{3}$ \\
Rupture velocity & $0.8 \times$ shear wave velocity \\
$Q(f)$ & $180 f^{0.45}$ \\
Moment magnitude & 7.0 \\
Average stress parameter & 50 bars \\
Kappa & 0.035 \\
Geometrical attenuation & If R<40, R ${ }^{-1}$, else $\mathrm{R}^{-0.5}$ \\
Sub-source duration & Sub-source rise time \\
Windowing function & Saragoni-Hart \\
Amplification function & Boore and Joyner $(1997)$ for $\mathrm{V}_{\mathrm{s} .30}=620 \mathrm{~m} / \mathrm{s}$ \\
Damping & $5 \%$ of critical damping \\
Number of EXSIM iterations & $30($ per site) \\
\hline
\end{tabular}


Directional Effect on Log PSAs of $25 \mathrm{~Hz}$ in Different Distances from Center of a Fault in E-W Direction

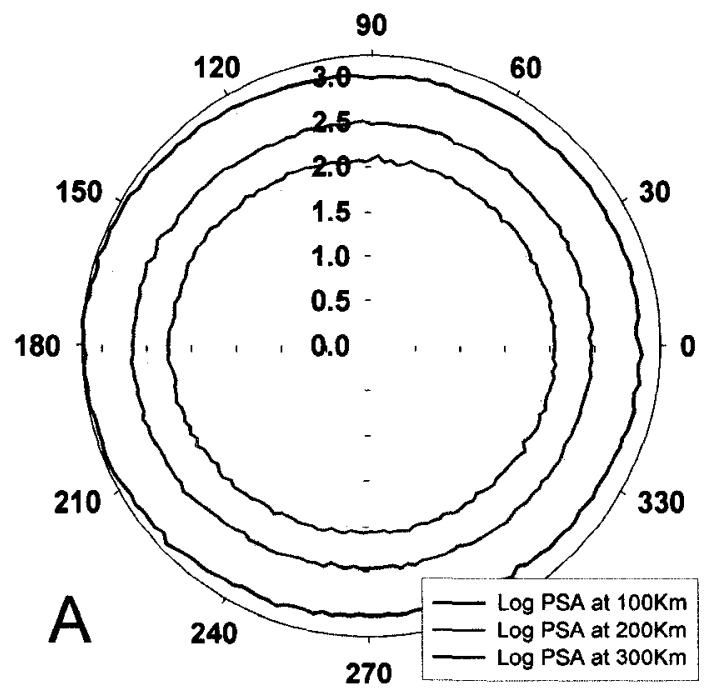

Directional Effect on Log PSAs of $1 \mathrm{~Hz}$ in Different Distances from Center of a Fault in E-W Direction

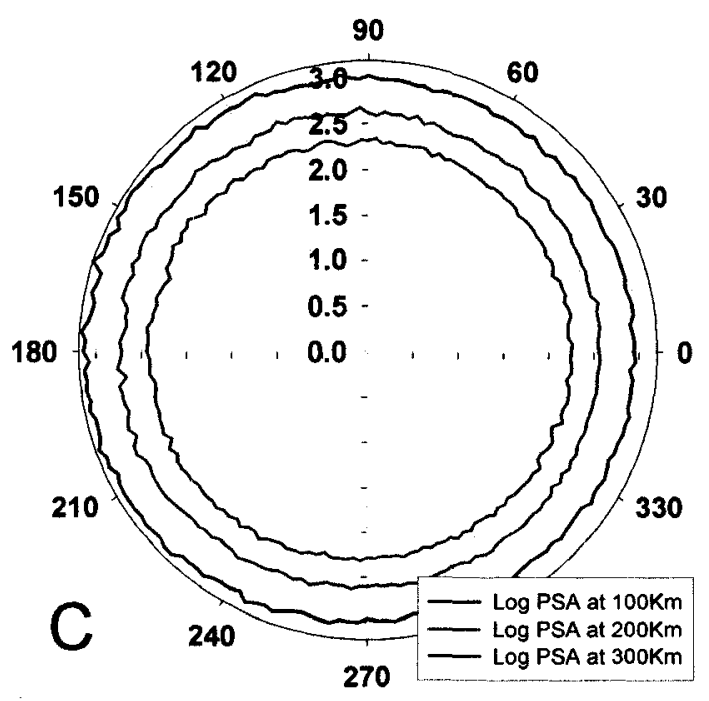

Directional Effect on Log PSAs of $5 \mathrm{~Hz}$ in Different Distances from Center of a Fault in E-W Direction

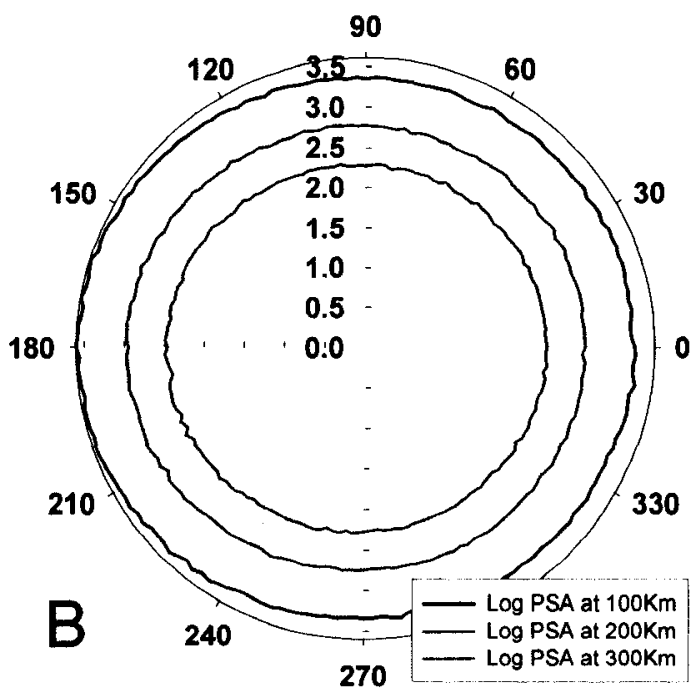

Directional Effect on Log PSAs of $0.2 \mathrm{~Hz}$ in Different Distances from Center of a Fault in E-W Direction

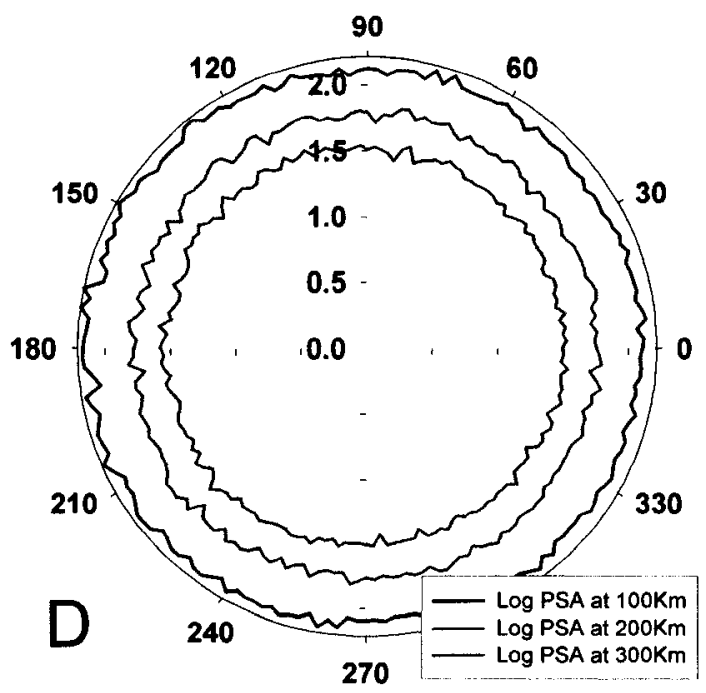

Figure (2.5) Log of PSA ordinates as a function of direction for geometry of Figure (2.1) and Table (2.2) derived for $100 \mathrm{Km}, 200 \mathrm{Km}$, and $300 \mathrm{Km}$ distances from center of fault as predicted by EXSIM for:(A) for $25 \mathrm{~Hz}$; (B) for $5 \mathrm{~Hz}$; (C) for $1 \mathrm{~Hz}$; and (D) for $0.2 \mathrm{~Hz}$. (Magnitude 6 with no extension of sub-source duration for distance is considered for the simulations). 
Directional Effect on Log PSAs of $25 \mathrm{~Hz}$ in Different
Distances from Center of a Fault in E-W Direction

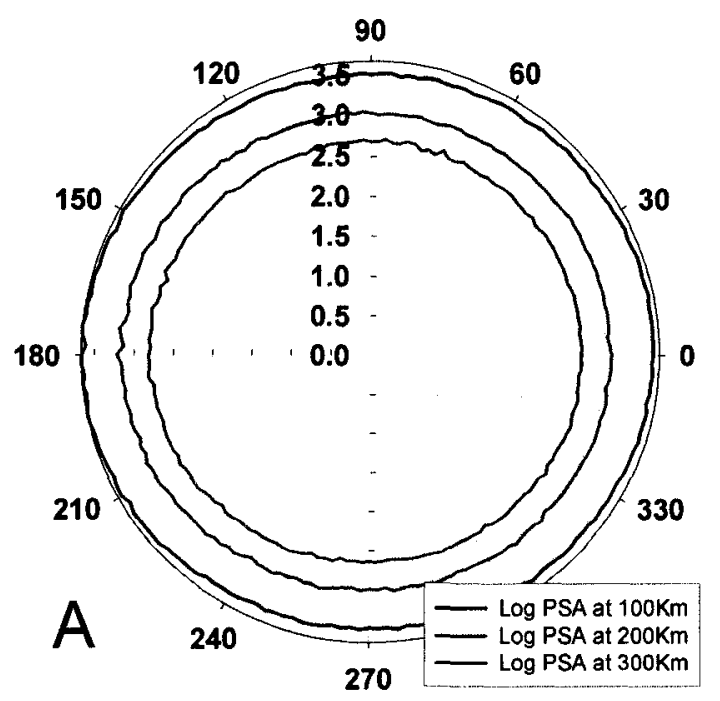

Directional Effect on Log PSAs of $1 \mathrm{~Hz}$ in Different Distances from Center of a Fault in E-W Direction

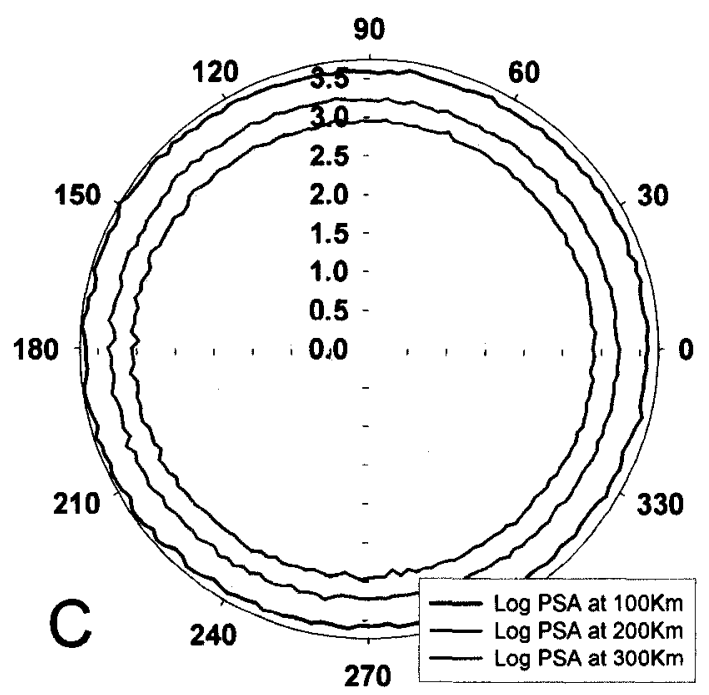

Directional Effect on Log PSAs of $5 \mathrm{~Hz}$ in Different Distances from Center of a Fault in E-W Direction

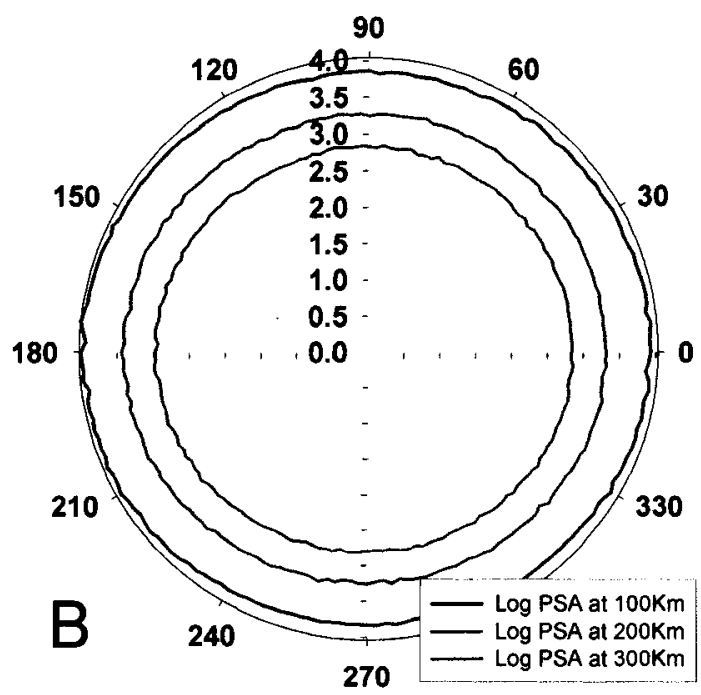

Directional Effect on Log PSAs of $0.2 \mathrm{~Hz}$ in Different Distances from Center of a Fault in E-W Direction

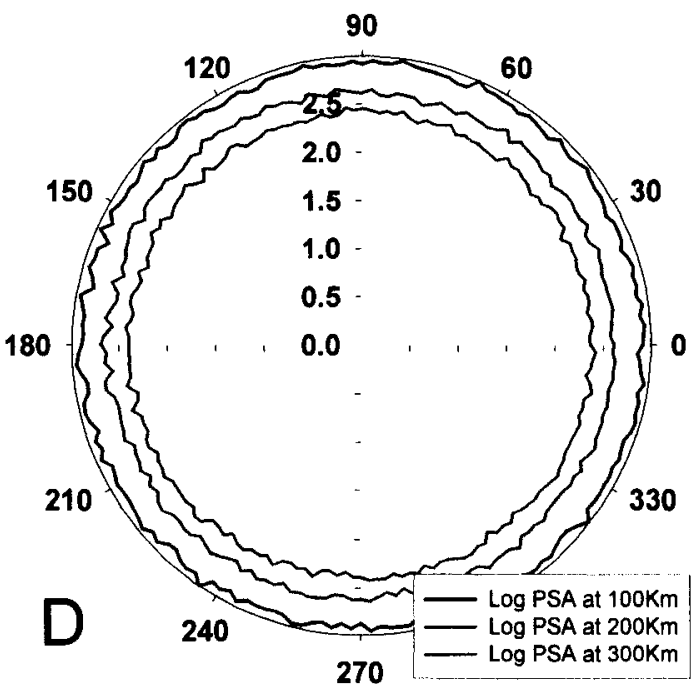

Figure (2.6) Log of PSA ordinates as a function of direction for geometry of Figure (2.1) and Table $(2.3)$ derived for $100 \mathrm{Km}, 200 \mathrm{Km}$, and $300 \mathrm{Km}$ distances from center of fault as predicted by EXSIM for:(A) for $25 \mathrm{~Hz}$; (B) for $5 \mathrm{~Hz}$; (C) for $1 \mathrm{~Hz}$; and (D) for $0.2 \mathrm{~Hz}$. (Magnitude 7 with no extension of sub-source duration for distance is considered for the simulations). 


\subsubsection{Simulation parameters and results}

Simulations are performed for 500 sites on a "racetrack" about the surface rupture of a vertical fault generating a M7.0 earthquake, at a distance of $15 \mathrm{~km}$ (Figure (2.7)). The dimensions of the fault are chosen as $40 \mathrm{~km} \times 19 \mathrm{~km}$ based on the empirical study of Wells and Coppersmith (1994). The hypocenter is assumed to be close to the center of the fault in the lower half; other simulation parameters are as given in Table (2.4). The average and standard deviation of the Fourier spectra (500 simulations) are calculated for different sub-source sizes. The chosen sub-source sizes are $\sim 1 \mathrm{~km}^{2}, \sim 4 \mathrm{~km}^{2}, \sim 9 \mathrm{~km}^{2}$, $\sim 16 \mathrm{~km}^{2}, \sim 25 \mathrm{~km}^{2}, \sim 36 \mathrm{~km}^{2}$, and $\sim 100 \mathrm{~km}^{2}$, although these exact values do not apply, since the surface area of the fault is constant and the number of sub-divisions in each direction must be an integer value.

Figure (2.8) shows the comparison of Fourier acceleration (FAS) average and FAS standard deviation for different sub-source sizes. It demonstrates that the stochastic finite fault modeling approach with a dynamic corner frequency (EXSIM) generates outputs whose FAS spectra on average, and the spread of FAS spectra around the average, are both sub-source size independent, except at very low frequencies.

\subsubsection{Discussion of sub-source size independence}

Considering that for each frequency and sub-source size there are 500 realizations of EXSIM output space generated with similar parameters, it is reasonable to assume that the elements of this set of 500 values are normally distributed. Considering that the averages and standard deviations of two normally distributed sets are equal, I conclude that these sets are identical (or they are different samples of an identical superset). Thus the spectral values of EXSIM simulations at short distances are not affected by subsource size, confirming the similar result reported by Motazedian and Atkinson (2005) for large distances. 
Table (2.4) Simulation parameters for sub-source size independence study

\begin{tabular}{cc}
\hline Parameter & Parameter value \\
\hline Fault orientation & Strike $90^{\circ}$, Dip $90^{\circ}$ \\
Depth of top & $0 \mathrm{~km}$ \\
Fault dimensions & Length $40 \mathrm{~km}$, Width $19 \mathrm{~km}$ \\
Number of sub-faults & Along length 10, Along width 5 \\
FFT points, sample interval & $8192,0.02 \mathrm{~s}$ \\
Shear wave velocity, Density & $3.5 \mathrm{~km} / \mathrm{s}, 2.8 \mathrm{~g} / \mathrm{cm}^{3}$ \\
Rupture velocity & $0.8 \times$ shear wave velocity \\
$Q(f)$ & $180 f^{0.45}$ \\
Moment magnitude & 7.0 \\
Average stress parameter & 50 bars \\
Kappa & 0.035 \\
Geometrical attenuation & If R $<40, \mathrm{R}^{-1}$, else $\mathrm{R}^{-0.5}$ \\
Sub-source duration & $\propto 0.05 \times$ distance $(\mathrm{sec})$. \\
Windowing function & Saragoni-Hart \\
Amplification function & Boore and Joyner $\left(1997\right.$ ) for $\mathrm{V}_{\mathrm{s} 30}=620 \mathrm{~m} / \mathrm{s}$ \\
Damping & $5 \%$ of critical damping \\
Number of EXSIM iterations & 1 (per site) \\
\hline
\end{tabular}

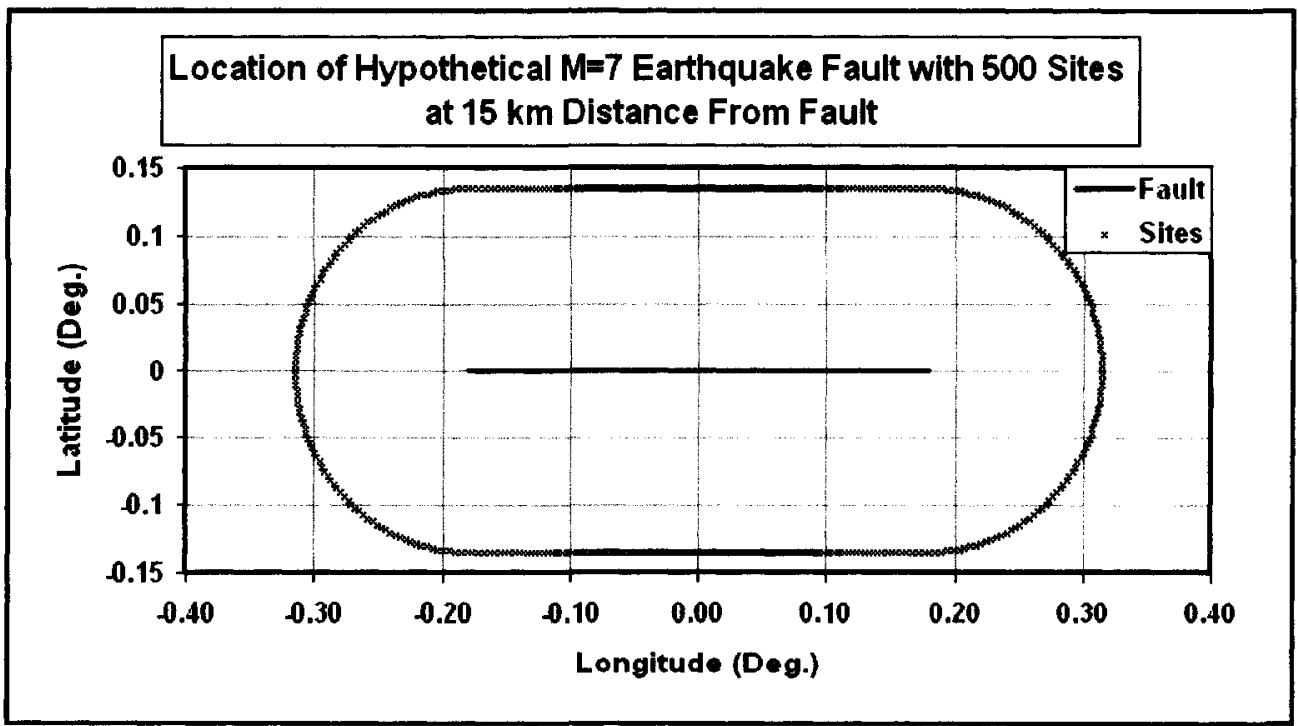

Figure (2.7) Surface trace of vertical fault for M7.0 earthquake, showing location of 500 sites at $15 \mathrm{~km}$ distance from fault. 

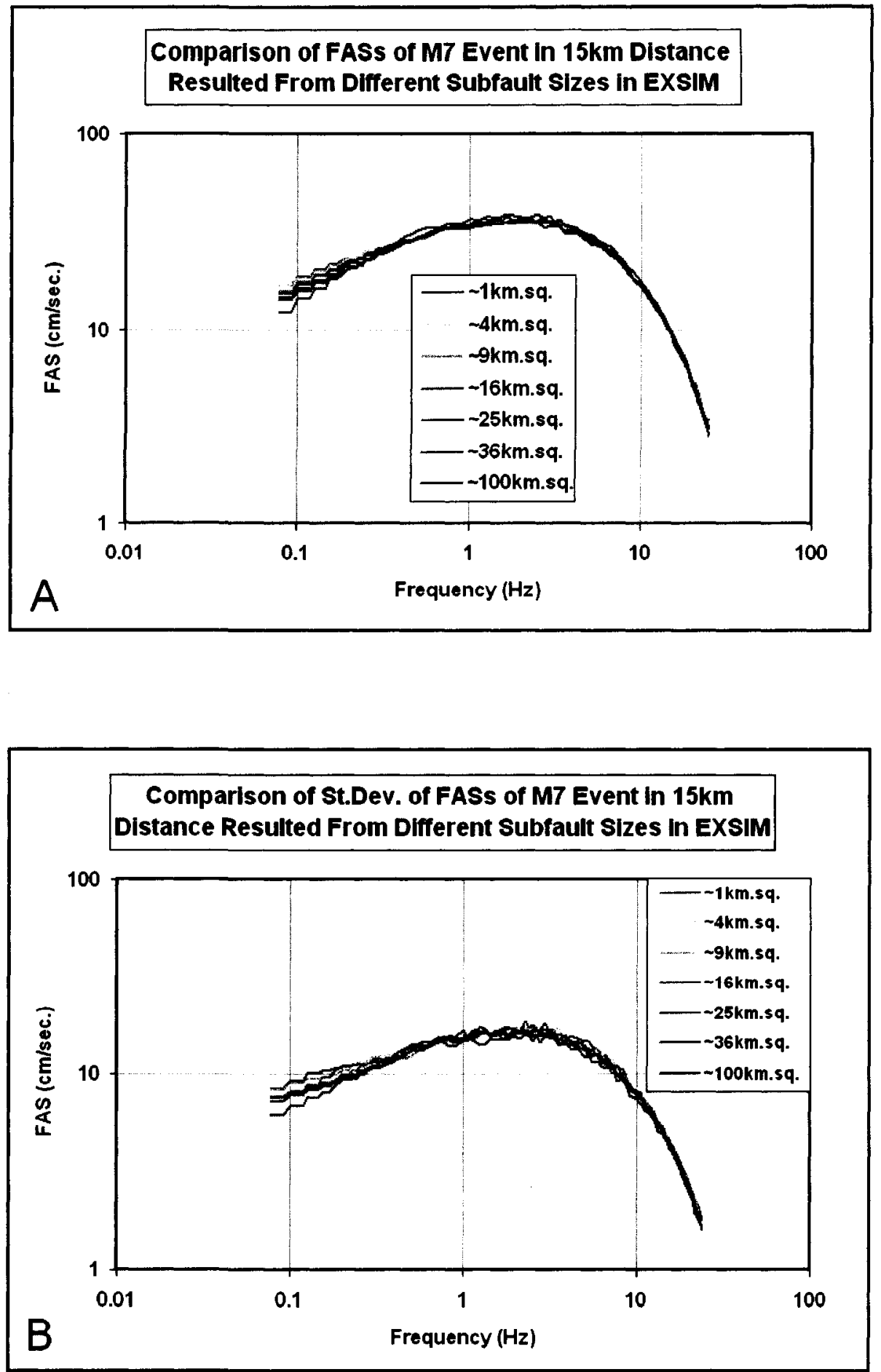

Figure (2.8) (A) Averages of Fourier acceleration spectra of 500 simulation sites for different selection of sub-source sizes. (B) Standard deviation of Fourier acceleration spectra of 500 simulation sites for different selection of sub-source sizes. 


\subsection{Spectral comparisons of different simulation algorithms}

\subsubsection{Why spectral comparisons?}

The stochastic methods discussed in this thesis generate time series of a scenario earthquake for a given source, path, and site characterization. These methods are designed to generate signals that on average carry the spectrum of an earthquake with the specified parameters. Because these methods are following different assumptions, their outputs will not be identical, and one can check their differences by comparing the resulting ground motion predictions. One way of performing comparisons between different methods (or different solutions) is to derive the average spectrum according to different methods and compare them. The Fourier spectrum and pseudo spectral acceleration (pseudo spectral acceleration, or PSA) are the types of spectra used for comparisons in this study. The average spectrum is derived by calculating the mean (arithmetic or geometric) of spectra over multiple simulations. The multiple simulations should be done over an array of sites that are equidistant from the trace of a vertical dipping fault, or its point-source equivalent.

\subsubsection{Comparing Fourier spectra of SMSIM (point-source) and EXSIM (extended source)}

In this section I examine the differences in predicted ground motion between point-source models, using the program SMSIM, and extended source, using the EXSIM program. Recall that SMSIM is a stochastic point-source modeling program developed by Boore (2000) and EXSIM is a stochastic finite-fault modeling program developed by Motazediana and Atkinson (2005). Fourier spectra are the basis of the comparisons. In these comparisons, the effect of three key parameters are studied: magnitude, distance, and stress parameter. The selected simulation parameter values are M5, M6, M7, and M8 for magnitude, $15 \mathrm{~km}$ and $300 \mathrm{~km}$ for distance, $50 \mathrm{bars}$ and 120 bars for stress parameter. 
In EXSIM simulations, magnitude affects the size of the fault generating the earthquake, while in SMSIM the sources of all magnitudes are treated as a point. The fault dimensions for use in EXSIM are given in Wells and Coppersmith (1994) for fault area and rupture length:

$$
\begin{aligned}
& \text { rupture length }=10^{0.69 M-3.22} \\
& \text { rupture area }=10^{0.91 M-3.49}
\end{aligned}
$$

where $\boldsymbol{M}$ is the moment magnitude. Wells and Coopersmith (1994) derived equations relating rupture dimensions to moment magnitude based on regression analysis of welldocumented and recorded earthquakes around the globe. Since the modeling studies here assume faults rupture the earth's surface, the rupture length of Equation (2.3) corresponds to surface rupture length. This introduces two minor imperfections. Wells and Coppersmith (1994) derived Equations (2.3) and (2.4) from regressing earthquake data in the magnitude range M5.2 to M8.1. Also they reported the surface rupture length is about $25 \%$ less than rupture length at depth. Since the closest distances from the fault to the site is $15 \mathrm{~km}$, treatment of the smallest magnitude is not optimal, although the overall results should not be affected to any significant extent.

The rupture surface is assumed to be vertical. For EXSIM modeling, an array of sites with constant distance from the trace of the fault and covering all azimuths is assumed. Since SMSIM is a point source modeler, its outputs are not sensitive to azimuth. In SMSIM the entire fault is assumed to be concentrated at a point, and the specified distances are those between the point source and the site. The stress parameter is directly applied to the simulation inputs for both programs.

The simulation parameters for the Fourier spectra comparisons of SMSIM and EXSIM are given in Table (2.5).

Before getting into details of Fourier spectra comparisons of scenarios in Table (2.5), two experiment results are demonstrated here. In the first experiment I assigned one subsource to the whole finite source and an identical location to sites when doing simulations 
with EXSIM. Other simulation parameters for both programs, SMSIM and EXSIM, are those of Table (2.5); magnitudes M5, M6, and M7 with stress equal to 50 bars at $300 \mathrm{~km}$ distance are considered. Since the number of sub-sources in EXSIM is "one" the finite source behaves like a point source, and the average Fourier spectra from either of methods show an exact match. Figure (2.9) shows the averages of 500 Fourier spectra for the three scenarios from SMSIM and EXSIM. In the second experiment, I assigned multiple sub-sources (30 sub-sources according to Table (2.5)) to the finite source and an identical location to sites when doing simulations with EXSIM. Similar to the previous experiment, other simulation parameters for both programs, SMSIM and EXSIM, are those of Table (2.5). Figure (2.10) shows the averages of 500 Fourier spectra from SMSIM and EXSIM for this experiment. When 30 subsources are used, there is a clear difference between the SMSIM and EXSIM predictions.

The average Fourier acceleration spectra of SMSIM and EXSIM simulations, resulting from different values of magnitude, distance, and stress parameters are compared in the following experiments. Figure (2.11) shows average Fourier spectra comparisons between SMSIM and EXSIM simulations for a 50-bar stress, at $15 \mathrm{~km}$. The simulation parameters are given in Table (2.5); and $\mathbf{M} 5, \mathbf{M} 6, \mathbf{M} 7$, and $\mathbf{M} 8$ magnitude scenarios are modeled. The red line is the average of 500 SMSIM simulations; small dots are individual spectra of 500 EXSIM simulations, and the blue line is the average of the 500 EXSIM simulations. At $15 \mathrm{~km}$ from the fault, with 50 bars of stress and moderate magnitude, up to 6, SMSIM and EXSIM generate similar average spectra. At larger magnitudes the effects of the finite fault become important, and the average predictions of the two methods diverge. For a M8 earthquake, the average of the SMSIM simulations spectrum touches the upper bound of EXSIM simulations spectra.

There is a very gentle sag in the EXSIM average spectra below $1 \mathrm{~Hz}$ at same magnitudes, which is suggestive of a double corner frequency spectral shape, but the effect is very weak. Note that the SMSIM simulations could be made very close to the EXSIM results 
using 2 devices: (i) a decreasing stress with increasing $M$; or (ii) an increase in "effective distance" at which to place the point source.

Figure (2.12) shows average Fourier spectra comparisons between SMSIM and EXSIM simulations at $15 \mathrm{~km}$ for a 120 bar stress drop, for M5, M6, M7, and M8. As in the 50-bar case, red and blue lines are average Fourier spectra of SMSIM and EXSIM respectively and dots are individual spectra ordinates of EXSIM simulations. The change of stress parameter from 50 bars to 120 bars does not make any significant changes in the average shape of SMSIM and EXSIM simulations spectra, except that spectra for both simulation methods are shifted up in amplitude.

Figure (2.13) shows average Fourier spectra comparisons between SMSIM and EXSIM simulations for 50 bars at $300 \mathrm{~km}$, for M5, M6, M7, and M8. The finite fault effects are reduced considerably at $300 \mathrm{~km}$ distance from the fault, so average Fourier spectra of simulations resulting from both methods, SMSIM and EXSIM, are showing a nearly perfect match in all magnitude ranges. By looking at Figure (2.13), we see that at magnitudes M5 and M6, EXSIM generates slightly higher amplitudes at high frequencies and slightly lower amplitudes at low frequencies. This can be explained by a higher highfrequency to low-frequency ratio of sub-sources compared to a point source; this ratio decreases when switching to higher magnitudes.

Figure (2.14) considers average Fourier spectra comparisons between SMSIM and EXSIM simulations for a stress parameter of 120 bars, at a distance of $300 \mathrm{~km}$ distance from the fault.. The relations between average spectra of SMSIM and EXSIM simulations remain the same as for lower stress values, but there is an overall shift in amplitudes. 
Table (2.5) Simulation parameters for SMSIM and EXSIM Fourier spectra comparisons

\begin{tabular}{|c|c|}
\hline Parameter & Parameter value \\
\hline Fault orientation & Strike $90^{\circ}$, Dip $90^{\circ}$ \\
\hline Depth of top & $0 \mathrm{~km}$ \\
\hline Fault dimensions & $\begin{array}{l}\text { A) Length } 2 \mathrm{~km} \text {, Width } 6 \mathrm{~km} \\
\text { B) Length } 8 \mathrm{~km} \text {, Width } 12 \mathrm{~km} \\
\text { C) Length } 40 \mathrm{~km} \text {, Width } 19 \mathrm{~km} \\
\text { D) Length } 200 \mathrm{~km} \text {, Width } 31 \mathrm{~km}\end{array}$ \\
\hline Number of sub-faults & $\begin{array}{l}\text { A) Along length 1, Along width } 3 \\
\text { B) Along length } 4 \text {, Along width } 6 \\
\text { C) Along length } 6 \text {, Along width } 3 \\
\text { D) Along length } 40 \text {, Along width } 6\end{array}$ \\
\hline FFT points, sample interval & $8192,0.02 \mathrm{~s}$ \\
\hline Shear wave velocity, Density & $3.5 \mathrm{~km} / \mathrm{s}, 2.8 \mathrm{~g} / \mathrm{cm}^{3}$ \\
\hline Rupture velocity & $0.8 \times$ shear wave velocity \\
\hline$Q(f)$ & $180 f^{0.45}$ \\
\hline Moment magnitude & $\begin{array}{l}\text { A) } 5.0 \\
\text { B) } 6.0 \\
\text { C) } 7.0 \\
\text { D) } 8.0\end{array}$ \\
\hline Average stress parameter & $\begin{array}{l}\text { I) } 50 \text { bars } \\
\text { II) } \quad 120 \text { bars }\end{array}$ \\
\hline Kappa & 0.035 \\
\hline Geometrical attenuation & If $\mathrm{R}<40, \mathrm{R}^{-1}$, else $\mathrm{R}^{-0.5}$ \\
\hline Sub-source duration & $\propto 0.05 \times$ distance $(\mathrm{sec})$. \\
\hline Windowing function & Saragoni-Hart \\
\hline Amplification function & Boore and Joyner (1997) for $\mathrm{V}_{\mathrm{s} 30}=620 \mathrm{~m} / \mathrm{s}$ \\
\hline Damping & $5 \%$ of critical damping \\
\hline Number of EXSIM iterations & 1 (per site) \\
\hline
\end{tabular}




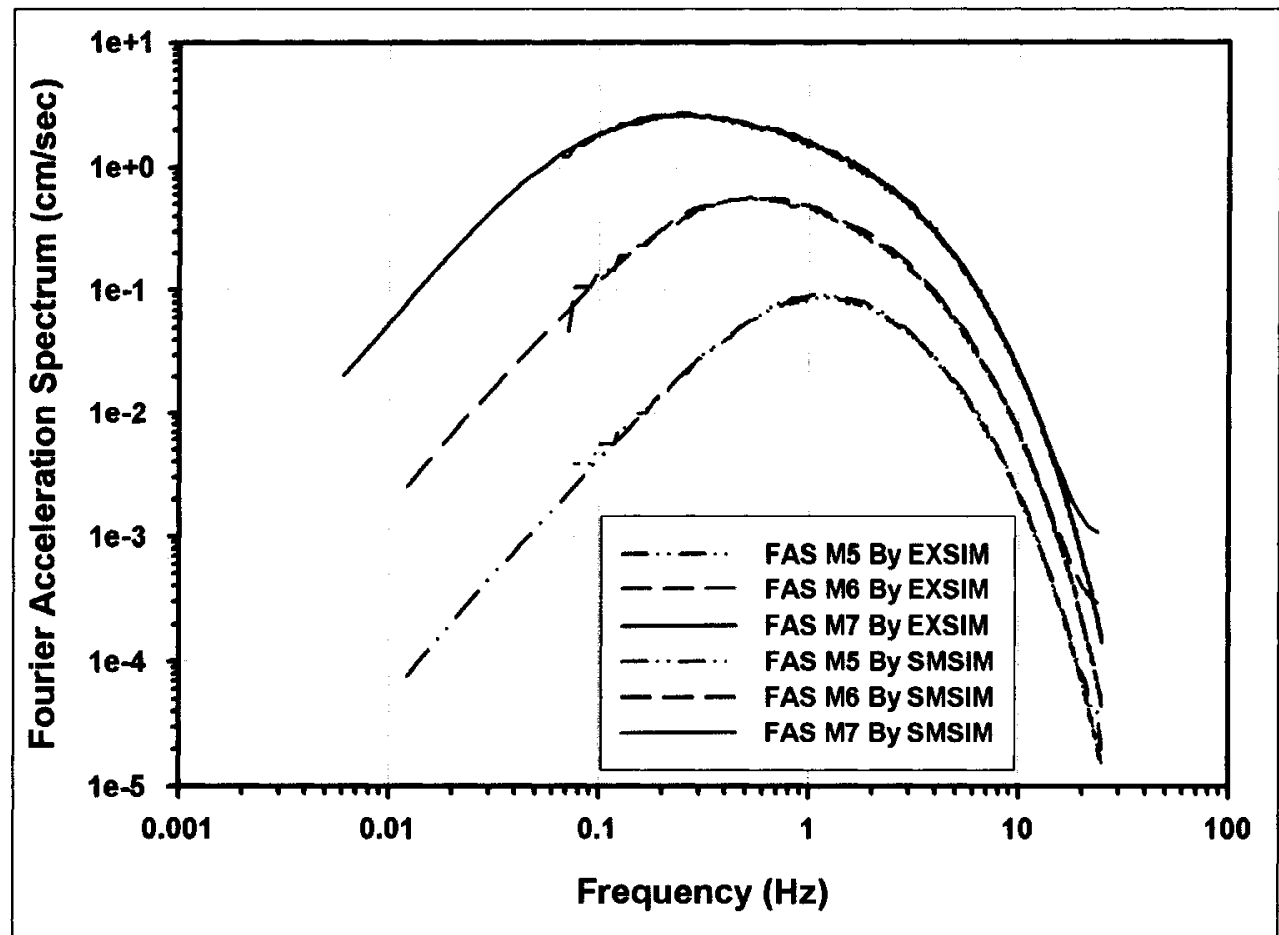

Figure (2.9) Averages of Fourier acceleration spectra of 500 simulations for a site located at $300 \mathrm{~km}$ distance from the fault at one point. Three scenarios with magnitudes M5, M6, and $\mathbf{M} 7$ are simulated using SMSIM and EXSIM. Simulation parameters are those of Table (2.5) except that the number of sub-sources in EXSIM simulations is one. 


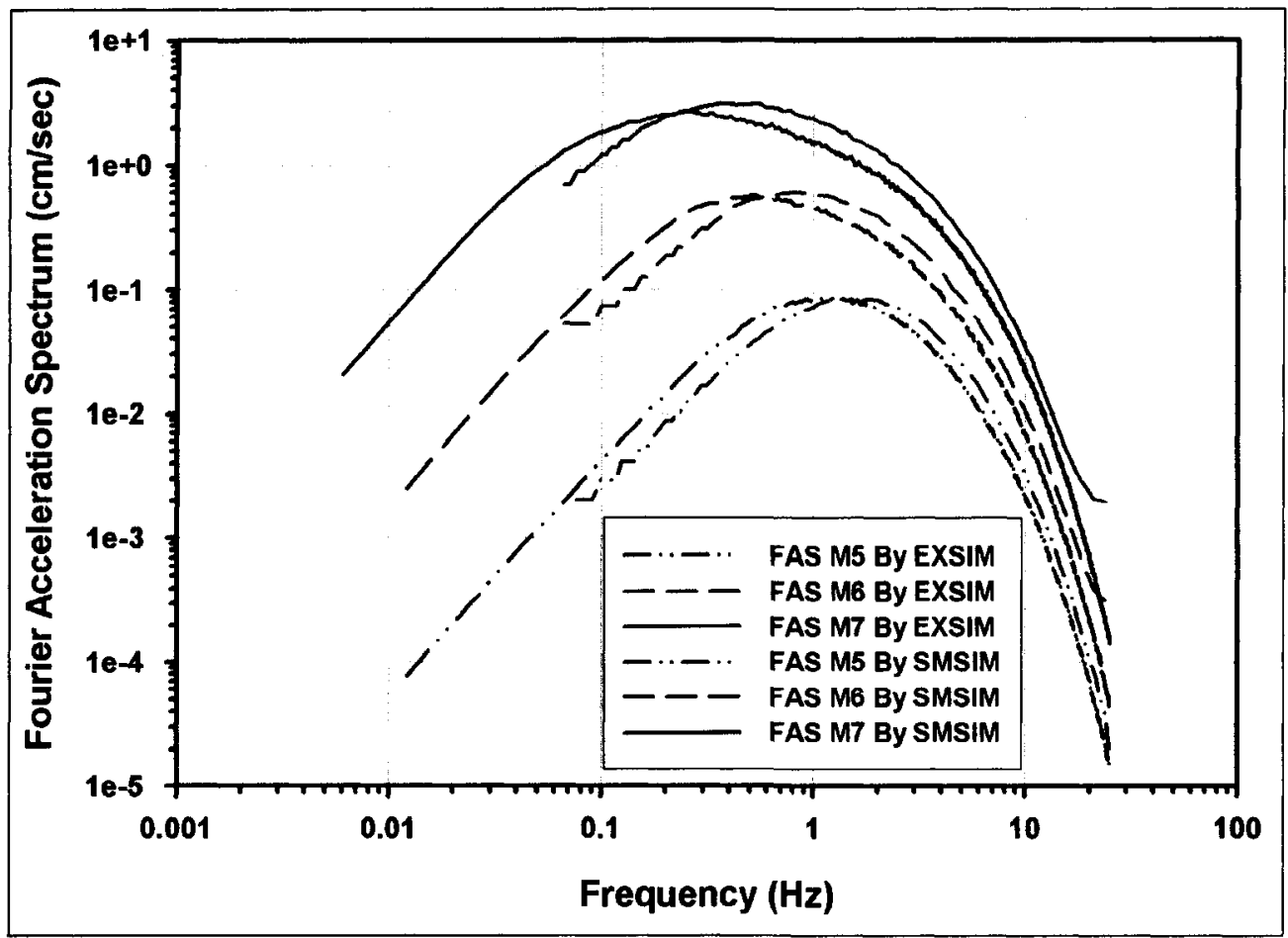

Figure (2.10) Averages of Fourier acceleration spectra of 500 simulations for a site located at $300 \mathrm{~km}$ distance from the fault at one point. Three scenarios with magnitudes M5, M6, and M7 are simulated using SMSIM and EXSIM. Simulation parameters are those of Table (2.5). Note that, according to table (2.5), the number of sub-sources in the simulations of this experiment is " 30 ". 
FAS Comparison of EXSIM and SMSIM (M=5)
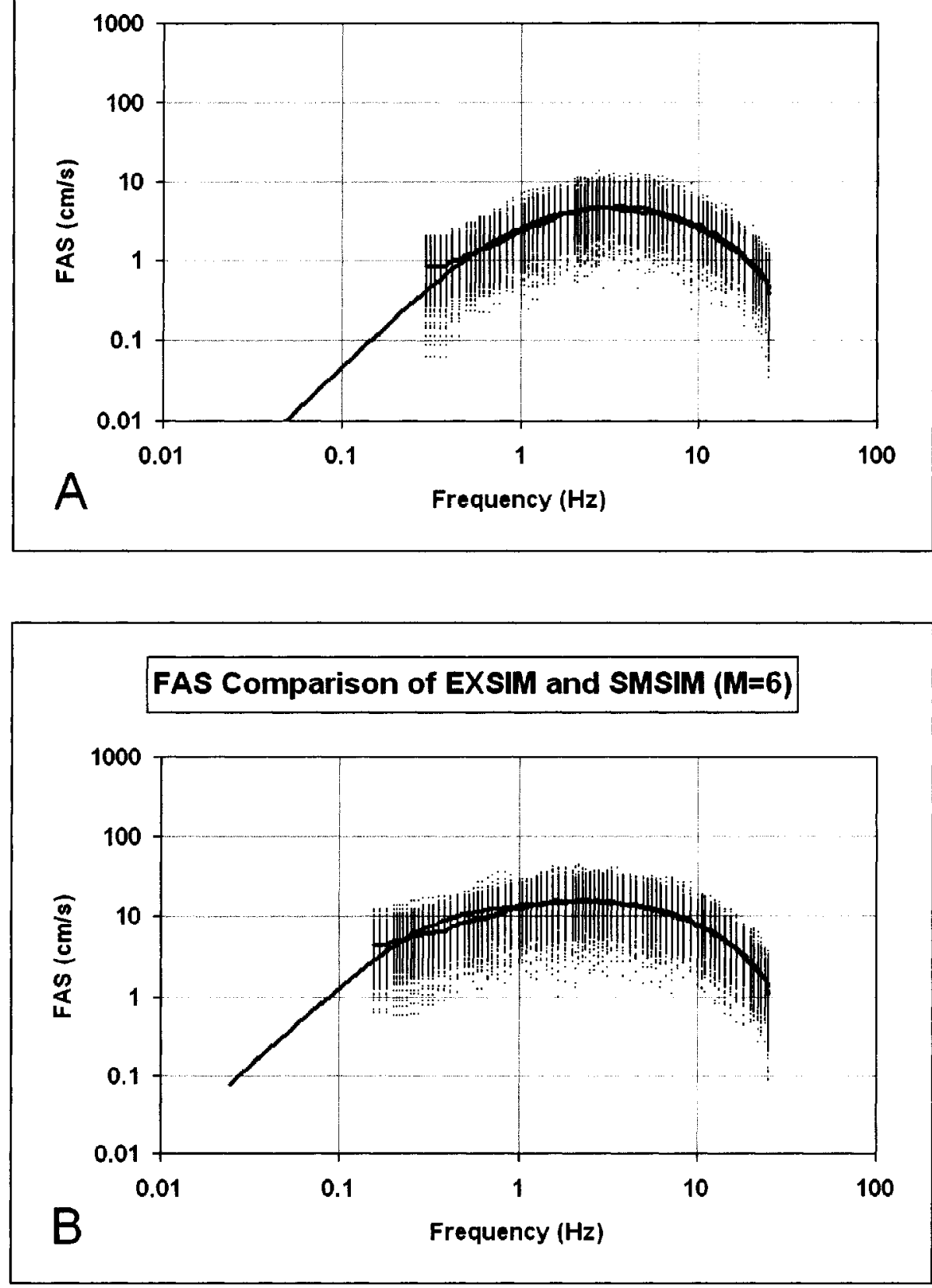

Figure (2.11) Average of Fourier acceleration spectra of 500 simulations for sites located at $15 \mathrm{~km}$ distance from fault and 50 bars of stress parameter for: (A) M5 and (B) M6 earthquakes. The dots are individual EXSIM output spectra, blue line is averages of EXSIM outputs' spectra, and red line is average of SMSIM outputs' spectra. 

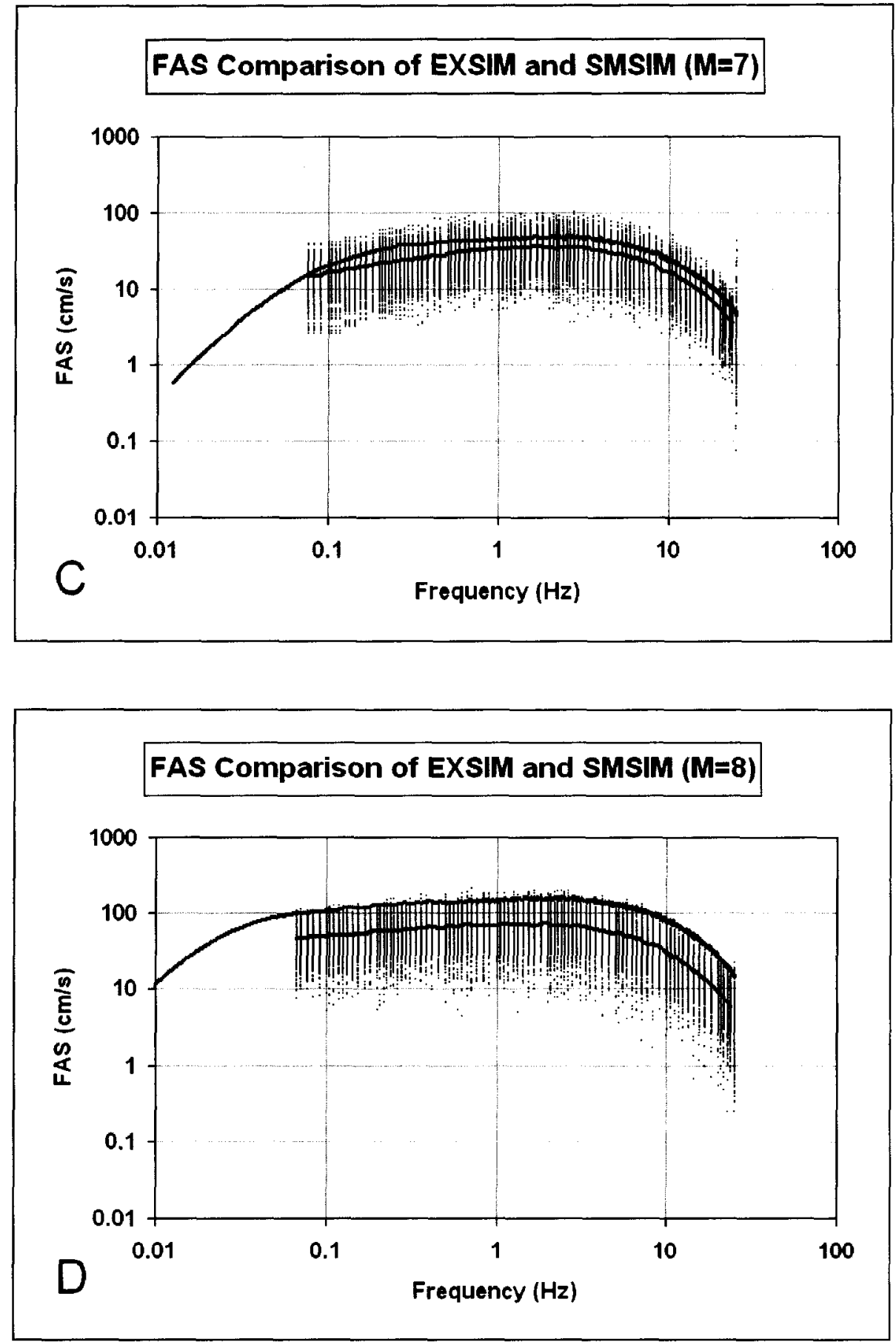

Figure (2.11) Average of Fourier acceleration spectra of 500 simulations for sites located at $15 \mathrm{~km}$ distance from fault and 50 bars of stress parameter for: (C) M7 and (D) M8 earthquakes. The dots are individual EXSIM output spectra, blue line is averages of EXSIM outputs' spectra, and red line is average of SMSIM outputs' spectra. 

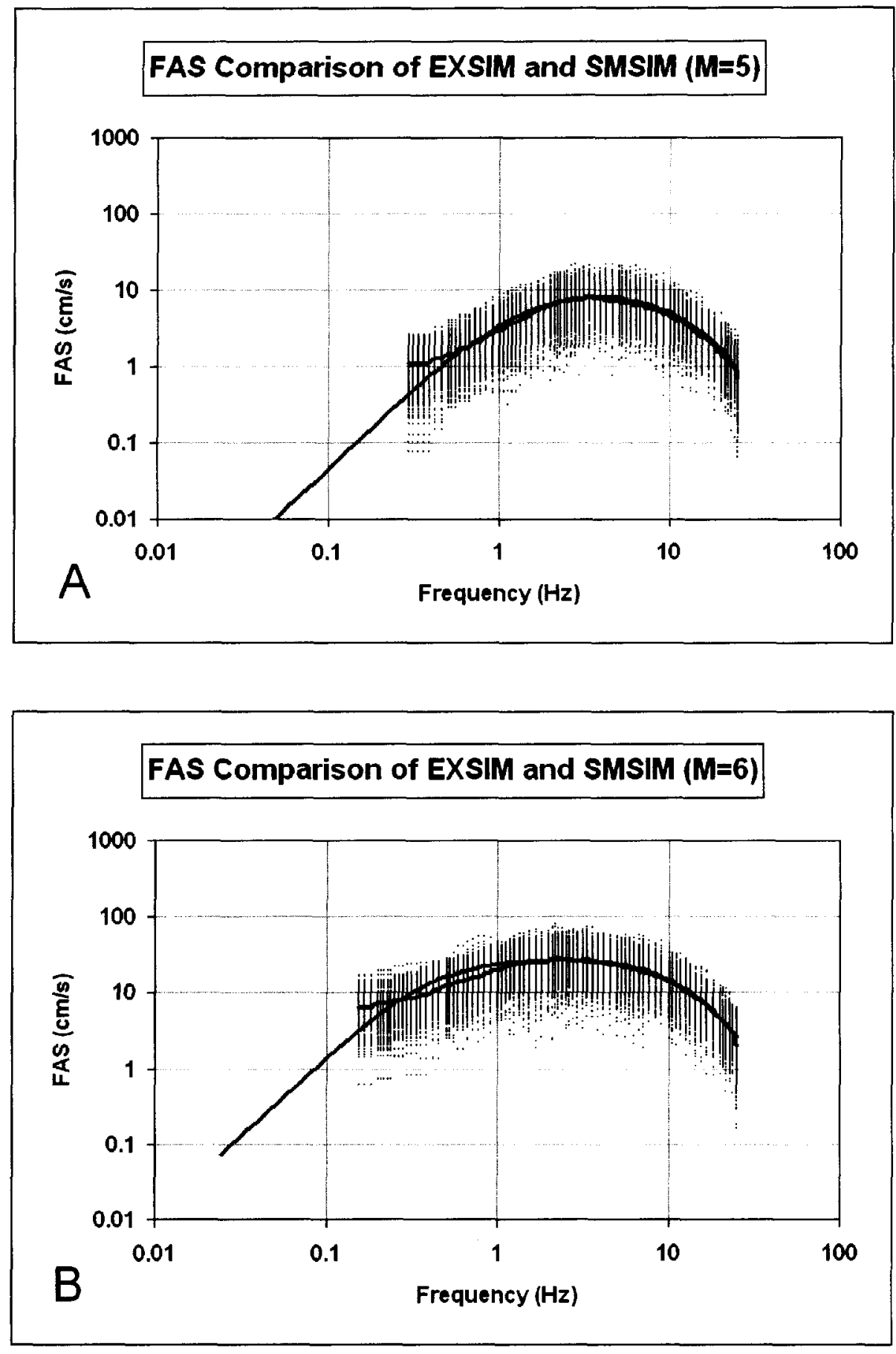

Figure (2.12) Average of Fourier acceleration spectra of 500 simulations for sites located at $15 \mathrm{~km}$ distance from fault and 120 bars of stress parameter for: (A) M5 and (B) M6 earthquakes. The dots are individual EXSIM output spectra, blue line is averages of EXSIM outputs' spectra, and red line is average of SMSIM outputs' spectra. 

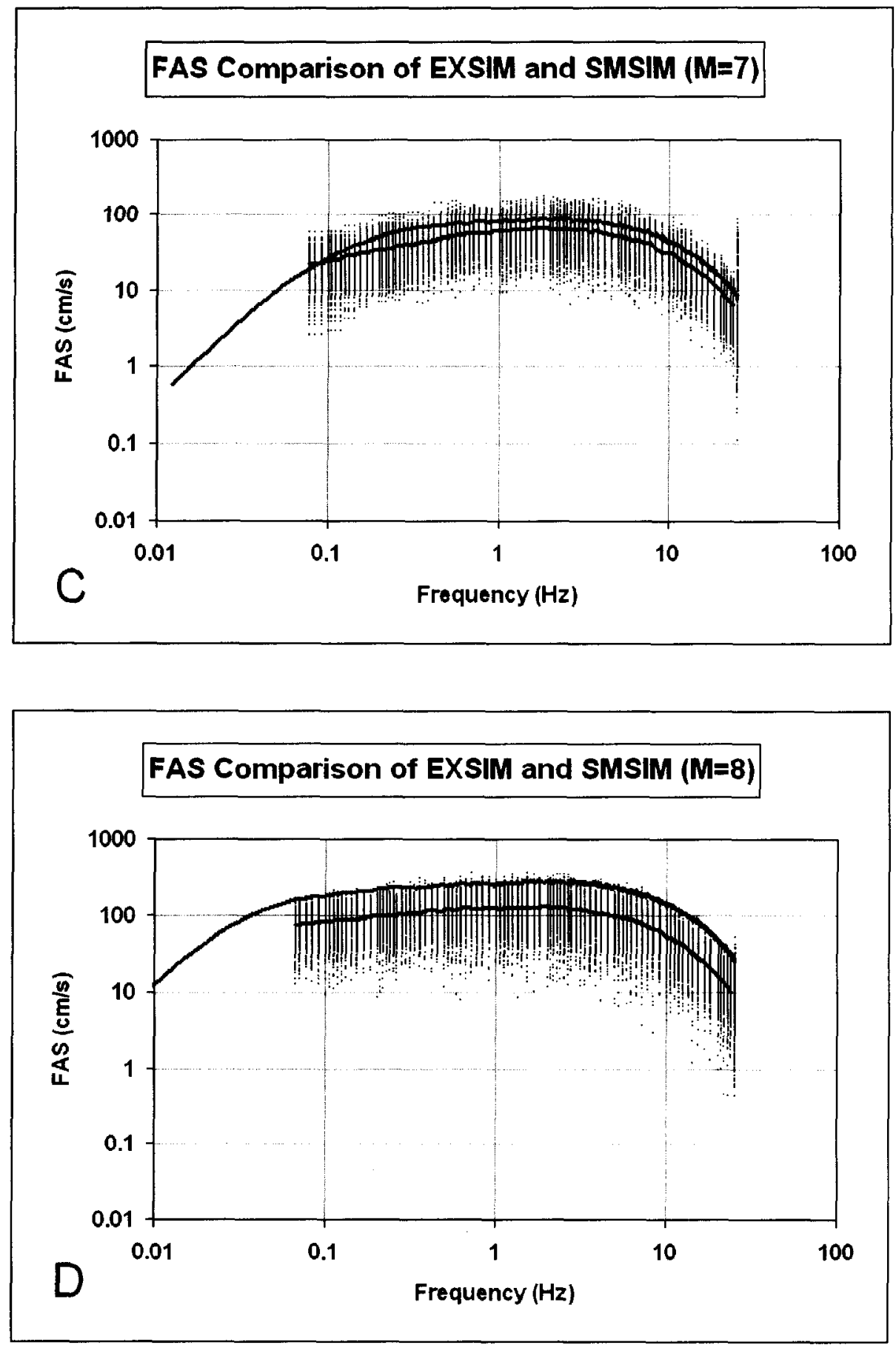

Figure (2.12) Average of Fourier acceleration spectra of 500 simulations for sites located at $15 \mathrm{~km}$ distance from fault and 120 bars of stress parameter for: (C) M7 and (D) M8 earthquakes. The dots are individual EXSIM output spectra, blue line is averages of EXSIM outputs' spectra, and red line is average of SMSIM outputs' spectra. 

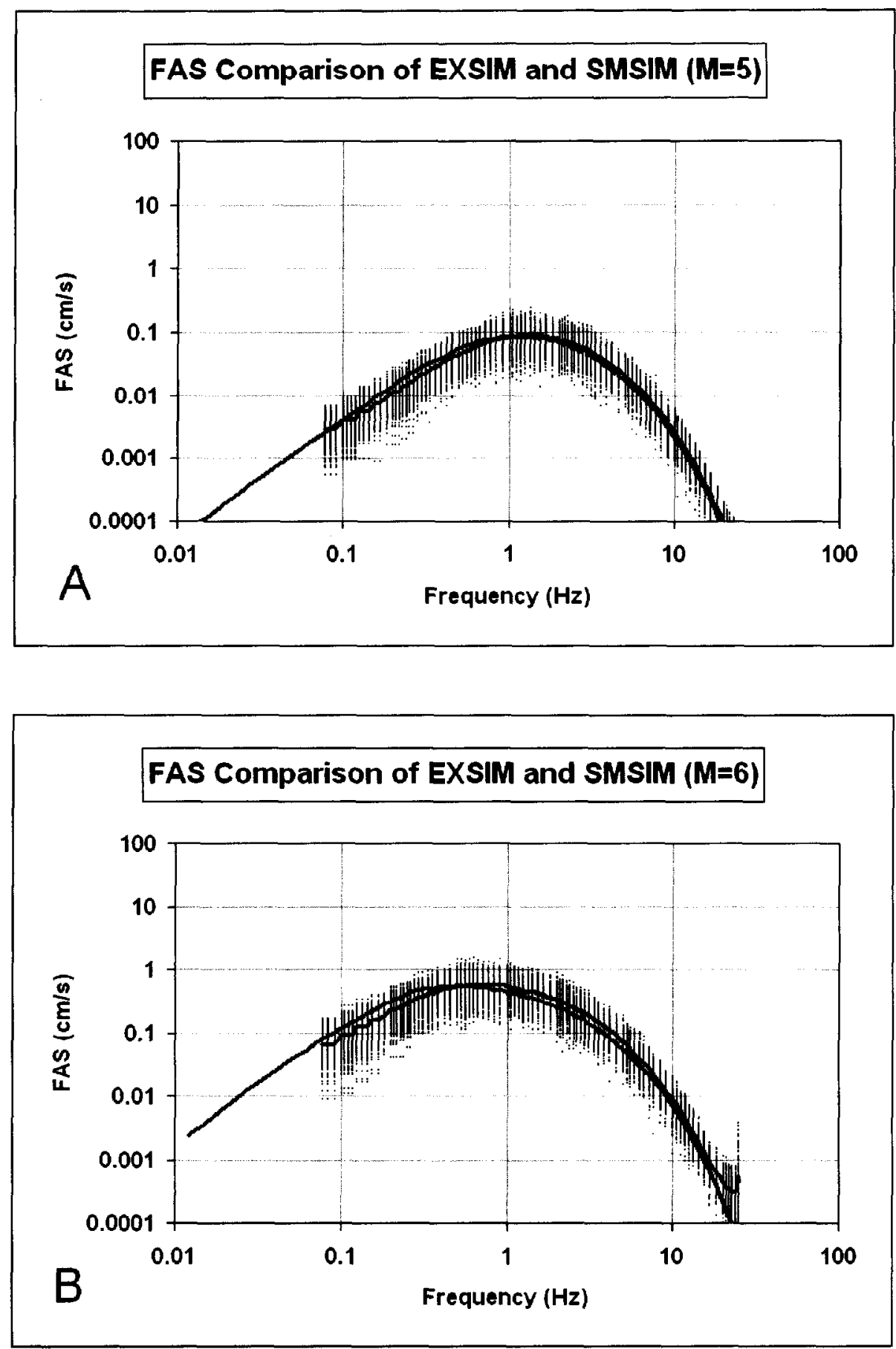

Figure (2.13) Average of Fourier acceleration spectra of 500 simulations for sites located at $300 \mathrm{~km}$ distance from fault and 50 bars of stress parameter for: (A) M5 and (B) M6 earthquakes. The dots are individual EXSIM output spectra, blue line is averages of EXSIM outputs' spectra, and red line is average of SMSIM outputs' spectra. 

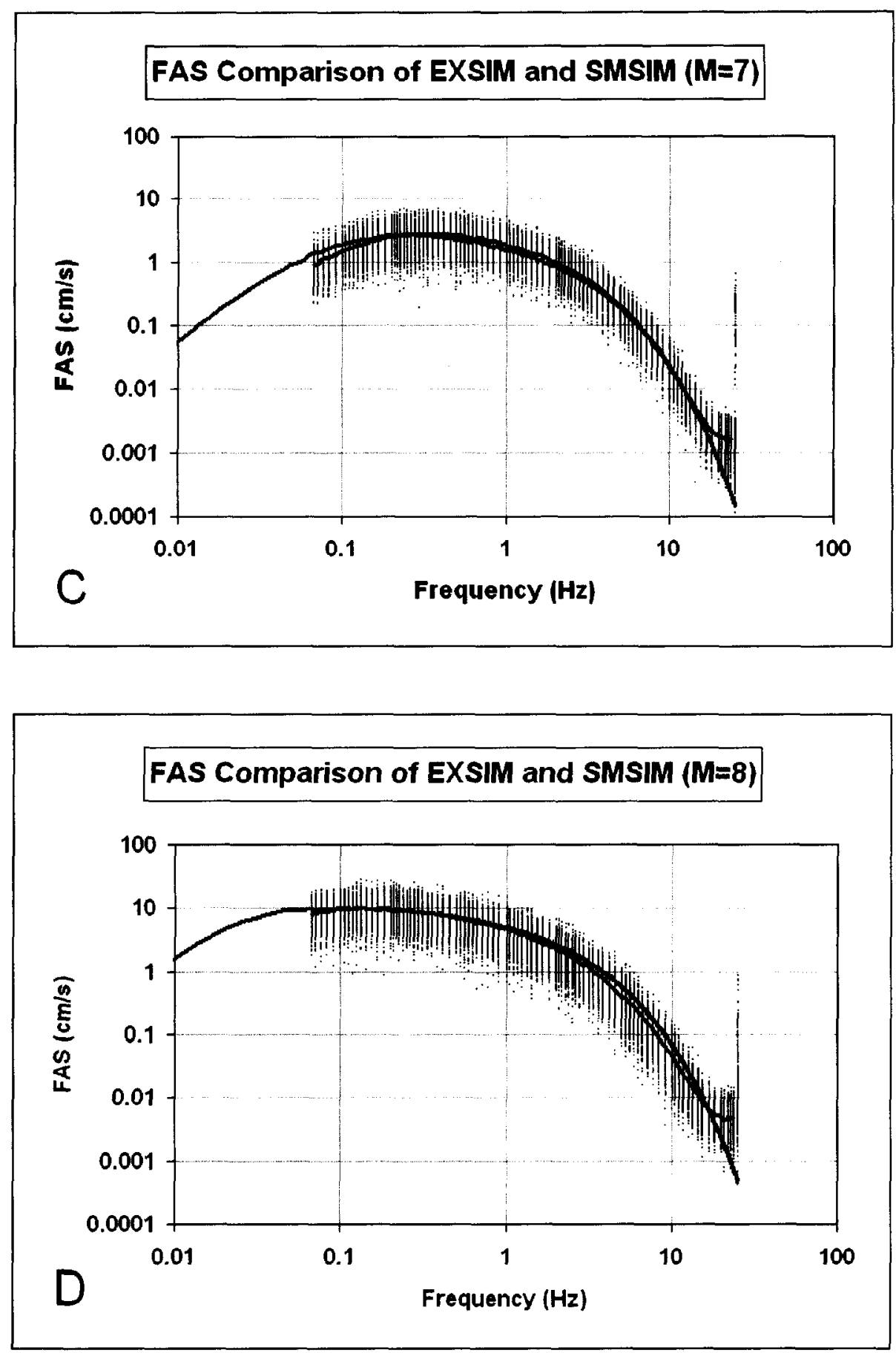

Figure (2.13) Average of Fourier acceleration spectra of 500 simulations for sites located at $300 \mathrm{~km}$ distance from fault and 50 bars of stress parameter for: (C) $\mathbf{M} 7$ and (D) $\mathbf{M} 8$ earthquakes. The dots are individual EXSIM output spectra, blue line is averages of EXSIM outputs' spectra, and red line is average of SMSIM outputs' spectra. 

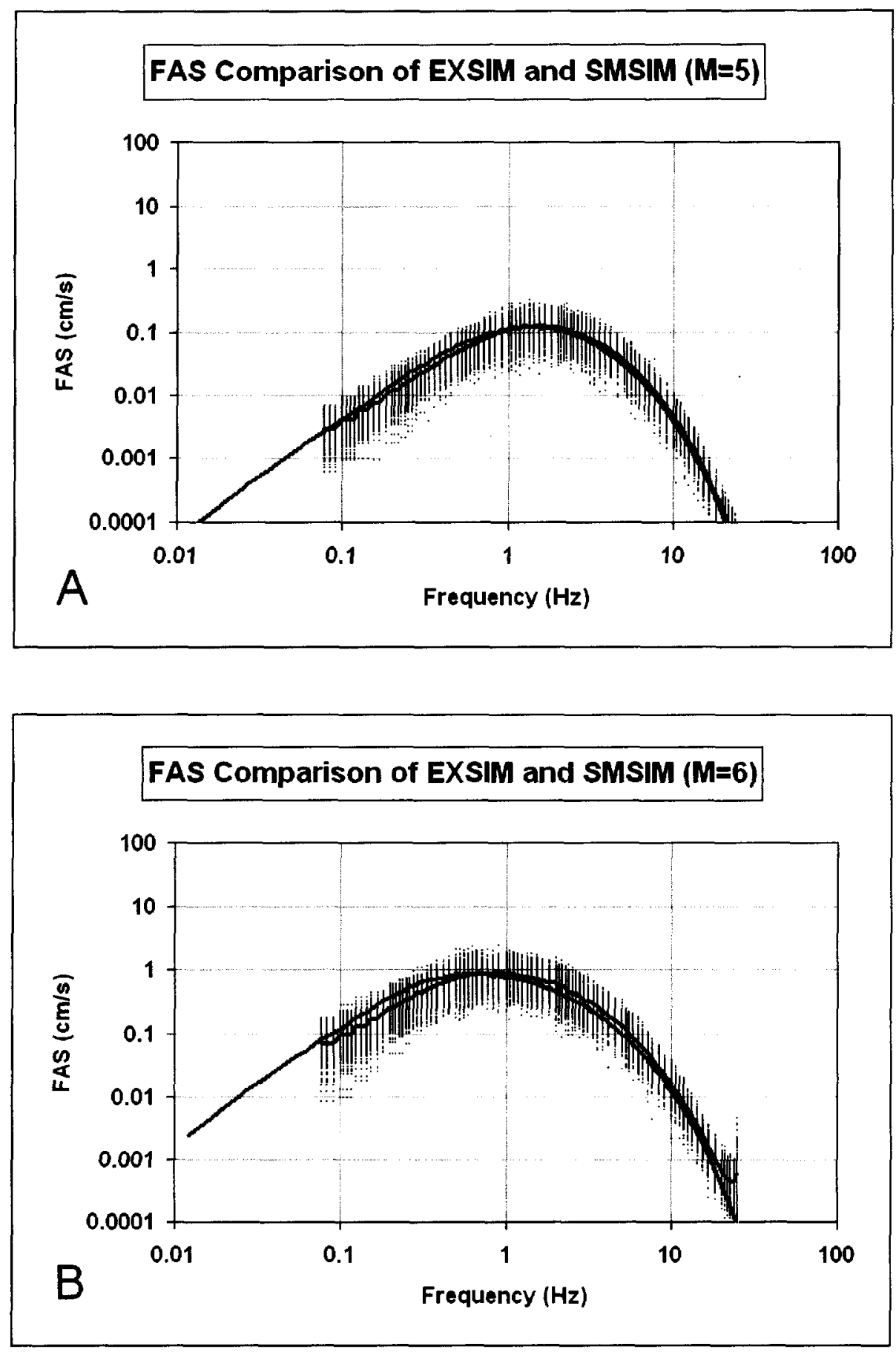

Figure (2.14) Average of Fourier acceleration spectra of 500 simulations for sites located at $300 \mathrm{~km}$ distance from fault and 120 bars of stress parameter for: (A) M5 and (B) M6 earthquakes. The dots are individual EXSIM output spectra, blue line is averages of EXSIM outputs' spectra, and red line is average of SMSIM outputs' spectra. 

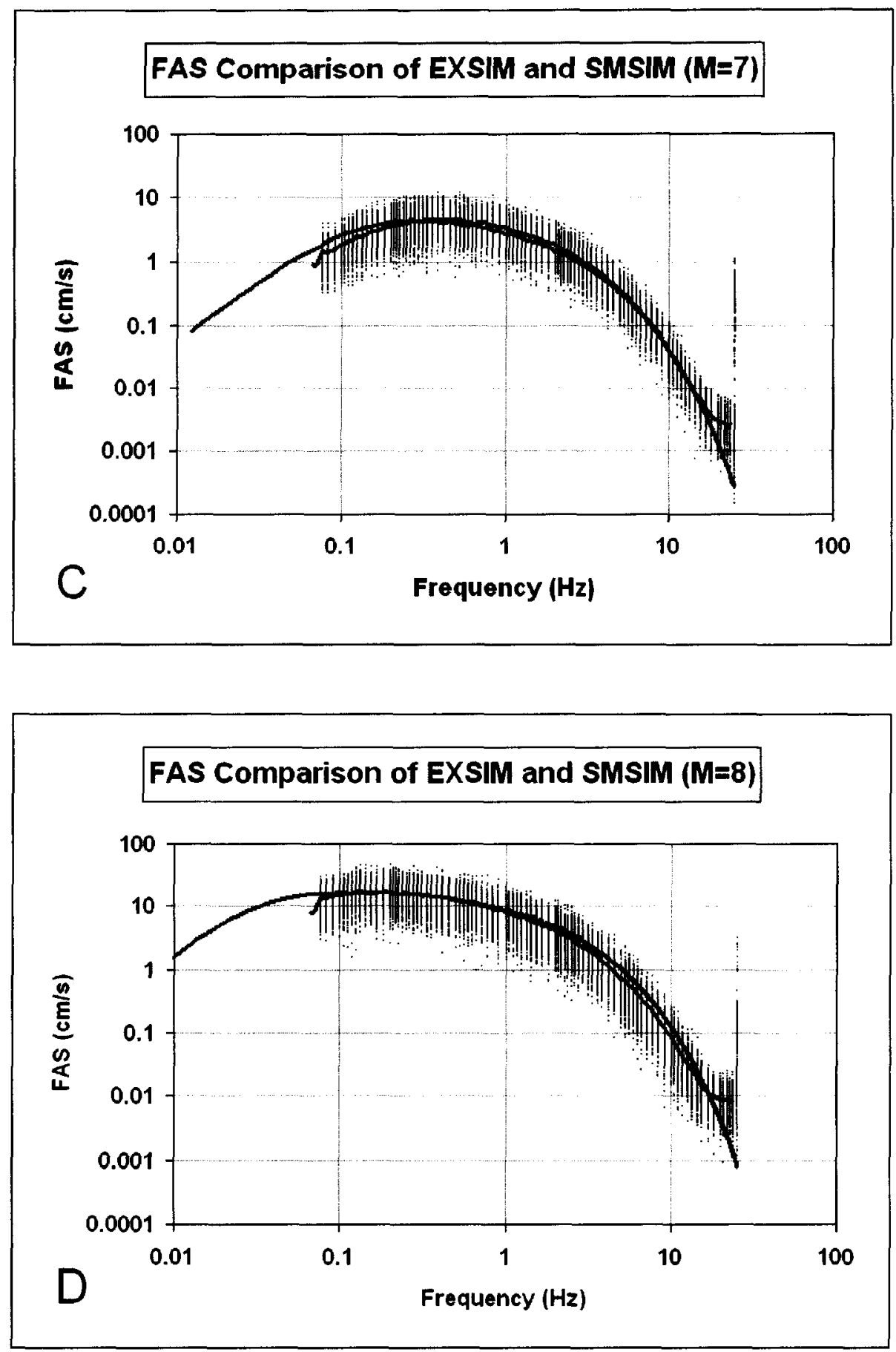

Figure (2.14) Average of Fourier acceleration spectra of 500 simulations for sites located at $300 \mathrm{~km}$ distance from fault and 120 bars of stress parameter for: (C) M7 and (D) M8 earthquakes. The dots are individual EXSIM output spectra, blue line is averages of EXSIM outputs' spectra, and red line is average of SMSIM outputs' spectra. 


\subsubsection{Comparing PSAs of SMSIM and EXSIM}

The average pseudo spectral acceleration of SMSIM and EXSIM simulations resulting from different values of magnitude, at short distance, are compared in the following experiments. The purpose of these experiments is to find out how the PSAs of the two simulation methods compare with each other, and to draw conclusions about the similarity of behavior of average PSAs and average FASs. 500 simulation results of point source modeling, SMSIM, and stochastic finite fault modeling, EXSIM, in four scenarios are used for PSA calculations. In each scenario and simulation method, the average PSA from 500 simulations are calculated. The simulation magnitudes are $\mathbf{M} 5, \mathbf{M} 6, \mathbf{M} 7$, and M8 for events at short distances, $15 \mathrm{~km}$ from point source or trace of a fault; the stress parameter is 120 bars. The remaining simulation parameters are as given in Table (2.5). The comparisons of PSA spectra of SMSIM and EXSIM averages for these magnitudes are shown in Figure (2.15). The red and blue lines are average PSAs of SMSIM and EXSIM respectively; dots are PSA ordinates of individual EXSIM simulations.

These comparisons show that a reasonable match exists between the average predictions of SMSIM and EXSIM for response spectra at low magnitudes, M5 and M6, while there is a considerable difference between the PSA predictions of these two methods at high magnitude, M7 and M8. The point source modeling approach, SMSIM, predicts higher PSA levels than EXSIM, and this difference is proportional to magnitude. This effect becomes more significant as the finite fault effect grows.

Comparisons of average PSAs of EXSIM and SMSIM at low magnitudes show the presence of a gentle double-corner sag at low frequencies in the EXSIM stochastic finite fault simulations.

Comparison of Figure (2.15) and Figure (2.12) demonstrates that the main features seen in Fourier spectra comparisons are projected into the response spectra. This explains the reversibility of application of PSAs and Fourier spectra in the inversion tool, introduced later. 

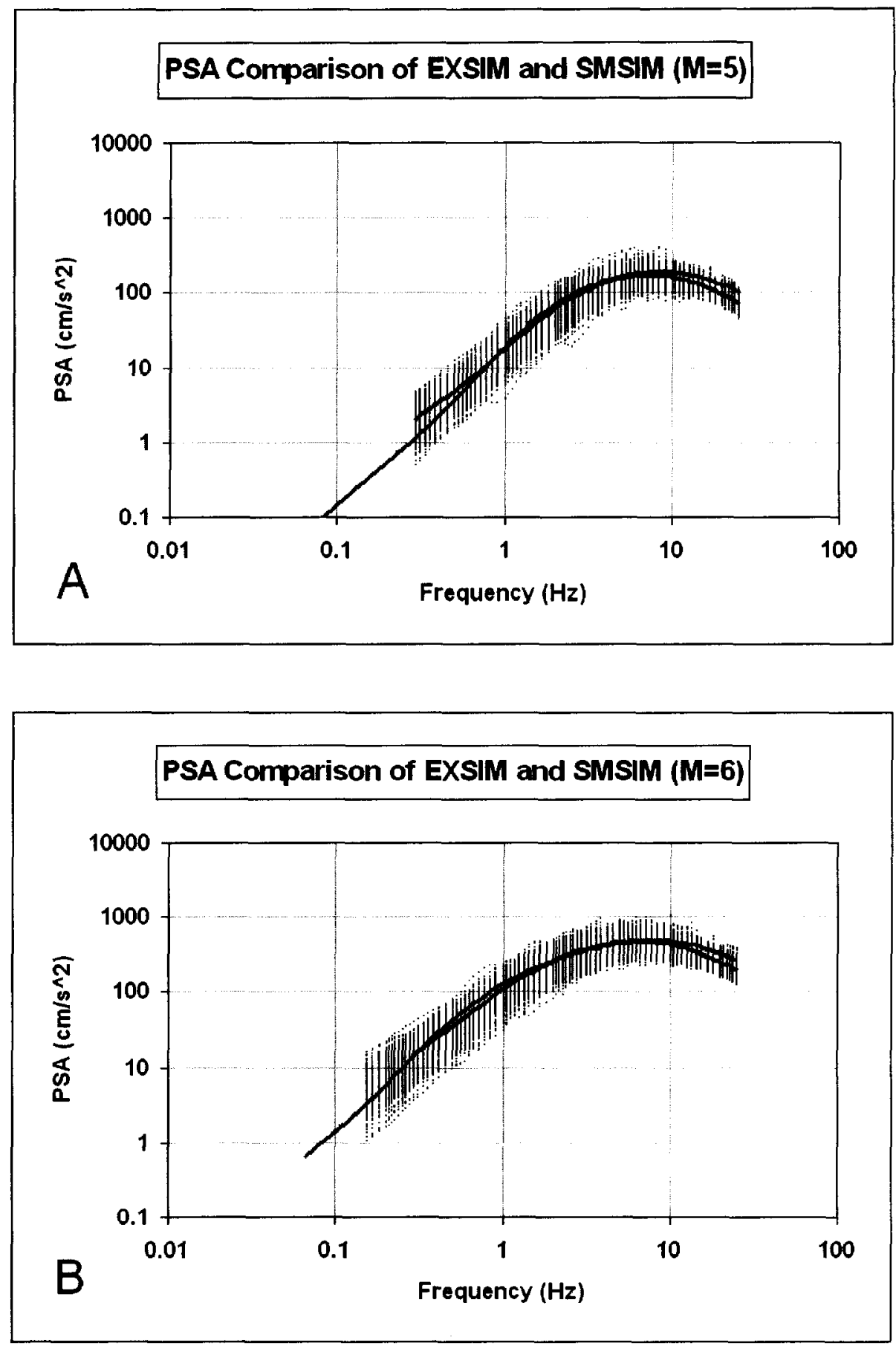

Figure (2.15) Average of pseudo spectral acceleration of 500 simulations for sites located at $15 \mathrm{~km}$ distance from fault and 120 bars of stress parameter for: (A) M5 and (B) M6 earthquakes. The dots are individual EXSIM output spectra, blue line is averages of EXSIM outputs' spectra, and red line is average of SMSIM outputs' spectra. 

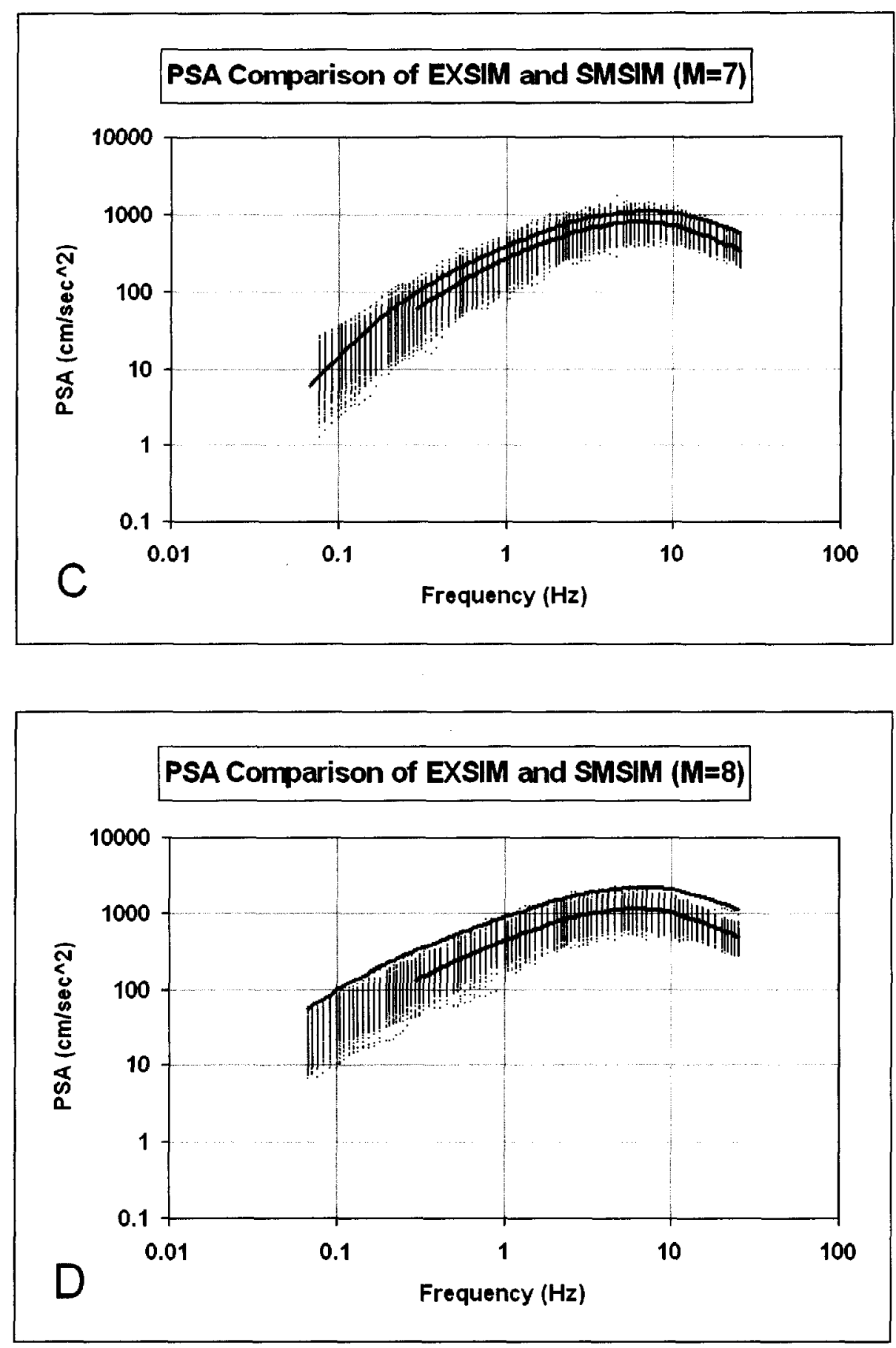

Figure (2.15) Average of pseudo spectral acceleration of 500 simulations for sites located at $15 \mathrm{~km}$ distance from fault and 120 bars of stress parameter for: (C) $\mathbf{M} 7$ and (D) $\mathbf{M} 8$ earthquakes. The dots are individual EXSIM output spectra, blue line is averages of EXSIM outputs' spectra, and red line is average of SMSIM outputs' spectra. 


\subsubsection{Comparing EXSIM with and without dynamic corner frequency}

As the fracture propagates on a fault surface and activates its sub-sources, the dynamic corner frequency approach assigns different value of corner frequency to each subsource, where the assigned corner frequency is a function of the total moment of all active sub-sources. In the stochastic finite fault modeling with dynamic corner frequency (Motazedian and Atkinson, 2005), two constraints control the shape (frequency distribution) and level of sub-source spectra. The first is that the dynamic corner frequency concept forces the method to introduce progressively decreasing corner frequency to sub-sources as the rupture progresses, affecting the frequency distribution. The second is conservation of energy, which is applied to balance the level of the subsources' spectra. Practically, the dynamic corner frequency approach assigns the corner frequency value of each sub-source based on the progressive fault size, or number of active sub-sources, at the time the fracture front reaches that sub-source. EXSIM is designed to allow the user to fix this number equal to unity and consequently disable the dynamic corner frequency function. It is important to differentiate between this mode of EXSIM functionality and FINSIM, since they are not the same even when the dynamic corner frequency aspect is disabled. In this mode, only the conservation of energy constraint is active. All sub-sources are activated based on their own moment and as a result they will have a high ratio of high frequency to low frequency content,; this characteristic will be projected in the overall synthetics' frequency content.

To demonstrate the effect of the dynamic corner frequency, an approach similar to previous spectral comparisons is adopted to compare average Fourier acceleration spectra. Four scenarios and two modes of functionality of EXSIM (with and without dynamic corner frequency) are considered. The scenario earthquakes are M5, M6, M7. and M8 with 50-bar stress at $15 \mathrm{~km}$ distance from the fault. For each case 500 simulations are performed around a vertical dipping fault and all Fourier spectra are averaged. The remaining simulation parameters are given in table (2.5). Figure (2.16) compares average Fourier spectra for the two modes of EXSIM. To make legends and titles of Figure (2.16) 
abbreviated, the ones with and without dynamic corner frequency are called "dynamic" and "static" respectively.

As expected, the version with the disabled dynamic corner frequency (static) predicts higher amplitudes at high frequencies and lower amplitudes at low frequencies compared to the one with dynamic corner frequency (dynamic). This behavior is pronounced in the M5 event because in small earthquakes the sub-sources are smaller and the corner frequency is large enough to fit in the plotted frequency range. In the larger earthquakes the corner frequencies of the sub-sources are small and thus the entire range of interest comes from the high frequency end of the sub-sources. Consequently, the average spectra corresponding to the disabled dynamic corner frequency (static) are higher than the average spectra when the dynamic corner frequency is invoked (dynamic). The dynamic corner frequency concept results in a slight lowering of high frequency spectral amplitudes for a given stress parameter, relative to the static case, because it shifts energy to lower frequencies. 

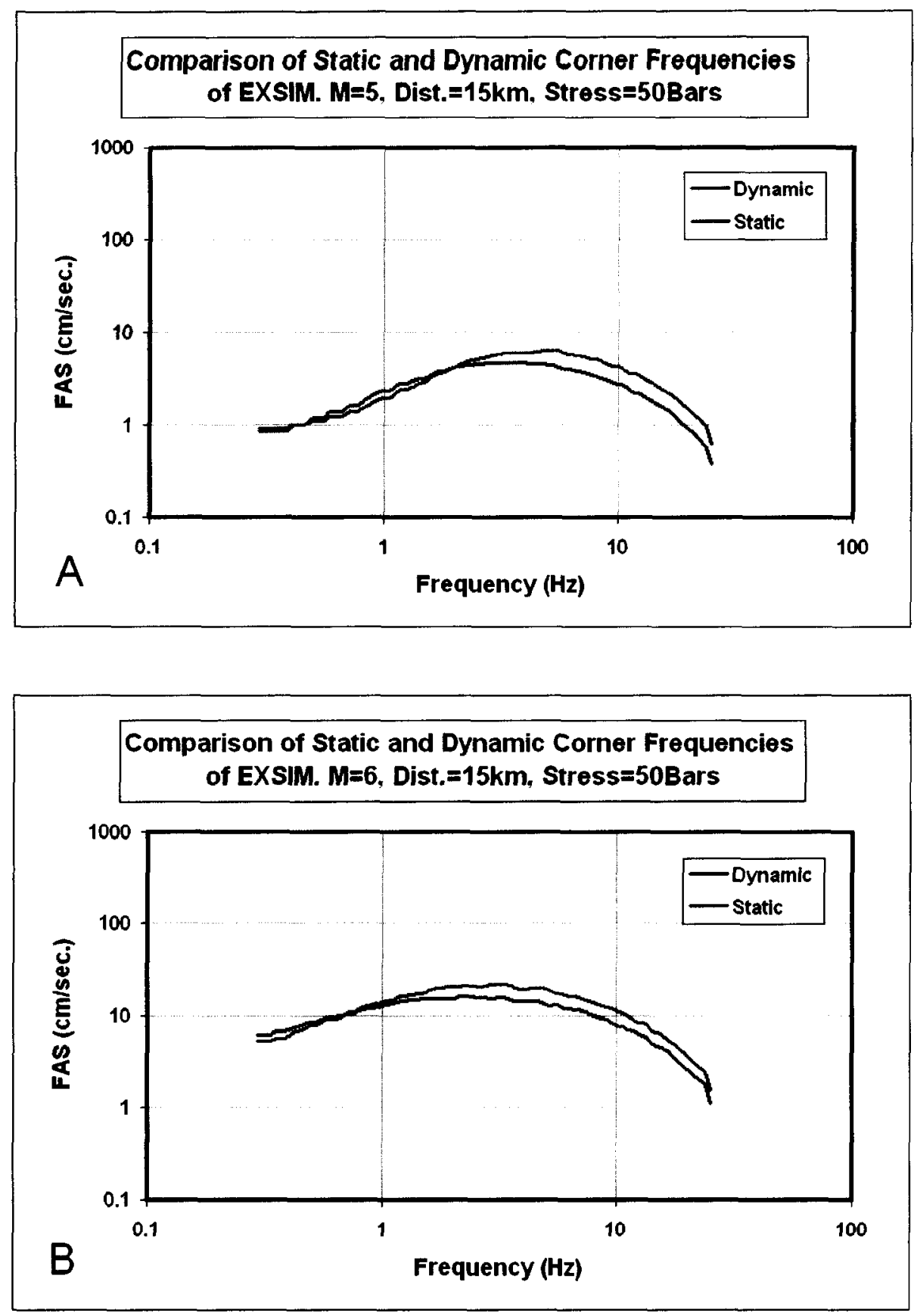

Figure (2.16) Average of Fourier acceleration spectra of 500 simulations for sites located at $15 \mathrm{~km}$ distance from fault and 50 bars of stress parameter for: (A) M5 and (B) M6 earthquakes. Blue line is average of Fourier spectra of EXSIM simulations with dynamic corner frequency and red line is average Fourier spectra of EXSIM simulations without dynamic corner frequency. 

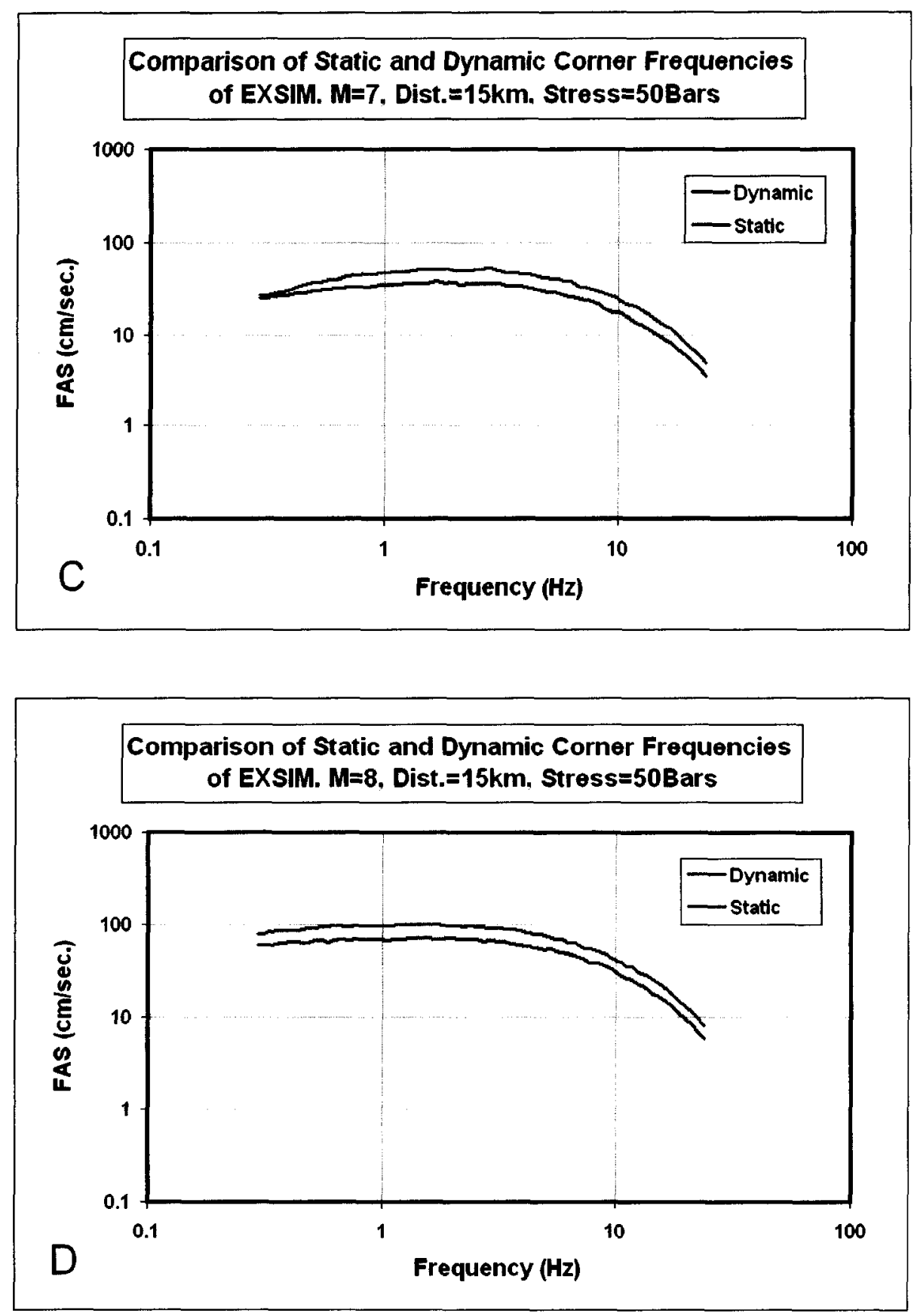

Figure (2.16) Average of Fourier acceleration spectra of 500 simulations for sites located at $15 \mathrm{~km}$ distance from fault and 50 bars of stress parameter for: (C) $\mathbf{M 7}$ and (D) M8 earthquakes. Blue line is average of Fourier spectra of EXSIM simulations with dynamic corner frequency and red line is average Fourier spectra of EXSIM simulations without dynamic corner frequency. 


\subsection{Comparing SMSIM, FINSIM, and EXSIM against data}

\subsubsection{Purpose}

The purpose of the experiments in this section is to compare the performance of the three stochastic waveform-modeling programs: SMSIM (Boore, 2005), FINSIM (Beresnev and Atkinson 1998), and EXSIM (Motazedian and Atkinson 2005) against data. The selected database is the compiled response spectra compiled for the PEER_NGA database (http://peer.berkeley.edu/nga/index.html). As previously noted, the PEER-NGA database consists of corrected strong ground motion records along with corresponding causative earthquakes' and time series/spectral parameters, including pseudo spectral accelerations, or PSAs. The approach taken is to select some reference magnitude and distance values; PSAs of NGA database are scaled to these magnitudes and distances using the Boore and Atkinson (2007) ground motion prediction equations to define scale factors. The scaling (described later) is minimal as data are selected to be close to the reference magnitude and distance. Then strong motion simulations are performed using the above-mentioned

three programs, for the reference magnitudes and distances. The response spectra of simulations are compared to the normalized PSA records of the NGA database, in order to draw conclusions about performance of the programs in different magnitude and distance ranges.

\subsubsection{Database}

The PEER NGA database (http://peer.berkeley.edu/nga/index.html) is used for this study. The records of the catalogue mainly originated from crustal earthquakes that occurred in tectonically active areas. There are 3551 records in the PEER database from 173 earthquakes. Events with fewer than 10 records are omitted from the database for our comparisons. The total number of the considered records is 2681 , from 26 earthquakes. The following database parameters are used in the comparisons: moment magnitude, $\mathrm{R}_{\mathrm{JB}}$ (Joyner-Boore distance, which is the minimum distance to the surface projection of the faulted surface, Abrahamson and Shedlock, 1997), $V_{S 30}$ (preferred average shear wave 
velocity in the upper 30 meters), and response spectral ordinates for 86 periods from $\mathrm{T}=0.04 \mathrm{~s}$ to $\mathrm{T}=10 \mathrm{~s}$, in CGS units, derived from geometrical averaging of horizontal components to obtain an orientation-independent measure of the mean horizontal component.

\subsubsection{Reference magnitudes and distances}

Reference magnitudes are considered to be in 0.2 magnitude steps in the range of M5.8 to M8.0. Four $\mathrm{R}_{\mathrm{JB}}$ reference distances are considered for this study: $1 \mathrm{~km}, 10 \mathrm{~km}, 30 \mathrm{~km}$, and $200 \mathrm{~km}$. These values are chosen as representatives of very close, close, intermediate, and far distances respectively. The magnitude-distance space is divided into a grid of subspaces with M5.8, M6.0, ... at $1 \mathrm{~km}, 10 \mathrm{~km}, 30 \mathrm{~km}$, and $200 \mathrm{~km}$ (Figure 2.17). Equidistant magnitude intervals of $\mathbf{M}=0.1$ from central magnitudes $(\mathbf{M} 5.8, \mathbf{M} 6.0, \ldots)$ are used for classifying event magnitudes. Events with distances less than $3.2 \mathrm{~km}$ are classified in the $1 \mathrm{~km}$ distance group; events with distances between $3.2 \mathrm{~km}$ and $17.3 \mathrm{~km}$ are classified in the $10 \mathrm{~km}$ distance group; events with distances between $17.3 \mathrm{~km}$ and $77.5 \mathrm{~km}$ are classified in the $30 \mathrm{~km}$ distance group; and events with distances more than $77.5 \mathrm{~km}$ are classified in the $200 \mathrm{~km}$ distance group. Figure (2.17) shows the scatter plot of the magnitude-distance distribution of records, as well as the assigned reference magnitudes and distances.

With this approach, all 2681 records are classified in 35 distinct groups, with a reference magnitude and distance value for each group (corresponding to the red squares in the Figure 2.17). Table (2.6) summarizes records that fall into the 35 magnitude-distance subspaces, for each of the 26 earthquakes. 


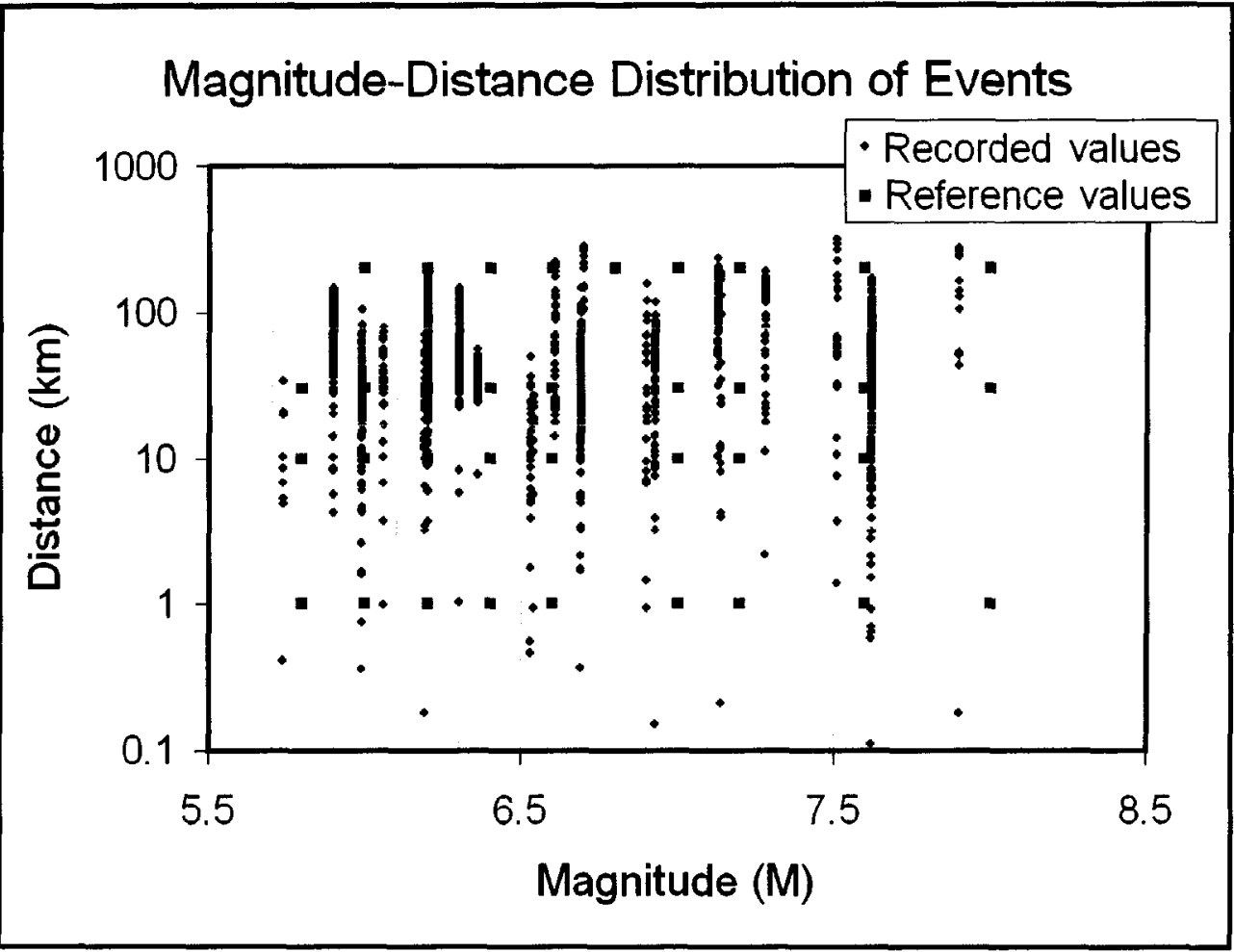

Figure (2.17) Magnitude-distance distribution of events used to evaluate simulation performance. Blue dots: magnitude and distance distribution of used records of NGA database, Red squares: reference magnitude and distance values. If a blue dot falls in a grey rectangle, the magnitude and distance of the red square in that rectangle is assigned to it and the corresponding PSA is corrected to this reference value. 


\subsubsection{Correction of recorded PSAs to reference level:}

After assigning the reference magnitude and distance of each record, the empirical ground-motion prediction equations of Boore and Atkinson (2007) are used to correct the amplitude of each record to the corresponding reference levels. These are minor adjustments because the record magnitude/distances are close to the reference. The BA2007 (Boore and Atkinson 2007) equations were derived by regressing the PSA data of the NGA database against four predictive variables: moment magnitude (M), JoynerBoore distance $\left(\mathrm{R}_{\mathrm{JB}}\right)$, average shear wave velocity of the upper 30 meters of the site $\left(\mathrm{V}_{\mathrm{S} 30}\right)$, and earthquake mechanism. The form of BA2007 equation is:

$$
\ln (\mathrm{Y})=\mathrm{F}_{\mathrm{M}}(\mathbf{M}, \mathrm{Mech})+\mathrm{F}_{\mathrm{D}}\left(\mathrm{R}_{\mathrm{JB}}, \mathbf{M}\right)+\mathrm{F}_{\mathrm{S}}\left(\mathrm{V}_{\mathrm{S} 30}, \mathrm{R}_{\mathrm{JB}}, \mathbf{M}\right)+\varepsilon \sigma_{\mathrm{T}}
$$

where $\mathrm{Y}$ is the ground motion parameter, say PGA or a PSA ordinate, $\mathrm{F}_{\mathrm{M}}$ is the source term, $F_{D}$ is the path term, $F_{S}$ is the site term, and $\varepsilon \sigma_{T}$ is the uncertainty band of the estimation (scatter of observations about the median). Simply put, Equation (2.5) predicts an average $\ln (\mathrm{Y})$ as a sum of the source, path, and site terms $\left(\mathrm{F}_{\mathrm{M}}, \mathrm{F}_{\mathrm{D}}\right.$, and $\left.\mathrm{F}_{\mathrm{S}}\right)$. BA2007 presented coefficients of the above equation for PGV, PGA, and 21 periods of PSA.

To correct the recorded PSA values for each record in the NGA database to the corresponding reference levels, the values of correction factors for each period are estimated using the following approach. I am looking for a value for factor " $\mathrm{A}$ ", that when multiplied by the recorded PSA ordinate $\left(\mathrm{Y}_{\text {rec }}\right)$ will generate the corresponding PSA ordinate value for the reference conditions $\left(\mathrm{Y}_{\mathrm{rcf}}\right)$.

$$
Y_{\text {ref }}(T)=A\left(M_{\text {rec }}, M_{\text {ref }}, R_{\text {JB, rec }}, R_{\mathrm{JB}, \text { ref }}, V_{S 30, \text { rec }}, V_{\text {S30,ref }}, M e c h, T\right) \times Y_{\text {rec }}(T)
$$

The value of "A" can be calculated as:

$$
\mathrm{A}\left(\mathbf{M}_{\mathrm{rec}}, \mathbf{M}_{\mathrm{ref}}, \mathrm{R}_{\mathrm{JB}, \mathrm{rec}}, \mathrm{R}_{\mathrm{JB}, \mathrm{re}}, \mathrm{V}_{\mathrm{S} 30, \mathrm{rec}}, \mathrm{V}_{\mathrm{S} 30, \mathrm{ref}}, \mathrm{Mech}, \mathrm{T}\right)=\mathrm{Y}_{\mathrm{rel}}(\mathrm{T}) / \mathrm{Y}_{\mathrm{rec}}(\mathrm{T})
$$

If we assume that the ratio of $\mathrm{Y}_{\text {ref }}(\mathrm{T})$ and $\mathrm{Y}_{\text {rec }}(\mathrm{T})$ can be predicted properly by Equation (2.5), the Equation (2.7) can be rewritten in the following form:

$$
\mathrm{A}(\ldots, \mathrm{T})=\mathrm{Y}_{\text {ref }}\left(\mathbf{M}_{\mathrm{ref}}, \mathrm{R}_{\mathrm{JB}, \mathrm{ref}}, \mathrm{V}_{\mathrm{S} 30, \mathrm{ref}}, \mathrm{Mech}, \mathrm{T}\right) / \mathrm{Y}_{\mathrm{rec}}\left(\mathbf{M}_{\mathrm{rec}}, \mathrm{R}_{\mathrm{IB}, \mathrm{rec}}, \mathrm{V}_{\mathrm{S} 30, \mathrm{rec}}, \mathrm{Mech}, \mathrm{T}\right)
$$


or

$$
\ln (A(\ldots, T))=F_{M, \text { ref }}(\ldots, T)+F_{D, \text { ref }}(\ldots, T)+F_{S, \text { ref }}(\ldots, T)-F_{M, \text { rec }}(\ldots, T)-F_{D, \text { rec }}(\ldots, T)-F_{S, r e c}(\ldots, T)
$$

Since the available constants of Equation (2.5) are given for just 21 periods, "A" values for desired intermediate periods are calculated by linear interpolation in $\ln (A)-\ln (T)$ space.

This procedure is applied to all of the ordinates of the 2681 records for our evaluation study. Then all $\ln \left(\mathrm{Y}_{\text {ref }}\right)$ values of an earthquake that fall into one distance group are averaged. So for each earthquake there are between 1 to 4 average PSAs, each corresponding to a reference distance. The number of average PSAs is 83 (Table 2.6), where each is the average of corrected records of a single earthquake to the reference distance and magnitude.

\subsubsection{SMSIM, FINSIM, and EXSIM simulations:}

There are 26 events in the NGA database that are used for evaluation study. The distribution of hypocentral depth and fault-upper-corner depth of these events are plotted as a function of earthquake magnitude in Figure (2.18), along with the best-fit lines to model this distribution. The depths predicted by the best lines are used to assign the corresponding depths in the simulations.

To obtain stable average PSA ordinates as predicted by each modeling approach (SMSIM, FINSIM, EXSIM), 500 simulations are performed at each reference magnitude and distance and their response spectra are averaged (simply or geometrically). Table (2.7) shows common simulation parameters applied to all three simulation approaches.

Table (2.8) shows all 35 available magnitude-distance scenarios that emerged from grouping the useful records of the NGA database (red squares in the Figure 2.17). 
Table (2.6) Information of extracted subset of NGA database used in this study including the number of records in each magnitude-distance range.

\begin{tabular}{|c|c|c|c|c|c|}
\hline Eq. ID & Earthhquake & $\begin{array}{l}\text { Reference } \\
\text { magnitude }\end{array}$ & $\begin{array}{l}\text { No. of used } \\
\text { records }\end{array}$ & $\begin{array}{c}\text { Reference } \\
\text { distance }\end{array}$ & $\begin{array}{l}\text { No. in reference } \\
\text { distance }\end{array}$ \\
\hline \multirow{4}{*}{0030} & \multirow{4}{*}{ San Fernando } & \multirow{4}{*}{6.6} & \multirow{4}{*}{44} & 1 & 1 \\
\hline & & & & 10 & 1 \\
\hline & & & & 30 & 24 \\
\hline & & & & 200 & 18 \\
\hline & \multirow{3}{*}{ Coyote Lake } & \multirow{3}{*}{5.8} & \multirow{3}{*}{10} & 1 & 1 \\
\hline \multirow[t]{3}{*}{0048} & & & & 10 & 5 \\
\hline & & & & 30 & 4 \\
\hline & \multirow{3}{*}{$\begin{array}{c}\text { Imperial } \\
\text { Valley-06 }\end{array}$} & \multirow{3}{*}{6.6} & \multirow{3}{*}{33} & 1 & 7 \\
\hline \multirow[t]{2}{*}{0050} & & & & 10 & 16 \\
\hline & & & & 30 & 10 \\
\hline \multirow{2}{*}{0068} & \multirow{2}{*}{ Irpinia, Italy-01 } & \multirow{2}{*}{7} & \multirow{2}{*}{12} & 10 & 4 \\
\hline & & & & 30 & 8 \\
\hline 0069 & Irpinia, Italy-02 & 6.2 & 10 & 10 & 2 \\
\hline & & & & 30 & 8 \\
\hline 0076 & Coalinga-01 & 6.4 & 46 & 10 & 2 \\
\hline & & & & $\begin{array}{c}30 \\
1\end{array}$ & 44 \\
\hline 0090 & Morgan Hill & 6.2 & 28 & 10 & $\begin{array}{l}1 \\
9\end{array}$ \\
\hline & & & & 30 & 9 \\
\hline & & & & 30 & 18 \\
\hline & & & & 10 & 3 \\
\hline 0101 & N. Palm Springs & 6 & 32 & 10 & 5 \\
\hline & & & & 30 & 23 \\
\hline 0103 & Chalfant & & & 200 & 1 \\
\hline 0103 & Valley-02 & 6.2 & 11 & 10 & 3 \\
\hline & & & & 30 & 8 \\
\hline & & & & & 6 \\
\hline 0113 & Whittier Narrows-01 & 6 & 116 & 10 & 35 \\
\hline & & & & 30 & 73 \\
\hline & & & & 200 & 2 \\
\hline 0116 & Superstition Hills-02 & 66 & 11 & & \\
\hline & & & & 10 & 3 \\
\hline & & & & 30 & 7 \\
\hline 0118 & & & & 10 & 15 \\
\hline 0118 & Loma Prieta & 7 & 83 & 30 & 56 \\
\hline & & & & 200 & 10 \\
\hline & & & & 1 & 1 \\
\hline & & & & 10 & 1 \\
\hline 0125 & Landers & 7.2 & 69 & 30 & 14 \\
\hline & & & & 200 & 53 \\
\hline & & & & 1 & 14 \\
\hline 0127 & Northridge-01 & 6.6 & 160 & 10 & 27 \\
\hline & & & & 30 & 102 \\
\hline & & & & 200 & 17 \\
\hline & & & & 1 & 3 \\
\hline 0129 & Kobe Japan & 7 & 12 & 10 & 1 \\
\hline & & & 12 & 30 & 4 \\
\hline & & & & 200 & 4 \\
\hline & & & & 1 & 1 \\
\hline 0136 & Kocaeli Turkey & 7.6 & 27 & 10 & 4 \\
\hline & Rocalli, lükey & & 27 & 30 & 13 \\
\hline & & & & 200 & 9 \\
\hline & & & & 1 & 20 \\
\hline 0137 & Chi-Chi Taiwan & 76 & 420 & 10 & 41 \\
\hline 0131 & Cni-chi, I alwan & 1.6 & 420 & 30 & 192 \\
\hline & & & & 200 & 167 \\
\hline & & & & 1 & 2 \\
\hline 0138 & Duzce Turkey & 7.2 & 22 & 10 & 6 \\
\hline 0130 & Duzce, furkey & 1.2 & $\angle 2$ & 30 & 4 \\
\hline & & & & 200 & 10 \\
\hline
\end{tabular}


Continuation of Table (2.6) Information of extracted subset of NGA database used in this study including the number of records in magnitude-distance ranges.

\begin{tabular}{|c|c|c|c|c|c|}
\hline Eq. ID & Earthhquake & $\begin{array}{l}\text { Reference } \\
\text { magnitude }\end{array}$ & $\begin{array}{l}\text { No. of used } \\
\text { records }\end{array}$ & $\begin{array}{c}\text { Reference } \\
\text { distance }\end{array}$ & $\begin{array}{l}\text { No. in reference } \\
\text { distance }\end{array}$ \\
\hline \multirow{3}{*}{0158} & \multirow{3}{*}{ Hector Mine } & \multirow{3}{*}{7.2} & \multirow{3}{*}{83} & 10 & 1 \\
\hline & & & & 30 & 19 \\
\hline & & & & 200 & 63 \\
\hline \multirow[t]{2}{*}{0168} & Nenana Mountain, & \multirow[t]{2}{*}{6.8} & \multirow[t]{2}{*}{36} & 200 & 36 \\
\hline & \multirow{3}{*}{ Denali, Alaska } & & & 1 & 1 \\
\hline \multirow[t]{2}{*}{0169} & & \multirow[t]{2}{*}{8} & \multirow[t]{2}{*}{24} & 30 & 3 \\
\hline & & & & 200 & 20 \\
\hline \multirow{3}{*}{0171} & \multirow{3}{*}{ Chi-Chi, Taiwan-02 } & \multirow{3}{*}{6} & \multirow{3}{*}{296} & 10 & 6 \\
\hline & & & & 30 & 139 \\
\hline & & & & 200 & 151 \\
\hline \multirow{4}{*}{0172} & \multirow{4}{*}{ Chi-Chi, Taiwan-03 } & \multirow{4}{*}{6.2} & \multirow{4}{*}{242} & 1 & 2 \\
\hline & & & & 10 & 6 \\
\hline & & & & 30 & 139 \\
\hline & & & & 200 & 95 \\
\hline \multirow{3}{*}{0173} & \multirow{3}{*}{ Chi-Chi, Taiwan-04 } & \multirow{3}{*}{6.2} & \multirow{3}{*}{242} & 10 & 2 \\
\hline & & & & 30 & 135 \\
\hline & & & & 200 & 105 \\
\hline \multirow[t]{3}{*}{0174} & \multirow[t]{3}{*}{ Chi-Chi, Taiwan-05 } & \multirow{3}{*}{6.2} & \multirow{3}{*}{322} & 30 & 126 \\
\hline & & & & 200 & 196 \\
\hline & & & & 1 & 2 \\
\hline \multirow{3}{*}{0175} & \multirow{3}{*}{ Chi-Chi, Taiwan-06 } & \multirow{3}{*}{6.4} & \multirow{3}{*}{290} & 10 & 2 \\
\hline & & & & 30 & 147 \\
\hline & & & & 200 & 139 \\
\hline
\end{tabular}

Table (2.7). Common parameter values used in the three simulation approaches

\begin{tabular}{cc}
\hline Parameter & Parameter value \\
\hline Number of stations & 500 \\
Sample interval & $0.02 \mathrm{~s}$ \\
Shear wave velocity, Density & $3.5 \mathrm{~km} / \mathrm{s}, 2.8 \mathrm{~g} / \mathrm{cm}^{3}$ \\
Rupture velocity & $0.8 \times$ shear wave velocity \\
$\mathrm{Q}(\mathrm{f})$ & $180 \times \mathrm{f}^{0.45}$ \\
Moment magnitude & Scenario dependent \\
Kappa & 0.035 \\
Geometrical attenuation & If $\mathrm{R}<40, \mathrm{R}^{-1}$, else $\mathrm{R}^{-0.5}$ \\
Sub-fault (or point source) & $\propto 0.05 \times$ distance $(\mathrm{sec}$.) \\
Windowing function & Saragoni-Hart \\
Amplification function & Boore and Joyner (1997) for \\
Damping & $5 \%$ of critical damping \\
\hline
\end{tabular}


For the point-source modeling approach (SMSIM), the $\mathrm{R}_{\mathrm{JB}}$ distance is combined with the expected hypocentral depth as predicted by the line fitted to the hypocentral depth distribution to predict hypocentral distance:

$$
R=\sqrt{R_{. / H}^{2}+h_{h y p}^{2}}
$$

where $\mathrm{R}_{\mathrm{JB}}$ is Joyner-Boore distance, $h_{h y p}$ is predicted by the best fit line on Figure (2.18), and $R$ is the distance used in SMSIM simulations. 500 simulations are performed for each of 35 scenarios of Table (2.8) and the resulting PSAs are averaged and prepared for comparisons with the corresponding database PSAs of Table (2.6).

For the two finite source approaches, additional factors need to be considered: a) JoynerBoore distance is defined as the closest distance from a site to the surface projection of the fault rupture (Abrahamson and Shedlock, 1997); b) the predictions will vary with fault geometry and azimuth. To obtain stable average predictions for a specified $M-R_{I B}$, a vertical fault with a specified upper corner depth is modeled in each case. The fault upper corner depths are taken from the best-fit line on Figure (2.18). For each of the 35 scenarios of Table (2.8), 500 sites spaced at equal Joyner-Boore distances from the surface rupture trace of the fault in all directions are defined (e.g., Figure 2.7). The predicted PSAs of each scenario are averaged for comparisons with the observed database PSAs.

The length and width of the faults are calculated for each magnitude using the Wells and Coppersmith (1994) relations (Equations 2.4 and 2.4). For FINSIM simulations, Equation (1.16) from Beresnev and Atkinson (2001) is used to define sub-source size (log $\Delta \mathrm{l}=0.4 \times \mathbf{M}-2$ ). The optimum values for sub-divisions along the length and width of the fault are chosen, so that sub-source length and width compare well with $\Delta \mathrm{l}$, and the number of sub-divisions is close to the ratio of fault area divided by the square of $\Delta \mathrm{l}$ (subsource area). 
Table (2.8). Reference magnitude-distance combinations of performed simulations.

\begin{tabular}{ccc}
\hline No & Magnitude $(\mathbf{M})$ & Distance $\left(\mathrm{R}_{\mathrm{JB}}\right)$ \\
\hline 1 & 5.8 & 1 \\
2 & 5.8 & 10 \\
3 & 5.8 & 30 \\
4 & 6.0 & 1 \\
5 & 6.0 & 10 \\
6 & 6.0 & 30 \\
7 & 6.0 & 200 \\
8 & 6.2 & 1 \\
9 & 6.2 & 10 \\
10 & 6.2 & 30 \\
11 & 6.2 & 200 \\
12 & 6.4 & 1 \\
13 & 6.4 & 10 \\
14 & 6.4 & 30 \\
15 & 6.4 & 200 \\
16 & 6.6 & 1 \\
17 & 6.6 & 10 \\
18 & 6.6 & 30 \\
\hline
\end{tabular}

\begin{tabular}{ccc}
\hline No & Magnitude $(\mathbf{M})$ & Distance $\left(\mathrm{R}_{\mathrm{JB}}\right)$ \\
\hline 19 & 6.6 & 200 \\
20 & 6.8 & 200 \\
21 & 7.0 & 1 \\
22 & 7.0 & 10 \\
23 & 7.0 & 30 \\
24 & 7.0 & 200 \\
25 & 7.2 & 1 \\
26 & 7.2 & 10 \\
27 & 7.2 & 30 \\
28 & 7.2 & 200 \\
29 & 7.6 & 1 \\
30 & 7.6 & 10 \\
31 & 7.6 & 30 \\
32 & 7.6 & 200 \\
33 & 8.0 & 1 \\
34 & 8.0 & 30 \\
35 & 8.0 & 200 \\
- & - & - \\
\hline
\end{tabular}

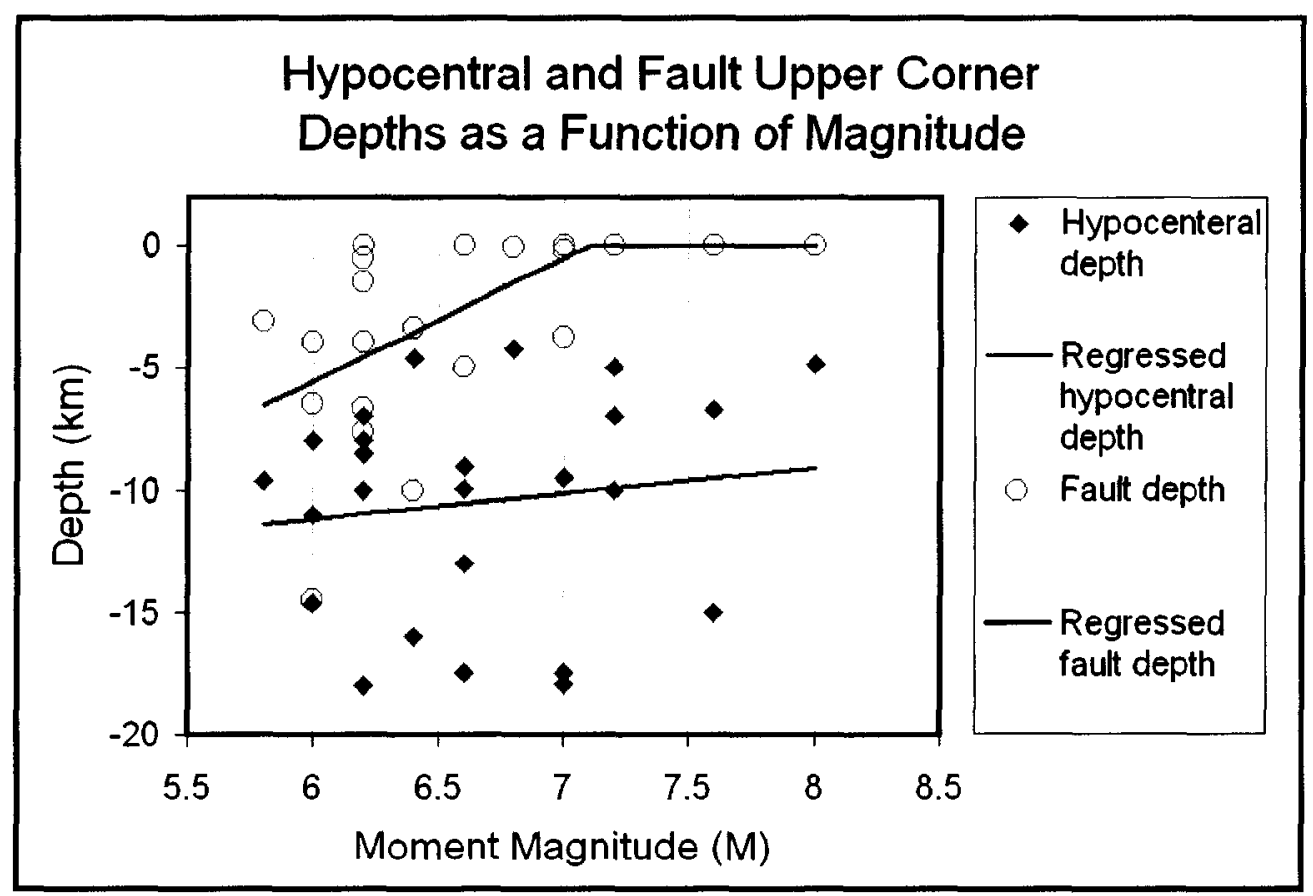

Figure (2.18) Distribution of hypocentral and fault upper corner depths of 26 events used in this study as well as their corresponding regressed lines. The regressed hypocentral and fault upper corner depths are used in point source and finite source simulations. 
In both finite source simulation methods, FINSIM and EXSIM, a random slip distribution is selected and the location of the hypocenter is modeled to be close to the center of the fault, in the lower half of the rupture area.

\subsubsection{Simulation results}

Figures (2.19) to (2.24) compare averages of corrected response spectra of 6 wellrecorded earthquakes with simulation PSAs: M6.2, 1999 Chi Chi earthquake aftershock; M6.6, 1994 Northridge earthquake; M7.0, 1989 Loma Prieta earthquake; M7.2, 1992 Landers earthquake; M7.6, 1999 Chi Chi earthquake; and M8.0, 2002 Denali earthquake. All three methods predict the average spectral shapes of the recorded ground motions reasonably well. The predictions seem to be better at short and intermediate distances, $10 \mathrm{~km}$ and $30 \mathrm{~km}$, than at very short or very large distances. There is significant disagreement between simulation PSAs and observed PSAs at large distances, which is suggestive of an inappropriate choice of the geometrical spreading function in the simulations. These results are shown in more detail by plotting the residuals of simulations for three earthquakes (where the residual is defined as log (observed PSA) $\log ($ predicted PSA)): M6.6, 1994 Northridge; M7.0, 1989 Loma Prieta; M7.2, 1992 Landers. These plots are provided in Appendix A). When the Chi Chi earthquake or its aftershocks are compared to simulation results (Figures 2.19 and 2.23), there is an excellent match between simulation and recorded PSAs. This can be explained by knowing that the attenuation in the Boore and Atkinson (2007) ground motion prediction equations was strongly influenced by the M7.6, 1999 Chi Chi earthquake data.

\subsubsection{Error measure}

This section evaluates the differences between observations and simulation predictions (model error) quantitatively. To quantify the difference between the PSA of a simulation and a record the Equation (2.11) is implemented: 


$$
\varepsilon\left(N_{1}, N_{2}\right)=\frac{1}{\left(N_{2}-N_{1}+1\right)} \sum_{i=N_{1}}^{N_{2}}\left|\log P S A\left(O_{i}\right)-\log P S A\left(S_{i}\right)\right|
$$

where "log PSA $\left(\mathrm{O}_{\mathrm{i}}\right)$ " is the $\log _{10}$ of $\mathrm{i}^{\text {th }}$ ordinate of PSA of record (processed database), and similarly " $\log \operatorname{PSA}\left(\mathrm{S}_{\mathrm{i}}\right)$ " is the $\log _{10}$ of $\mathrm{i}^{\text {th }}$ ordinate of PSA of simulation, $N_{l}$ and $N_{2}$ are ordinate numbers of PSAs for which the comparison is performed, and $\varepsilon$ is the measure of error. Each ordinate number corresponds to a specific frequency in the $0.1 \mathrm{~Hz}$ to $25 \mathrm{~Hz}$ range.

\subsubsection{Error measurement results}

The error measure " $\varepsilon$-value" of Equation (2.11) is used to evaluate the simulation methods. The error values averaged over the $1 \mathrm{~Hz}-10 \mathrm{~Hz}$ frequency range are given in Table (2.9) for each simulation approach. Also, "E-values" calculated for three frequencies: $3.333 \mathrm{~Hz}(0.3 \mathrm{sec}.) ; 1 \mathrm{~Hz}(1 \mathrm{sec}$.$) ; and 0.333 \mathrm{~Hz}(3 \mathrm{sec}$.) are given in Table $(2.10)$.

Averages and scatter of the errors presented in Table (2.9) are given in Figure (2.25). Overall, the EXSIM program has the best performance in $1 \mathrm{~Hz}-10 \mathrm{~Hz}$ frequency range, if the scatter and average of error measure " $\varepsilon$-value" is considered.

The information in Tables (2.9) and (2.10) is summarized in Figures (2.26) to (2.29) for each frequency range separately. Different " $\varepsilon$-value" subsets are averaged for each of simulation methods to make comparisons. The number of available records for calculation of " $\varepsilon$-value", distance, and magnitude are the criteria for choosing the subsets. Comparing averages of " $\varepsilon$-values" in selected sub-sets provides an overview of the performance of the simulation programs in different magnitude and distance ranges. Average errors are very uncertain when the number of records is small; I therefore consider " $\varepsilon$-values" averages when the "number of used records" is 3 or more. There are 62 " $\varepsilon$-values" in each frequency range and simulation method that meet this condition (of 83 total). Average " $\varepsilon$-values" in each of four distance ranges are also calculated. The number of " $\varepsilon$-values" for averaging in $1 \mathrm{~km}, 10 \mathrm{~km}, 30 \mathrm{~km}$, and $200 \mathrm{~km}$ distances are 17 . 
23,25 , and 18 respectively. Set "A" graphs of Figures (2.26) to (2.29) compare average " $\varepsilon$-values" of these six sub-sets $(83,62,17,23,25,18)$ for three simulation methods and four frequencies. Set "B" graphs of Figures (2.26) to (2.29) compare average " $\varepsilon$-values" in magnitude bins for three simulation methods and four frequencies. The information for plotting Figures (2.26) to (2.29) are those of Table (2.9) for $1 \mathrm{~Hz}-10 \mathrm{~Hz}$ frequency range and Table (2.10) for $0.333 \mathrm{~Hz}, 1 \mathrm{~Hz}$, and $3.333 \mathrm{~Hz}$ frequencies.

The averages of errors ( $\varepsilon$-values) over all 83 values, and the subset of 62 records with more than 3 observations, are lower for EXSIM at all distances and frequencies than for the other two programs, demonstrating better performance of EXSIM in all tested frequencies. When performance at different magnitudes is considered, again EXSIM shows better performance on average, although at large magnitudes and frequencies greater than $1 \mathrm{~Hz}$, FINSIM shows better performance than the other methods.

Comparisons in Figures (2.26) to (2.29) show the performance of methods under various magnitude and distance ranges. Figures (2.26) to (2.29) may be used as a guide to judge the preferred simulation method for a given scenario (magnitude-distance). An important issue is whether the observed differences between average " $\varepsilon$-values" of the different simulation methods are the result of random variability, rather than applicability of a specific simulation method. Most statistically strong tests are parametric and rely on parameters of the data with an assumption about its distribution. For example, analysis of variance can be implemented to find out to a degree of certainty if columns of a table belong to statistically different populations, but such a procedure needs valid assumption about normality of distribution of the data in columns of the table. Since the " $\varepsilon$-values" in the columns of the tables do not follow a normal, or any specific distribution, it is better to use a kind of non-parametric test that does not make any assumption for the distribution of the data. In this study, for comparison of two columns of "$\varepsilon$-values" I chose the Kolmogorov-Smirnov test (D'Agostino and Stephens, 1986; Kolmogorov, 1950; Kolmogorov and Shiriayev, 1992) because this test is robust and powerful. It is robust because it performs well under a wide range of distributional assumptions and it is 
powerful because it has the ability to detect a difference when that difference actually exists (NIST/SEMATECH, 2006).

In this study, four frequency ranges are studied separately: $0.333 \mathrm{~Hz}, 1 \mathrm{~Hz}, 3.333 \mathrm{~Hz}$, and $1 \mathrm{~Hz}-10 \mathrm{~Hz}$ ranges. For each frequency range, six subsets of " $\varepsilon$-value" data resulted from the three simulation methods. The six subsets are: all $\varepsilon$-values, " $\varepsilon$-values" determined by at least 3 records, " $\varepsilon$-values" determined with records in $1 \mathrm{~km}, 10 \mathrm{~km}, 30 \mathrm{~km}$, and $200 \mathrm{~km}$ distance classes. For each frequency range and subset, " $\varepsilon$-values" from SMSIM, FINSIM, and EXSIM methods are mutually compared; a hypothesis test of the distribution similarity of the parent sets (that we believe " $\varepsilon$-value" columns are subsets of parent sets) is performed. The result of the test is 1 if the hypothesis that the parent distributions are identical is rejected; it is 0 if this hypothesis cannot be rejected. The hypothesis is rejected if the test is significant at the $5 \%$ level. Table (2.11) summarizes the Kolmogorov-Smirnov test results. When the results of Table (2.11) are considered, combined with Figures (2.26) to (2.29), the conclusions about the preference of one simulation method to the other for a scenario will be reliable. Goodness of fit tests, like Kolmogorov-Smirnov, are not performed for " $\varepsilon$-values" of magnitude bins because the number of elements in each magnitude bin is too small to represent the parent set distribution.

Table (2.11) shows that differences between simulation methods are recognizable mainly when 83 or 62 records for averaging " $\varepsilon$-values" are used. Except at low frequencies $(0.333 \mathrm{~Hz})$, the EXSIM " $\varepsilon$-value" distribution seems to be different from the " $\varepsilon$-value" distributions of FINSIM and SMSIM. Figures (2.26) to (2.29) show that in general EXSIM average " $\varepsilon$-values" are lower than those of FINSIM and SMSIM. Consequently it is reasonable to accept that EXSIM has better performance than the other two programs at high frequencies. At low frequency $(0.333 \mathrm{~Hz})$ the FINSIM average " $\varepsilon$-values" are higher, and significantly different from those of other two programs. This suggests that FINSIM is not a good choice when low frequency components are required. 
An alternative way to compare simulations against real data is implementation of residuals. Appendix A provides residual comparison plots for three implemented simulation methods in this thesis. Since the residuals, like " $\varepsilon$-values", are calculated for different magnitudes, distances, and frequencies, a number of plots are generated to compare residuals of simulation methods as a function of magnitude and frequency for different distances and earthquakes. Comparisons of residual plots further confirm the results obtained from studies of " $\varepsilon$-values".

\subsubsection{Discussion and conclusion on three program comparisons}

The average error measures ( $\varepsilon$-values) of three simulation techniques and database records are plotted in different reference magnitudes and distances. The finite source modeling technique, EXSIM, performs reasonably well in most magnitudes, distances, and frequencies. When the magnitude is large, FINSIM generates reasonable simulations although this result is not confirmed statistically because it comes from just one or two earthquakes.

Table (2.12) can be used to guide the selection of appropriate simulation method. Table (2.12) shows that it is not clear which method works better than the others at low frequencies; this may be because all three methods are reasonably fine for simulation of high frequencies, and weak at low frequencies. From the residual analysis in Appendix A. Table (A.1) shows the portions of magnitude/distance/frequency space where each simulation method results in the minimum residual. Table (A.1) is very similar to Table (2.12), and suggests the same conclusions. Overall, EXSIM provides the best model performance. 

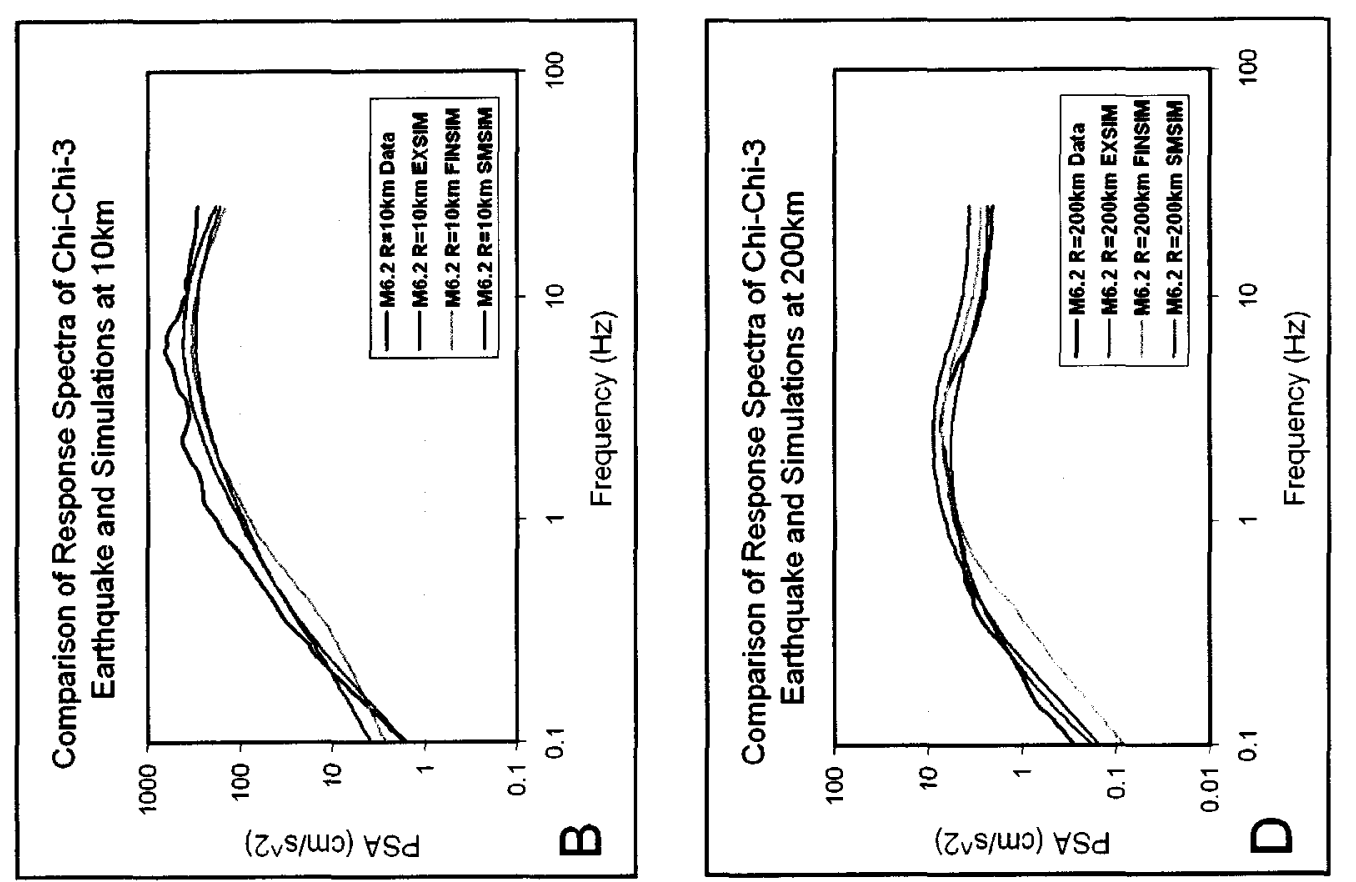

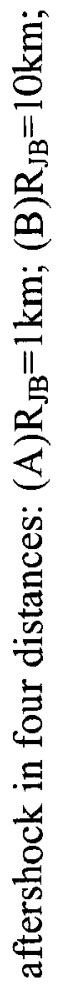

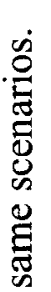

䔅

छ
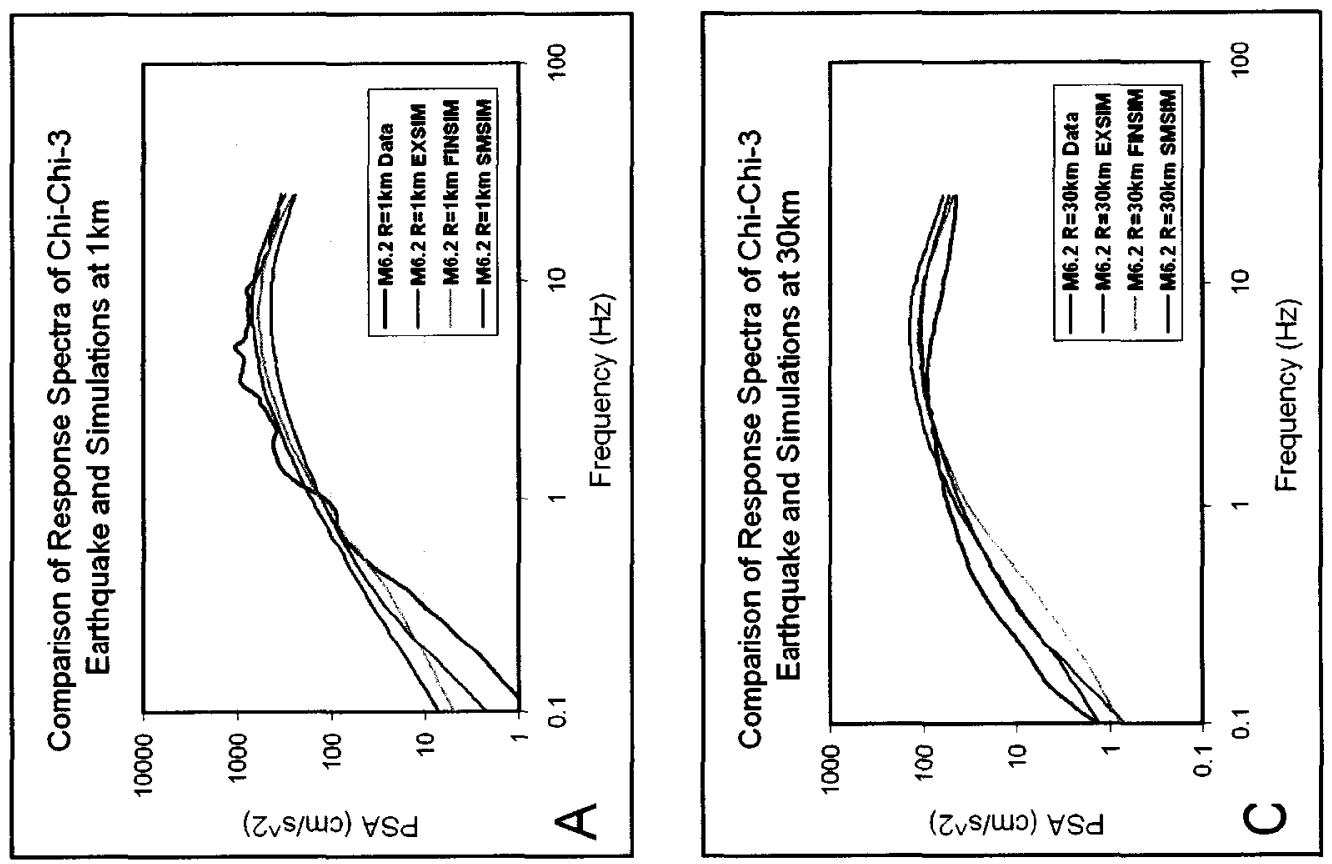

合

离

चु

苍

충

范

品

을

䧽 

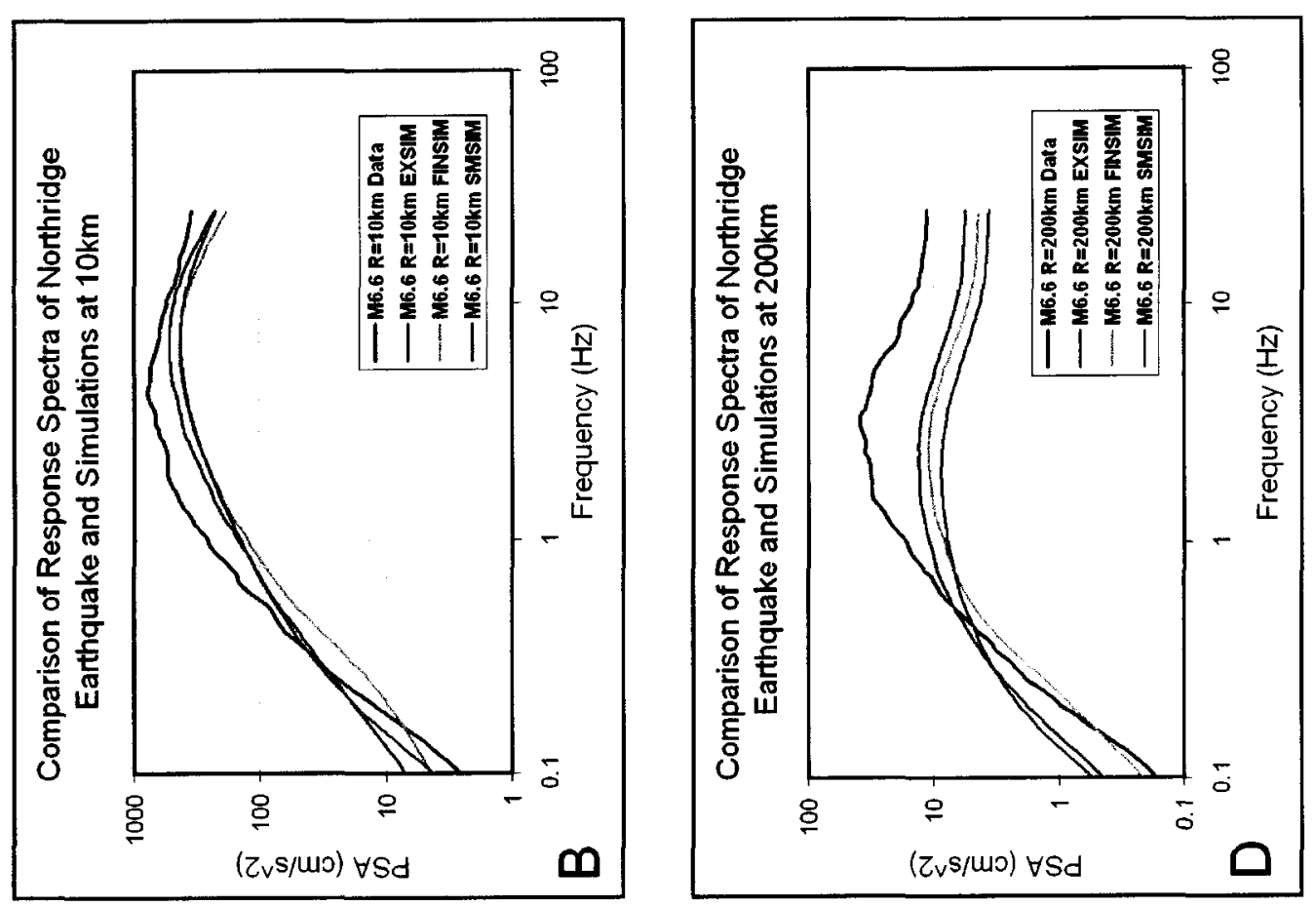

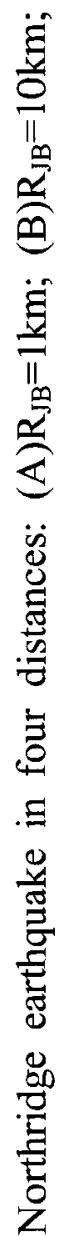
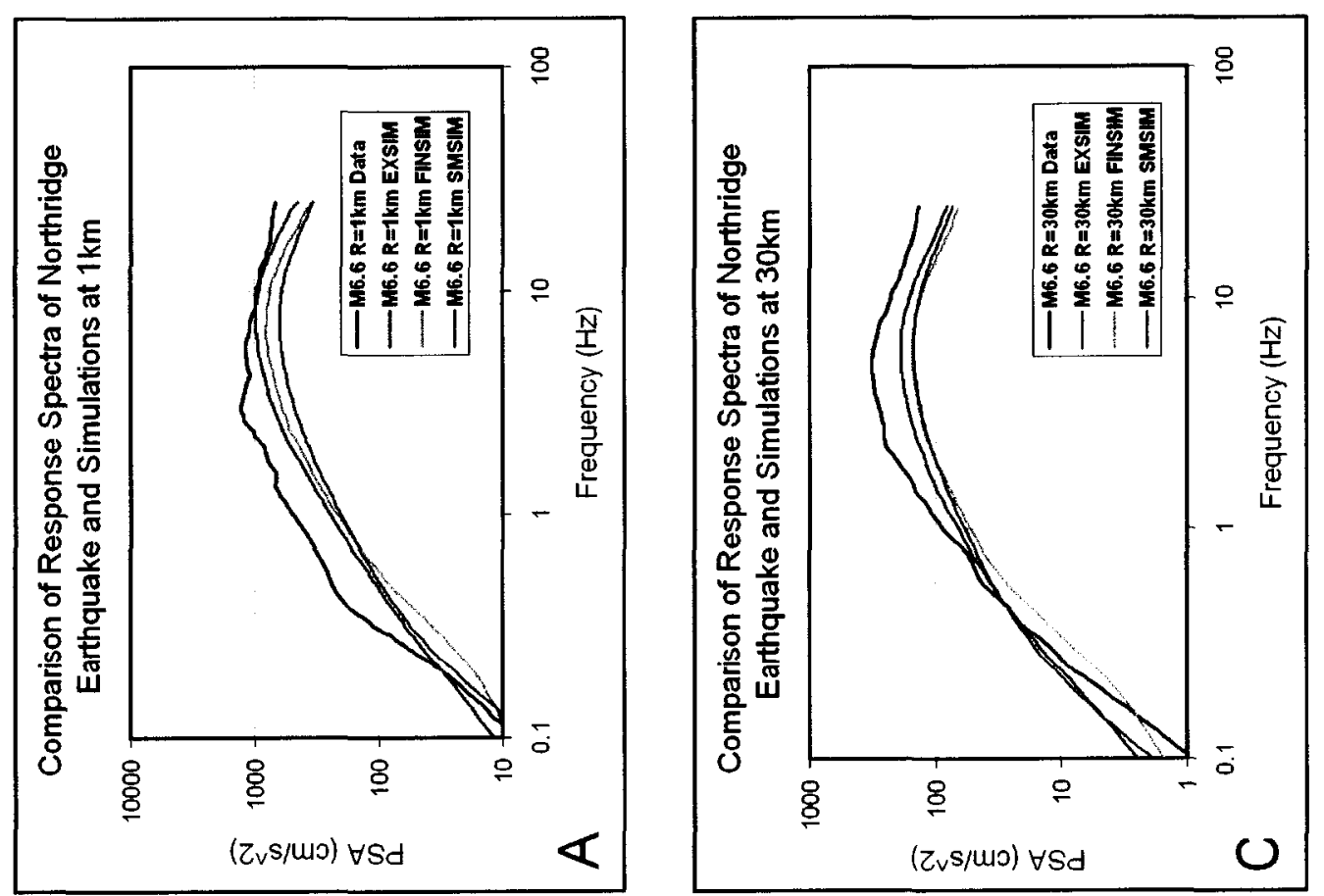

2

ब ह

응 등

$\sum^{0} \frac{\pi}{\vec{\Xi}}$

4

\&

岕

응

冚

융

$4 \quad 1$

○

离

ণิ

¿ํ

总 
$m$
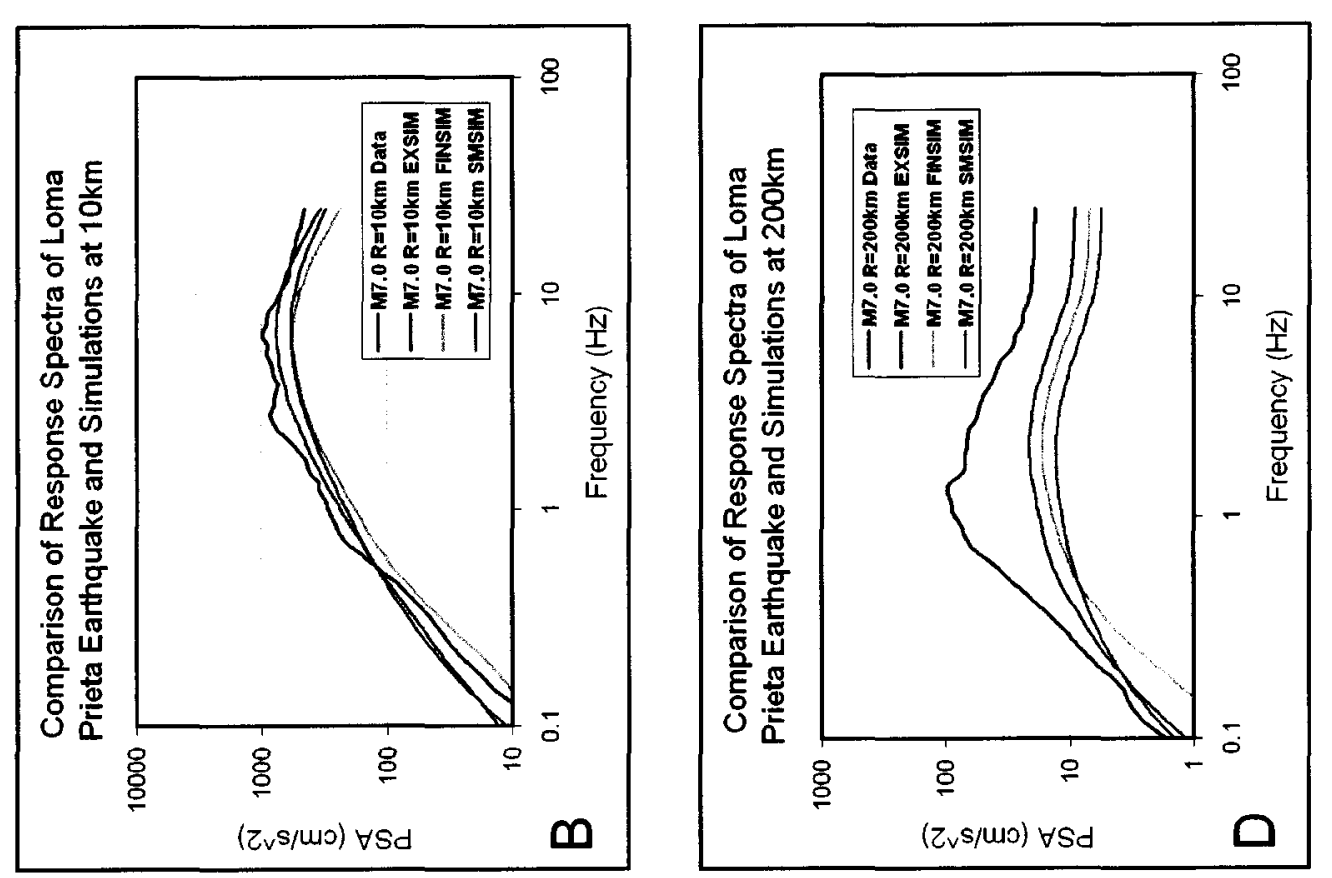

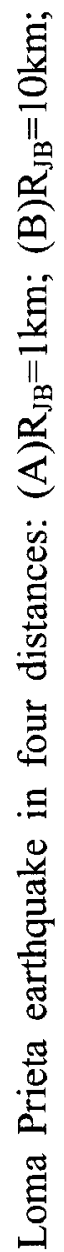

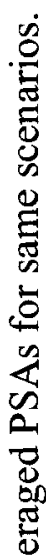

क
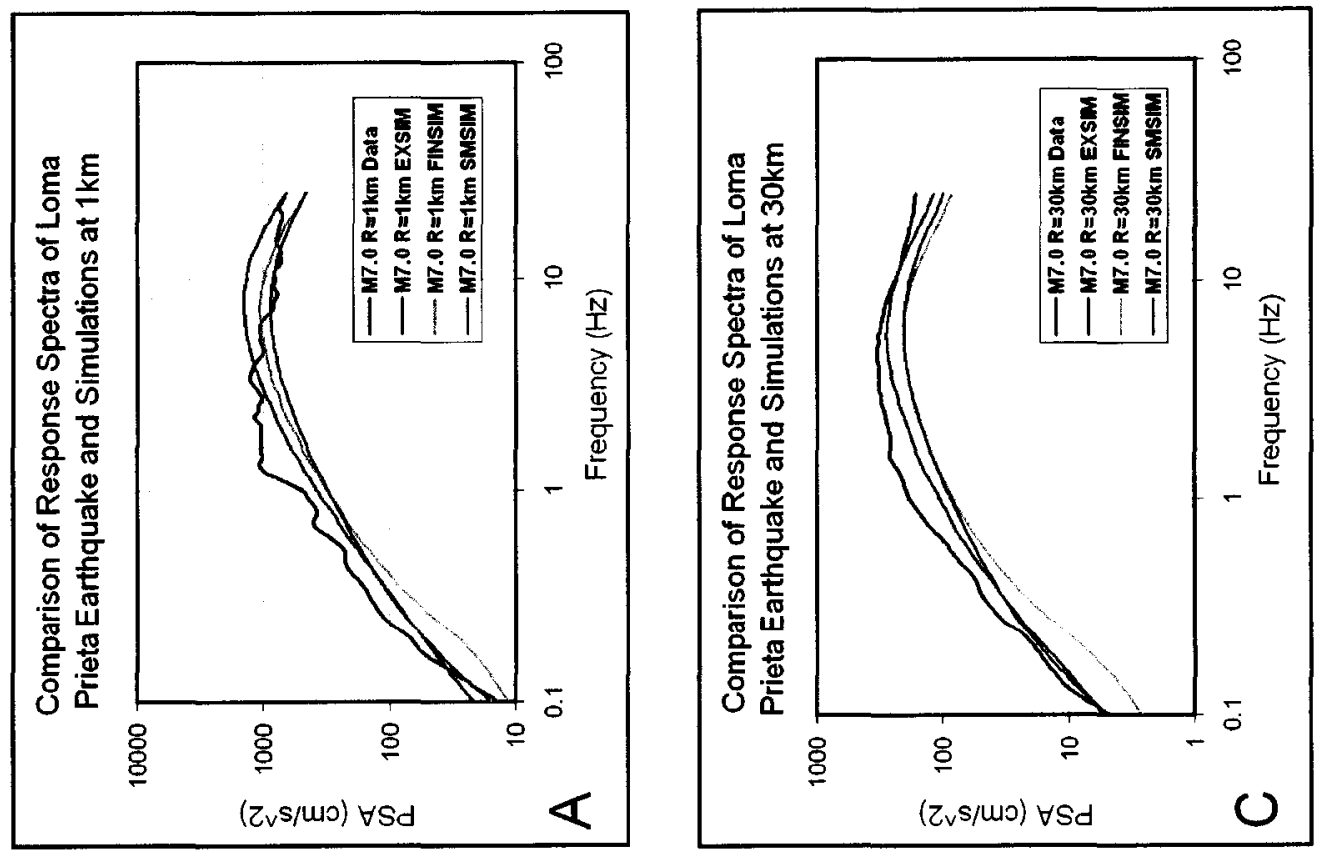

$\sum^{0}$

늘

出

응

志是

○

$4 \stackrel{0}{\circ}$

$\stackrel{2}{\widetilde{2}}$

营

$\widehat{\vec{\lambda}} \ddot{\tilde{g}}$

त्

莺 

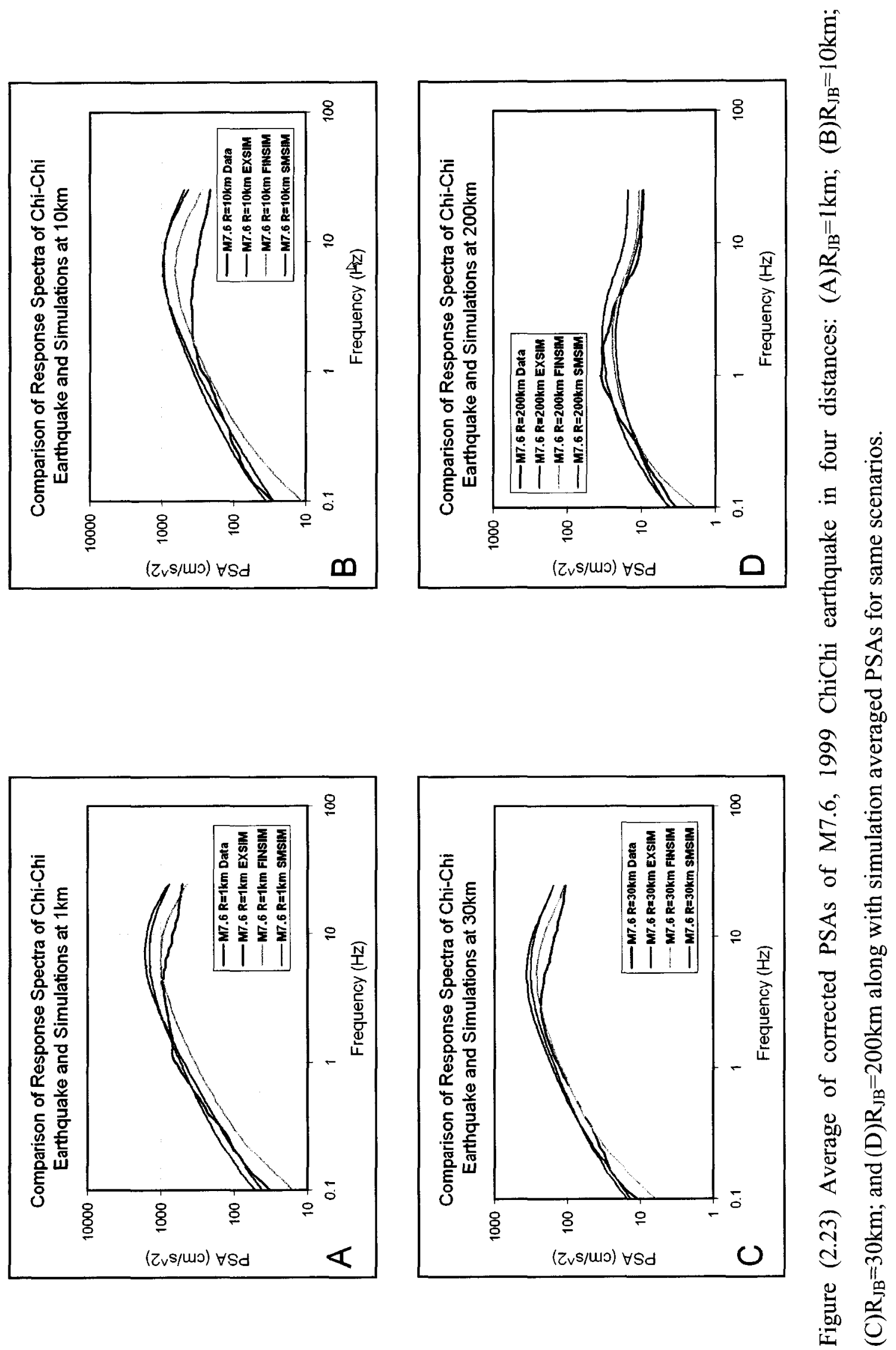

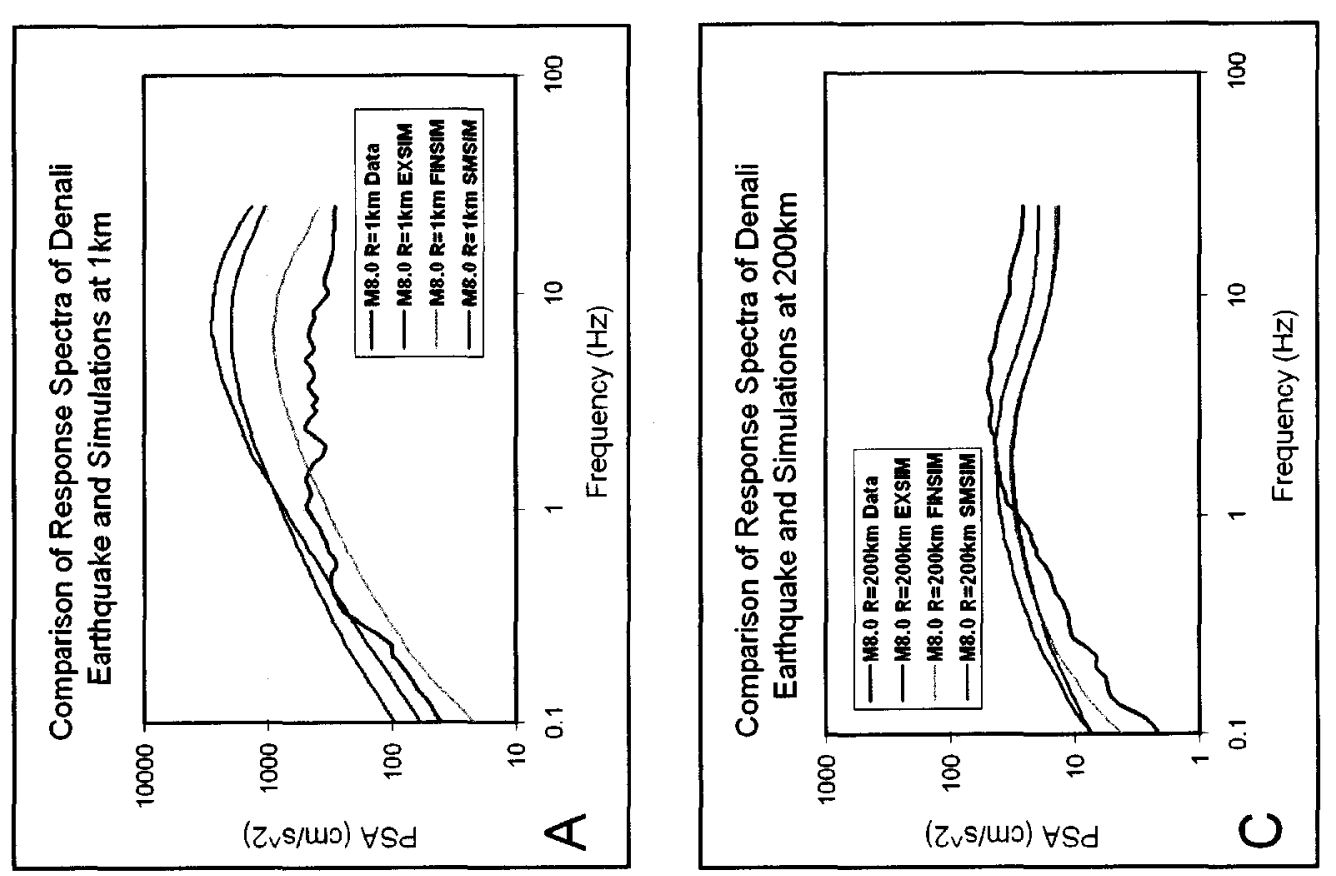

ธิธ

范

ᄋे

$\sum^{\infty} \frac{\infty}{0}$

$4 \frac{2}{0}$

赵

क

횽

总

क क

प

跑 $\frac{00}{\pi}$

究

营 
Table (2.9). Information and number of records of used earthquakes in Magnitudedistance ranges. Last three columns are calculated error " $\varepsilon$ " between average PSAs of corrected database and SMSIM, FINSIM, and EXSIM simulations in $1 \mathrm{~Hz}-10 \mathrm{~Hz}$ frequency range.

\begin{tabular}{|c|c|c|c|c|c|c|c|c|}
\hline Eq. ID & Earthhquake & $\begin{array}{l}\text { Reference } \\
\text { magnitude }\end{array}$ & $\begin{array}{l}\text { No. of used } \\
\text { records }\end{array}$ & $\begin{array}{c}\text { Reference } \\
\text { distance }\end{array}$ & $\begin{array}{l}\text { No. in reference } \\
\text { distance }\end{array}$ & $\begin{array}{c}\text { SMSIM } \\
\text { error }\end{array}$ & $\begin{array}{l}\text { FINSIM } \\
\text { Error }\end{array}$ & $\begin{array}{c}\text { EXSIM } \\
\text { error }\end{array}$ \\
\hline \multirow{4}{*}{0030} & \multirow{4}{*}{ San Fernando } & \multirow{4}{*}{6.6} & \multirow{4}{*}{44} & 1 & 1 & 0.76937 & 0.68349 & 0.61327 \\
\hline & & & & 10 & 1 & 0.26962 & 0.25555 & 0.23731 \\
\hline & & & & 30 & 24 & 0.12337 & 0.12619 & 0.07248 \\
\hline & & & & 200 & 18 & 0.22908 & 0.13510 & 0.09793 \\
\hline \multirow{3}{*}{0048} & \multirow{3}{*}{ Coyote Lake } & \multirow{3}{*}{5.8} & \multirow{3}{*}{10} & 1 & 1 & 0.54432 & 0.49174 & 0.33351 \\
\hline & & & & 10 & 5 & 0.29897 & 0.32016 & 0.12609 \\
\hline & & & & 30 & 4 & 0.25551 & 0.27978 & 0.11844 \\
\hline \multirow{3}{*}{0050} & \multirow{3}{*}{$\begin{array}{l}\text { Imperial } \\
\text { Valley-06 }\end{array}$} & \multirow{3}{*}{6.6} & \multirow{3}{*}{33} & 1 & 7 & 0.22735 & 0.14146 & 0.07826 \\
\hline & & & & 10 & 16 & 0.10980 & 0.11346 & 0.03774 \\
\hline & & & & 30 & 10 & 0.15177 & 0.15467 & 0.07213 \\
\hline \multirow{2}{*}{0068} & \multirow{2}{*}{ Irpinia, Italy-01 } & \multirow{2}{*}{7} & \multirow{2}{*}{12} & 10 & 4 & 0.10529 & 0.10954 & 0.17487 \\
\hline & & & & 30 & 8 & 0.03926 & 0.04135 & 0.08733 \\
\hline 0069 & Irpinia Italy-02 & 6.2 & 10 & 10 & 2 & 0.20256 & 0.22308 & 0.21309 \\
\hline 0009 & IIpinia, Ialy-UL & 0.2 & 10 & 30 & 8 & 0.07840 & 0.08147 & 0.04507 \\
\hline 0076 & Coalinga-01 & 6.4 & 46 & 10 & 2 & 0.38133 & 0.38213 & 0.29335 \\
\hline 0010 & Guallinga-o I & 0.4 & 40 & 30 & 44 & 0.28690 & 0.29085 & 0.19818 \\
\hline & & & & 1 & 1 & 0.65555 & 0.56193 & 0.49130 \\
\hline 0090 & Morgan Hill & 6.2 & 28 & 10 & 9 & 0.13037 & 0.12253 & 0.03860 \\
\hline & & & & 30 & 18 & 0.13304 & 0.13180 & 0.05411 \\
\hline & & & & 1 & 3 & 0.41947 & 0.39522 & 0.24833 \\
\hline 0101 & N Palm Springs & 6 & 32 & 10 & 5 & 0.05889 & 0.08377 & 0.09565 \\
\hline 0101 & N. Palm springs & 6 & 32 & 30 & 23 & 0.28702 & 0.35745 & 0.17786 \\
\hline & & & & 200 & 1 & 0.20716 & 0.18570 & 0.14305 \\
\hline 0103 & Chalfant & 62 & & 10 & 3 & 0.24796 & 0.24013 & 0.14370 \\
\hline 0103 & Valley-02 & b. 2 & 11 & 30 & 8 & 0.09949 & 0.07932 & 0.04979 \\
\hline & & & & 1 & 6 & 0.24243 & 0.21818 & 0.07129 \\
\hline 0113 & Whittier & & & 10 & 35 & 0.16327 & 0.21697 & 0.05554 \\
\hline 0773 & Narrows-01 & 6 & 176 & 30 & 73 & 0.31547 & 0.38590 & 0.20631 \\
\hline & & & & 200 & 2 & 0.59192 & 0.57311 & 0.40463 \\
\hline & & & & 1 & 1 & 0.19446 & 0.13908 & 0.12878 \\
\hline 0116 & $\begin{array}{l}\text { Superstition } \\
\text { Hills-02 }\end{array}$ & 6.6 & 11 & 10 & 3 & 0.26266 & 0.26632 & 0.19054 \\
\hline & & & & 30 & 7 & 0.20143 & 0.20434 & 0.11290 \\
\hline & & & & 1 & 2 & 0.20938 & 0.17513 & 0.15644 \\
\hline 0118 & & & 83 & 10 & 15 & 0.18625 & 0.21729 & 0.09772 \\
\hline 0118 & Loma Prieta & $r$ & 83 & 30 & 56 & 0.24557 & 0.24921 & 0.12632 \\
\hline & & & & 200 & 10 & 0.67677 & 0.57122 & 0.46491 \\
\hline & & & & 1 & 1 & 0.10842 & 0.14254 & 0.09884 \\
\hline 0125 & Landers & 72 & 69 & 10 & 1 & 0.14820 & 0.16292 & 0.19396 \\
\hline $01 \angle 0$ & Landers & 1.2 & 69 & 30 & 14 & 0.06886 & 0.12912 & 0.04434 \\
\hline & & & & 200 & 53 & 0.40965 & 0.35664 & 0.18648 \\
\hline & & & & 1 & 14 & 0.34346 & 0.25758 & 0.18741 \\
\hline & & & & 10 & 27 & 0.26662 & 0.27028 & 0.19450 \\
\hline 0127 & Northridge-01 & 6.6 & 160 & 30 & 102 & 0.29123 & 0.29413 & 0.20270 \\
\hline & & & & 200 & 17 & 0.57582 & 0.48030 & 0.39331 \\
\hline & & & & 1 & 3 & 0.27396 & 0.21725 & 0.15221 \\
\hline 0129 & Kobe, Japan & 7 & 12 & 10 & 1 & 0.27167 & 0.29748 & 0.20855 \\
\hline & & & & 30 & 4 & 0.17128 & 0.17491 & 0.06498 \\
\hline & & & & 200 & 4 & 0.65613 & 0.55058 & 0.44427 \\
\hline & & & & 1 & 1 & 0.38593 & 0.21514 & 0.40347 \\
\hline 0136 & Kocaeli Turkey & 76 & 27 & 10 & 4 & 0.27665 & 0.10854 & 0.26248 \\
\hline 0136 & kосаёl, rurkey & 1.0 & 27 & 30 & 13 & 0.02422 & 0.06918 & 0.06620 \\
\hline & & & & 200 & 9 & 0.39862 & 0.35718 & 0.21764 \\
\hline & & & & 1 & 20 & 0.13416 & 0.10861 & 0.16492 \\
\hline 0137 & Chi-Chi, Taiwan & 76 & 420 & 10 & 41 & 0.29531 & 0.14496 & 0.28113 \\
\hline 0131 & Cni-chi, talwan & 1.0 & $4 \angle 0$ & 30 & 192 & 0.12723 & 0.06651 & 0.17728 \\
\hline & & & & 200 & 167 & 0.09852 & 0.08220 & 0.12659 \\
\hline
\end{tabular}


Continuation of Table (2.9). Information and number of records of used earthquakes in Magnitude-distance ranges. Last three columns are calculated error " $\varepsilon$ " between average PSAs of corrected database and SMSIM, FINSIM, and EXSIM simulations in $1 \mathrm{~Hz}-10 \mathrm{~Hz}$ frequency range.

\begin{tabular}{|c|c|c|c|c|c|c|c|c|}
\hline Eq. ID & Earthhquake & $\begin{array}{l}\text { Reference } \\
\text { magnitude }\end{array}$ & $\begin{array}{l}\text { No. of used } \\
\text { records }\end{array}$ & $\begin{array}{l}\text { Reference } \\
\text { distance }\end{array}$ & $\begin{array}{l}\text { No. in reference } \\
\text { distance }\end{array}$ & $\begin{array}{l}\text { SMSIM } \\
\text { error }\end{array}$ & $\begin{array}{l}\text { FINSIM } \\
\text { Error }\end{array}$ & $\begin{array}{l}\text { EXSIM } \\
\text { error }\end{array}$ \\
\hline \multirow{4}{*}{0138} & \multirow{4}{*}{ Duzce, Turkey } & \multirow{4}{*}{7.2} & \multirow{4}{*}{22} & 1 & 2 & 0.22933 & 0.17652 & 0.40231 \\
\hline & & & & 10 & 6 & 0.10475 & 0.07570 & 0.15578 \\
\hline & & & & 30 & 4 & 0.32413 & 0.26387 & 0.43733 \\
\hline & & & & 200 & 10 & 0.09293 & 0.04752 & 0.13024 \\
\hline \multirow{3}{*}{0158} & \multirow{3}{*}{ Hector Mine } & \multirow{3}{*}{7.2} & \multirow{3}{*}{83} & 10 & 1 & 0.09994 & 0.16912 & 0.09356 \\
\hline & & & & 30 & 19 & 0.08240 & 0.14267 & 0.03665 \\
\hline & & & & 200 & 63 & 0.42695 & 0.37393 & 0.20378 \\
\hline \multirow{3}{*}{0168} & \multirow{3}{*}{$\begin{array}{c}\text { Nenana } \\
\text { Mountain, } \\
\text { Alaska }\end{array}$} & \multirow[b]{2}{*}{6.8} & \multirow[b]{2}{*}{36} & & & & & \\
\hline & & & & 200 & 36 & 0.40791 & 0.30891 & 0.21091 \\
\hline & & & \multirow{3}{*}{24} & 1 & 1 & 0.52036 & 0.20548 & 0.60066 \\
\hline \multirow[t]{2}{*}{0169} & \multirow[t]{2}{*}{ Denali, Alaska } & \multirow[t]{2}{*}{8} & & 30 & 3 & 0.18994 & 0.05520 & 0.16990 \\
\hline & & & & 200 & 20 & 0.21227 & 0.20027 & 0.10395 \\
\hline \multirow{4}{*}{0171} & \multirow{4}{*}{$\begin{array}{l}\text { Chi-Chi, } \\
\text { Taiwan-02 }\end{array}$} & \multirow{4}{*}{6} & \multirow{3}{*}{296} & 10 & 6 & 0.06736 & 0.05097 & 0.15752 \\
\hline & & & & 30 & 139 & 0.10115 & 0.16922 & 0.06377 \\
\hline & & & & 200 & 151 & 0.31707 & 0.29827 & 0.12978 \\
\hline & & & \multirow{4}{*}{242} & 1 & 2 & 0.25064 & 0.15738 & 0.09856 \\
\hline \multirow{3}{*}{0172} & \multirow{3}{*}{$\begin{array}{l}\text { Chi-Chi, } \\
\text { Taiwan-03 }\end{array}$} & \multirow{3}{*}{6.2} & & 10 & 6 & 0.27459 & 0.26676 & 0.17033 \\
\hline & & & & 30 & 139 & 0.08300 & 0.10787 & 0.14522 \\
\hline & & & & 200 & 95 & 0.05907 & 0.03946 & 0.13524 \\
\hline \multirow{3}{*}{0173} & \multirow{3}{*}{$\begin{array}{l}\text { Chi-Chi, } \\
\text { Taiwan-04 }\end{array}$} & \multirow{3}{*}{6.2} & \multirow{3}{*}{242} & 10 & 2 & 0.30905 & 0.30121 & 0.21001 \\
\hline & & & & 30 & 135 & 0.02688 & 0.04742 & 0.11808 \\
\hline & & & & 200 & 105 & 0.11394 & 0.04130 & 0.07327 \\
\hline \multirow{3}{*}{0174} & \multirow{3}{*}{$\begin{array}{l}\text { Chi-Chi, } \\
\text { Taiwan-05 }\end{array}$} & 62 & 322 & 30 & 126 & 0.33303 & 0.33179 & 0.22804 \\
\hline & & 0.2 & $3 \angle 2$ & 200 & 196 & 0.47741 & 0.39525 & 0.29021 \\
\hline & & & & 1 & 2 & 0.39235 & 0.30700 & 0.22222 \\
\hline 0175 & Chi-Chi, & 64 & 290 & 10 & 2 & 0.09354 & 0.09674 & 0.05300 \\
\hline & Taiwan-06 & 0.4 & 290 & 30 & 147 & 0.18623 & 0.19018 & 0.09404 \\
\hline & & & & 200 & 139 & 0.25815 & 0.17575 & 0.07731 \\
\hline
\end{tabular}


2

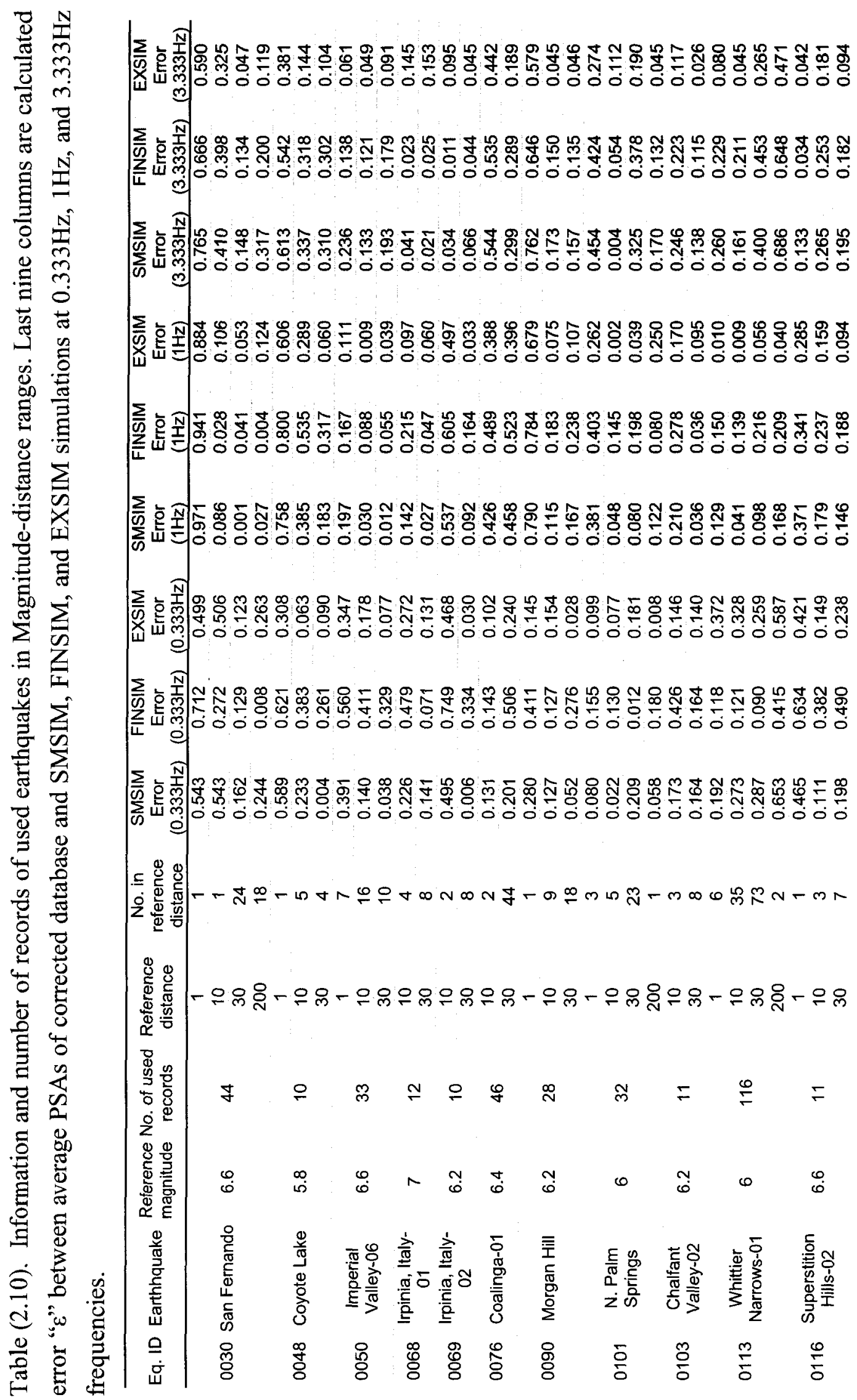




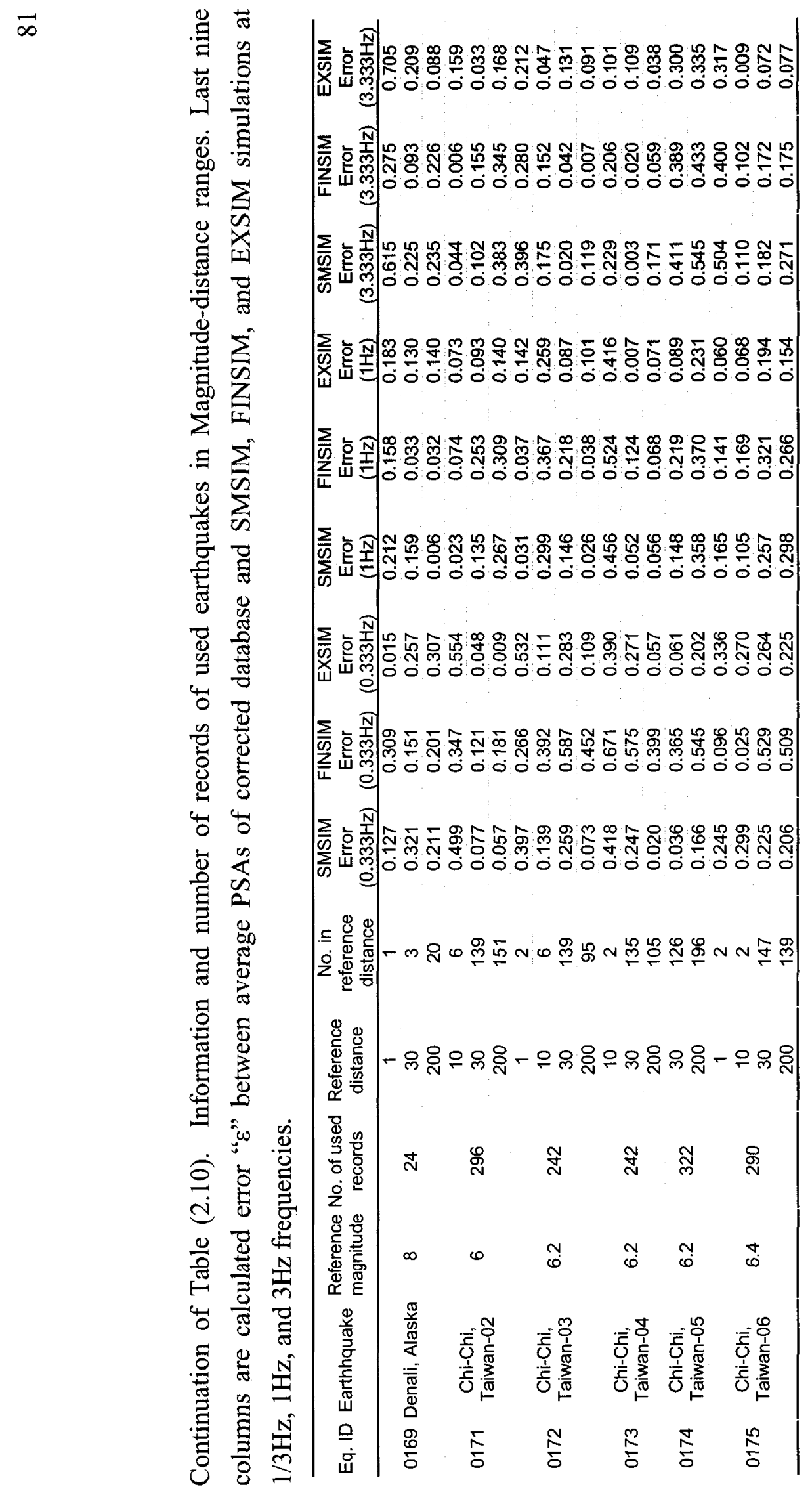




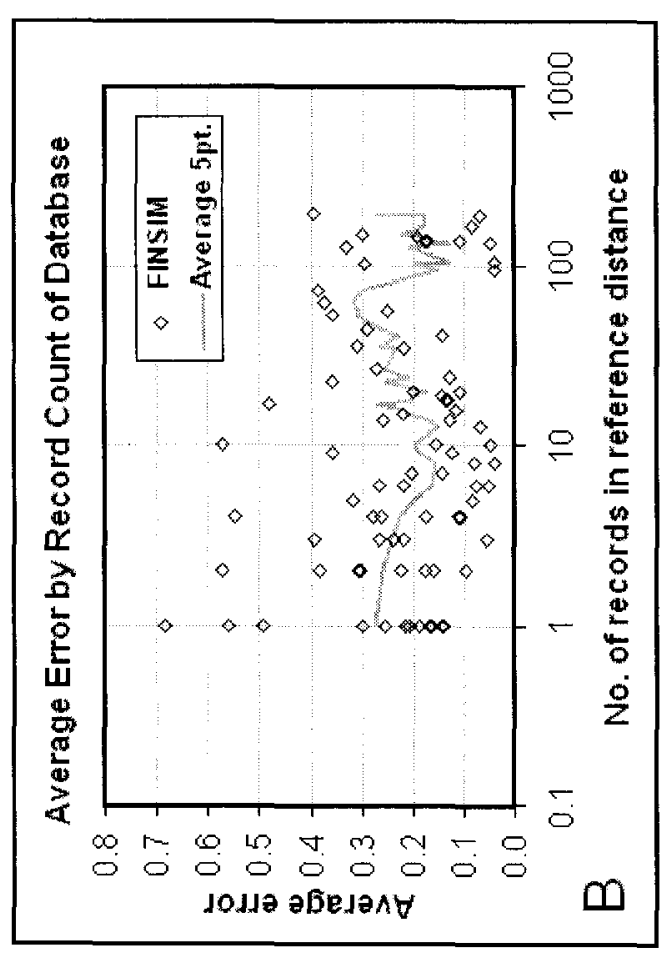

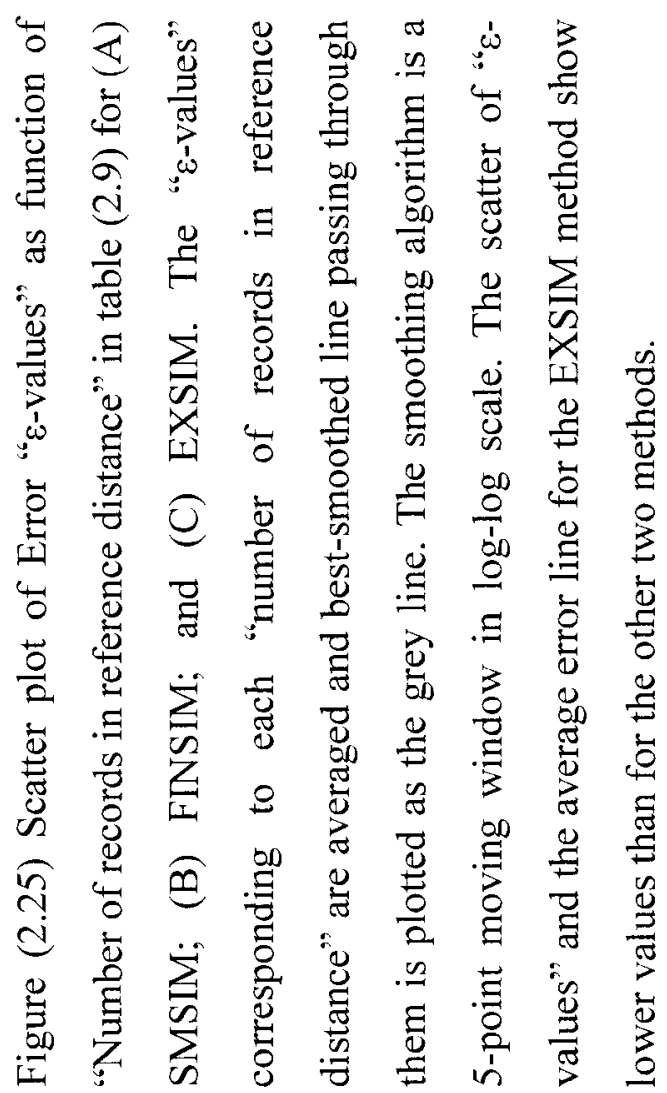
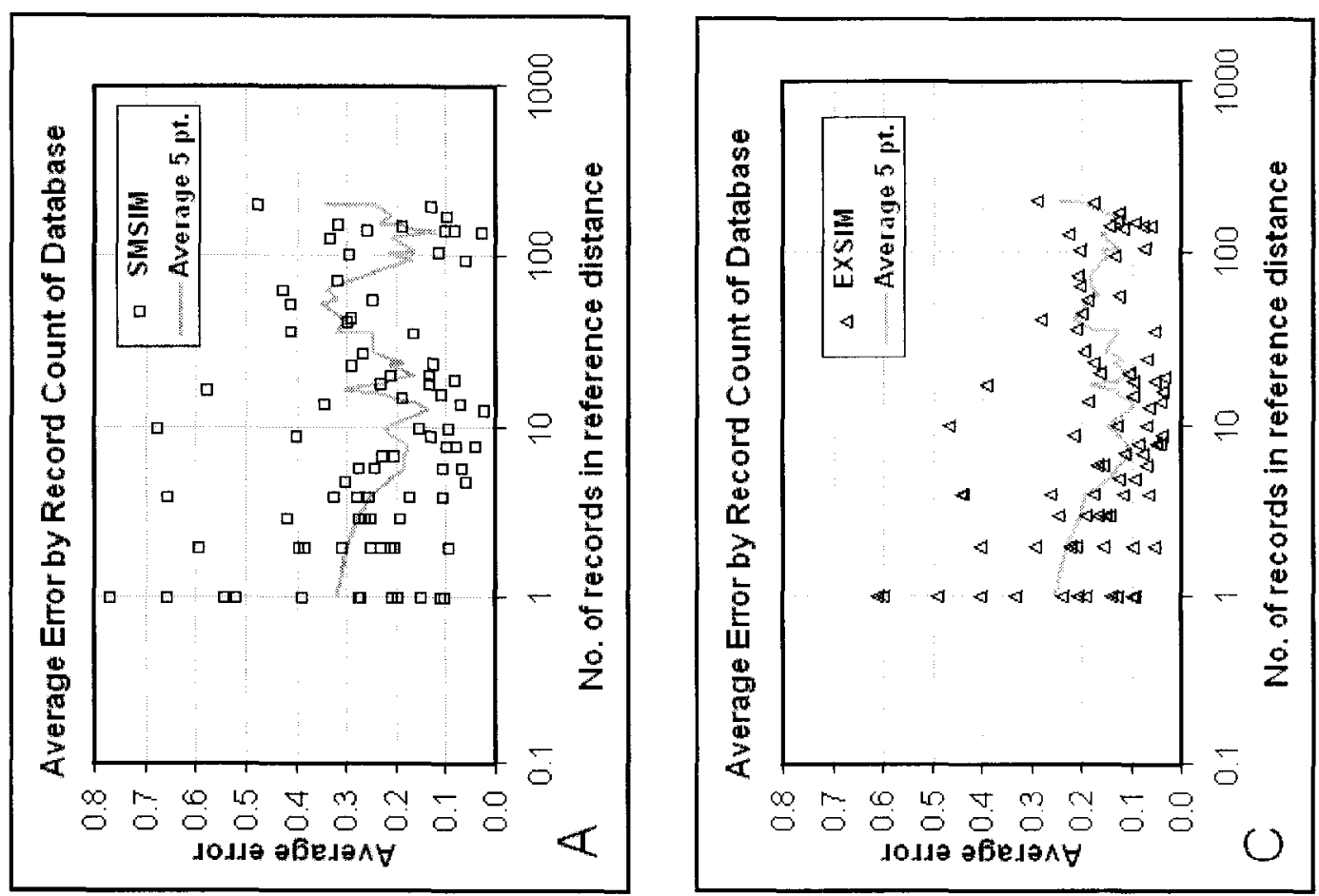

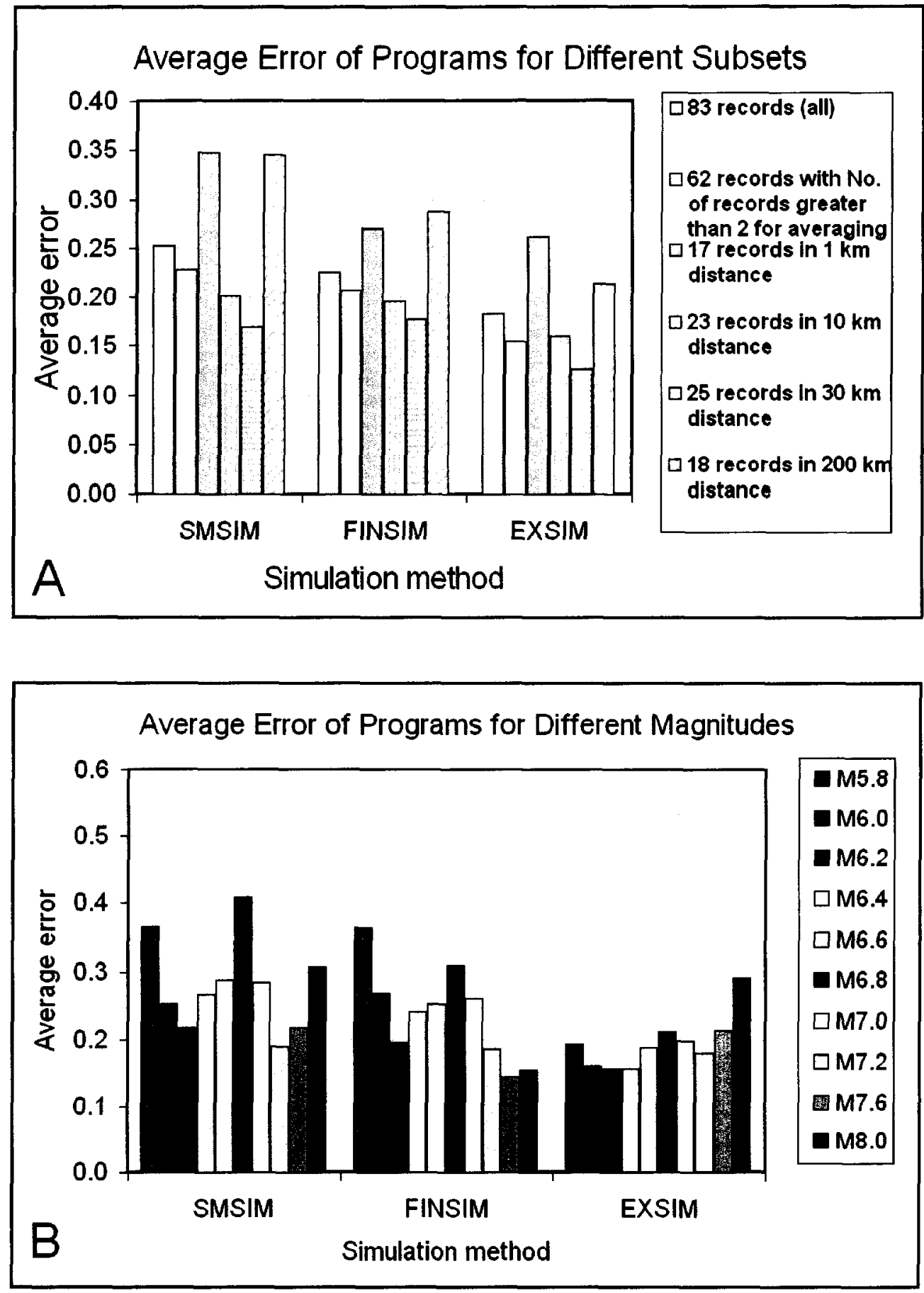

Figure (2.26) Comparisons of averages of sub-sets of " $\varepsilon$-values" derived from three different simulation methods in $1 \mathrm{~Hz}-10 \mathrm{~Hz}$ frequency range. (A) All $\varepsilon$-values, " $\varepsilon$-values" determined by at least 3 records, " $\varepsilon$-values" determined with records in $1 \mathrm{~km}, 10 \mathrm{~km}$, $30 \mathrm{~km}$, and $200 \mathrm{~km}$ distance classes; (B) " $\varepsilon$-values" determined with records in different magnitude classes. Data in last three columns of Table (2.9) are used. 

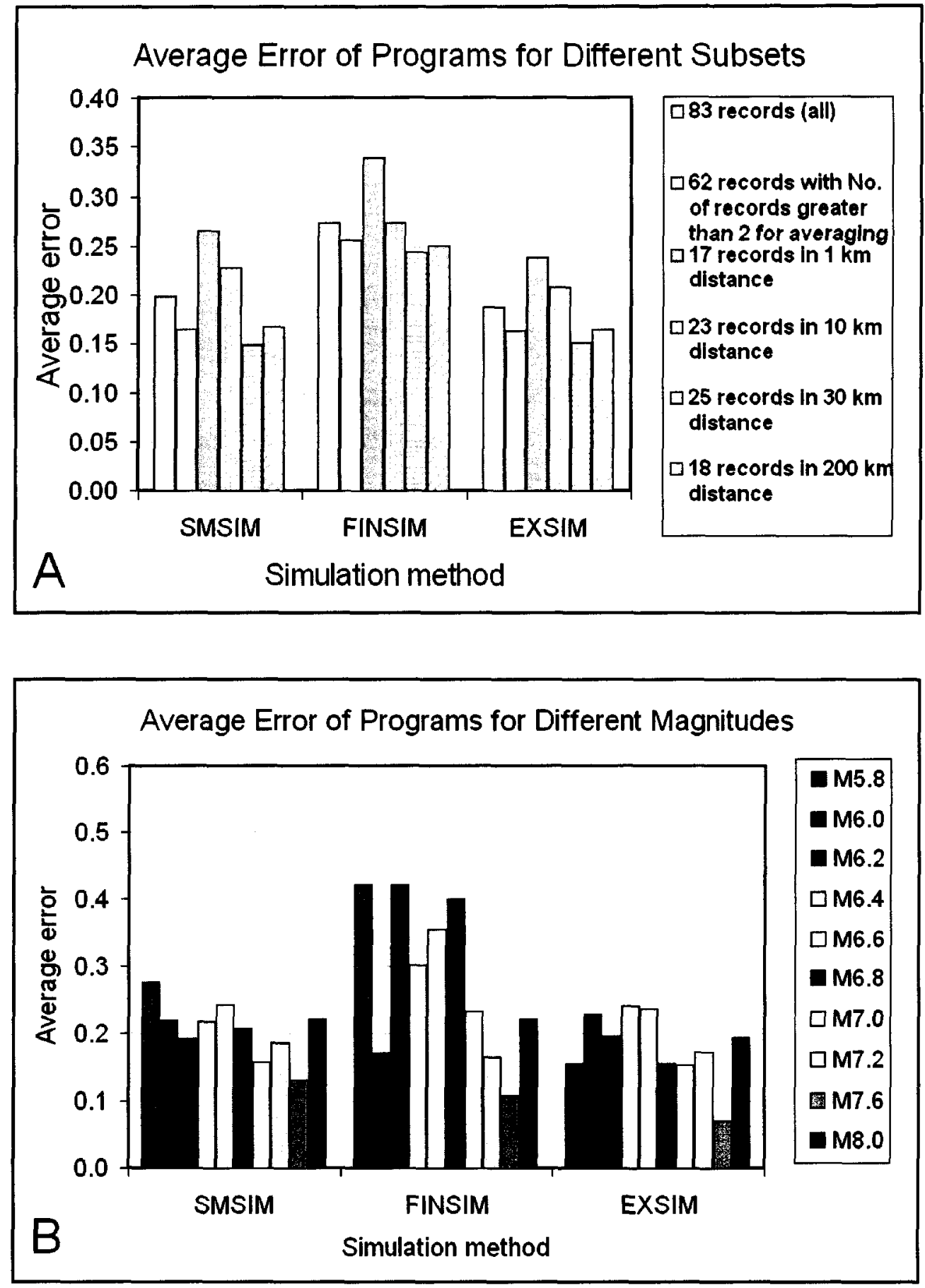

Figure (2.27) Comparisons of averages of sub-sets of " $\varepsilon$-values" derived from three different simulation methods at $0.333 \mathrm{~Hz}$ frequency. (A) All $\varepsilon$-values, " $\varepsilon$-values" determined by at least 3 records, " $\varepsilon$-values" determined with records in $1 \mathrm{~km}, 10 \mathrm{~km}$, $30 \mathrm{~km}$, and $200 \mathrm{~km}$ distance classes; (B) " $\varepsilon$-values" determined with records in different magnitude classes. Data of Table (2.10) in yellow shaded columns are used. 

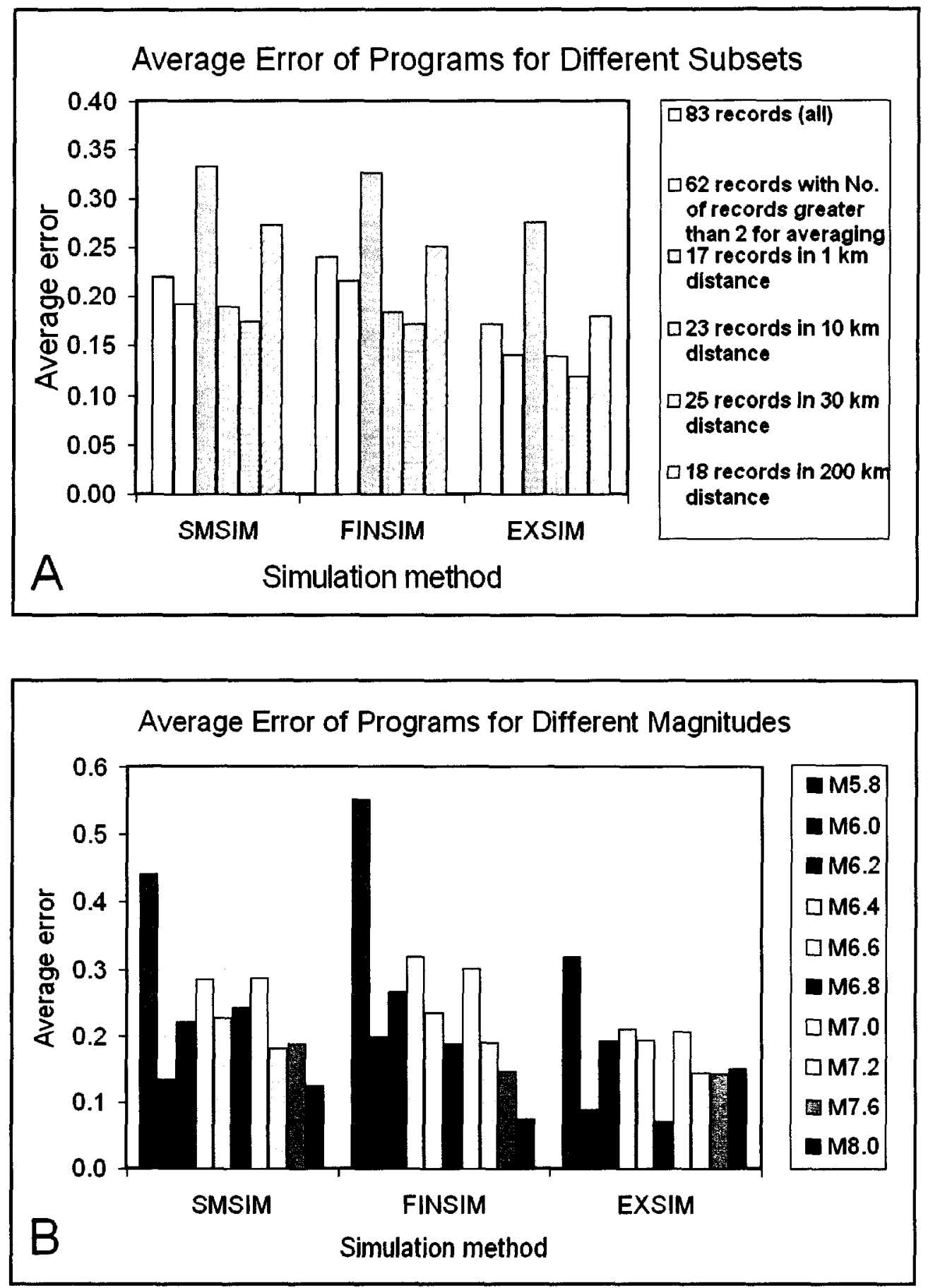

Figure (2.28) Comparisons of averages of sub-sets of " $\varepsilon$-values" derived from three different simulation methods at $1 \mathrm{~Hz}$ frequency. (A) All $\varepsilon$-values, " $\varepsilon$-values" determined by at least 3 records, " $\varepsilon$-values" determined with records in $1 \mathrm{~km}, 10 \mathrm{~km}, 30 \mathrm{~km}$, and $200 \mathrm{~km}$ distance classes; (B) " $\varepsilon$-values" determined with records in different magnitude classes. Data of Table (2.10) in blue shaded columns are used. 

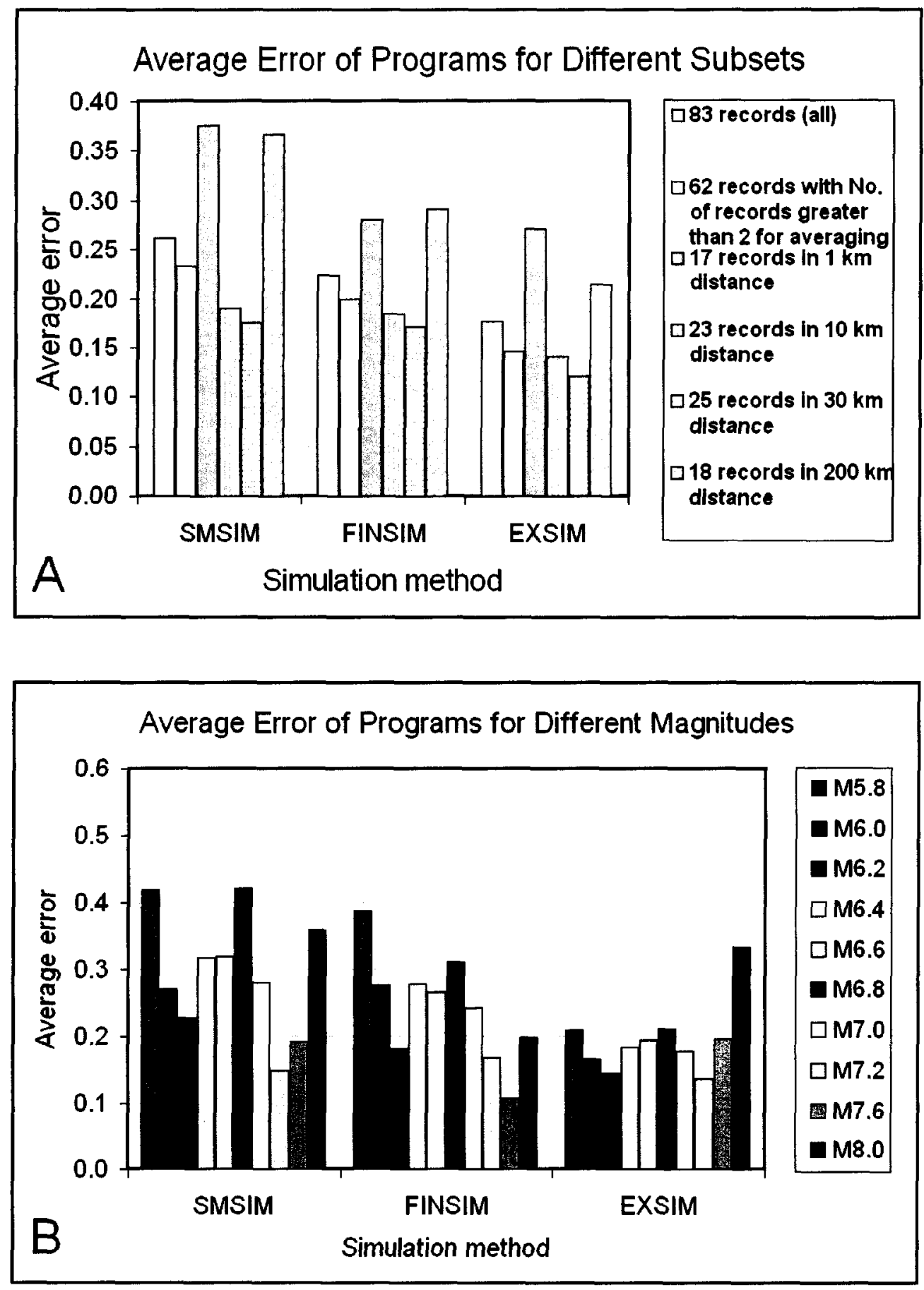

Figure (2.29) Comparisons of averages of sub-sets of " $\varepsilon$-values" derived from three different simulation methods at $3.333 \mathrm{~Hz}$ frequency. (A) All $\varepsilon$-values, " $\varepsilon$-values" determined by at least 3 records, " $\varepsilon$-values" determined with records in $1 \mathrm{~km}, 10 \mathrm{~km}$, $30 \mathrm{~km}$, and $200 \mathrm{~km}$ distance classes; (B) " $\varepsilon$-values" determined with records in different magnitude classes. Data of Table (2.10) in green shaded columns are used. 


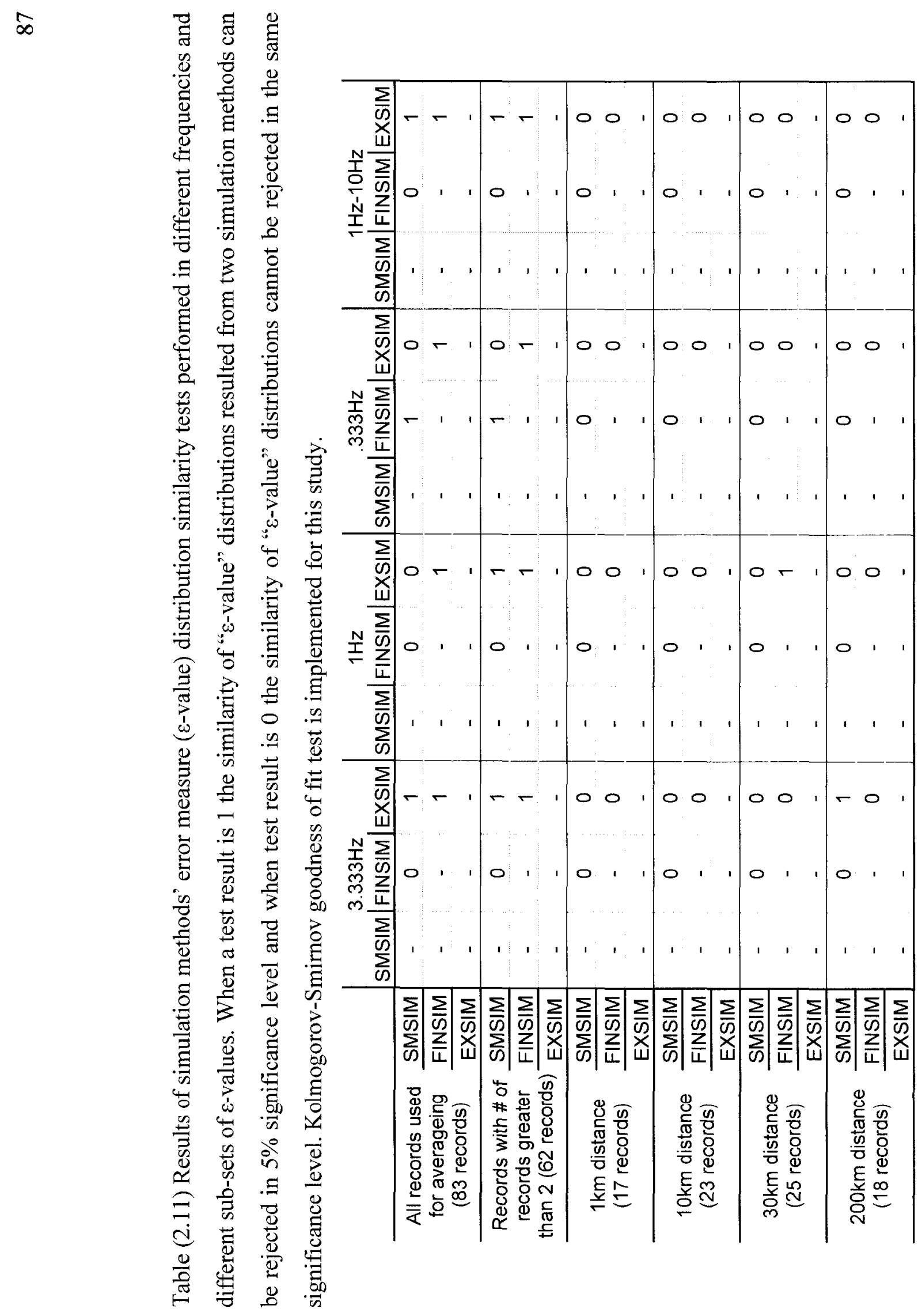


$\infty$

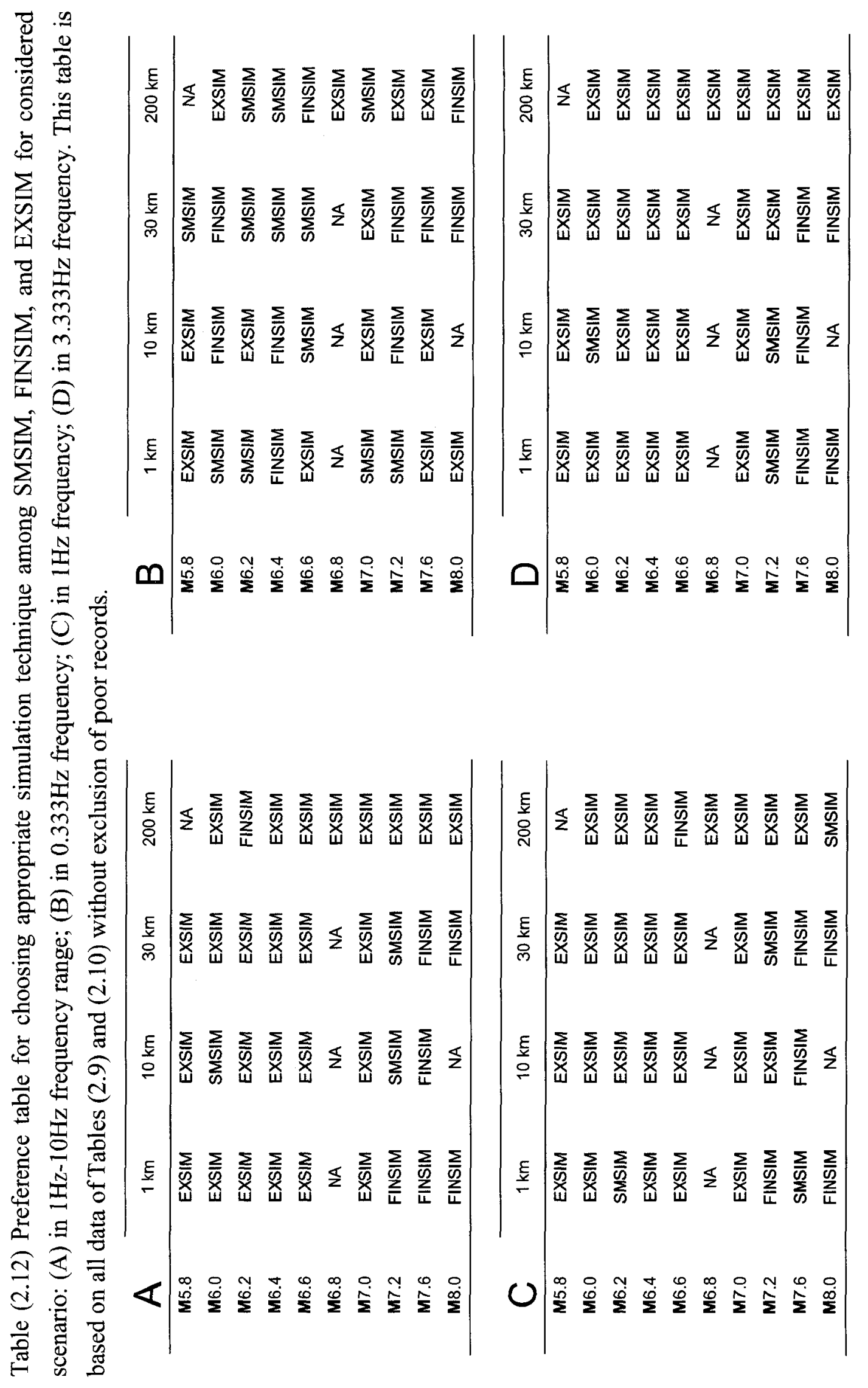




\section{Chapter 3: Extended applications of stochastic finite fault modeling}

\subsection{Introduction}

Two enhancements to stochastic finite fault modeling techniques were developed. This chapter describes these developments and their significance. The enhancements introduce the capability to model variability of the rupture velocity and the stress parameter across the fault plane. Theoretical and analytical studies have shown that variability of the stress parameter on the fault surface, and variability of rupture velocity, play important roles in determining the characteristics of strong ground motion records (Bernard and Herrero, 1994; Boore, 1983 and 2003; Doornbos, 1984; Hisada, 2001; Papageorgiou and Aki, 1983 and 1983). All stochastic finite fault techniques developed to date (Schneider et al, 1993; Beresnev and Atkinson, 1998; Motazedian and Atkinson, 2005) assume a uniform value for the stress parameter and fault rupture velocity for all sub-sources. In this section, I present an extension of EXSIM that introduces variability of stress parameter and rupture velocity, while satisfying theoretical constraints on these parameters. Reasonable ranges of stress and rupture velocities are applied in tests of the improved models; performance is tested by means of a strong motion measurement criterion, applied after forward modeling.

\subsection{Implementation of variable rupture velocity in EXSIM}

EXSIM assumes a constant rupture velocity on the modeled fault surface. Variable rupture velocity is introduced to EXSIM to make the method more realistic. Variable rupture velocity affects two parameters in the EXSIM simulations. One is the rise time of sub-sources and the other is the delay time between excitation of the hypocenter and a specific sub-source. 


\subsubsection{Modification of rise time}

Rise time in EXSIM appears in two equations. First it appears in Equation (1.1), which relates point source duration to the rise time of a sub-source. Its second appearance is in Equation (1.17); a fraction of sub-source rise time is added to the delay time of subsource activation. The rise time in EXSIM is defined as:

$$
T=\frac{d l \times d w}{\pi \times v}
$$

where $T$ is the rise time of the sub-source, $d l$ and $d w$ are sub-source length and width respectively, and $v$ is the rupture velocity. To generalize Equation (3.1) to a case where each sub-source has a unique rupture velocity, it is necessary to calculate this equation for each sub-source separately:

$$
T_{i j}=\frac{d l \times d w}{\pi \times v_{i j}}
$$

where $T_{i j}$ and $v_{i j}$ are the rise time and rupture velocity of the sub-source in the $i^{\text {th }}$ column and $\mathrm{j}^{\text {th }}$ row of the fault plane array. Equation (3.2) is applied in EXSIM, along with necessary changes to the code for proper treatment of this change.

\subsubsection{Modification of rupture delay time}

Rupture velocity variability affects the delay time of a sub-source, $\Delta t_{i j}$, which is the time required for the rupture to reach the center of a sub-source from the hypocenter. This section describes modification of rupture delay times of sub-sources in EXSIM in order to simulate variable rupture velocity. When rupture velocity is constant, the length of a line connecting the hypocenter and the center of a sub-source is calculated, and divided by the rupture velocity to get the sub-source delay time:

$$
\Delta t_{i j}=\frac{\sqrt{\left(\left(i-i_{0}\right) \times d l\right)^{2}+\left(\left(j-j_{0}\right) \times d w\right)^{2}}}{v}
$$

where $i$ and $i_{0}$ are indices of the sub-source and hypocenter along the fault length, $j$ and $j_{0}$ are indices of the sub-source and hypocenter along the fault width, $d l$ and $d w$ are the 
length and width of the sub-source, and $v$ is rupture velocity. The illustrations of these quantities are shown in Figure (3.1). In Equation (1.17), delay time is written as $\Delta t_{i}$ where $i$ stands for the index of the sub-source. Here two indices are introduced to be more specific regarding the element location along the fault length and width.

The modified version of EXSIM to model variable rupture velocity allows every subsource to have a unique rupture velocity, $v_{i j}$; consequently Equation (3.3) does not apply. A simplifying assumption for solving this problem is that the rupture gets to a sub-source along a straight line connecting the hypocenter to the center of the sub-source in question. To find the delay time, lengths of segments of the line that connects the hypocenter to the center of a sub-source, and fall within each sub-source, are calculated and divided by the corresponding rupture velocities. The sum of these values is considered as the modified delay time according to the following relation:

$$
\Delta t_{l j}=\sum_{m, n} \frac{X\left(m, n, i, j, i_{0}, j_{0}, d l, d w\right)}{v_{m n}}
$$

$X\left(m, n, i, j, i_{0}, j_{0}, d l, d w\right)$ is the length of the line segment that connects the center of the subsource at column $i$ and row $\mathrm{j}$ to the one at column $i_{n}$ and row $j_{0}$, and lies in the sub-source at column $m$ and row $n$; $d l$ and $d w$ are the sub-source's length and width, $v_{m n}$ is the rupture velocity of the sub-source at column $m$ and row $n$, and the summation is over all sub-sources. Figure (3.1) illustrates the geometry of these parameters graphically. $X\left(m, n, i_{j}, i_{0}, j_{0}, d l, d w\right)$ is calculated stepwise by starting to move along the rake of the line connecting the hypocenter to the center of the sub-source in question, and finding the coordinates of consecutive intersections with each sub-sources' borders. The subroutine that calculates $X\left(m, n, i, j, i_{0}, j_{0}, d l, d w\right)$ is implemented in the modified EXSIM, along with necessary modifications of the code for this implementation. 


\subsubsection{Rupture velocity variability experiments}

In this section some experiments with the modified EXSIM for variable rupture velocity are described. The purpose of these experiments is to demonstrate the significance of rupture velocity variability on EXSIM results in different frequency ranges.

Theoretical studies suggest that spectral levels should be proportional to the rate of change of rupture velocity (e.g., Dong and Papageogiou, 2002; Sato, 1994). The rupture velocity variability experiments with EXSIM are aimed to explore the degree of match between the results of the modified EXSIM and this theoretical expectation. These experiments are performed in three frequency ranges to demonstrate that the conclusions are not frequency dependent. The measured quantity is the change of the value of the average $\log P S A$ in a range of frequencies, with changes of $D_{r}$ in Equation (2.1), when switching from EXSIM to the extended EXSIM (where $D_{r}$ is the average of $\log P S A \mathrm{~s}$ in a range of frequencies).

The design of the experiment is to perform simulations for a grid of stations around a hypothetical fault for two scenarios. One scenario is with variable rupture velocity and the other is with uniform rupture velocity. The differences of average $\log P S A$ s of the two scenarios are plotted as maps around the fault, using the kriging method (Isaaks and Srivastava, 1989; Armstrong, 1998; Chiles and Delfiner, 1999). These maps are compared against the variability maps of the same simulation results, also derived from kriging. The areas where the variability maps show comparable values to those driven by changes in rupture velocity are considered places with insensitivity to rupture velocity. In other words, we only consider rupture velocity important if it produces an effect that is larger than that of random variability. These comparisons are performed in three frequency ranges, as described below.

The parameters and geometry of the simulations are given in Table (3.1); they are those of the M6.0, 2004 Parkfield earthquake. Simulations are performed for 441 sites around the fault. The trace of the fault and distribution of stations around it are shown in Figure (3.2). To capture the details of the effects of the variability of rupture velocity, a dense 
station distribution is considered at close distances from the fault, while a sparse distribution is sufficient for stations at distance. For distances less than $10 \mathrm{~km}$ from the fault, station intervals are $2 \mathrm{~km}$. For distances between $10 \mathrm{~km}$ and $20 \mathrm{~km}$, station intervals are $5 \mathrm{~km}$, and beyond that the intervals are $10 \mathrm{~km}$. It is assumed that the variability of rupture velocity is distributed laterally with values between 0.6 to 0.9 times the shear wave velocity. In the uniform rupture velocity scenario its value is taken equal as 0.8 times the shear wave velocity. In both scenarios all other parameters are identical, and the slip distribution is assumed to be uniform.

The changes of: (i) $\log P S A$ at $25 \mathrm{~Hz}$, (ii) the average $\log P S A$ from $2.5 \mathrm{~Hz}-20 \mathrm{~Hz}$, and (iii) the average $\log P S A$ from $0.45 \mathrm{~Hz}-10 \mathrm{~Hz}$ are calculated by subtracting the $D_{r}$ values of modeling with uniform rupture velocity from those of modeling with variable rupture velocity, based on the Equations (3.5) and (3.6):

$$
\begin{array}{r}
E=\log P S A(25 H z)_{\text {Variable rupture }}-\log P S A(25 H z)_{\text {Uniform rupture }} \\
E=\frac{1}{N_{f 2}-N_{f 1}+1} \sum_{N=N_{f 1}}^{N_{f 2}}\left(\log P S A(N)_{\text {Variable rupture }}\right)- \\
\frac{1}{N_{f 2}-N_{f 1}+1} \sum_{N=N_{f 1}}^{N_{f 2}}\left(\log P S A(N)_{\text {Uniform rupture }}\right)
\end{array}
$$

where $E$ is the changes in (average) $\log P S A$ and $N_{f}$ is the index number corresponding to frequency $f$. Equation (3.5) is used for calculation of changes in $\log P S A$ at $25 \mathrm{~Hz}$ (this is essentially the $\log$ of PGA) and Equation (3.6) is used for calculation of changes in average $\log P S A$ in a range of frequencies.

Figures (3.3), (3.4), and (3.5) show the distribution of changes of average $\log P S A$ values for $25 \mathrm{~Hz}, 2.5 \mathrm{~Hz}$ to $20 \mathrm{~Hz}$, and $0.45 \mathrm{~Hz}$ to $10 \mathrm{~Hz}$ frequency ranges, plotted up to $20 \mathrm{~km}$ from the fault. The maps of Figures (3.3) to (3.5) are based on simulation results of sites in Figure (3.2). These maps are prepared by the kriging interpolation method for $100 \mathrm{~m} \times 100 \mathrm{~m}$ pixel sizes and smoothed by averaging over a circular moving window with $1 \mathrm{~km}$ diameter. 
Figures (3.3) to (3.5) seem to be very similar regardless of the frequency range chosen for averaging of $\log P S A$ s. Table (3.2) is the correlation coefficient list of Figures (3.3), (3.4) and (3.5), calculated based on values of 18108 interpolated points in each map. This similarity shows that it is possible to take the forward modeling results of a few frequency ranges as the overall picture of the effect of variable rupture velocity for all frequencies.

Figures (3.3) to (3.5) show that the (average) $\log P S A$ is reduced around areas of low fault rupture velocity and increased around areas of high fault rupture velocity. Also, (average) $\log P S A$ is increased close to the areas where rupture velocity changes from 0.8 to 0.6 times of shear wave velocity. The area of decreased (average) $\log P S A \mathrm{~s}$ is considerable in the three figures. The contour lines separate areas of positive and negative changes, showing that overall decrease of (average) $\log P S A \mathrm{~s}$ is more prominent than its increase, from a spatial point of view. This might be due to the fact that the average rupture velocity on the fault surface is not identical in the two scenarios and its effect is reflected in the form of large areas of decreased (average) $\log P S A$ s compared to areas of increased value. Although there are observable changes in the (average) $\log P S A$ values by switching from uniform rupture velocity to variable velocity, the overall effect is very small (ranging from -0.070 to $0.034 \log$ units in the most extreme case of $25 \mathrm{~Hz}$ ), suggesting the necessity to check the significance of these observed changes.

We consider that the observed changes are insignificant if the E-values are indistinguishable from those expected due to randomness. The kriging interpolation method assigns a standard deviation of interpolation error to each point. The standard deviations of the E-value maps are compared to the absolute of E-values (derived from calculating absolute value of Figures (3.3) - (3.5)). Figures (3.6) to (3.8) are the same as Figures (3.3) to (3.5), but plotted just in areas where absolute E-values are higher than the standard deviation of E-values. It is considered that the areas with absolute values of the changes that exceed the standard deviations are areas of significant E-values. Because Figures (3.6) to (3.8) are filtered versions of Figures (3.3) to (3.5), they are highly 
correlated and show almost the same picture. An important point is that all significant changes correspond to areas with decreased (average) $\log P S A$ values. This is probably because of larger absolute values of changes in these areas (it should be considered that the uncertainty introduced by interpolation is a function of the separation of observation points and spatial density of simulation sites, which is equal for both positive and negative changes).

Figures (3.3) to (3.5) demonstrate the average $\log P S A$ changes as a result of switching from uniform rupture velocity to variable rupture velocity. The method shows a slight increase in $E$-values close to the areas where rupture velocity is higher than the uniform velocity value. Also there is a small increase in $E$-values close to the areas of rupture velocity change, as expected from theoretical/analytical studies. There is also a decrease in $E$-values close to the areas where rupture velocity is lower than the uniform rupture velocity value. The amplitude and expanse of increased/decreased $E$-value areas depend on increase/decrease of average rupture velocity relative to uniform rupture velocity. The high degree of similarity of Figures (3.3) to (3.5) suggests that the results of this study are almost insensitive to selected frequency ranges and this allows generalizing the results of three frequency ranges to all frequencies. Taking into account the uncertainties associated with evaluation of average $\log P S A$ changes, Figures (3.6) to (3.8) show that there is no significant increase in the average $\log P S A$ close to areas of rupture velocity change. This means that although EXSIM predicts as increase in spectral levels close to the areas of rupture velocity change, this increase is not significant. EXSIM also predicts high or low spectral levels close to high or low rupture velocity areas, which is less frequently emphasized in the literature. The results of these experiments suggest that although EXSIM differentiates between uniform and variable rupture velocity implementations, the level of changes is insignificant if reasonable ranges of rupture velocities are adopted. In summary, EXSIM results are not sensitive enough to variability of rupture velocity to warrant modifications of EXSIM to consider this effect. 


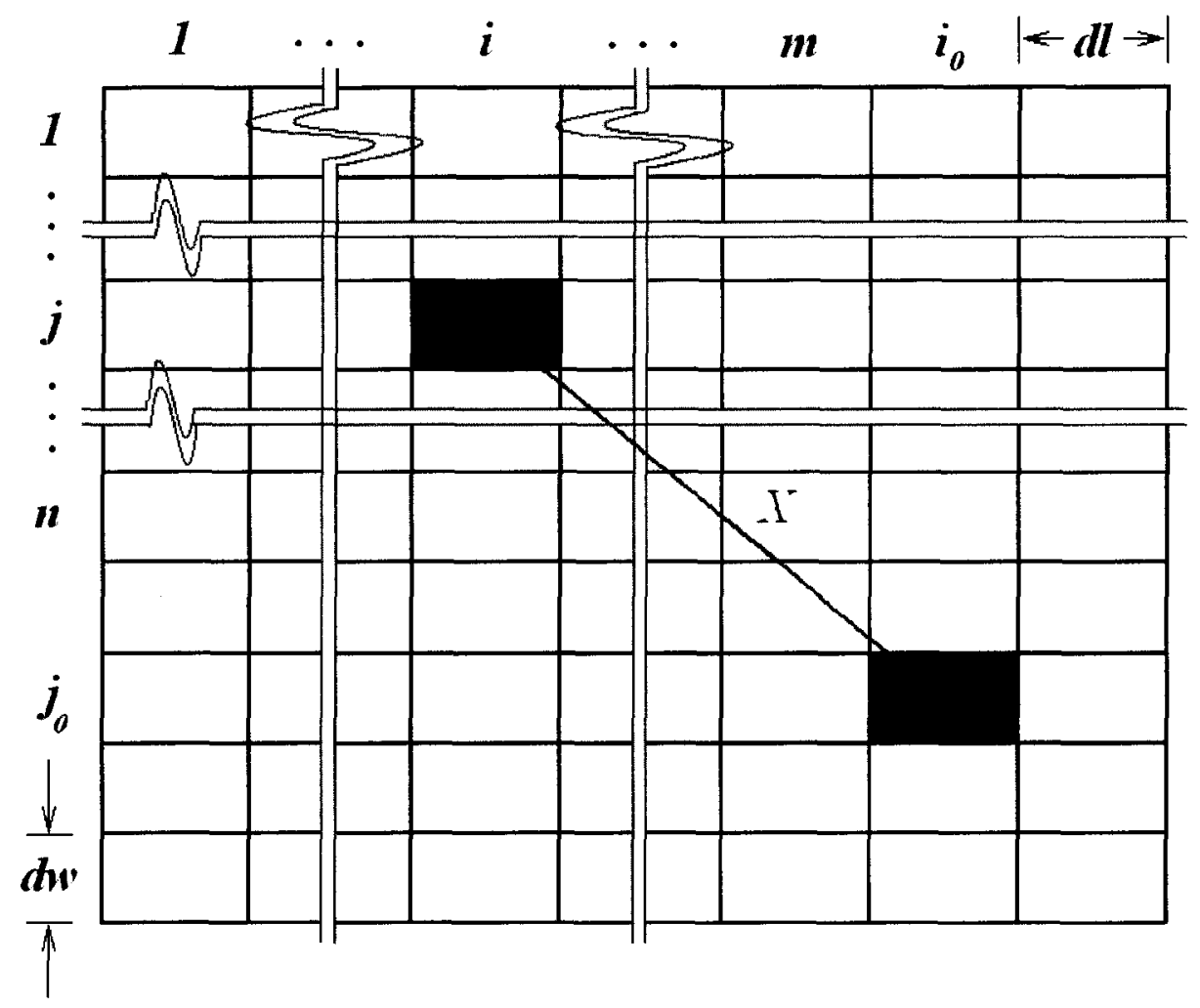

Figure (3.1) Earthquake fault subdivisions into sub-sources illustrating the parameters and geometry for calculating the delay time of sub-source activation when variable rupture velocity applies. The red element is the hypocentral sub-source and green element is the sub-source at column $i$ and row $j$, for which delay time, $\Delta t_{i j}$, is needed. The line connecting the centers of the two elements is shown in pink. A segment of this line that lies in the element at column $m$ and row $n$ is $X\left(m, n, i, j, i_{0}, j_{0}, d l, d w\right)$ and is shown in blue. Rupture velocity $v_{i j}$ is attributed to each sub-source and $\Delta t_{i j}$ is calculated using Equation (3.4). 


\section{Location of Fault and Distribution of Sites for Simulation}

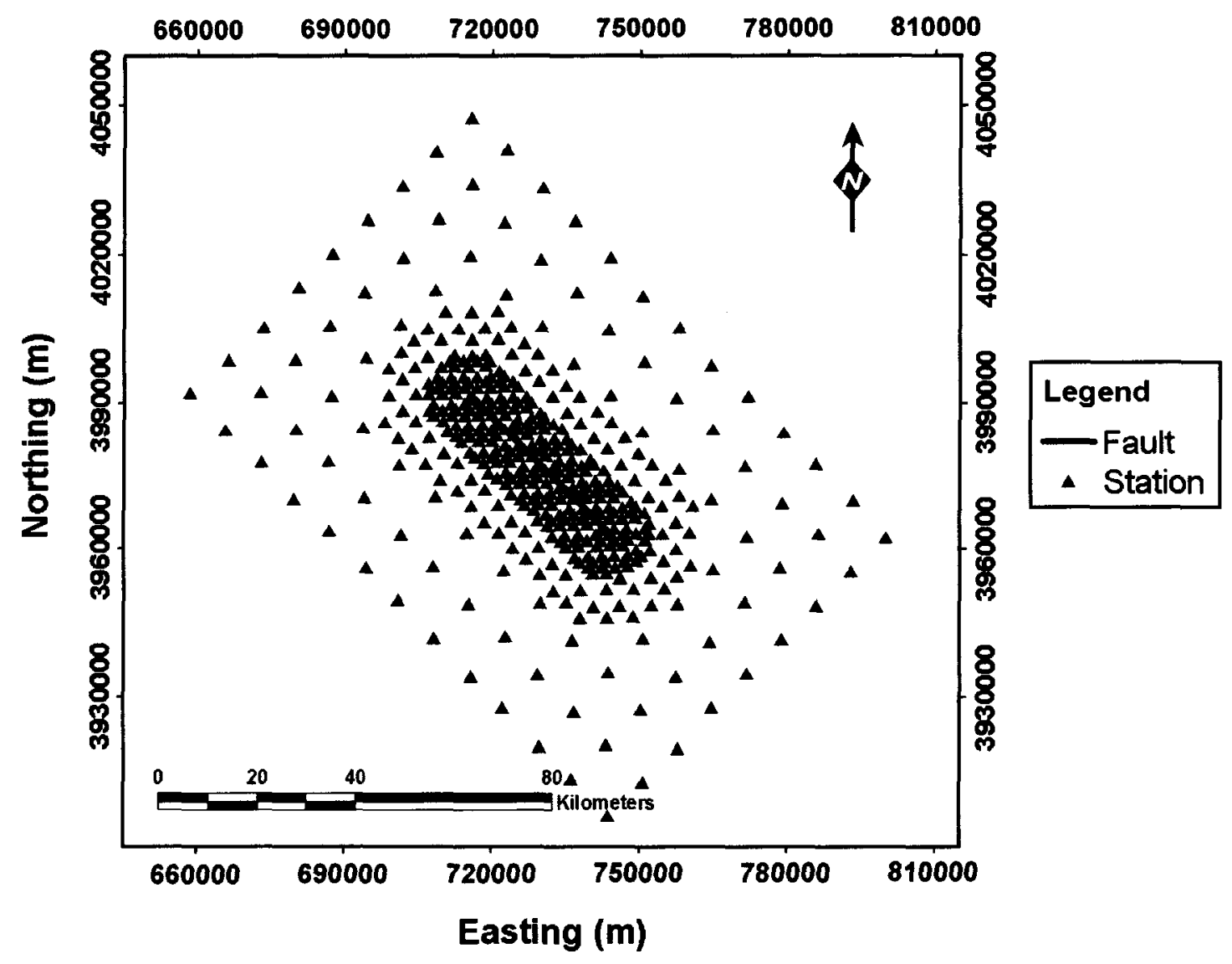

Figure (3.2). Distribution of simulation sites around the trace of the fault for forward modeling in the study of the effect of variable rupture velocity. 
Table (3.1) Common simulation parameters for 441 sites for studying the effect of variable rupture velocity.

\begin{tabular}{|c|c|}
\hline Parameter & Parameter value \\
\hline Fault orientation & Strike $136.9^{\circ}, \operatorname{Dip} 83^{\circ}$ \\
\hline Depth of top & $0 \mathrm{~km}$ \\
\hline Latitude and longitude of fault & $36.047^{\circ} \mathrm{N}, 120.609^{\circ} \mathrm{W}$ \\
\hline Fault dimensions & Length $40 \mathrm{~km}$, Width $13 \mathrm{~km}$ \\
\hline Number of sub-faults & Along length 10 , Along width 3 \\
\hline FFT points, sample interval & $8192,0.02 \mathrm{~s}$ \\
\hline Shear wave velocity, Density & $3.5 \mathrm{~km} / \mathrm{s}, 2.8 \mathrm{~g} / \mathrm{cm}^{3}$ \\
\hline Rupture velocity & $\begin{array}{l}0.8 \times \text { shear wave velocity } \\
\text { Variable }\end{array}$ \\
\hline$Q(f)$ & $180 f^{0.45}$ \\
\hline Moment magnitude & 6.0 \\
\hline Average stress parameter & 50 bars \\
\hline Kappa & 0.035 \\
\hline Geometrical attenuation & If $\mathrm{R}<40, \mathrm{R}^{-1}$, else $\mathrm{R}^{-0.5}$ \\
\hline Sub-source duration & $\propto 0.05 \times$ distance $(\mathrm{sec})$. \\
\hline Windowing function & Saragoni-Hart \\
\hline Amplification function & Boore and Joyner $(1997)$ for $\mathrm{V}_{\mathrm{s} 30}=620 \mathrm{~m} / \mathrm{s}$ \\
\hline Damping & $5 \%$ of critical damping \\
\hline Number of EXSIM iterations & 10 (per site) \\
\hline
\end{tabular}

Table (3.2) The correlation coefficients of Figures (3.3), (3.4), and (3.5). Frequencies correspond to the frequency ranges in which the figures are developed.

\begin{tabular}{cccc}
\hline & $25 \mathrm{~Hz}$ & $2.5 \mathrm{~Hz}-20 \mathrm{~Hz}$ & $0.45 \mathrm{~Hz}-10 \mathrm{~Hz}$ \\
\hline $25 \mathrm{~Hz}$ & 1.0000 & & \\
$2.5 \mathrm{~Hz}-20 \mathrm{~Hz}$ & 0.8654 & 1.0000 & \\
$0.45 \mathrm{~Hz}-10 \mathrm{~Hz}$ & 0.8343 & 0.9027 & 1.0000 \\
\hline
\end{tabular}




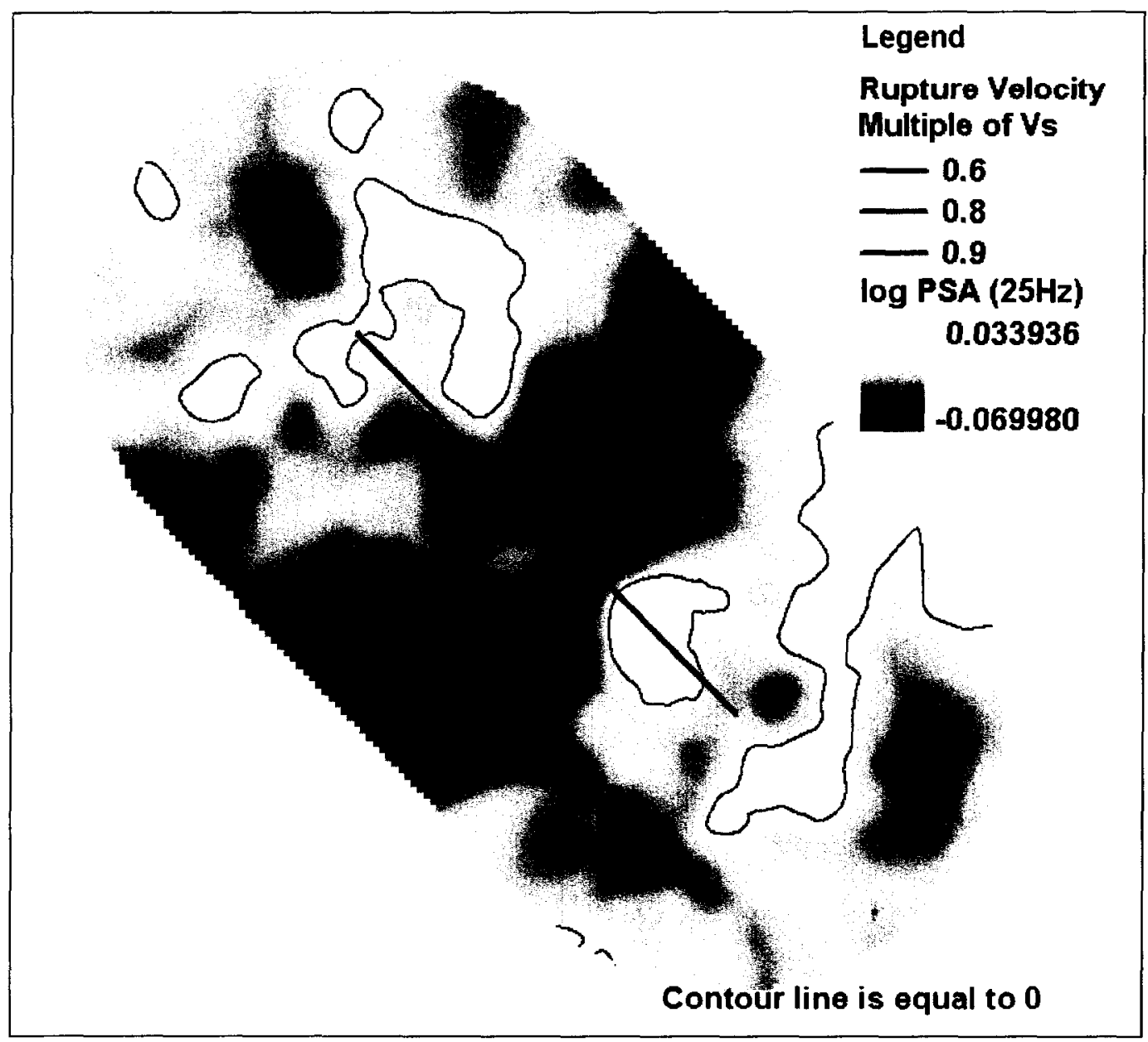

Figure (3.3). The map of the changes of $\log P S A$ values ( $E$-values) at $25 \mathrm{~Hz}$ plotted for $20 \mathrm{~km}$ distance from fault. The variability of rupture velocity on the fault surface is shown by different colors (blue, green, red). Contour lines represent locations with no changes of $\log P S A$ by switching from uniform rupture velocity to variable rupture velocity. 


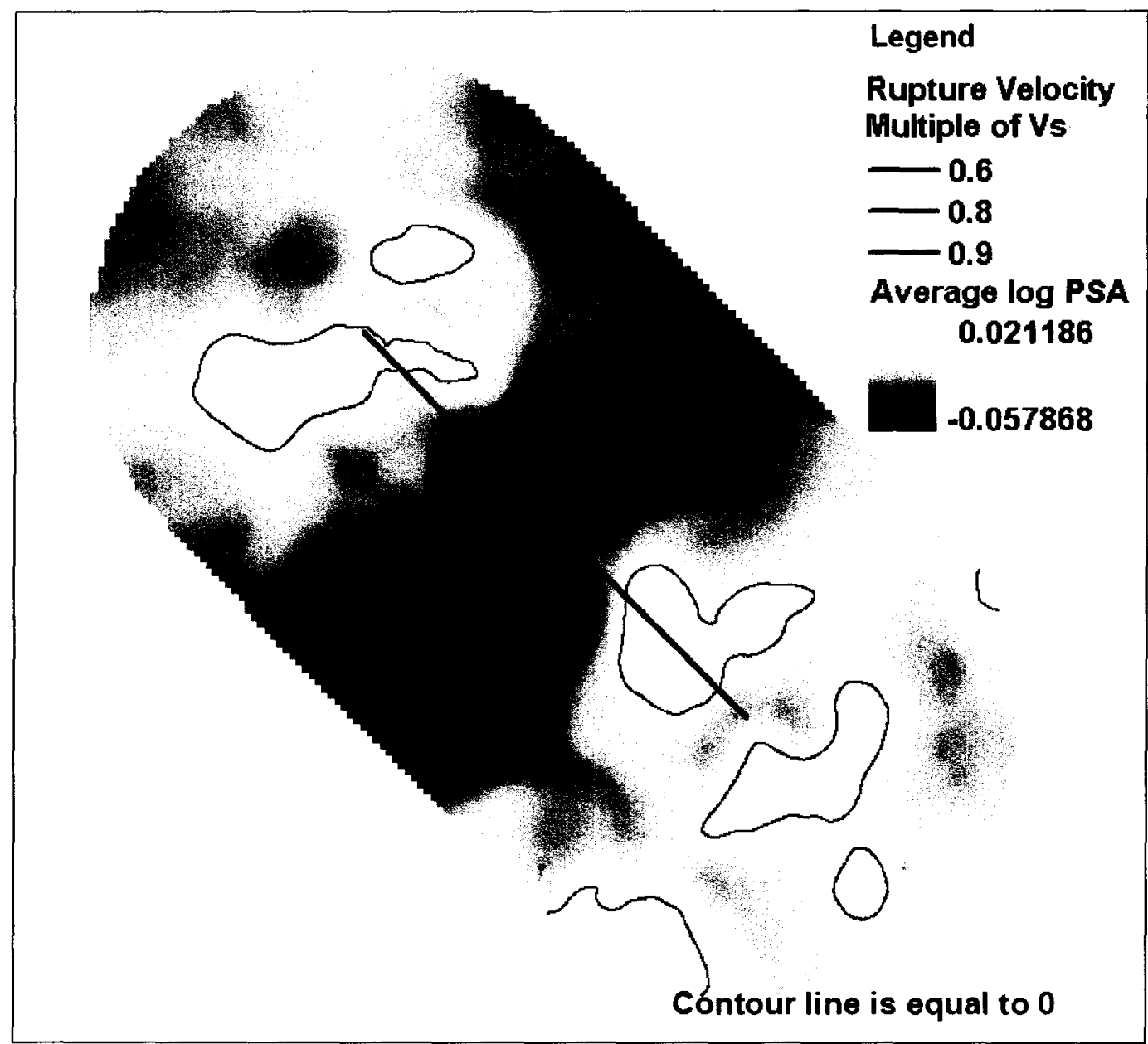

Figure (3.4). Map of changes of average $\log P S A$ values ( $E$-values) in the $2.5 \mathrm{~Hz}$ to $20 \mathrm{~Hz}$ range plotted for $20 \mathrm{~km}$ distance from fault. The variable rupture velocity on the fault surface is shown by different colors (blue, green, red). Contour lines represent locations with no changes of average $\log P S A$ by switching from uniform rupture velocity to variable rupture velocity. 


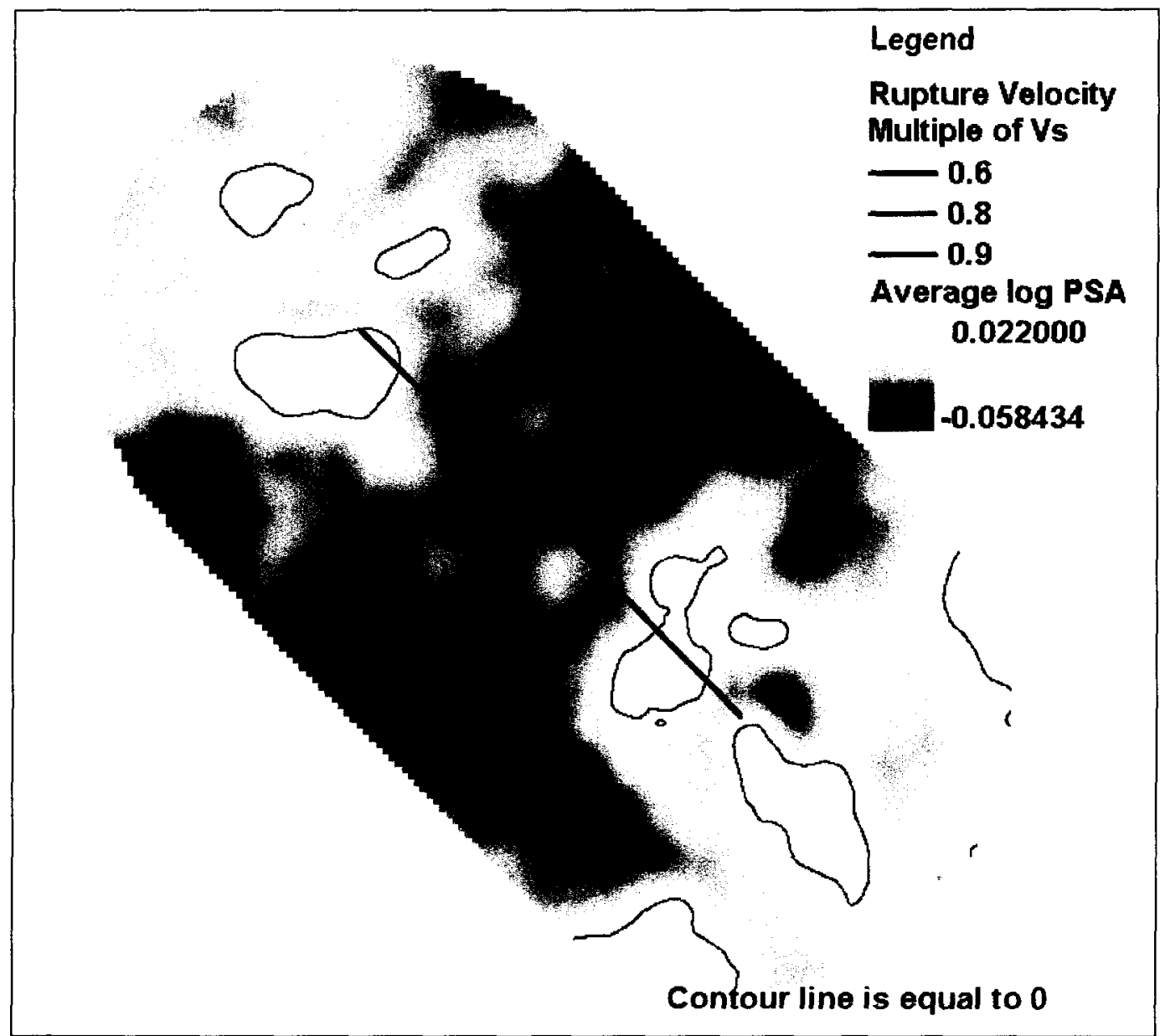

Figure (3.5). Map of changes of average $\log P S A$ values ( $E$-values) in the $0.45 \mathrm{~Hz}$ to $10 \mathrm{~Hz}$ range plotted for $20 \mathrm{~km}$ distance from fault. The variable rupture velocity on the fault surface is shown by different colors (blue, green, red). Contour lines represent locations with no changes of average $\log P S A$ by switching from uniform rupture velocity to variable rupture velocity. 


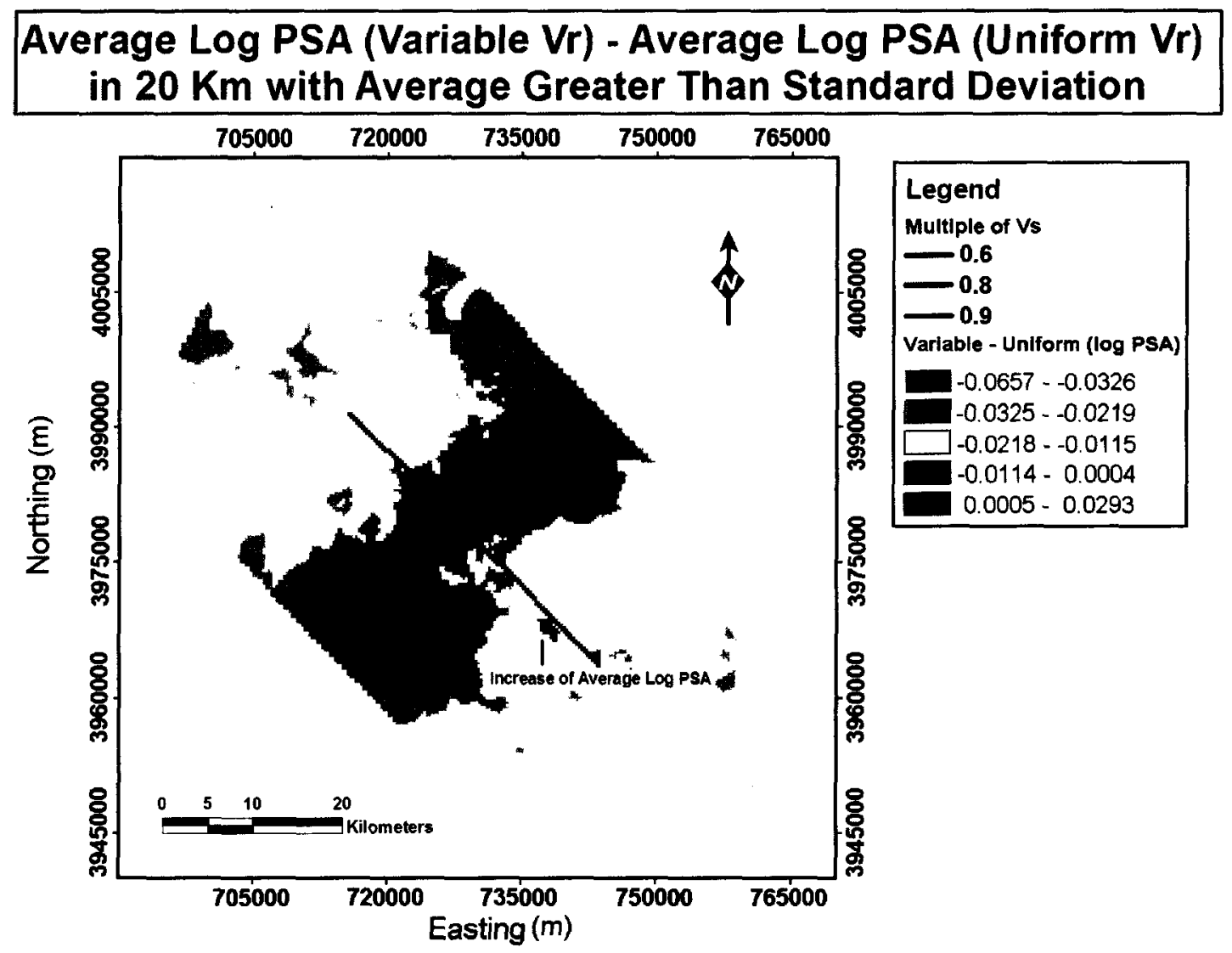

Figure (3.6). Map of significant changes of $\log P S A$ values ( $E$-values) at $25 \mathrm{~Hz}$ plotted for $20 \mathrm{~km}$ distance from fault (it is considered significant if the absolute value of a point is more than the kriging standard deviation value at that point). The variability of rupture velocity on the fault surface is shown by different colors (blue, green, red). There is one spot in which $\log P S A$ increased significantly as a result of switching to variable rupture velocity. 


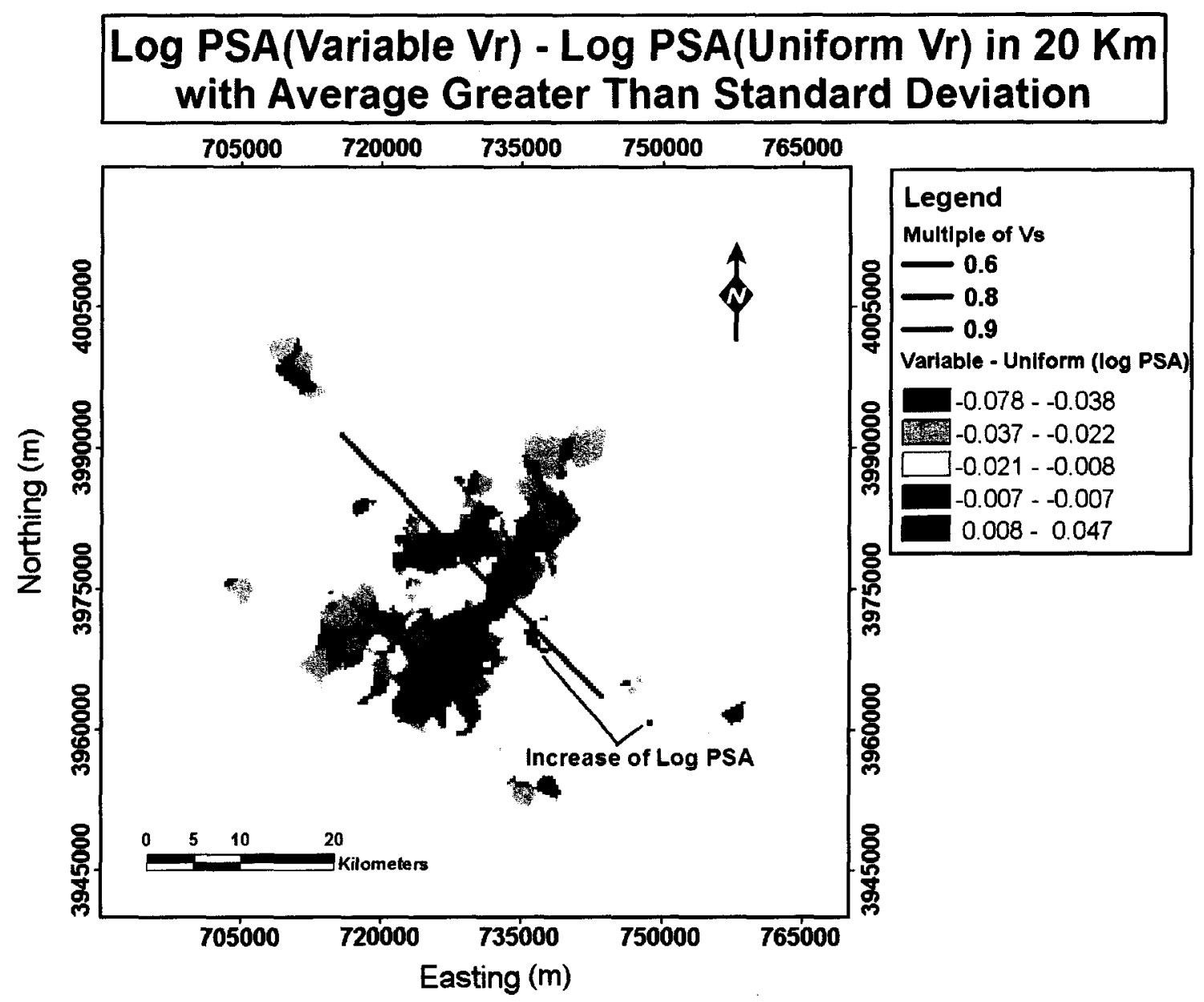

Figure (3.7). Map of significant changes of average $\log P S A$ values ( $E$-values) in the $2.5 \mathrm{~Hz}$ to $20 \mathrm{~Hz}$ range plotted for $20 \mathrm{~km}$ distance from fault (it is considered significant if the absolute value of a point is more than the kriging standard deviation value at that point). The variability of rupture velocity on the fault surface is shown by different colors (blue, green, red). There are two spot in which $\log P S A$ increased significantly as a result of switching to variable rupture velocity. 


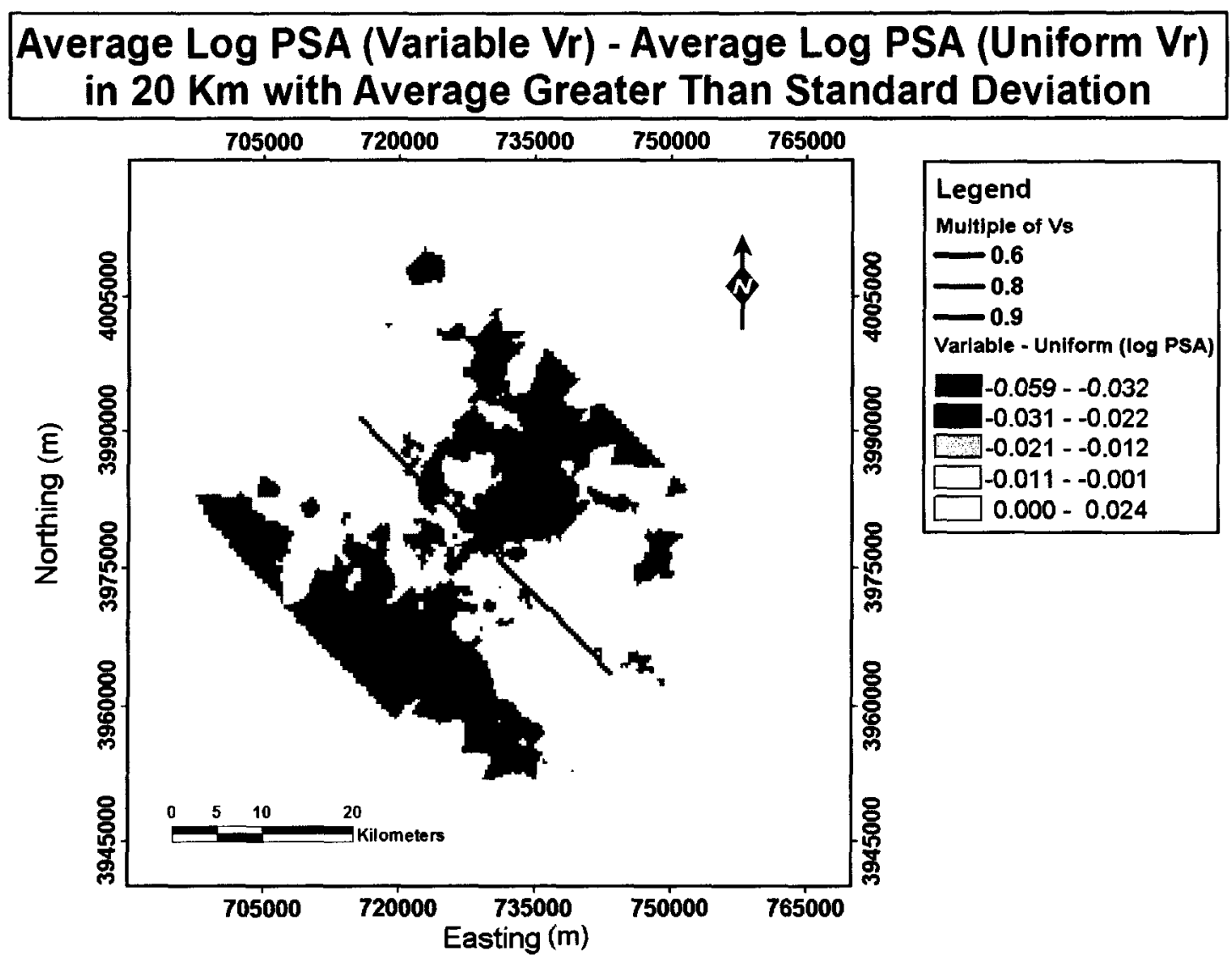

Figure (3.8). Map of significant changes of average $\log P S A$ values (E-values) in the $0.45 \mathrm{~Hz}$ to $10 \mathrm{~Hz}$ range plotted for $20 \mathrm{~km}$ distance from fault (it is considered significant if the absolute value of a point is more than the kriging standard deviation value at that point). The variability of rupture velocity on fault surface is shown by different colors (blue, green, red). 


\subsection{Implementation of variable stress parameter in EXSIM}

The original version of EXSIM assumes that the stress on the fault surface controlling the strength of high frequency radiation is distributed uniformly. I modified EXSIM to enable the modeling of variability of stress parameter across the fault surface. The nonuniform distribution of stress parameter on fault surface may affect the spectral levels of records. For example, Boore (1983) showed that the high frequency spectral level of a point source is proportional to stress raised to the $2 / 3$ power. This section describes the assumptions and formulations applied to introduce the concept of a variable stress parameter distribution to stochastic finite fault modeling through EXSIM. The effect of stress concentrations or asperities (Aki, 1984, on average $\log$ PSA levels is studied. Finally, the effect of variable stress parameter distribution in finite fault modeling with EXSIM is shown to be equivalent to modeling a variable slip distribution.

\subsubsection{Assumptions and formulations of variable stress parameter concept}

There are several constraints to be considered in the modeling of variable stress parameter on the fault surface. The modified EXSIM modeling approach follows the stochastic finite-fault method of Motazedian and Atkinson (2005), except that weights are assigned to sub-sources such that the stress parameter on each sub-source is the product of the weight and the average stress parameter of the fault. It is assumed that the following conditions should be satisfied: (1) the low frequency portion of the spectrum should be independent of stress, because the moment determines the zero-frequency level of the spectrum; (2) as per the Brune source model, the high frequency amplitude level should be proportional to the sub-event stress parameter raised to the power 2/3 (Brune, 1970; Boore, 1983, 2003); (3) if the stress parameter of a sub-source approaches zero, the contribution of that sub-source to the time history should approach zero $\left(\Delta \sigma_{i j} \rightarrow 0 \Rightarrow p_{i j} \rightarrow 0 \Rightarrow P_{i j} \rightarrow 0 \Rightarrow X_{i j}(f) \rightarrow 0\right)$; and (4) if the stress parameter of a sub-source equals the average value for the fault, the sub-source stress correction factor $\left(X_{i j}\right)$ should be unity. 
A functional form that multiplies the spectral components of the $\mathrm{ij}^{\text {th }}$ sub-source and satisfies the above conditions is:

$$
X_{i j}(f)=\frac{1+\frac{f^{2}}{f_{C_{i j}}^{2}}}{1+\frac{f^{2}}{P_{i j} \times f_{C_{i j}}^{2}}}
$$

where $X_{i j}(f)$ is the multiplicative sub-source stress correction factor for a sub-source in the $i^{\text {th }}$ row and $\mathrm{j}^{\text {th }}$ column, $f$ is frequency, $f_{C i j}$ is corner frequency of the sub-source as derived per Motazedian and Atkinson (2005) in Equation (1.19), and $P_{i j}$ is the weighting factor:

$$
P_{i j}=\frac{N p_{i j}^{2 / 3}}{\sum_{i} \sum_{i} p_{i j}^{2 / 3}}
$$

where $N$ is the total number of sub-faults and $p_{i j}$ is the ratio of a sub-source's stress parameter to the average stress parameter of the fault ( $i$ and $j$ are the indexes of the column and row of the sub-source, respectively):

$$
p_{i j}=\frac{\Delta \sigma_{i j}}{\overline{\Delta \sigma}}
$$

where $\Delta \sigma_{i j}$ is the stress parameter of a sub-fault and $\overline{\Delta \sigma}$ is the average stress parameter of the fault. Based on the above correction factors, the time history of the simulated trace will be of the form:

$$
A(t)=\sum_{i, j} \boldsymbol{F}^{-1}\left(X_{i j}(f) \times A_{i j}(f)\right)
$$

where $A(t)$ is the accelerogram generated by the entire fault, $X_{i j}(f)$ is the spectrum stress correction factor calculated in Equation (3.7), $A_{i j}(f)$ is the sub-fault spectrum given by Equation (3.11), and $\boldsymbol{F}^{-1}$ denotes the inverse Fourier transform. The sub-source spectrum is:

$$
A_{i j}(f)=\boldsymbol{F}\left(H_{i j} \times Y_{i j}(t)\right)
$$

where $H_{i j}$ is a normalization factor for the $\mathrm{ij}^{\text {th }}$ sub-source that results from the consideration of the conservation of energy, $Y_{i j}(t)$ is the signal of the ij ${ }^{\text {th }}$ sub-source 
activation based on Equation (1.17), and $\boldsymbol{F}$ is the Fourier transform. The normalization factor $H_{i j}$ is calculated by equating high frequency energies radiated from all sub-sources with total high frequency energy of the earthquake (see Equation (1.20)).

\subsubsection{Stress parameter variability experiments}

Forward modeling experiments are conducted to check the significance of stress parameter variability on the spectral outputs of the modified EXSIM. The $D_{r}$ values for a grid of points around a hypothetical fault of Figure (3.2), selected to have the Parkfield fault geometry, are calculated after simulation. The simulation parameters are those given in Table (3.1). The $D_{r}$ values in these experiments are average log $P S A$ maps for $25 \mathrm{~Hz}$, $2.5 \mathrm{~Hz}-20 \mathrm{~Hz}$, and $0.45 \mathrm{~Hz}-10 \mathrm{~Hz}$ frequency ranges as shown in Figures (3.9), (3.10), and (3.11). The maps of Figures (3.9) to (3.11) are prepared by the kriging interpolation method for $100 \mathrm{~m} \times 100 \mathrm{~m}$ pixel sizes after calculating $D_{r}$ values for the sites of Figure (3.2). In all three cases the same slip and stress parameter distributions are implemented: uniform slip and uniform stress, except for a patch in the southeast end of the fault having 5 times larger than average stress (it is shown in yellow).

As expected, the high $D_{r}$ values are concentrated around the high stress parameter end of the fault. Figures (3.9) to (3.11) are very similar and depict almost the same pattern of $D_{r}$. (average $\log P S A$ ) in the area, suggesting the effect is similar over a wide range of frequencies. To show the degree of similarity of these three maps in a more quantitative way, the correlation coefficients of the three maps are presented in the Table (3.3). The correlation coefficients are based on the values of 18108 interpolated points in each map. The strong correlation coefficients between these maps suggest interchangeability of the used frequency ranges. So if $0.45 \mathrm{IIz}-10 \mathrm{Iz}$ is used for calculating the average Log PSAs, the overall picture will not be that much different from that which would be obtained by averaging other frequency ranges, as long as calculations are for frequencies above the earthquake corner frequency. I conclude that a concentration of the stress parameter in part of the fault, as modeled by the modified EXSIM, makes significant changes in the 
spatial distribution of predicted ground motions; the spatial pattern is not sensitive to frequency range, for frequencies above the event corner frequency.

\subsubsection{Stress parameter variability versus slip variability}

Both slip and stress parameter distributions affect the spectral levels of simulated traces for stations around a fault; the equivalence of these two parameters is explored in this section. Equations (3.7) to (3.11) and Figures (3.9) to (3.11) show that high frequency spectral levels are affected by the stress parameter distribution of sub-sources. If all parameters of a simulation remain identical for all sub-sources, except for the stress parameter, the closer is the location of a site to a high stress sub-source, the higher will be the average spectral level $\left(D_{r}\right.$ level).

In this section, I perform an experiment to compare the effects of slip and stress parameter distributions. Two sets of simulations are carried out for the array of stations in Figure (3.2). The simulation parameters are those given in Table (3.1). The fault is subdivided into 30 sub-sources, ten along length and three along width, similar to that of Figure (2.1). The stress parameter of a sub-source is multiplied by five in the first simulation set, while the slip of the same sub-source is multiplied by five in the second set. Predicted ground motions for the two sets are then compared.

In the first set of simulations slip is assumed to be uniform on the fault surface. The stress is assumed to be identical for all sub-sources except for one in the middle row at the southeast end of the fault, which has a value five times larger than average. The average $\log P S A \mathrm{~s}$ of the simulations ( $D_{r}$ values) in the $0.45 \mathrm{~Hz}$ to $10 \mathrm{~Hz}$ frequency range are calculated for the 441 sites of Figure (3.2), interpolated for 8200 points in the area using the kriging method, and plotted in Figure (3.12). In the second set of simulations a similar approach is adopted, with variable slip. In this case the stress parameter is assumed to be uniformly distributed on the fault surface. The slip is assumed to be identical for all subsources except for one in the middle row in the southeast end of fault, which has a value 
five times larger than average. The $D_{r}$ values are calculated as in the first simulation and plotted in Figure (3.12).

The correlation coefficients of 8200 interpolated average $\log P S A \mathrm{~s}$ ( $D_{r}$ values) of the two sets of simulations is $0.9946 ; D_{r}$ values in both sets are in the range of 1.4 to 2.6. The correlation coefficient and the range of $D_{r}$ values of the two sets of simulations suggest that both slip and stress affect the spatial distribution of average $\log P S A \mathrm{~s}$ in a similar way, and to a similar degree.

The effect of variable slip seems to be slightly larger than that of variable stress. Figure (3.13) shows an empirical cumulative distribution function of average $\log P S A$ s for the two sets of simulations. It is evident that average $\log P S A$ s resulting from slip variability are larger than those of stress variability. A Kolmogorov-Smirnov test shows that the two empirical cumulative distributions are different at almost any significance level. This difference is suggesting that slip variability has a statistically more significant effect than stress variability, considering average $\log P S A$ as the measurement criterion. So slip and stress parameter distributions have similar effects on simulation results, but slip variability has a slightly stronger effect than stress parameter.

This difference can be explained by the way the increase of moment and the increase of stress parameter affect the high frequency spectral level of a sub-source. If the slip of a sub-source is multiplied by some factor then the moment of the sub-source increases by the same factor (Equation (1.18)), and a proportional change affects the spectrum of the sub-source (Equation (1.5)). Before the calculation of the overall time history, the normalization factor " $\mathrm{H}$ " modifies the effect of the multiplicative factor (Equation (1.17)). If the same factor multiplies the stress level of the same sub-source, its $2 / 3$ power will affect the high frequency level of that sub-source (Equation (3.8)). This explains the reason for the overall similarity and slight differences in the size of the slip and stress variability effects.

Since the high frequency components of accelerograms recorded close to high stress parameter sub-sources are amplified, theoretically it is possible to use the records of 
nearby stations to infer the stress parameter distribution on the fault surface during an earthquake from variable-stress stochastic finite-fault modeling. If the slip distribution on the fault surface is not known, the approach would be to assign a uniform slip to the model and assume all variability results from stress parameter variability on the fault surface.

\subsection{Note on Equation (3.8)}

After preparation of this thesis it was discovered that the correct form of Equation (3.8) should be:

$$
P_{i j}=\left(\frac{N p_{i j}}{\sum_{i} \sum_{j} p_{i j}}\right)^{2 / 3}
$$

where all the parameters are as explained before. This correction makes a subtle change to the values of the weighting factors, $P_{i j}$. To check the effect of this change, I simulated a fault with 30 sub-sources, having a random distribution of $p$ values, following either a normal or uniform distribution. In both cases, the calculated $P_{i j}$ values are affected by only a few percent by the change in formula.

Considering that the $P_{i j}$ values are multiplied by sub-sources' spectra at the high frequency end of spectra, while a smaller factor is multiplied at lower frequencies, it is clear that this correction will not change simulation results significantly. I conclude that the stochastic finite fault modeling method should be corrected to apply Equation (3.12) instead of Equation (3.8), but the overall simulation results will not change and consequently the developed inversion tool will not be affected. 


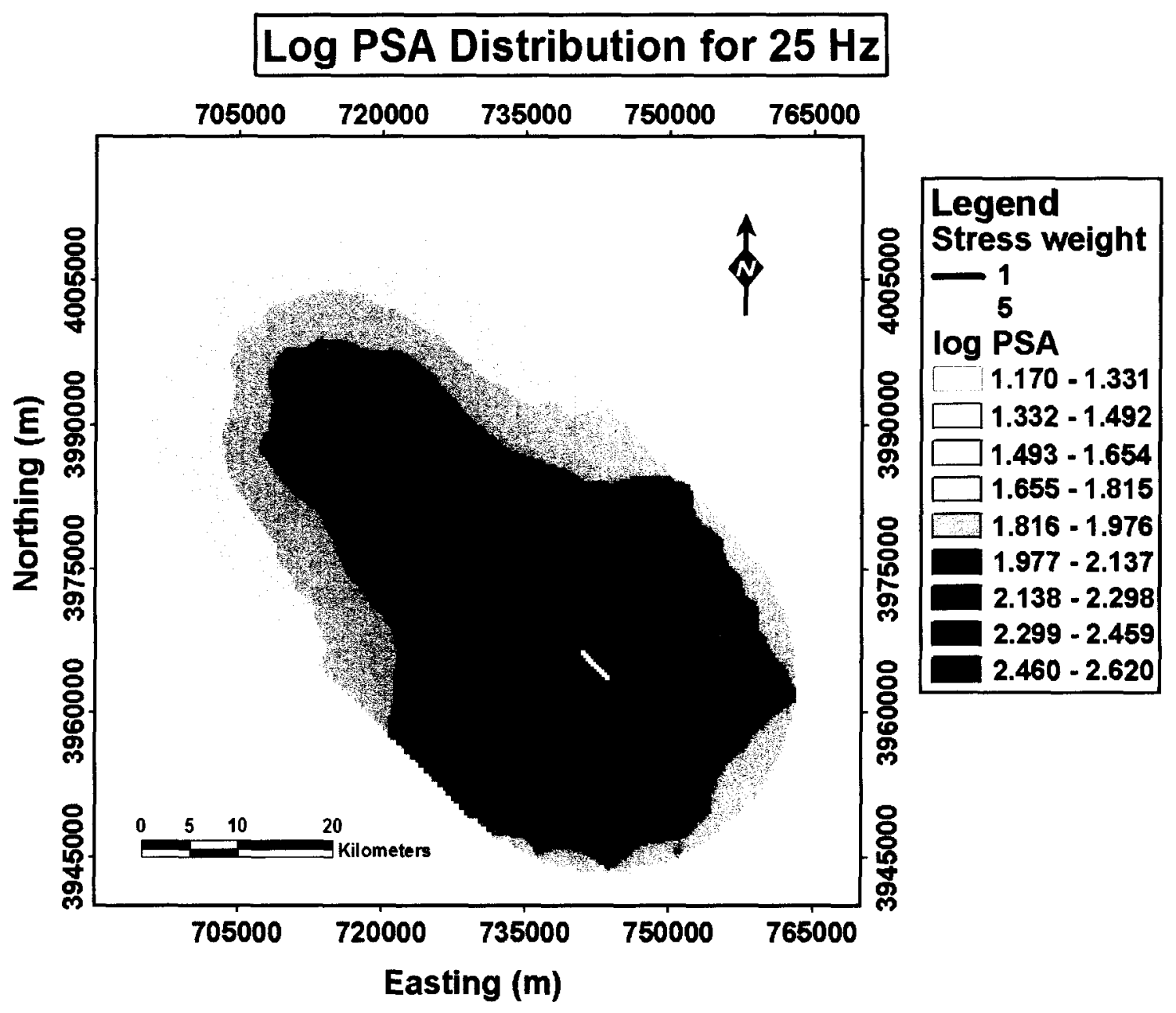

Figure (3.9). Map of $\log P S A$ values ( $D_{r}$ values) at $25 \mathrm{~Hz}$ plotted for $20 \mathrm{~km}$ distance from the fault. The modeled fault has uniform slip and stress parameter distribution except for one sub-source in middle row at the southeast end of the fault that has stress parameter equal to 5 times that of the other sub-sources (its location is shown with yellow color). The high $D$, values are concentrated around the area of high stress parameter centers. 


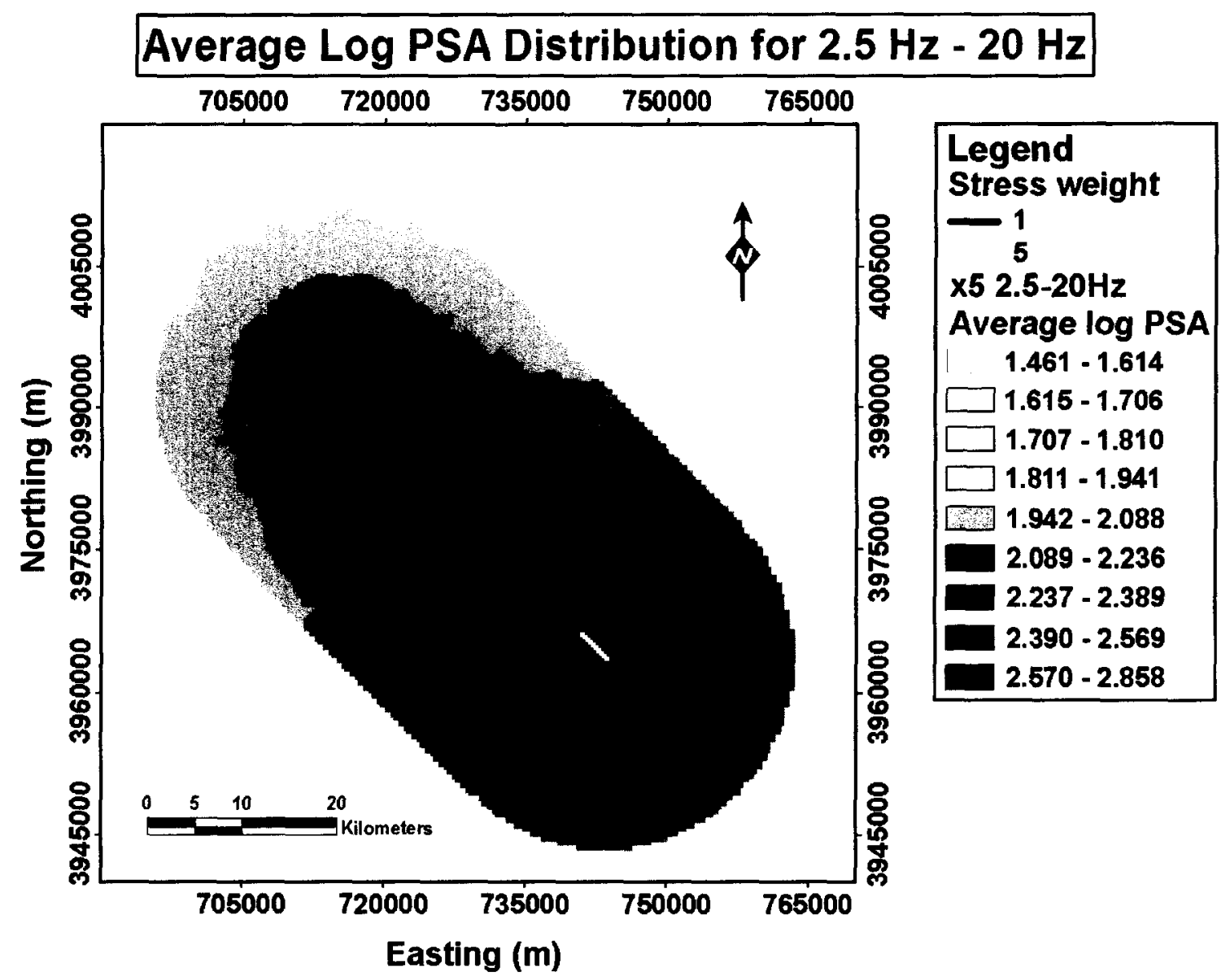

Figure (3.10). The map of average $\log P S A$ values ( $D_{r}$ values) in the $2.5 \mathrm{~Hz}$ to $20 \mathrm{~Hz}$ plotted for $20 \mathrm{~km}$ distance from the fault. The modeled fault has uniform slip and stress parameter distribution except for one sub-source in middle row at the southeast end of the fault that has stress parameter equal to 5 times that of the other sub-sources (its location is shown with yellow color). The high $D_{r}$ values are concentrated around the area of high stress parameter centers. 


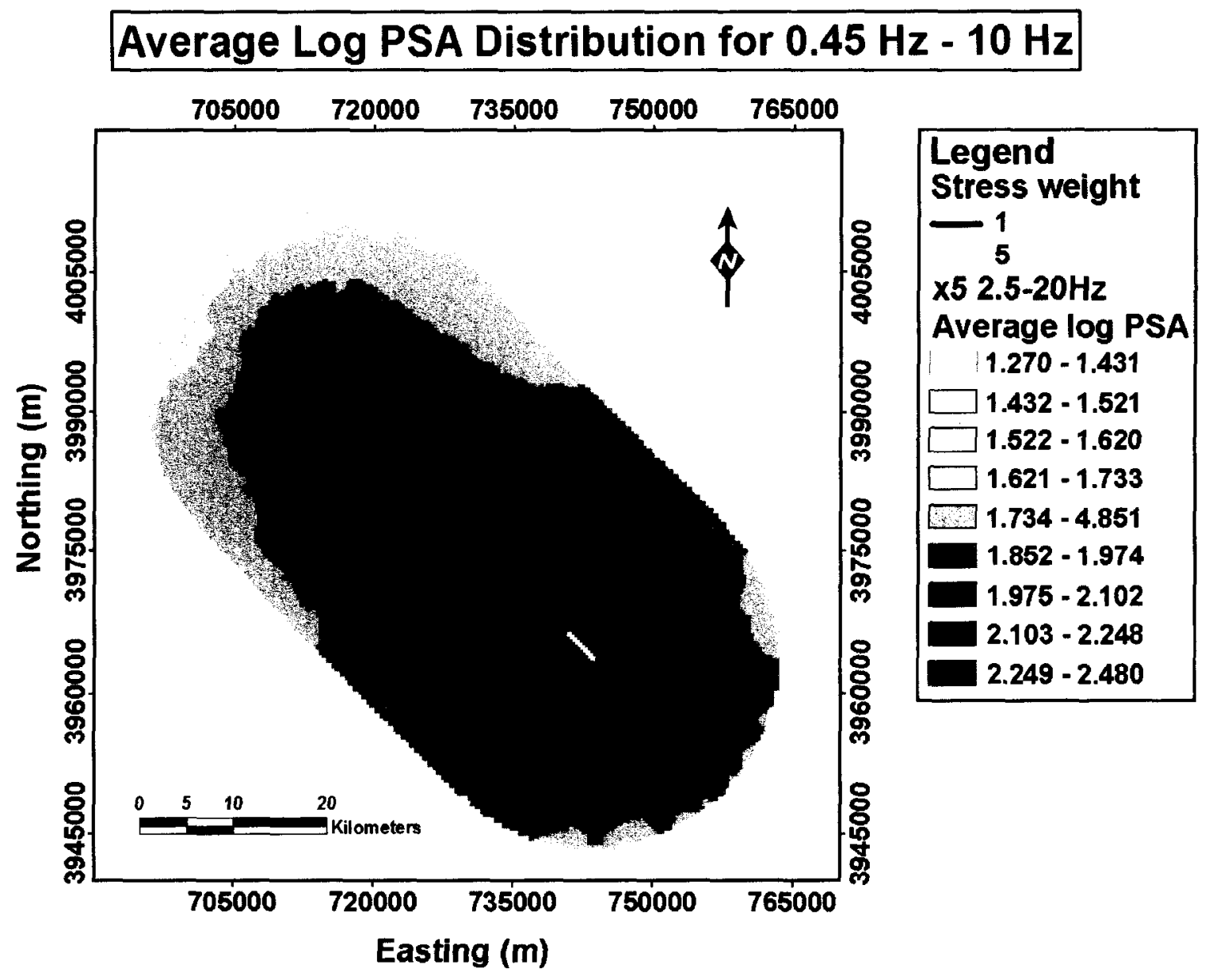

Figure (3.11). The map of average $\log P S A$ values $\left(D_{r}\right.$ values) in the $0.45 \mathrm{~Hz}$ to $10 \mathrm{~Hz}$ plotted for $20 \mathrm{~km}$ distance from the fault. The modeled fault has uniform slip and stress parameter distribution except for one sub-source in middle row at the southeast end of the fault that has stress parameter equal to 5 times that of the other sub-sources (its location is shown with yellow color). The high $D_{r}$ values are concentrated around the area of high stress parameter centers. 


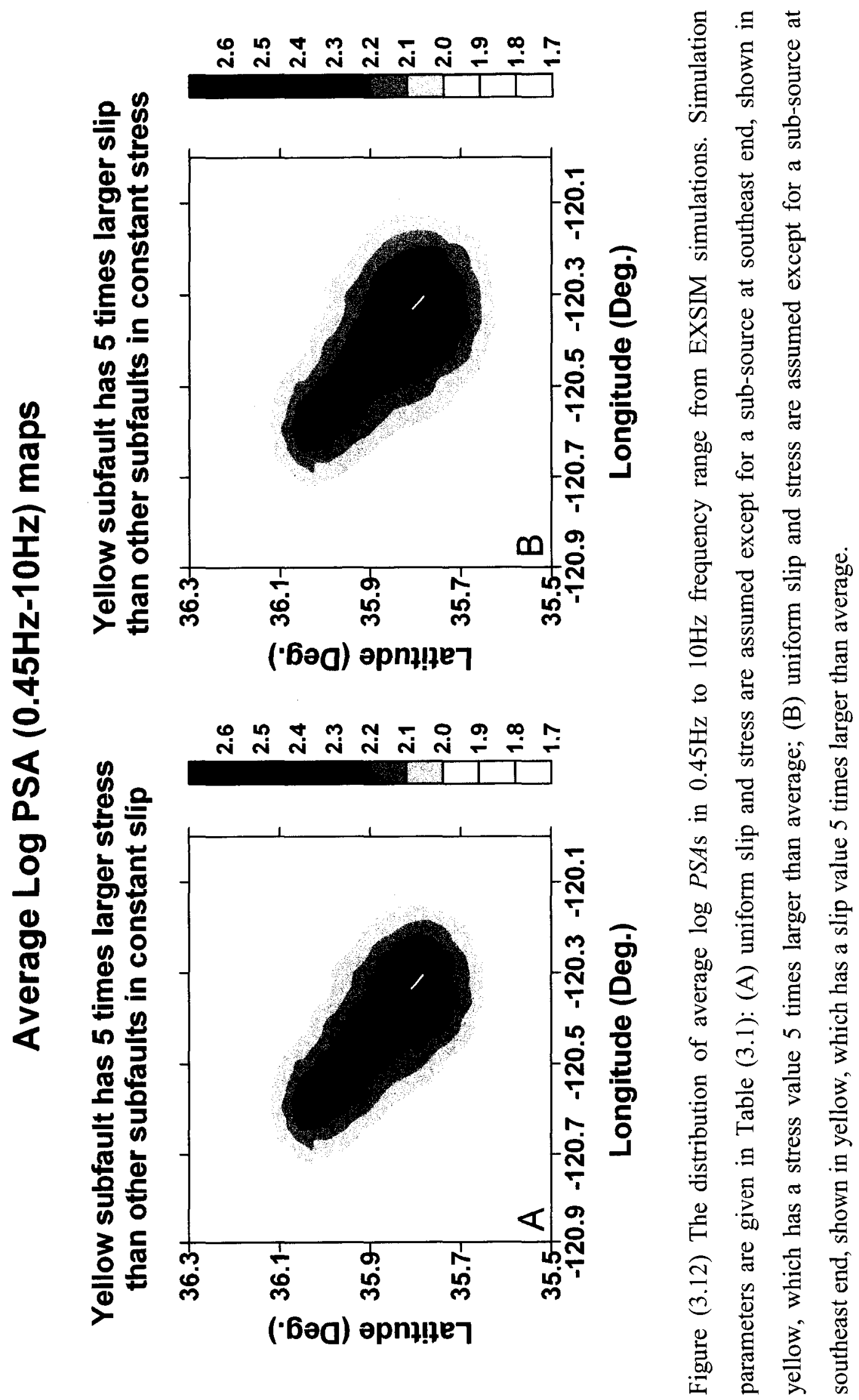


Table (3.3) The correlation coefficients of Figures (3.9), (3.10), and (3.11). Frequencies correspond to the frequency ranges in which the figures are developed.

\begin{tabular}{cccc}
\hline & $25 \mathrm{~Hz}$ & $2.5 \mathrm{~Hz}-20 \mathrm{~Hz}$ & $0.45 \mathrm{~Hz}-10 \mathrm{~Hz}$ \\
\hline $25 \mathrm{~Hz}$ & 1.0000 & & \\
$2.5 \mathrm{~Hz}-20 \mathrm{~Hz}$ & 0.9956 & 1.0000 & \\
$0.45 \mathrm{~Hz}-10 \mathrm{~Hz}$ & 0.9802 & 0.9874 & 1.0000 \\
\hline
\end{tabular}

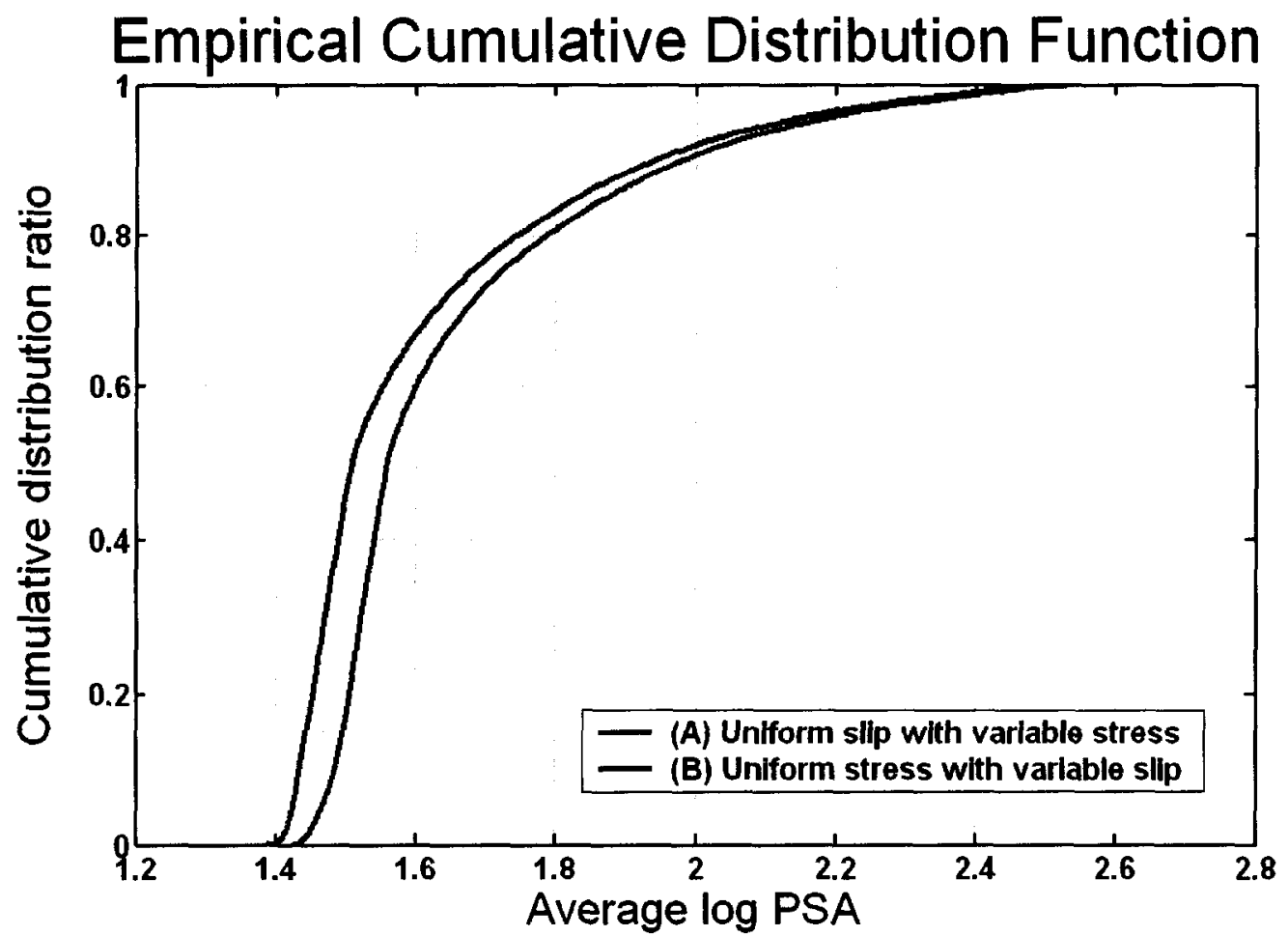

Figure (3.13) The empirical cumulative distribution function of the average log $P S A$ s of 8200 points in Figure (3.12). Blue line corresponds to uniform slip and non-uniform stress parameter scenario in Figure (3.12.A). Red line corresponds to uniform stress parameter and non-uniform slip scenario in Figure (3.12.B). 


\section{Chapter 4: Inversion program development and performance}

\subsection{Introduction}

The improved version of EXSIM enabling variable stress parameter distribution modeling is incorporated with an analytical inversion algorithm. The purpose of this development is to make a tool for inverting the response spectra of records to deduce the stress parameter distribution on the earthquake causative fault surface. In this chapter, a formulation for joining the EXSIM forward modeler with an inversion algorithm is developed, and the corresponding objective functions are introduced. The effects of the geometrical parameters of source and site distributions and EXSIM modeling parameters on the performance of the developed inversion tool are studied. Then an algorithm is developed to improve the performance of the developed inversion tool. Finally the response spectra of M6.0, 2004 Parkfield earthquake records are inverted to derive the stress parameter distribution on the earthquake fault surface and the results are compared to the slip distribution results of other researchers.

The sensitivity of the response spectra of simulated signals to stress parameter distribution, as established in previous chapters, allows development of an inversion tool to derive the stress parameter distribution on the fault surface, based on the response spectra of available records. The inversion problem is a multi-parameter non-linear one, where the stress parameters of sub-sources are the parameters; it is solved iteratively by the combined least squares and gradient estimation methods of Levenberg-Marquardt (Marquardt, 1963; Lee and Stewart 1981; Draper and Smith 1998). The measure of error, or the objective function, in the inversion process is the difference between the observed and simulated logarithm of response spectra, averaged over the high frequency part of the spectrum. This approach first assigns uniform slip and stress parameters to the fault, using the Motazedian and Atkinson (2005) simulation method (EXSIM), and finds their optimum average values for a specific event (based on modeling of the recorded ground motions). Then by keeping the slip uniformly distributed, the inversion program searches for the stress parameter distribution that provides the best possible match of the response 
spectra of simulations to the response spectra of observations, at high frequencies. The outcome of this approach might be leakage of some slip (moment) effect into stress space, since there is no exact boundary between the low and high frequency spectral ranges. Because the high-frequency spectral level is mainly affected by the stress parameter, the slip (or moment) leakage issue is considered to be unimportant. So the inversion is carried out in an iterative way to minimize the measure of error in each step using a hybrid approach that includes both enumerative (grid search) and LevenbergMarquardt methods.

The effects of the following parameters on the performance of the developed inversion tool are studied: dip angle of the fault, the distribution of stations around the fault, the distribution and sizes of asperities on the fault, and the number of EXSIM trials used to define average motions. The effect of the initial guess of parameters on the inversion process is studied too. These parameters are selected because they have significant effects on the EXSIM simulations and/or inversion performance.

Other tests are also carried out to check the performance of the inversion tool and an improvement is applied that accelerates the inversion process. In general, inversion processes do not result in unique outcomes and the solutions are reached via iterative refinement of an initial guess (of parameter values). The appropriate choices of initially guessed values for parameters may result in a fast inversion process with reasonable results for some algorithms (Tarantola, 1987; 2005; Lee and Stewart, 1981; Menke, 1984). The chosen analytical inversion algorithm, Levenberg-Marquardt (Marquardt. 1963; Lee and Stewart 1981; Draper and Smith 1998), is less sensitive to the correctness of the initial guess than other algorithms. This algorithm generally converges to reasonable results from an un-informed initial guess quickly, and this characteristic of the Levenberg-Marquardt approach is checked in this study. These properties and corresponding tests further justify the implementation of Levenberg-Marquardt as the main inversion algorithm in this study. A study is conducted to show the evolution of errors in successive inversion iterations. Based on knowledge gained from these tests and 
experiments, an algorithm is designed so that when linked to the inversion tool it accelerates the inversion process.

The M6, 2004 Parkfield earthquake data are inverted to obtain the stress parameter distribution, and these results are compared to slip distribution results of other research studies. The Parkfield area had been expecting the occurrence of an M6 earthquake since 1987. Consequently a large number of seismometers were distributed in an area close to the fault to capture very near-source ground-motion data upon occurrence of the expected earthquake. The M6 Parkfield earthquake occurred in September 2004, providing the scientific community with a large number of earthquake records at close distances to the fault. The close vicinity of the recording stations to the rupture and their large number provide ideal data for testing the performance of the developed inversion tool with real data. The inversion program results are compared with slip distribution results of other researchers and, as I will show, there is considerable similarity between them. It is a reasonable assumption to consider shear stress and shear strain as proportional quantities. Thus similarity of stress parameter distribution from this study with slip distribution of other studies can be considered as validation of the inversion solutions of this study.

\subsection{Development of inversion tool for stress parameter distribution}

This section describes the inversion tool for finding the best stress parameter distribution on the fault surface. First, we introduce the error measures used in the formulation of the inversion process. Then we describe the approach to inverting average log PSA residuals (observed $\log$ PSA - simulated $\log$ PSA) to obtain the stress parameter distribution on the fault surface. We demonstrate an inversion short cut that is based on assuming a functional form for the objective function to be minimized, and find its coefficients empirically. The rationale for the short cut is to reduce the number of EXSIM simulations (and the processing time) necessary for the construction of the Jacobian matrix (Lee and Stewart, 1981) in each iteration of the inversion. 


\subsubsection{Error measurements to evaluate model performance}

The inversion process used in this study finds an optimum stress parameter distribution on the fault surface that minimizes the difference between response spectra (PSAs) of observed and simulated accelerograms. These differences are defined by error equations. Two types of error measures are used in this study, both of which utilize the logarithm of PSA ordinates to avoid potential biases arising from pronounced local effects that may occur at some stations. The first measure, $\Xi$, is the average value of $\Xi_{j}$ over all stations, where $\Xi_{\mathrm{j}}$ is the average absolute error of the $\log$ PSA ordinates of the simulation, with respect to the observation at station $\mathrm{j}$ :

$$
\Xi_{j}=\frac{1}{N} \sum_{i=1}^{N}\left|\log P S A\left(O_{i j}\right)-\log P S A\left(S_{i j}\right)\right|
$$

where $\operatorname{PSA}\left(S_{i j}\right)$ is the $5 \%$-damped pseudo-acceleration ordinate of a simulated signal at the $\mathrm{i}^{\text {th }}$ frequency for the $\mathrm{j}^{\text {th }}$ station, $P S A\left(O_{i j}\right)$ is the corresponding PSA ordinate of observed signal, and $N$ is the total number of frequencies. Averaging over $K$ stations results in:

$$
\Xi=\frac{1}{K} \sum_{j=1}^{K} \Xi_{j}
$$

The reason for separating the $\Xi$ calculation into two equations is that the numbers of spectral frequencies $(N)$ are not equal for all stations. This measure is used in the beginning of any inversion iteration to find the appropriate average stress parameter value.

An alternative definition of average error is $\Xi_{i}$, where instead of summing error over frequencies as in Equation (5), it is done over stations. The result is an average error spectrum:

$$
\Xi_{i}=\frac{1}{K} \sum_{j=1}^{K}\left|\log P S A\left(O_{i j}\right)-\log P S A\left(S_{i j}\right)\right|
$$

This measure is used to demonstrate how the error evolves as a function of frequency during the inversion; its implementation will be shown later. 
The second error measure, $\Theta$, is an average of square values of $\varepsilon_{j}$ over all stations, where $\varepsilon_{\mathrm{j}}$ is the average error of $\log P S A$ ordinates of the simulation compared to the observation over a specified frequency range at station $\mathrm{j}$. Calculation of $\varepsilon_{j}$ is performed for frequencies higher than double the earthquake corner frequency, between frequency numbers $N_{l}$ to $N_{2}$ as:

$$
\varepsilon_{j}=\frac{-1}{\left(N_{2}-N_{1}+1\right)} \sum_{i=N_{1}}^{N_{2}}\left(\log P S A\left(O_{i j}\right)-\log P S A\left(S_{i j}\right)\right)
$$

where $N_{l}$ and $N_{2}$ are the lower and upper ordinate number of the considered frequency range, respectively. The lower frequency $\left(N_{l}\right)$ is assumed to be about twice the value of the fault's corner frequency, while the upper frequency $\left(N_{2}\right)$ is the maximum frequency available in the simulations and records. Our approach for selection of $N_{2}$ was to choose the smallest of the maximum available frequencies among all records, which makes the programming easier. Forward modeling experiments of chapter three show that the average $\log P S A \mathrm{~s}$ are not sensitive to the choice of frequency range; for example, if we restrict consideration to just very high frequencies (say, 4 times the corner frequency instead of 2), we obtain very similar results. The negative sign in Equation (4.4) is for consistency with forthcoming equations. Then:

$$
\Theta=\frac{1}{K} \sum_{j=1}^{K} \varepsilon_{j}^{2}
$$

The need for defining two sets of error measures arises due to different optimization approaches for the determination of the average stress parameter on the fault, and the stress parameter distribution of sub-faults. The determination of average stress parameter follows an enumerative optimization method. In this method, combinations of possible parameters are searched over a grid of parameter values so as to result in a minimum objective function value. $\Xi$ is a good candidate for the objective function; parameter values providing the minimum $\Xi$ value are the best choice for all stations and all frequencies. The stress parameter distribution determination follows a calculus-based optimization method (Goldberg 1988). In this method, we need the error at each station as well as its sign. The measure $\varepsilon_{\mathrm{j}}$ is a good candidate for this purpose and meets these 
conditions. As shown in the next section the inversion tool formulation is based on the minimization of $\Theta$.

\subsubsection{Formulation of inversion tool}

The formulation of multivariable nonlinear inversion in seismology is a classic in the literature (Aster et al. 2005, Gubbins 2004, Menke 1989, Lee and Stewart 1981, Tarantola 2005, 1987, Parker 1994). We follow the Lee and Stewart (1981) formulation, using the Levenberg-Marquardt inversion algorithm (Marquardt 1963). This method is selected because it combines beneficial aspects of Gauss-Newton and gradient methods, while avoiding some of their weaknesses. The advantages of the selected approach are: (i) the solution converges quickly, so we will be able to avoid many additional EXSIM simulations; (ii) in many cases an uninformed standard guess works well, so that there is a high chance of a converging solution being found for an initial guess based on a uniform stress parameter distribution. This property of the LM algorithm makes inclusion of additional information for an appropriate initial guess unnecessary.

The iterative optimization equation is:

$$
\delta(\Delta \sigma)=-\left[A^{T} A+\lambda I\right]^{-1} A^{T} \varepsilon
$$

where $\delta(\Delta \sigma)$ is the stress parameter adjustment vector and $\varepsilon$ is the residual vector obtained over a range of frequencies for the recording stations; its elements are calculated using Equation (4.4). $A$ is the Jacobian matrix, $\lambda$ is the Levenberg-Marquardt adjustable parameter, and $I$ is the identity matrix. All elements refer to the current iteration. The Jacobian matrix is calculated using the following equation:

$$
A=\left[\begin{array}{cccc}
\frac{\partial \varepsilon_{1}}{\partial(\Delta \sigma)_{1}} & \frac{\partial \varepsilon_{1}}{\partial(\Delta \sigma)_{2}} & \cdots & \frac{\partial \varepsilon_{1}}{\partial(\Delta \sigma)_{m}} \\
\frac{\partial \varepsilon_{2}}{\partial(\Delta \sigma)_{1}} & \frac{\partial \varepsilon_{2}}{\partial(\Delta \sigma)_{2}} & \cdots & \frac{\partial \varepsilon_{2}}{\partial(\Delta \sigma)_{m}} \\
\vdots & \vdots & \ddots & \vdots \\
\frac{\partial \varepsilon_{n}}{\partial(\Delta \sigma)_{1}} & \frac{\partial \varepsilon_{n}}{\partial(\Delta \sigma)_{2}} & \cdots & \frac{\partial \varepsilon_{n}}{\partial(\Delta \sigma)_{m}}
\end{array}\right]
$$


Each element of the Jacobian matrix gives the variation of the residual at a station, per unit variation of one sub-fault's stress parameter.

Initially, a guessed value of the stress parameter distribution is assumed; the uniform stress parameter distribution with the best average value for the fault is a good choice. Then the values of Jacobian matrix elements, errors at each station, and overall error based on Equation (4.5) are calculated. Also an initial value is assigned to $\lambda$, say 0.001 . By substituting these values in Equation (4.6) the correction stress parameter vector, $\delta(\Delta \sigma)$, is derived. By applying the stress correction to the initial guess a new stress parameter distribution is derived, and processed the same way. The procedure repeats until the stress parameter distribution has converged to the best value. If the overall error in a given step $\Theta$ reduces, then the value of $\lambda$ will be multiplied by a number less than unity, to provide for a larger weight of the Gauss-Newton method compared to the gradient method. Conversely, if $\Theta$ increases in a step, then $\lambda$ is multiplied by a number greater than unity, to provide larger weight to the gradient method compared to the Gauss-Newton method. We followed Press et al., (1992) and used 10 and 1/10 for the multipliers of $\lambda$, for more than unity, and less than unity, respectively. The iterations repeat for some predefined number of times, or as long as the error reduction is significant. The calculation of the inverse matrix of Equation (4.6) is performed using Gauss-Jordan elimination and a subroutine by Press et al. (1992) is used for this purpose. The results are not sensitive to the selected method for this calculation.

\subsubsection{Functional form of objective function and Jacobian matrix elements}

The inversion process includes the calculation of elements of the Jacobian matrix, which is the derivative of the error at a site relative to the stress parameter of each individual sub-source. Calculation of the matrix elements would require that the number of EXSIM runs be at least two times the number of sub-faults, times the number of stations, for each iteration. This is a large number of EXSIM runs, which would make application of this approach time consuming on a $\mathrm{PC}$. We solve this problem as follows. 
Each component of the Jacobian matrix is the derivative of a summation from Equation (4.4) for a station with respect to stress parameter, i.e. $A_{A_{k}}=\frac{\partial \varepsilon_{j}}{\partial(\Delta \sigma)_{k}}$ where $i_{j}$ is the value in Equation (4.4) and $(\Delta \sigma)_{\mathrm{k}}$ is the stress parameter in the $\mathrm{k}^{\text {th }}$ sub-source. Because the recorded trace's response spectrum is not changing, then:

$$
\begin{gathered}
A_{j k}=\frac{\partial \varepsilon_{j}}{\partial(\Delta \sigma)_{k}}=\frac{\partial}{\partial(\Delta \sigma)_{k}}\left(\frac{1}{\left(N_{2}-N_{1}+1\right)} \sum_{i=N_{1}}^{N_{2}}\left(\log _{10} P S A\left(S_{i j}\right)\right)\right) \quad \text { or } \\
A_{j k}=\left.\frac{\partial}{\partial(\Delta \sigma)_{k}} \overline{\log _{10}\left(P S A\left(S_{j}\right)\right)}\right|_{f_{1}} ^{f_{2}}
\end{gathered}
$$

Equation (4.8) shows that element $A_{j k}$ of the Jacobian matrix is equal to the derivative with respect to stress parameter of the average simulated $\log P S A$ at station $\mathrm{j}$. We exploit this observation to develop the following inversion short cut.

Simulations are performed for an earthquake at a range of distances, and for varying values of uniform stress parameter. The simulation parameters are the same as those used for the entire fault simulation, except that the fault is defined to be the size of a subsource and is assigned a correspondingly small magnitude (based on sub-source size). Since these simulations are performed for a sub-source, their results are equivalent to point source simulations such as those derived by Boore (2000). The calculated $\left.\overline{\log _{10}(P S A(S))}\right|_{f_{1}} ^{f_{2}}$ results using the values of Table (4.1) are shown in Figures (4.1) and (4.2). Figures (4.1) and (4.2) show that there is a linear relationship between $\left.\overline{\log _{10}(P S A(S))}\right|_{f_{1}} ^{f_{2}}$ and $\log _{10}(\Delta \sigma)$ of a sub-source, and between $\left.\overline{\log _{10}(P S A(S))}\right|_{f_{1}} ^{f_{2}}$ and log distance. The linear relationship between $\left.\overline{\log _{10}(P S A(S))}\right|_{f_{1}} ^{f_{2}}, \log _{10}(\Delta \sigma)$, and $\log _{10} \mathrm{R}$ is expressed as:

$$
\left.\overline{\log _{10}(P S A(S))}\right|_{f_{1}} ^{f_{2}}=c_{1} \log _{10} R+c_{2} \log _{10} \Delta \sigma+c_{3}
$$

where $R$ is the distance from the center of sub-fault, and $c_{1}, c_{2}$, and $c_{3}$ are constants that are determined from linear multiple regression. The derivative of Equation (4.9) with respect to stress parameter will be proportional to $A_{j k}$, where the proportionality constant is assumed to be a function of distance. The reason for such a proportionality is that 
$\left.\overline{\log _{10}(P S A(S))}\right|_{f_{1}} ^{f_{2}}$ at a station from a fault is not equal to the algebraic sum of $\left.\overline{\log _{10}(P S A(S))}\right|_{f_{1}} ^{f_{2}}$ of sub-faults, in which case $A_{j k}$ would not be a function of distance, $\mathrm{R}_{\mathrm{jk}}$. Based on this reasoning the elements of the Jacobian matrix are:

$$
A_{j k}=\frac{\mathcal{c}_{2} \cdot R_{j k}^{\alpha}}{(\Delta \sigma)_{k} \times \ln 10}
$$

where $c_{2}$ is the coefficient from Equation (4.9), $R_{j k}$ is the ratio of the distance between station $\mathrm{j}$ and sub-source $\mathrm{k}$ divided by unit distance, $\ln$ is logarithm in natural base, $(\Delta \sigma)_{\mathrm{k}}$ is the stress parameter of the $\mathrm{k}^{\text {th }}$ sub-source, and $\alpha$ has the value of $c_{/} / 2$ (Equation 4.9). The value of $a$ was determined empirically by checking various values in the range of $c_{l}$, in order to reproduce the Jacobian matrix values of Equation (4.8).

\subsubsection{Inversion procedure}

The program for inversion starts by assuming a uniform stress parameter distribution, then performs a grid search to find the optimum average stress parameter that minimizes $\Xi$, based on recorded horizontal-component PSA values. Then it uses $\varepsilon_{j}$ (Equation 4.4) of stations to construct the Jacobian and data matrices of the inverse problem. It also calculates the $\Theta$ error for the current stress parameter distribution. After solution of the inverse matrix, the stress parameter correction vector, $\delta(\Delta \sigma)$, is derived and applied to the stress parameter distribution. At this time the first iteration is complete.

In the beginning of the second iteration, the new stress parameter distribution is treated as weights, normalized by the average stress. Then the program does a grid search for the optimum average stress parameter for this distribution. The new optimum average stress parameter is then multiplied by the normalized weights, providing a revised initial guess of the stress parameter distribution, which is used for derivation of the stress parameter correction vector. The error $\Theta$ is then calculated again. If the value of $\Theta$ is lower than it was in the previous step, then the $\lambda$ value in Equation (4.6) is multiplied by a number smaller than unity (we used 0.1) for the next step, and the new solution is accepted as a 
successful iteration. If the value of $\Theta$ is higher than it was in the previous stage, then the $\lambda$ value in Equation (4.6) is multiplied by a number larger than unity (we used 10) for the repeating step; in this case the new solution is not accepted as a successful iteration, and is discarded. The same iteration is repeated from the stress parameter distribution of the previous step, but with the new larger $\lambda$ value. This procedure is repeated for at least ten iterations, or the number of times that the program can run successfully, whichever is smaller.

\subsection{Model performance studies}

It is useful to evaluate the performance of the inversion program under ideal conditions in order to draw conclusions about its applicability and self-consistency. The cases studied here consider the Parkfield fault dimensions, strike, and depth, but evaluate the effect of different dip angles, station geometries, size and distribution of asperities, initial guess, and EXSIM trials. The parameters in Table (4.2) are used for forward modeling. For a given stress parameter distribution and station distribution, we use forward modeling to generate a synthetic PSA dataset, which is then input as data to the inversion routine. The output of the inversion routine is then compared to the initial assigned stress parameter distribution (called the target distribution). The similarity of the target and output stress parameter distributions is a measure of the program's performance under the given conditions.

The effects of number of stations, number of EXSIM iterations per station (to smooth the predicted PSA), fault dip angle, station distribution relative to asperity location, and initial guess in the inversion input are studied. The way that the program identifies multiple asperities with different sizes is also considered.

Forward modeling is performed for a grid of symmetrically distributed stations around the fault as shown in Figure (4.3). We chose a dense array of 328 stations, to allow the inversion program to have sufficient independent equations to resolve the parameters (stress parameter of sub-faults). Forward simulations are performed for the above station 
distribution using EXSIM, given the parameters of Table (4.2), with an assumed stress parameter distribution and fault dip angle. The correlation coefficient is considered as a measure of similarity between the inversion outputs and target stress parameter distribution. A reduction in the value of error measure $\Xi$ is considered to indicate improvement of the solution with additional iterations.

As an example the forward modeling results of parameters in Table (4.2) and target stress parameter distribution of Figure (4.4a) are entered to the inversion program. The inversion program starts from a uniform stress parameter distribution and modifies the distribution iteratively. Figure (4.4) shows the succession of the derivation of target distribution from uniform distribution. The successful iterations in the graphs of Figure (4.4), and the following figures, are the ones during which the error value " $(-)$ " reduces.

\subsubsection{Effect of EXSIM iterations and fault dip angle}

In this sub-section the effects of fault dip angle and EXSIM iterations ("EXSIM iterations" is the number of EXSIM simulation trials used for obtaining an average PSA at each site under the considered scenario) on the obtained results are studied. More emphasis is on the comparisons of different dip angles' effects.

In this experiment, the assumed (target) stress parameter is uniform on the fault except for one sub-fault, which has a stress parameter 5 times larger than that of the other subfaults. We consider fault dip angles of $5^{\circ}, 25^{\circ}, 45^{\circ}$, and $83^{\circ}$ (the actual dip angle of the Parkfield fault) with 10 EXSIM simulation trials to generate smooth PSA's. Figures (4.5), (4.6), and (4.7) show 4 snapshots of the evolution of solutions from uniform stress parameter distribution to the target distribution for $5^{\circ}, 25^{\circ}$, and $45^{\circ}$ of fault dip angles. The evolution of solutions for $83^{\circ}$ fault dip angle is shown in Figure (4.4). The simulation parameters are those of Table (4.2). The inversion iterations of each case continued until no improvement in the solutions appeared, or the inversion program stopped. In each snapshot the correlation coefficient of the result with the target distribution is presented. The correlation coefficient of the final solution of each fault dip angle scenario is given in Table (4.3). Table (4.3) shows that for steep fault dip angles the correlation coefficient of 
target distribution and inversion output can be poor, suggesting low resolution of asperities found by the inversion program for near vertical faults.

solution at each iteration is shown for four fault dip angles: $5^{\circ}, 25^{\circ}, 45^{\circ}$, and $83^{\circ}$, in Figure (4.8). This graph shows that: (i) in 10 inversion iterations the error reduces considerably; (ii) in the case of $83^{\circ}$ and $45^{\circ}$ fault dip angles the $\Xi$ value approaches its asymptotic value in 10 inversion iterations; (iii) the larger the fault dip angle, the poorer is the convergence of the solution, and the more ambiguous is the ultimate result; and (iv) gentle fault dip angles result in a solution that continues to improve with further iterations.

In a new set of experiments, for $25^{\circ}$ and $83^{\circ}$ fault dip angles, forward modeling and inversions are carried out with the same parameters except that 30 EXSIM iteration for smoothing PSAs replaced the 10 iterations used in the previous experiment. Error $\Xi$ values for $25^{\circ}$ and $83^{\circ}$ fault dip angles are plotted in Figure (4.9). This graph compares application of 10 or 30 EXSIM simulations per station on the error $\Xi$ in different iterations. 30 EXSIM iterations can result in a slightly lower error $\Xi$ for steep fault dip angles (in this case $83^{\circ}$ ), but in general 10 EXSIM trials is sufficient to establish stable average expected values of PSA against which real or simulated data can be compared.

The inversions of synthetic data with varying fault dip angles show that loss of resolution in finding the high stress parameter patches on the fault increases gradually with increasing fault dip angle. This can be attributed to the range of angles over which subfaults can be seen as a function of dip angle. The higher the dip angle, the smaller is the angular coverage of stations around sub-faults, and the lower is the likelihood of finding the effect of individual sub-faults distinctively using the inversion method.

\subsubsection{Multiple asperities}

Since the PSA of a record at a site is not the algebraic sum of the PSAs of individual subfaults, it is important to find out if the presence of a high-stress-parameter asperity eliminates the effect of a neighboring low-stress-parameter asperity, and to what extent multiple asperities might be detected. We tested the ability of the inversion program to distinguish multiple asperities by carrying out three experiments. The simulation parameters are those given in the Table (4.2) and the array of stations are those of Figure 
(4.3). The chosen fault dip angle in these experiments is $5^{\circ}$ to minimize the unwanted effect of fault dip angle.

In the first experiment two asperities of equal size are located on the fault surface with considerable separation. EXSIM forward modeling outputs resulting from this distribution are entered to the inversion program to check its performance in the identification and evaluation of the size of these asperities. Figure (4.10) shows the target distribution, the evolution of the solution and the performance of the inversion program. It turns out that the program finds the location of two asperities quickly.

In another experiment, one asperity is added between the two asperities of the previous experiment to make the distances between asperities smaller. The purpose of this experiment is to check if the program is capable of distinguishing individual asperities located at close distances. Figure (4.11) shows the target distribution and program solutions. These results demonstrate the program's ability to distinguish closely-located asperities. Figure (4.11) shows that the two asperities that are close to each other are assigned slightly smaller stress than the other one in the southeast, although in the target distribution they are all similar.

In the third experiment of this sub-section, different stress parameters are assigned to the three asperities of the previous experiment. The purpose of this experiment is to test the ability of the inversion program to evaluate the stress level of asperities. Figure (4.12) shows the target distribution with 3 high-stress-parameter patches and the solutions derived from the program. Improvement of the solution after 2, 7, and 15 inversion iterations are shown in Figures (4.12b) to (4.12d). The program successfully found the location and relative magnitude of the high-stress-parameter sub-sources in the initial iterations after starting from a uniform distribution (given a good coverage of stations around the fault and a gentle dip angle). It is interesting that for multiple asperities, the solution is reached in fewer inversion iterations compared to single asperity cases.

We conclude that the program has the capability to distinguish multiple asperities and evaluate their stress level reasonably well, if there is proper station coverage around the fault. The solution is converging to the answer quickly if the real distribution contains multiple stress parameter patches. 
Table (4.1) Simulation parameters for the derivation of $D_{r}$ values $\left(\left.\overline{\log _{10}(P S A(S))}\right|_{f_{1}} ^{f_{2}}\right)$ for a range of distances and stress parameters of a sub-source.

\begin{tabular}{cc}
\hline Parameter & Parameter value \\
\hline FFT points, sample interval & $8192,0.02 \mathrm{~s}$ \\
Shear wave velocity, Density & $3.5 \mathrm{~km} / \mathrm{s}, 2.8 \mathrm{~g} / \mathrm{cm}^{3}$ \\
Rupture velocity & $0.8 \times$ shear wave velocity \\
$Q(f)$ & $180 f^{0.45}$ \\
Moment magnitude & 5.0 \\
Average stress parameter & Variable \\
Kappa & 0.035 \\
Geometrical attenuation & If $\mathrm{R}<40, \mathrm{R}^{-1}$, else $\mathrm{R}^{-0.5}$ \\
Sub-source duration & $\propto 0.05 \times$ distance $(\mathrm{sec})$. \\
Windowing function & Saragoni-Hart \\
Amplification function & Boore and Joyner $(1997)$ for $\mathrm{V}_{\mathrm{s}, 30}=620 \mathrm{~m} / \mathrm{s}$ \\
Damping & $5 \%$ of critical damping \\
Number of EXSIM trials & 10 \\
\hline
\end{tabular}

Table (4.2) Simulation parameters for model performance studies. These values are M6, 2006 Parkfield earthquake parameters.

\begin{tabular}{cc}
\hline Parameter & Parameter value \\
\hline Fault orientation & Strike $137^{\circ}$, Dip $83^{\circ}$ \\
Depth of top & $0 \mathrm{~km}$ \\
Latitude and longitude of fault & $36.047^{\circ} \mathrm{N}, 120.609^{\circ} \mathrm{W}$ \\
Fault dimensions & Length $40 \mathrm{~km}$, Width $13 \mathrm{~km}$ \\
Number of sub-faults & Along length 10, Along width 3 \\
FFT points, sample interval & $8192,0.02 \mathrm{~s}$ \\
Shear wave velocity, Density & $3.5 \mathrm{~km} / \mathrm{s}, 2.8 \mathrm{~g} / \mathrm{cm}^{3}$ \\
Rupture velocity & $0.8 \times$ shear wave velocity \\
$Q(f)$ & $180 f^{0.45}$ \\
Moment magnitude & 6.0 \\
Average stress parameter & Variable \\
Kappa & 0.035 \\
Geometrical attenuation & If R $<40, \mathrm{R}^{-1}$, else $\mathrm{R}^{-0.5}$ \\
Sub-source duration & $\propto 0.05 \times$ distance $(\mathrm{sec})$. \\
Windowing function & Saragoni-Hart \\
Amplification function & Boore and Joyner $(1997)$ for $\mathrm{V}_{\mathrm{s} 30}=620 \mathrm{~m} / \mathrm{s}$ \\
Damping & $5 \%$ of critical damping
\end{tabular}




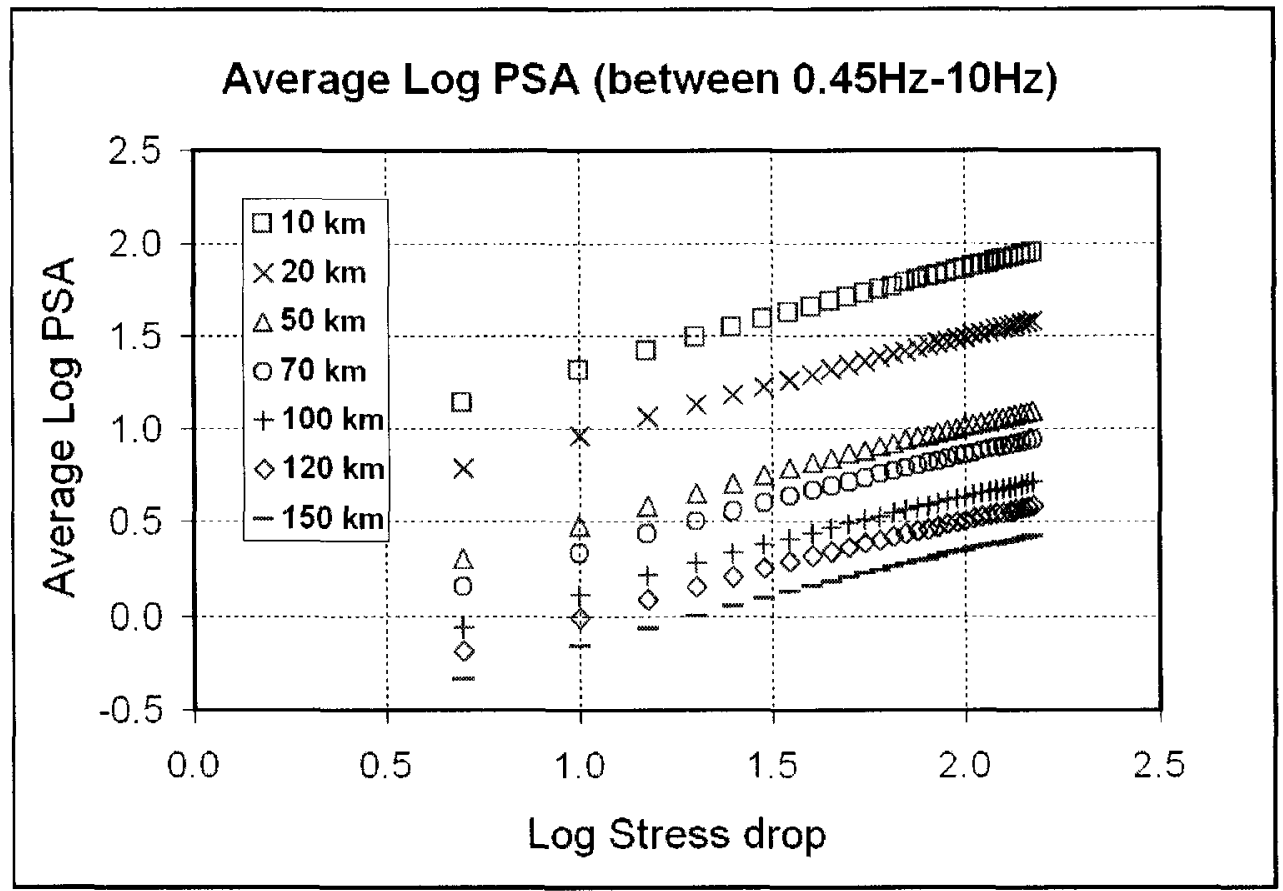

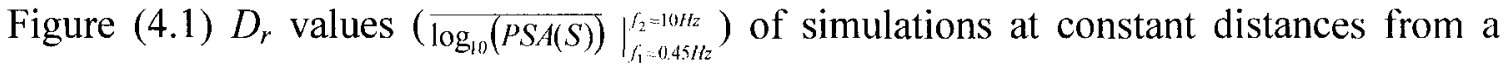
sub-source for varying stress parameters.

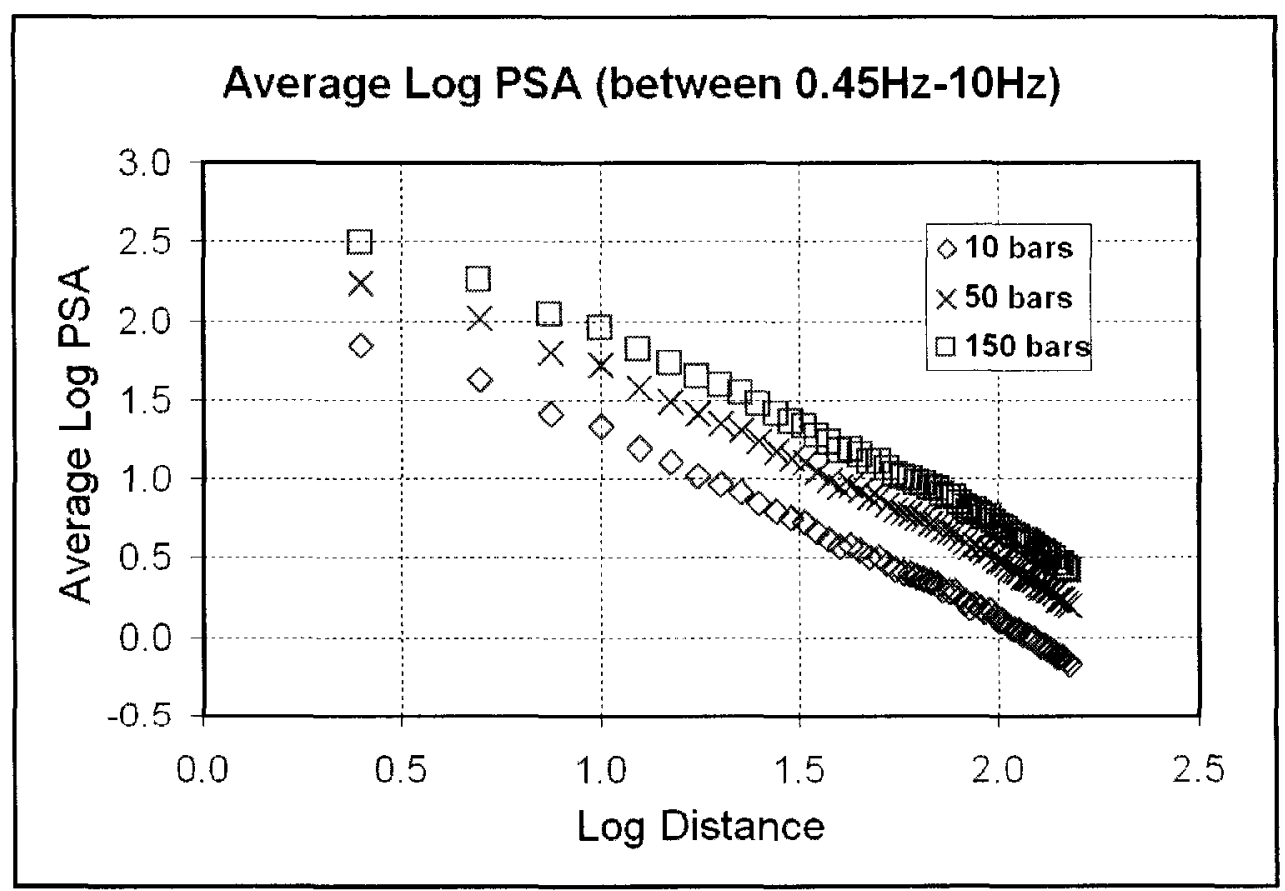

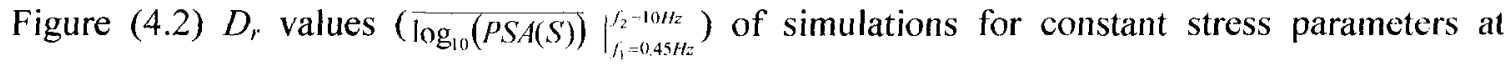
varying distances from a sub-fault.For the above experiment, the error, $\Xi$ of the inversion 


\section{Distribution of Hypothetical Stations Around Fault}

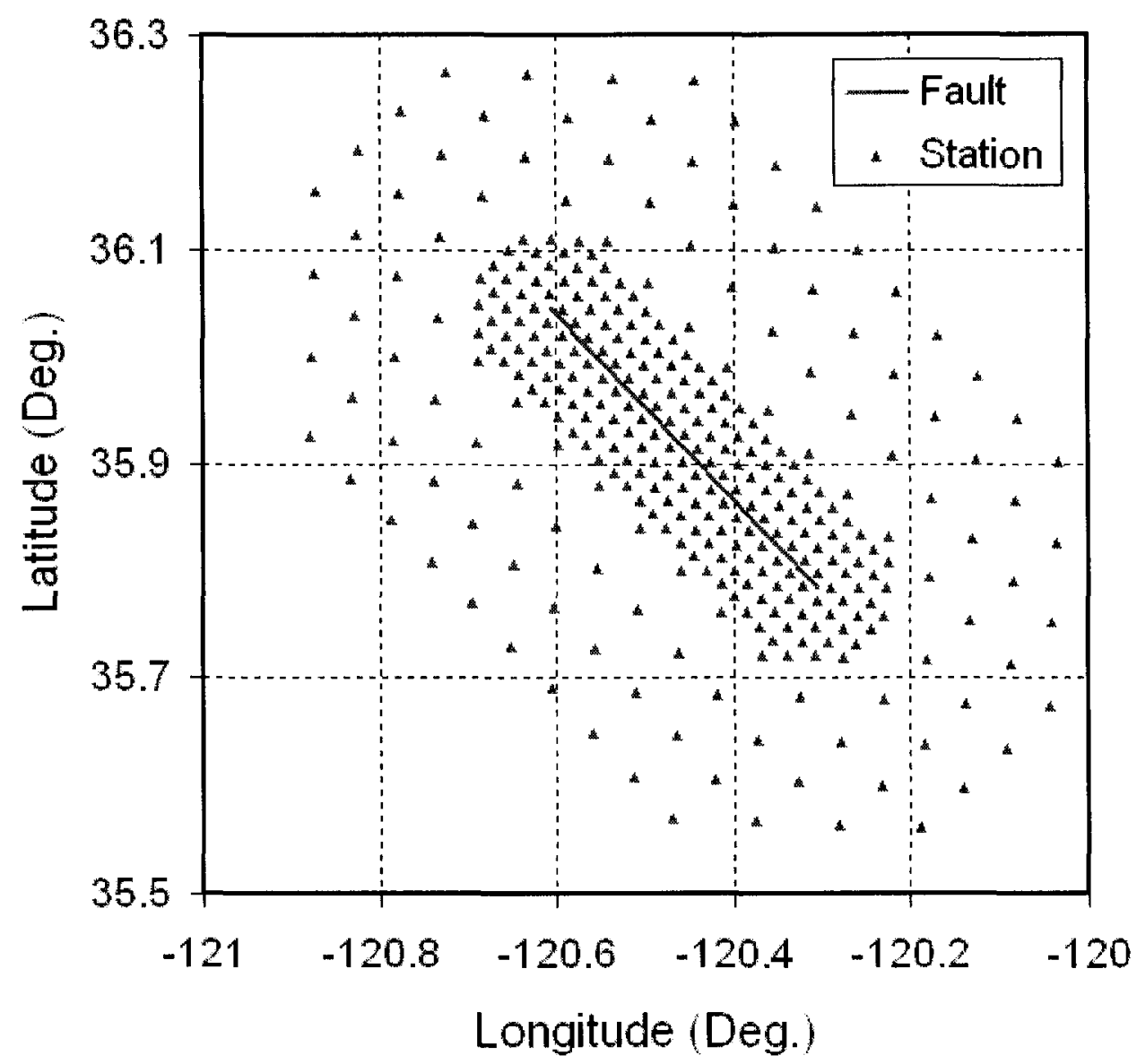

Figure (4.3). Distribution of hypothetical stations around the fault. Simulation outputs for these stations are used as data for inversion program.

Table (4.3) The correlation coefficients of inversion results with target distributions for different fault dip angles. The parameters of both forward and inverse modeling are those of Table (4.2).

\begin{tabular}{cc}
\hline Fault dip angle & Correlation coefficient \\
\hline $5^{\circ}$ & 0.957 \\
$25^{\circ}$ & 0.961 \\
$45^{\circ}$ & 0.704 \\
$83^{\circ}$ & 0.384
\end{tabular}


A- Target stress distribution:

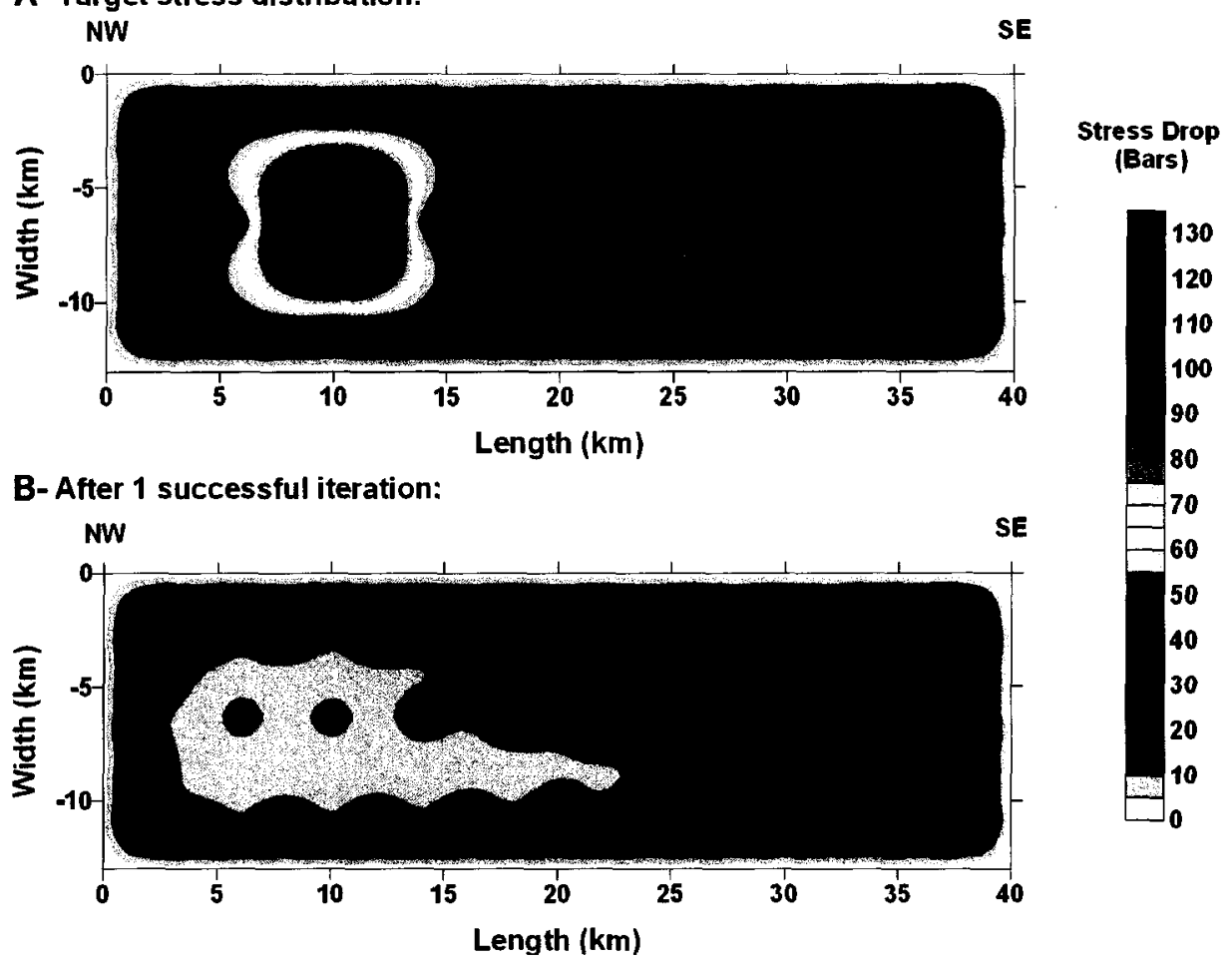

C-After 2 successful iterations:

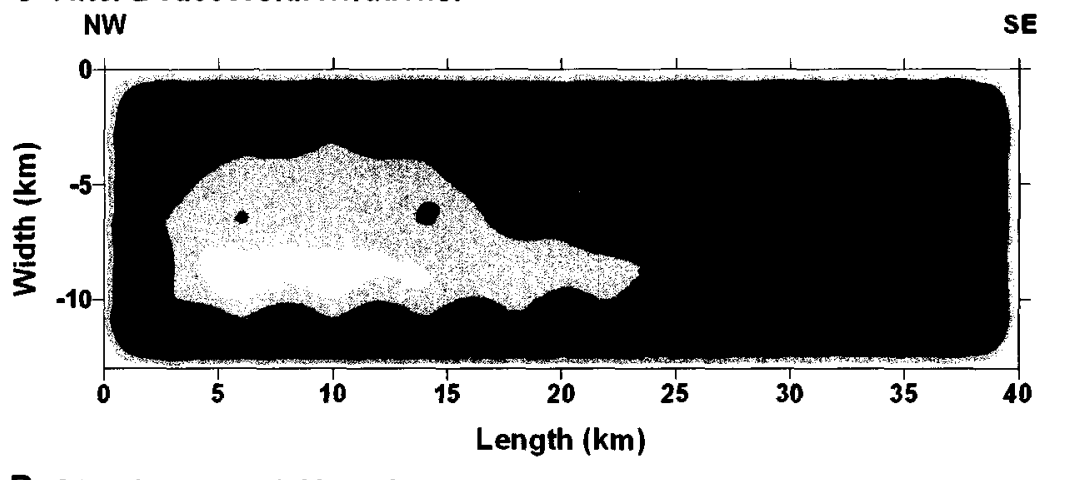

Stress Drop

(Bars)

D-After 3 successful iterations:
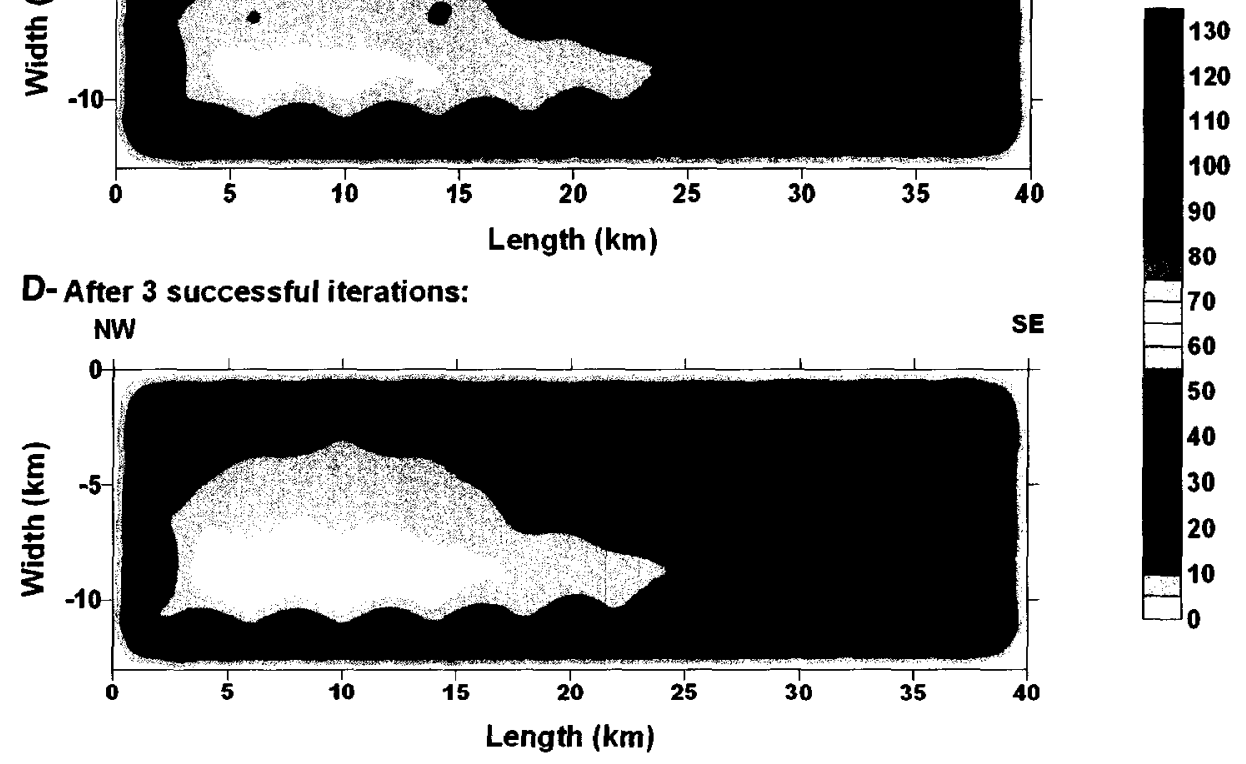

Figure (4.4) The succession of inversion program solutions for the derivation of target stress parameter distribution in the specified inversion steps. Fault dip angle is $83^{\circ}$ and 10 EXSIM trials are used for averaging PSAs. 
E-After 4 successful iterations:

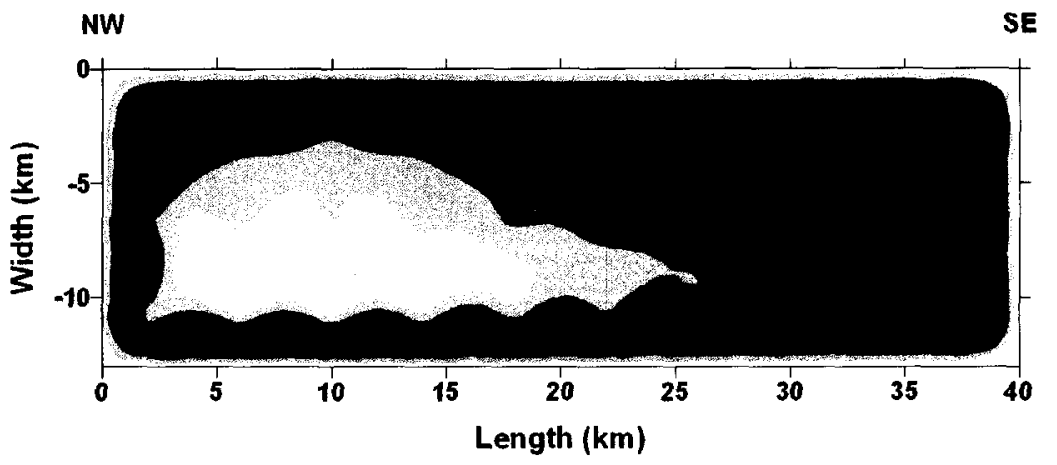

Stress Drop

(Bars)

F-After $\mathbf{5}$ successful iterations:
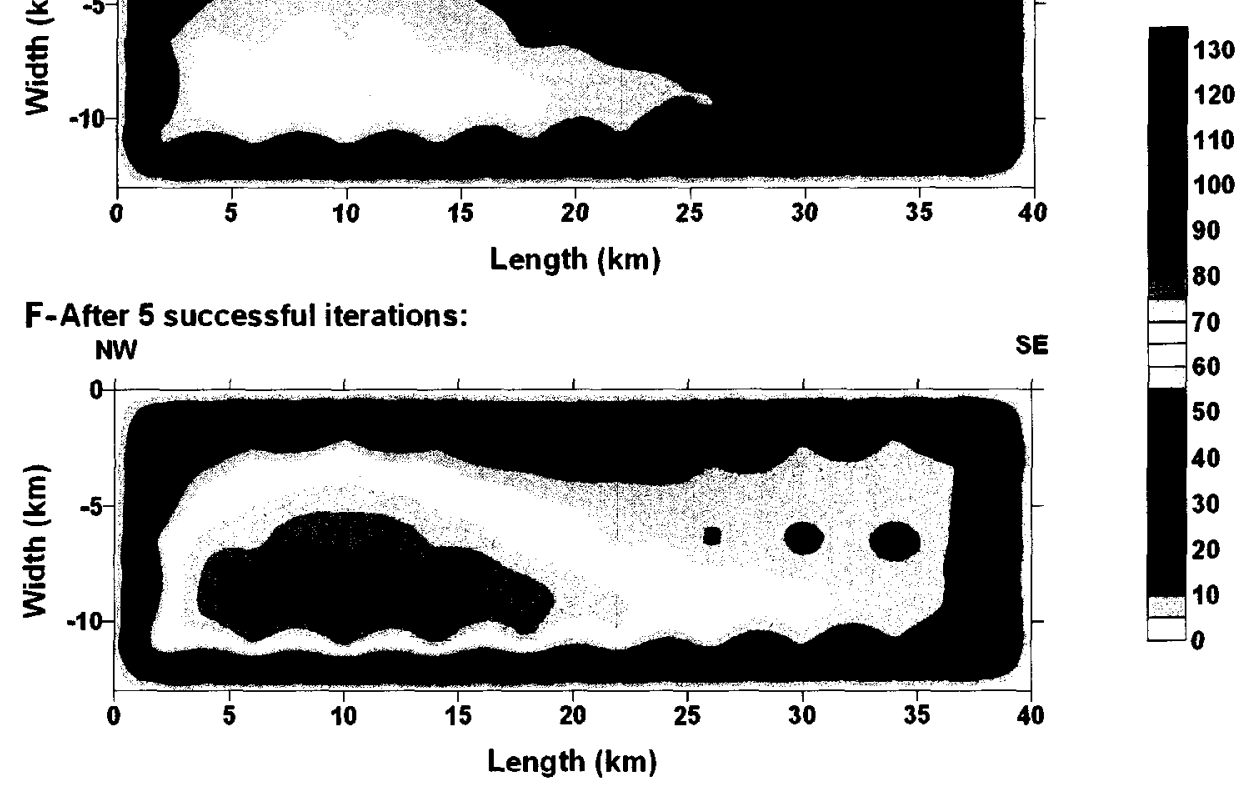

G-After 6 successful iterations:

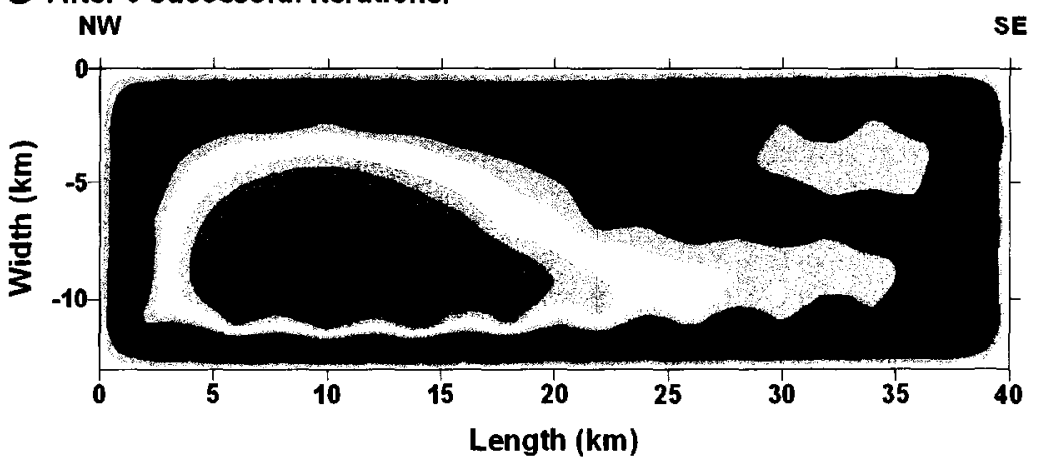

Stress Drop (Bars)

H-After 7 successful iterations:
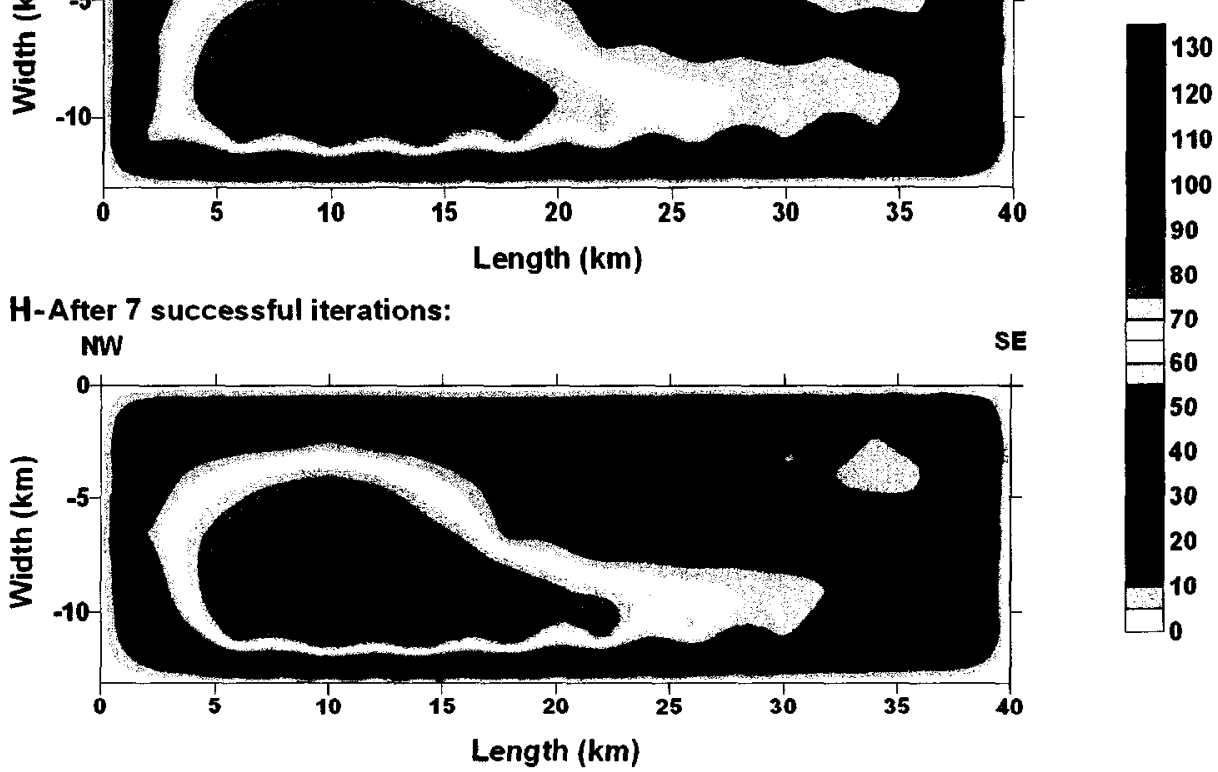

Figure (4.4) The succession of inversion program solutions for the derivation of target stress parameter distribution in the specified inversion steps. Fault dip angle is $83^{\circ}$ and 10 EXSIM trials are used for averaging PSAs. 


\section{I- After 8 successful iterations:}

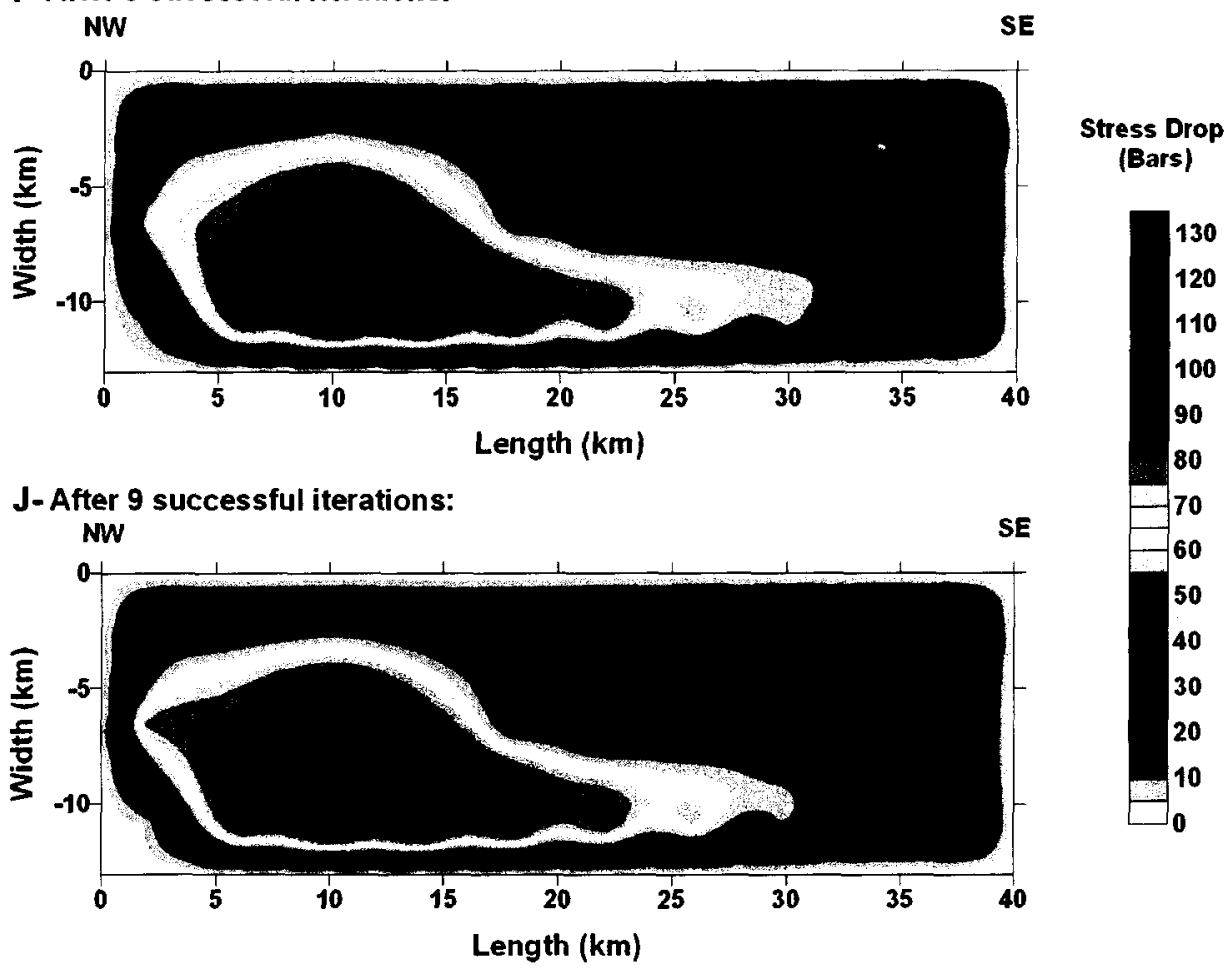

Figure (4.4) The succession of inversion program solutions for the derivation of target stress parameter distribution in the specified inversion steps. Fault dip angle is $83^{\circ}$ and 10 EXSIM trials are used for averaging PSAs.. 
A-Target:

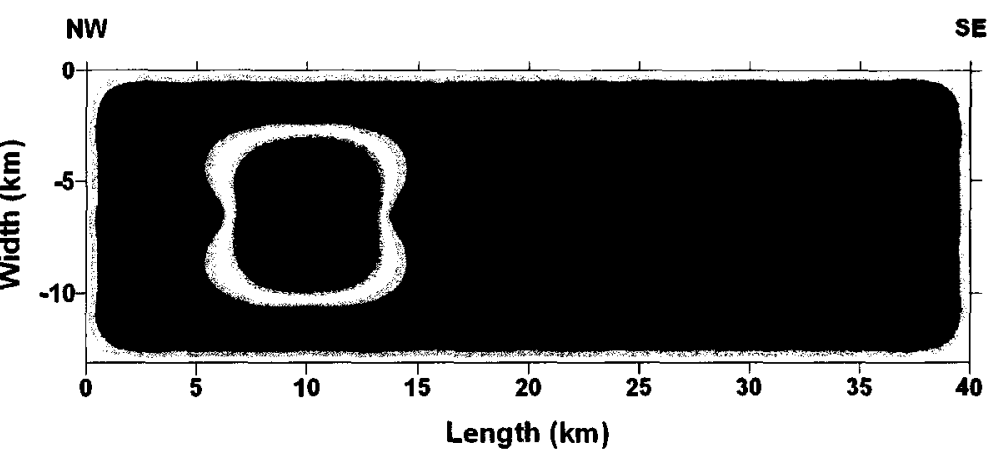

Stress Drop

(Bars)

B-After 5 successful iterations:
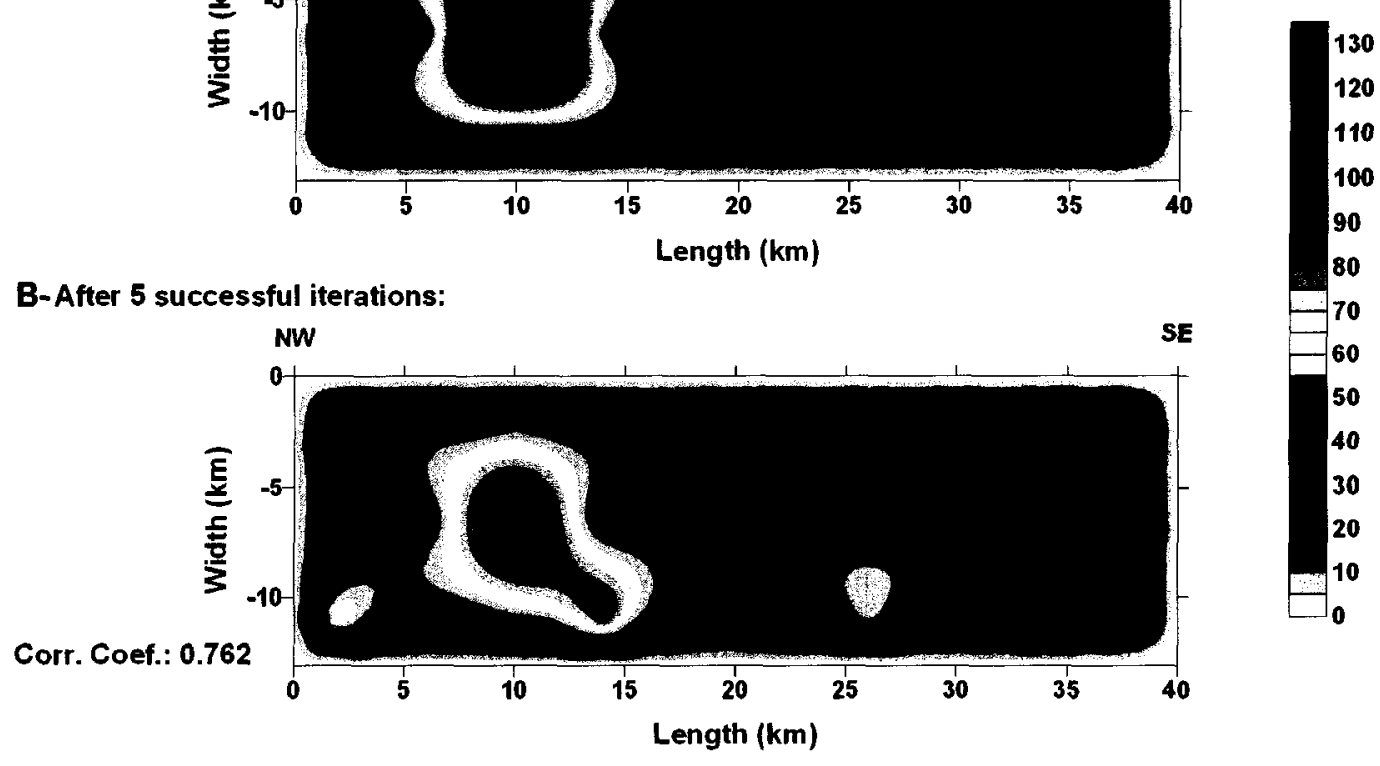

$=70$

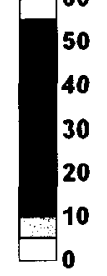

C-After 10 successful iterations:

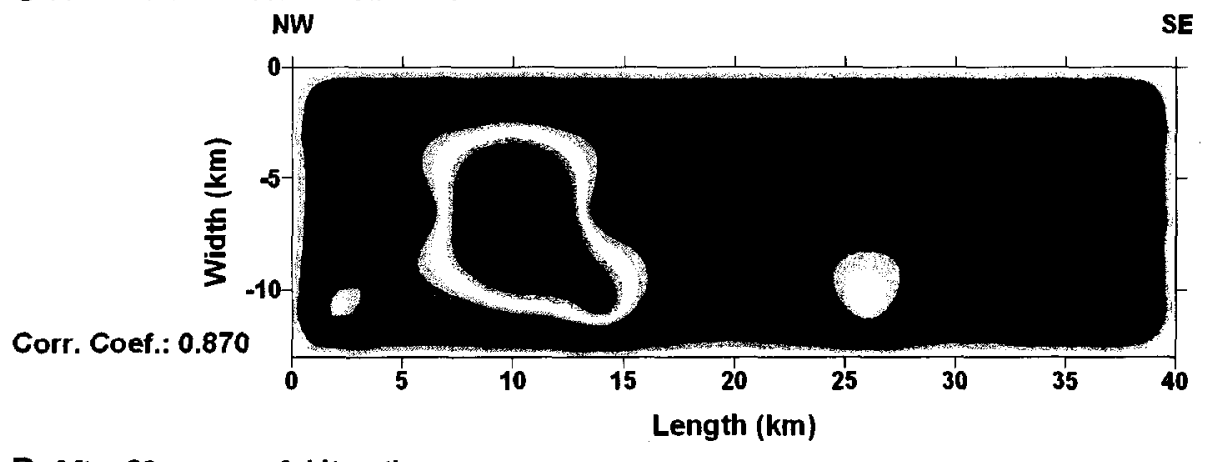

Stress Drop (Bars)

D-After 20 successful iterations:
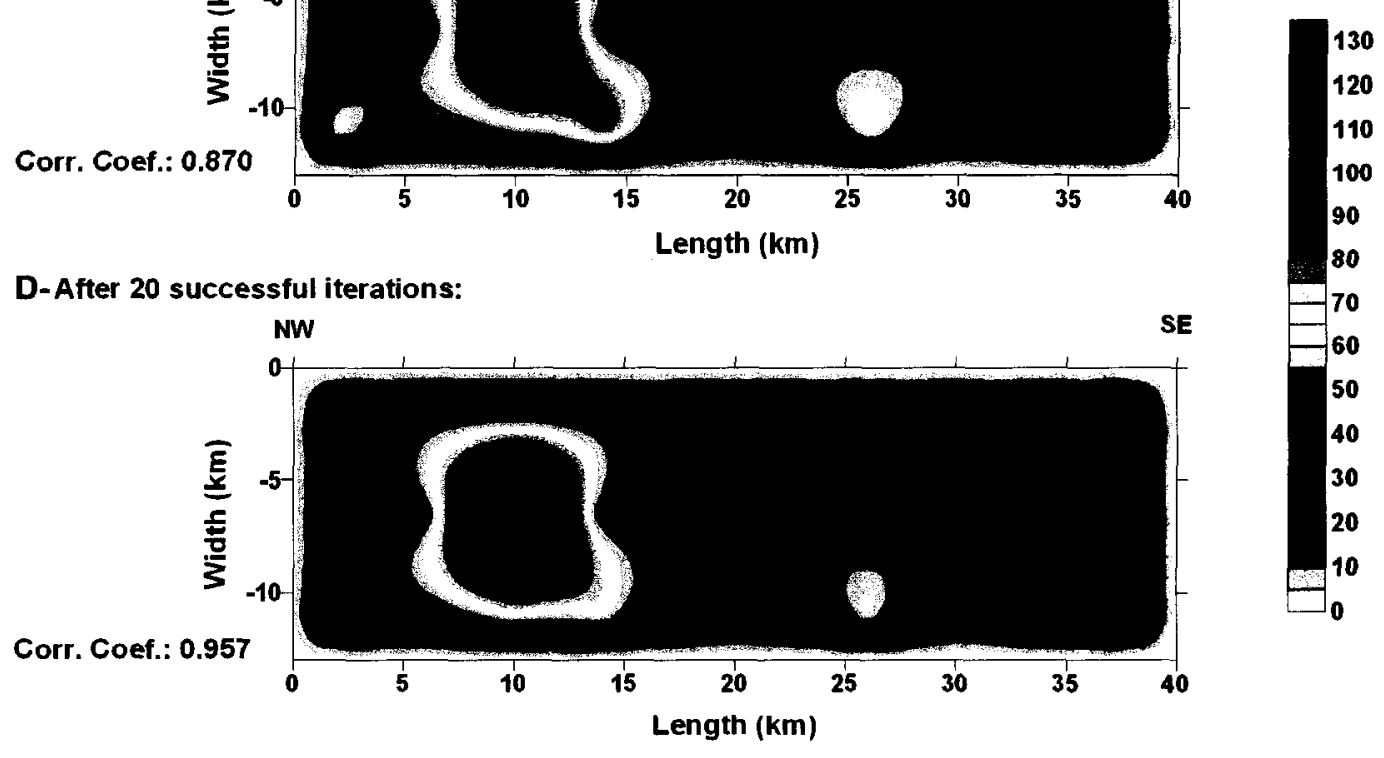

Figure (4.5) The succession of inversion program solutions for the derivation of target stress parameter distribution in the specified inversion steps. Fault dip angle is $5^{\circ}$ and 10 EXSIM trials are used for averaging PSAs. 
A-Target:

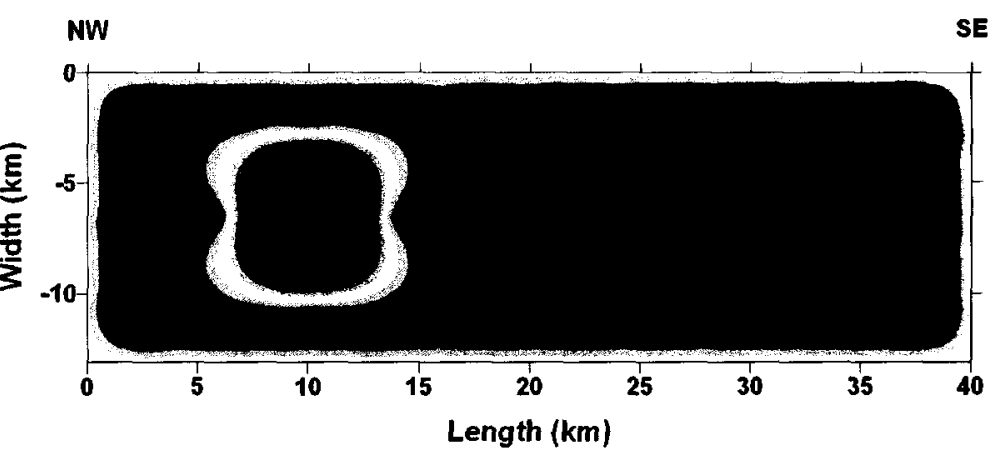

Stress Drop

(Bars)

B-After 5 successful iterations:
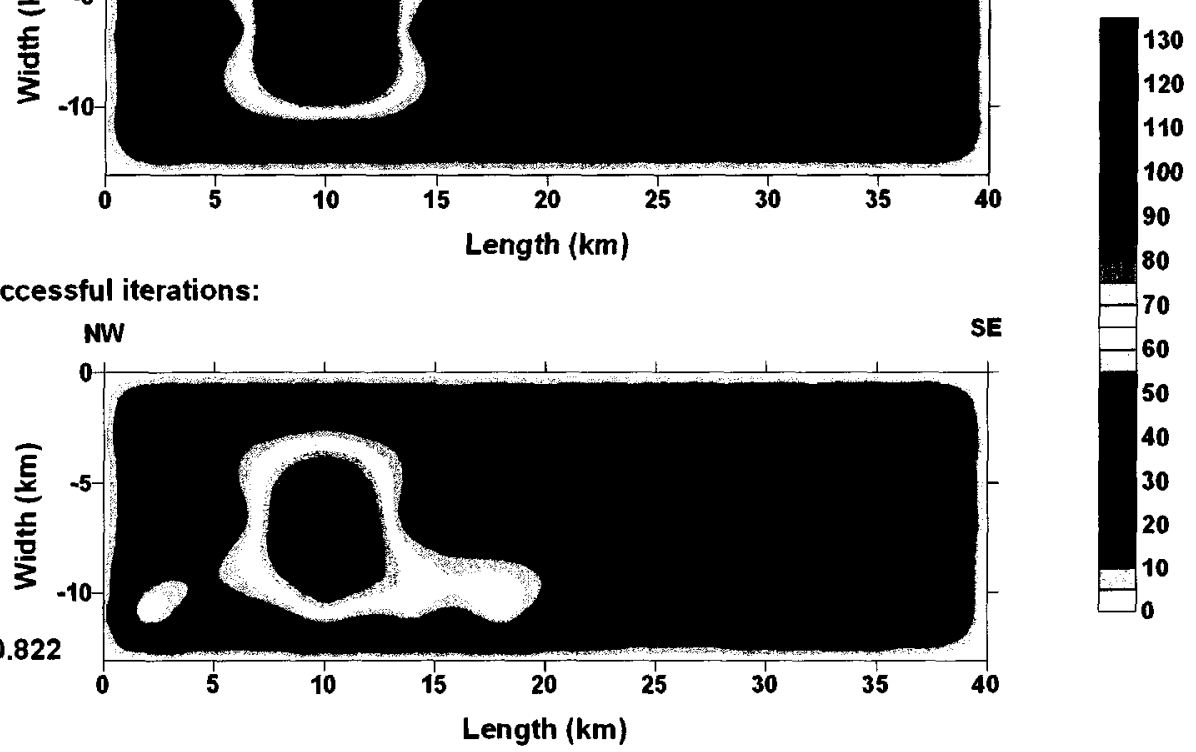

Corr. Coef.: 0.822

Length (km)

C-After 10 successful iterations:

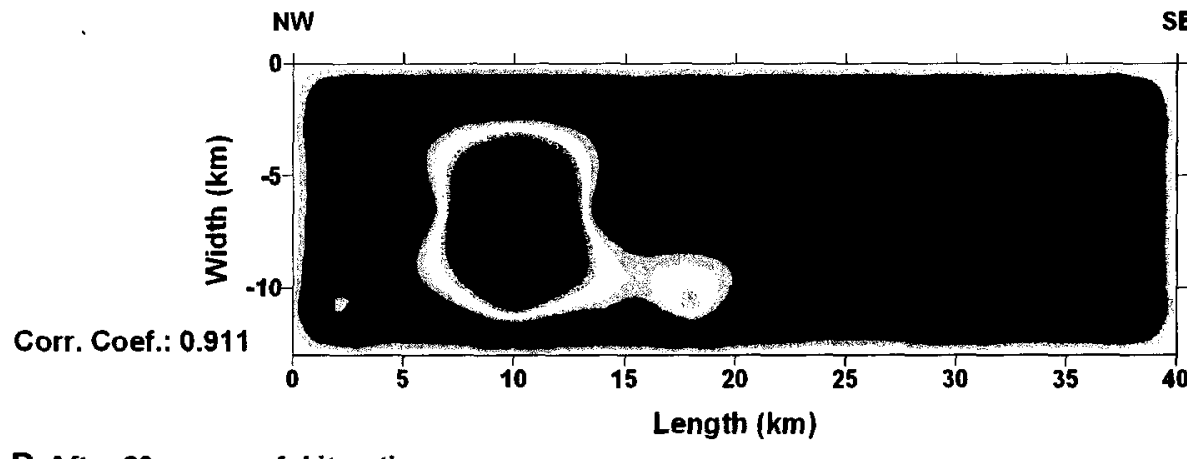

D-After 20 successful iterations:

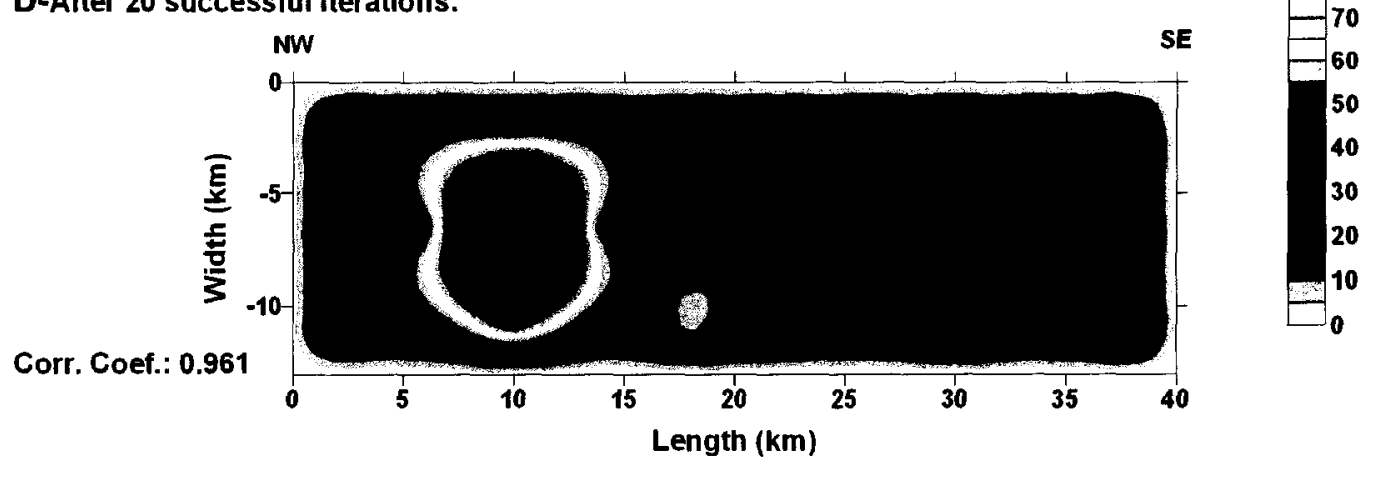

Figure (4.6) The succession of inversion program solutions for the derivation of target stress parameter distribution in the specified inversion steps. Fault dip angle is $25^{\circ}$ and 10 EXSIM trials are used for averaging PSAs. 
A-Target:

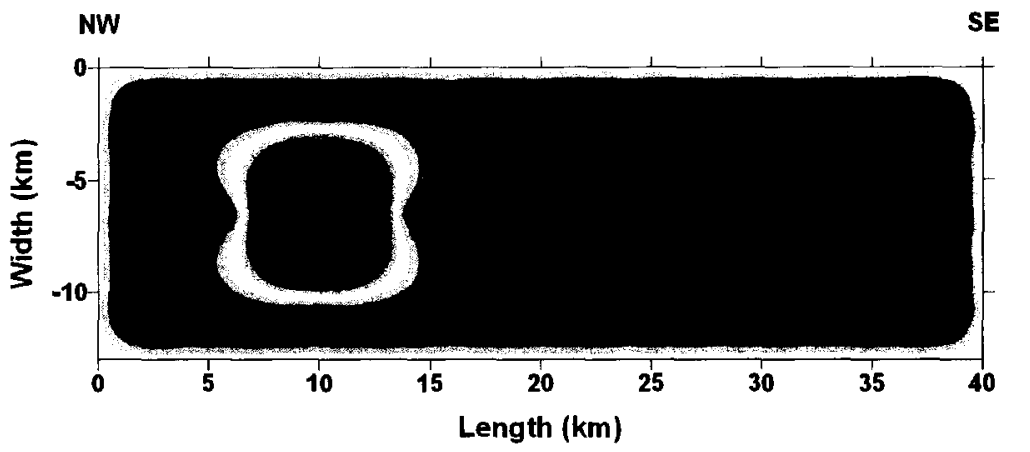

Stress Drop

(Bars)

B-After 5 successful iterations:

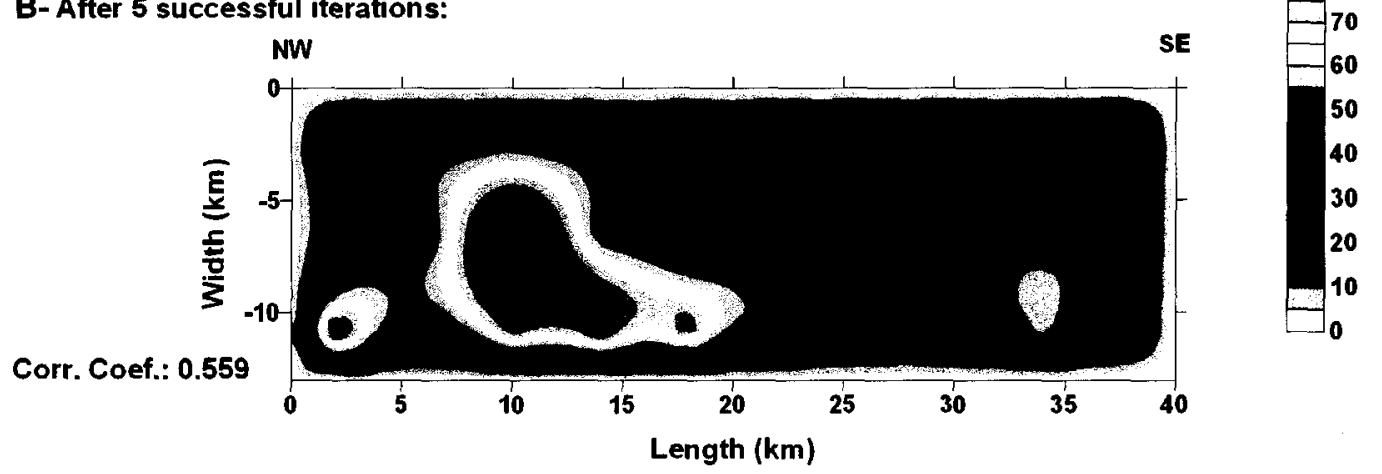

C-After 10 successful iterations:

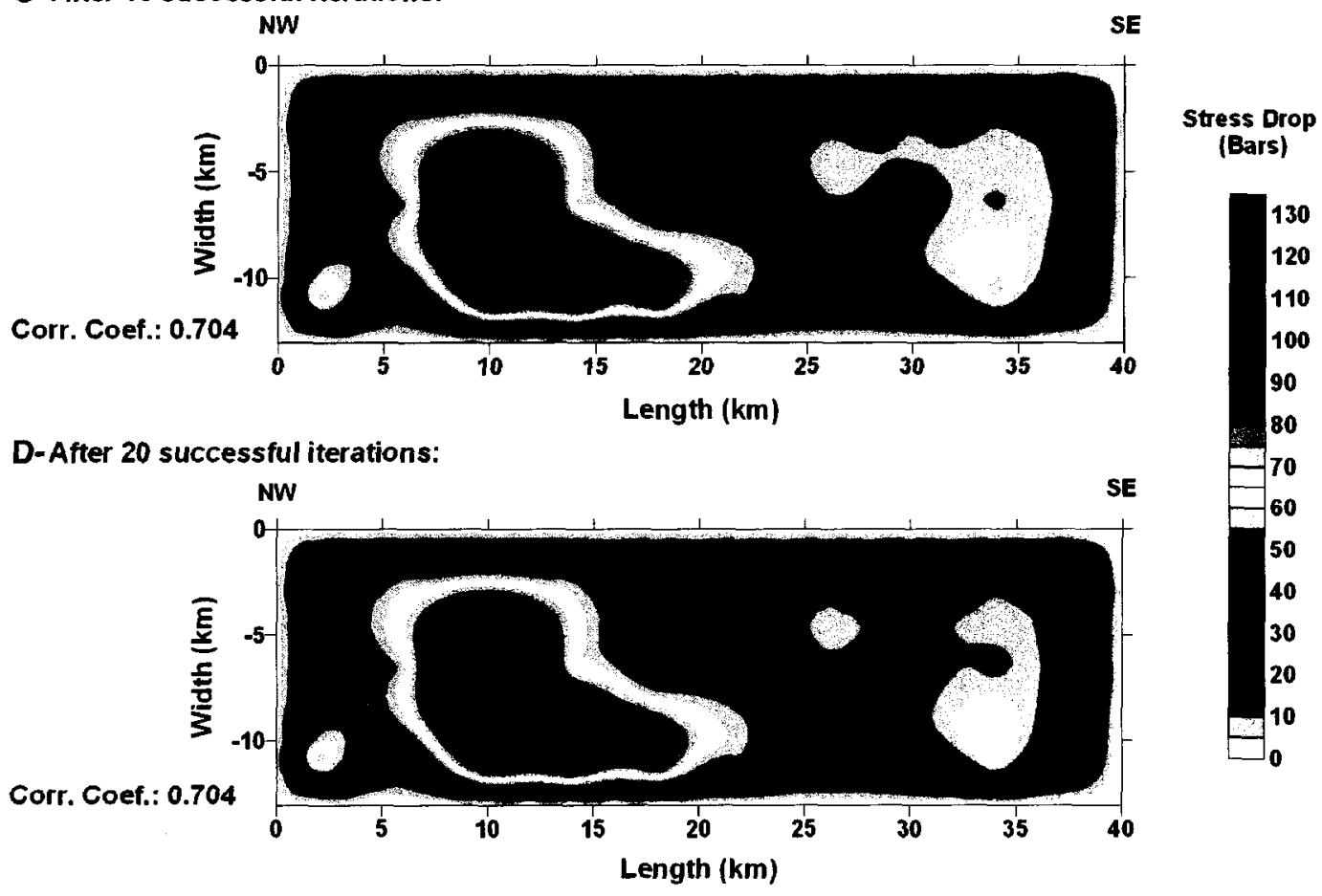

Figure (4.7) The succession of inversion program solutions for the derivation of target stress parameter distribution in the specified inversion steps. Fault dip angle is $45^{\circ}$ and 10 EXSIM trials are used for averaging $P S A$ s. 


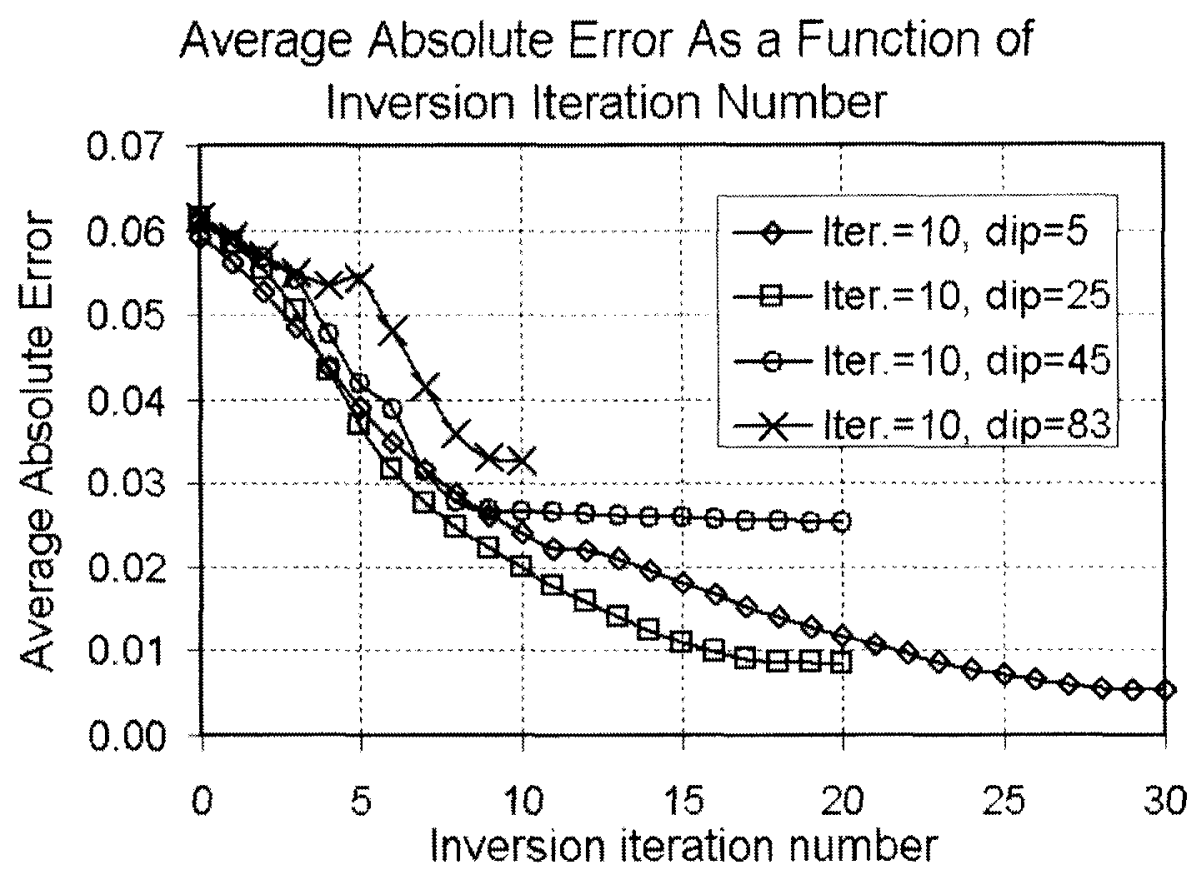

Figure (4.8) Evolution of error $\Xi$ by inversion iteration number for four fault dip angles. Ten EXSIM iterations were applied to both forward modeling and inversion procedures.

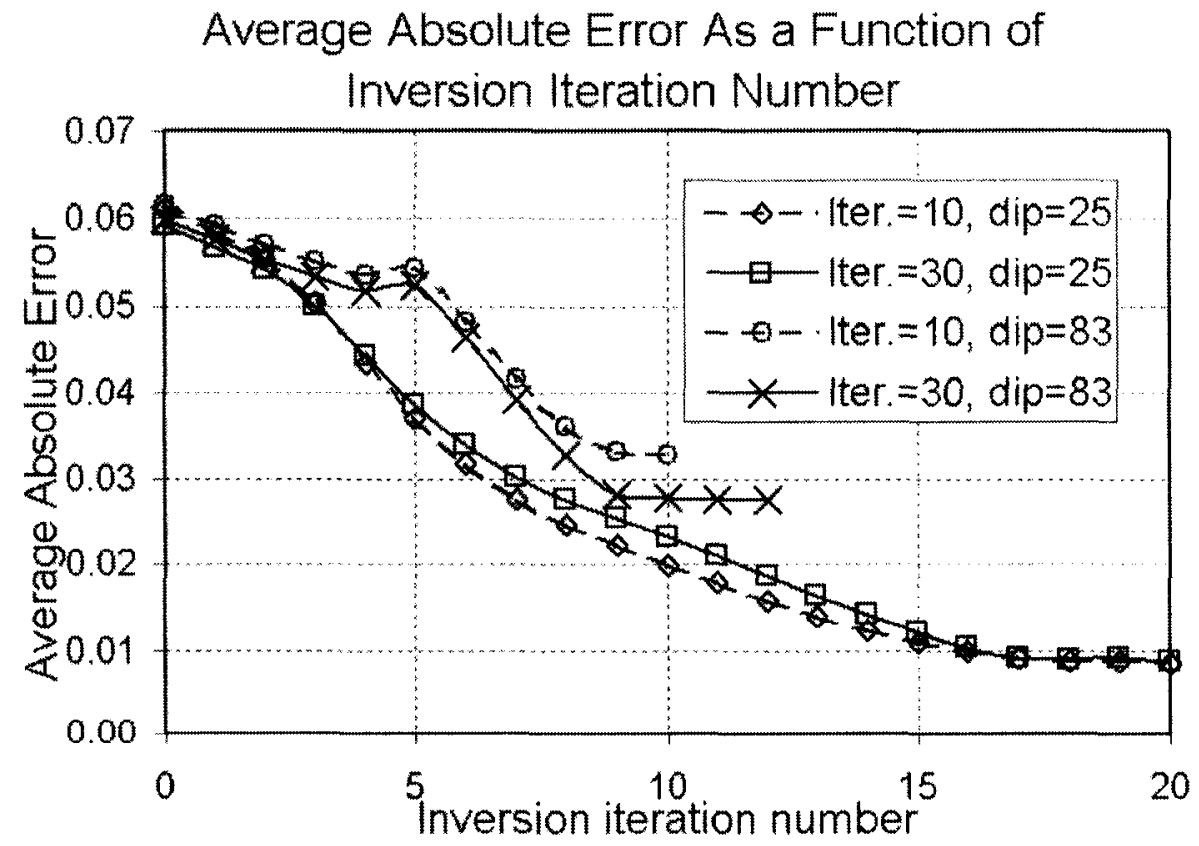

Figure (4.9) Evolution of error $\Xi$ by inversion iteration for two fault dip angles. Ten and thirty EXSIM iterations were applied to both forward modeling and inversion procedures. 
A-Target:

B-After 2 successful iterations:

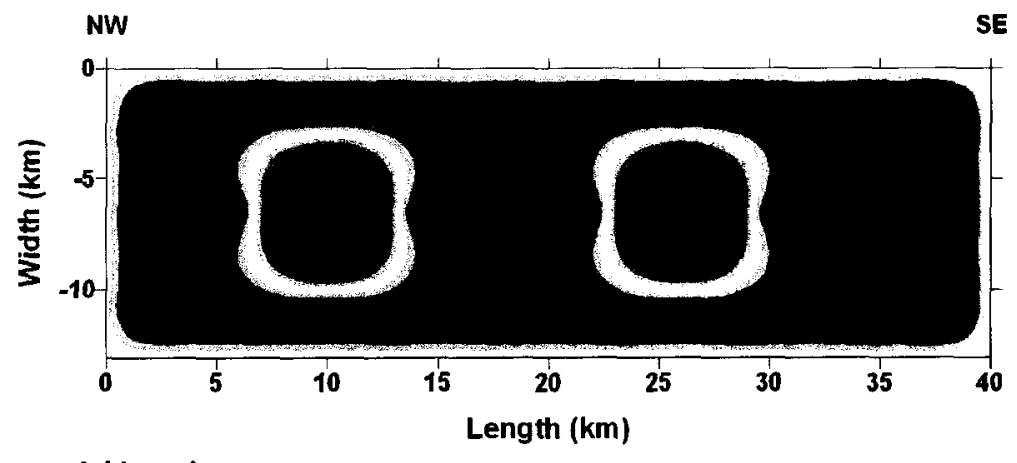

Stress Drop (Bars)
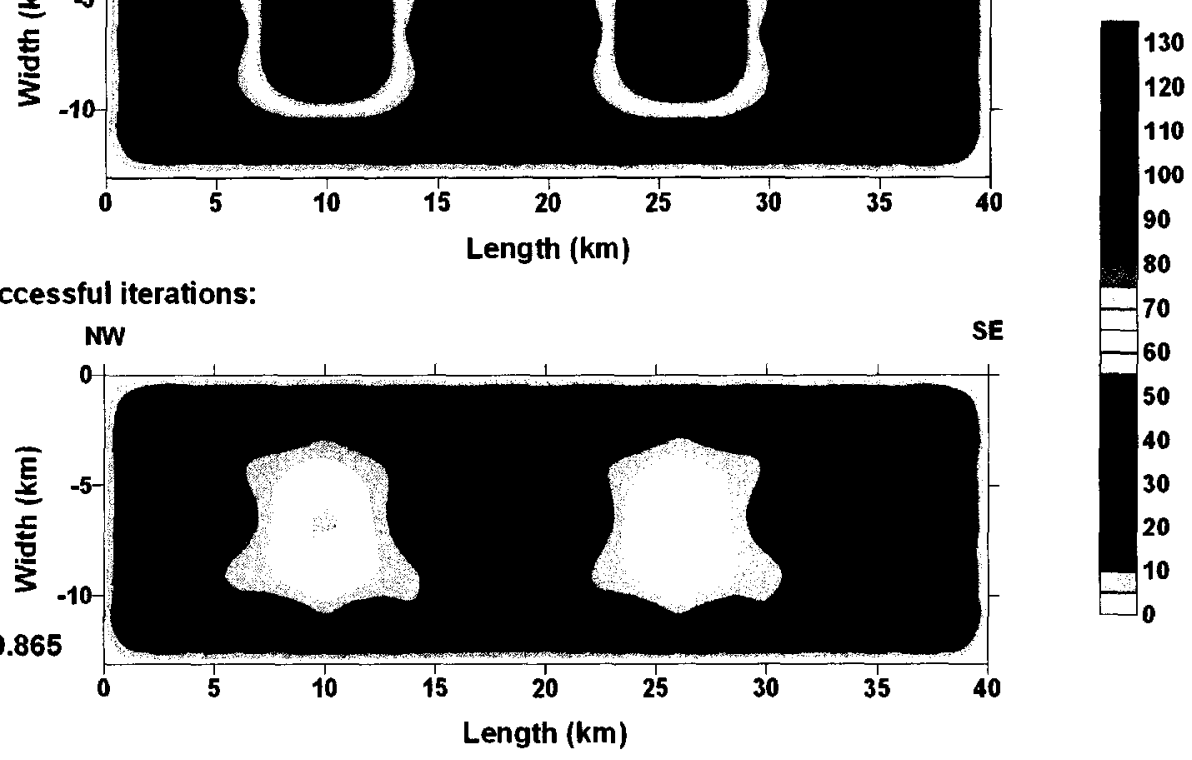

Corr. Coef.: 0.865

$$
\text { Length (km) }
$$

C-After 7 successful iterations:

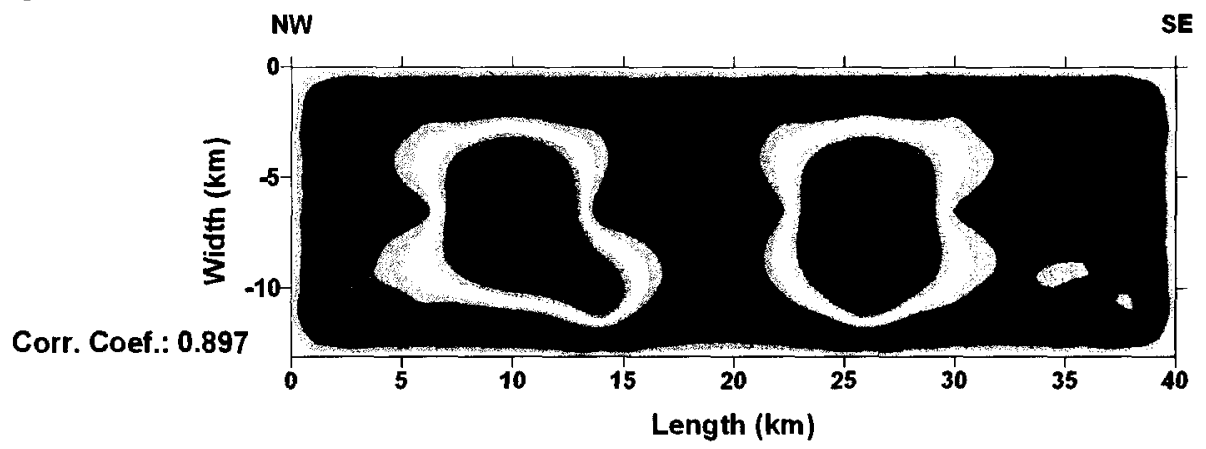

D-After 13 successful iterations:
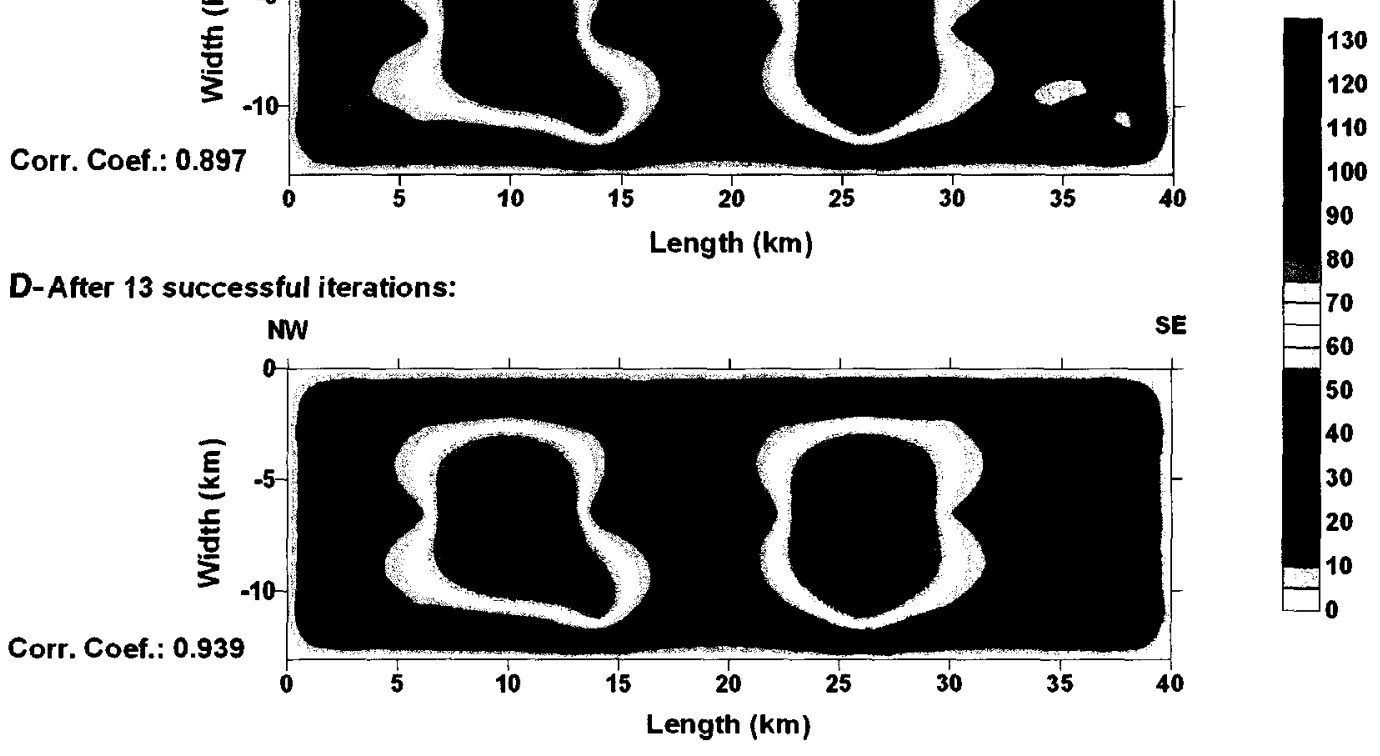

Figure (4.10) The succession of inversion program solutions for the derivation of target stress parameter distribution in the specified inversion steps. The program successfully finds the location and the approximate size of asperities. 
A-Target:

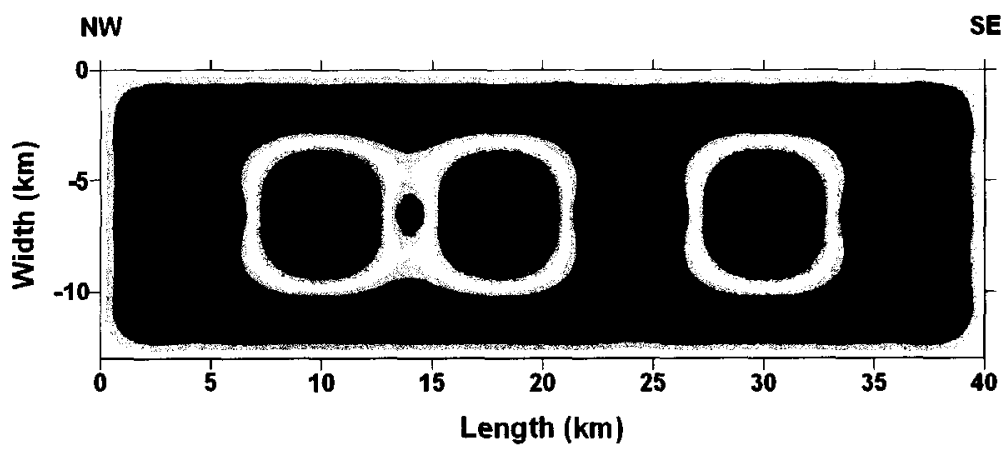

B-After 2 successful iterations:

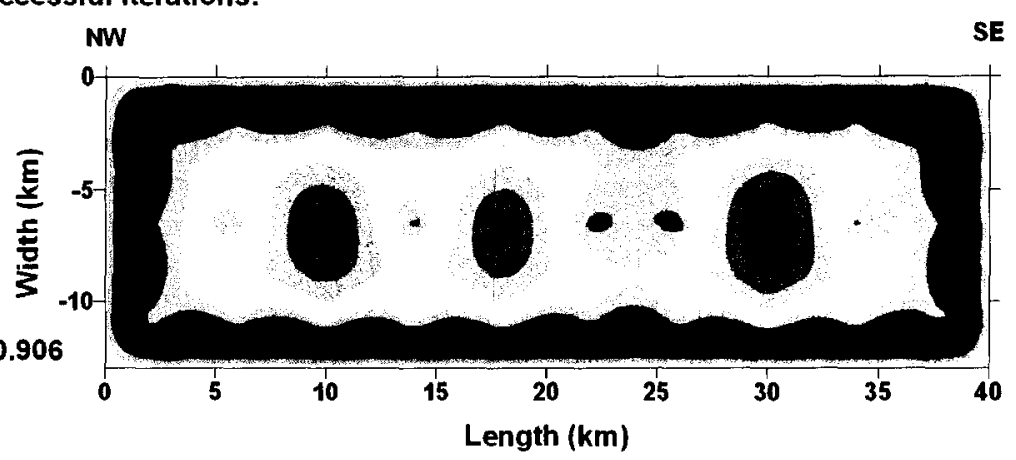

Stress Drop (Bars)

Corr. Coef.: 0.906

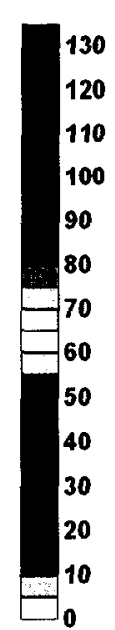

C-After 8 successful iterations:

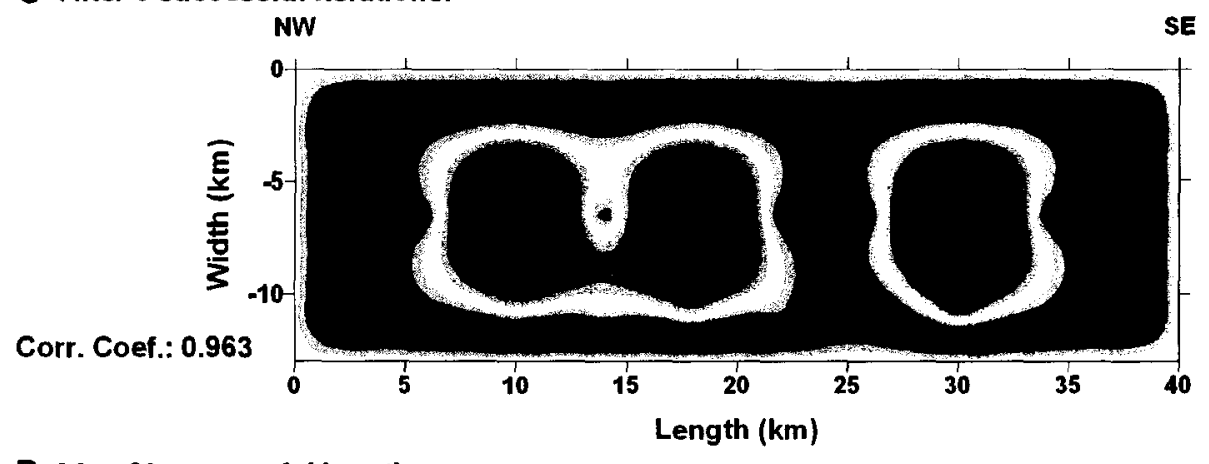

Stress Drop (Bars)

D-After 20 successful iterations:
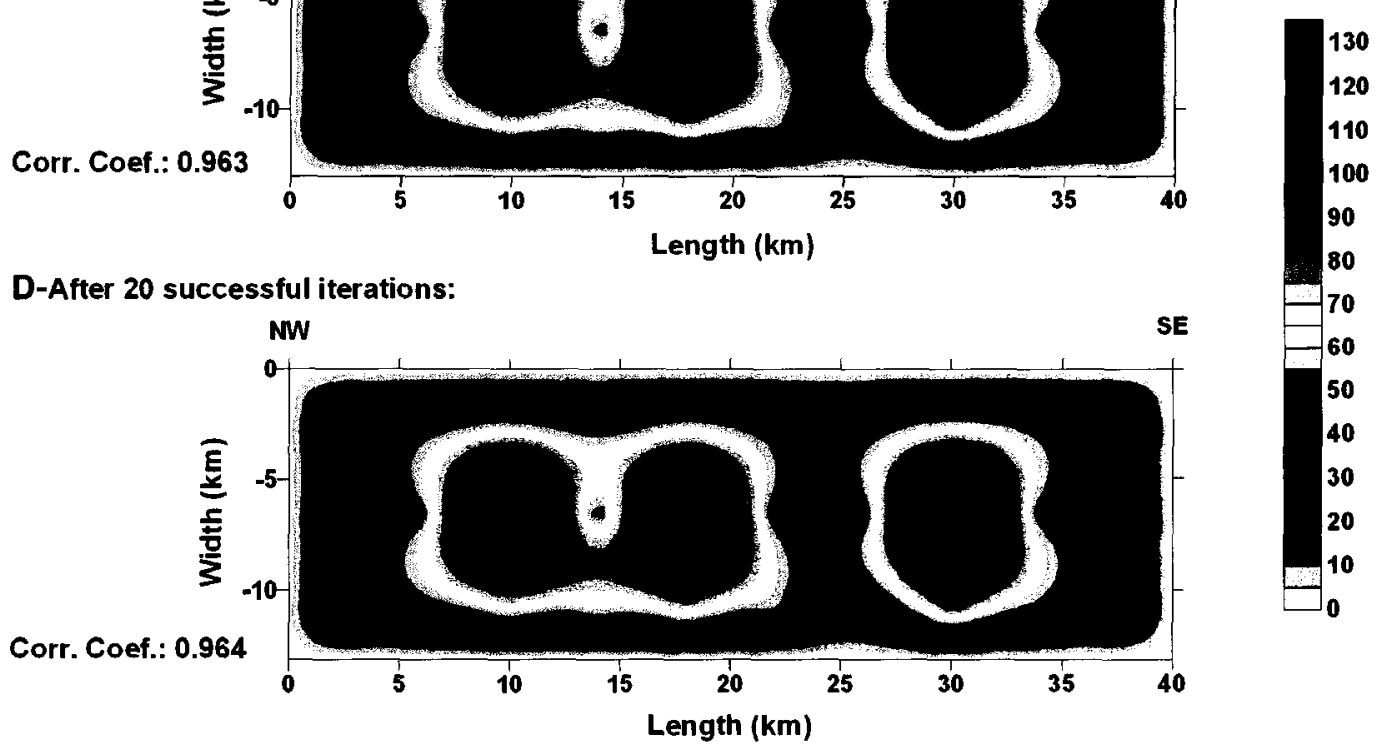

Figure (4.11) The succession of inversion program solutions for the derivation of target stress parameter distribution in the specified inversion steps. The program successfully finds the location and the approximate size of asperities. 
A-Target:

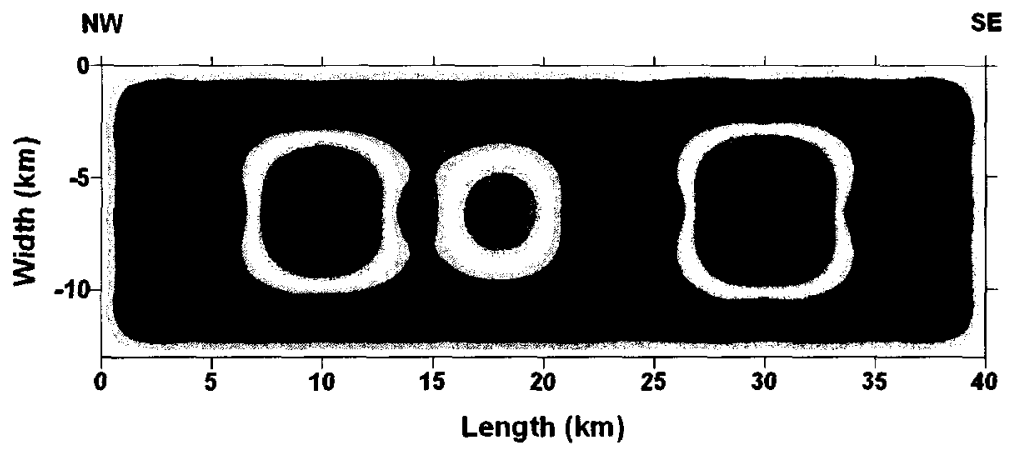

Stress Drop (Bars)

B-After 2 successful iterations:
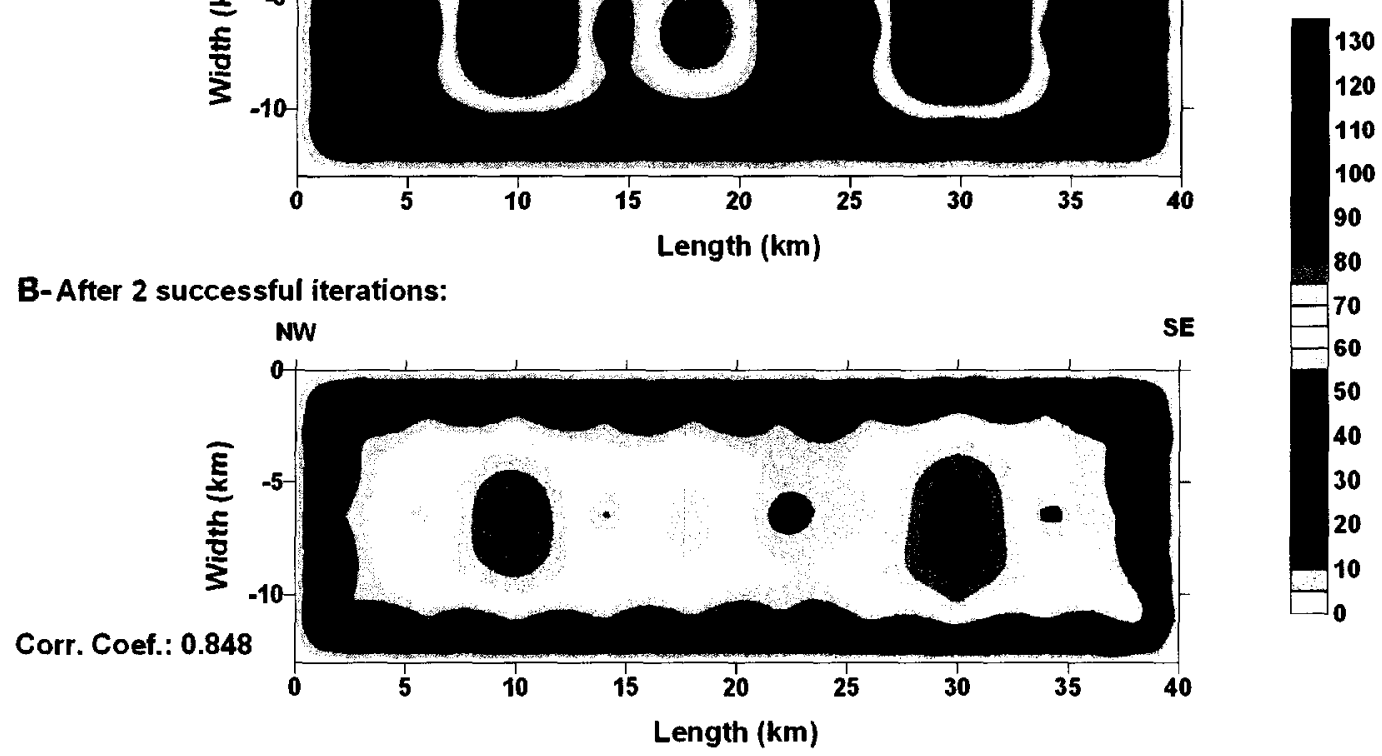

C-After 7 successful iterations:
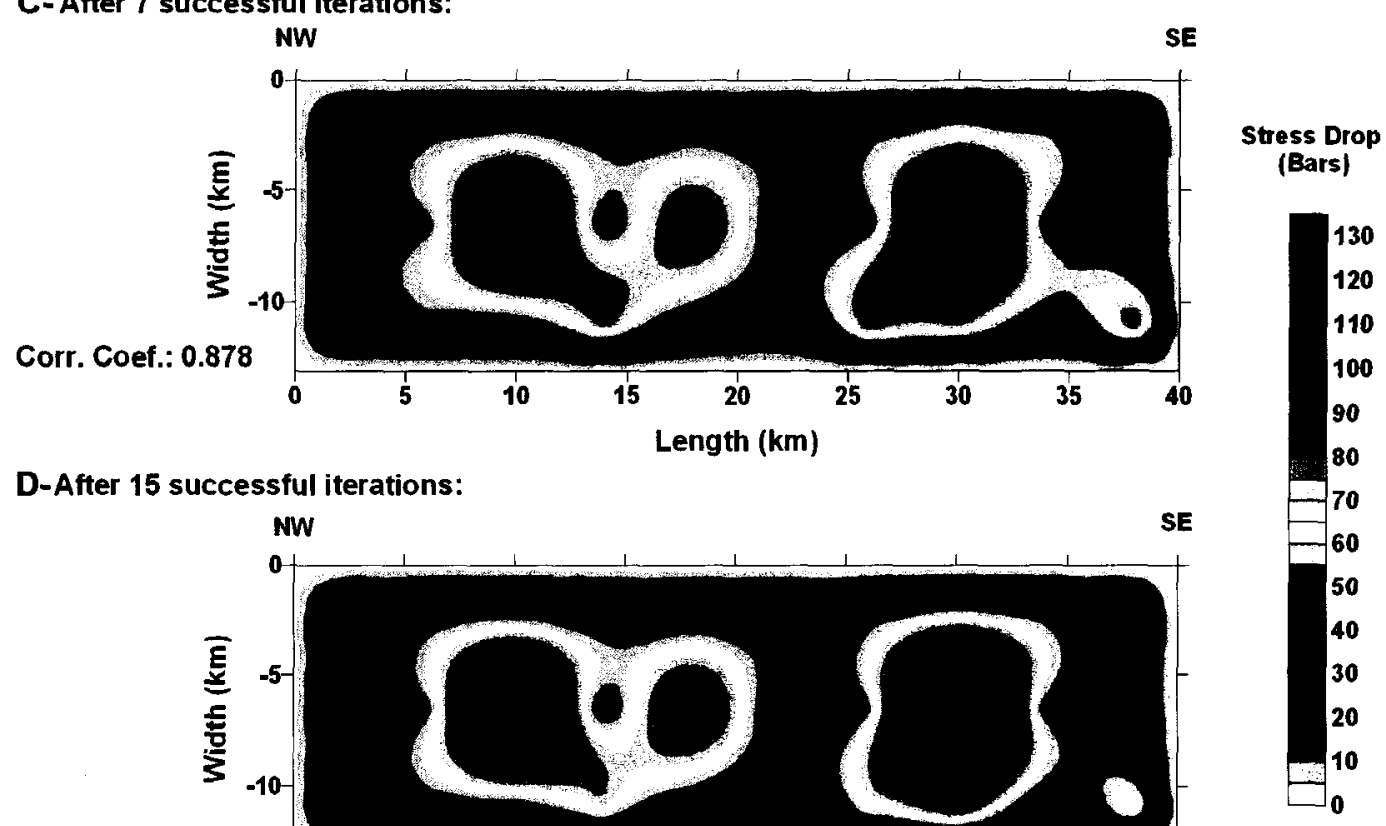

D-After 15 successful iterations:

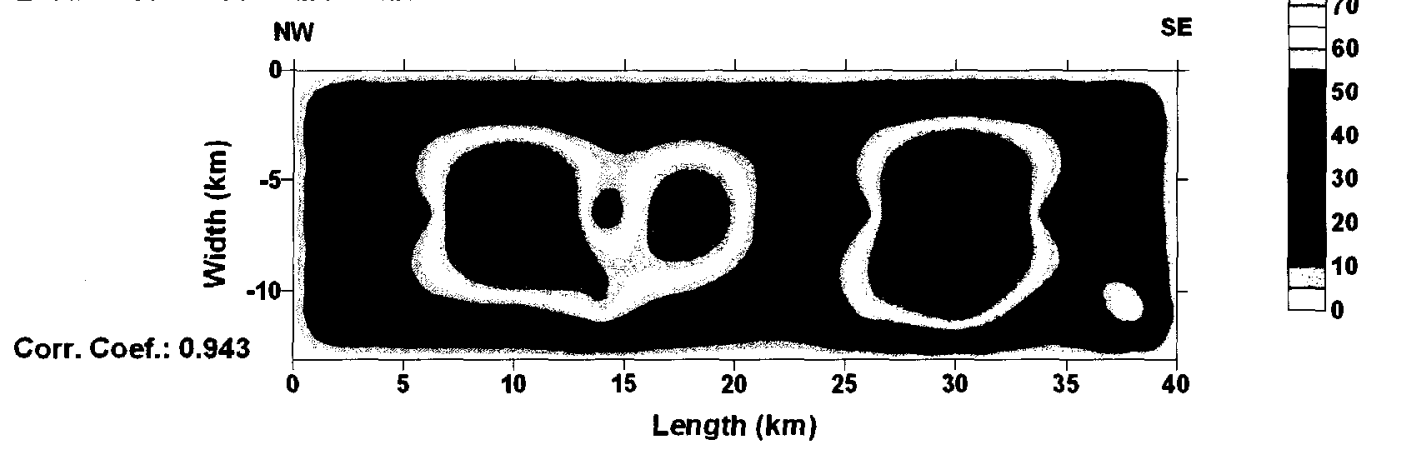

Figure (4.12) The succession of inversion program solutions for the derivation of target stress parameter distribution in the specified inversion steps. The program successfully finds the relative size of asperities. 


\subsubsection{Effect of initial guess}

The previous experiments consider a uniform stress parameter distribution as the initial guess for inversion. One of the reasons for choosing the Levenberg-Marquardt inversion method is its high success probability when starting from uniform values of parameters. However, the initial guess plays an important role in nonlinear multi-parameter estimation techniques. To check how it impacts our approach we also considered the effects of three different distributions for the initial guess: (i) very accurate initial guess; (ii) slightly inaccurate initial guess; and (iii) completely wrong initial guess. The effects of initial guess for above cases were studied by inputting forward modeling result into inversion program considering the parameters of Table (4.2) and $5^{\circ}$ of fault dip angle. The findings are as follows:

(i) Figure (4.13) shows target distribution, initial guess (which is uniform distribution), and a solution reached after 5 iterations by the inversion program for the experiment. In another experiment the target distribution remains the same, but the initial guess is somewhat close to the target distribution as shown in Figure (4.14). The comparisons of distributions reached after 5 iterations in Figures (4.13) and (4.14) show a better solution for the second experiment, or Figure (4.14).

When the initial guess is accurate, the solution does not change fundamentally from the initial guess, but it reaches the target solution within the first few iterations. The correlation coefficient, which is a measure of similarity between solutions and the target distribution, shows that a good initial guess can push the solution towards the target very quickly, and such a guess is more effective than assuming a uniform distribution.

(ii) Figure (4.15) shows target distribution, initial guess (which is uniform distribution), and resulting stress parameter distribution after 5 inversion iterations for an experiment. Figure (4.16) shows results of a companion experiment in which the target distribution is the same as before, but the initial guess is an inaccurate approximation of the target distribution. The inversion program improved the solution but the inaccuracies remained after 5 iterations. Comparisons of inversion products of Figures (4.15) and (4.16) suggest better results for the first experiment, or Figure (4.15). 
When the initial guess is inaccurate but carries some important features of the target distribution, then the inversion program improves the solution in every iteration, but the inaccuracies of the initial guess are not recovered within the first initial iterations. The correlation coefficient is smaller in second experiment than in first experiment in each iteration, suggesting that a uniform stress parameter distribution is a better choice than an inaccurate distribution as an initial guess.

(iii) The experiment for checking the response of the inversion procedure to an entirely wrong initial guess is done in two ways. We considered two wrong initial guesses: (a) a very poor initial guess, where the guessed asperity is in the wrong place, and as large in amplitude as the actual main asperity; and (b) a poor initial guess, where the guessed asperity is in the wrong place, but is much smaller than the actual asperity. Figures (4.17) and (4.18) show target distributions, initial guesses, and the ultimate outputs of inversion program tests for very poor and poor initial guesses respectively. The inversion program failed after a successful iteration when started from a very poor initial guess (an asperity in the wrong place and large in size). The program only succeeded in finding the correct location of the main asperity in this case. However, in the case of a poor initial guess where the asperity is in the wrong place but small in size, the program succeeded to clear the wrong location of the asperity, and found its correct location and size eventually. The performance of the inversion program when dealing with very poor and poor initial guesses is demonstrated in Figures (4.17) and (4.18). The experiments show that if the initial guess is a very poor one, the inversion procedure may fail to continue iteratively toward the correct solution, but if the initial guess is just somewhat poor, then the program can converge toward the right distribution in the long run.

Overall, we conclude that the uniform distribution is probably the best initial guess unless we have sufficient information from the intensity distribution or time evolution of amplitudes in records to make a perfect initial guess, which is seldom the case in real applications. 
A-Target:

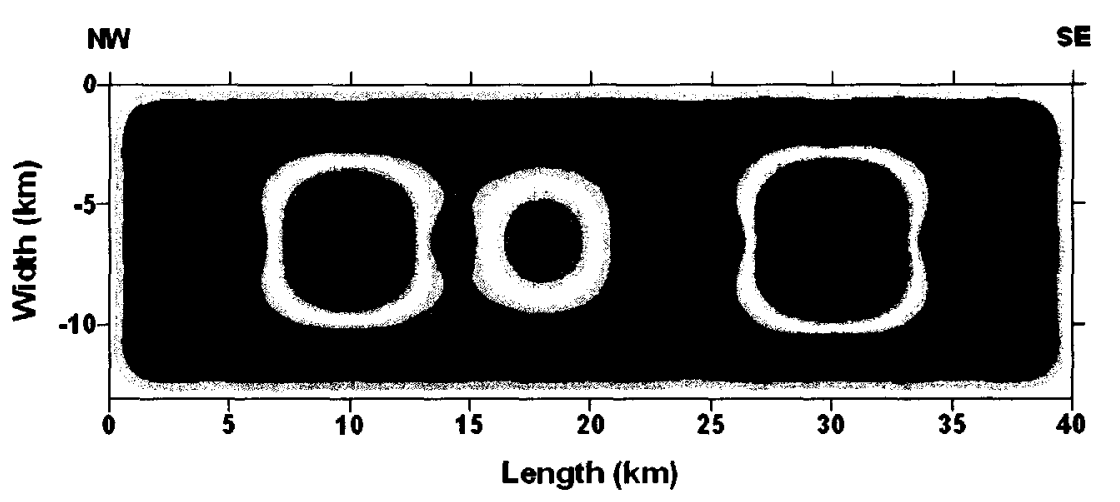

B-Initial Guess:

Stress Drop

(Bars)
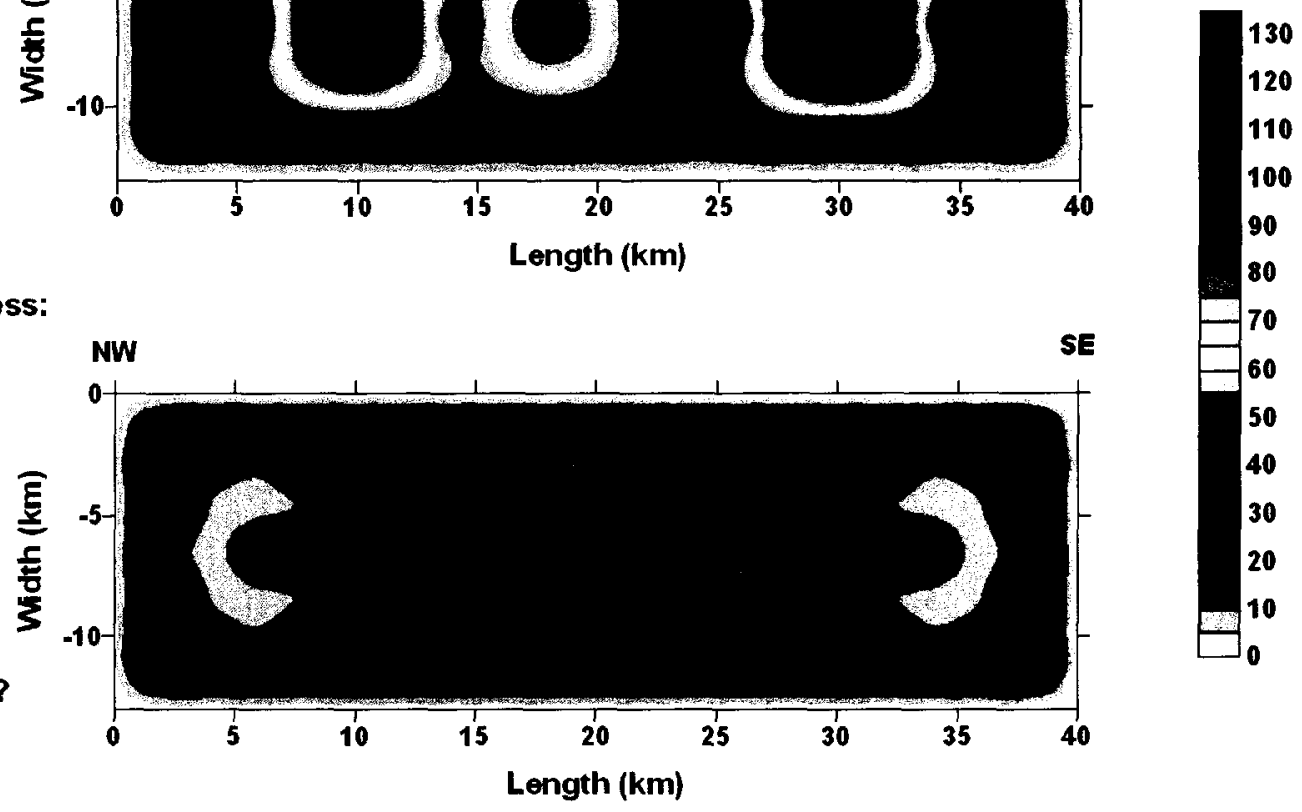

Corr. Coef.: ?

$$
\text { Length (km) }
$$

C-After 5 successful iterations:

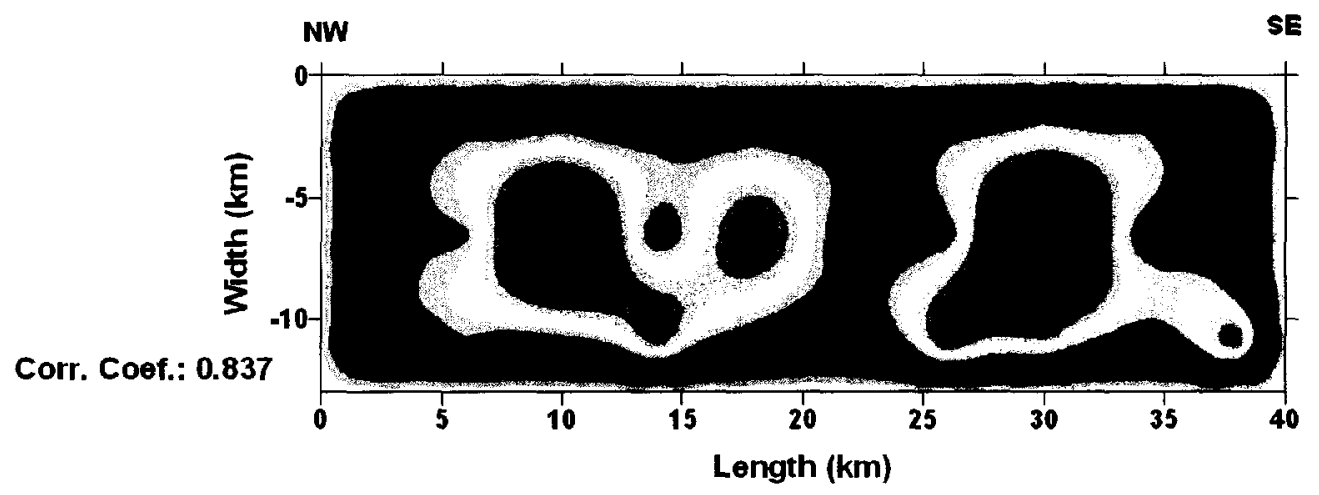

Figure (4.13) (A) Target stress parameter distribution; (B) Initial guess, which is uniform; (C) Stress parameter distribution reached after 5 inversion iterations (compare with Figure 4.14). 
A-Target:

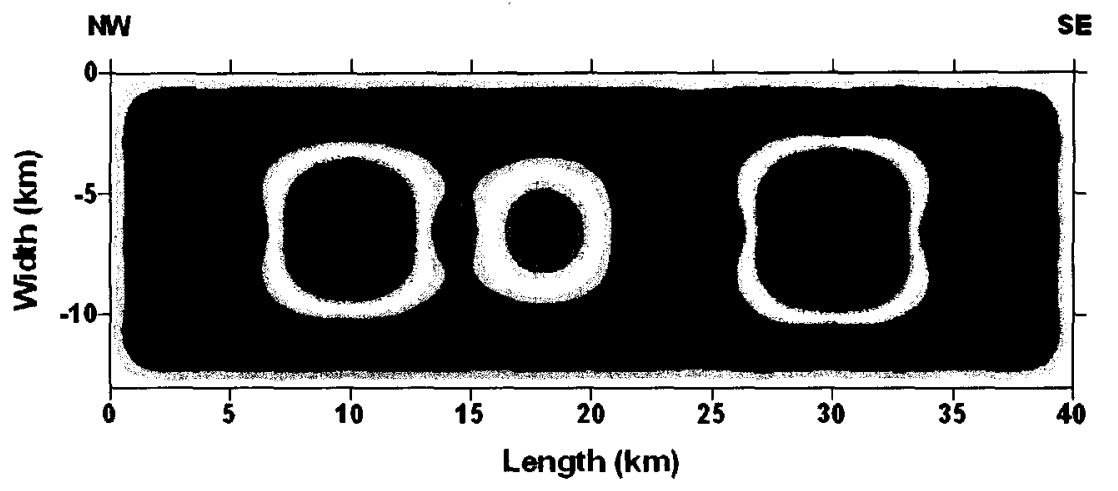

Stress Drop

(Bars)

\section{B-Initial Guess:}
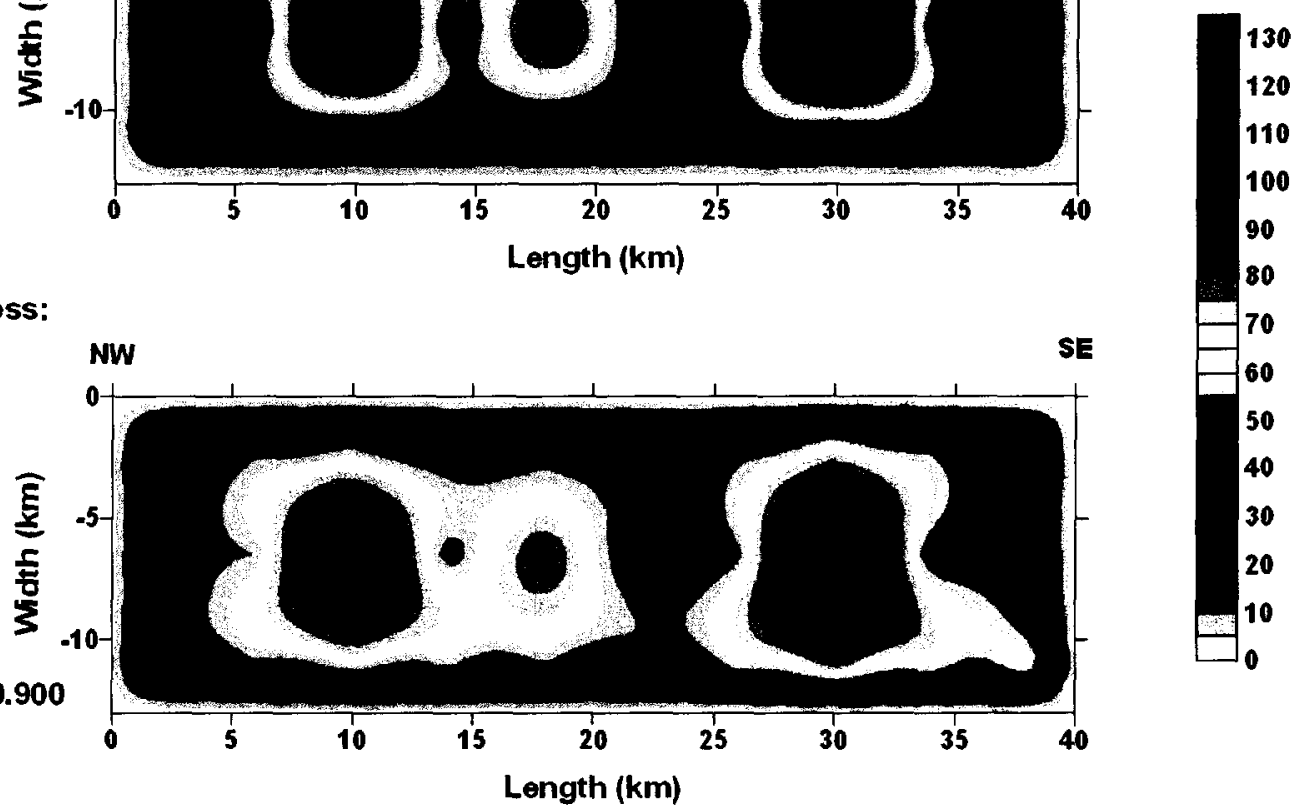

Corr. Coef.: 0.900

C-After 5 successful iterations:

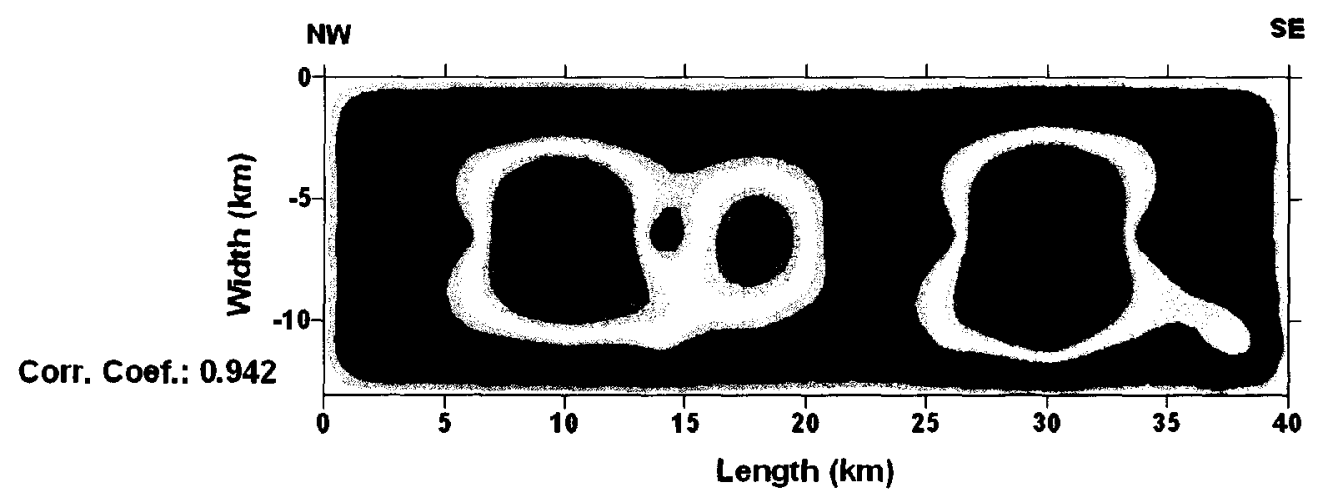

Figure (4.14) (A) Target stress parameter distribution; (B) Initial guess, which is accurate; (C) Stress parameter distribution reached after 5 inversion iterations (compare with Figure 4.13). 
Target:

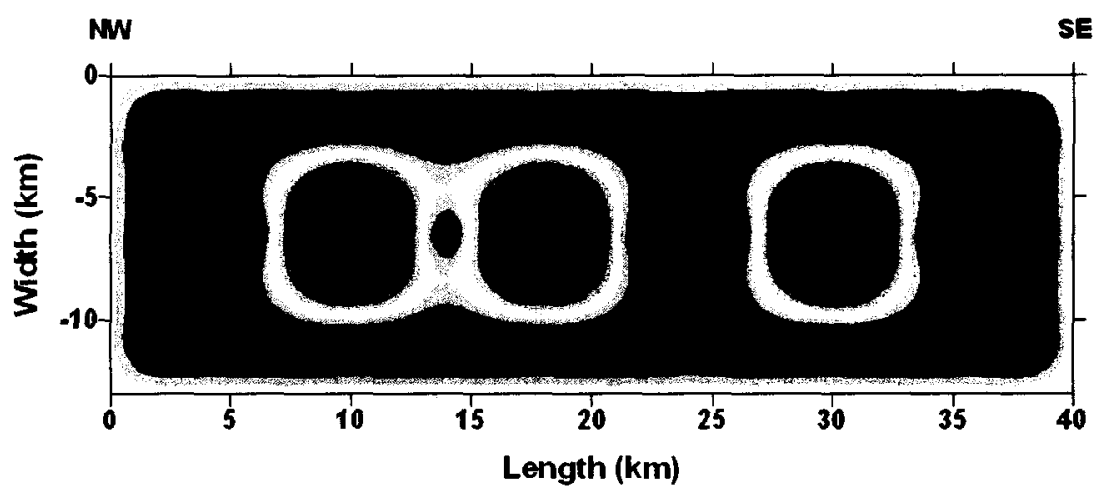

Stress Drop

(Bars)

Initial Guess:

Corr. Coef.: ?
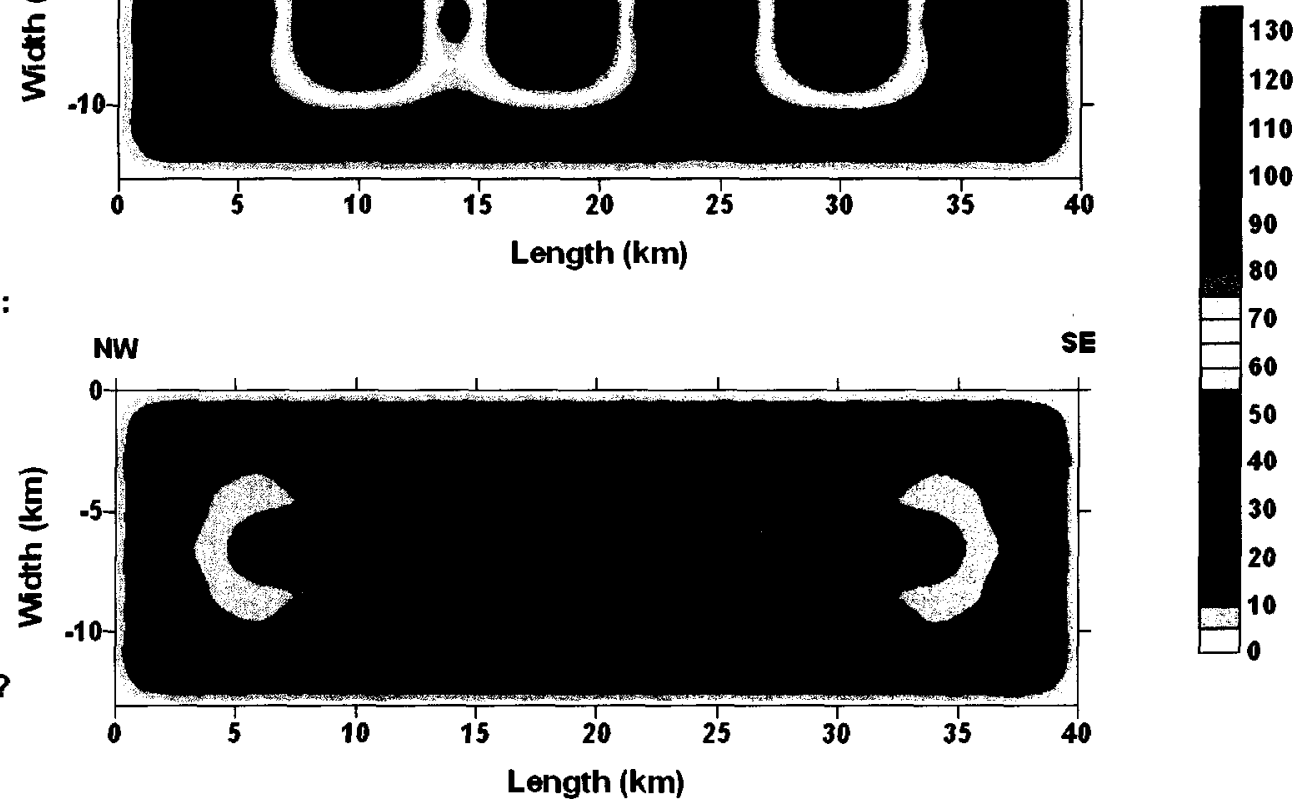

After 5 successful iteration:

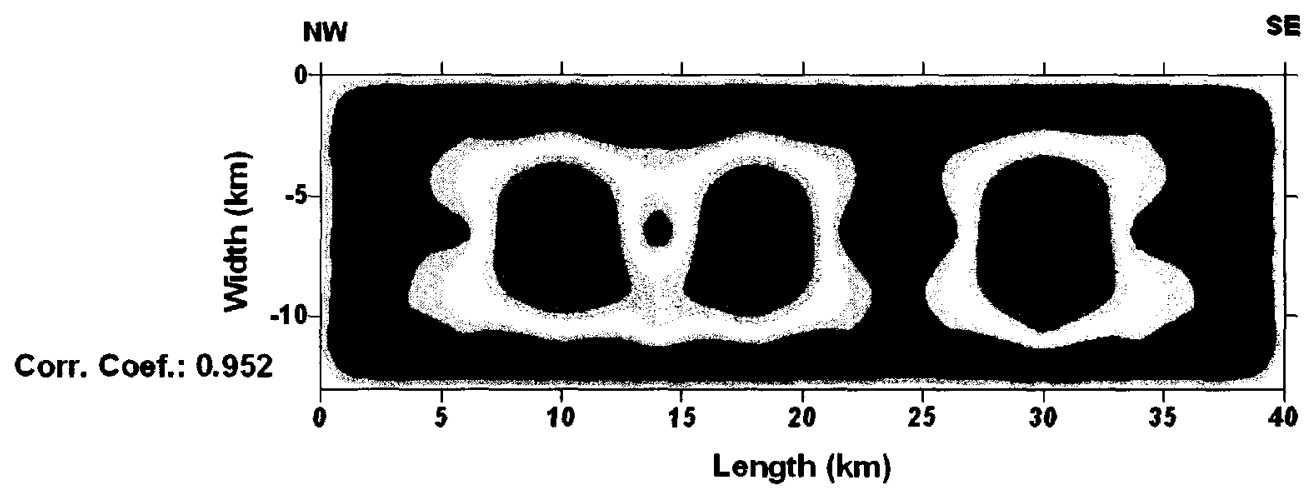

Figure (4.15) (A) Target stress parameter distribution; (B) Initial guess, initial guess, which is uniform; (C) Stress parameter distribution reached after 5 inversion iterations (compare with Figure 4.16). 
A-Target:

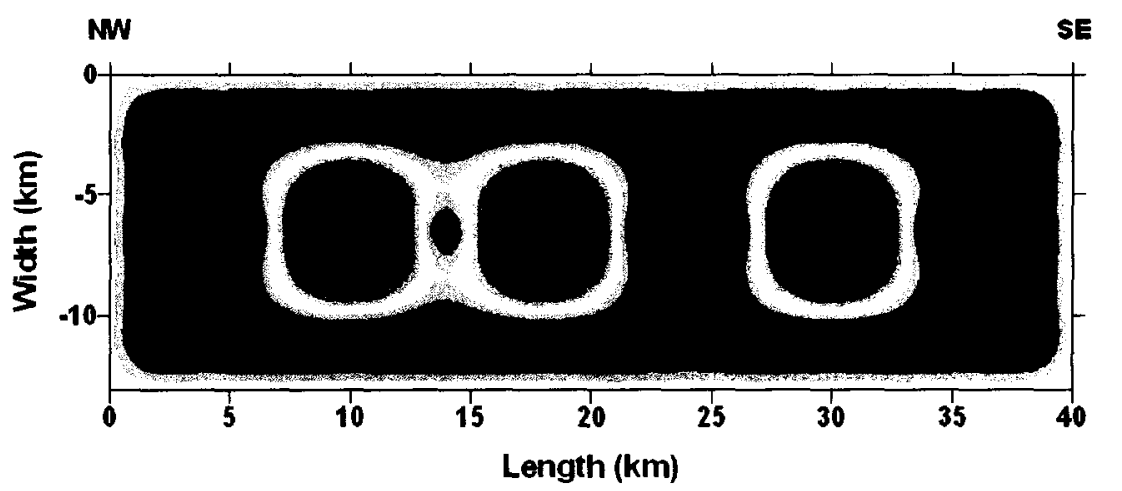

Stress Drop

(Bars)

B-Initial Guess:
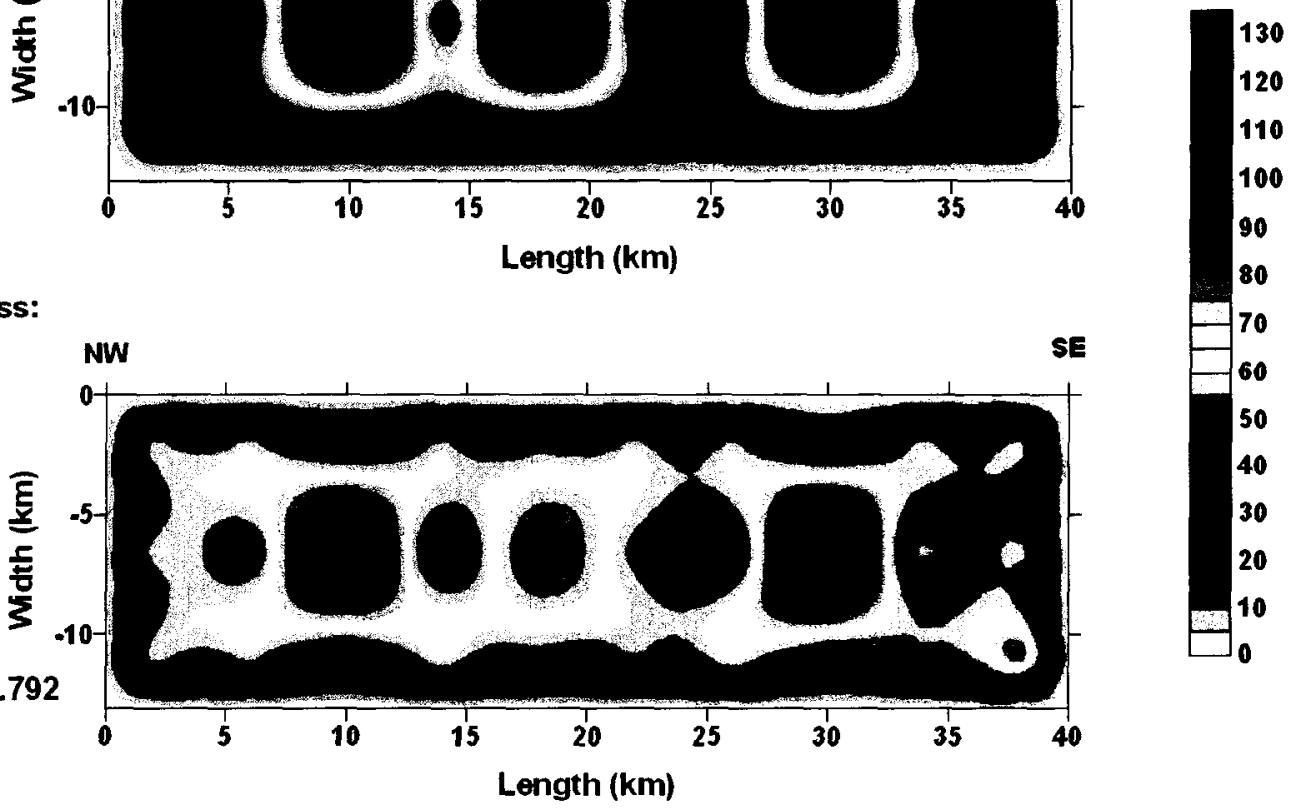

Corr. Coef.: 0.792

C-After 5 successful iterations:

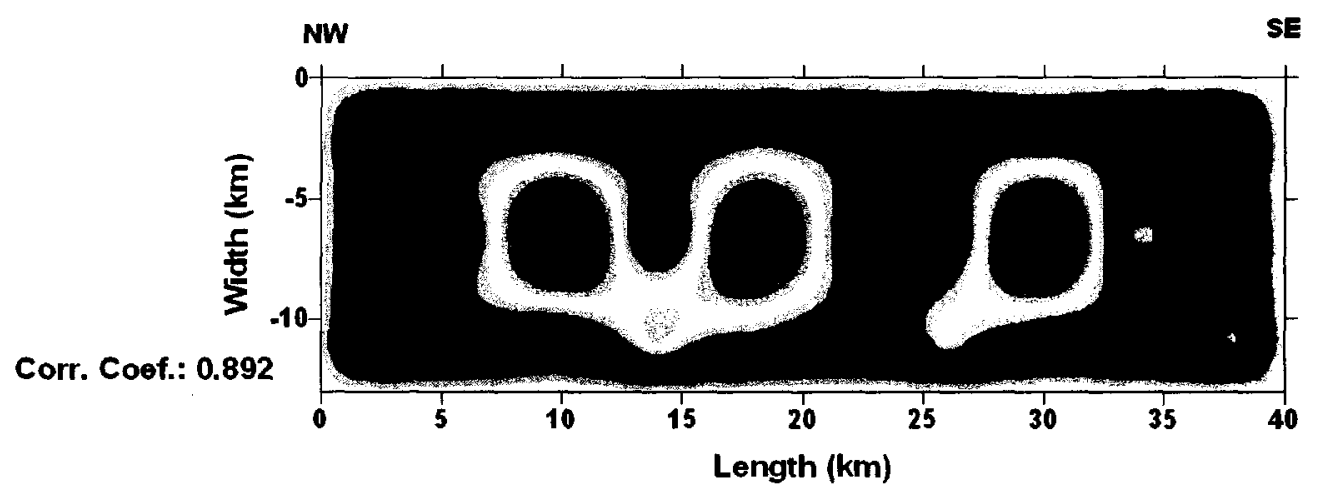

Figure (4.16) (A) Target stress parameter distribution; (B) Initial guess, which is inaccurate; (C) Stress parameter distribution reached after 5 inversion iterations (compare with Figure 4.13). 


\section{A-Target:}

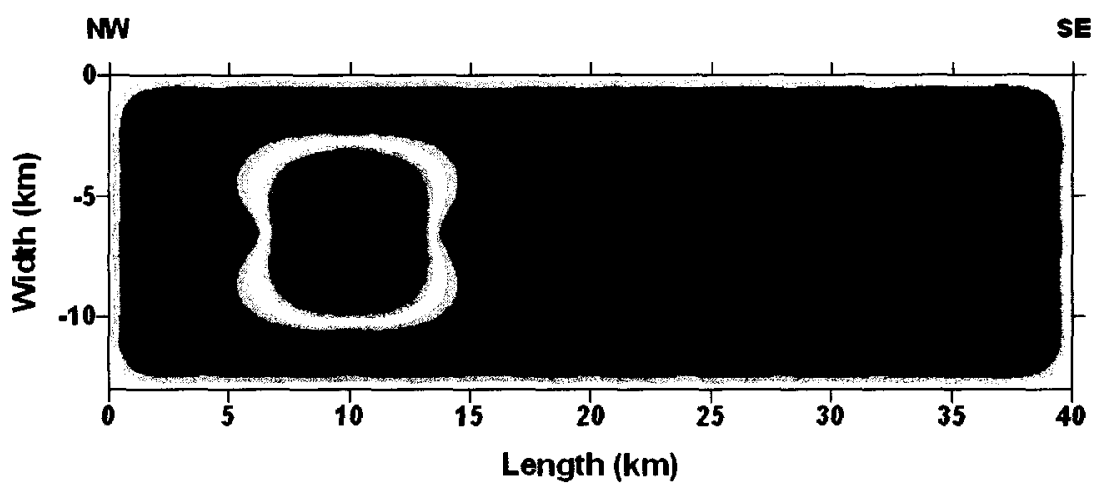

Stress Drop (Bars)

B-Initial Guess:
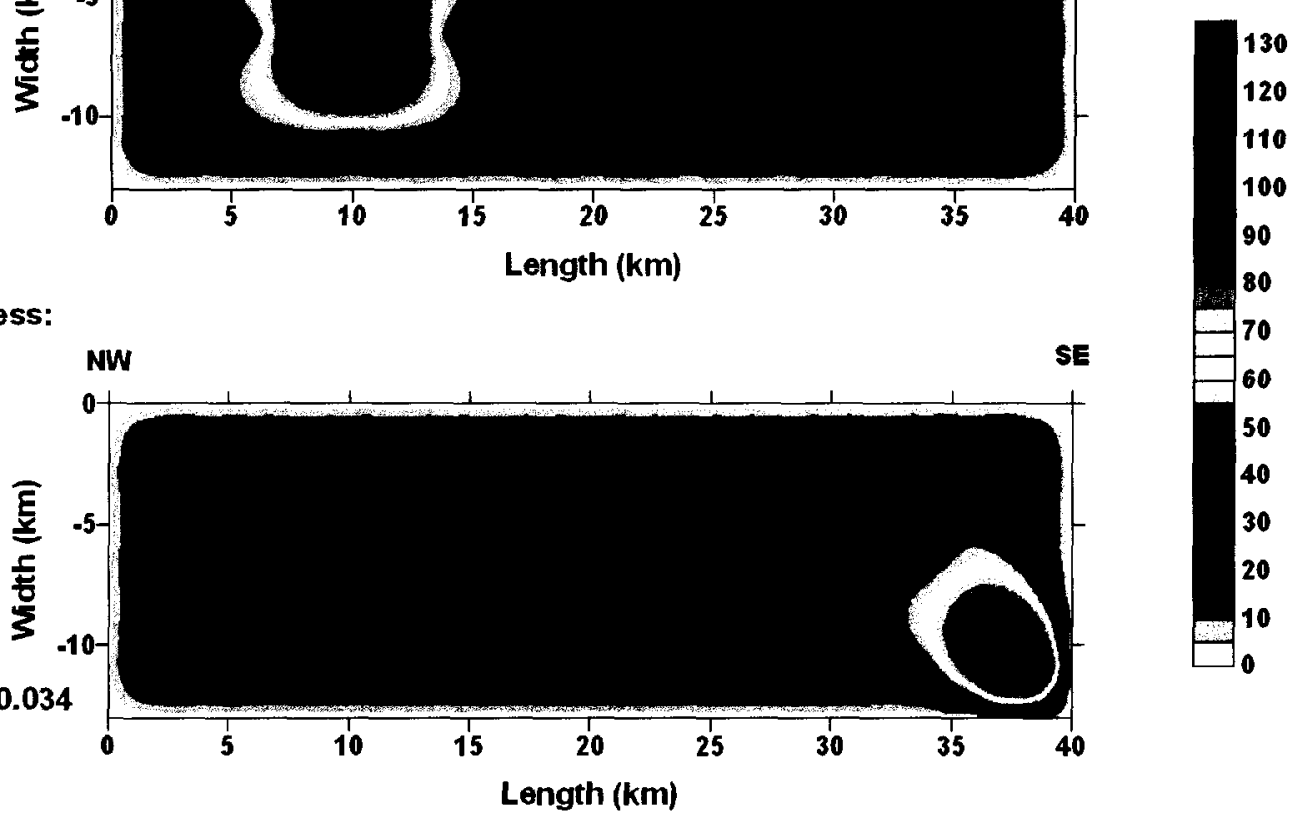

Corr. Coef.: -0.034

ccessful):
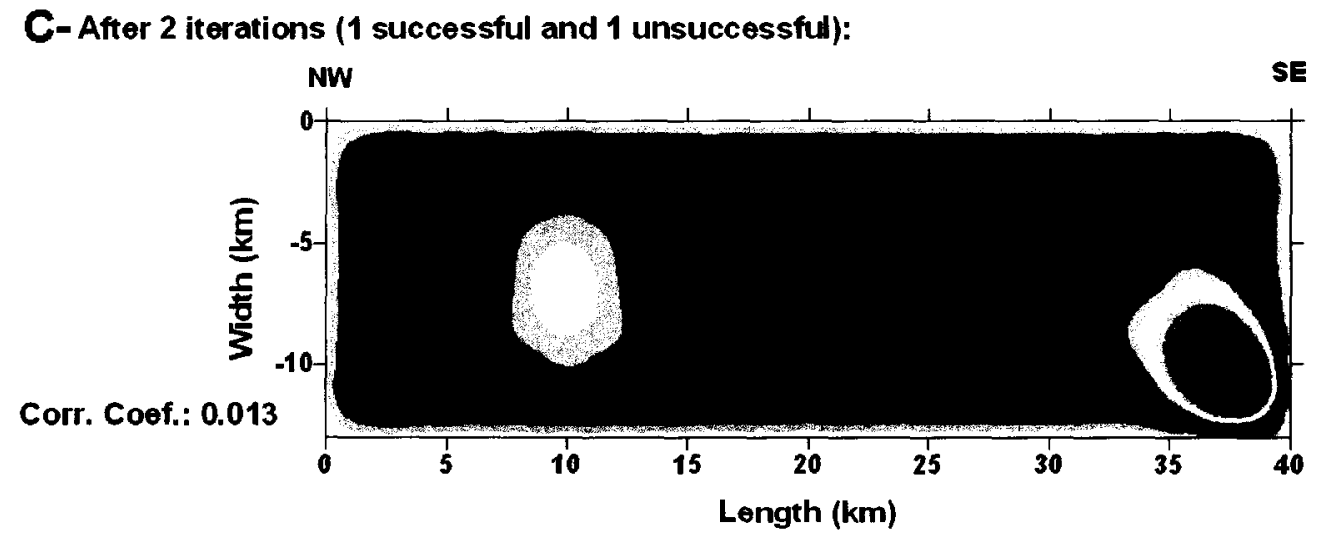

Figure (4.17) (A) Target stress parameter distribution; (B) Initial guess, which is a very poor guess with wrong place and large size of asperity; (C) Ultimate stress parameter distribution reached by inversion program. 


\section{A-Target:}

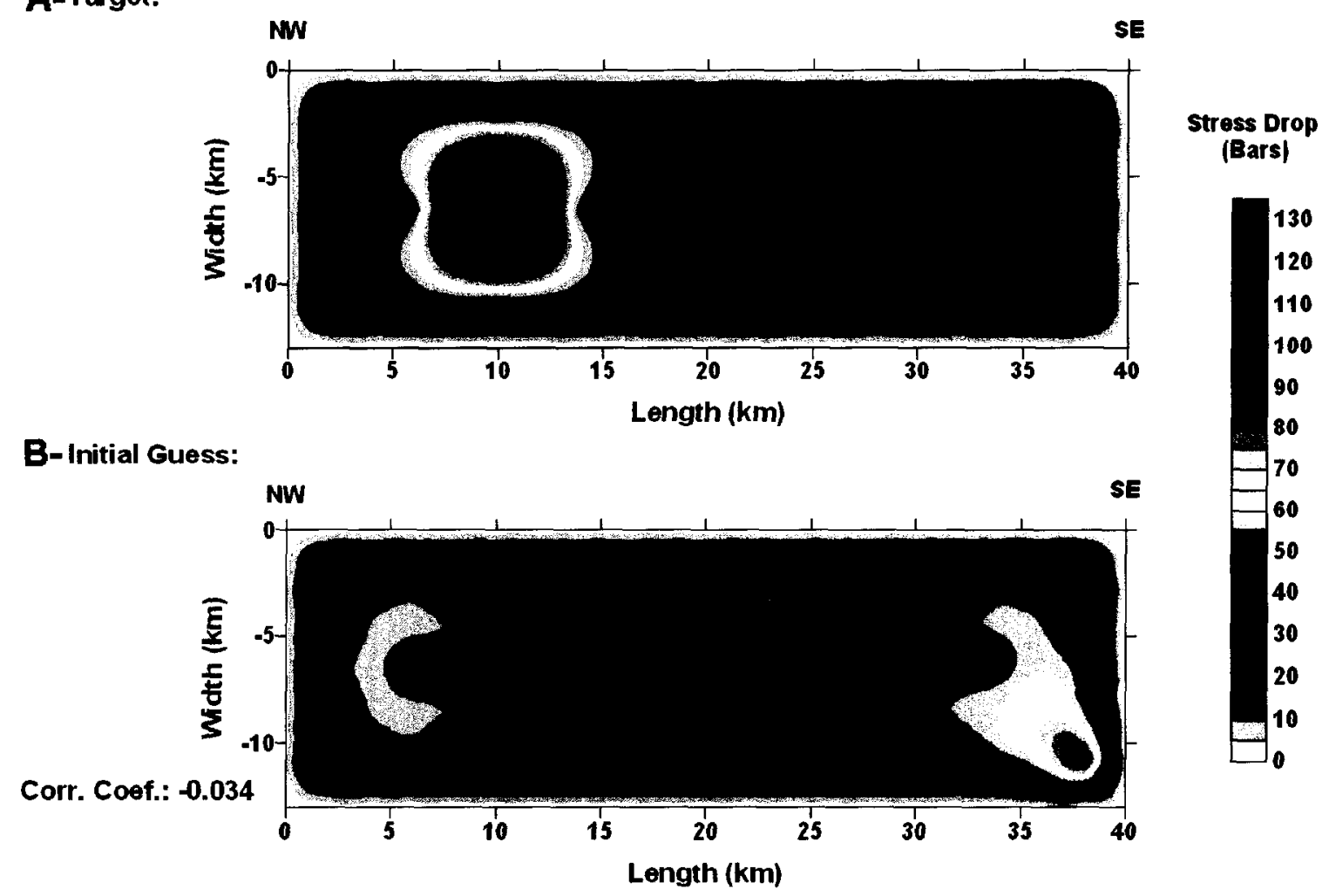

C-After 35 successful iterations:

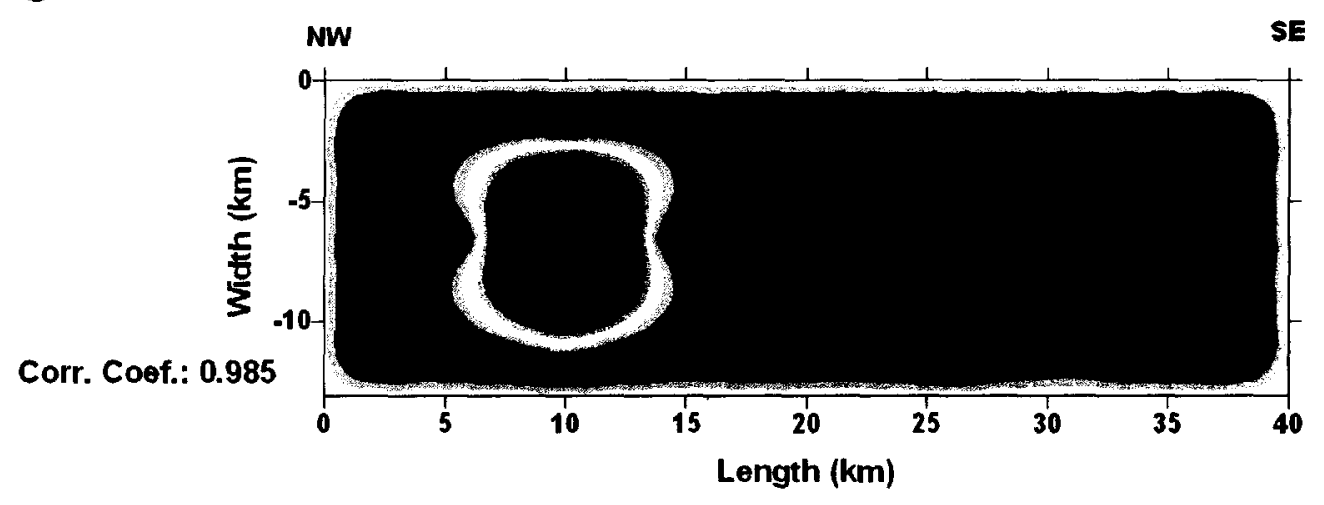

Figure (4.18) (A) Target stress parameter distribution; (B) Initial guess, which is a poor guess with wrong place and small size of asperity; (C) Ultimate stress parameter distribution reached by inversion program. 


\subsubsection{Effect of station distribution and asperity location}

Station distribution plays an important role in locating asperities, since from Equation (4.10) we know that elements of the Jacobian matrix are distance dependent (depending on the distance from the center of the sub-source to the recording site). Furthermore, a sparse distribution of stations around a sub-source makes the data insufficient for the unique determination of stress parameter in that sub-source. To study the effect of asymmetric station distribution around fault, we chose the actual station distribution around the 2006 Parkfield earthquake causative fault (Figure 4.19). Fault dip angle and EXSIM trial numbers in both forward modeling and inversion are $83^{\circ}$ and 10 , respectively. The other parameter settings of EXSIM are those in Table (4.2). Figure (4.19) shows that the station distribution around the southeast end of the fault is much denser than in the northwest end. Forward modeling and inversions are performed for 10 different scenarios, in which we assigned one high-stress-parameter sub-source (for each scenario) to lie in a location in the mid row of the fault from northeast to southwest. Graphs $(4.20 \mathrm{~A})$ to $(4.20 \mathrm{~J})$ show the target distribution and the inversion program's ultimate solutions for the $1^{\text {st }}$ to $10^{\text {th }}$ scenarios respectively. In Case 1 the high-stress subsource is at the northwest end of the fault, and in successive cases the high-stressparameter sub-source is switched towards the southeast, in one sub-source intervals. The stress of the high-stress-parameter sub-source is fixed at 5 times the value of the other sub-sources.

The solutions obtained after the $3^{\text {rd }}$ scenario $\left(3^{\text {rd }}\right.$ location of high-stress sub-source) start to be meaningful. By the $10^{\text {th }}$ scenario (high-stress sub-source in southwest), the solution is excellent, as measured by a high value of correlation coefficient, and good $\Xi$ value reduction. Since the Parkfield fault is almost vertical, the overall resolving power of the program is not high. The inversion program fails after the $1^{\text {st }}$ iteration in the first scenario (high-stress in northwest) because of lack of a sufficient number of recording sites around that sub-source (there is just one station above the high stress parameter sub-source and nothing else for some distance). We allowed the inversion program to run for up to 50 iterations for some cases, although the solution improvement in all cases is very small after the $10^{\text {th }}$ iteration. As the asperity is moved towards the southeast, where the station distribution is denser, the ability of the program improves markedly, as shown in Figure 
(4.20). We conclude that if the fault dip angle is steep, then the high-stress-drop asperity may hide itself in an area of very low station density. However, even if there are a few stations around the asperity, the method can still find its most probable location horizontally (but not vertically) for steeply dipping faults. 


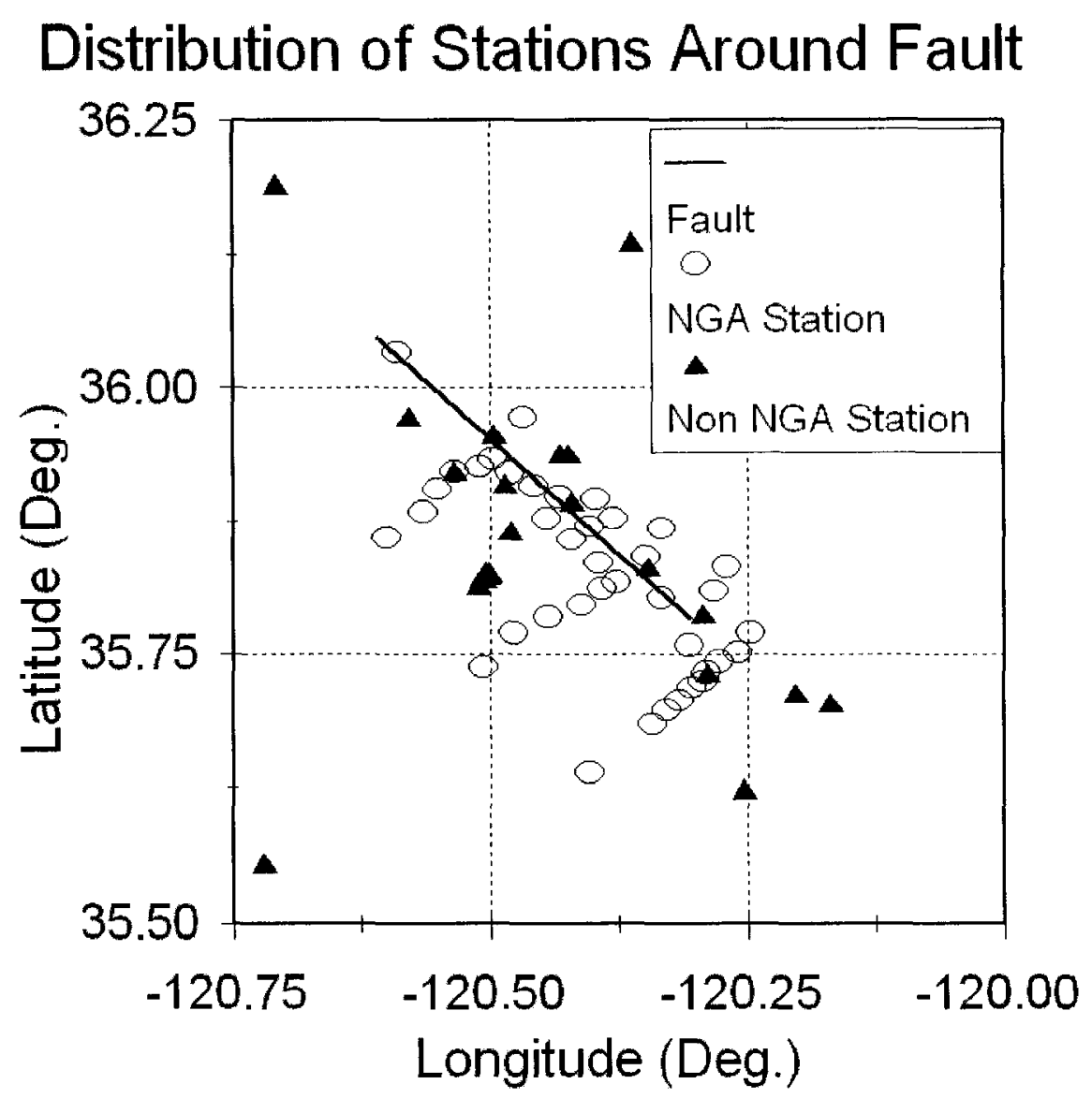

Figure (4.19) Distribution of stations around the Parkfield fault with known (NGA) and unknown (non-NGA) site types. 

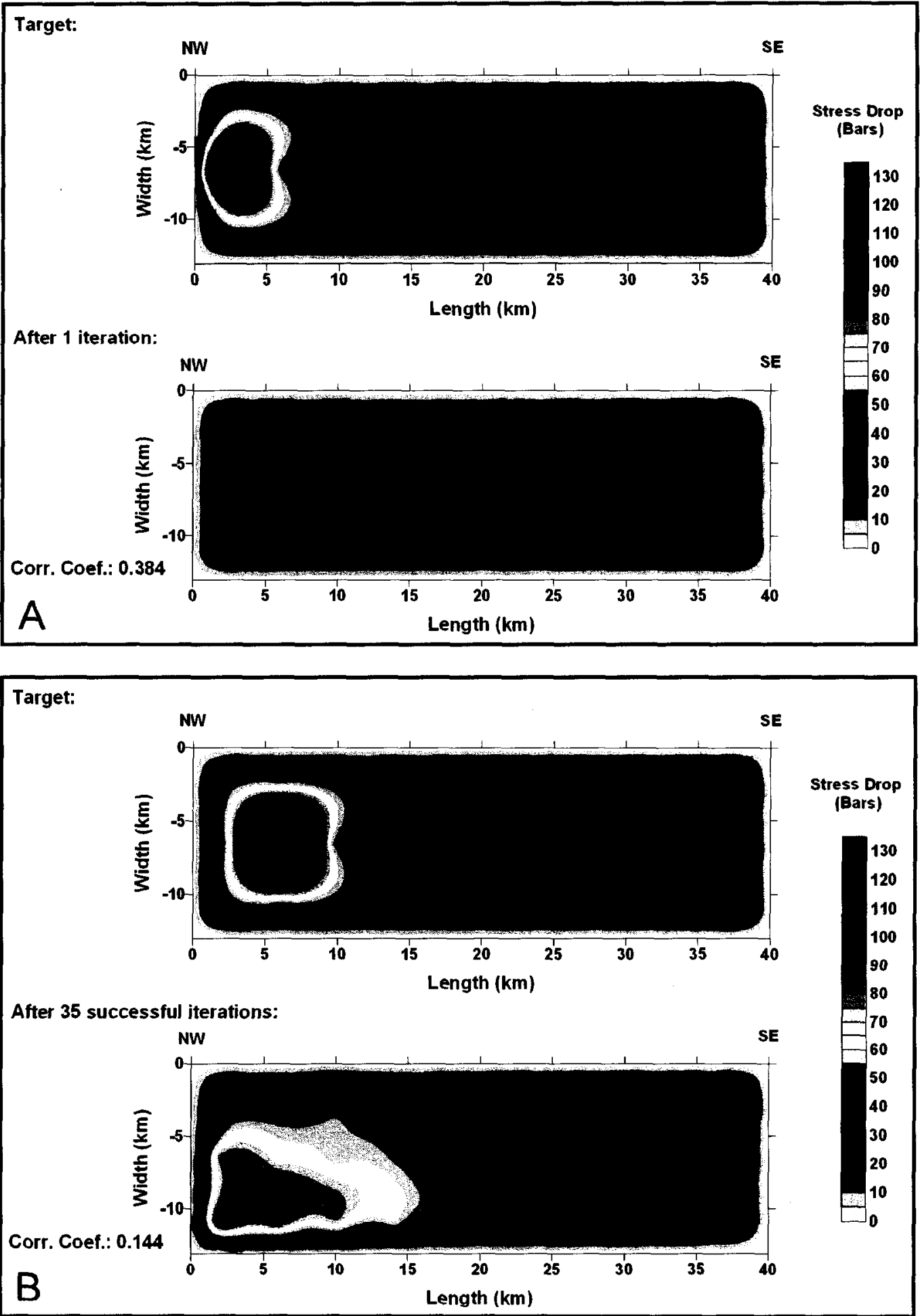

Figure (4.20) Target stress parameter distributions and corresponding inversion results when forward modeling and inversions are performed for the actual station distribution of the Parkfield earthquake. Differences in solutions are a result of the non-uniform station distribution. 

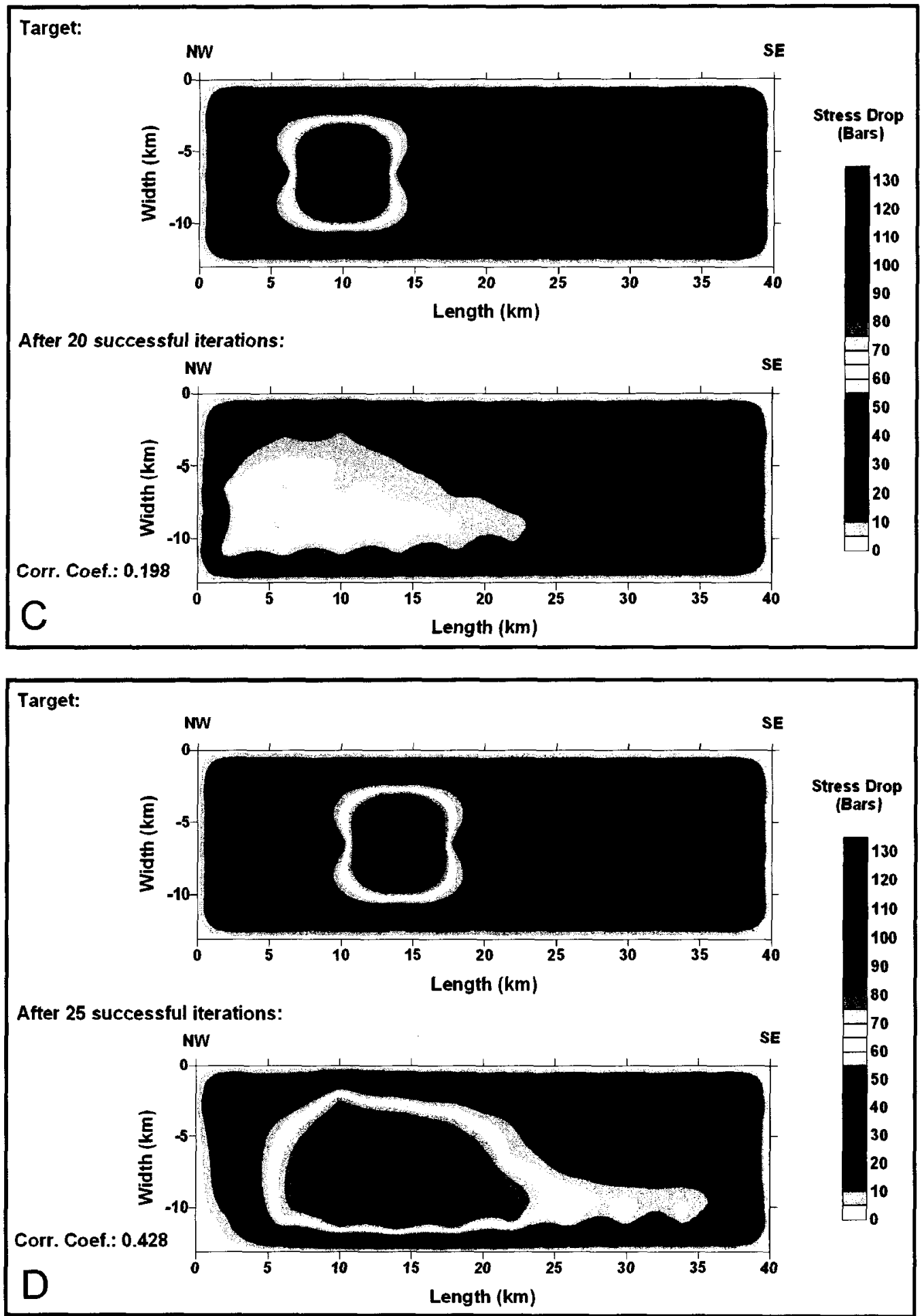

Figure (4.20) Target stress parameter distributions and corresponding inversion results when forward modeling and inversions are performed for the actual station distribution of the Parkfield earthquake. Differences in solutions are a result of the non-uniform station distribution. 

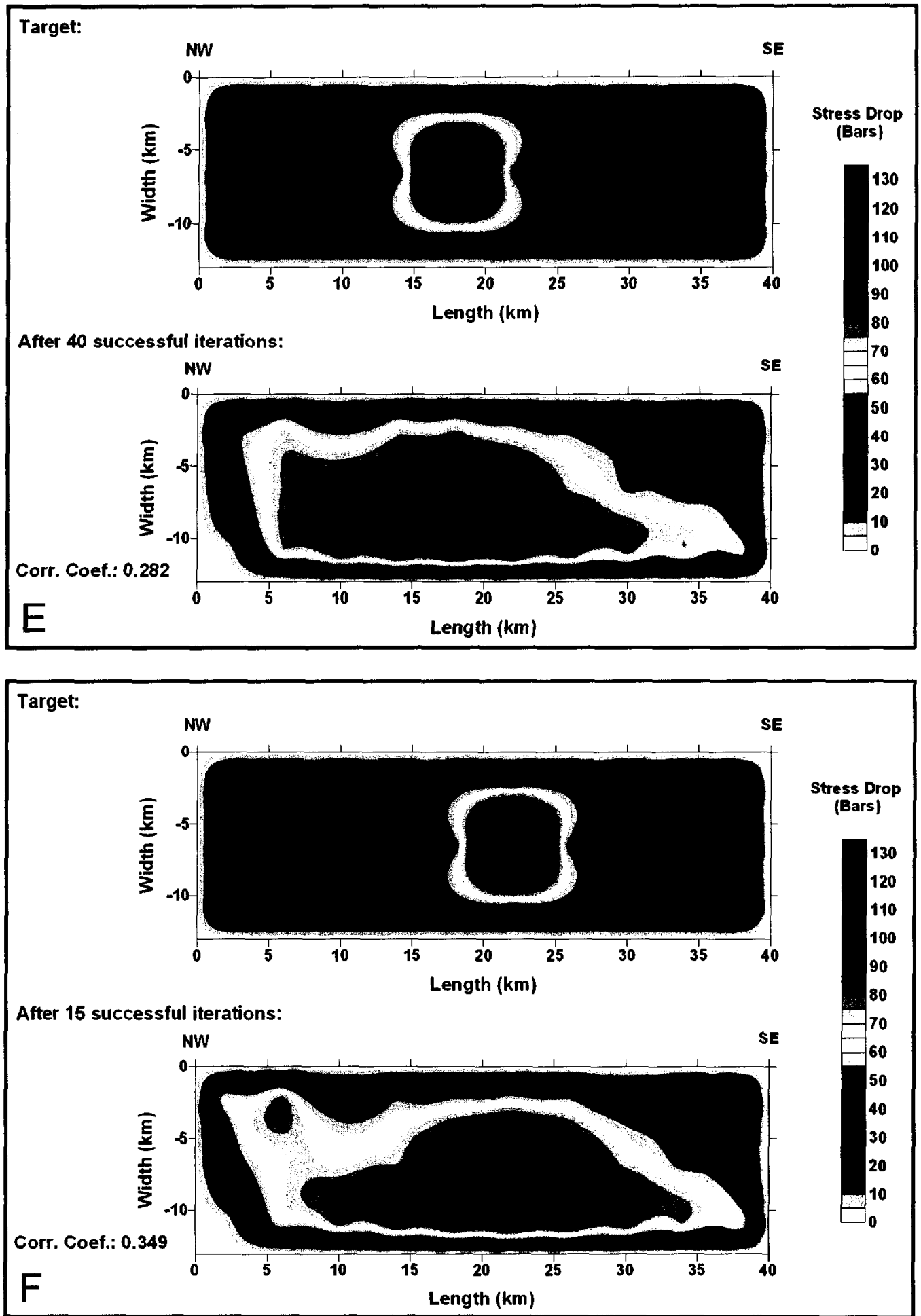

Figure (4.20) Target stress parameter distributions and corresponding inversion results when forward modeling and inversions are performed for the actual station distribution of the Parkfield earthquake. Differences in solutions are a result of the non-uniform station distribution. 

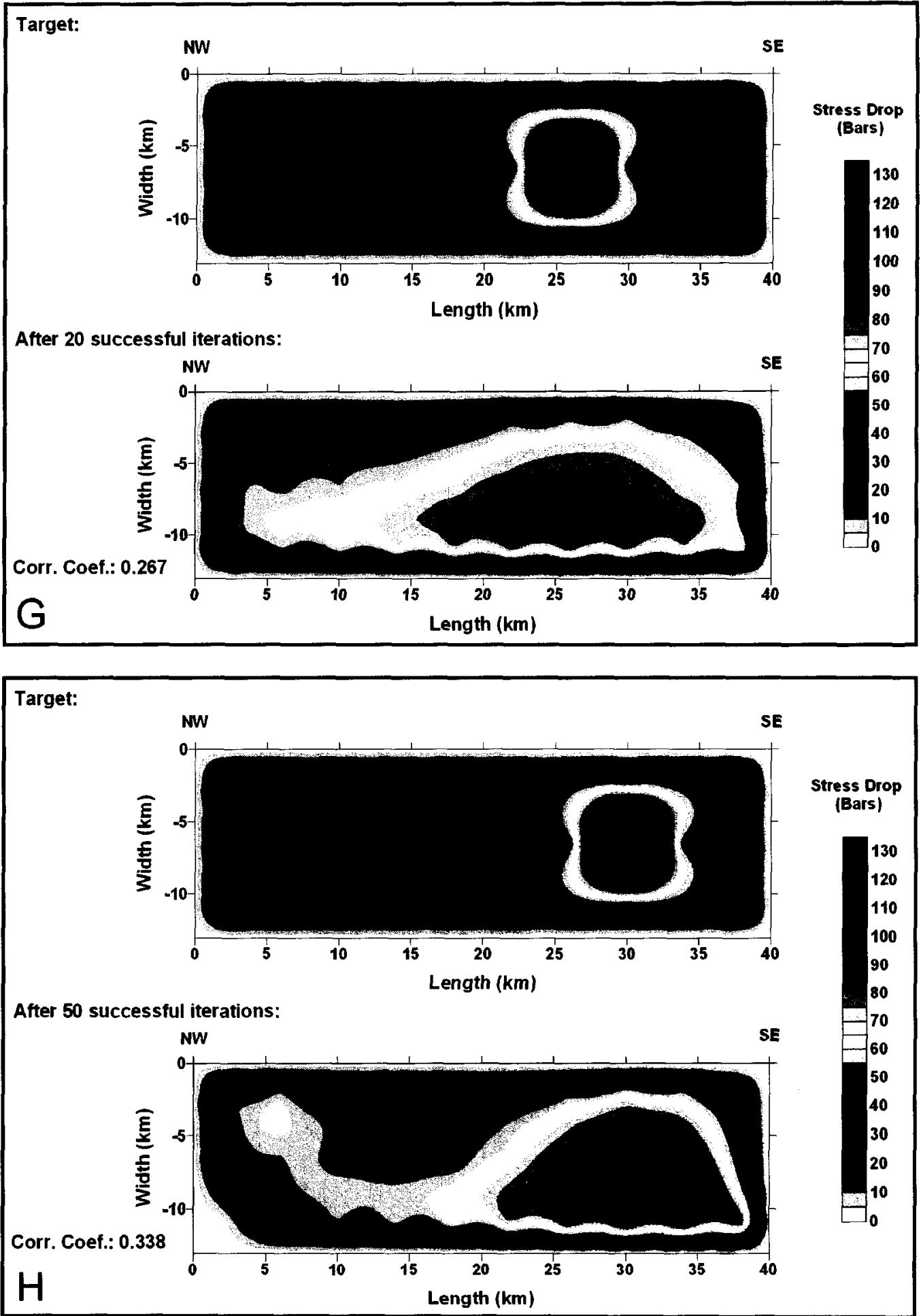

Figure (4.20) Target stress parameter distributions and corresponding inversion results when forward modeling and inversions are performed for the actual station distribution of the Parkfield earthquake. Differences in solutions are a result of the non-uniform station distribution. 

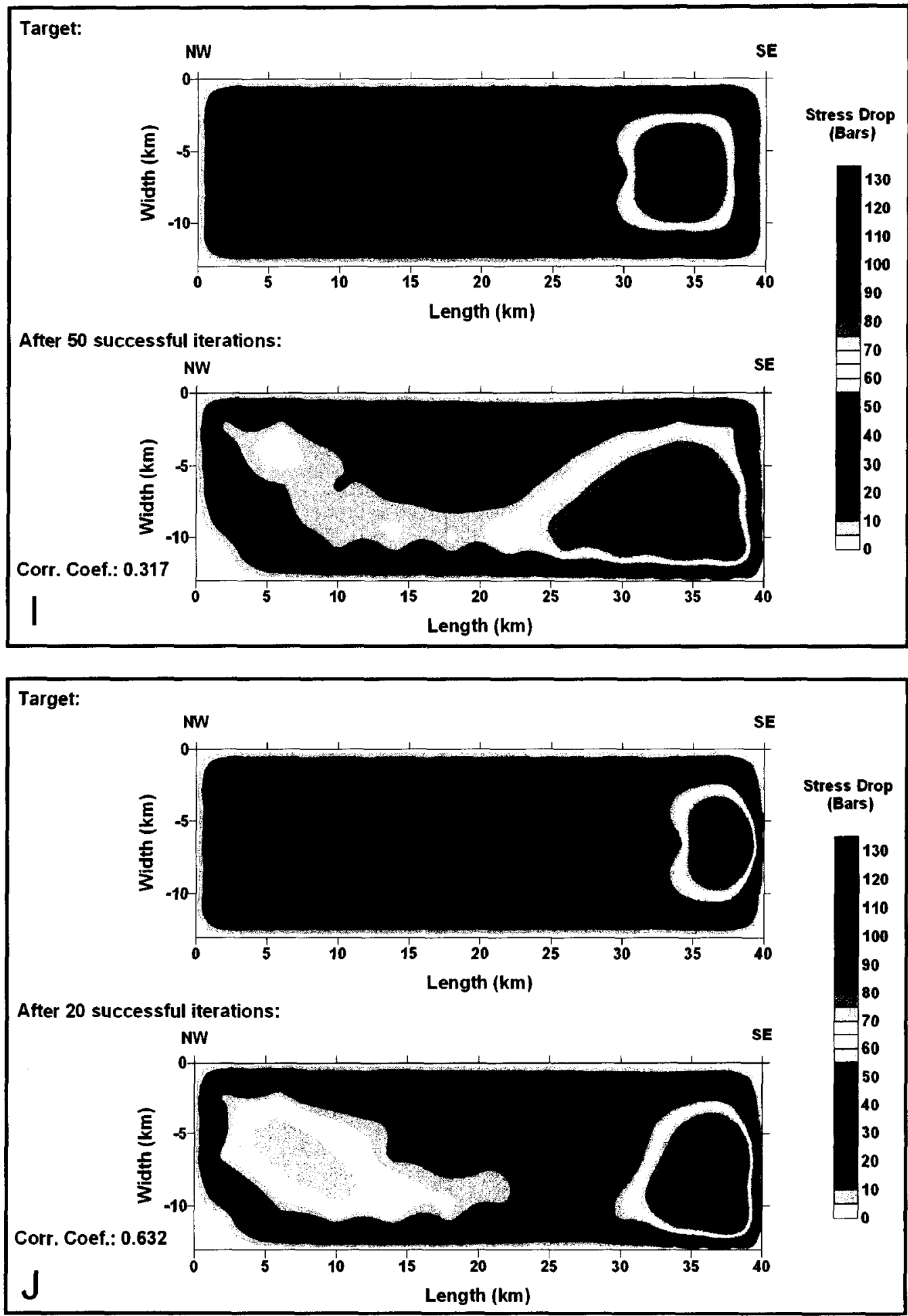

Figure (4.20) Target stress parameter distributions and corresponding inversion results when forward modeling and inversions are performed for the actual station distribution of the Parkfield earthquake. Differences in solutions are a result of the non-uniform station distribution. 


\subsubsection{A note on resolution}

The abovementioned approach for doing performance studies is a simple version of the checkerboard-test implemented in velocity structure studies. The checkerboard test is an experimental test mainly used to find the resolution of tomographic inversion studies. In this test it is assumed that every other element of the grid under study has high velocity (a checkerboard pattern). Forward modeling is performed for the checkerboard model, and the model predictions are then fed to the inversion tool to check if the checkerboard-like velocity structure in the model can be retrieved. The degree of the similarity of the solution with the initial model is considered as a measure of quality or resolution of inversion for the given velocity grid and source/receiver geometries.

A similar approach could be adopted to study the resolution power of our inversion tool, assigning high stress parameter concentrations to every other sub-source, doing forward modeling for recording sites, and inverting forward modeling results for the derivation of stress parameter distribution on the fault surface. However, for our study the checkerboard test is not a realistic check on resolution for the following reasons: (a) the tests performed above suggest that, for realistic station distributions, the method is only capable of finding distinct concentrations of the stress parameter, and will tend to spread this stress level over neighboring sub-sources - thus we would not expect it to be capable of resolving a checkerboard pattern; (b) since the stations are always on the surface and the formulation of the problem assigns lower weight to farther sub-sources, the deep grid elements will always suffer from lower resolution. The performance studies in this chapter confirm these expectations (e.g., see Figures 4.4, 4.20). Consequently if a packed grid of alternating high-stress and low-stress is implemented as the target distribution, the spreading of the high-stress concentrations over neighboring sub-sources will mix together and result in a washed-out picture of this distribution. This method is only capable of handling the more typical case that is observed on earthquake faults, in which there is a small number of significant asperities to be determined. It is therefore 
reasonable to focus on results of performance studies with simple target distributions, as given in section 4.3 .

\subsection{Accelerating the inversion program}

A new algorithm is designed and the corresponding program is linked to the inversion program to accelerate its convergence speed. Previous studies in this chapter showed that (i) an accurate initial guess can accelerate inversion program speed and (ii) in most cases the inversion program finds the location of asperities in its initial iterations (e.g., Figures $4.5,4.10,4.11,4.12)$. When the inversion program finds location of asperities, it tries to refine the solution iteratively by amplifying stress levels of asperity locations. The idea behind accelerating the inversion program is to develop an algorithm that takes the outputs of the inversion program resulting in its initial steps, amplifies the suggested stress levels of asperities, and feeds these partially processed data back into the inversion program for further refinement. We call this program: "Contrast amplifier".

\subsection{1 "Contrast amplifier" program}

A program called "Contrast amplifier" is developed to amplify the stress level of asperities resulting from the initial iterations of the inversion program. The program converts the stress parameter distribution on the fault surface resulting from the initial steps of the inversion process, and then enters the converted distribution as an input (or initial guess) to the inversion program in the coming iteration. Figure (4.21) shows the flow chart of the program. Its algorithm is as follows:

(1) Stress parameter distribution from the output of inversion program after initial iterations is assigned to the array " $a$ ".

(2) Every element of "a" is raised to power "exp" and assigned to a new array "b".

(3) The average slope (it will really be slope, if $\alpha \times \exp =1$ ) towards the center of a subsource is calculated (sub-source with indices $i$ and $j$ ) and assigned to array "c". The value of $c_{i j}$ (an element of array "c") is calculated using the following equation: 


$$
c_{i j}=\frac{\sum_{m=-1 n=-1}^{1} \sum_{i j}^{1}\left(b_{i j}^{\alpha}-b_{(i+m)(j+n)}^{\alpha}\right)\left(1-\delta_{m n}\right)\left(1-\delta_{-m n n}\right)}{\sum_{m=-l n=-1}^{1} \sum^{1}\left(1-\delta_{m n}\right)\left(1-\delta_{-m n}\right)}
$$

where $\delta_{m n}$ is the Kronecker Delta function, and is equal to 1 when $\mathrm{m}$ and $\mathrm{n}$ are equal and zero otherwise.

(4) " $c$ " is normalized to have an average equal to the average of "a". The minimum of " $c$ " is subtracted from all "c" elements (this is done if the smallest element of "c" has negative value). The new array's average is added to each element and raised to power "exp". Then each element of this new array is divided by the whole array average, and multiplied by the average of array " $a$ ".

(5) The locations of sub-sources with maxima of " $b$ " are identified by means of derivatives. The sub-sources that are local maxima are assigned a value of 1 and all other sub-sourced are assigned a value of 0 . These values are assigned to array "hill".

(6) In this step the program finds the modified stress parameter distribution, with the contrasts amplified. In this stage if a sub-source's indices correspond to a zero in array "hill", or ratio of "c" divided by average "c" is less than $\beta$ the value of corresponding element of array " $c$ " is assumed to be the average of array " $a$ ". Otherwise the element of array "c" is not modified.

(7) The array "c"is printed as output.

The purpose of this procedure is to amplify the level of the local minima on the fault surface, while keeping the average intact. So we call it "contrast amplifier". Quick review of parameter values of the program were checked and the values: $\exp =2, \alpha=0.5$, and $\beta=2$ are found by trial and error to produce the desired behavior.

\subsubsection{Application and performance of "Contrast amplifier" program}

The application and performance of the "contrast amplifier" program is checked in two sets of experiments. In the first set of experiments the output of the inversion program is 
used and stress parameter distributions after the $1^{\text {st }}, 2^{\text {nd }}$, or $n^{\text {th }}$ iterations are fed to the "contrast amplifier" (CA) program. By the target distribution, stress parameter distribution after $\mathrm{n}^{\text {th }}$ iteration, outputs of CA program, and renewing the correlation coefficients, it will be possible to evaluate improvement of results by application of the program. In the second set of experiments a further step is taken: the stress parameter distribution after an initial iteration of the inversion program is entered into the CA program and its output is used in the inversion program again. The levels of errors along with correlation coefficients of inversion program outputs in each iteration are compared in the two cases (In one case the inversion program starts from uniform stress and goes toward target stress parameter distribution while in the second case the output of the inversion program after initial iterations is sent to the CA program, and the CA program output is put back into the inversion program for further inversion steps).

Figure (4.22) correspond to dip angle of $5^{\circ}$, where the inversion program is working properly and its output after first iteration is entered into CA program. Figure (4.22) demonstrates the performance of CA program in filtering the fuzzy image of stress parameter distribution to a reasonable estimate of the target distribution. Since increasing fault dip angle affects inversion program by reducing the resolution of determined asperity locations in initial iterations, the CA program may wander in the very first inversion iterations until the inversion program succeeds in making a fuzzy picture of the real stress concentrations with correct asperity locations. Figure (4.23) corresponds to an experiment with $45^{\circ}$ fault dip angle. In this experiment the target distribution is the one used for forward modeling, and the modeling outputs are entered to the inversion program. Outputs of the inversion program in successive iterations are processed using the CA program and the results are compared with inversion outputs by means of correlation coefficients. The outputs of the inversion program and the processed results of the CA program for the first, third, and eighth iterations as well as the target distribution are presented in Figure (4.23). This experiment shows that some difficulties may arise for the CA program. When the image of asperities from the inversion program's initial trials is faint, the CA program cannot improve resolution properly by increasing contrast. As 
the dip angle of modeled fault and/or station distances increases or the number of stations decreases, the inaccuracies of the inversion program increase. Consequently, stress parameter distribution changes only slightly after each iteration and the improvements after initial iterations are not sufficient or even in the right direction to produce reasonable results, when processed by the CA program. So for steep fault dip angles, more than one inversion iteration will be needed to produce a stress parameter distribution appropriate for processing with the CA program. Figure (2.24) compares correlation coefficients of target distribution with inversion outputs and their processed products by the CA program, for up to 10 inversion iterations in this experiment. Figure (2.24) demonstrates that until the third iteration, the inversion program results are too low in quality to be improved by the CA program. However after the third iteration the processed distributions from the CA program show more similarity with the target distribution than the unamplified inversion outputs.

In another experiment a successful application of the CA program is demonstrated. With the target distribution of the previous example (Figure 4.23 ) and a $25^{\circ}$ fault dip angle, the inversion program is allowed to run to obtain the stress parameter distribution. In a parallel experiment the output of the inversion program after its first iteration is processed using the CA program and the result is put back into the inversion program for continuation. Then the error value $\Xi$ of these two inversion processes are plotted for up to 11 iterations in Figure (4.25). The values and evolution of $\Xi$ values suggest that proper use of the CA program can result in improved solutions that could be implemented by the inversion program, and we could thus skip many additional iterations of the inversion process.

When the number of stations is sufficient and the dip of the fault is gentle, the inversion program performs properly. So the stress parameter distribution after initial iterations will be clean enough to be improved properly by the CA program. In such cases application of the CA program output in the inversion program will result in a reduced number of inversion iterations needed to reach a solution for the stress parameter distribution. 
(1)

(2)

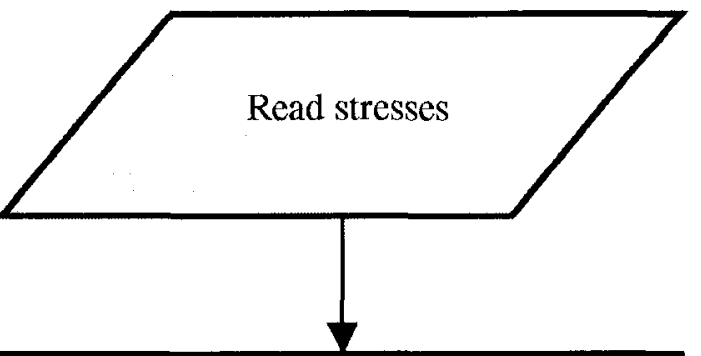

Raise stresses to power "exp"

(3)

(4)

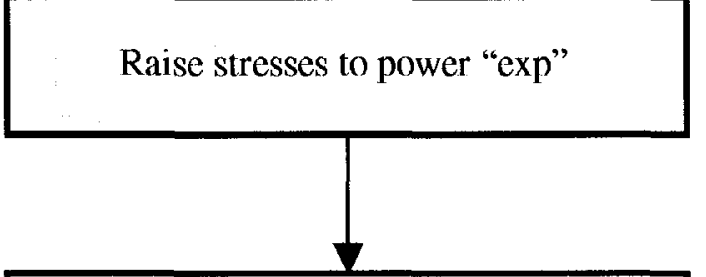

Calculate average derivative toward point with power "alpha" and assign to " $\mathrm{c}_{\mathrm{ij}}$ "

(5)

Find maximum sub-faults of "a $a_{i j}$ "

(6)

(7)

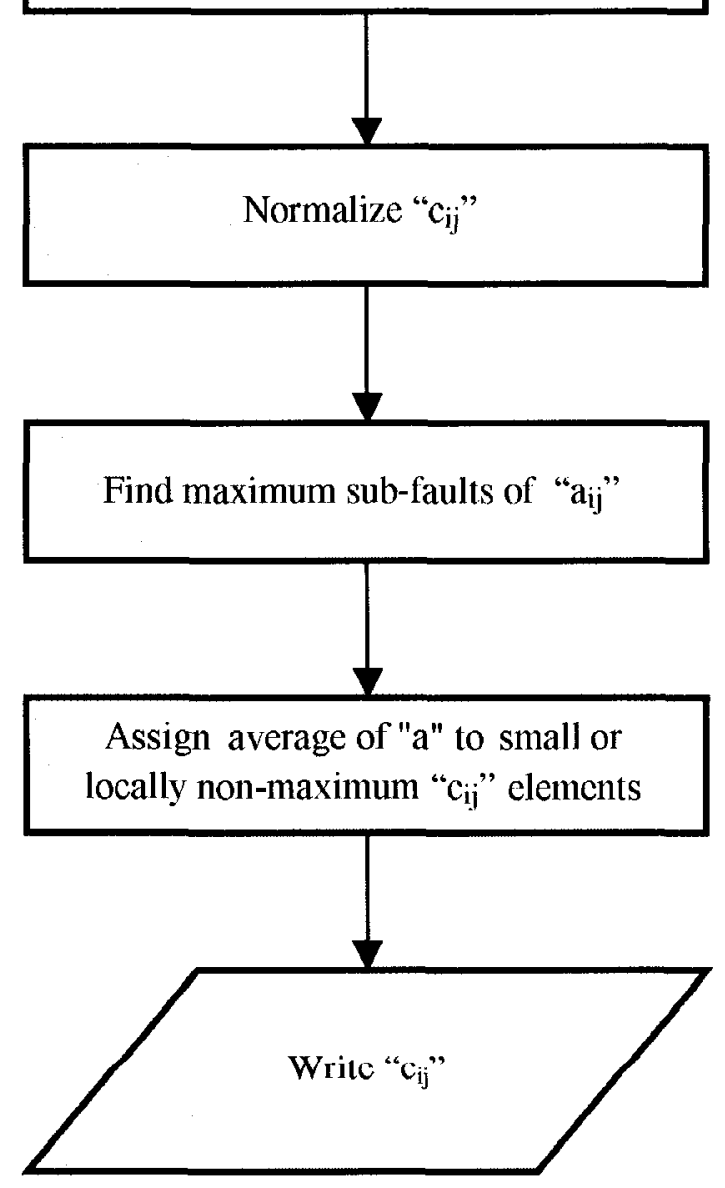

Figure (4.21) Flow chart of "Contrast amplifier" program. 
A- Target:

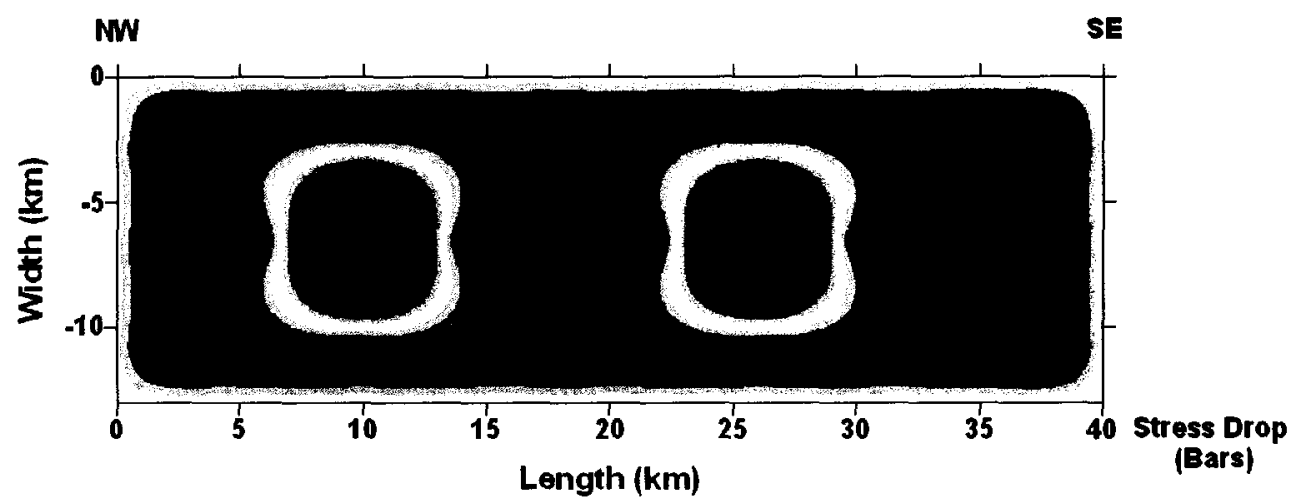

B- After 1 successful iteration:
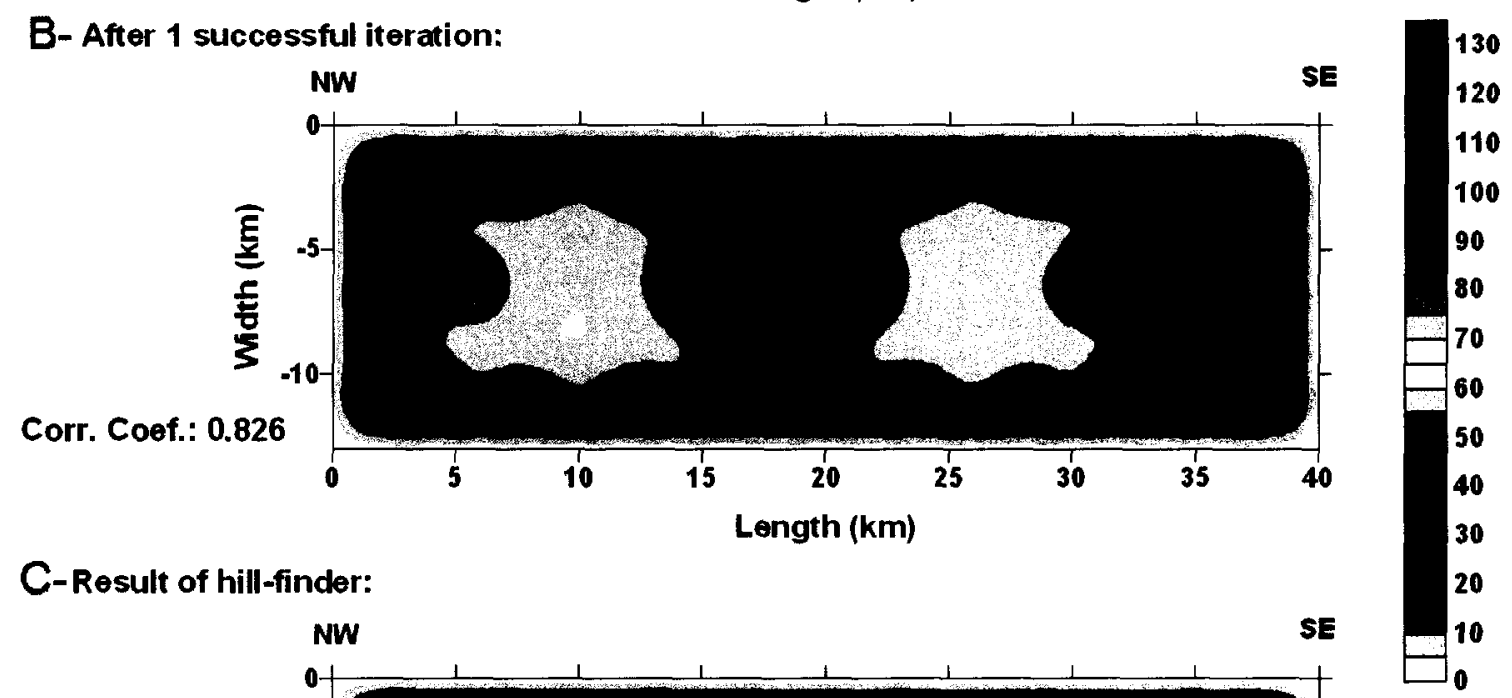

C-Result of hill-finder:

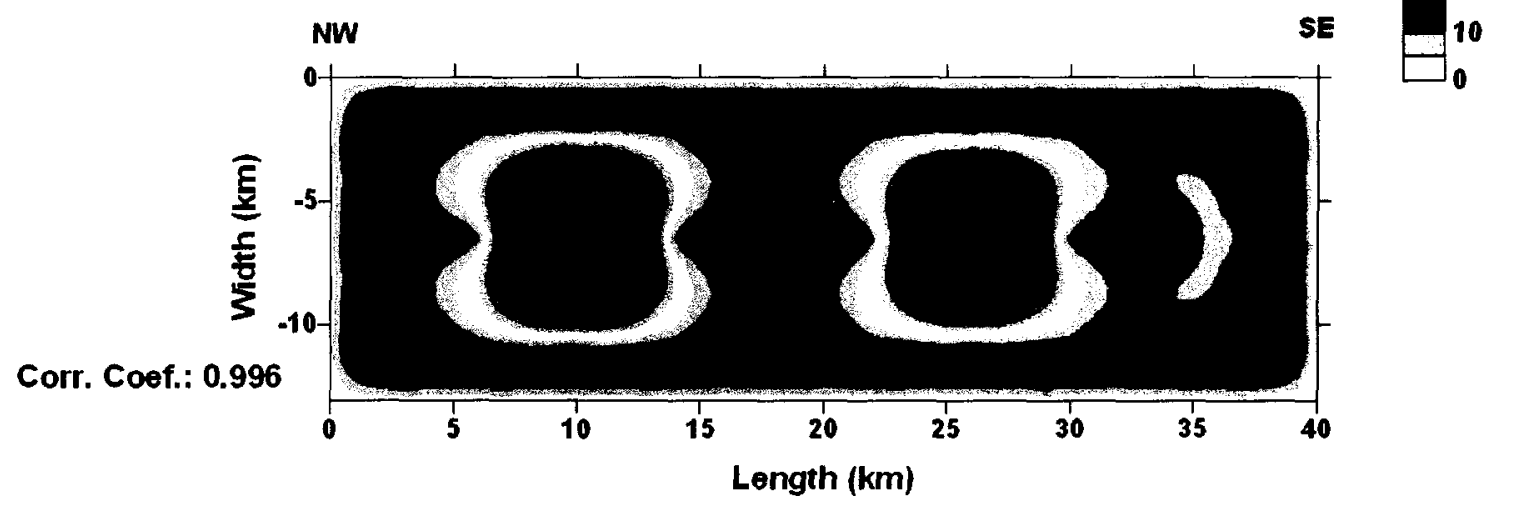

Figure (4.22) (A) target stress parameter distribution; (B) output of inversion program after 1 iteration; (C) CA program output from processing distribution in (B) as input. Fault dip angle is 5 degrees. 
A-Target:

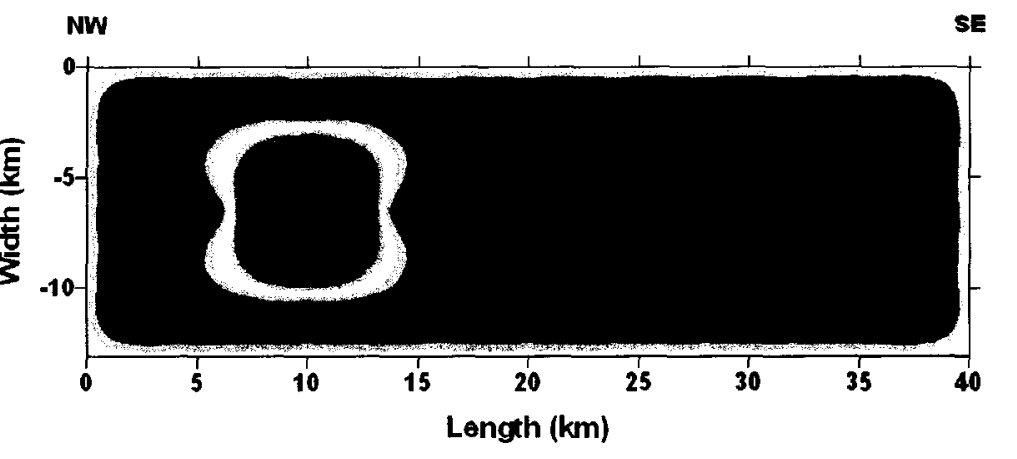

Stress Drop (Bars)

B-After 1 successful iteration:
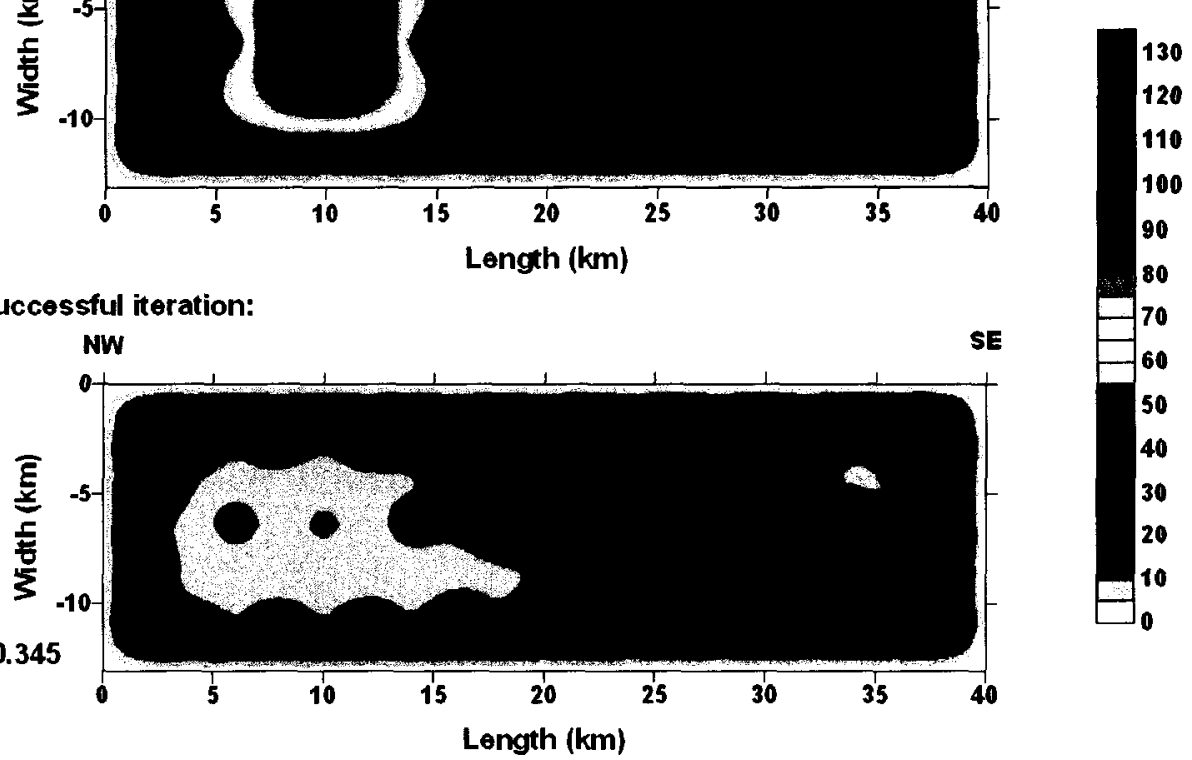

Corr. Coef.: 0.345

ation:

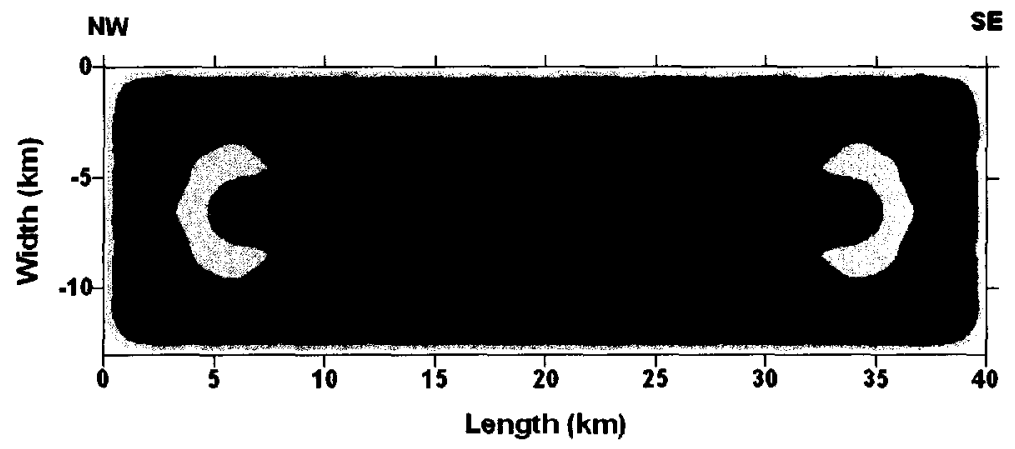

Figure (4.23) (A) target stress parameter distribution; (B) output of inversion program after 1 iteration; (C) CA program output from processing distribution in (B) as input. Fault dip angle is 45 degrees. 
D-After 3 successful iterations:

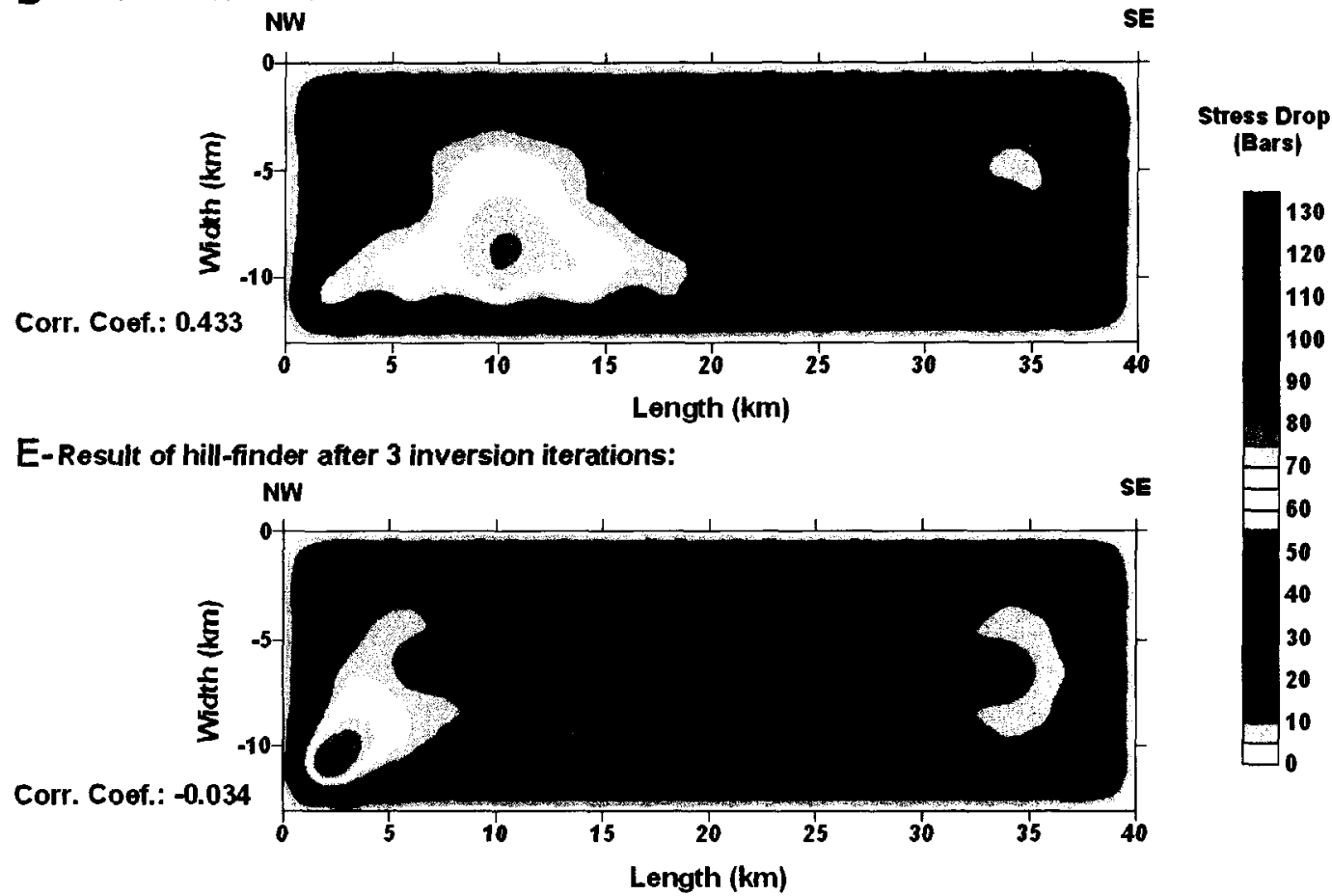

F- After 8 successful iterations:

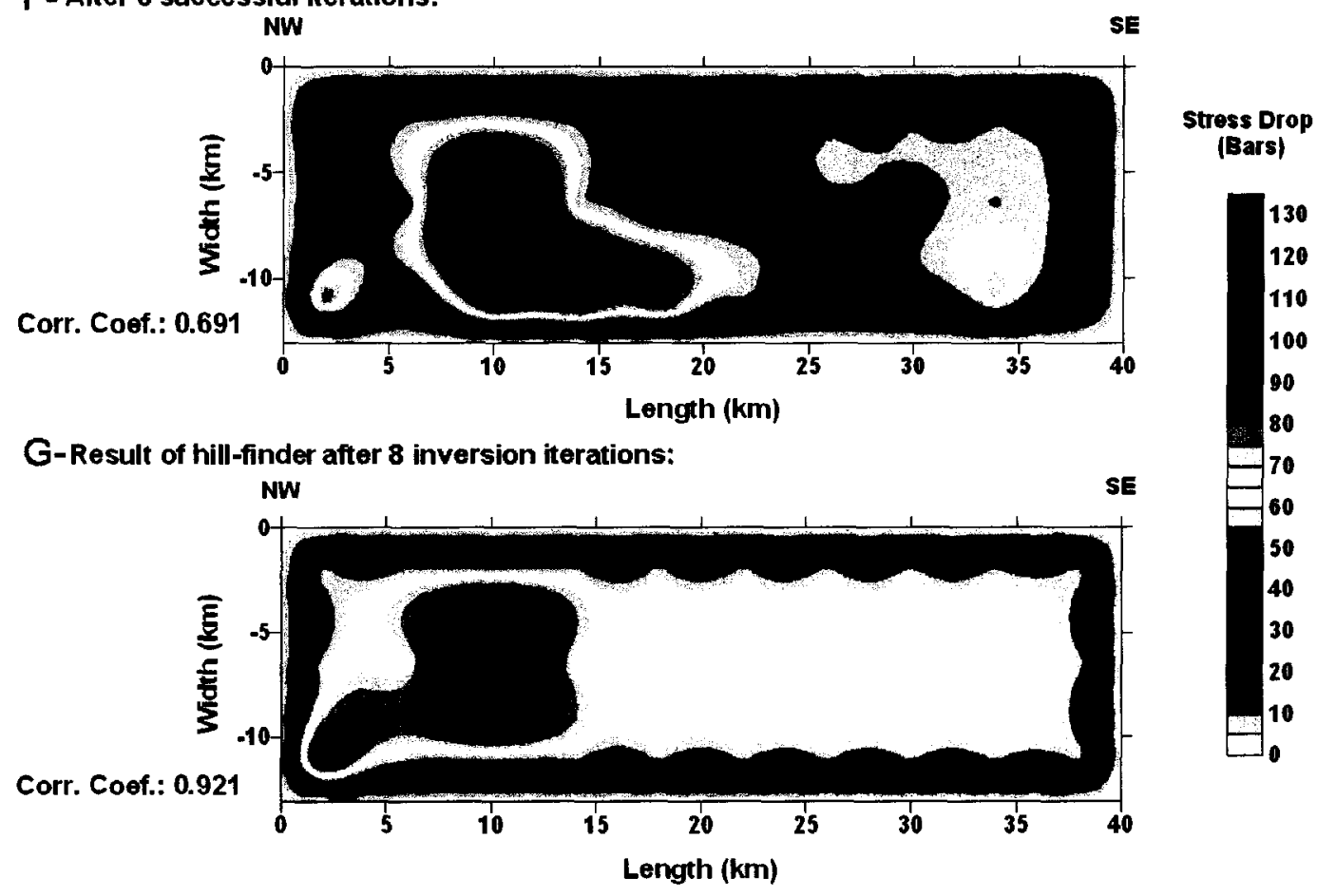

Figure (4.23) (D) output of inversion program after 1 iteration; (E) CA program output from processing distribution in (D) as input; (F) output of inversion program after 1 iteration; (G) CA program output from processing distribution in (F) as input; Fault dip angle is 45 degrees. 


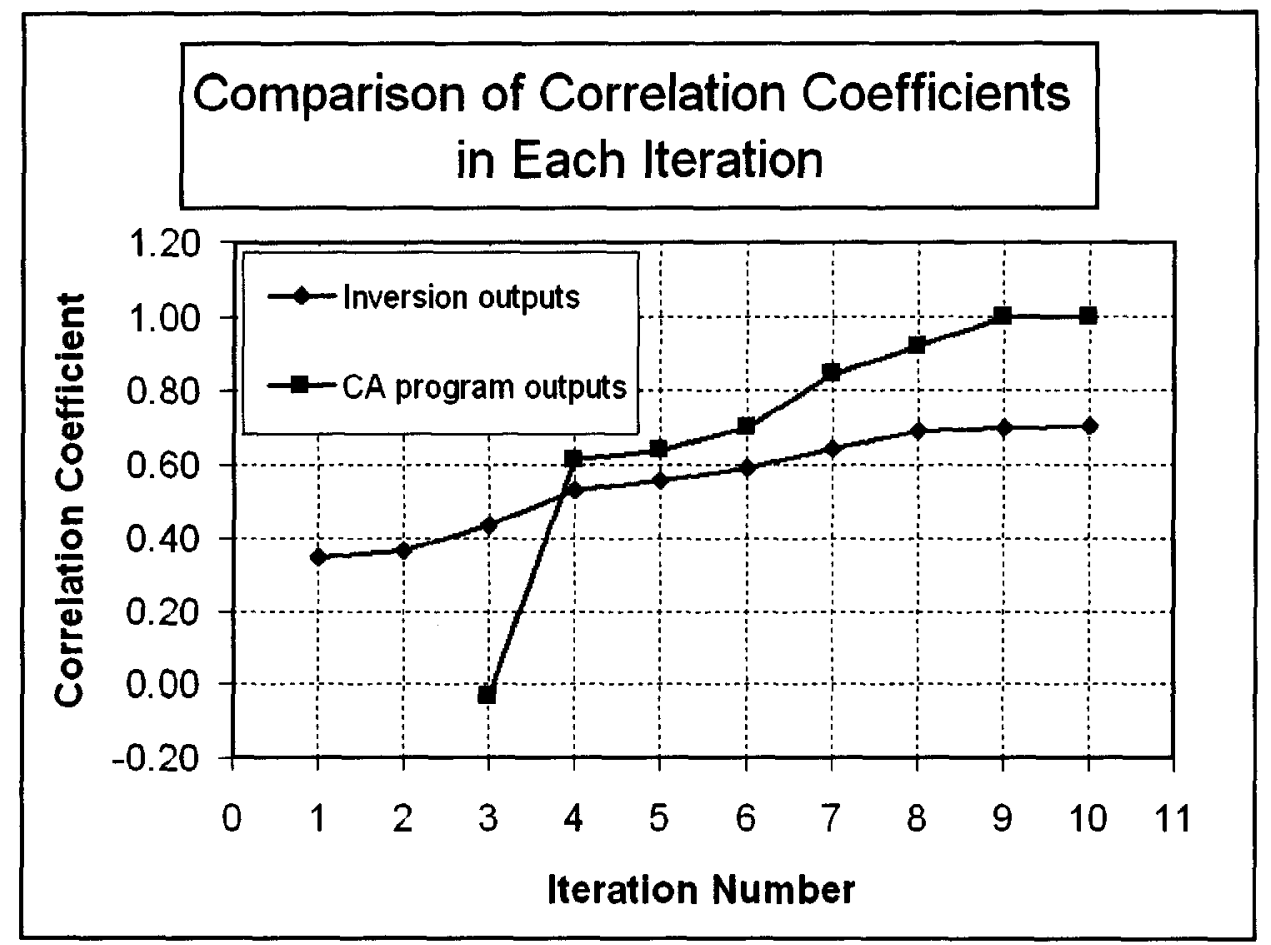

Figure (4.24) (Red) The evolution of the correlation coefficient of target distribution with inversion program outputs and (Blue) the evolution of the correlation coefficient of the target distribution with same distributions after modification by CA program, in successive iterations. The fault dip angle is $45^{\circ}$. 


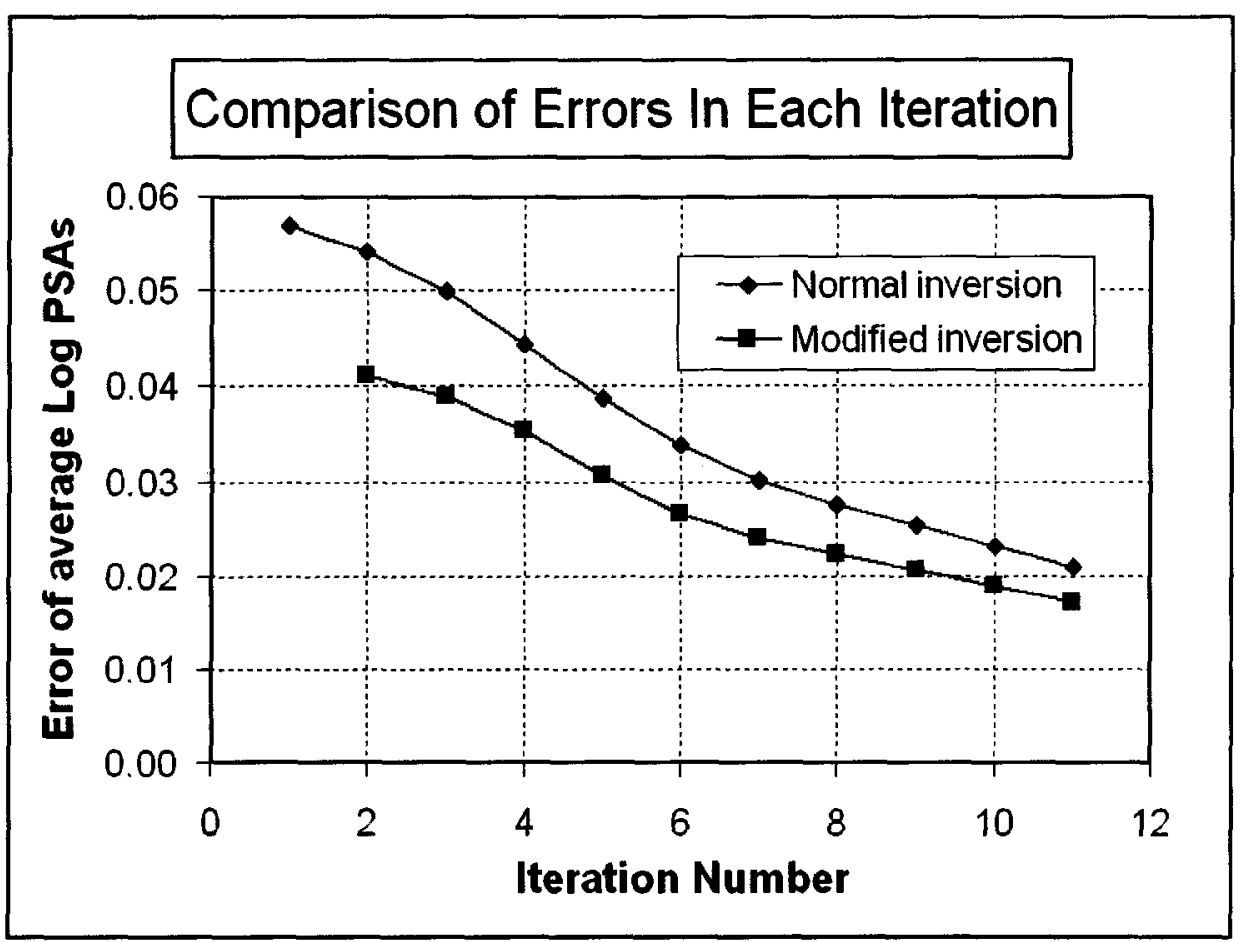

Figure (4.25) Evolution of error $\Xi$ with successive inversion iterations. Target distribution is same as that of Figure (4.23) but with $25^{\circ}$ of fault dip. (Red) ordinary inversion and (blue) the distribution of the first inversion iteration is processed by CA program and plugged into inversion program for further iterations. 


\subsection{Application of Method to the Parkfield Earthquake}

The M6.0 2004 Parkfield earthquake provided strong motion data from more than 85 stations, mostly at very close distances to the causative fault. Response spectra of horizontal components from these data are compared with simulations based on our modified version of stochastic finite-fault modeling, for the same stations. A stress parameter distribution on the fault surface is sought that minimizes the overall differences between the high-frequency pseudo-acceleration (PSA) ordinates of the simulated records and observations. The seeking process is performed by means of the LevenbergMarquardt inversion method (Marquardt 1963, Lee and Stewart 1981, Draper and Smith 1998 ) as described above. The PSAs of recorded data are first corrected for site response to the equivalent values for a uniform $V_{S 30}=620 \mathrm{~m} / \mathrm{s}$ site type, before performing the inversion. The procedure of site correction is explained next. (Note: an alternative approach for site correction would be to modify the synthetic records during EXSIM simulations for every site and then compare site-specific simulated spectra with recorded ones in the inversion process; we opted to correct spectra of the recorded signals to a common reference level for programming reasons).

\subsubsection{Data used and site corrections}

The list of stations used in this study is given in Table (4.4); the distribution of stations around the Parkfield fault is shown in Figure (4.19). The list is extracted from the COSMOS database (Squibb et al., 2004; Archuleta et al., 2003; http://db.cosmoseq.org/scripts/default.plx). The total number of stations is 87 , among which 46 are listed in the NGA database (Pacific Earthquake Engineering Research, New Generation Attenuation Relation Project 2005, http://peer.berkeley.edu/nga/index.html). The stations in the NGA database (http://peer.berkeley.edu/nga/flatfile.html) contain $\mathrm{V}_{\mathrm{S30}}$ (the average shear wave velocity in the upper 30 meters) and site type. The $V_{530}$ values of Table (4.4) are extracted from the preferred $\mathrm{V}_{\mathrm{S} 30}$ column of the NGA database, as based on USGS Northridge assignments (Borcherdt and Fumal 2002) and CGS assignments (Wills and 
Silva, 1998; Wills et al., 2000). Other stations with unknown site type were assigned NEHRP class " $D$ " with average $V_{S 30}=255 \mathrm{~m} / \mathrm{s}$; this value is a reasonable average for soil sites in the area. In the error calculations and inversions the data from the non-NGA stations "Hog Canyon" and "Priest Valley" were omitted because of poor bandwidth.

The recorded signals' response spectra are first corrected for soil response to produce the corresponding spectra for $\mathrm{V}_{\mathrm{S} 30}=620 \mathrm{~m} / \mathrm{s}$ (generic rock, Boore and Joyner 1997) site conditions. This is done in two steps. First, using Equation (4.12) from Boore and Atkinson (2006) the PHAr (Peak horizontal acceleration for $\mathrm{V}_{\mathrm{S} 30}=620 \mathrm{~m} / \mathrm{s}$ ) that is expected at each station for $\mathbf{M}=6$ is calculated:

$$
\text { Ln PHAr }=\mathrm{F}_{\mathrm{M}}(\mathbf{M})+\mathrm{F}_{\mathrm{D}}\left(\mathrm{r}_{\mathrm{j} b}, \mathbf{M}\right)+\mathrm{F}_{\mathrm{S}}\left(V_{s 30}, \mathbf{M}, \mathrm{r}_{\mathrm{jb}}\right)+\varepsilon \sigma_{T}
$$

where $F_{M}(M)$ is a magnitude scaling term, $F_{D}\left(r_{j b}\right)$ is a distance scaling term, and $F_{S}\left(V_{S 30}\right.$, $\left.\mathbf{M}, \mathrm{r}_{\mathrm{j} b}\right)$ is the site scaling term. These three terms can be calculated based on values of $\mathrm{V}_{\mathrm{s} 30}, \mathbf{M}, \mathrm{r}_{\mathrm{jb}}$ (Joyner-Boore distance), and constants presented in Boore and Atkinson (2006). The constants are different for PHArs larger and smaller than $0.06 \mathrm{~g}$ due to nonlinearity of soil response. Then the PHAr values are used in Equation (4.13) from Choi and Stewart (2005) to find the nonlinear amplification factors of each site:

$$
\ln \left(F_{i j}\right)=c \times \ln \left(\frac{V_{s 30_{i j}}}{V_{r e f}}\right)+b \times \ln \left(\frac{P H A_{r_{i j}}}{0.1}\right)
$$

where $F_{i j}$ is the amplification factor needed to multiply PHAr to predict the PHA (or response spectral ordinates) for a site with a given $\mathrm{V}_{\mathrm{S} 30} . b, c$, and $V_{r e f}$ are coefficients given in tables presented by Choi and Stewart (2005). Note that Boore and Atkinson (2006) adopted a slightly smoothed version of Choi and Stewart's result in their derivation of empirical ground motion relations in the NGA project (www.peer.berkeley.edu). By dividing the ordinates of the acceleration response spectrum at each station by the corresponding amplification factor, the site-corrected $P S A$ s are derived; these are the equivalent $P S A$ values for a site with $\mathrm{V}_{\mathrm{S} 30}=620 \mathrm{~m} / \mathrm{s}$. 


\subsubsection{Inversion of Recorded Parkfield Data}

The first step in the modeling of stress parameter distribution on the Parkfield fault is to determine the best uniform-stress model, using the response spectra of all horizontal components of stations (Table 4.4), with motions for all sites corrected to $\mathrm{V}_{\mathrm{S} 30}=620 \mathrm{~m} / \mathrm{s}$. The basic parameters of the simulation are typical for California and follow previous applications (Motazedian and Atkinson 2005); they are given in Table (4.2). The parameters to be determined are the stress parameter and pulsing percentage. These are allowed to vary on a grid of parameters, with stress from 10-100 bars and pulsing percentage from $10 \%-100 \%$. The calculated error measurement is $\Xi$ (Equation 4.2). Figure (4.26) plots the model errors; the optimal values are stress $\sim 50$ bars and pulsing percentage $\sim 20 \%$. Plotting the errors for each station at a specific frequency shows the level of over/underestimation of the model at different stations around the fault; this is $\varepsilon_{j}$ in Equation (4.4), for a given frequency index $\left(\mathrm{N}_{1}=\mathrm{N}_{2}=\mathrm{N}\right)$. The geographical distribution of $\varepsilon_{\mathbf{j}}$ around the fault for $5 \mathrm{~Hz}$ is shown in Figure (4.27). From Figure (4.22) we infer a high-stress patch near the southeast end of the fault (causing the observed positive residuals).

We inverted the recorded data ( 85 stations corrected to $V_{\mathrm{S} 30}=620 \mathrm{~m} / \mathrm{s}$ ) to obtain the stress parameter distribution of the M6 2004 Parkfield earthquake. The available response spectra in the range of $0.45 \mathrm{~Hz}$ to $10 \mathrm{~Hz}$ are used as input data to the inversion procedure; this frequency range is considered to be a reasonable choice for the high-frequency range for the M6 Parkfield earthquake based on the overall fault corner frequency (which is $0.2 \mathrm{~Hz})$.

The inversion program starts from a uniform stress parameter distribution and stops after just 4 successful iterations of the inversion program to produce the derived distribution. The error $\Xi$ of the solution is $8.3 \%$ lower than that of the initial guess. Average error spectra, the absolute errors at each frequency averaged over all stations, are shown in Figure (4.28). Figure (4.29) shows the initial and two derived stress parameter distributions on the fault. In the derivation of stress parameter distribution, another 
experiment sub-divides the fault surface into 12 elements along length and 3 elements along width $(12 \times 3)$ as shown in Figure (4.29C). The two distributions, one resulting from $10 \times 3$ and another from $12 \times 3$ sub-divisions on the fault surface, suggest no important differences between solutions, except that the one with a larger number of sub-sources $(12 \times 3)$ provides more details about the derived solution. It is important to note that there are limitations on the size of the sub-divisions of a fault. If the selected sub-source sizes are large, then one will lose resolution. If the selected sub-source sizes are small, then the number of parameters will increase, and depending on the number of records and the geometry of problem, one may lose precision, encounter non-unique answers, or may not reach a solution at all.

As was shown in Figure (4.27), the uniform stress parameter distribution caused large underestimation of the $\left.\overline{\log _{10}(P S A(S))}\right|_{f_{1}=0.45 \mathrm{~Hz}} ^{f_{2}=10 \mathrm{~Hz}}$ at the southeast end of the fault, small underestimation at the middle and northwest end of the fault, and overestimation in between these points. Modification of the stress parameter distribution to that of Figure (4.29) compensates these errors reasonably; this distribution is consistent with our visual judgment of errors in Figure (4.27).

Overall, the inversion clearly indicates that a high stress parameter patch is located in the southeast end of the Parkfield fault, at depths greater than $4 \mathrm{~km}$. The relative locations of the hypocenter and the important asperity is in good agreement with the observation by Mai et al., $(2005,2003)$, that the hypocenter is close to high-stress patches but not in the center of them. Also, the stress parameter distribution of Figure (4.29) derived by our inversion method has many similarities to the slip distribution derived by Langbein et al., (2005) and Liu et al., (2006). Langbein et al., (2005) used both strong motion and GPS data for their solution; Liu et al., (2006) inverted strong-motion seismograms to obtain space-time distribution model of co-seismic slip on the fault. Their inversion for rupture model yielded non-unique solutions; they presented a total of 10 possible solutions, along with an average solution. Figure (4.30) shows two representative solutions, and the 
average solution (it was redrawn using the information provided in the Liu et al. electronic supplement). The comparisons of Figures (4.29) and (4.30) show that the two researchers recognized almost the same concentrations of stress/slip on the fault surface. The implicit assumption made in this comparison is that high-slip areas correspond to high-stress-drop areas. This assumption is reasonable based on proportionality of stress and strain.

\subsection{Conclusion}

To make it possible to calculate the stress parameter distribution on the fault surface from the observed response spectra of an earthquake, an inversion tool is developed based on the combination of the modified EXSIM modeler and the Levenberg-Marquardt inversion method. The inversion generally converges quickly from an initial assumed uniform stress parameter distribution.

We examined the ability of the inversion tool to recover an input stress parameter distribution under various conditions and conclude that:

(i) Gentle fault dip angles allow an accurate determination of the stress parameter distribution, while steep fault dip angles limit its resolution. With gentle fault dip angles, the stations "see" the sub-faults across a fuller range of angles and distances, and this makes the equations for the inversion distinct. The method performs very well if the fault dip angle is less than $45^{\circ}$, although it performs reasonably well with dip angles more than $45^{\circ}$, at least in terms of locating the horizontal position of asperities.

(ii) The method has the capability to resolve multiple asperities, with as little as one subfault distance between them. It can also find the relative stress parameter levels of the asperities. With multiple asperities the solution converges faster than is the case for a single asperity.

(iii) If the initial guess of the stress parameter distribution is an accurate one, the solution will converge with few inversion iterations. If an accurate initial guess is not obvious, 
then the uniform distribution provides a reasonable initial guess. If the initial guess is a strong asperity in the incorrect location, then the program may fail to converge; otherwise it will be able to push the answer toward the real distribution eventually.

(iv) As is the case in other inversion techniques, a dense station distribution around the fault will result in high accuracy and precision of the high-stress asperities. If the station distribution is sparse, or there are no stations near the asperity, then the program may fail to find an asperity, or provide it with low resolution.

A program called "Contrast amplifier" was developed to accelerate the process of inversion. The idea is that the inversion program can find the location of stress concentrations in its initial iterations, if the dip angle of the fault is gentle or sufficient data from an appropriate number of stations are available. It is possible to amplify stress levels of these initially-determined stress centers to find a stress parameter distribution which is a better approximation of the target distribution. The "Contrast amplifier" program is designed and developed to process the stress parameter distribution from inversion initial steps. By inputting the CA processed distribution to the inversion program, the inversion process will be accelerated since the input distribution is much closer to the target distribution than the inversion output in the initial iterations.

The horizontal components of the M6 2004 Parkfield earthquake recorded at 85 locations are used in a test application of the inversion method. First, the response spectra of the records are corrected to equivalent values for a site condition of $V_{s 30}=620 \mathrm{~m} / \mathrm{s}$. Then the corrected PSAs are inverted for the stress parameter distribution on the fault surface. The inversion of the Parkfield data clearly demonstrates the presence of an asperity in the southeast end of the fault at depths between $4 \mathrm{~km}$ to $8 \mathrm{~km}$. This is in agreement with the conclusion of Mai et al. $(2003,2005)$ that the hypocenter and the main asperity (area of large slip) are very close to each other.

We may deduce the stress parameter distribution on the fault surface based on strongmotion observations from stations around the fault. With a reasonable number and distribution of stations around a fault, the method is able to find the asperities, although a 
steep fault dip angle limits the resolution of the depth of the asperities. The ability to interpret the strong-motion observations in terms of source property variability along the fault should lead to improved understanding of the driving mechanisms behind groundmotion observations and their spatial variability. It does not lead to improved predictive capabilities for future earthquakes in the short-term, but may in the long-term as information on stress parameter distributions is compiled for a large number of events. 
Table (4.4) List of stations used for M6, 2004 Parkfield earthquake study.

\begin{tabular}{|c|c|c|c|c|c|c|c|}
\hline No. & Station & Owner* & $\begin{array}{c}\text { In NGA } \\
\text { list }\end{array}$ & $\begin{array}{l}\text { Site } \\
\text { type }\end{array}$ & $\mathrm{V}_{\mathrm{S} 30}$ & LAT & LON \\
\hline 1 & Big Sur, CA - Pfeiffer State Park & CSMIP & No & ? & $?$ & 36.252 & -121.782 \\
\hline 2 & Buttonwillow, CA - Hwy 58 \& Wasco & CSMIP & No & ? & ? & 35.403 & -119.449 \\
\hline 3 & Cambria, CA - Hwy 1 Caltrans Bridge Grnds & CSMIP & No & ? & ? & 35.593 & -121.124 \\
\hline 4 & Capitola , CA - Fire Station & CSMIP & Yes & D & 288 & 36.973 & -121.953 \\
\hline 5 & $\begin{array}{l}\text { Coalinga, CA - Slack Canyon; Hidden Valley } \\
\text { Ranch }\end{array}$ & CSMIP & Yes & $\mathrm{C}$ & 684 & 36.034 & -120.590 \\
\hline 6 & Coalinga, CA - Fire Station & USGS & No & $?$ & ? & 36.137 & -120.363 \\
\hline 7 & Fresno, CA - NSMP Office & USGS & No & $?$ & ? & 36.744 & -119.728 \\
\hline 8 & Fresno, CA - VA Medical Center & USGS & No & ? & ? & 36.773 & -119.781 \\
\hline 9 & Greenfield, CA - Police Station & CSMIP & No & ? & ? & 36.321 & -121.243 \\
\hline 10 & Hog Canyon, CA & NCSN & No & $?$ & ? & 35.867 & -120.480 \\
\hline 11 & Hollister, CA - South St and Pine Dr & CSMIP & Yes & $\mathrm{C}$ & 370 & 36.848 & -121.397 \\
\hline 12 & Hollister, CA - Airport Building \# 3 & USGS & Yes & D & 271 & 36.891 & -121.404 \\
\hline 13 & Hollister, CA - City Hall Annex & USGS & Yes & D & 274 & 36.851 & -121.402 \\
\hline 14 & King City, CA - 2-story hospital & CSMIP & No & $?$ & ? & 36.206 & -121.132 \\
\hline 15 & King City, CA - Canal \& Reich & CSMIP & No & ? & $?$ & 36.207 & -121.131 \\
\hline 16 & Moss Landing, CA - Hwy 1 \& Dolan & CSMIP & No & ? & ? & 36.807 & -121.778 \\
\hline 17 & Cholame 1E & CSMIP & Yes & D & 338 & 35.743 & -120.277 \\
\hline 18 & Cholame 2E & CSMIP & Yes & $\mathrm{C}$ & 376 & 35.751 & -120.259 \\
\hline 19 & Cholame $2 \mathrm{~W}$ & CSMIP & Yes & D & 184 & 35.733 & -120.290 \\
\hline 20 & Cholame 3E & CSMIP & Yes & $\mathrm{C}$ & 376 & 35.770 & -120.247 \\
\hline 21 & Cholame $3 \mathrm{~W}$ & CSMIP & Yes & D & 338 & 35.724 & -120.294 \\
\hline 22 & Cholame $4 \mathrm{AW}$ & CSMIP & Yes & D & 338 & 35.707 & -120.316 \\
\hline 23 & Cholame $4 \mathrm{~W}$ & CSMIP & Yes & $\mathrm{C}$ & 438 & 35.718 & -120.304 \\
\hline 24 & Cholame 5SW & CSMIP & Yes & D & 289 & 35.697 & -120.328 \\
\hline 25 & Cholame $6 \mathrm{~W}$ & CSMIP & Yes & D & 338 & 35.684 & -120.342 \\
\hline 26 & Cholame $12 \mathrm{~W}$ & CSMIP & Yes & $\mathrm{C}$ & 408 & 35.639 & -120.404 \\
\hline 27 & Fault Zone 1 & CSMIP & Yes & D & 338 & 35.758 & -120.307 \\
\hline 28 & Fault Zone 3 & CSMIP & Yes & $\mathrm{C}$ & 370 & 35.803 & -120.334 \\
\hline 29 & Fault Zone 4 & CSMIP & Yes & D & 338 & 35.836 & -120.395 \\
\hline 30 & Fault Zone 6 & CSMIP & Yes & $\mathrm{C}$ & 438 & 35.859 & -120.420 \\
\hline 31 & Fault Zone 7 & CSMIP & Yes & $\mathrm{C}$ & 370 & 35.871 & -120.404 \\
\hline 32 & Fault Zone 8 & CSMIP & Yes & $\mathrm{C}$ & 376 & 35.878 & -120.381 \\
\hline 33 & Fault Zone 9 & CSMIP & Yes & $\mathrm{C}$ & 438 & 35.879 & -120.445 \\
\hline 34 & Fault Zone 11 & CSMIP & Yes & $\mathrm{C}$ & 376 & 35.896 & -120.398 \\
\hline 35 & Fault Zone 12 & CSMIP & Yes & D & 338 & 35.899 & -120.433 \\
\hline 36 & Fault Zone 14 & CSMIP & Yes & D & 338 & 35.908 & -120.458 \\
\hline 37 & Fault Zone 15 & CSMIP & Yes & $\mathrm{C}$ & 376 & 35.921 & -120.481 \\
\hline 38 & Gold Hill & USGS & No & $?$ & $?$ & 35.833 & -120.346 \\
\hline 39 & Gold Hill $1 \mathrm{~W}$ & CSMIP & Yes & D & 338 & 35.818 & -120.378 \\
\hline 40 & Gold Hill 2E & CSMIP & Yes & D & 338 & 35.843 & -120.348 \\
\hline 41 & Gold Hill 2W & CSMIP & Yes & $\mathrm{C}$ & 376 & 35.812 & -120.391 \\
\hline 42 & Gold Hill 3E & CSMIP & Yes & $\mathrm{C}$ & 370 & 35.870 & -120.334 \\
\hline 43 & Gold Hill $3 W$ & CSMIP & Yes & $\mathrm{C}$ & 438 & 35.796 & -120.411 \\
\hline 44 & Gold Hill 4W & CSMIP & Yes & $\mathrm{C}$ & 438 & 35.785 & -120.444 \\
\hline 45 & Gold Hill 5W & CSMIP & Yes & $\mathrm{C}$ & 438 & 35.770 & -120.477 \\
\hline 46 & Gold Hill 6W & CSMIP & Yes & $\mathrm{C}$ & 438 & 35.738 & -120.507 \\
\hline 47 & Hwy 46/Cholame Creek Bridge & CSMIP & No & ? & $?$ & 35.733 & -120.289 \\
\hline 48 & Stone Corral $1 \mathrm{E}$ & CSMIP & No & $?$ & ? & 35.788 & -120.294 \\
\hline
\end{tabular}




\begin{tabular}{|c|c|c|c|c|c|c|c|}
\hline 9 & Stone Corral 2E & CSMIP & Yes & $\mathrm{C}$ & 376 & 35.810 & -120.282 \\
\hline 50 & Stone Corral $3 \mathrm{E}$ & CSMIP & Yes & $\mathrm{C}$ & 376 & 35.833 & -120.270 \\
\hline 51 & Temblor & CSMIP & No & $?$ & ? & 35.705 & -120.169 \\
\hline 52 & Vineyard Cyn $1 \mathrm{~W}$ & CSMIP & Yes & $\mathrm{C}$ & 376 & 35.934 & -120.497 \\
\hline 53 & Vineyard Cyn $2 \mathrm{E}$ & CSMIP & Yes & $\mathrm{C}$ & 712 & 35.973 & -120.467 \\
\hline 54 & Vineyard Cyn $2 \mathrm{~W}$ & CSMIP & Yes & D & 338 & 35.927 & -120.509 \\
\hline 55 & Vineyard Cyn $3 \mathrm{~W}$ & CSMIP & Yes & D & 297 & 35.922 & -120.534 \\
\hline 56 & Vineyard Cyn $4 \mathrm{~W}$ & CSMIP & Yes & $\mathrm{C}$ & 376 & 35.905 & -120.551 \\
\hline 57 & Vineyard Cyn $5 \mathrm{~W}$ & CSMIP & Yes & $\mathrm{C}$ & 376 & 35.885 & -120.565 \\
\hline 58 & Vineyard Cyn $6 \mathrm{~W}$ & CSMIP & Yes & $\mathrm{C}$ & 438 & 35.861 & -120.600 \\
\hline 59 & Donna Lee & USGS & No & $?$ & $?$ & 35.939 & -120.425 \\
\hline 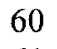 & Eades & USGS & No & $?$ & $?$ & 35.894 & -120.421 \\
\hline 61 & Froelich & USGS & No & ? & $?$ & 35.911 & -120.486 \\
\hline 62 & Jack Canyon & USGS & No & ? & $?$ & 35.713 & -120.203 \\
\hline 63 & Joaquin Canyon & USGS & No & $?$ & $?$ & 35.940 & -120.432 \\
\hline 64 & Middle Mountain & USGS & No & ? & ? & 35.958 & -120.496 \\
\hline 65 & Red Hills & USGS & No & $?$ & $?$ & 35.624 & -120.254 \\
\hline 66 & Stockdale Mtn & USGS & No & ? & $?$ & 35.973 & -120.579 \\
\hline 77 & Vineyard Canyon & USGS & No & ? & ? & 35.923 & -120.534 \\
\hline 68 & Work Ranch & USGS & No & $?$ & $?$ & 35.814 & -120.511 \\
\hline 69 & Point Buchon, CA - Los Osos & CSMIP & No & $?$ & $?$ & 35.274 & -120.885 \\
\hline 70 & Coalinga, CA - Priest Valley & CSMIP & No & $?$ & $?$ & 36.191 & -120.708 \\
\hline 71 & Salinas, CA - City Yard - John \& Work & CSMIP & Yes & $\mathrm{D}$ & 271 & 36.671 & -121.642 \\
\hline 72 & Salinas, CA - County Hosp Grounds & CSMIP & Yes & D & 271 & 36.697 & -121.634 \\
\hline 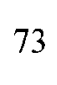 & $\begin{array}{l}\text { San Luis Obispo, Ca - City Recreation Bldg - } \\
864\end{array}$ & USGS & Yes & $\mathrm{C}$ & 712 & 35.285 & -120.661 \\
\hline 74 & San Luis Obispo, CA - Lopez Lake Grounds & CSMIP & No & $?$ & $?$ & 35.208 & -120.457 \\
\hline 75 & Templeton, CA - Hospital Grounds & CSMIP & No & $?$ & ? & 35.556 & -120.720 \\
\hline 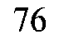 & USGS Parkfield Dense Seismograph Array 01 & USGS & No & $?$ & $?$ & 35.821 & -120.507 \\
\hline 77 & USGS Parkfield Dense Seismograph Array 02 & USGS & No & $?$ & $?$ & 35.822 & -120.506 \\
\hline 78 & USGS Parkfield Dense Seismograph Array 03 & USGS & No & $?$ & $?$ & 35.821 & -120.505 \\
\hline 79 & USGS Parkfield Dense Seismograph Array 05 & USGS & No & $?$ & $?$ & 35.824 & -120.503 \\
\hline 80 & USGS Parkfield Dense Seismograph Array 06 & USGS & No & ? & ? & 35.824 & -120.503 \\
\hline 81 & USGS Parkfield Dense Seismograph Array 07 & USGS & No & $?$ & $?$ & 35.824 & -120.503 \\
\hline 82 & USGS Parkfield Dense Seismograph Array 08 & USGS & No & ? & ? & 35.825 & -120.501 \\
\hline 83 & USGS Parkfield Dense Seismograph Array 09 & USGS & No & $?$ & $?$ & 35.826 & -120.501 \\
\hline 34 & USGS Parkfield Dense Seismograph Array 10 & USGS & No & ? & ? & 35.828 & -120.500 \\
\hline 85 & USGS Parkfield Dense Seismograph Array 11 & USGS & No & ? & ? & 35.826 & -120.502 \\
\hline 86 & USGS Parkfield Dense Seismograph Array 12 & USGS & No & $?$ & $?$ & 35.827 & -120.504 \\
\hline 87 & USGS Parkfield Dense Seismograph Array 13 & USGS & No & ? & $?$ & 35.827 & -120.505 \\
\hline
\end{tabular}




\section{Total Average Error Of Spectra Comparisons}

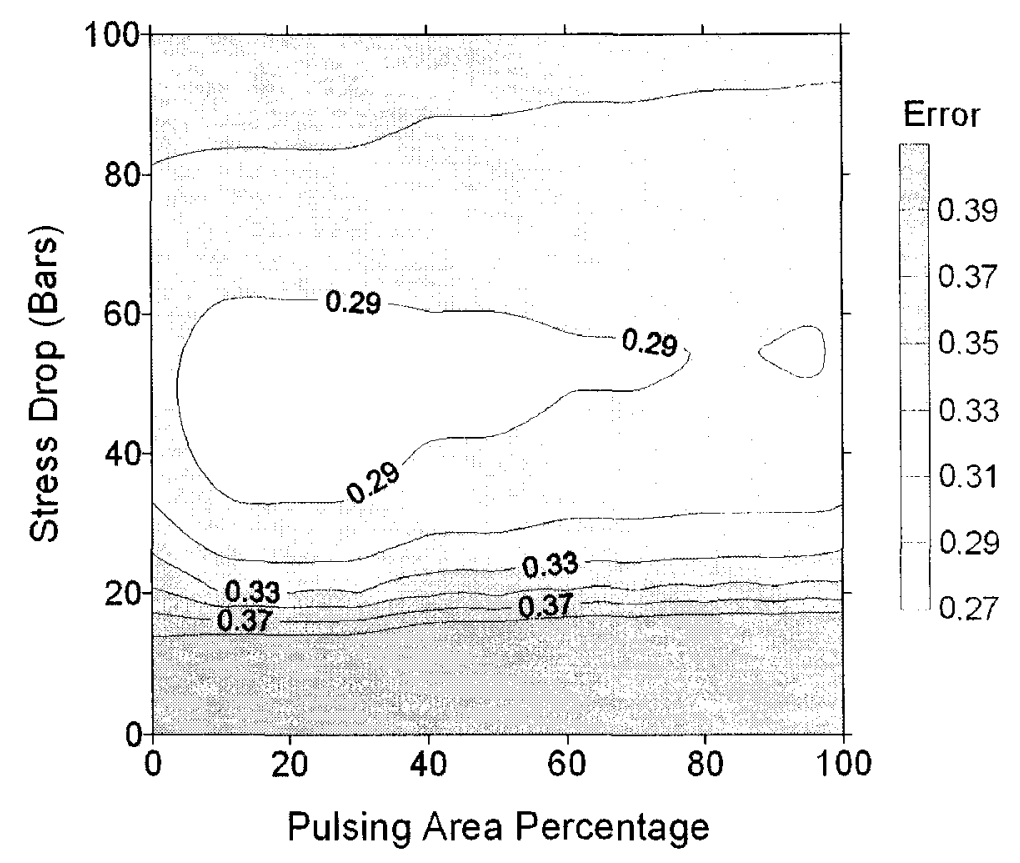

Figure (4.26) Error $\Xi$ (in $\log _{10}$ units) for different values of pulsing area and uniform stress parameter. Best match with data is for 50 bars, with $20 \%$ pulsing area.

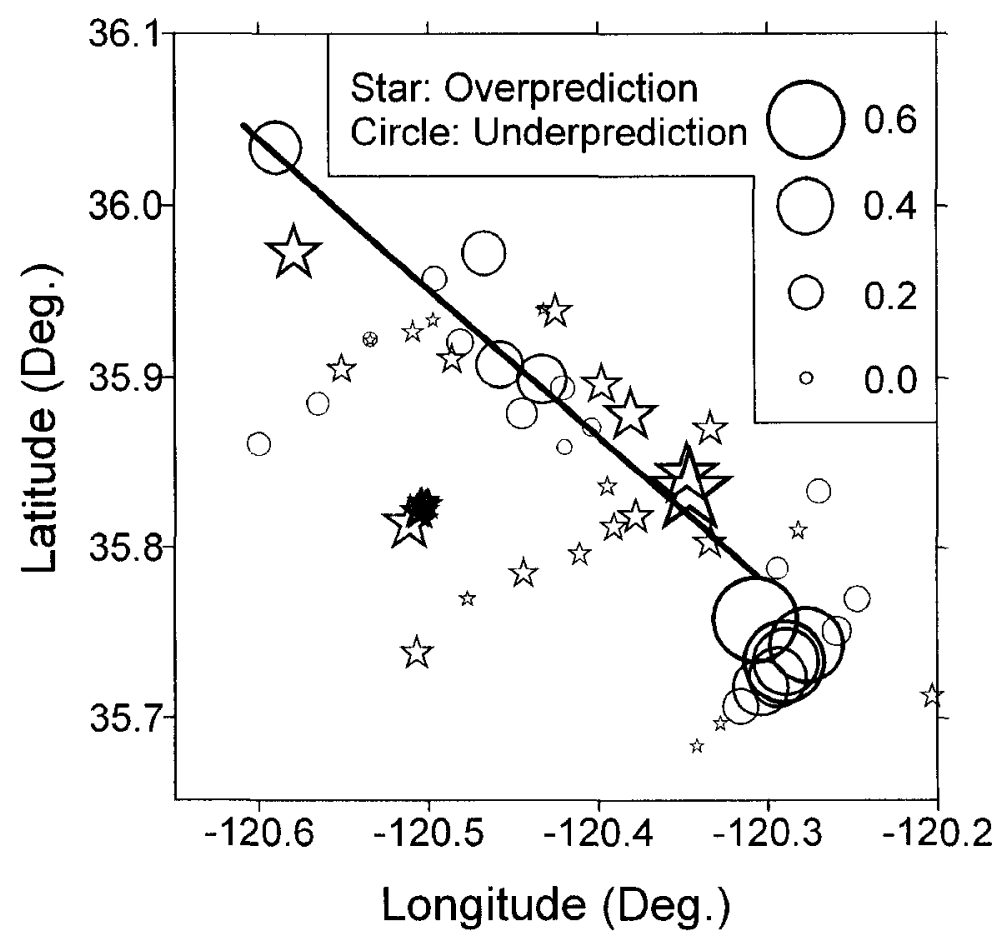

Figure (4.27) Residuals of simulations at each station $\varepsilon_{j}$ around the fault, for PSA at $5 \mathrm{~Hz}$ (in $\log _{10}$ units). 


\section{Average Errors Over All Stations For Two Models As} A Function of Frequency. 85 Stations 2 Components

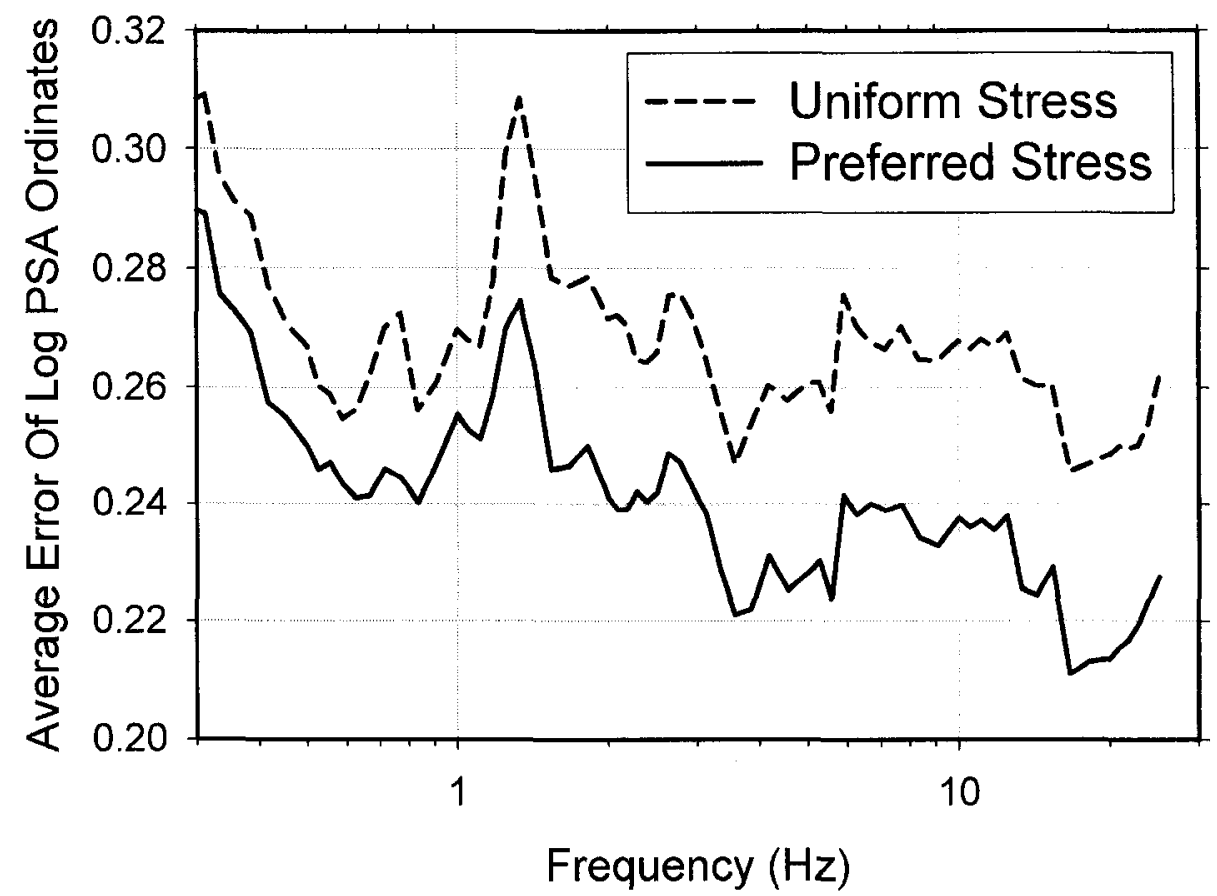

Figure (4.28) Average error $\left(\Xi_{\mathrm{i}}\right)$ at all stations as a function of frequency, for uniform stress parameter distribution (dashed) and preferred stress parameter distribution of Figure (4.24) (solid). 
A- Initial guess, uniform distribution:
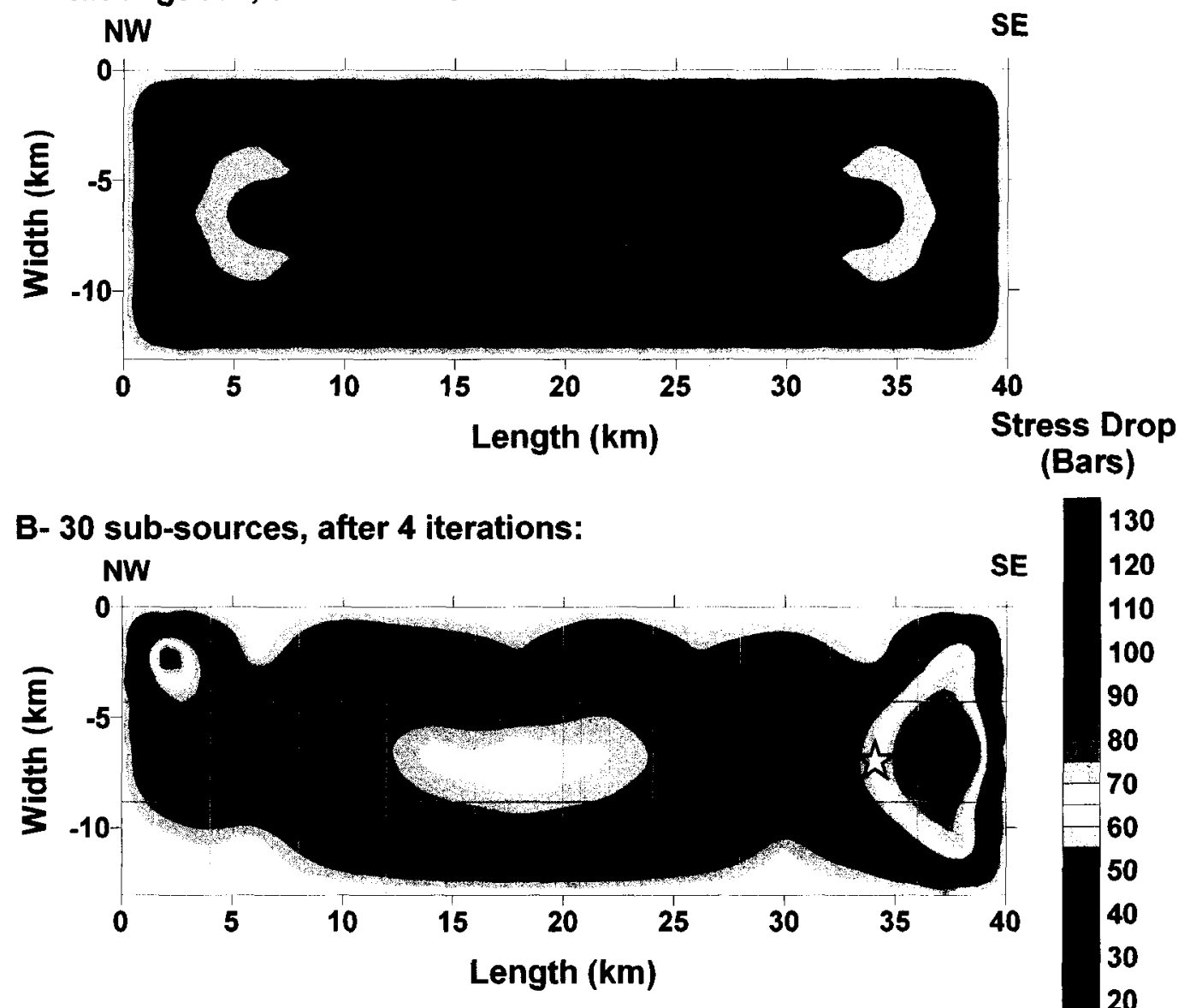

B- 30 sub-sources, after 4 iterations:

C- 36 sub-sources, after 7 iterations:

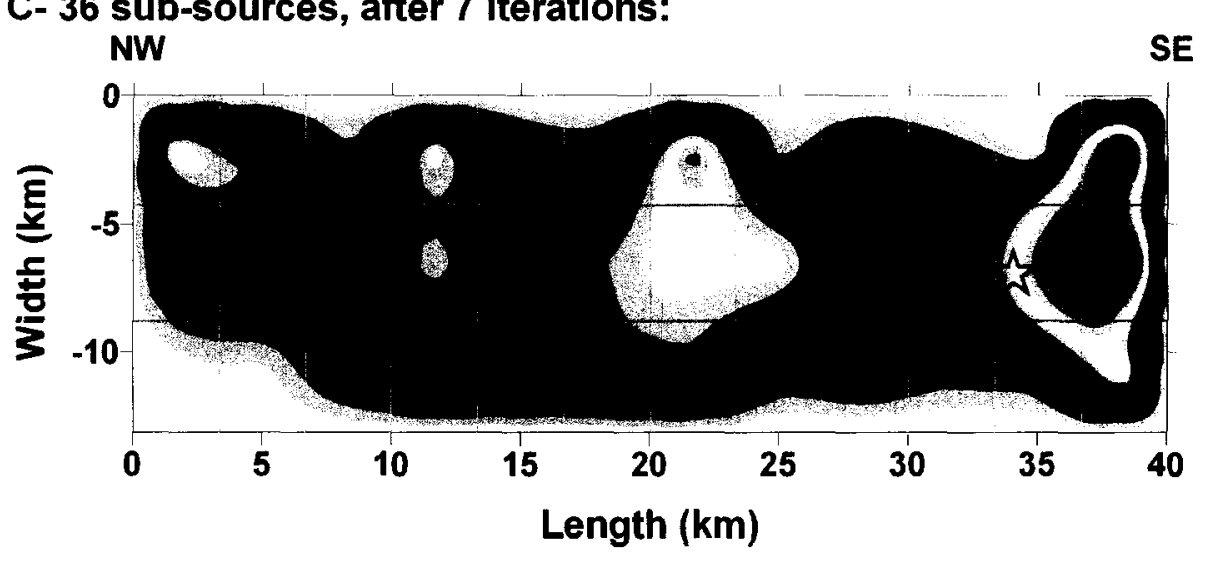

Figure (4.29) (A) Initial guess; (B and C) stress parameter distributions derived for M6, 2004 Parkfield earthquake. The fault surface is sub-divided into 30 and 36 sub-sources and corresponding inversion solutions are presented in (B) and (C) respectively. The solutions are reached after 4 and 7 inversion iterations in (B) and (C) with no further error reduction thereafter. Grids show the size of sub-sources and the star is the hypocenter. 
a-Liu et al., 2006, slip model \#3:

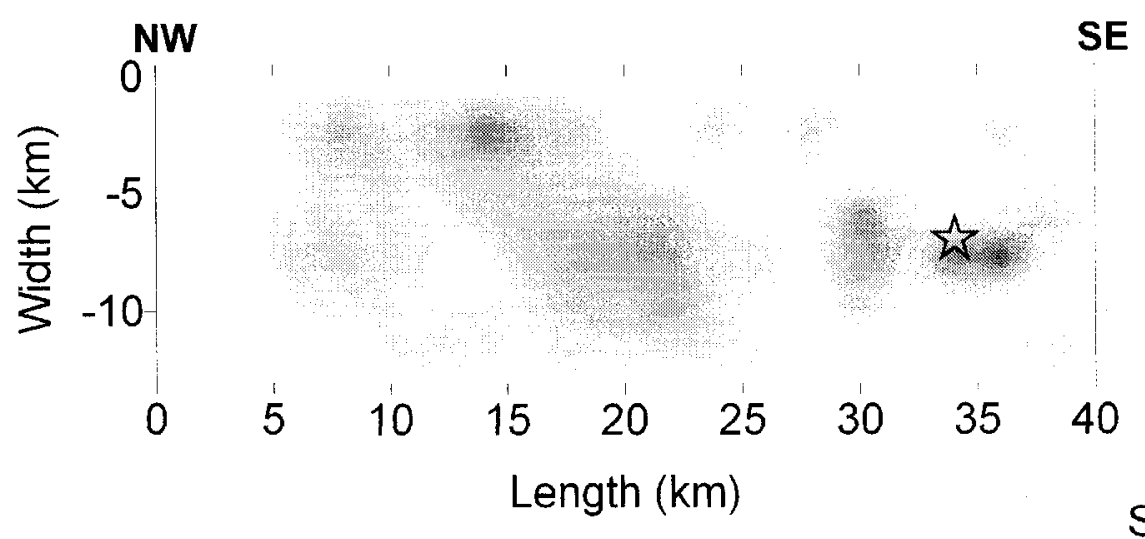

b-Liu et al., 2006, slip model \#5:

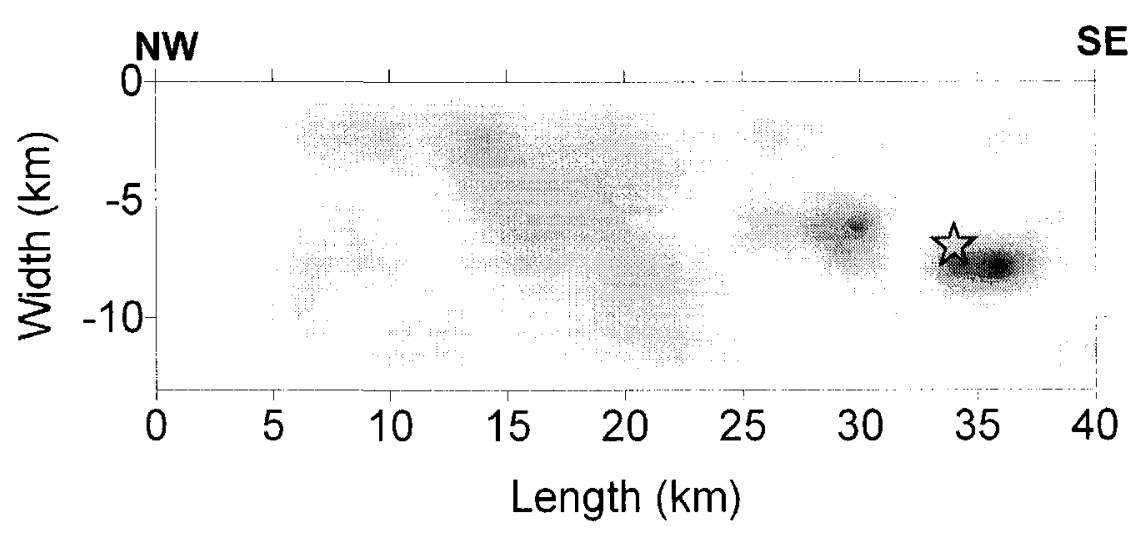

c-Liu et al., 2006, average of 10 slip models:

$\operatorname{Slip}(m)$

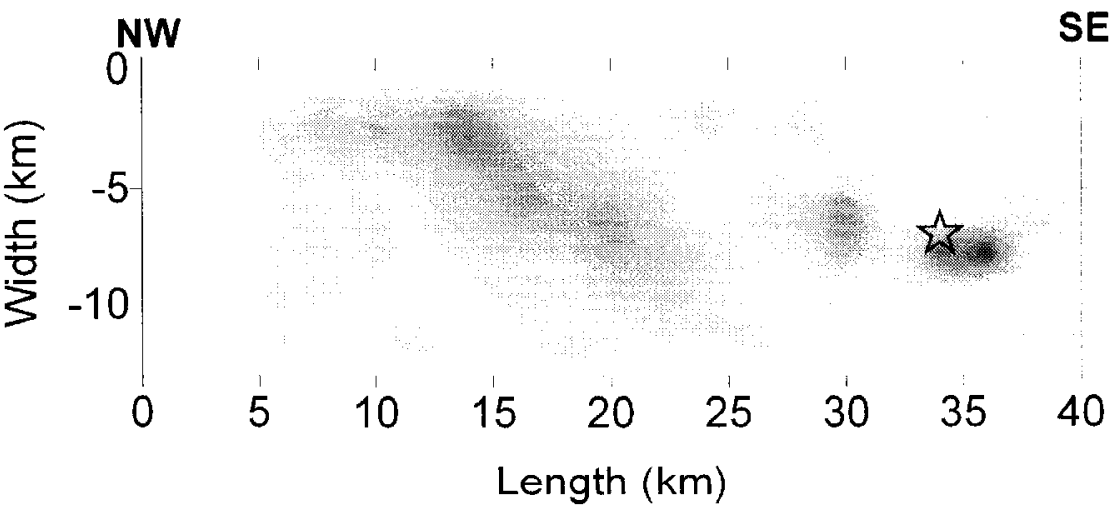

Figure (4.30). Slip distribution on the Parkfield fault surface derived from strong-motion seismographic data (from Liu et al., (2006)); (a) solution number 3; (b) solution number $5 ;(\mathrm{c})$ average of 10 solutions. 


\section{Chapter 5: Implementation of inversion tool for three events}

\subsection{Introduction}

In this chapter the developed inversion tool is implemented for the derivation of the stress parameter distribution on the fault surface of three major California earthquakes; the results are compared to slip distributions derived from other studies. In the previous chapter, after development of an inversion tool I deduced the stress parameter distribution of the M6.0, 2004 Parkfield earthquake by inverting response spectral data of the recorded strong motions. The current study is an extension of that work for three other major California earthquakes (M7.1, 1989 Loma Prieta earthquake; M7.2, 1992 Landers earthquake; and M6.7, 1994 Northridge earthquake). The same formulation and developed error criteria are implemented here. A new criterion, spatial error, is introduced and its evolution is studied in this chapter. For each earthquake, three different numbers of sub-sources (or sizes) are considered. The evolution of errors is presented. The final results for each earthquake are compared to one another and to slip distributions of other researchers. This study further validates the stress-distribution inversion method, explores its limitations, and develops an initial database of stress parameter distributions of earthquakes.

\subsection{Implemented error measures and criteria}

In this chapter, two additional quantities are introduced that measure error improvements as function of location and frequency. These are used to allow us to see more visually what happens to error in the inversion process. The purpose of the detailed consideration of spatial and frequency-dependence of error evolution (from initial guess to final solution) is to obtain deeper insight into the study results and demonstrate the inversion procedure performance.

To get a view of spatial distribution of error the absolute values of Equation (4.4), $\left|\varepsilon_{j}\right|$, are mapped and the changes of this quantity from initial guess to final solution are calculated and plotted: 


$$
\begin{aligned}
\Psi_{j}=-\Delta\left|\varepsilon_{j}\right| & =\left|\frac{1}{\left(N_{2}-N_{1}+1\right)} \sum_{i=N_{1}}^{N_{2}}\left(\log P S A\left(O_{i j}\right)-\log P S A\left(S_{i j}\right)\right)\right|_{\text {inititul guess }}- \\
& \left|\frac{1}{\left(N_{2}-N_{1}+1\right)} \sum_{i=N_{1}}^{N_{2}}\left(\log P S A\left(O_{i j}\right)-\log P S A\left(S_{i j}\right)\right)\right|
\end{aligned}
$$

The $\varepsilon_{j}$ value is calculated using Equation (4.4), which is equal to the terms inside the absolute value bars of Equation (5.1). The subscript " $\mathrm{j}$ " appears because this value is calculated for each site separately and the map is prepared by kriging of the $\Psi_{j}$ values in the area.

To show the error improvement at different frequencies, the graphs of Equation (4.3), calculated for initial guess and final solution, are compared with each other. Here the same relation is presented to complete the list of error measures and criteria equations implemented in this part of study:

$$
\Xi_{i}=\frac{1}{K} \sum_{j=1}^{K}\left|\log P S A\left(O_{i j}\right)-\log P S A\left(S_{i j}\right)\right|
$$

In this equation $\Xi_{i}$ is the average absolute error at the $i^{\text {th }}$ frequency averaged over all stations. This quantity can be calculated for the initial guess (uniform stress), last solution, or a solution after any number of successful iterations, and plotted against frequency on the same graph.

\subsection{Data preparation and modeling parameters}

Horizontal-component response spectra data of three California earthquakes (M7.1, 1989 Loma Prieta earthquake; M7.2, 1992 Landers earthquake; and M6.7, 1994 Northridge earthquake) were extracted from the NGA database (Pacific Earthquake Engineering Research, New Generation Attenuation Relation Project 2005, http://peer.berkeley.edu/nga/index.html). The $\mathrm{V}_{\mathrm{S} 30}$ values are extracted from the preferred $\mathrm{V}_{\mathrm{S} 30}$ column of the NGA database, as based on USGS Northridge assignments (Borcherdt and Fumal 2002) and CGS assignments (Wills and Silva, 1998; Wills et al., 2000). The recorded signals' response spectra are first corrected for soil response to produce the 
corresponding spectra for $\mathrm{V}_{\mathrm{s} 30}=620 \mathrm{~m} / \mathrm{s}$ (NEHRP B/C boundary) site conditions, based on the site factor correction given by Boore and Atkinson (2007). The correction procedure follows that explained in section (4.5) except that the most recent version of ground motion prediction equations (Boore and Atkinson, 2007 versus Boore and Atkinson 2006) are implemented in the correction of PSAs. The difference in correction factors (Boore and Atkinson, 2007 versus Boore and Atkinson 2006) is negligible and thus this is not important.

The simulation parameters of the three earthquakes in this study are summarized in Table (5.1). Since all response spectra are corrected to $\mathrm{V}_{\mathrm{s} 30}=620 \mathrm{~m} / \mathrm{s}$, the crustal and site amplification functions in the modified version of the EXSIM modeler are considered identical for all sites and earthquakes. The assumed path parameters are also considered identical. The only parameters that vary from one earthquake to another are the source parameters (including the moment magnitude), fault/sub-source geometry, and stress parameter distribution.

\subsection{Inversion results}

\subsubsection{M7.1, 1989 Loma Prieta earthquake}

The geometry of the M7.1, 1989 Loma Prieta earthquake is given in Table (5.1), as taken from Wald et al., (1991).

\subsubsection{Case 1}

Response spectral information of 83 stations is used for derivation of the coseismic stress parameter distribution on the fault surface. The locations of the stations around the fault are shown in Figure (5.1) (from the PEER-NGA database). The number of sub-sources in Case 1 , for which the derivation of stress parameter is performed, is $32(8 \times 4)$. The number of data points is more than twice the number of the sub-sources. Considering that PSA of a station and its geometry relative to all sub-sources is different from those of 
other stations, and knowing that we have 83 stations and 32 sub-sources in this case, it seems reasonable to assume that the problem is an overdetermined one, and thus will have solutions based on the least squares error criterion (minimizing $\Theta$ in Equation 4.5).

The stress parameter distribution derived from our study is shown in Figure (5.2). This ultimate solution is derived after 4 successful inversion iterations starting from a uniform stress parameter distribution. The assigned average stress parameter of the initial guess was 50 bars; and it evolved to an average of 60 bars at the end. The evolutions of average absolute error $(\Xi)$ and average error of $\left|\varepsilon_{j}\right|$ values over all stations $(\Phi)$ with successive inversion iteration number are shown in Figure (5.3). In the beginning of an iteration the minimization of $\Xi$ assigns an optimum average stress parameter to the solution and then minimization of $\Theta$, by Levenberg-Marquardt procedure, finds an improved stress parameter distribution on the fault surface. Figure (5.3) compares the evolution of two measures of error that characterize the mechanics of the inversion process. Figure (5.3) shows that both error measures decrease with increasing iteration number, implying that on average response spectra of simulations at all stations get closer to the response spectra of actual records as we iterate.

Figure (5.4) shows average absolute error as function of frequency $\left(\Xi_{\mathrm{i}}\right)$ for the initial guess and the final solution after 4 iterations. Since the stress parameter is proportional to the high frequency level of the spectrum, and the inversion tool optimizes stress parameter distribution on the fault surface, it is expected for the method to optimize just the high frequency content of the signals. Figure (5.4) shows the success of the method in reducing the error level $\Xi_{\mathrm{i}}$ of simulations at high frequencies while leaving the low frequency level without any change.

Figure (5.5) shows absolute average error difference $\left(\Psi_{\mathrm{j}}\right)$ of the initial guess and final solution as a function of location. The $\Psi$ values of Equation (5.1) are calculated for each individual station and averaging is performed over the $0.45 \mathrm{~Hz}$ to $10 \mathrm{~Hz}$ frequency interval. According to equation (5.1), a positive value of $\Psi$ corresponds to solution improvement at a point by switching from the initial guess to a given solution, while a 
negative value corresponds to the solution degrading at that point. For all of the points in the blue area, the implementation of the modified stress parameter distribution on the fault surface (Figure 5.2) resulted in an improved match of simulation PSAs to recorded PSAs. On the contrary, for all of the points in the red area the implementation of the modified stress parameter distribution on the fault surface resulted in a poorer match. Figure (5.5) shows that the number of stations showing reduction of error as a result of implementation of the modified stress parameter distribution is greater than the number of stations showing an increase of error. This suggests that the average error is reduced, although the use of the modified stress parameter distribution in modeling reduced the accuracy of the PSAs of simulations for some records.

An important point to note is that the map of Figure (5.5) is prepared by the kriging method, so the obtained values are valid in areas of considerable data density. Accordingly the reliable values of the map are the areas located on a band extending from northwest to southeast. This band covers more blue area than red. Also, some of the blue spots that cover the fault trace result from nearby stations in that area, while this is not the case for red spots on the fault trace.

The application of the inversion program for the M7.1, 1989 Loma Prieta earthquake resulted in a stress parameter distribution with three stress concentrations on the fault surface as shown in Figure (5.2). Both average absolute error $(\Xi)$ and average error $(\Phi)$ iteratively decrease during the inversion process (Figure 5.3), suggesting that with the chosen station distribution and the sub-division of the fault to sub-sources, the program performs successfully. Figure (5.4) compares average errors of all stations $\left(\Xi_{i}\right)$ in each frequency between application of uniform and modified stress parameter distributions, showing that on average the error $\Xi_{i}$ reduced considerably by switching from uniform stress parameter distribution to the derived stress parameter distributions. Figure (5.5) shows the spatial distribution of error changes $(\Psi)$ that resulted from switching to the derived stress parameter distribution from the uniform stress parameter distribution. Most of the stations experienced positive $\Psi$. These are mainly located in the northwest side of 
the fault. The graphs (5.3) to (5.5) show that the inversion program improves the solution for stress parameter distribution by reducing the overall errors at all stations.

\subsubsection{Case 2}

Response spectral information of 29 stations is used for the derivation of the coseismic stress parameter distribution on the fault surface. The locations of the nearby stations around the fault, between latitudes $36.8^{\circ} \mathrm{N}$ and $37.4^{\circ} \mathrm{N}$, that are used for this case, are shown in Figure (5.6). The number of sub-sources in the case 2 is $24(6 \times 4)$. The number of data points is not much more than the number of the sub-sources. This suggests that the solutions derived from the inversion program may have poor quality. This might be because (i) the number of stations is not large and (ii) clustered stations generate a set of equations with slight differences in error only, which will not be capable of resolving the stress of individual sub-sources.

The stress parameter distribution derived from our study is shown in Figure (5.7). This solution is derived after 40 successful inversion iterations starting from a uniform distribution. The assigned average stress parameter of the initial guess was 50 bars; it evolved to an average of 70 bars at the end of the $40^{\text {th }}$ iteration.

The evolution of average absolute error $(\Xi)$ and average error $(\Phi)$ with successive inversion iteration number is shown in Figure (5.8). Figure (5.8) shows that the average error $(\Phi)$ decreases continuously in the process of inversion, although its reduction is not considerable after the $4^{\text {th }}$ iteration. Except for a jump at the $3^{\text {rd }}$ iteration where the average stress on the fault surface increased, average absolute error $(\Xi)$ reduced slowly with successive inversion iterations. Overall Figure (5.8) shows reduction of error with successive inversion iterations for both error criteria.

Figure (5.9) shows average absolute error as function of frequency $\left(\Xi_{i}\right)$ for the initial guess and the solution after the $40^{\text {th }}$ iteration. Figure (5.9) does not show a reasonable success of the method in reducing the error level $\Xi_{i}$ of simulations at high frequencies. 


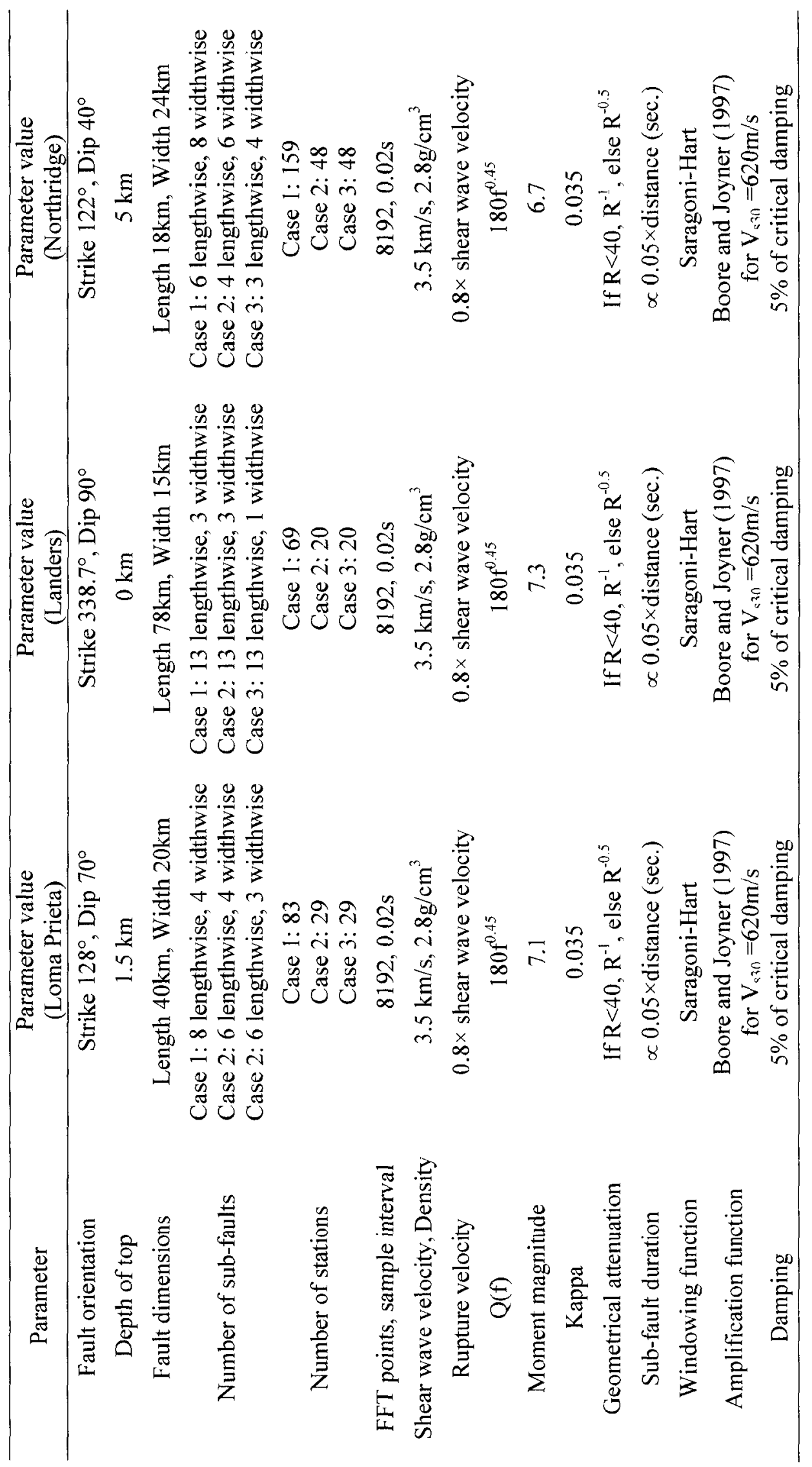




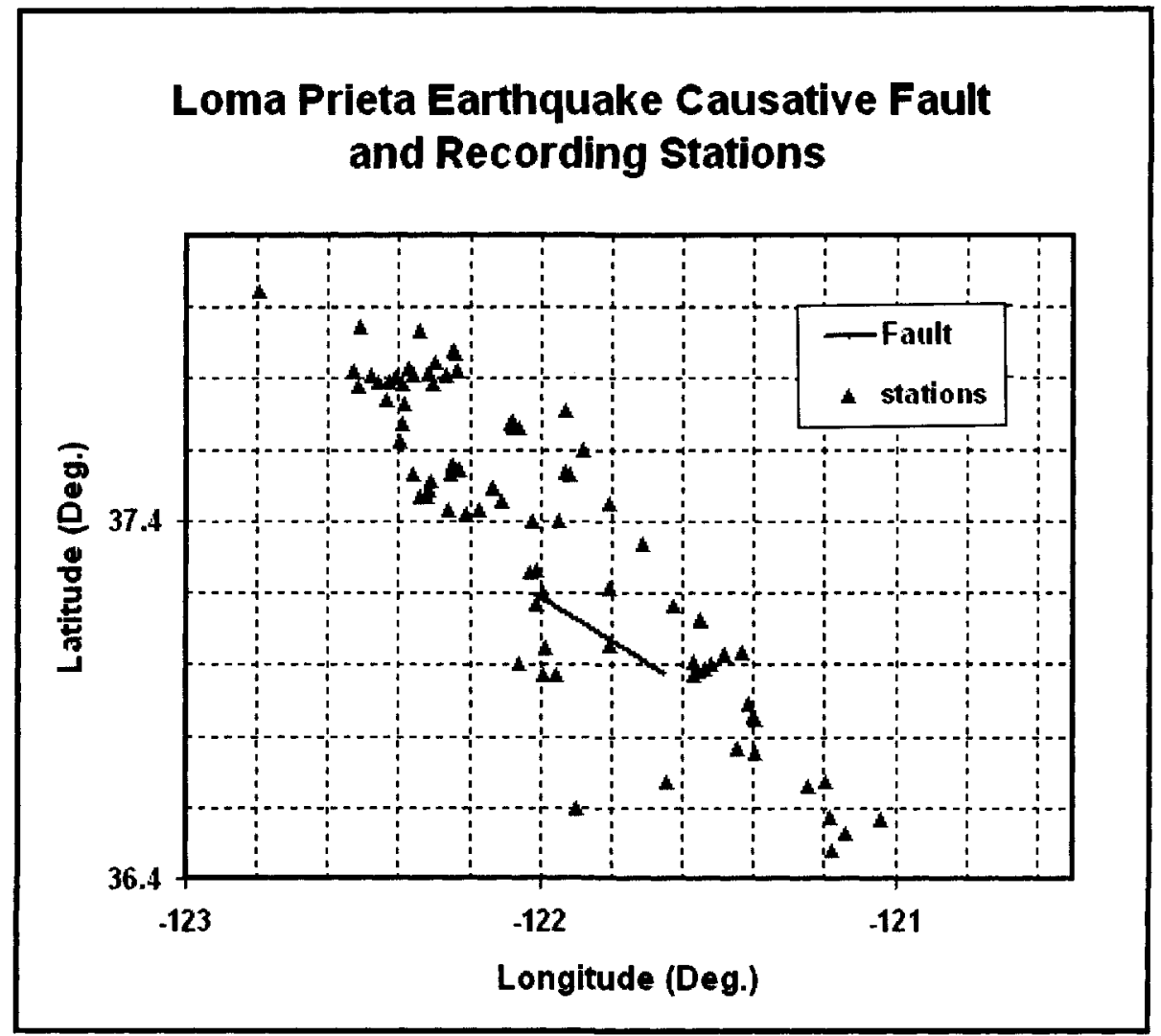

Figure (5.1) Distribution of stations used for Loma Prieta earthquake and the earthquake fault location in the case 1 .

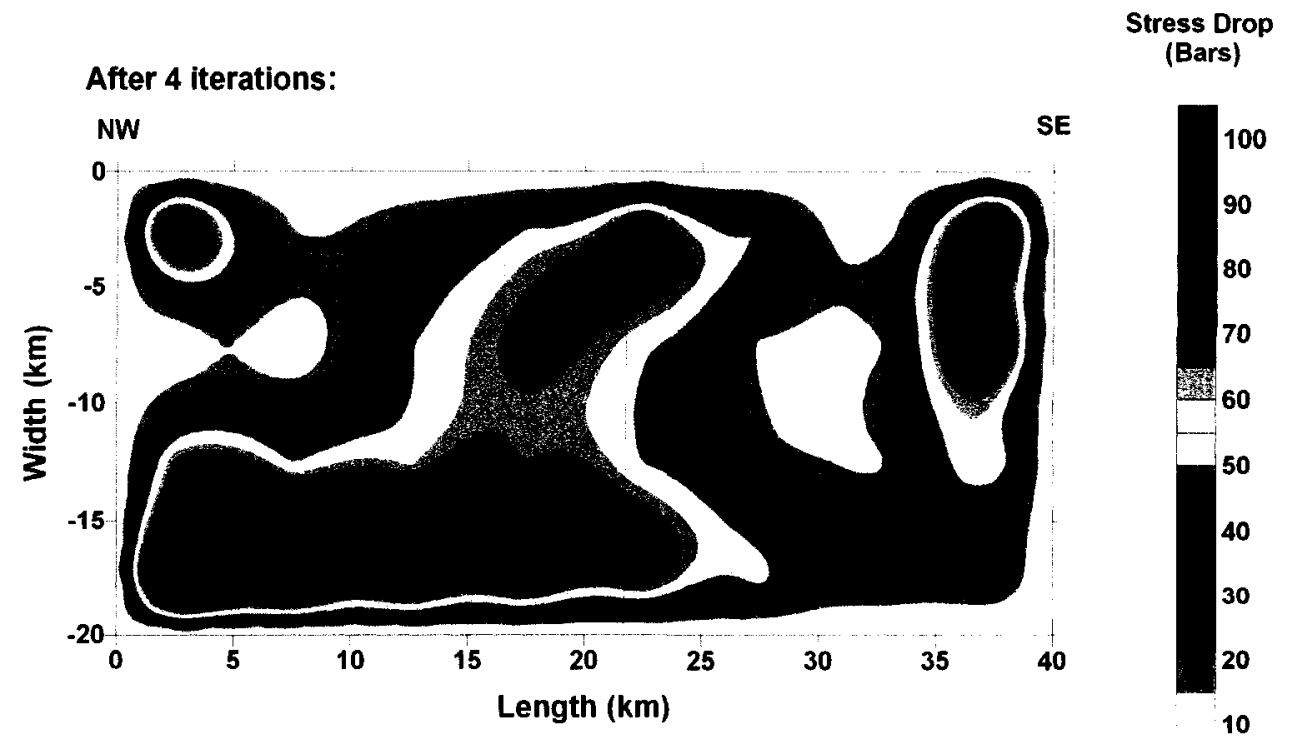

Figure (5.2) Derived stress parameter distribution on the Loma Prieta fault in the case 1. Star shows the location of hypocenter. 


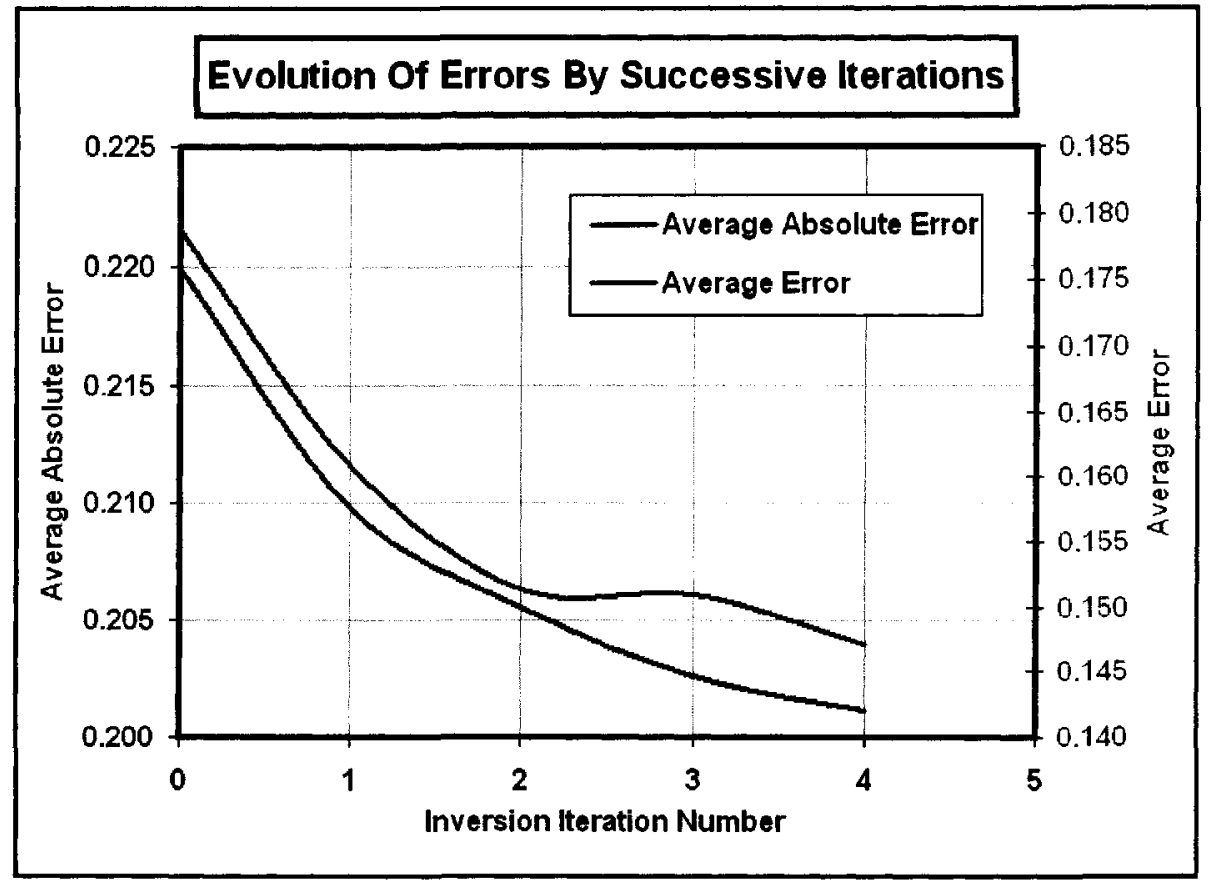

Figure (5.3) Evolution of average absolute error $(\Xi)$ and average error $(\Phi)$ with successive inversion iteration in the case 1 of Loma Prieta study.

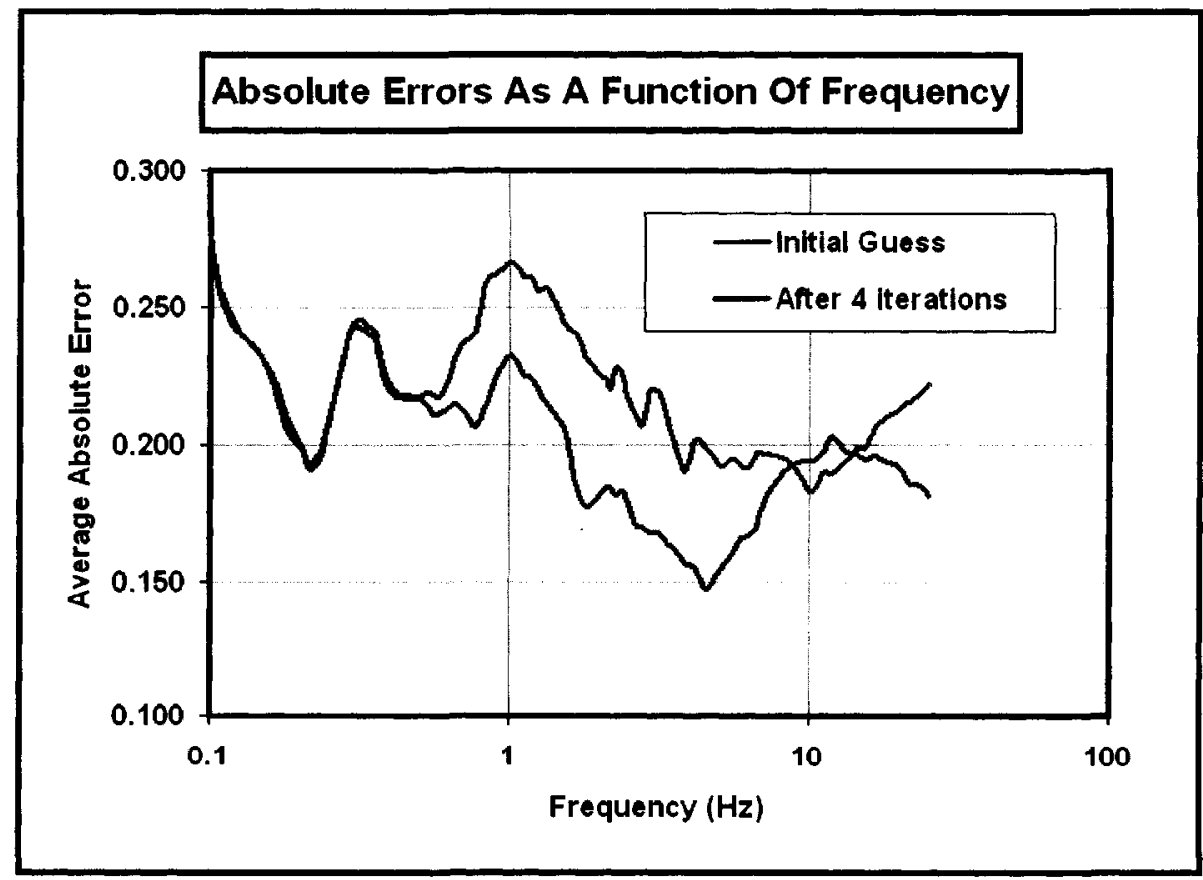

Figure (5.4). Average absolute error $\left(\Xi_{\mathrm{i}}\right)$ as a function of frequency in the case 1 of Loma Prieta inversion. 


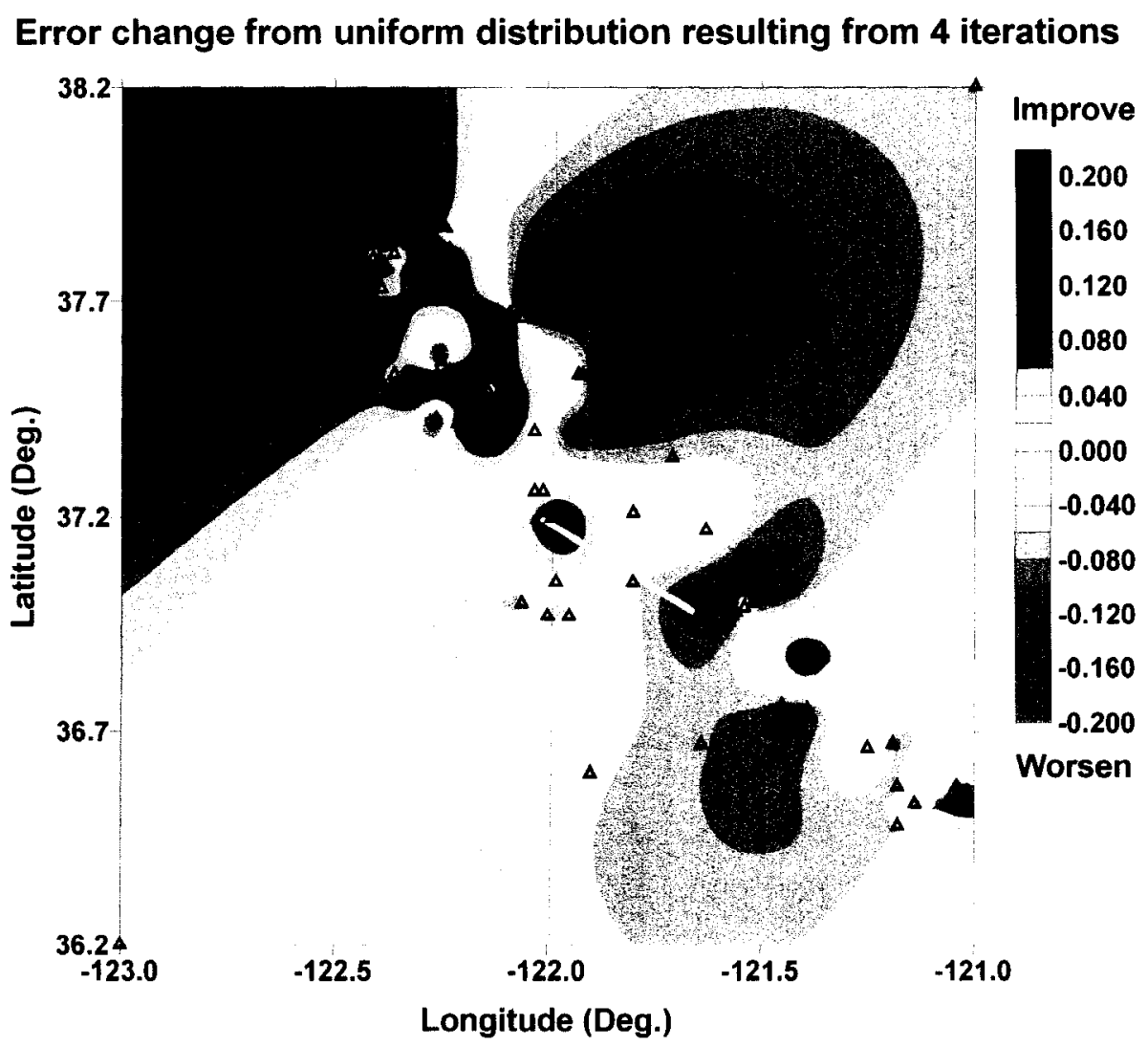

Figure (5.5). Spatial distribution of error improvement criterion $\Psi_{j}$ from initial guess to final solution after 4 inversion iterations in the case 1 of Loma Prieta study. Triangles are location of stations and the yellow solid line is the location of the top of the fault. 


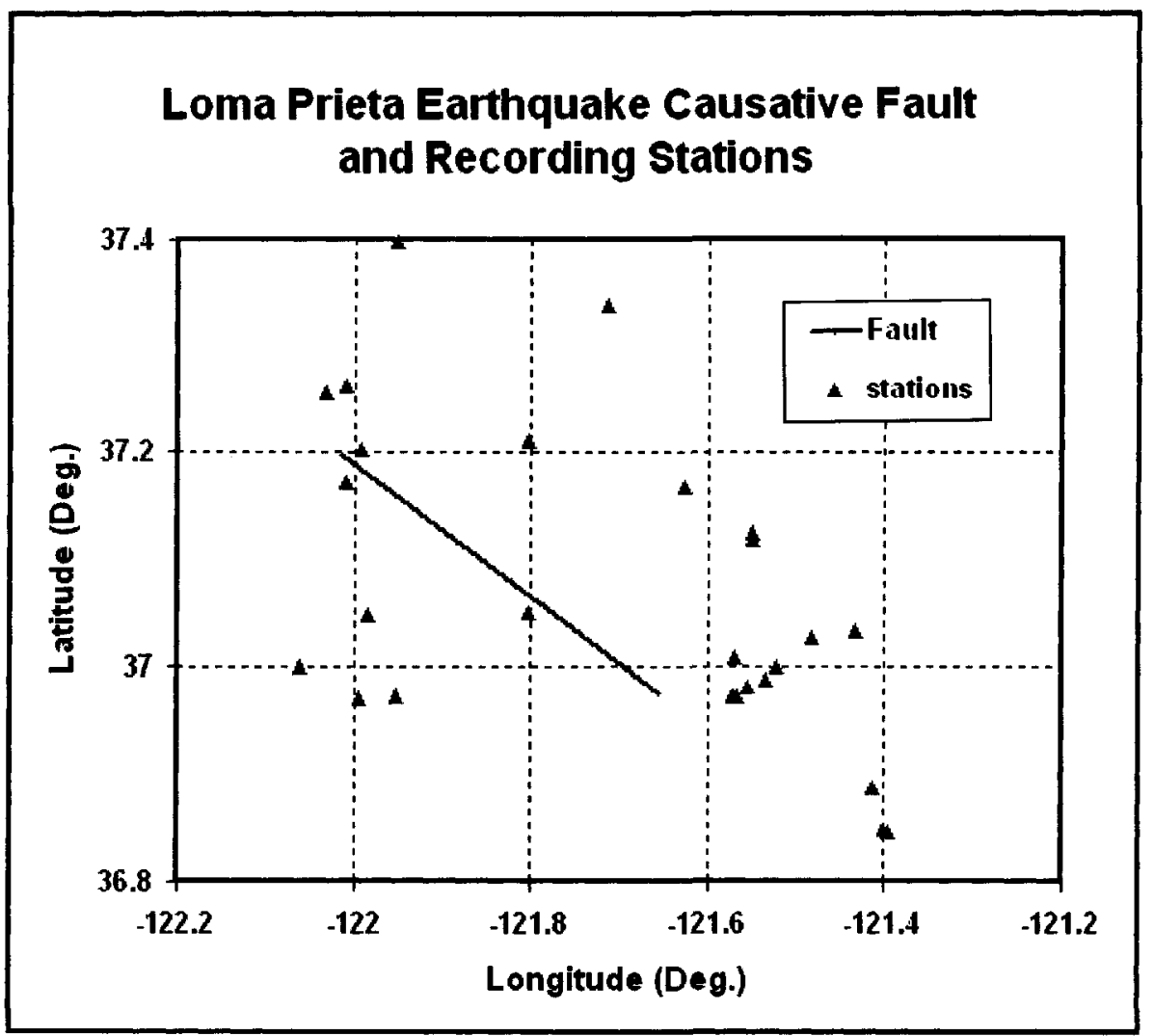

Figure (5.6) Distribution of stations used for Loma Prieta earthquake and the earthquake fault location in Cases 2 and 3.

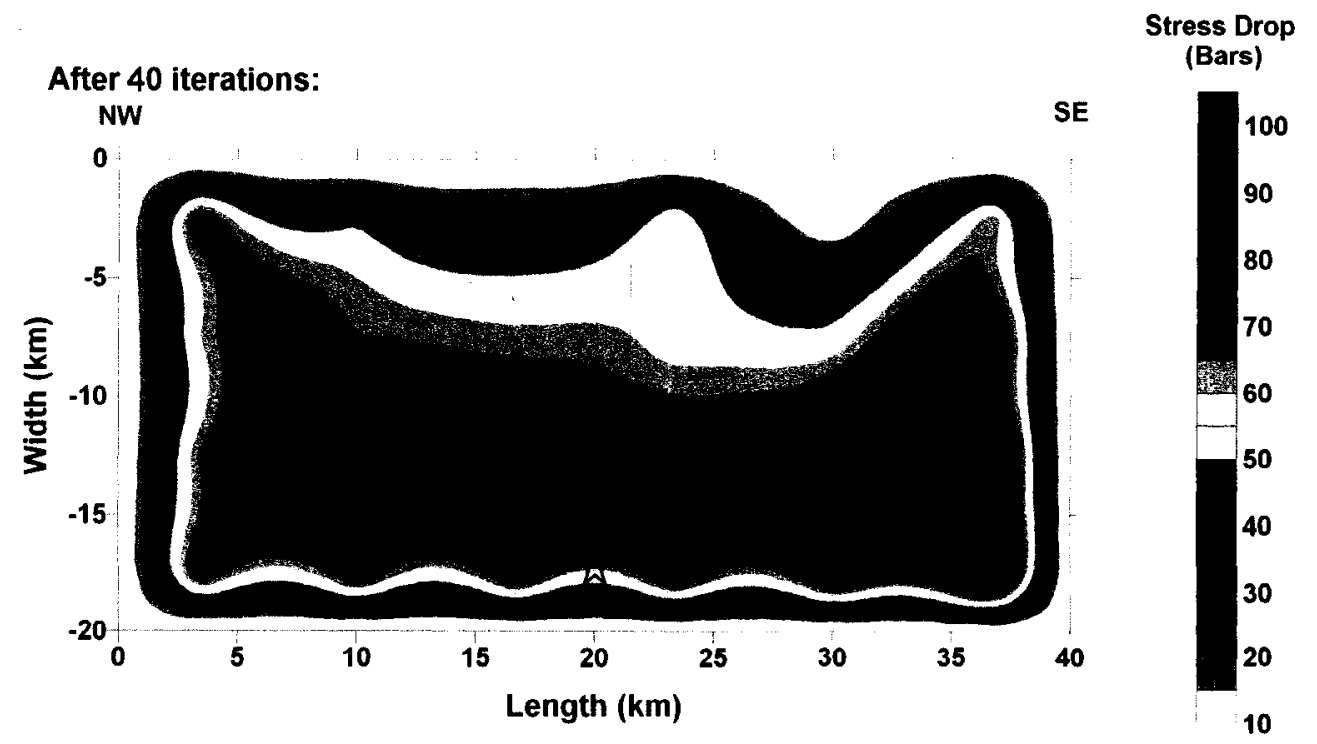

Figure (5.7) Derived stress parameter distribution on the Loma Prieta fault in Case 2. Star shows the location of hypocenter. 


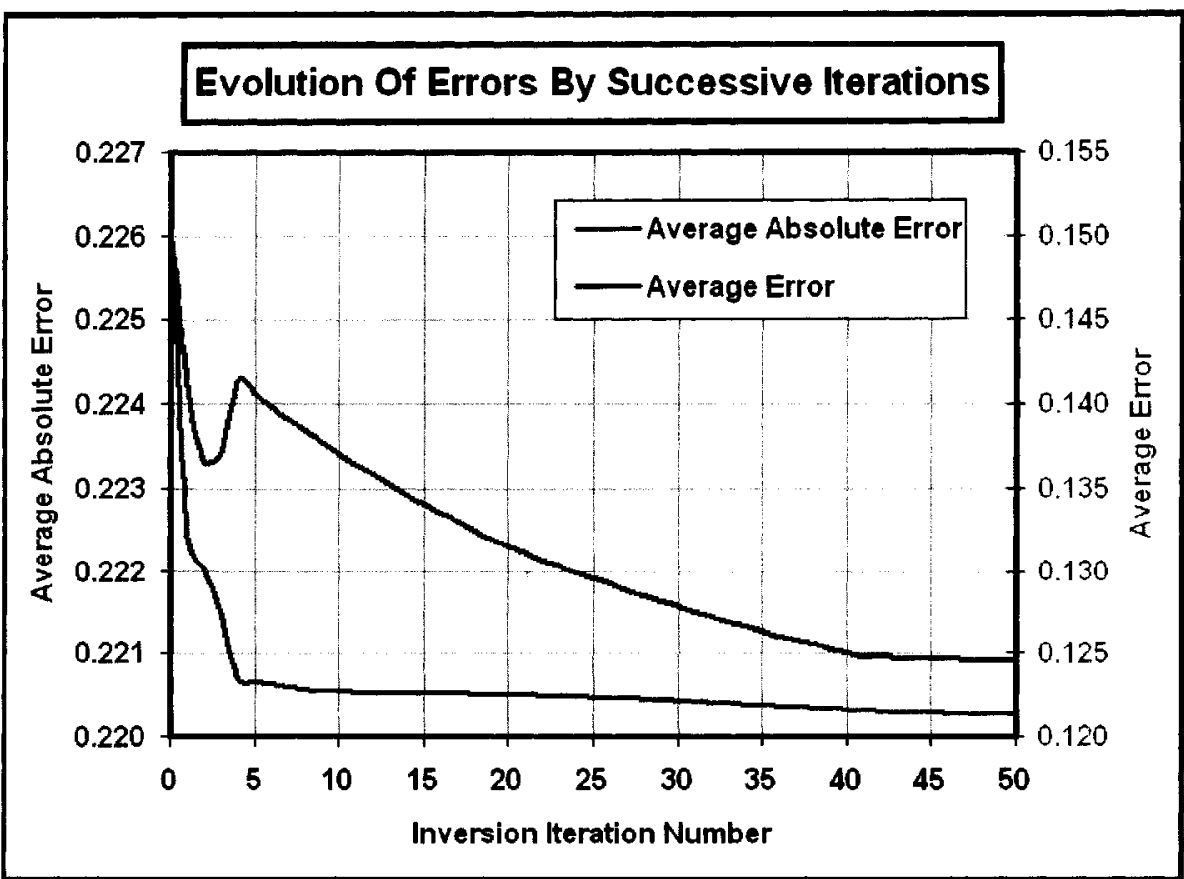

Figure (5.8) Evolution of average absolute error $(\Xi)$ and average error $(\Phi)$ with successive inversion iteration in Case 2 of Loma Prieta study.

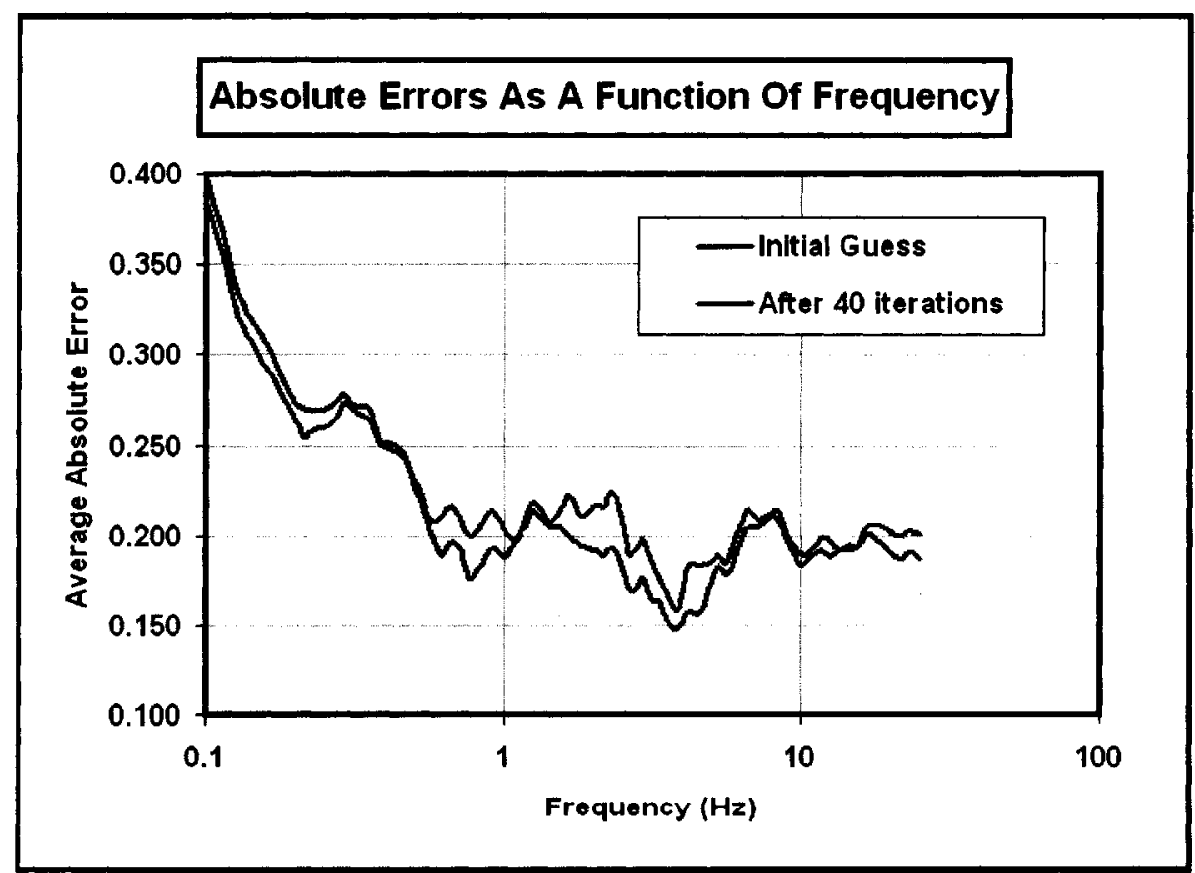

Figure (5.9). Average absolute error $\left(\Xi_{\mathrm{i}}\right)$ as a function of frequency in Case 2 of Loma Prieta inversion. 
This problem can be attributed to the insufficient number of stations for resolving the stress level of each sub-source using this method.

Figure (5.10) shows the absolute average error difference $\left(\Psi_{\mathrm{j}}\right)$ of the initial guess and final solution after the $40^{\text {th }}$ iteration as a function of location. The $\Psi$ values are calculated for each individual station over the $0.45 \mathrm{~Hz}$ to $10 \mathrm{~Hz}$ frequency interval. Figure $(5.10)$ shows that the number of stations that show reduction of error for the modified stress parameter distribution is greater than the number of stations that show an increase of error. This suggests that the average error is reduced, overall. Again it is important to note that the colored map is valid for areas around the location of the stations.

The application of the inversion program for M7.1, 1989 Loma Prieta earthquake in case 2 resulted in a stress parameter distribution with a stress concentrations on the fault surface in the south east end of the fault in the depth (Figure 5.7). Average error (Ф) decreases iteratively during the inversion process (Figure 5.8) but its trend is not significant after the $4^{\text {th }}$ iteration. On the other hand, the average absolute error $(\Xi)$ shows a continuous decrease with successive iterations except in the $3^{\text {rd }}$ iteration. These error reduction curves show that the inversion program did its job properly ( $\Phi$ and $\Xi$ are the parameters that the program reduces by changing the stress parameter distribution). Figure (5.9) shows no significant reduction in errors in any frequency range $\left(\Xi_{i}\right)$ by switching from the application of uniform to the modified stress parameter distributions. Figure (5.10) shows that the most of the stations experienced positive $\Psi$ but there are no stations that experienced strong negative $\Psi$ by switching from the uniform distribution to the derived distribution. This is again suggesting that the inversion program performed well. Putting together the results of Figures (5.8) to (5.10) shows that the program performed well, but the small number of stations and the clustering of some of them resulted in lack of sufficient data for proper resolution of the stress of sub-sources. In other words, the two error criteria $\Phi$ and $\Xi$ reduced in the inversion process while the error measure $\Xi_{i}$ did not show any signs of improvement in the same process, suggesting 
that the program reduced those errors that are in its control $(\Phi$ and $\Xi)$ but failed to reduce the other error measure $\left(\Xi_{\mathrm{i}}\right)$.

\subsubsection{Case 3}

Response spectra information of the same 29 stations of Case 2 is used but in Case 3 the number of sub-faults is $18(6 \times 3)$. The number of data points is not much more than the number of the sub-sources. This suggests that like Case 2 the solutions derived from the inversion program may be poor.

The stress parameter distribution derived from our study is shown in Figure (5.11). This solution is derived after 25 successful inversion iterations starting from a uniform distribution. The assigned average stress parameter of initial guess was 50 bars and it evolved to an average of 70 bars at the end of the $25^{\text {th }}$ iteration.

The evolution of average absolute error $(\Xi)$ and average error $(\Phi)$ with successive inversion iteration number is shown in Figure (5.12). Both error measures decrease with increasing iteration number, implying that on average the response spectra of simulations at all stations gets closer to the response spectra of actual records. But the improvements of error criteria, both $\Xi$ and $\Phi$, becomes very slow after the $4^{\text {th }}$ iteration.

Figure (5.13) shows the average absolute error as a function of frequency $\left(\Xi_{i}\right)$ for the initial guess and the solution after $25^{\text {th }}$ iteration. Figure (5.13) does not show a reasonable success of the method in reducing the error level $\Xi_{\mathrm{i}}$ of simulations at high frequencies. This problem can be attributed to the insufficient number of stations for resolving the stress level of each sub-source using this method.

Figure (5.14) shows absolute average error difference $\left(\Psi_{j}\right)$ of the initial guess and the final solution as a function of location. The spatial distribution of $\Psi$ for case 3 is very similar that for case 2 .

The application of the inversion program for the M7.1, 1989 Loma Prieta earthquake in case 3 resulted in a stress parameter distribution with a stress concentrations on the fault 
surface in the southeast end of the fault at depth (Figure 5.11). Average error (D) and average absolute error $(\Xi)$ decrease iteratively during inversion process (Figure 5.12) but their trend is not significant after the $4^{\text {th }}$ iteration. Figure (5.13) shows no significant reduction in errors in any frequency range $\left(\Xi_{\mathrm{i}}\right)$ by switching from the application of the uniform to the derived stress parameter distributions. Overall, the program performed well, but the small number of stations and the clustering of some of them resulted in lack of sufficient data for proper resolution of the stresses of sub-sources.

\subsubsection{Loma Prieta earthquake slip model from other studies}

There are many study results for the slip distribution of the Loma Prieta earthquake fault (Beroza, 1991; Emolo and Zollo, 2005; Steidl et al., 1991; Su et al., 1994; Wald et al., 1991). For example Steidl et al., (1991) inverted strong motion waveforms for slip distribution on the fault surface; their results (downloaded from http://www.seismo.ethz.ch/srcmod/) are replotted in Figure (5.15). Two areas of Figure (5.15) are outlined with dashed lines and show the locations of asperities derived from the Steidl et al., 1991 study. Comparing Figure (5.15) to the results of our study, Figure (5.2) (using all stations), shows considerable similarity between the slip distribution and stress parameter distribution, derived from different methods. If we assume a uniform rigidity on the fault surface, then high slip on the fault surface will correspond to high stress over the same area. This suggests that the slip and stress parameter distributions should be similar.

Wald et al., (1991) also found two main patches of slip and stress, with the largest slip/stress patch located at the northwest side of the fault. Their study showed proportionality of slip and stress for the Loma Prieta earthquake high-slip patches. The finding of two asperities near the edge of the fault by Wald et al., (1991) is in agreement with the findings of this study. The second result of Wald et al., (1991), regarding proportionality of slip and stress for the Loma Prieta earthquake, confirms the validity of 
that assumption as implemented in this study for the comparison of stress and slip distributions.

\subsubsection{M7.3, 1992 Landers earthquake}

The geometry of the M7.3, 1992 Landers earthquake is given in Table (5.1), as taken from Wald and Heaton (1994).

\subsubsection{Case 1}

Response spectral information of 69 stations is used for the derivation of coseismic stress parameter distribution on the fault surface. The locations of the stations around the fault are shown in Figure (5.16) (PEER NGA database). The number of sub-sources in Case 1 is $39(13 \times 3)$. The number of data points is thus more than twice the number of the subsources, and so the problem is almost an overdetermined one. However, the clustering of stations at the west side of the fault, the large distances of many stations from the fault, and the large size of the fault/sub-sources will reduce the resolution of the solution.

The stress parameter distribution derived from our study is shown in Figure (5.17). This solution is derived after 20 successful inversion iterations, starting from a uniform distribution. The assigned average stress parameter of the initial guess was 50 bars; it evolved to an average of 70 bars at the end. The evolution of average absolute error $(\Xi)$ and average error $(\Phi)$ with successive inversion iteration number is shown in Figure (5.18). Figure (5.18) shows that both error measures decrease with increasing iteration number, implying that on average response spectra of simulations at all stations get closer to the response spectra of records. However the evolution of these error criteria is negligible after the $6^{\text {th }}$ iteration. 


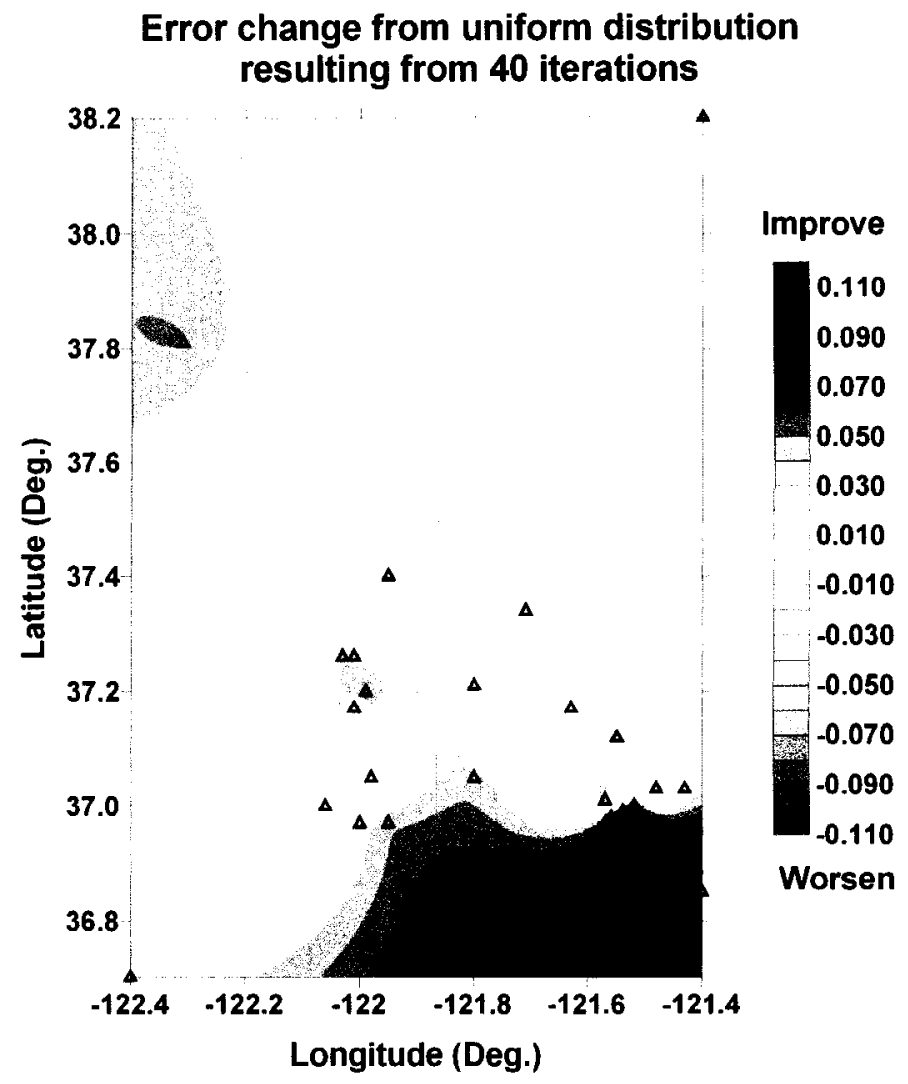

Figure (5.10). Spatial distribution of error improvement criterion $\Psi_{\mathrm{j}}$ from initial guess to final solution after 40 inversion iterations in Case 2 of the Loma Prieta study. Crosses are location of stations and the yellow solid line is the location of the top of the fault.

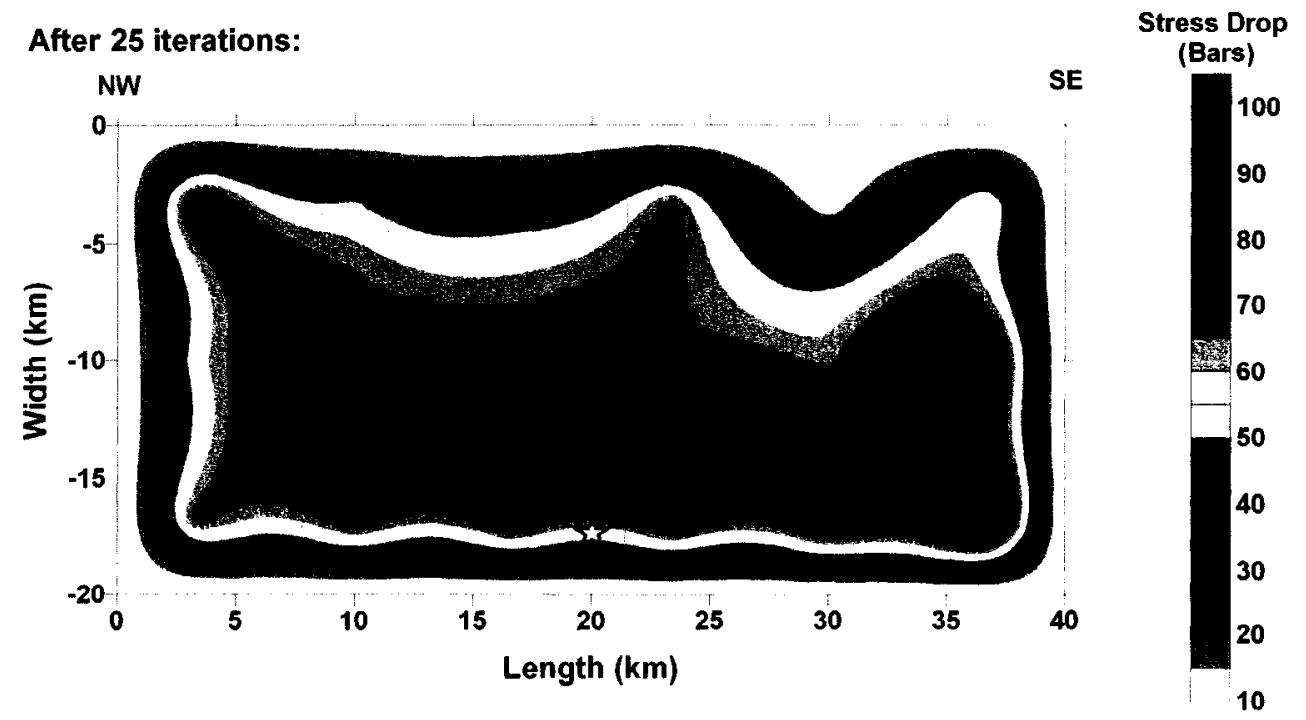

Figure (5.11) Derived stress parameter distribution on the Loma Prieta fault in Case 3. Star shows the location of hypocenter. 


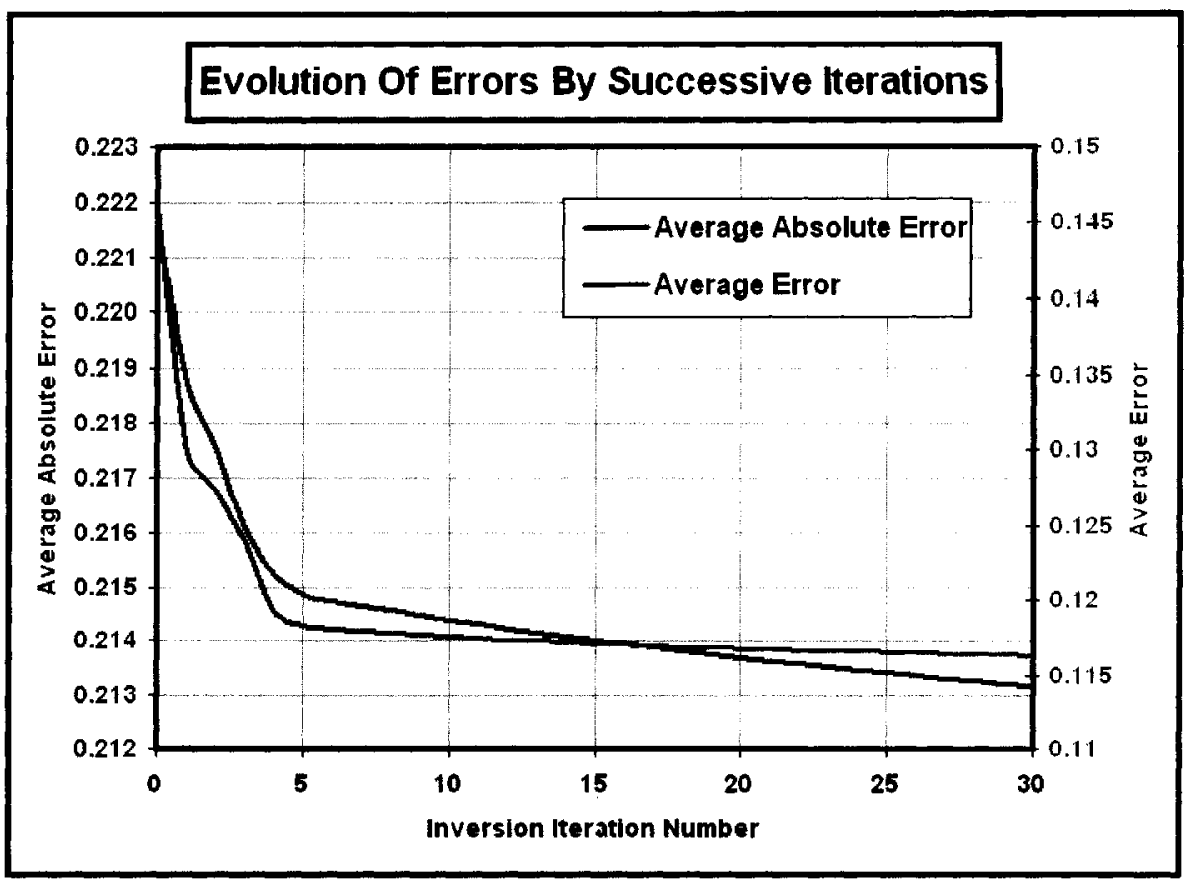

Figure (5.12) Evolution of average absolute error $(\Xi)$ and average error $(\Phi)$ with successive inversion iteration in Case 3 of Loma Prieta study.

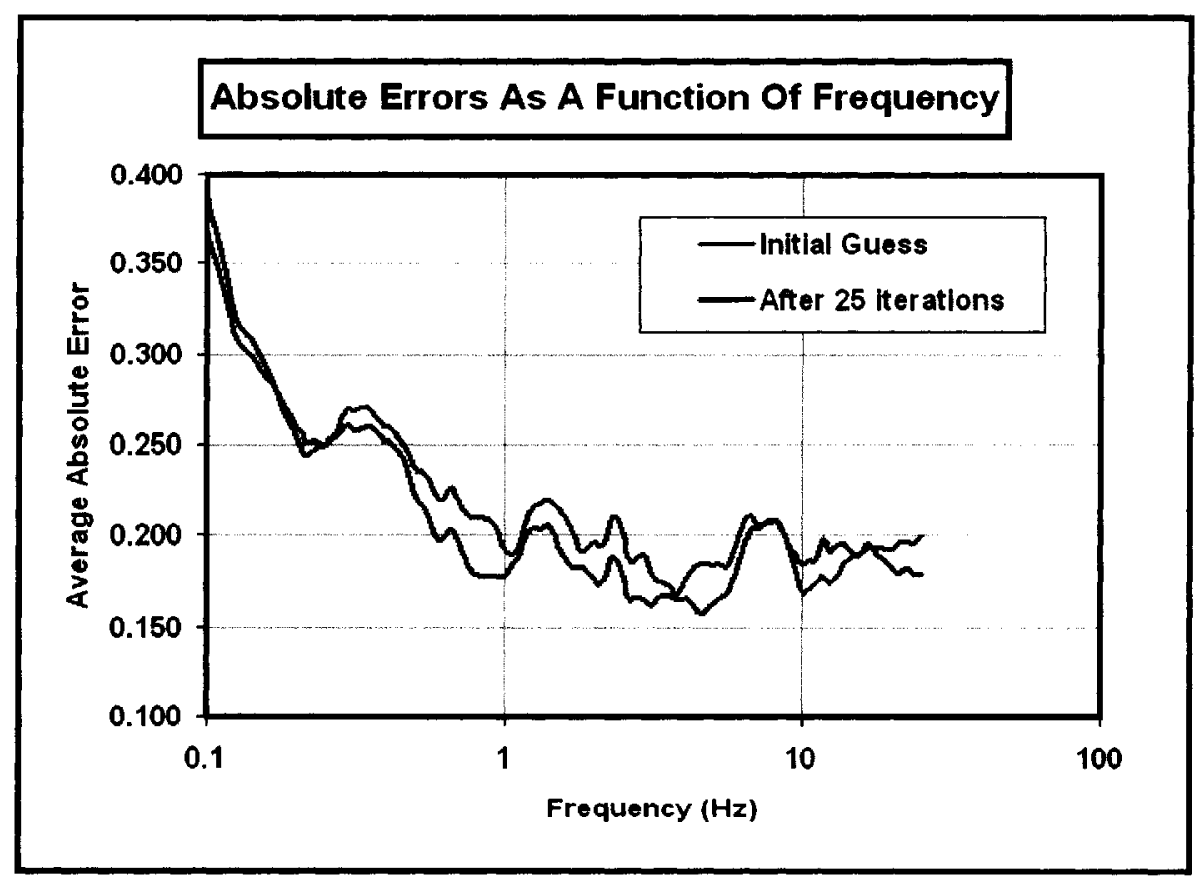

Figure (5.13). Average absolute error $\left(\Xi_{i}\right)$ as a function of frequency in Case 3 of Loma Prieta inversion. 


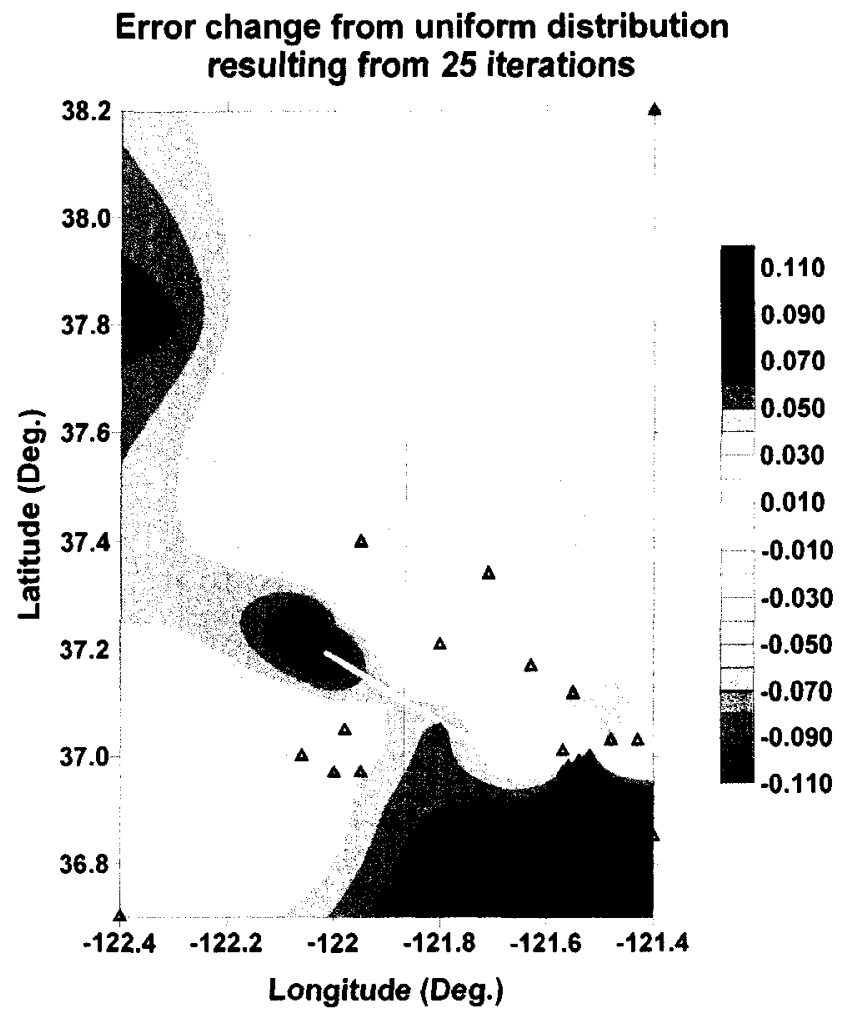

Figure (5.14). Spatial distribution of error improvement criterion $\Psi_{\mathrm{j}}$ from initial guess to final solution after 25 inversion iterations in Case 3 of Loma Prieta study. Crosses are location of stations and the yellow solid line is the location of the top of the fault.

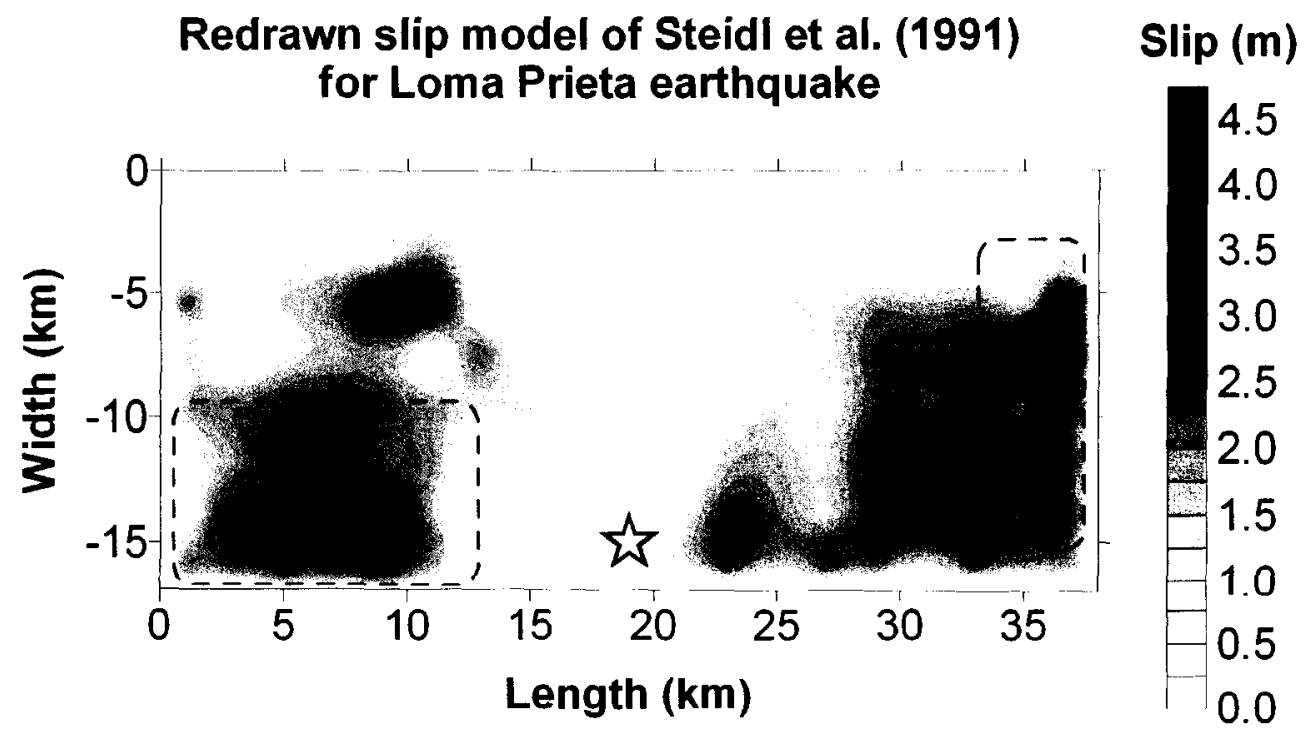

Figure (5.15). Slip distribution of Loma Prieta earthquake derived from strong motion waveform inversion by Steidl et al. (1991). Redrawn using data at http://www.seismo.ethz.ch/srcmod/. 
Figure (5.19) shows the average absolute error as a function of frequency $\left(\Xi_{i}\right)$ for the initial guess and the final solution after 20 iterations. Figure (5.19) shows the success of the method in reducing the error level $\Xi_{\mathrm{i}}$ of simulations at high frequencies while leaving the low frequency error level unchanged.

Figure (5.20) shows the absolute average error difference $\left(\Psi_{j}\right)$ of the initial guess and final solution as a function of location, over the $0.22 \mathrm{~Hz}$ to $10 \mathrm{~Hz}$ frequency interval. Figure (5.20) shows that the number of stations that show reduction of error as a result of the modified stress parameter distribution (blue areas) is greater than the number of stations that show increase of error (red areas).

The application of the inversion program for the M7.3, 1992 Landers earthquake resulted in a solution where the stress amplitude on the fault surface increases from the hypocenter towards the fault center as shown in Figure (5.17). Both average absolute error $(\Xi)$ and average error $(\Phi)$ iteratively decrease during inversion process (Figure 5.18) suggesting that with the chosen station distribution and the sub-division of the fault to sub-sources, the program performs successfully. Figures (5.18) to (5.20) show that the inversion program improves the solution for stress parameter distribution by reducing the overall errors at all stations.

\subsubsection{Case 2}

Response spectral information of only 20 stations is used for the derivation of the coseismic stress parameter distribution on the fault surface in Case 2. The locations of the nearby stations around the fault, that are those used for this case, are shown in Figure (5.21). The number of sub-faults in Case 2 is again $39(13 \times 3)$. The number of data points is less than the number of the sub-sources. This suggests that the problem is an underdetermined one and no reasonable solution can be derived from inversion program.

The stress parameter distribution derived from this study is shown in Figure (5.22). This solution is derived after 31 successful inversion iterations starting from a uniform 
distribution. It looks like a uniform distribution with a few minor disturbances. The assigned average stress parameter of the initial guess was 50 bars and it didn't evolve to another value at the end. The inversion program runs and generates a solution, although this solution cannot be accepted as an answer since the problem is underdetermined and an infinite number of similar solutions may exist. The study of error measures $\Xi, \Phi, \Xi_{\mathrm{i}}$, and $\Psi_{\mathrm{j}}$ demonstrates that the resulting solution of Figure (5.22) is not an answer.

The evolution of average absolute error $(\Xi)$ and average error $(\Phi)$ with successive inversion iteration number is shown in Figure (5.23). Figure (5.23) shows that only average error $(\Phi)$ decreases continuously in the process of inversion and the amount of decrease is only $\sim 4 \%$.

Figure (5.24), showing average absolute error as a function of frequency $\left(\Xi_{i}\right)$ for the initial guess and the solution after 31 iterations, again suggests no difference between the results of the initial guess and the derived solution.

Figure (5.25), showing performance as a function of location, shows that just one station, located very close (or almost on top of fault), experienced considerable reduction of error as a result of implementation of the modified stress parameter distribution; other stations don't experience large (positive or negative) $\Psi$ values.

All these results suggest that the program is unable to solve an underdetermined problem, although the Levenberg-Marquardt algorithm finds distributions that reduce the error measure $\Phi$ by a small amount. The output is reasonable in this case in that a stable result that is little different from the starting point is returned. 


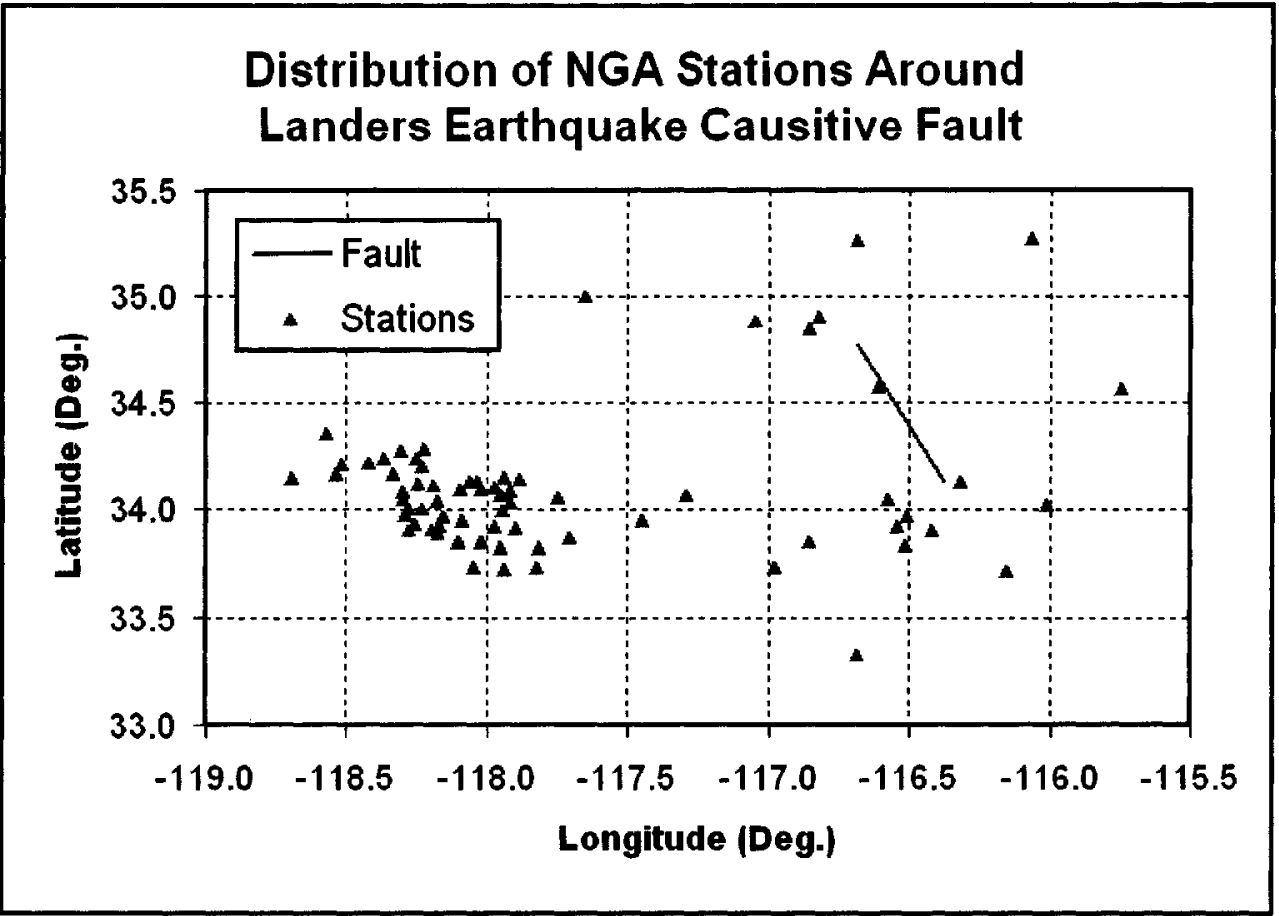

Figure (5.16) Distribution of stations used for Landers earthquake and the earthquake fault location in Case 1.

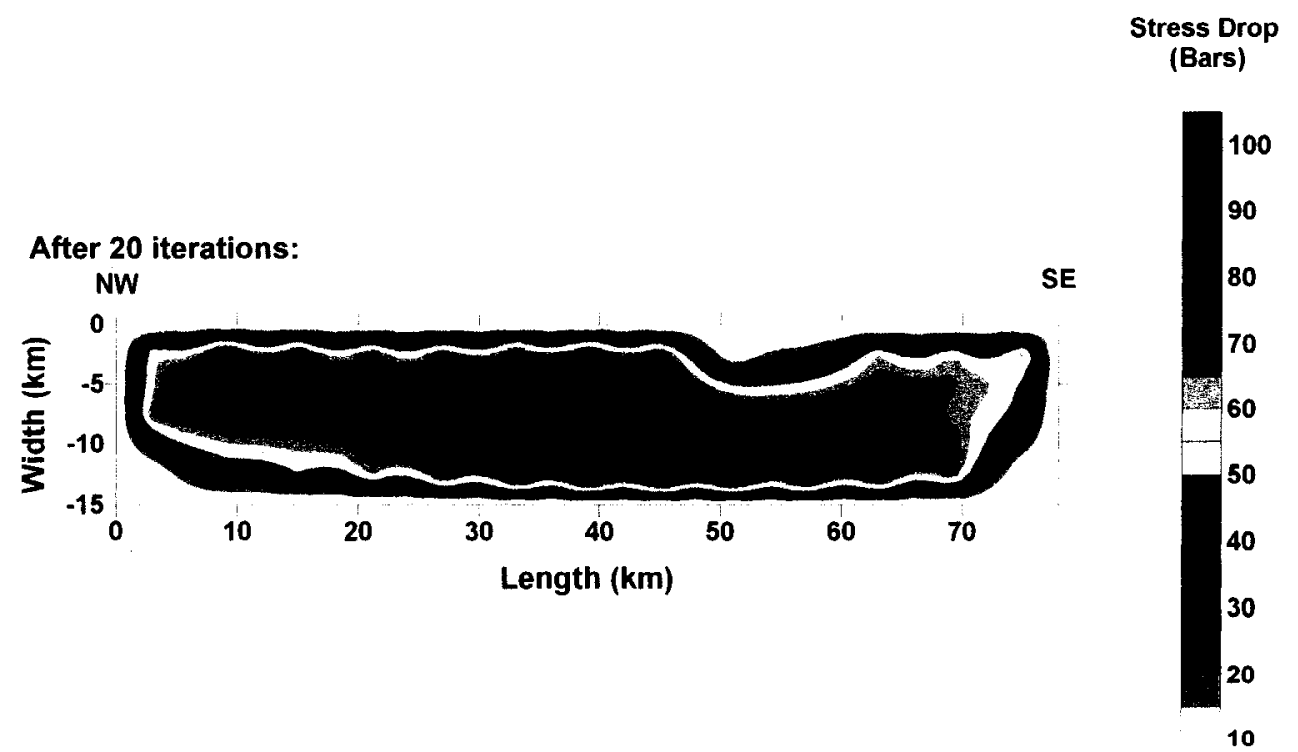

Figure (5.17) Derived stress parameter distribution on the Landers earthquake causative fault after $20^{\text {th }}$ iteration in Case 1. Star shows the location of hypocenter. 


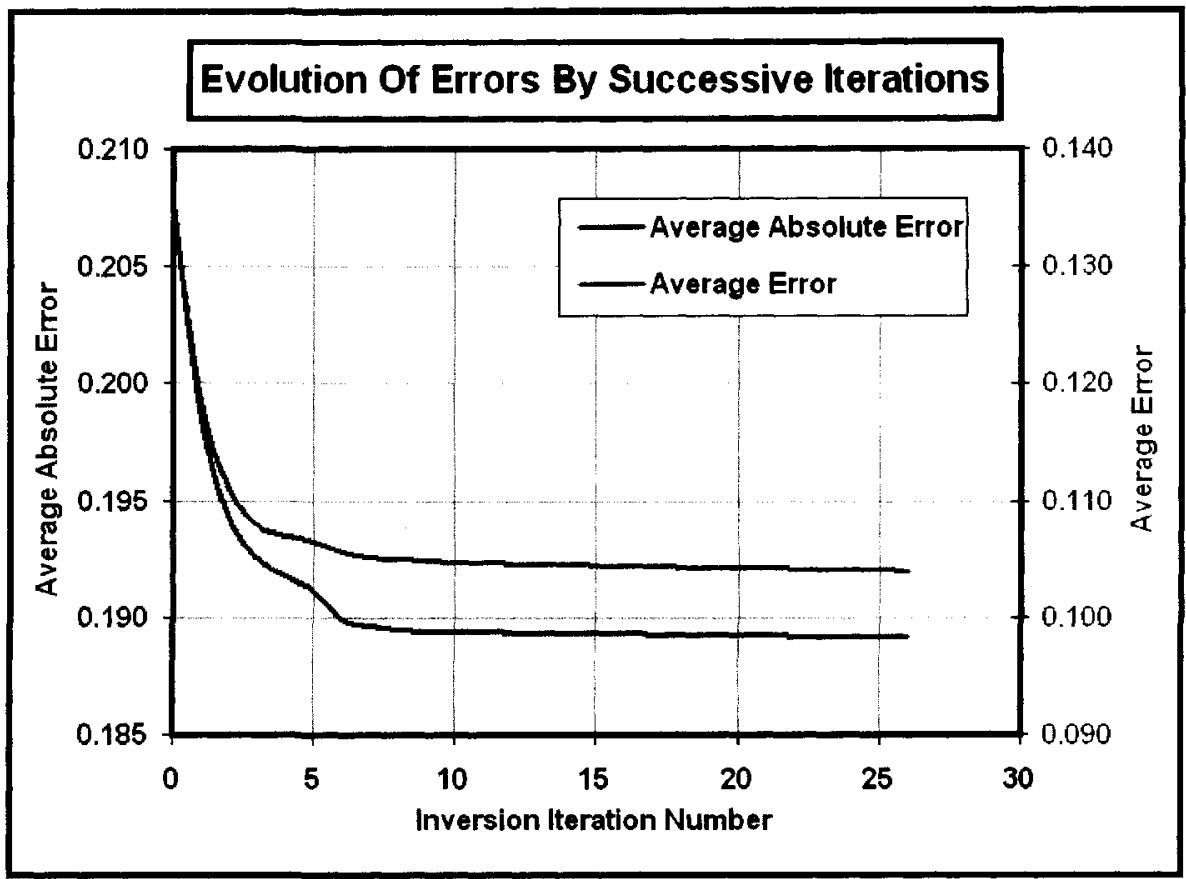

Figure (5.18) Evolution of average absolute error $(\Xi)$ and average error $(\Phi)$ with successive inversion iteration in Case 1 of Landers study.

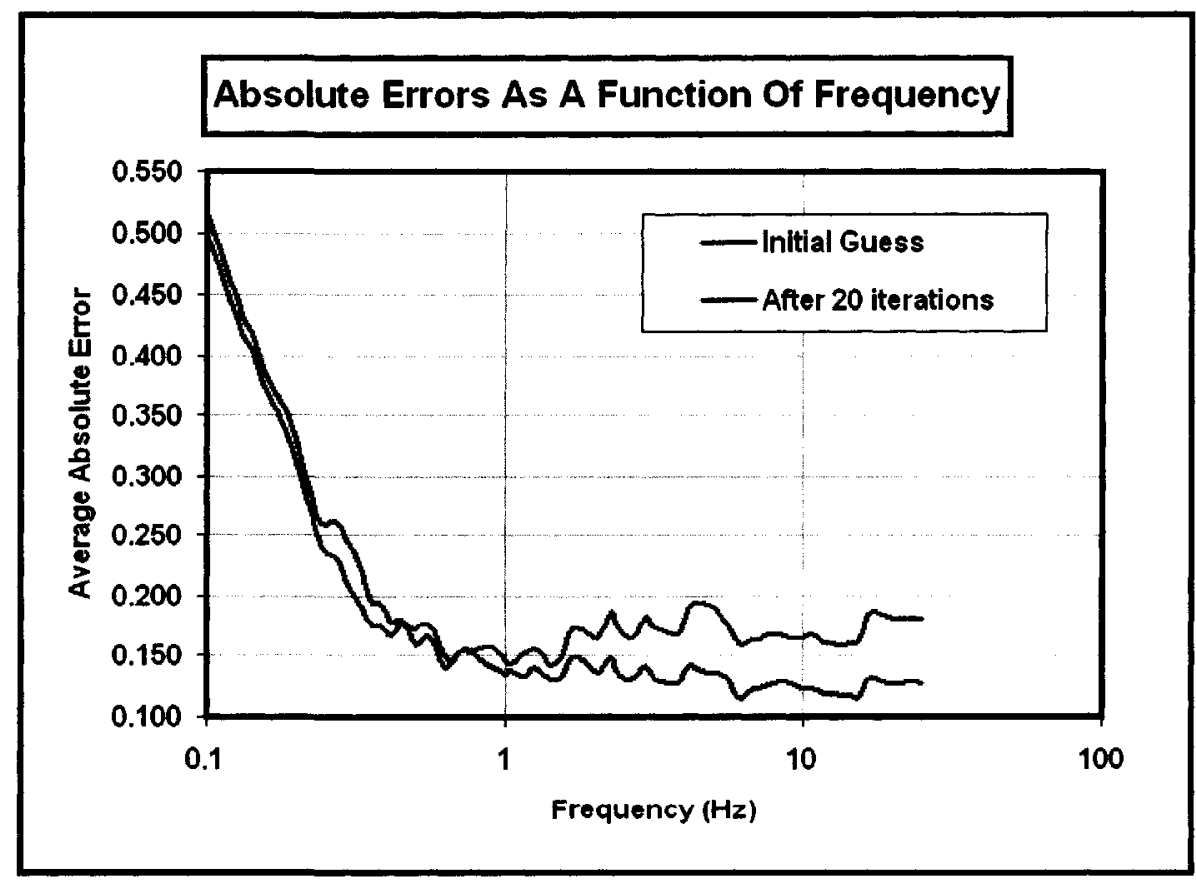

Figure (5.19). Average absolute error $\left(\Xi_{i}\right)$ as a function of frequency in Case 1 of Landers inversion. The results correspond to $\Xi_{\mathrm{i}}$ of initial guess and derived distributions. 
Error change from uniform distribution resulting from 20 iterations

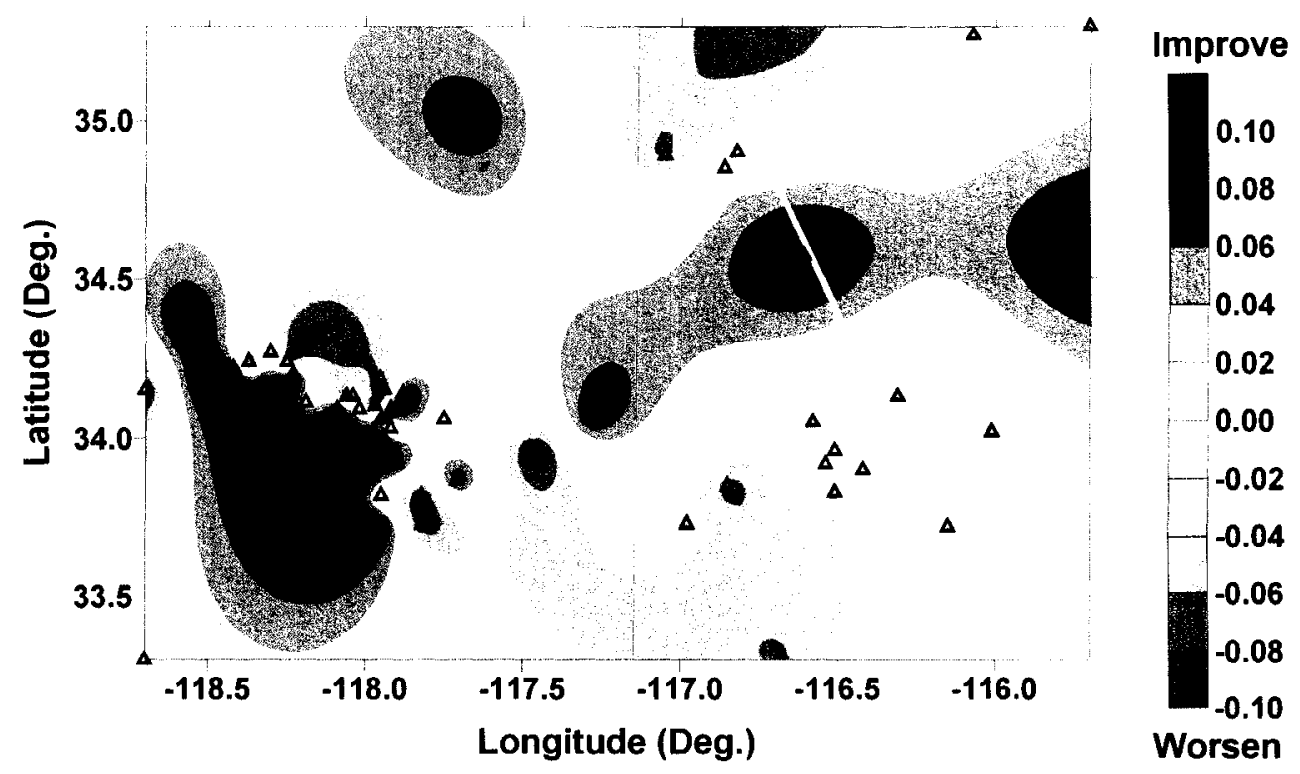

Figure (5.20). Spatial distribution of error improvement criterion $\Psi_{\mathrm{j}}$ from initial guess to final solution after 20 inversion iterations in Case 1 of Landers study. Crosses are location of stations and the yellow solid line is the location of the top of the fault.

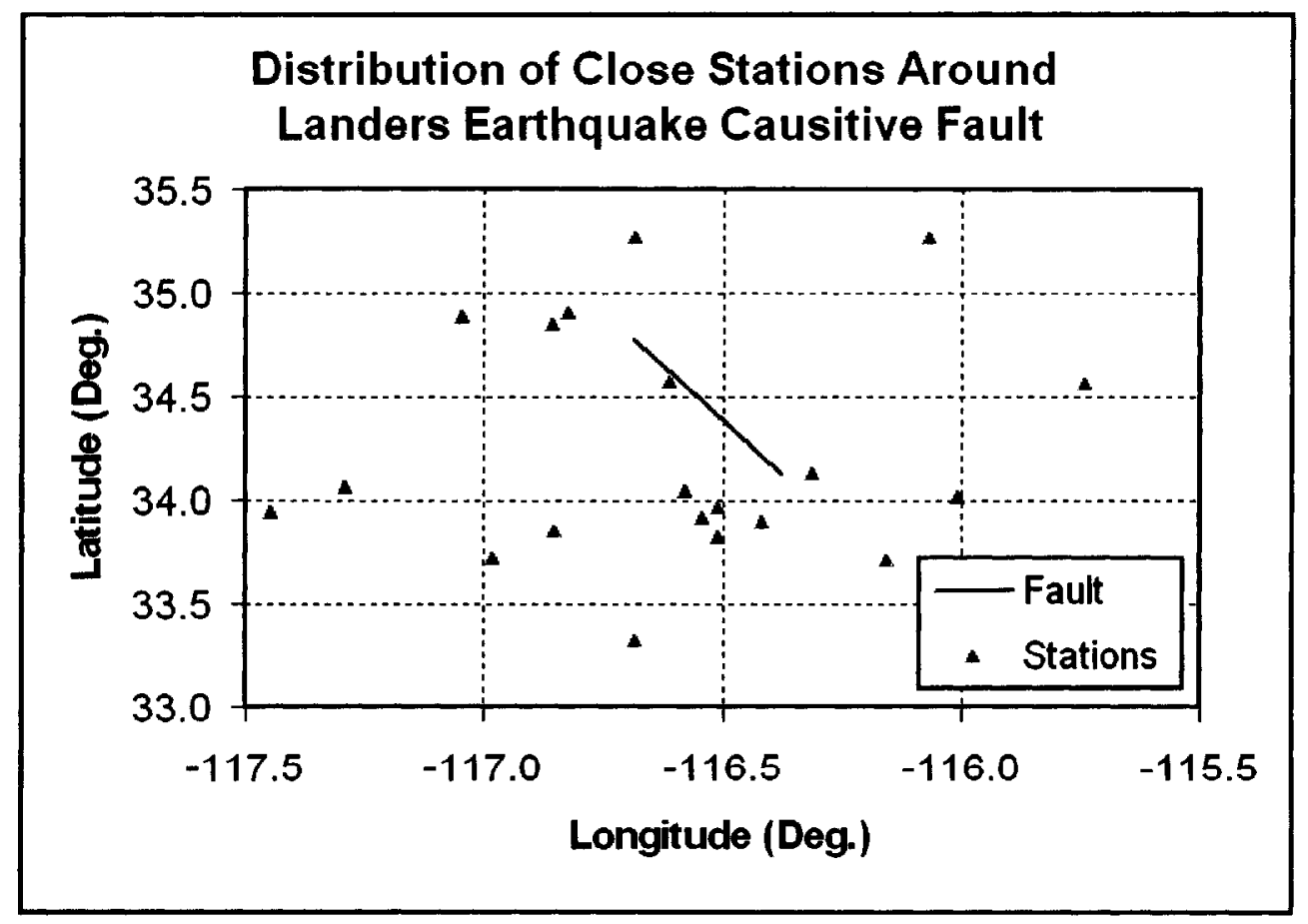

Figure (5.21) Distribution of stations used for Landers earthquake and the earthquake fault location in Cases 2 and 3. 


\subsubsection{Case 3}

Response spectra of the 20 near-fault stations of Case 2 are used, but the number of subsources in the Case 3 , is reduced to $13(13 \times 1)$. The number of data points is still not much more than the number of the sub-sources, so the solution may be poor, but is at least not under-determined.

The stress parameter distribution derived in Case 3 is shown in Figure (5.26). This solution is derived after 16 successful inversion iterations starting from a uniform distribution. The assigned average stress parameter of the initial guess was 50 bars, and it didn't evolve to another value.

The evolution of average absolute error $(\Xi)$ and average error $(\Phi)$ with successive inversion iteration number is shown in Figure (5.27). Figure (5.27) shows that only average error $(\Phi)$ decreased continuously in the process of inversion; the amount of decrease is only $\sim 4 \%$. Average absolute error $(\Xi)$ remains almost constant, but with a detailed view it is seen that $\Xi$ reduces in the first two iterations and then increases continuously with a very small trend. Overall, Figure (5.27) does not show any meaningful reduction of the error criteria with successive inversion iterations.

Figure (5.28) shows average absolute error as function of frequency $\left(\Xi_{\mathrm{i}}\right)$ for the initial guess and the solution after 16 iterations; there is no success of the method in reducing error level. This problem can be attributed to insufficient data. Figure (5.29) shows absolute average error reduction $\left(\Psi_{\mathrm{j}}\right)$ in map view.

The application of the inversion program for the M7.3, 1992 Landers earthquake in Case 3 resulted in a stress parameter distribution with main stress concentrations on the fault surface around the hypocenter and the center of the fault (Figure 5.26). Results of Figures (5.27) to (5.29) show that the program didn't perform well, and the errors $\Xi$ and $\Xi_{\mathrm{j}}$ didn't improve in the process of inversion. This problem could be related to the relatively small ratio of the number of the recording stations to the number of the sub-sources. Also the 
problem could be related to the relatively large distances of the stations from the fault. The experiments suggest we obtain reasonable solutions when the ratio of the number of the recording stations to the number of sub-sources is about two or more, even when some stations are clustered spatially.

\subsubsection{Landers earthquake slip model from other studies}

Several studies have derived a slip distribution for the Landers earthquake fault surface (Cohee and Beroza, 1994; Su et al., 1994; Cotton and Compillo, 1995; Hernandez et al., 1999; Wald and Heaton, 1994). Figure (5.30) shows the redrawn slip distribution solution of Cohee and Beroza (1994), derived from inversion of near-source displacement seismograms. The Cohee and Beroza (1994) solution found increasing amplitude of slip on the fault surface from the epicenter, progressing towards the center of the fault, and then a sudden decrease in the amplitude of slip from the center of the fault towards the northwest. Comparison of Figures (5.17) and (5.30) shows a reasonable degree of similarity between the Case 1 solution of this study (using all stations) and the Cohee and Beroza (1994) solution, suggesting their compatibility if the proportionality of slip and stress parameter on the fault surface is assumed.

Studies by different groups show that the Landers earthquake was the result of activation of a chain of faults (Cohee and Beroza, 1994; Freymueller et al., 1994; Hauksson et al., 1993; Wald and Heaton, 1994). The fault segments are the Landers/Johnson Valley faults, the Homestead Valley fault, and the Camp Rock/ Emerson fault, from southeast to northwest respectively. The hypocenter is located in the Landers/Johnson Valley faults segment and the largest slip occurred in the Homestead Valley fault segment. In this study the maximum stress parameter release coincides with the Homestead Valley fault segment. 


\subsubsection{M6.7, 1994 Northridge earthquake}

The geometry of the M6.7, 1994 Northridge earthquake is given in Table (5.1), as taken from Wald et al., (1996).

\subsubsection{Case 1}

Response spectral information of 159 stations is used for the derivation of the coseismic stress parameter distribution on the fault surface. The locations of the stations around the fault are shown in Figure (5.31) (PEER-NGA database). The number of sub-sources in Case 1 is $48(6 \times 8)$. The number of data points is more than twice the number of the subsources, so the problem is an overdetermined one. The distribution of stations close to the fault is not dense, although the stations provide some reasonable spatial coverage in all directions around the fault. We may thus expect to obtain an acceptable solution from the inversion program in this case.

The stress parameter distribution derived from our study is shown in Figure (5.32). This solution is derived after 4 successful inversion iterations, starting from a uniform distribution. There was no significant improvement with additional inversion iterations and the solution after the $4^{\text {th }}$ iteration is considered final. The assigned average stress parameter of the initial guess was 50 bars, which evolved to an average of 80 bars at the end.

The evolution of average absolute error $(\Xi)$ and average error $(\Phi)$ with successive inversion iteration number is shown in Figure (5.33). Figure (5.33) shows that both error measures decrease continuously and significantly with increasing iteration number, implying that on average response spectra of simulations at all stations get closer to the response spectra of records. 


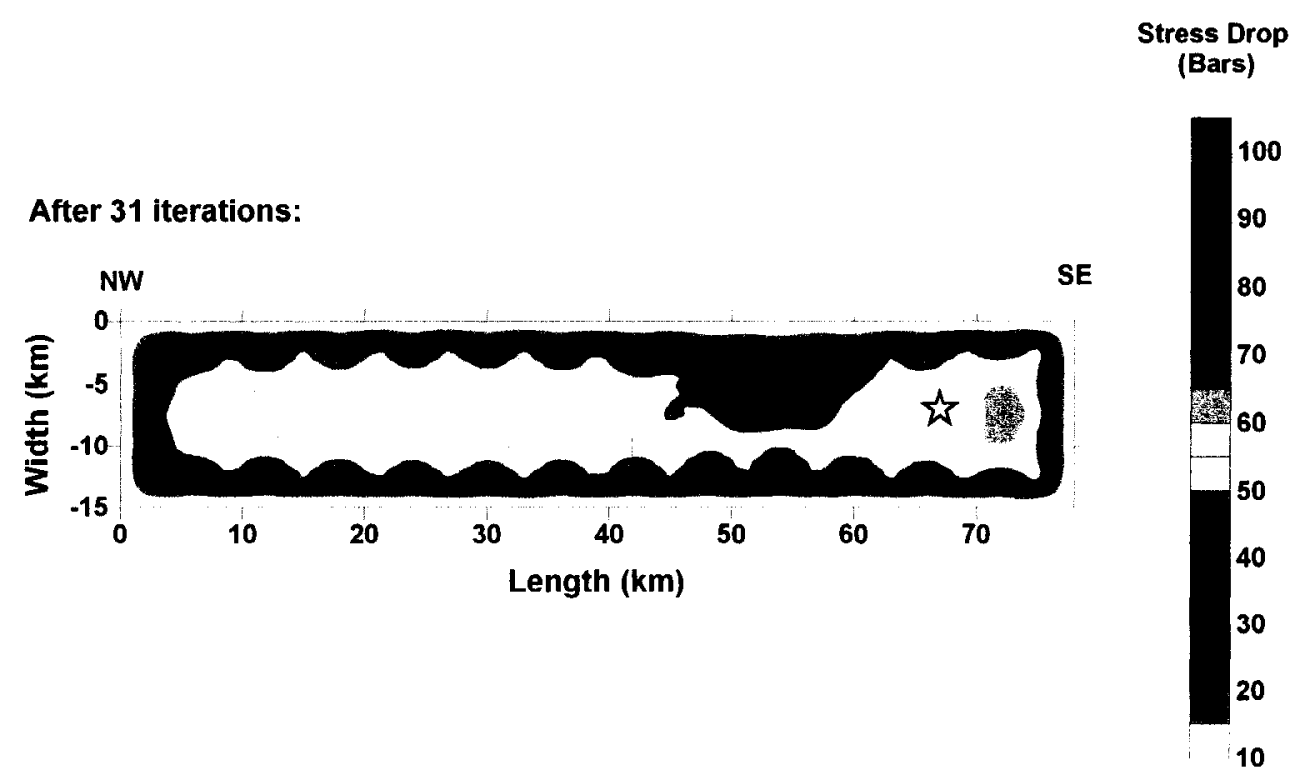

Figure (5.22) Derived stress parameter distribution on the Landers earthquake causative fault after $31^{\text {th }}$ iteration in Case 2. Star shows the location of hypocenter.

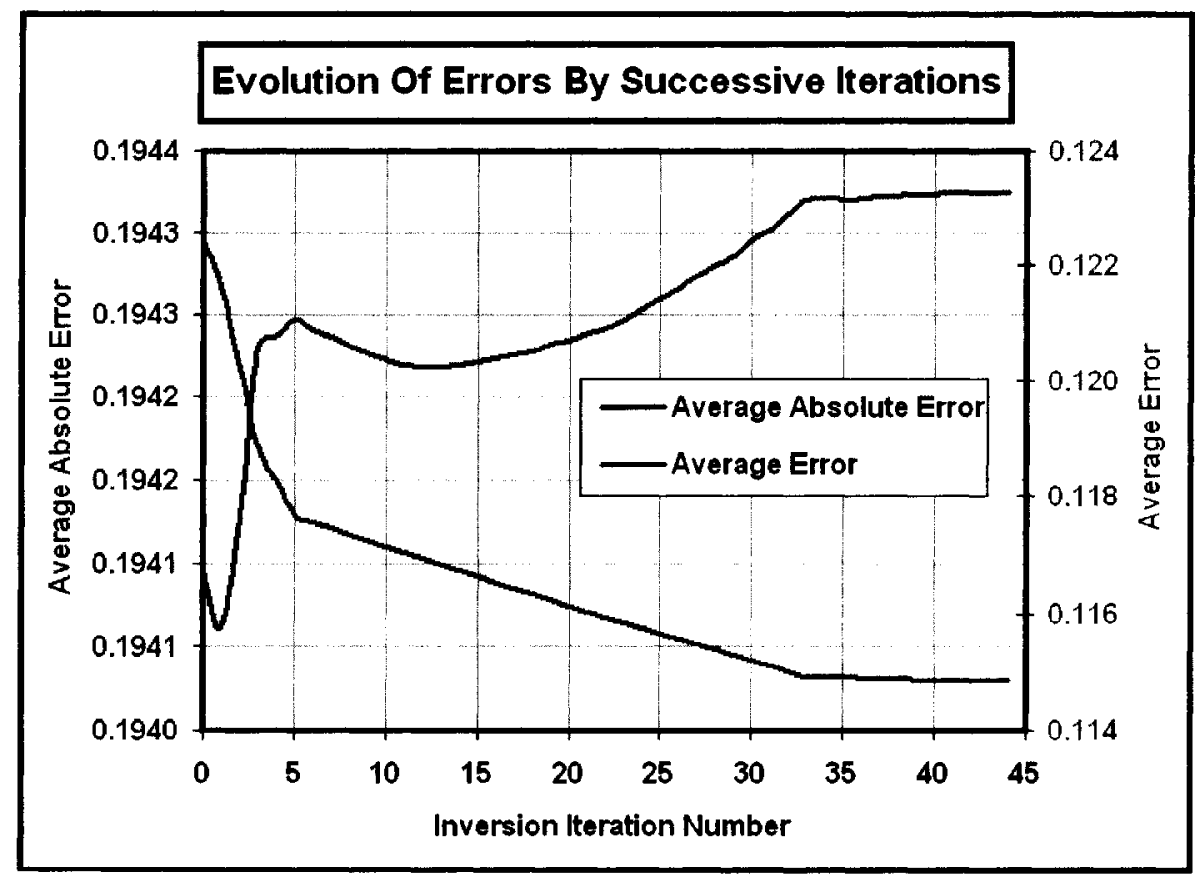

Figure (5.23) Evolution of average absolute error $(\Xi)$ and average error $(\Phi)$ with successive inversion iteration in Case 2 of Landers study. 


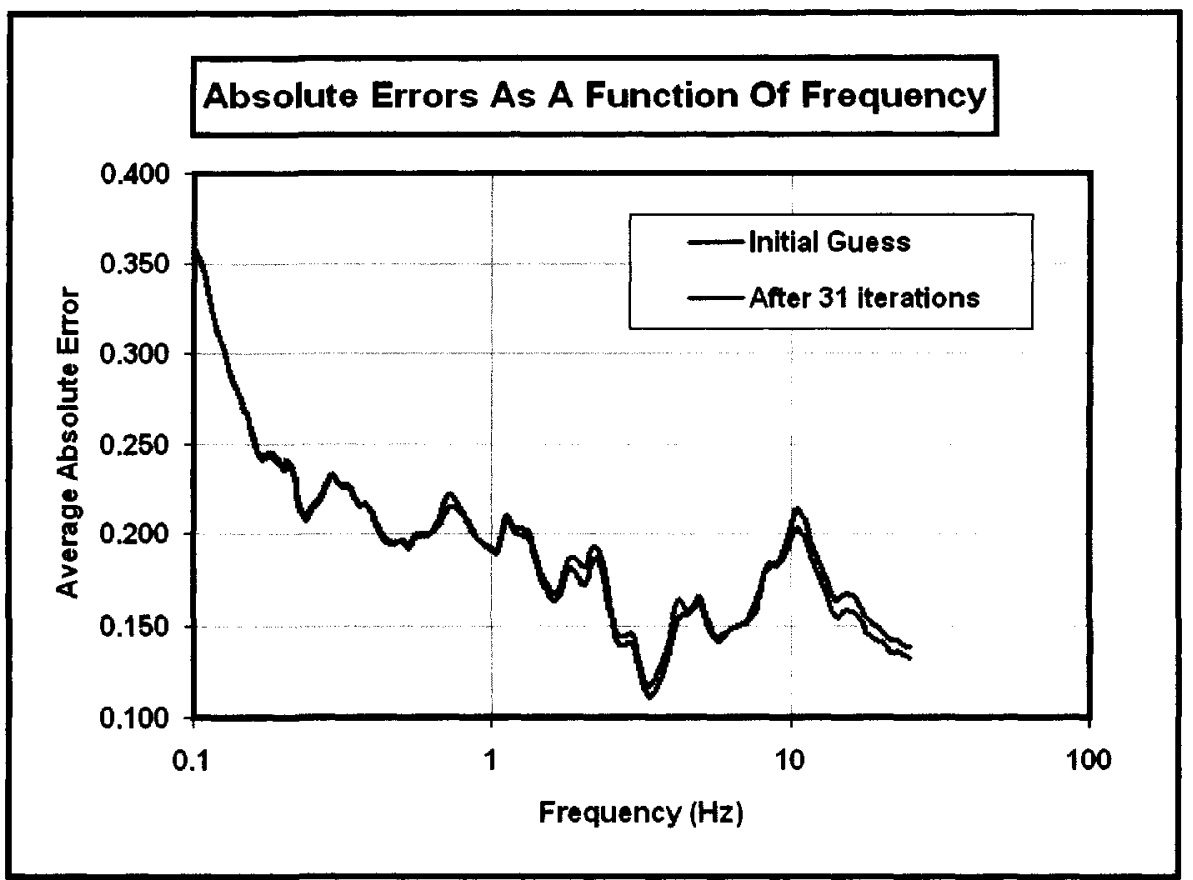

Figure (5.24). Average absolute error $\left(\Xi_{\mathrm{i}}\right)$ as a function of frequency in Case 2 of Landers inversion. The results correspond to $\Xi_{\mathrm{i}}$ of initial guess and derived distributions.

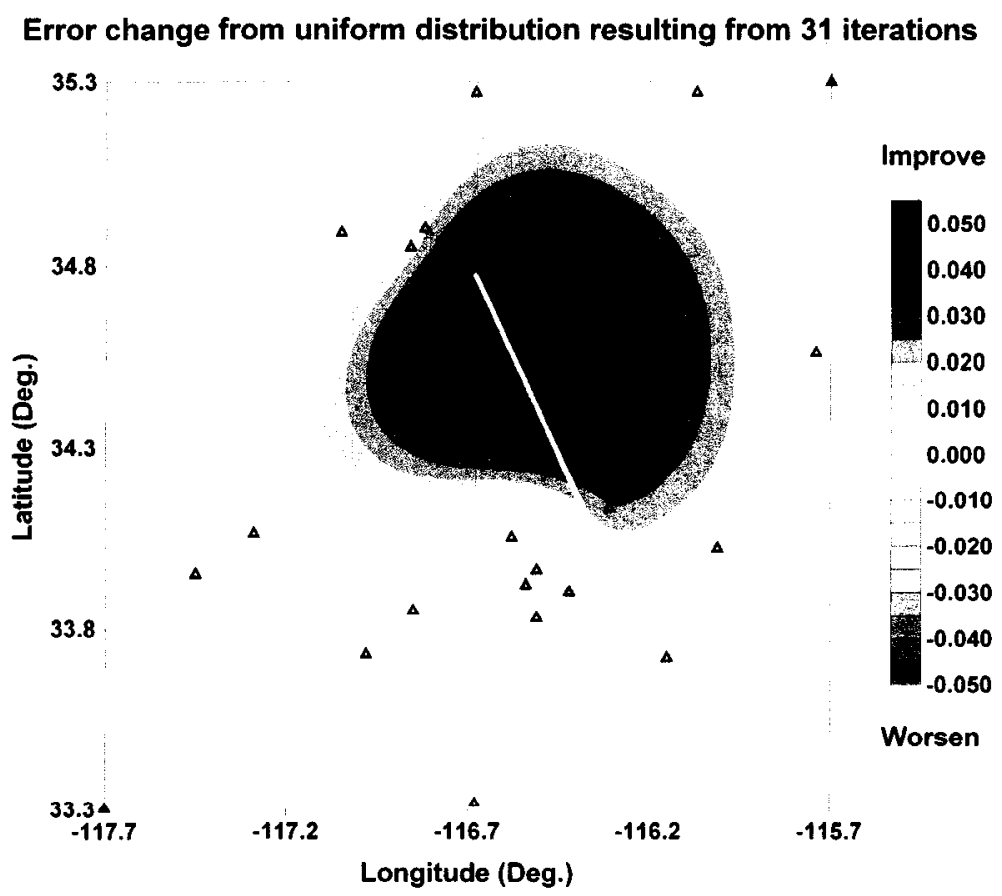

Figure (5.25). Spatial distribution of error improvement criterion $\Psi_{\mathrm{j}}$ from initial guess to final solution after 31 inversion iterations in Case 2 of Landers study. 


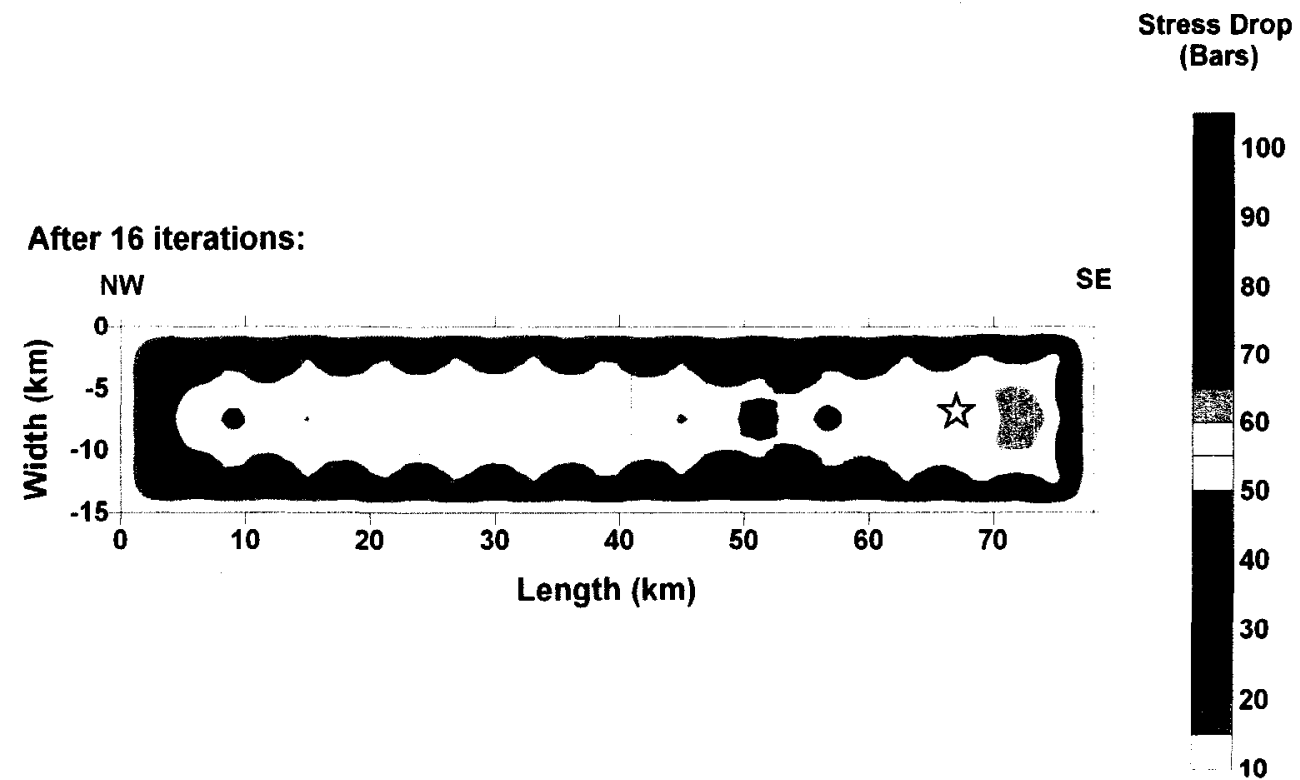

Figure (5.26) Derived stress parameter distribution on the Landers earthquake causative fault after $16^{\text {th }}$ iteration in Case 3 . Star shows the location of hypocenter.

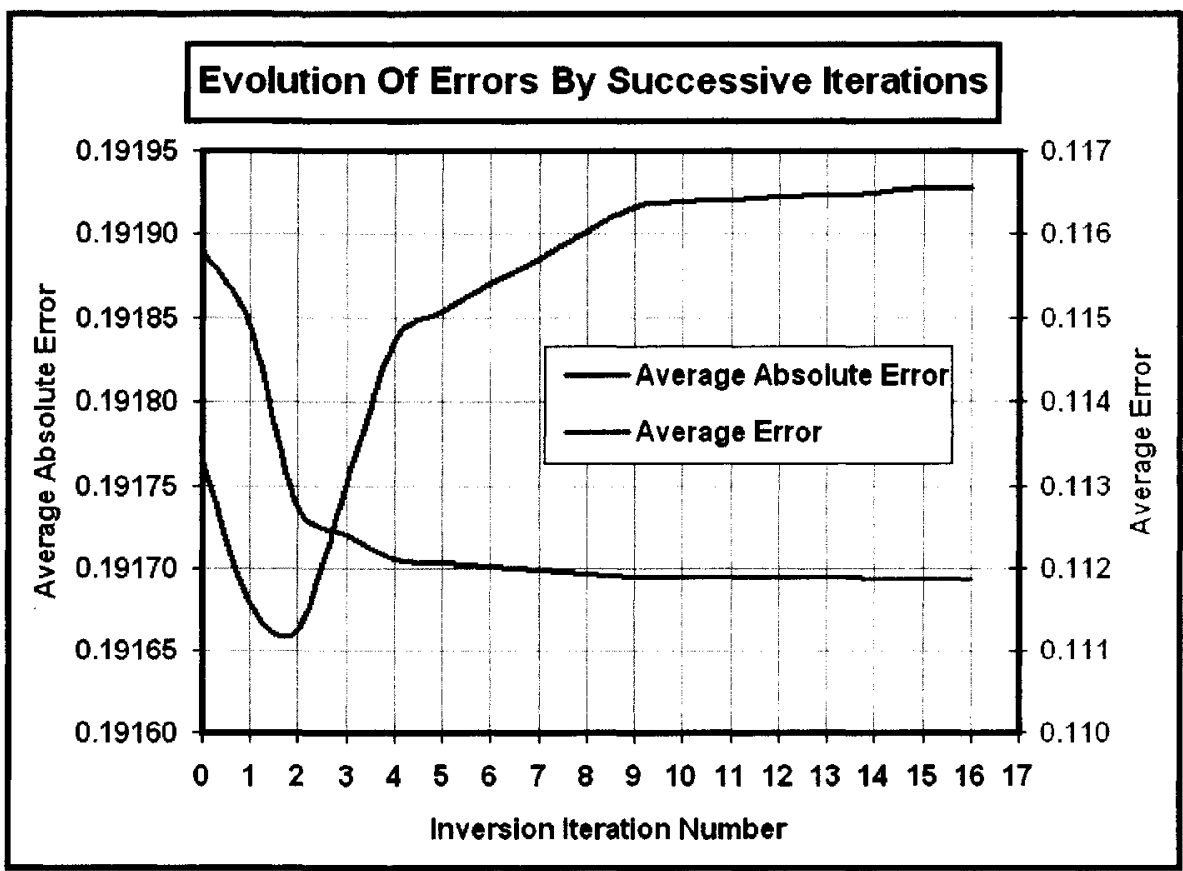

Figure (5.27) Evolution of average absolute error $(\Xi)$ and average error $(\Phi)$ with successive inversion iteration in Case 3 of Landers study. 


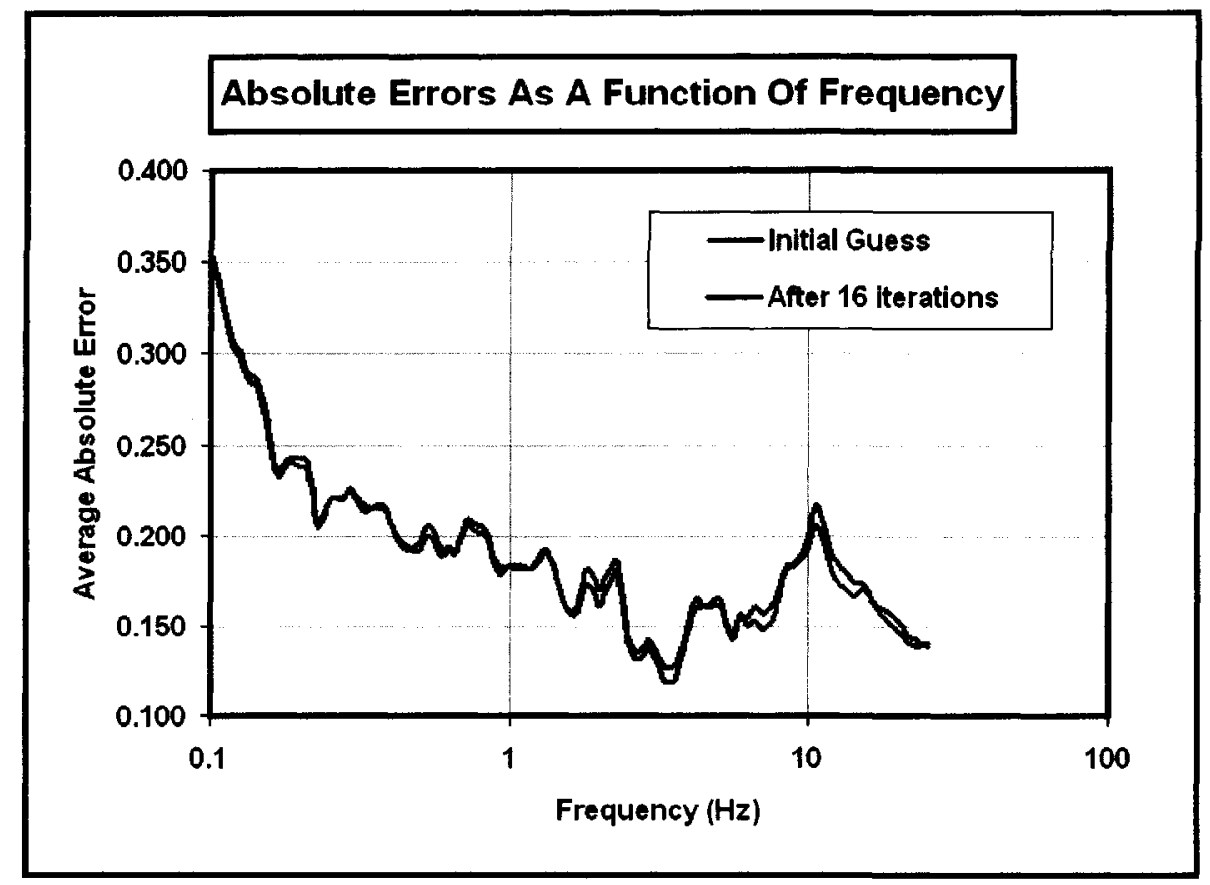

Figure (5.28). Average absolute error $\left(\Xi_{\mathrm{i}}\right)$ as a function of frequency in Case 3 of Landers inversion. The results correspond to $\Xi_{\mathrm{i}}$ of initial guess and derived distributions.

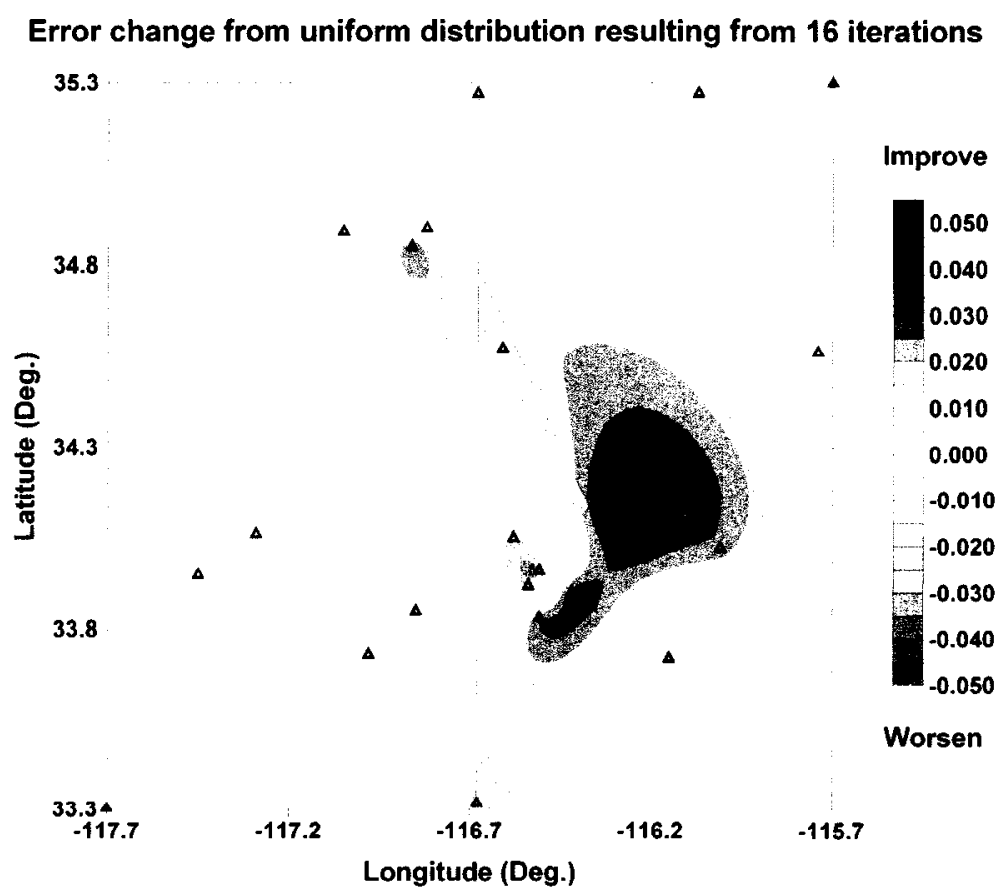

Figure (5.29). Spatial distribution of error improvement criterion $\Psi_{j}$ from initial guess to final solution after 16 inversion iterations in Case 3 of Landers study. 


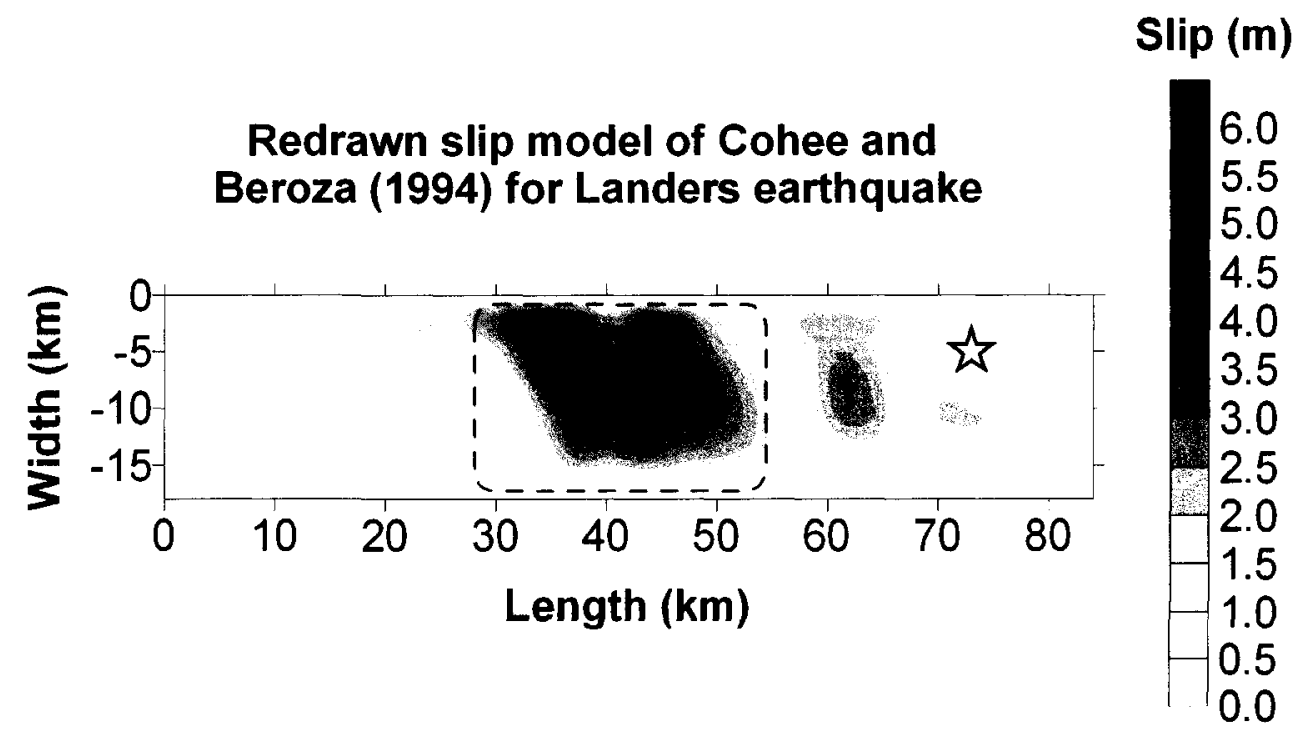

Figure (5.30). Slip distribution of Landers earthquake derived from inversion by Cohee and Beroza, (1994). Redrawn using data at http://www.seismo.ethz.ch/srcmod/.

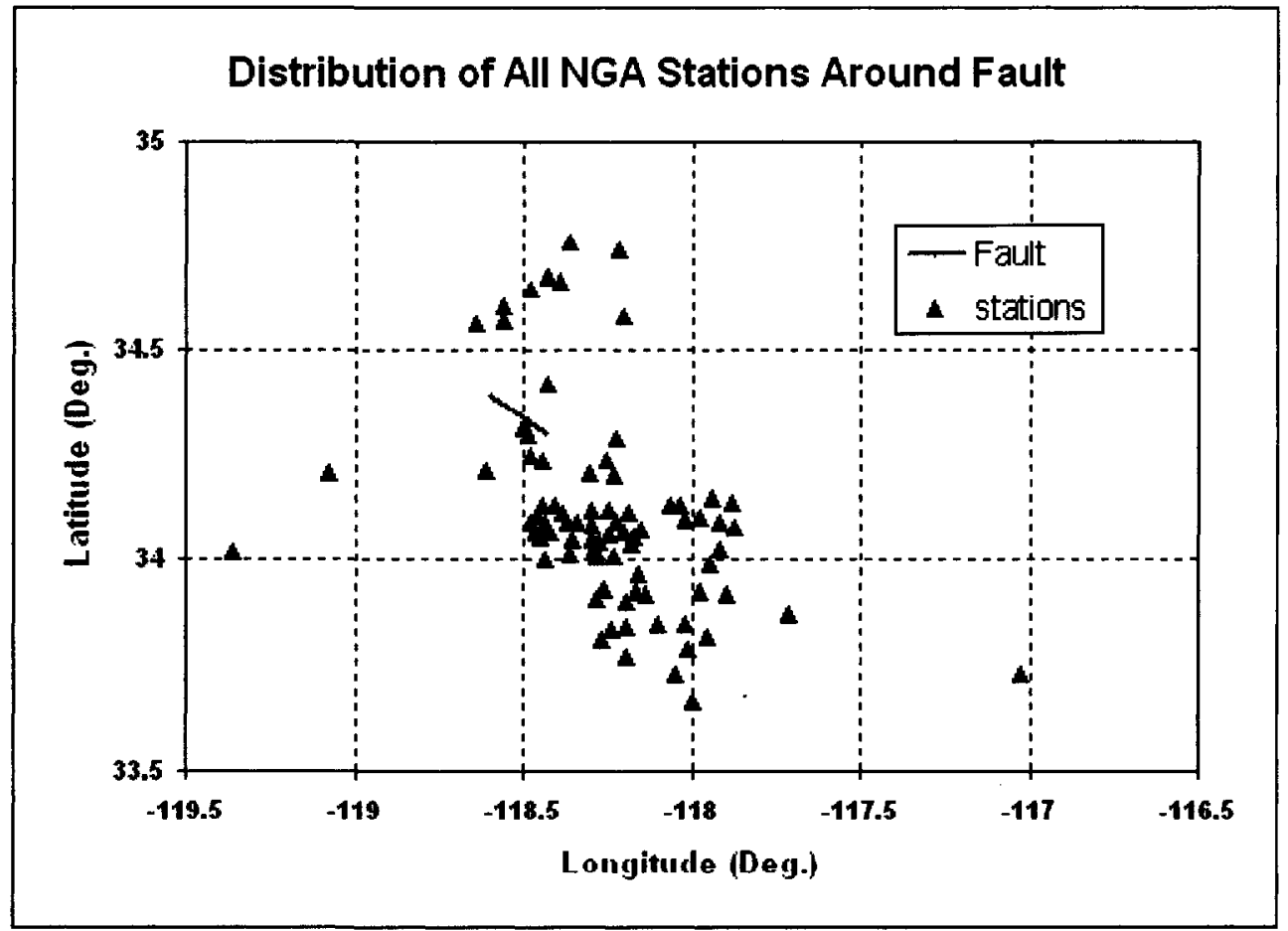

Figure (5.31) Distribution of stations used for Northridge earthquake and the earthquake fault location in Case 1. Only stations of NGA database are used for this study. 
Figure (5.34) shows average absolute error as a function of frequency $\left(\Xi_{\mathrm{i}}\right)$ for the initial guess and the final solution after the $4^{\text {th }}$ iteration. Figure (5.34) shows the success of the method in reducing error level $\Xi_{i}$ of simulations at high frequencies, while leaving the low frequency error level unchanged.

Figure (5.35) shows the map view of the absolute average error difference $\left(\Psi_{j}\right)$. The number of the stations that show reduction of error exceed those that show an increase of error.

The application of the inversion program for the M6.7, 1994 Northridge earthquake resulted in a solution with two major and two minor concentrations of stress or asperities, as shown in Figure (5.32). Figures (5.33) to (5.35) show that the inversion program is successful at reducing the overall errors at all stations.

\subsubsection{Case 2}

Response spectra of only 48 stations are used as shown in Figure (5.36). The nearby stations, which within $40 \mathrm{~km}$ of the fault, are used for this case, as shown in Figure (5.21). The number of sub-sources in Case 2 is $24(4 \times 6)$. The number of data points is still more than twice the number of the sub-faults, so the problem is again overdetermined. The distribution of stations close to the fault is not dense, although the stations provide reasonable spatial coverage in all directions around the fault. So it is expected to obtain an acceptable solution from the inversion program.

The stress parameter distribution derived from our study is shown in Figure (5.37). This solution is derived after 4 successful inversion iterations starting from a uniform distribution. There was no significant improvement with additional inversion iterations and the solution after the $4^{\text {th }}$ iteration is considered final. The assigned average stress parameter of initial guess was 50 bars and it evolved to an average of 80 bars at the end. There are two major areas of stress concentration. 
Error graphs are shown in Figures (5.38) to (5.40). Both average absolute error ( $\Xi$ ) and average error $(\Phi)$ iteratively decrease during inversion process (Figure 5.38) suggesting that with the given station distribution and the sub-division of the fault to sub-sources, the program performs successfully.

\subsubsection{Case 3}

In Case 3, I again use information from just 48 stations, (Figure (5.36)), but the number of sub-sources is reduced to $12(3 \times 4)$. This problem is thus overdetermined.

The derived stress parameter distribution for Case 3 is shown in Figure (5.41). This solution is derived after 7 successful inversion iterations starting from a uniform distribution. There was no significant improvement after the $4^{\text {th }}$ iteration, but the solution after the $7^{\text {th }}$ iteration is considered the final one. The assigned average stress parameter of the initial guess was 50 bars; it evolved to an average of 110 bars at the end. This is different from the 80 bars derived from the previous two cases; it may be associated with the large sub-source sizes assigned to fault subdivisions in this case. Figures (5.42) to (5.44) show error evolution for this case. 


\section{After 4 iterations:}

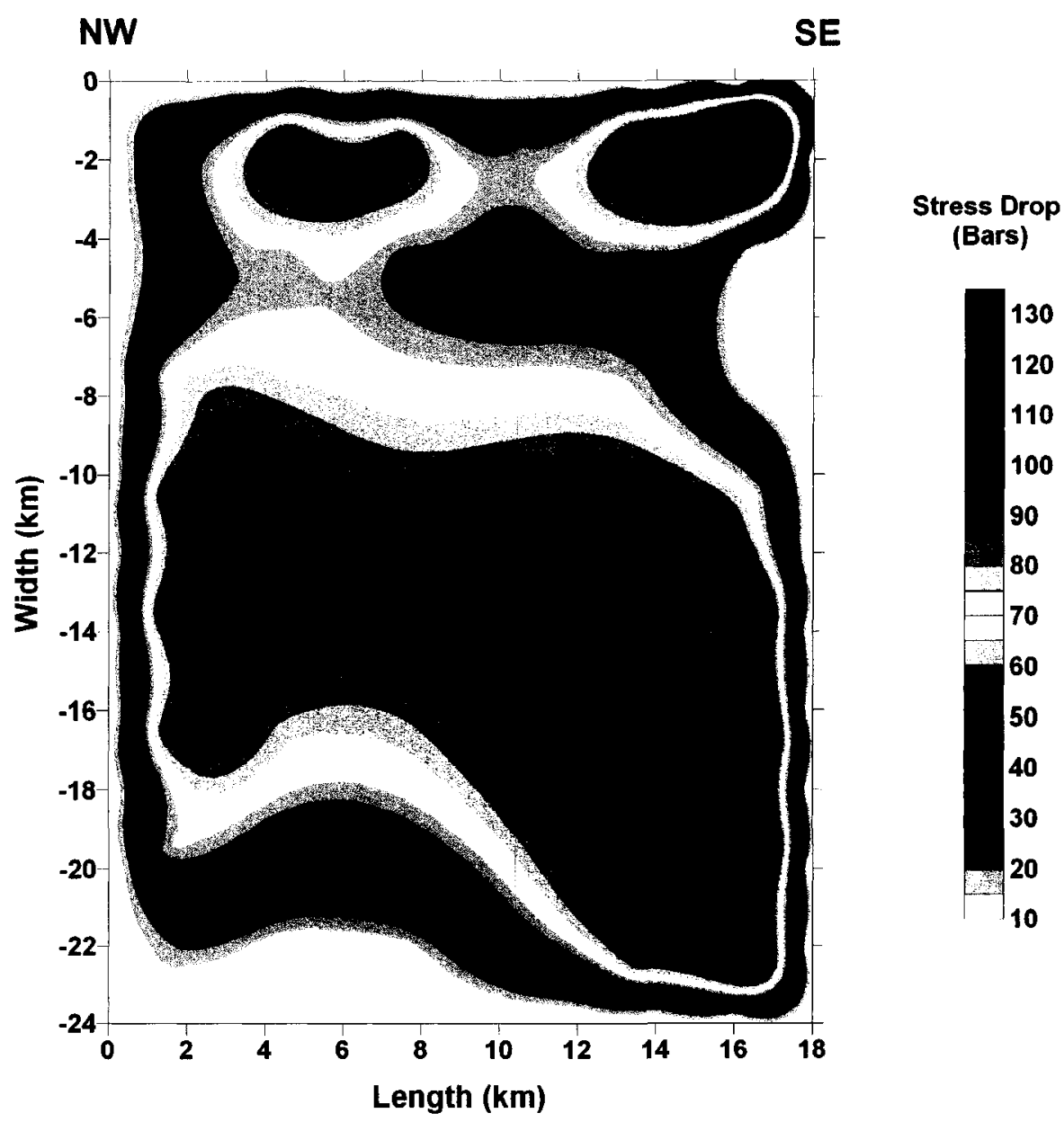

Figure (5.32) Derived stress parameter distribution on the Northridge earthquake causative fault after $4^{\text {th }}$ iteration in Case 1 . Star shows the location of hypocenter. 


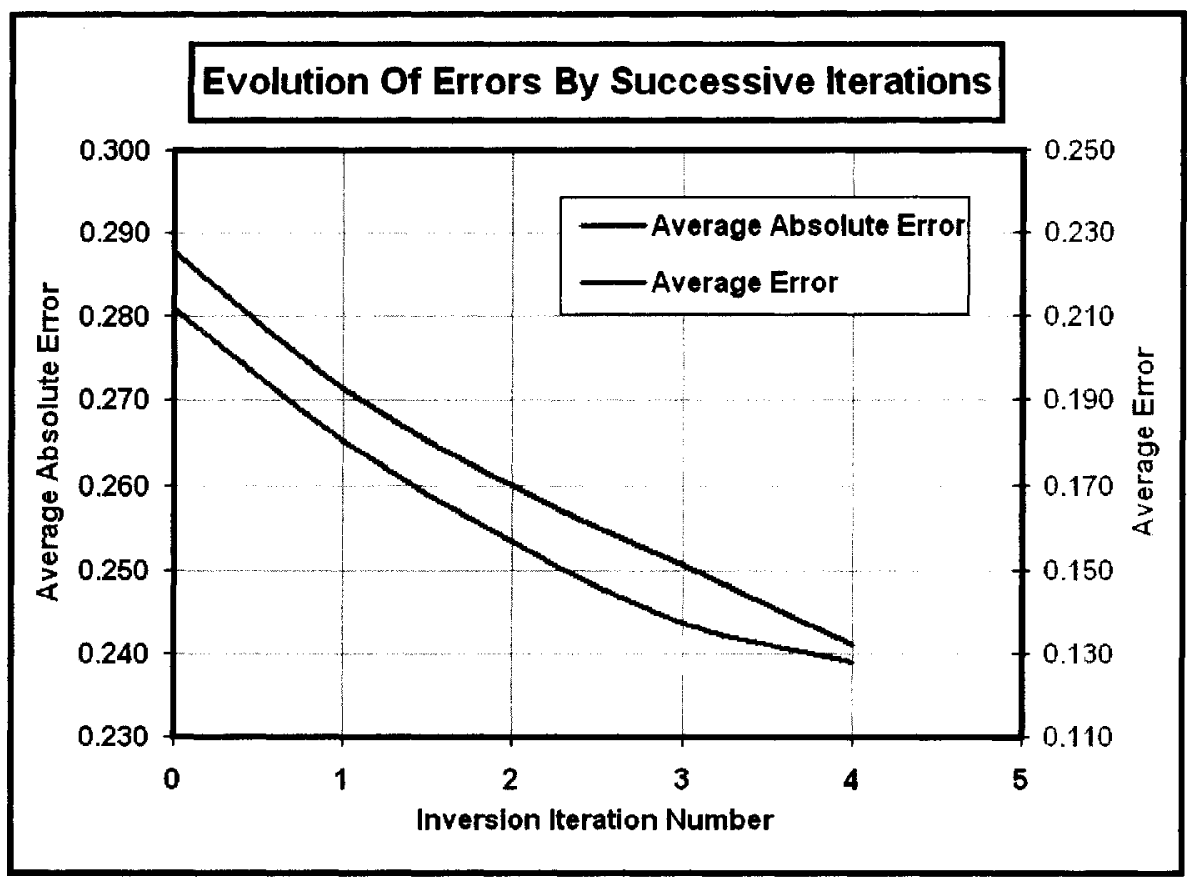

Figure (5.33) Evolution of average absolute error $(\Xi)$ and average error $(\Phi)$ with successive inversion iteration in Case 1 of Northridge study.

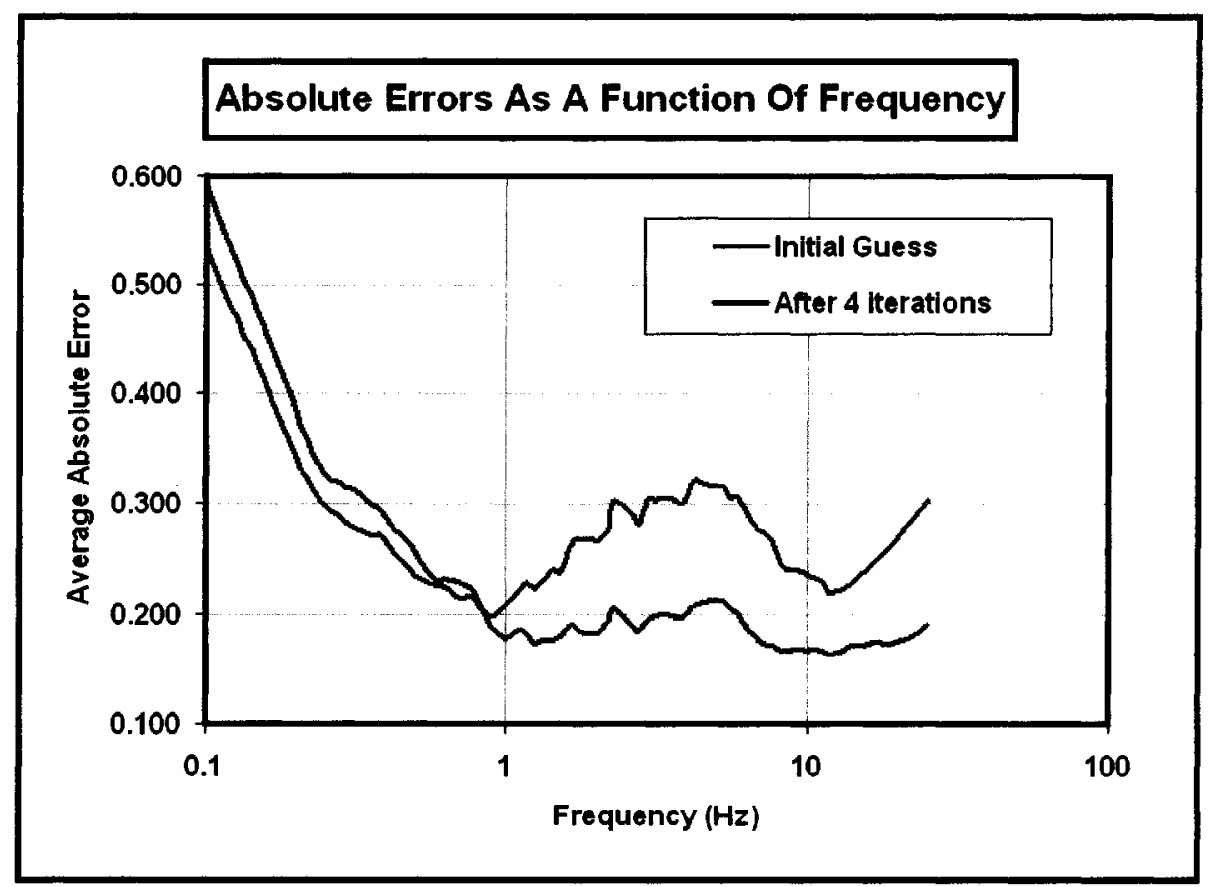

Figure (5.34). Average absolute error $\left(\Xi_{i}\right)$ as a function of frequency in Case 1 of Northridge inversion. The results correspond to $\Xi_{i}$ of initial guess and derived distributions. 


\section{Error change from uniform distribution resulting from 4 iterations}

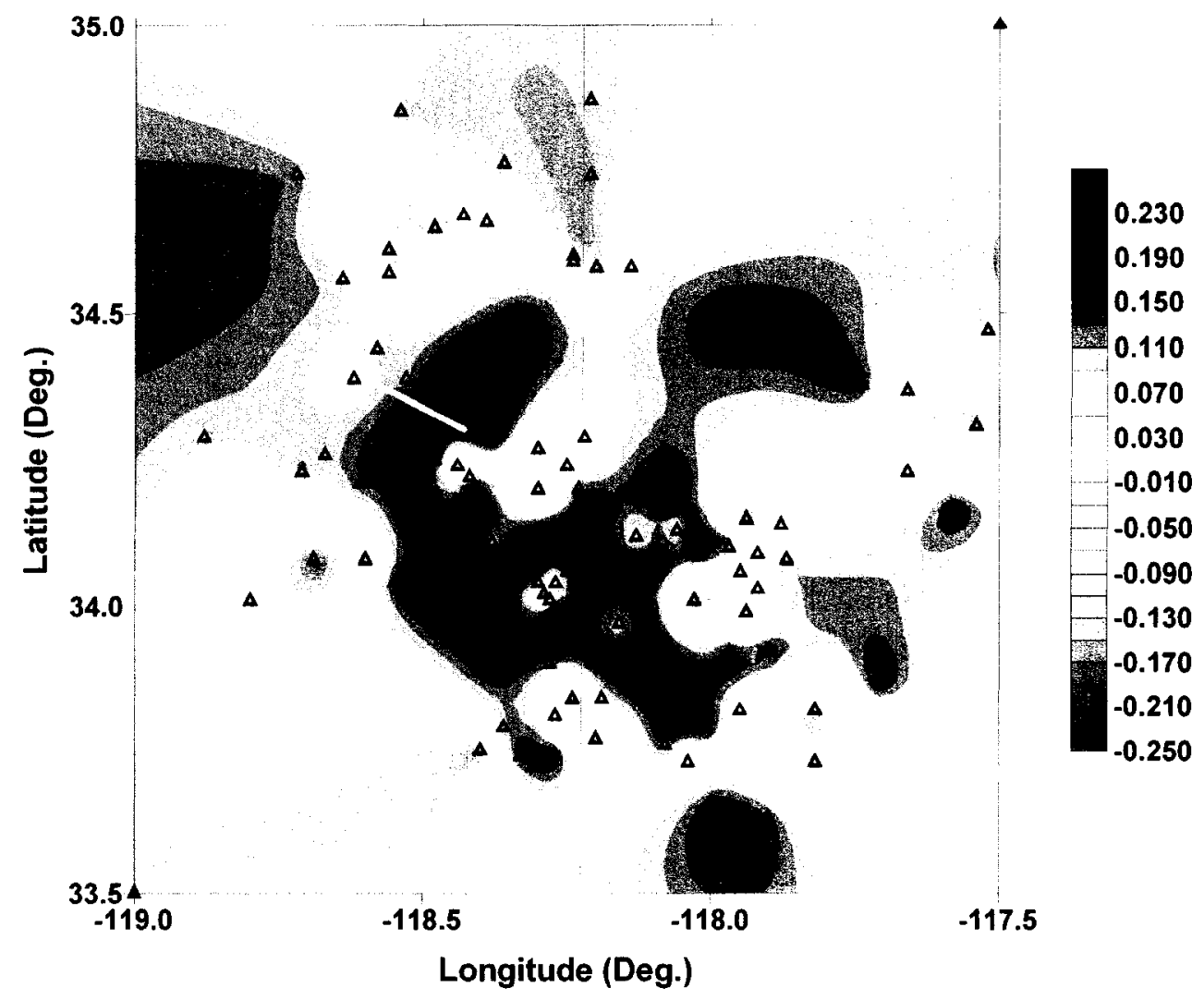

Figure (5.35). Spatial distribution of error improvement criterion $\Psi_{\mathrm{j}}$ from initial guess to final solution after 4 inversion iterations in Case 1 of Northridge study. Crosses are location of stations and the yellow solid line is the location of the top of the fault. 


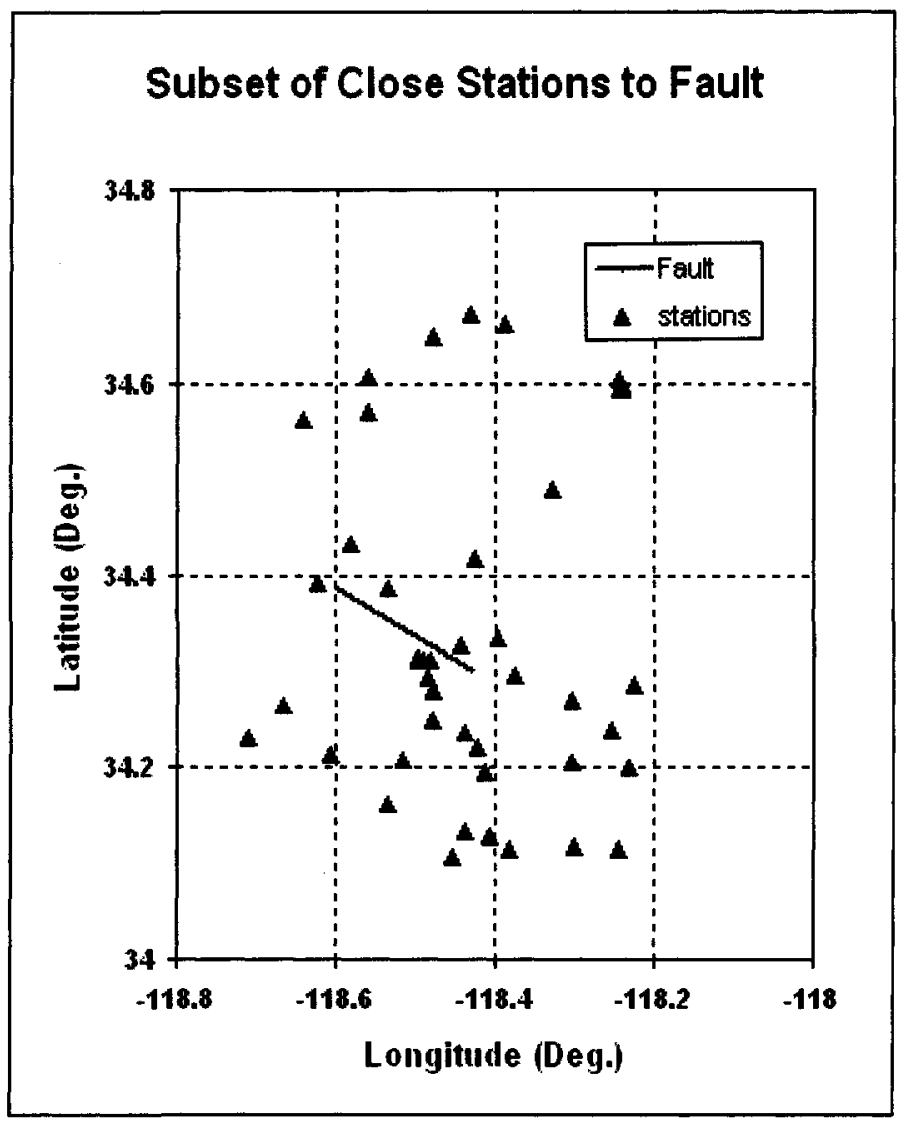

Figure (5.36) Distribution of stations used for Northridge earthquake and the earthquake fault location in Cases 2 and 3. 


\section{After 4 iterations:}

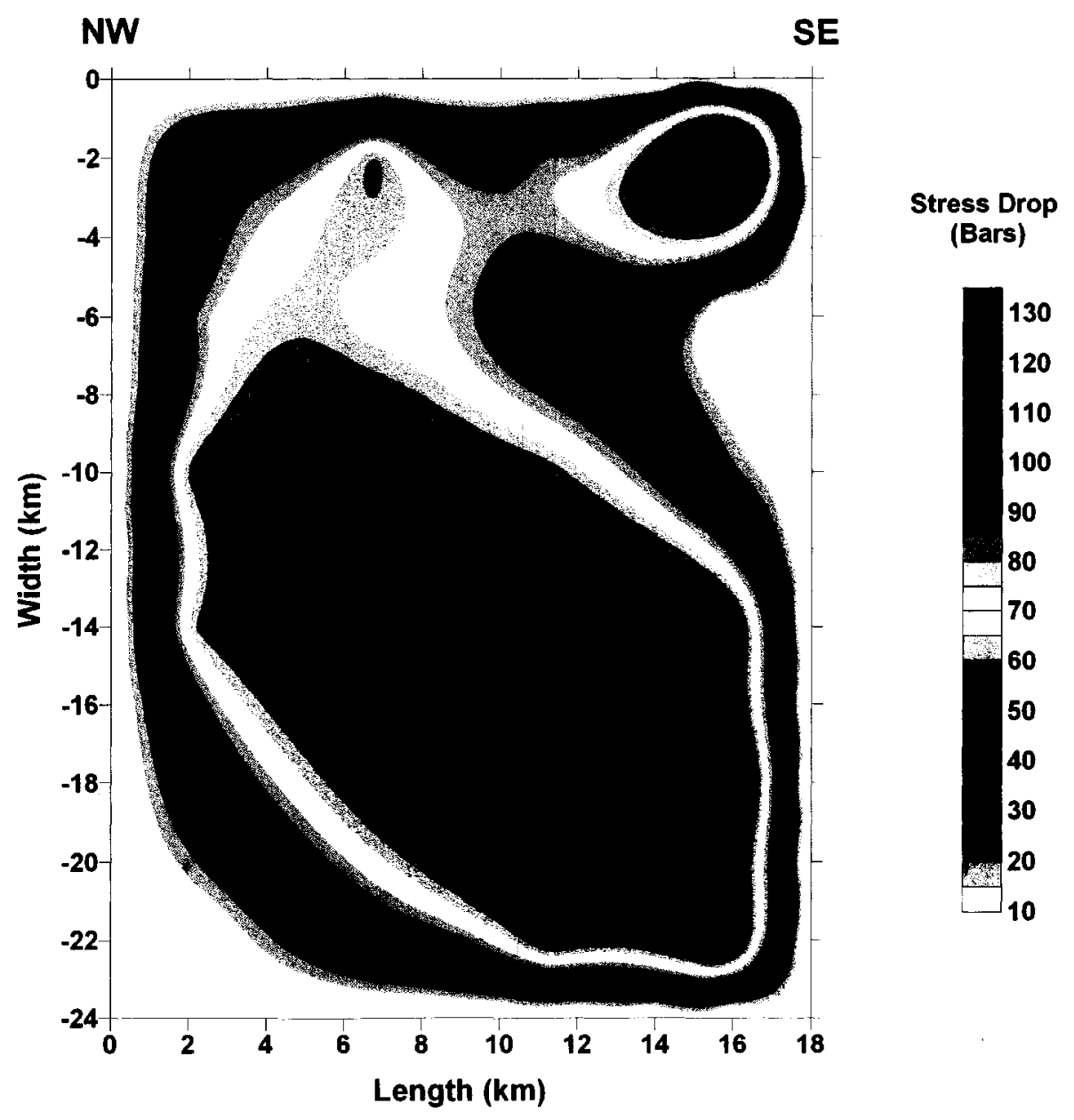

Figure (5.37) Derived stress parameter distribution of the Northridge earthquake causative fault after the $4^{\text {th }}$ iteration in Case 2 . Star shows the location of hypocenter. 


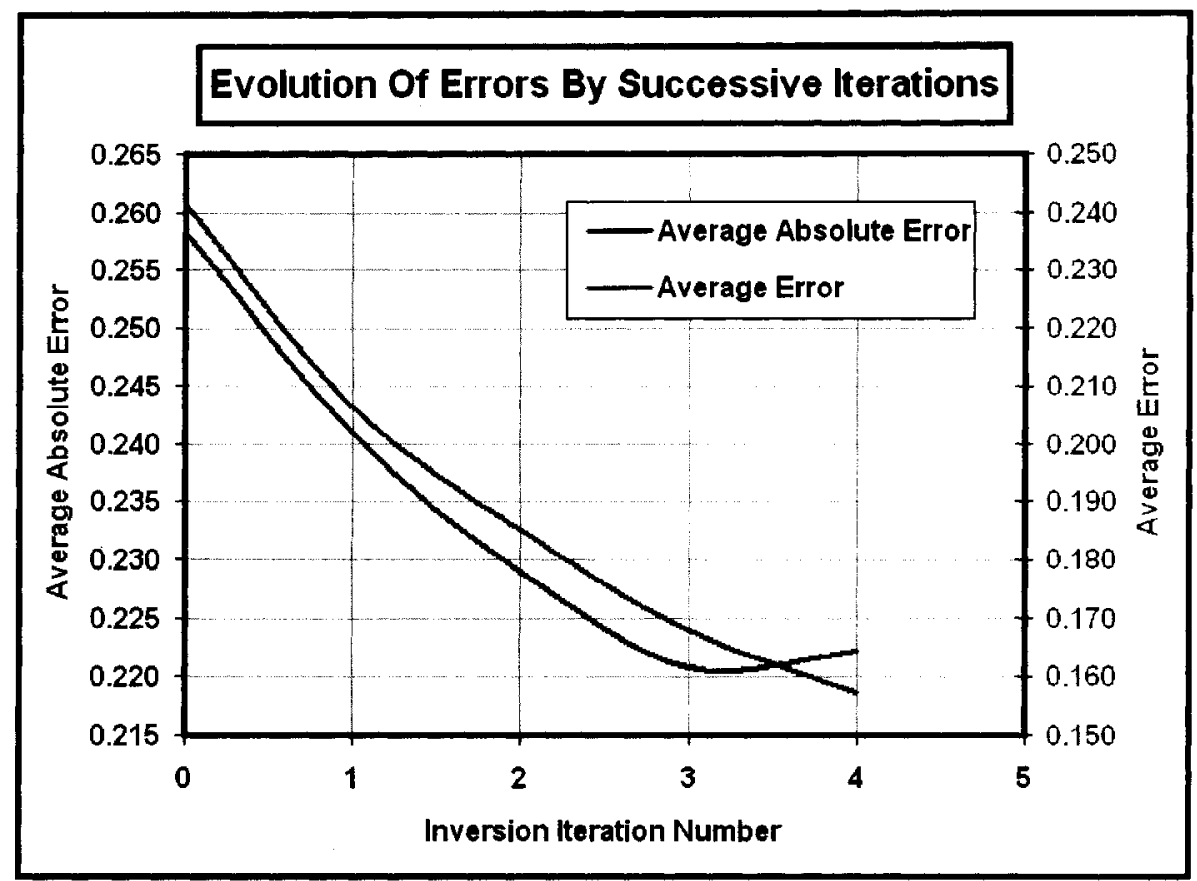

Figure (5.38) Evolution of average absolute error $(\Xi)$ and average error $(\Phi)$ with successive inversion iteration in Case 2 of the Northridge study.

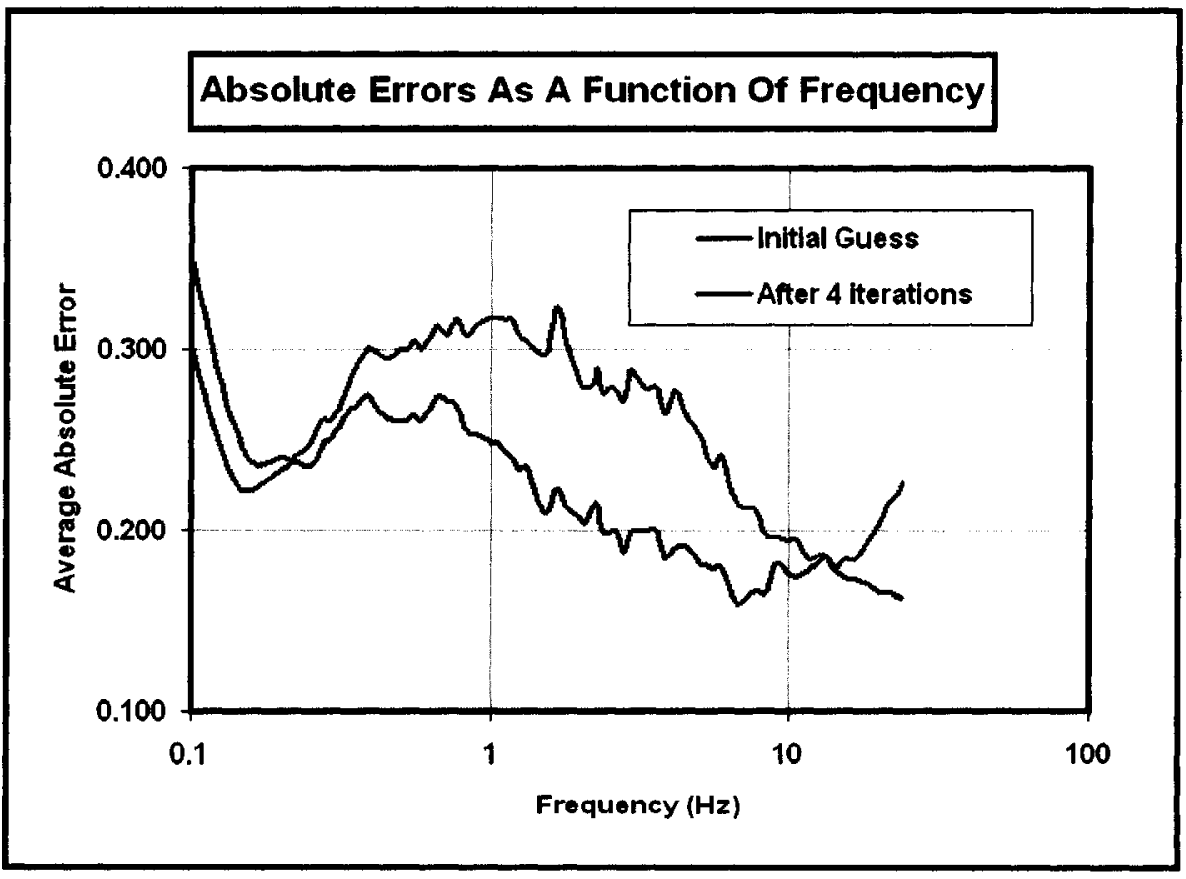

Figure (5.39). Average absolute error $\left(\Xi_{\mathrm{i}}\right)$ as a function of frequency in Case 2 of the Northridge inversion. The results correspond to $\Xi_{\mathrm{i}}$ of initial guess and derived distributions. 
Error change from uniform distribution resulting from 4 iterations

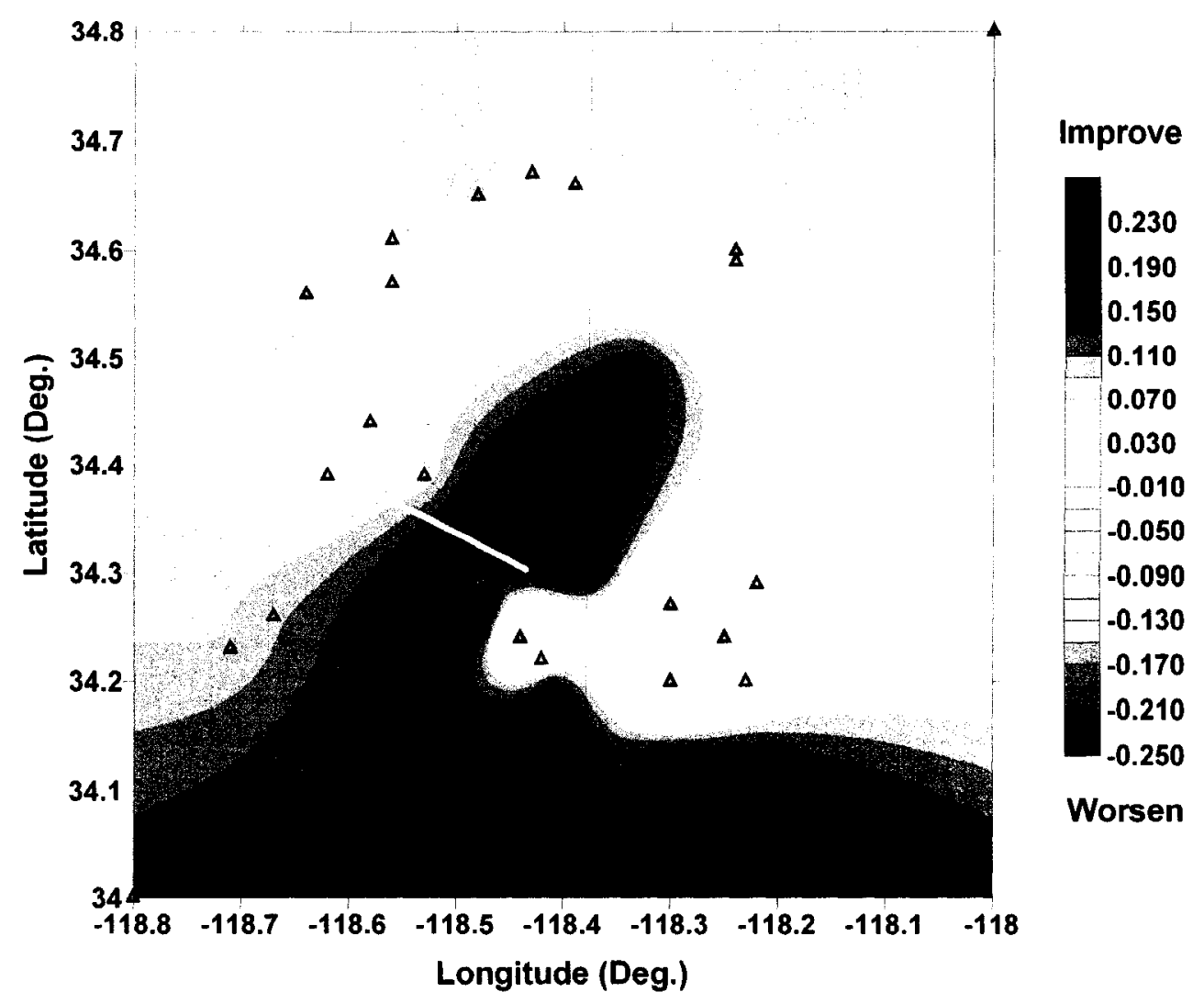

Figure (5.40). Spatial distribution of error improvement criterion $\Psi_{\mathrm{j}}$ from initial guess to final solution after 4 inversion iterations in Case 2 of Northridge study. Crosses are location of stations and the yellow solid line is the location of the top of the fault. 
The application of the inversion program for M6.7, 1994 Northridge earthquake resulted in a solution with two major areas of concentrations of stress or asperities as shown in Figure (5.41). Both average absolute error $(\Xi)$ and average error $(\Phi)$ iteratively decrease during the inversion process (Figure 5.42) suggesting that with the given station distribution and the sub-division of the fault to sub-sources, the program performs successfully. Figure (5.43) compares average errors of all stations $\left(\Xi_{\mathrm{i}}\right)$ in each frequency between application of the uniform and the modified stress parameter distributions after 7 iterations, showing that on average the error $\Xi_{i}$ reduced considerably when switching from the uniform to the derived stress parameter distributions. The main problem with the solution in case 3 is its coarse sub-source size, that results in lower resolution of the final result as well as affecting the calculated optimum average stress parameter; which is about 30 bars larger than the results of previous two cases. Case 1 is the best scenario as it uses all of the available information.

\subsubsection{Northridge earthquake slip model from other studies}

Slip distribution studies for the Northridge earthquake have been conducted by several investigators (Dreger, 1994; Hartzell, et al., 1996; Hudnut et al., 1996; Shen et al., 1996; Wald et al., 1996; and Zeng and Anderson, 1996) and the overall picture of all these studies are very similar. In the comparisons of this part, the Wald et al., (1996) solutions are picked because they provided solutions based on inversion of strong-motion data as well as combined inversion of strong-motion, teleseismic, and geodetic data. Figure (5.45A) shows the redrawn solution of Wald et al., (1996), as inverted from a combination of strong motion, teleseismic, and geodetic data. The overall distribution of slip amplitude on the fault surface derived by Wald et al., (1996) has considerable similarities with the stress parameter distribution of our study (compare Figures 5.32 and 5.45). An alternative Wald et al., (1996) solution derived only from the strong motion data, redrawn in Figure (5.45B), has even more similarities with our results, as might be 
expected based on the commonality of data. This is again in accordance with the assumption of general proportionality of stress and slip on the fault surface.

\subsection{Conclusions}

Case studies in this chapter show that the inversion tool introduced in this thesis is capable of deriving stress parameter distribution on an earthquake fault surface from $P S A$ data of records of the earthquake. Experiments show that the program performs well if: (i) the number of stations recording earthquake ground motion is sufficiently high compared to the number of sub-sources comprising the modeled fault (something in the range of double the number of sub-sources, or more); (ii) the stations provide a reasonable spatial coverage around the earthquake causative fault without too much clustering; and (iii) there are sufficient stations at close distances to the earthquake causative fault. Experiments of this chapter show that the program generates an answer even if these conditions are not met, but poor compliance with these conditions will result in a poor but stable solution.

In all three earthquakes studied here, the derived stress parameter distribution shows considerable similarity to slip distributions derived by other research groups for the same earthquakes. The similarity of the stress and slip distributions is attributed to proportionality of shear stress and strain, as noted by Wald et al., (1991). The similarity of distributions noted here suggests that the method applied in this study is a viable tool for derivation of the stress parameter distribution. It can thus be applied to other earthquakes to prepare a database of stress parameter distributions for past earthquakes. By understanding the stress parameter distributions of past events, we can ultimately improve predictions of the expected ground motions and their variability for future earthquakes. As the stochastic model is a widely-used tool for such ground-motion predictions, it forms a convenient basis to characterize our understanding of the salient parameters that control strong ground motion. 


\section{After 7 iterations:}

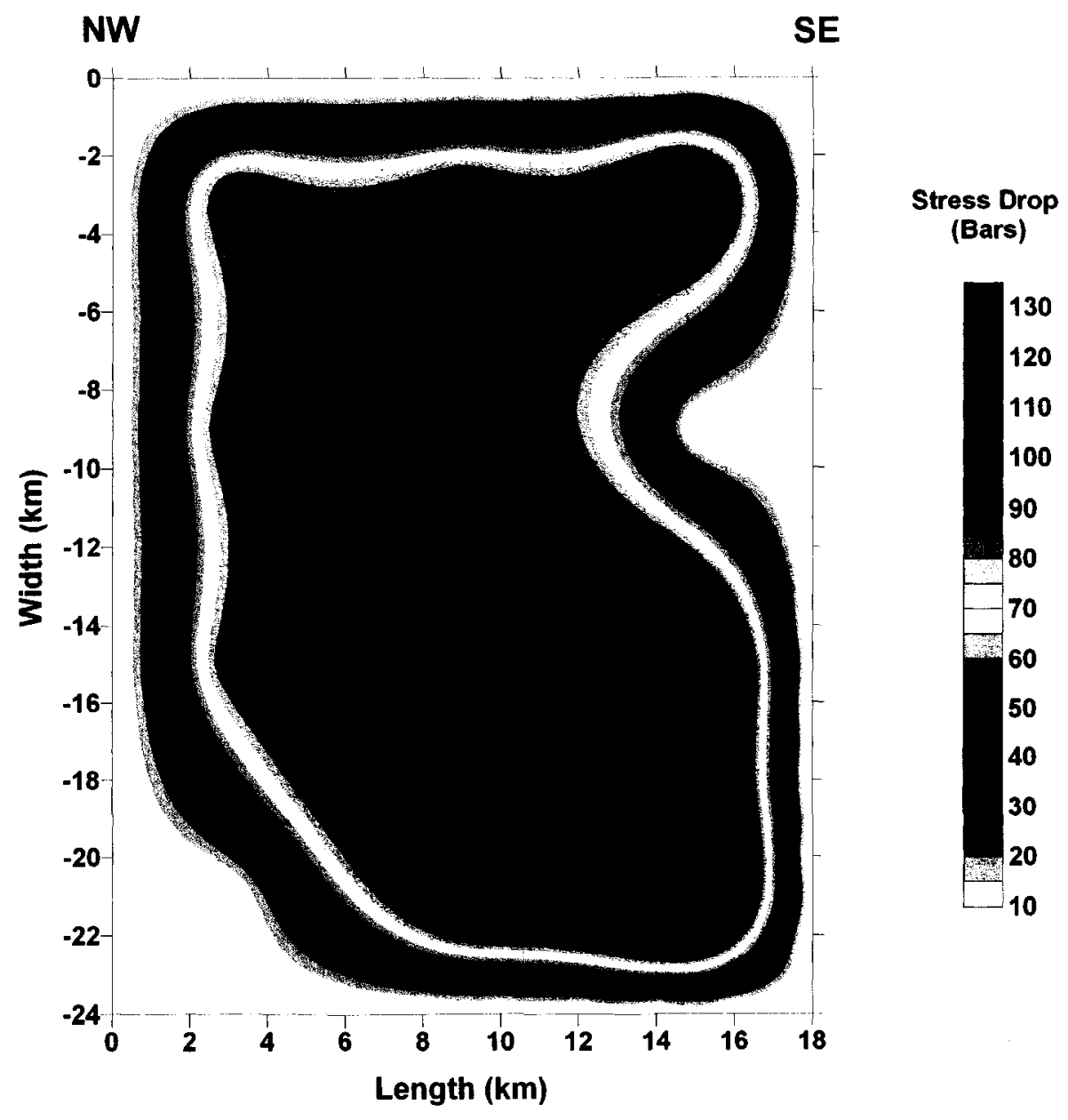

Figure (5.41) Derived stress parameter distribution of the Northridge earthquake causative fault after the $7^{\text {th }}$ iteration in case 3 . Star shows the location of hypocenter. 


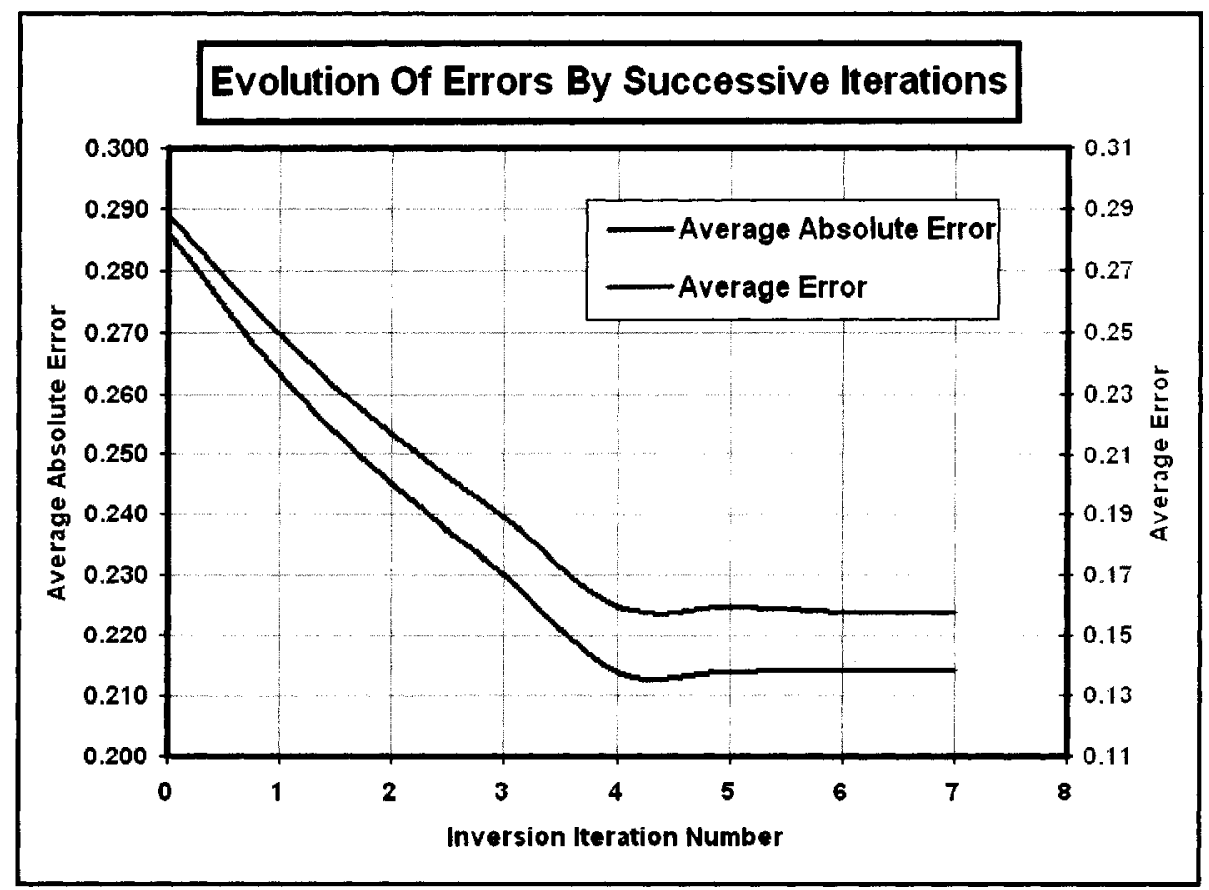

Figure (5.42) Evolution of average absolute error $(\Xi)$ and average error $(\Phi)$ with successive inversion iteration in case 3 of the Northridge study.

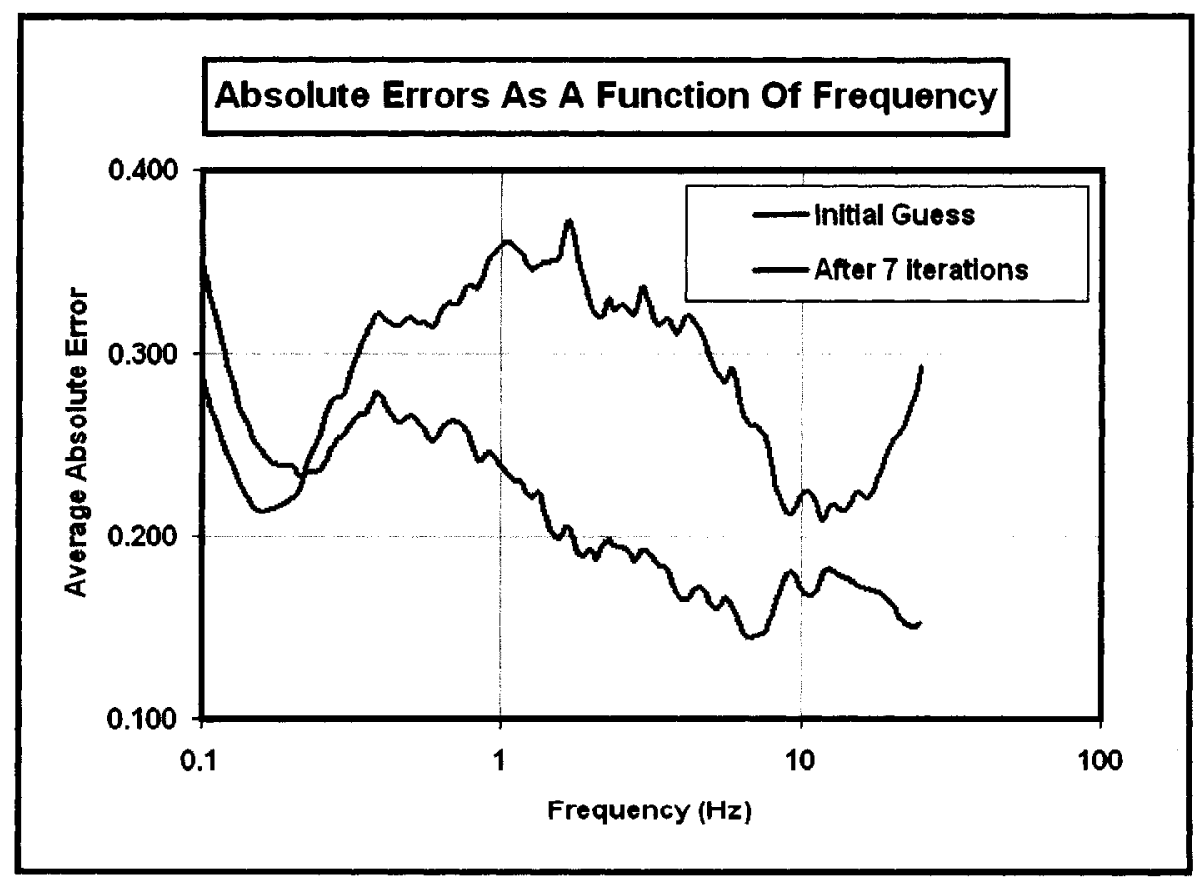

Figure (5.43). Average absolute error $\left(\Xi_{\mathrm{i}}\right)$ as a function of frequency in Case 3 of the Northridge inversion. The results correspond to $\Xi_{i}$ of initial guess and derived distributions. 


\section{Error change from uniform distribution resulting from 7 iterations}

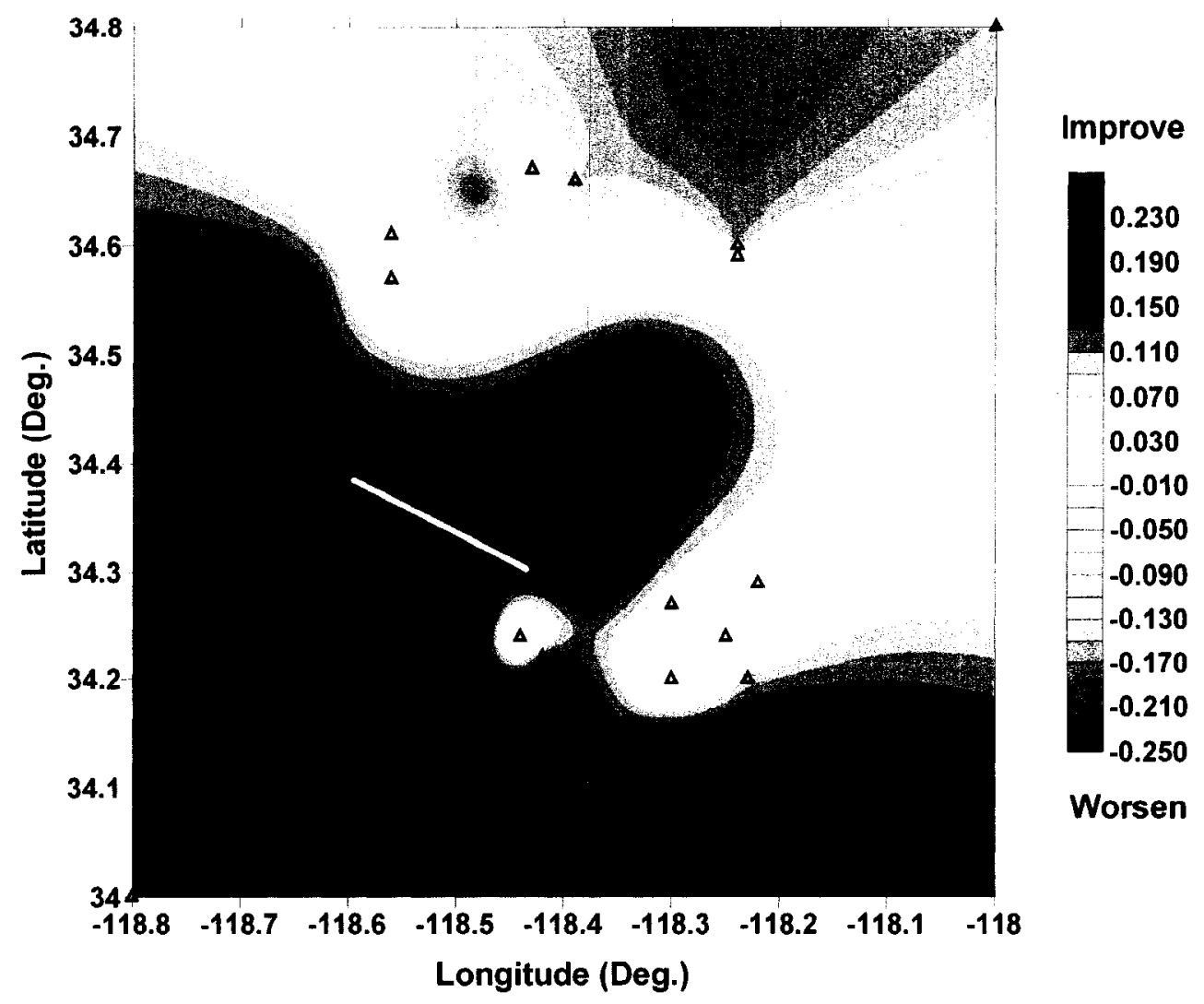

Figure (5.44). Spatial distribution of error improvement criterion $\Psi_{\mathrm{j}}$ from initial guess to final solution after 7 inversion iterations in Case 3 of the Northridge study. Crosses are location of stations and the yellow solid line is the location of the top of the fault. 


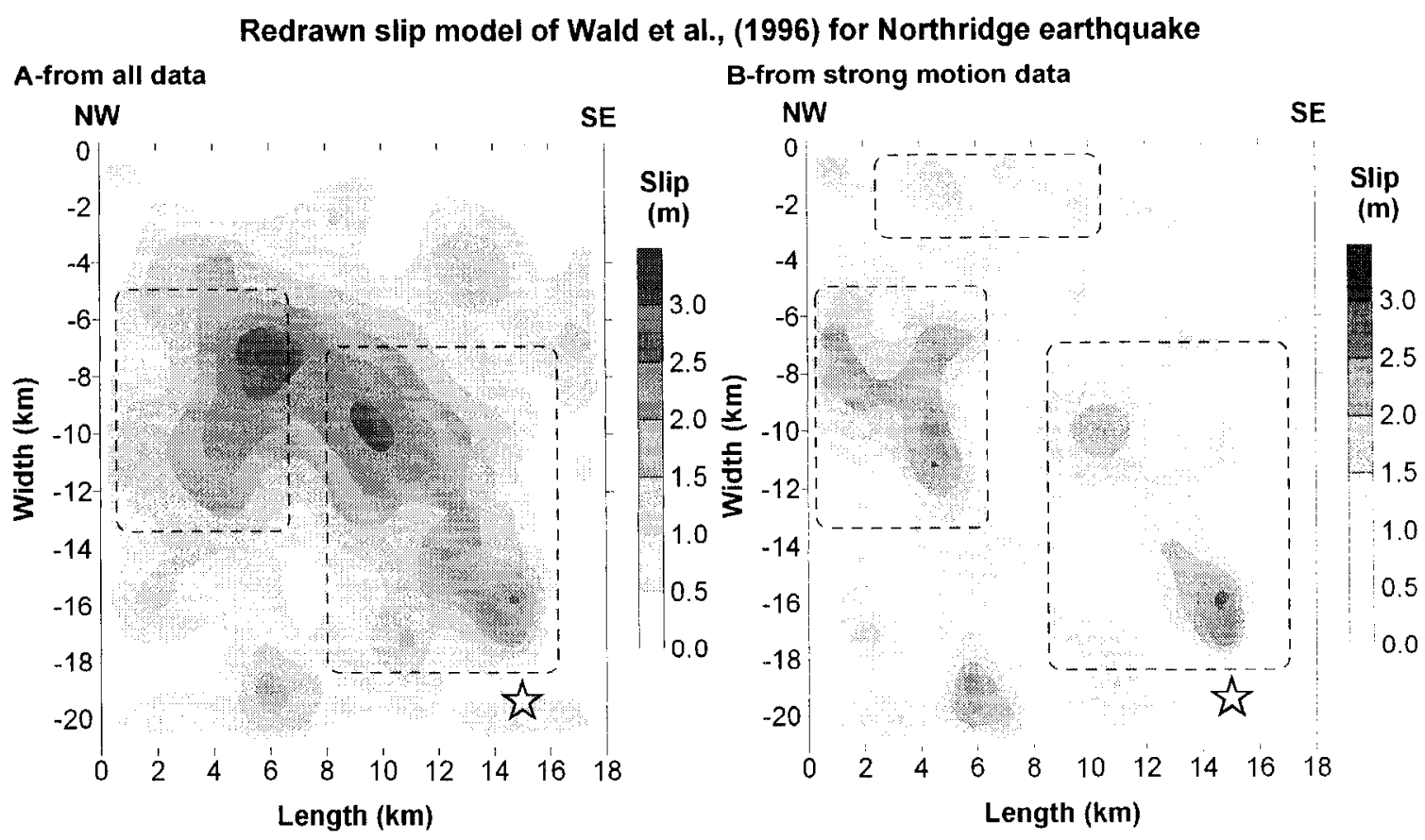

Figure (5.45) Slip distribution of Northridge earthquake derived from inversion by Wald et al., (1996). Redrawn using data at http://www.seismo.ethz.ch/srcmod/. 


\section{Chapter 6: Summary and Conclusions}

In this work, I overviewed the development of common stochastic modeling techniques from point source to finite-fault approaches. Using the EXSIM stochastic finite fault model of Motazedian and Atkinson (2005) as a starting point, I performed forward modeling experiments to explore the performance of EXSIM in modeling finite source characteristics, in sub-source size dependence, and in modeled accelerogram spectral properties. I also compared EXSIM to two similar alternative approaches (FINSIM and SMSIM); I did not attempt to compare EXSIM to all stochastic modeling approaches, so this is a limited comparison. Having established its overall performance, I modified EXSIM to take into account the effects of variable rupture velocity and variable stress parameter on the earthquake fault surface, and studied the significance of these modifications. I combined the modified EXSIM, that has the capability to model variable stress parameter, with inversion algorithms, and developed an inversion tool that uses the response spectra of recorded accelerograms to derive the stress parameter distribution on the fault surface. Performance studies provided insight into the parameters that affect the performance of the inversion tool. Then the inversion tool was applied to the data of the M6.0, 2004 Parkfield earthquake, and the stress parameter distribution on the fault surface was derived. The inversion tool was also applied to derive the stress parameter distribution on the fault surface of three major California earthquakes; the results were compared with slip distributions derived from other studies. This process provided understanding of the performance of the inversion tool when applied to real data, highlighted the program's capabilities and limitations, and initiated a stress parameter distribution database for previous earthquakes.

The main results of these studies are summarized below. 


\subsection{EXSIM finite fault model}

EXSIM (Motazedian and Atkinson, 2005) is a stochastic finite fault-modeling program that evolved from the SMSIM (Boore, 2000) stochastic point source model and the FINSIM (Beresnev and Atkinson, 1998) stochastic finite fault modeling techniques. The idea behind stochastic finite fault modeling is to include finite fault effects such as rupture propagation, directivity, and source-receiver geometry, in the context of a stochastic model of ground motion.

There are many versions of stochastic modeling techniques. Some of them combine a stochastic source with an empirical model of path (Silva et al., 1989; Chin and Aki, 1991; Schneider et al., 1993); others combine a stochastic source with empirical attenuation and duration models, or with a synthetic model of path effects (Zeng et al., 1994; Yu et al., 1995). In this study, I focus on comparisons of three inherently-similar versions of stochastic techniques: SMSIM (Boore, 2000), FINSIM (Beresnev and Atkinson, 1998). and EXSIM (Motazedian and Atkinson, 2005). These versions all combine a stochastic source model with an empirical path model.

The EXSIM program is capable of modeling directivity. When unilateral faulting is modeled by EXSIM, the log of spectral ordinates and their averages in a frequency range ( $D_{r}$ values) show directional dependence on the rupture direction. Its maximum is in the direction of fracture propagation and the minimum is away from the fracture propagation direction. There is a problem associated with EXSIM when modeling directivity; specifically, it predicts a stronger directivity effects for high frequency ordinates than low frequencies, with almost no directivity associated with periods of a few seconds or more. This behavior is the natural consequence of the modeling of a stochastic finite source as an array of stochastic point sources, but it is in contrast to expectations based on empirical studies. Future work is required to improve the treatment of directivity in stochastic finite-fault modeling. 
A good feature of EXSIM is that its results are independent from sub-source sizes in a large range of sub-source sizes and large range of frequencies, even at short distances, where finite source effects are significant.

Furthermore, the comparisons of Fourier spectra and pseudo spectral accelerations of a point source model (SMSIM) and EXSIM demonstrate that if one sub-source is assigned to EXSIM (which is the case with small earthquakes), the results will be identical to those of the point source model.

The spectral shapes and amplitudes (both Fourier spectrum and response spectrum) of motions generated by EXSIM and SMSIM are very similar at large distances. At short distances, the similarity is limited to events of small to moderate magnitude. At large magnitude and short distances, SMSIM predicts higher spectral levels than EXSIM for a given stress parameter. The spectral shapes of EXSIM and SMSIM motions differ slightly, in that EXSIM spectra feature a very gentle double corner frequency source spectral shape (although the EXSIM subsource spectra are simple omega-squared spectra).

Three stochastic simulation methods - SMSIM, FINSIM, and EXSIM - are compared against the NGA database (Pacific Earthquake Engineering Research, New Generation Attenuation Relation Project 2005, http://peer.berkeley.edu/nga/index.html) for events over a wide range of magnitude, distance, and frequency. All three methods are capable of generating reasonable synthetics. The finite source modeling technique of EXSIM is preferable because on average it performs better than the other two at most magnitudes. distances, and frequencies. At low frequencies, $(f \leq 0.3 \mathrm{~Hz})$ there is almost no preference amongst the methods; this might be due to the generally poor performance of stochastic methods at these frequencies.

Stochastic finite fault modeling techniques provide better simulation results than a stochastic point source approach. Allowing the spatial variability of source parameters on the fault surface is a further improvement. Possibilities for further refinements could be the introduction of variable slip velocity, non-flat fault geometry, and segmented fault 
geometry. It is also possible to work on improvement of the technique to make it capable of simulating the directivity effect and other intermediate-to-low frequency effects in a more realistic way.

\subsection{Variable stress modeling}

Two improvements are applied to EXSIM to enable it to model variable rupture velocity and spatially variable stress parameter on the earthquake fault surface. The implementation of variable rupture velocity, within a reasonable range of values, does not significantly affect simulated ground motions. By contrast, a spatially variable stress parameter on the fault surface has significant impact, over a reasonable range of stress parameter values.

The original EXSIM is capable of modeling variable slip distribution on the fault surface. The behavior of variable slip in the original EXSIM is highly similar to the behavior of variable stress parameter distribution in the modified EXSIM. In this sense, variable slip and variable stress may be considered interchangeable in EXSIM. As the stochastic method is primarily focused on high frequencies, I choose to consider stress the controlling model parameter rather than slip.

\subsection{Inversion tool}

The modified EXSIM, with the capability to model variable stress parameter distribution, is combined with enumerative and analytical inversion algorithms to develop an inversion tool. The inversion tool starts from a uniform stress parameter distribution and performs a grid search (enumerative inversion algorithm) to find the average stress parameter, then runs a Levenberg-Marquardt algorithm (analytical inversion algorithm) to assign weights to stresses of each sub-source; after completion of this cycle the program repeats the same procedure until a solution is reached and no significant solution 
improvement occurs. This inversion tool is developed to deduce the stress parameter distribution on the fault surface by inverting response spectra of recorded accelerograms.

A set of model performance studies established the following conclusions regarding performance of the inversion tool:

(i) If the number of EXSIM trials for averaging the predicted response spectra for a given stress parameter distribution is $\geq 10$, then the inversion tool will reach a reasonable answer. Setting this number to values greater than 10 will reduce the speed of the inversion tool without any improvement in the obtained solution.

(ii) Gentle fault dip angles allow an accurate determination of the stress parameter distribution, while steep fault dip angles limit its resolution. With gentle fault dip angles, the stations "see" the sub-faults across a fuller range of angles and distances, and this makes the equations for the inversion distinct. The method performs very well if the fault dip angle is less than $45^{\circ}$, although it performs reasonably well with dip angles more than $45^{\circ}$, at least in terms of locating the horizontal position of asperities.

(iii) The method has the capability to resolve multiple asperities, with as little as one subsource distance between them. It can also find the relative stress parameter levels of the asperities. With multiple asperities the solution converges faster than is the case for a single asperity.

(iv) If the initial guess of the stress parameter distribution is an accurate one, the solution will converge with few inversion iterations. If an accurate initial guess is not obvious, then the uniform distribution provides a reasonable initial guess. If the initial guess is a strong asperity in the incorrect location, then the program may fail to converge; otherwise it will be able to push the answer toward the real distribution eventually.

(v) As is the case in other inversion techniques, a dense station distribution around the fault will result in high accuracy and precision of the high-stress asperities. If the station distribution is sparse, or there are no stations near the asperity, then the program may fail to find an asperity, or provide it with low resolution. 
A program called "Contrast amplifier" was designed and developed to process the stress parameter distribution from the inversion initial steps. By inputting the distribution processed by "Contrast amplifier" to the inversion program, the inversion process will be accelerated, because the new input distribution is much closer to the target distribution than the inversion output in the initial iterations.

\subsection{Variable stress modeling of California earthquakes}

The horizontal components of the M6 2004 Parkfield earthquake recorded at 85 locations are used in a test application of the inversion method. First, the response spectra of the records are corrected to equivalent values for a site condition of $V_{\mathrm{s} 30}=620 \mathrm{~m} / \mathrm{s}$. Then the corrected PSAs are inverted for the stress parameter distribution on the fault surface. The inversion of the Parkfield data clearly demonstrates the presence of an asperity in the southeast end of the fault at depths between $4 \mathrm{~km}$ to $8 \mathrm{~km}$. This is in agreement with the conclusion of Mai et al. $(2003,2005)$ that the hypocenter and the main asperity (large slip area) are very close to each other.

Increasing the number of sub-sources (making them smaller) can increase the resolution of a solution and show more details of the stress parameter distribution on the fault surface, if the problem remains over-determined and solvable. From a theoretical point of view, the program is capable of generating a solution while the program remains overdetermined or even-determined; this means that the number of sub-sources cannot exceed the number of recording stations. From a practical point of view, the experiments with three California earthquake solutions demonstrate that the inversion program can generate reasonable solutions if the number of stations is about twice the number of subsources. Even if we have an over-determined problem, having a small number of stations reduces the quality of solutions, or may make them meaningless. This is because of some common problems with real data, such as the spatial clustering of stations, their large distances from the fault, or poor azimuthal coverage of stations around the fault. These 
conditions render some of the constraining equations repeated or trivial, and as a result the inversion program may fail.

The stress parameter distribution of three other major California earthquakes (M7.1, 1989 Loma Prieta; M7.3, 1992 Landers; and M6.7, 1994 Northridge) are obtained using the developed inversion tool. In all three earthquakes, the stress parameter distribution shows considerable similarity to slip distributions derived by other research groups for the same earthquakes. The similarity of the stress and slip distributions is attributed to proportionality of shear stress and strain, as noted by Wald et al., (1991). The similarity of distributions noted here suggests that the method applied in this study is a viable tool for derivation of the stress parameter distribution, and it can be applied to other earthquakes to prepare a database of stress parameter distributions for past earthquakes. By understanding the stress parameter distributions of past events, we can ultimately improve predictions of the expected ground motions and their variability for future earthquakes. As the stochastic model is a widely-used tool for such ground-motion predictions, it forms a convenient basis to characterize our understanding of the salient parameters that control strong ground motion. 


\section{Appendix A}

\section{Residual study of SMSIM, FINSIM, and EXSIM}

In chapter 2 , the performance of three simulation methods are compared against 2681 records of NGA database by means of average absolute error of Log PSAs or " $\varepsilon$-values". In this appendix, I extend the performance comparisons of SMSIM, FINSIM, and EXSIM by implementing residual (or bias) comparisons. The residual is defined as $\log ($ observed $)-\log$ (predicted). The average residual at any frequency when considering a number of stations is defined as:

$$
\varepsilon_{1}=\frac{1}{K} \sum_{j=1}^{K}\left(\log P S A\left(O_{i j}\right)-\log P S A\left(S_{i j}\right)\right)
$$

where $\varepsilon_{1}$ is the residual at one frequency averaged over all stations, $\mathrm{K}$ is the number of stations, $\log P S A\left(O_{i j}\right)$ is $\log$ of observed pseudo spectral acceleration ordinate at $i^{\text {th }}$ frequency and $\mathrm{j}^{\text {th }}$ station, and $\log P S A\left(S_{i j}\right)$ is $\log$ of simulation pseudo spectral acceleration ordinate at $\mathrm{i}^{\text {th }}$ frequency and $\mathrm{j}^{\text {th }}$ station. This quantity is calculated for three abovementioned simulation methods and three earthquakes: M6.6, 1994 Northridge; M7.0, 1989 Loma Prieta; M7.2, 1992 Landers; in Figures (A.1) to (A.3). The comparisons of residuals ( $\varepsilon_{1}$-values) show that on average EXSIM performs the best in most frequencies and distances: eg. $10 \mathrm{~km}, 30 \mathrm{~km}$, and $200 \mathrm{~km}$ from the fault. The results for very short distances, $1 \mathrm{~km}$ from the fault, are not reliable because all three earthquakes had a very small number of records for averaging in this distance bin.

One may also consider the average residual over all stations within a specified frequency range:

$$
\varepsilon_{2}\left(N_{1}, N_{2}\right)=\frac{1}{K\left(N_{2}-N_{2}+1\right)} \sum_{j=1}^{K} \sum_{i=N_{1}}^{N_{2}}\left(\log P S A\left(O_{i j}\right)-\log P S A\left(S_{i j}\right)\right)
$$

where $\varepsilon_{2}$ is the residual averaged over all stations, for all frequencies with ordinate numbers $N_{l}$ to $N_{2}$, and all other parameters are as above. These residuals " $\varepsilon_{2}$-values" are plotted for three simulation methods as a function of magnitude for distances $1 \mathrm{~km}, 10 \mathrm{~km}$, $30 \mathrm{~km}$, and $200 \mathrm{~km}$ from the fault in Figures (A.4) to (A.7) respectively. In the preparation 
of Figures (A.4) to (A.7), four different frequency ranges are considered: $0.3 \mathrm{~Hz}, 1 \mathrm{~Hz}$, $3 \mathrm{~Hz}$, and $1 \mathrm{~Hz}-10 \mathrm{~Hz}$. These figures demonstrate that on average EXSIM has the minimum high frequency residuals (or bias) for magnitudes less than $\sim \mathbf{M} 7.2$.

Finally, the values of $\left|\varepsilon_{2}\right|$ are calculated for the three simulation methods; the one with the smallest $\left|\varepsilon_{2}\right|$ value is assigned to the corresponding spot in magnitude and distance space, as shown in Table (A.1). Table (A.1) demonstrates that at high-frequencies, EXSIM simulations generate minimum residuals at most magnitudes and distances. 
$\stackrel{\infty}{\sim}$
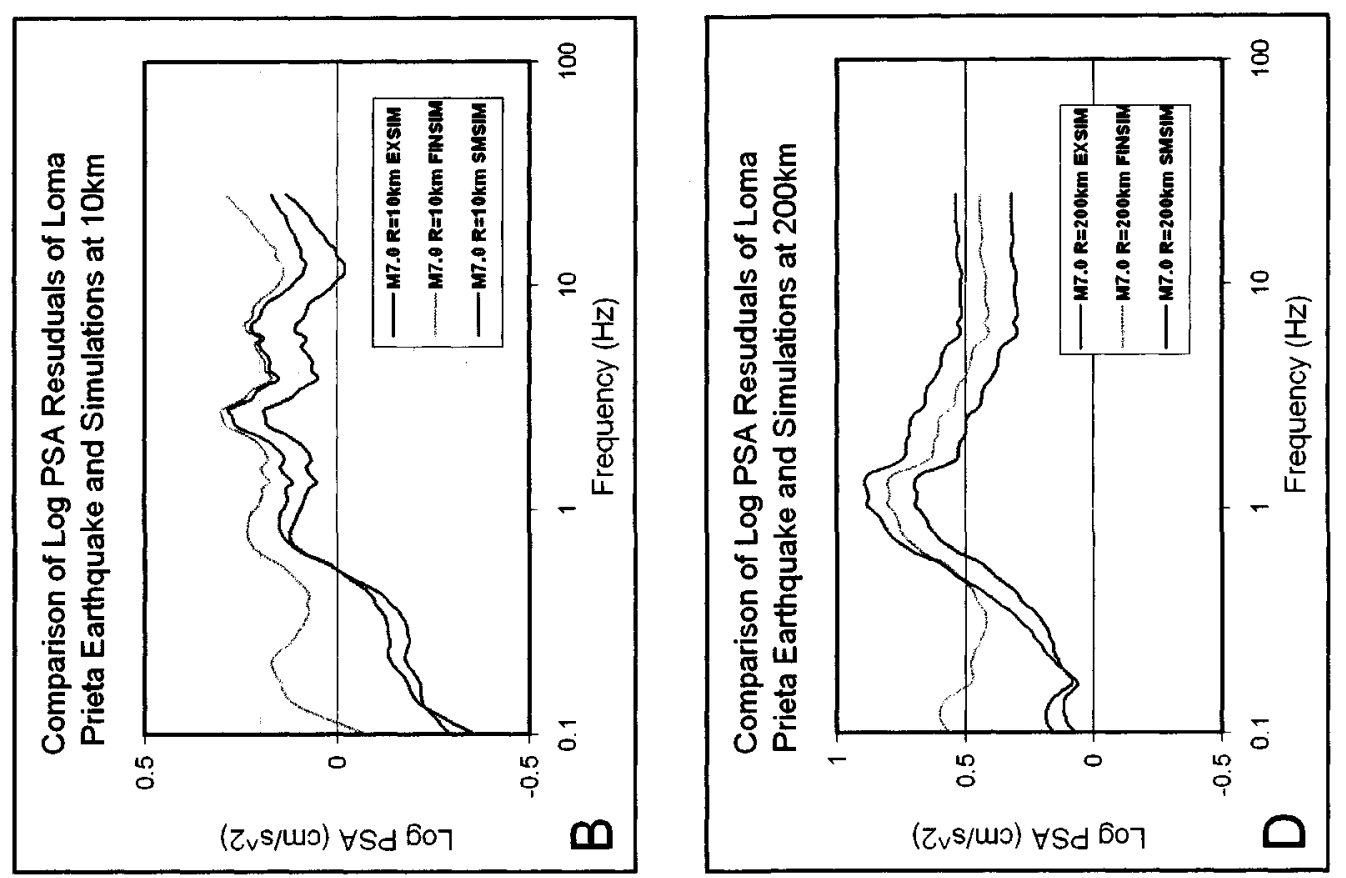

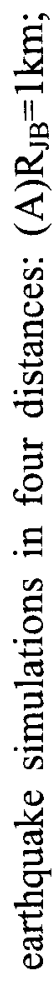

悉

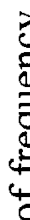

के
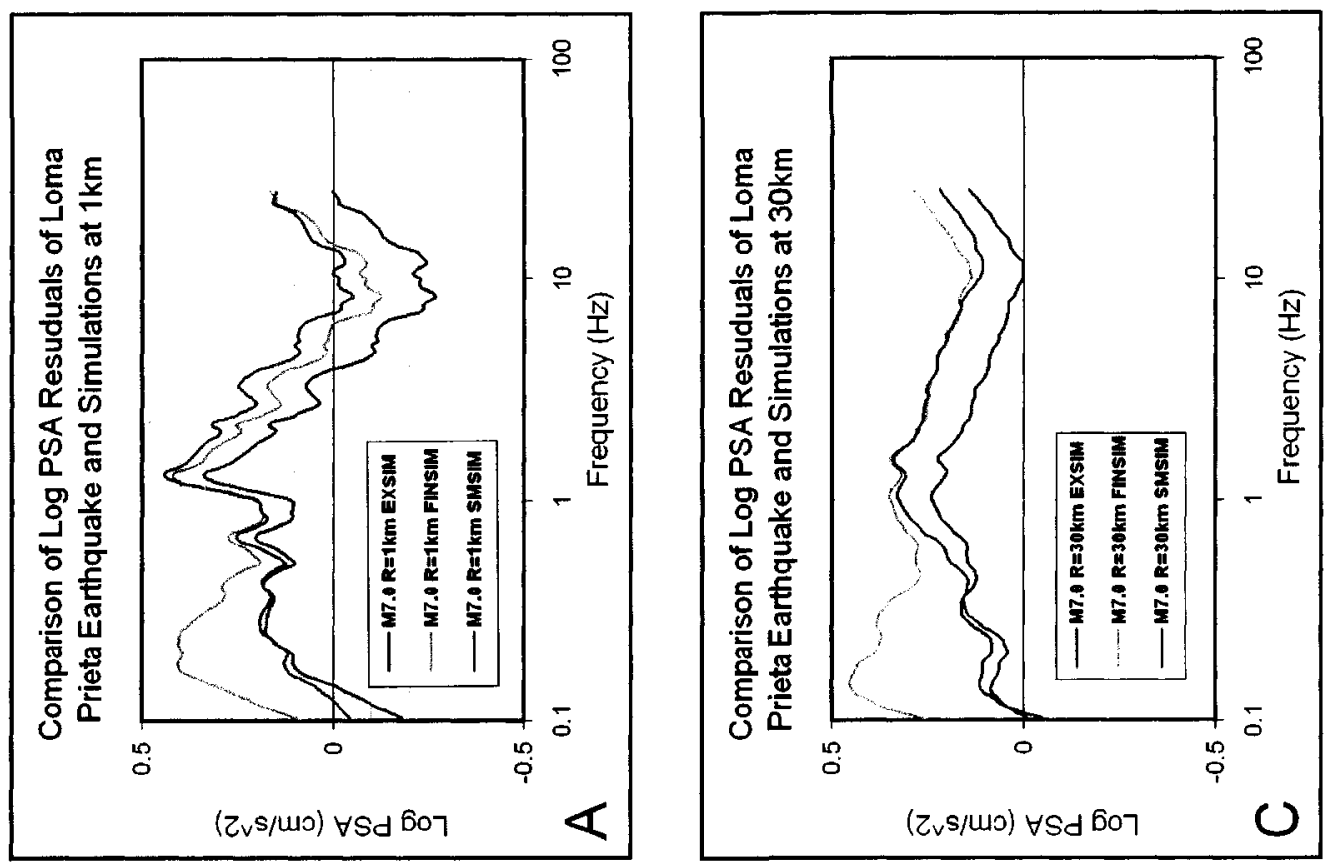

욤

$\sum^{4}$

)

㫕

an

$\stackrel{5}{\circ}$

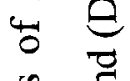

क छ

莺 总

य

跑

$\geqslant \bigcup$

\&

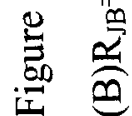


กิ
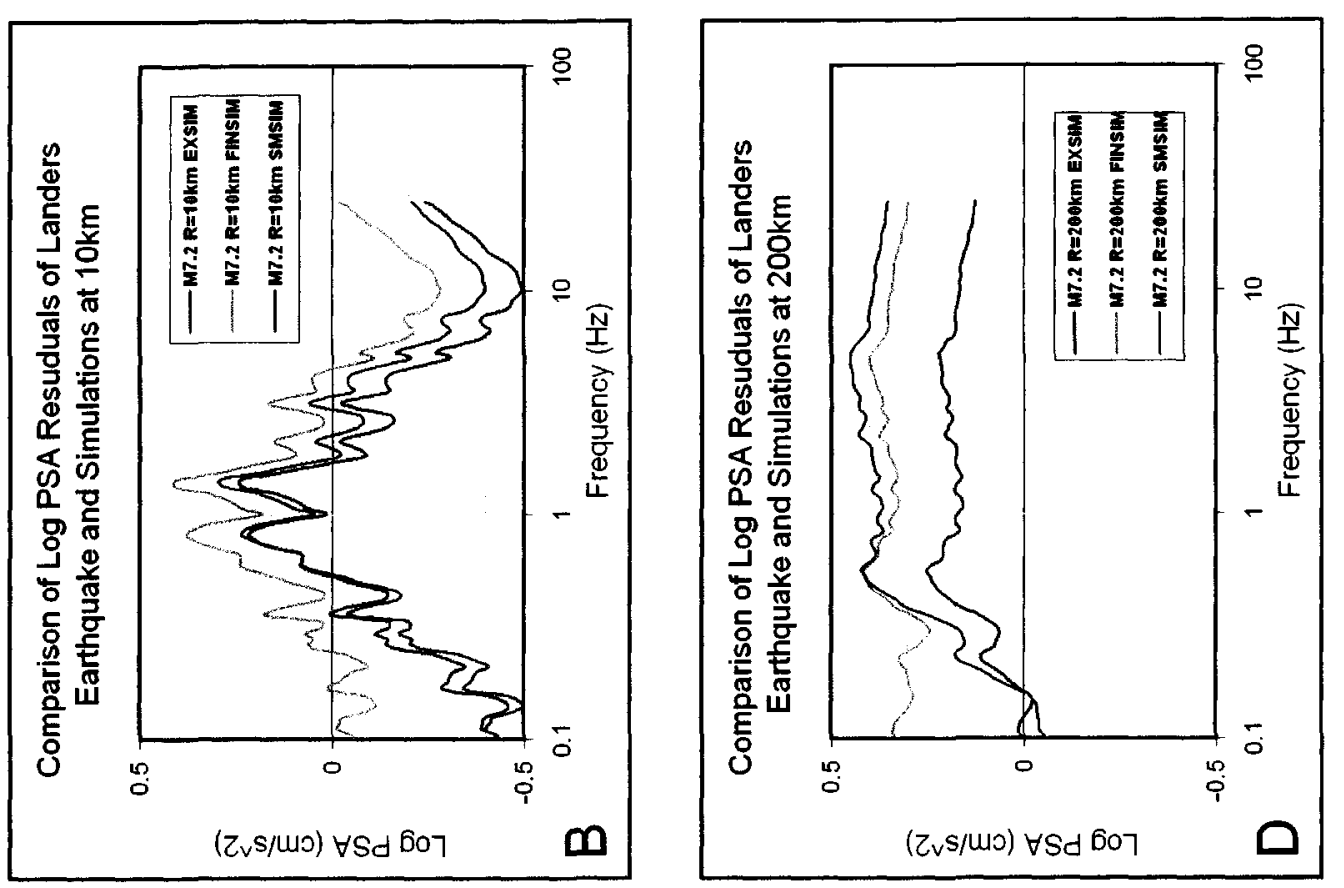

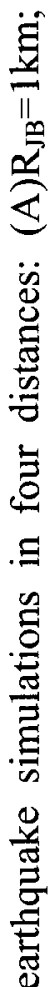

离

它

ริ

ร
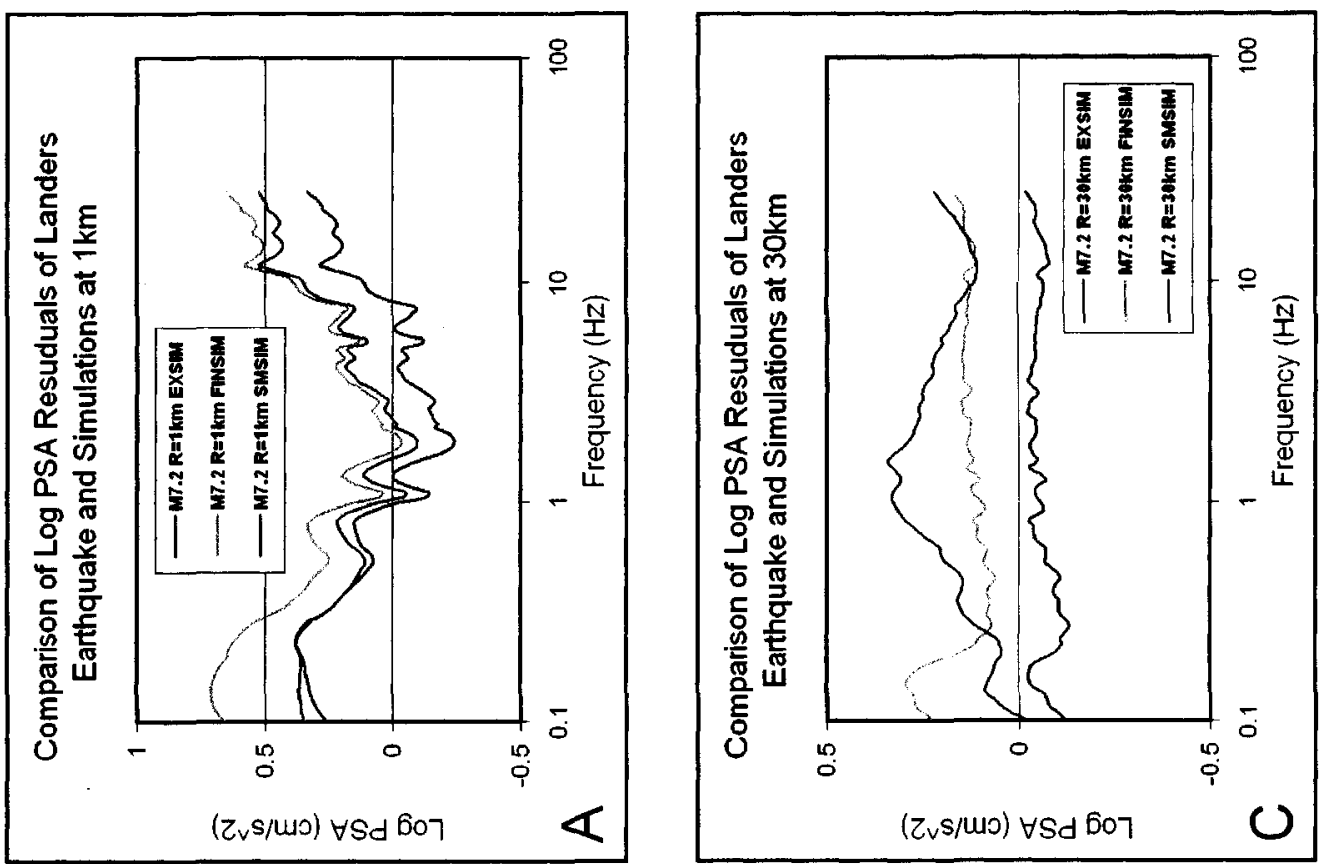

तi

$\sum \sum$

4

选 咅

on

党

을

焉

总

步

跑急

ก

ণิ

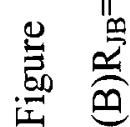


导
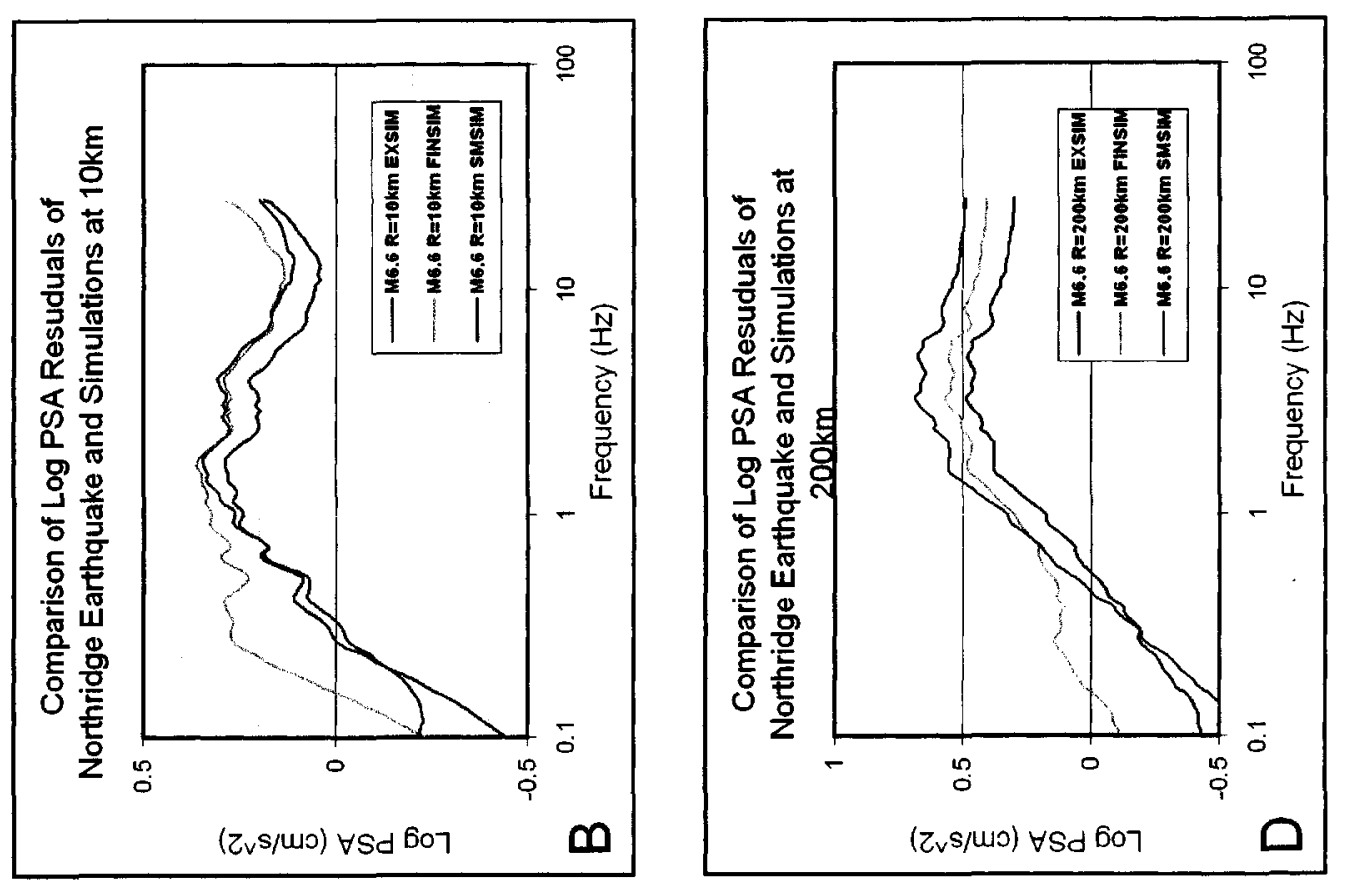

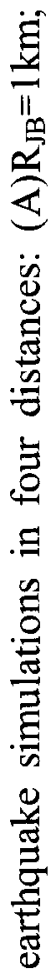

总

己

ठ
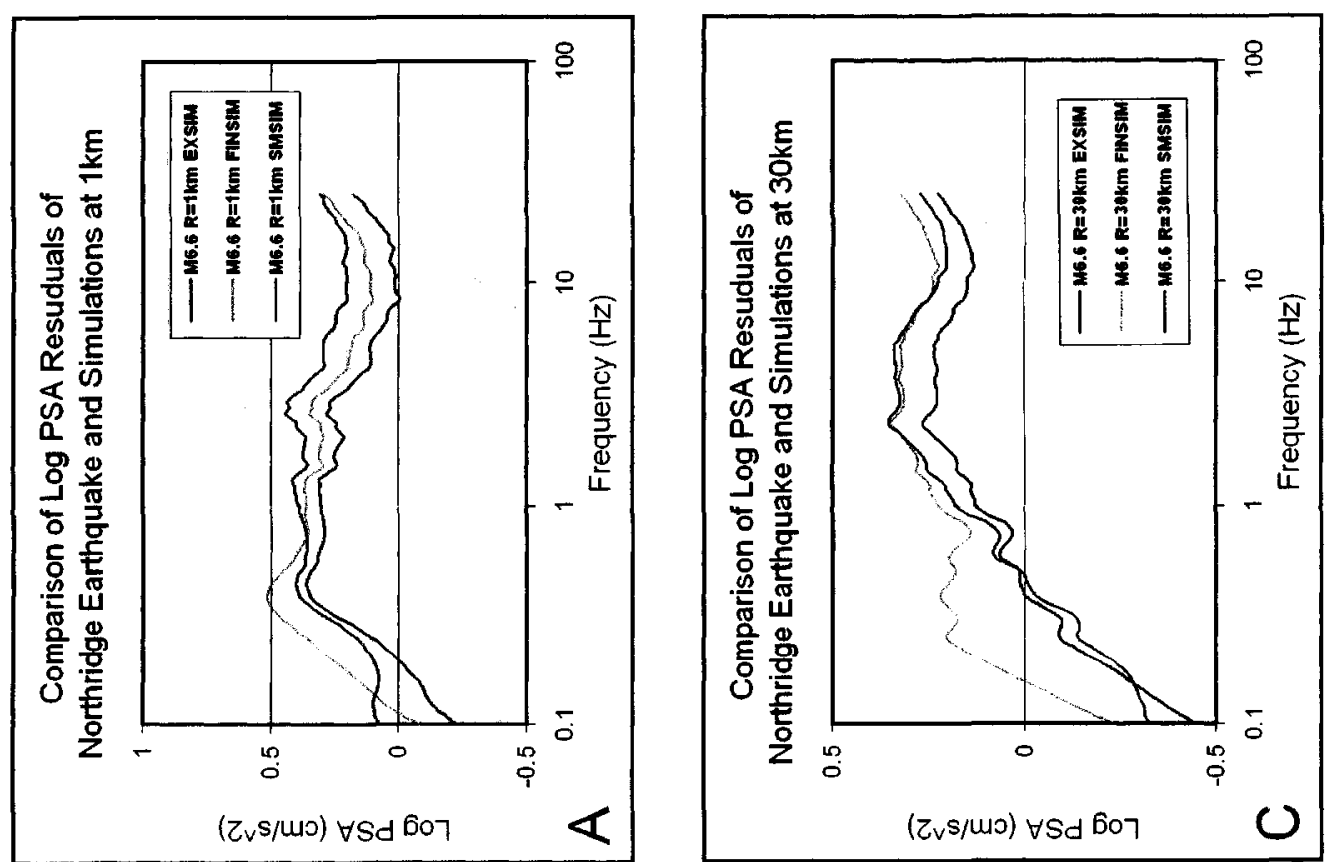

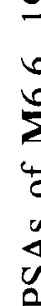

ڤ̊.

$\sum$ a

造

a

$\stackrel{0}{1}$

4 ?

卷

寻

总

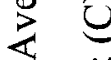

§

营 

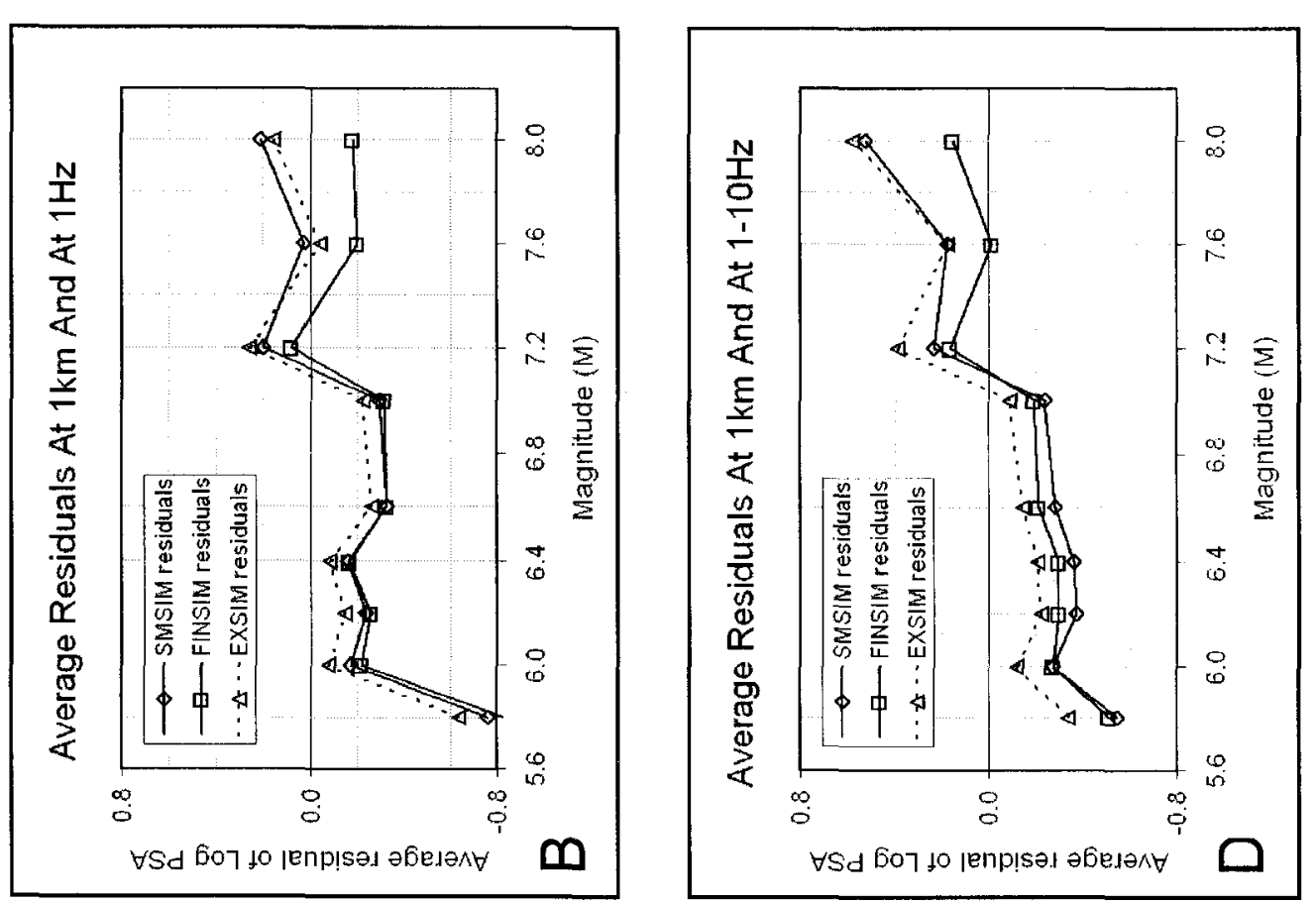

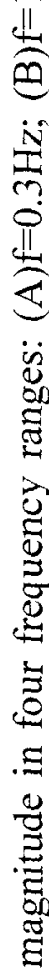

4

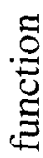
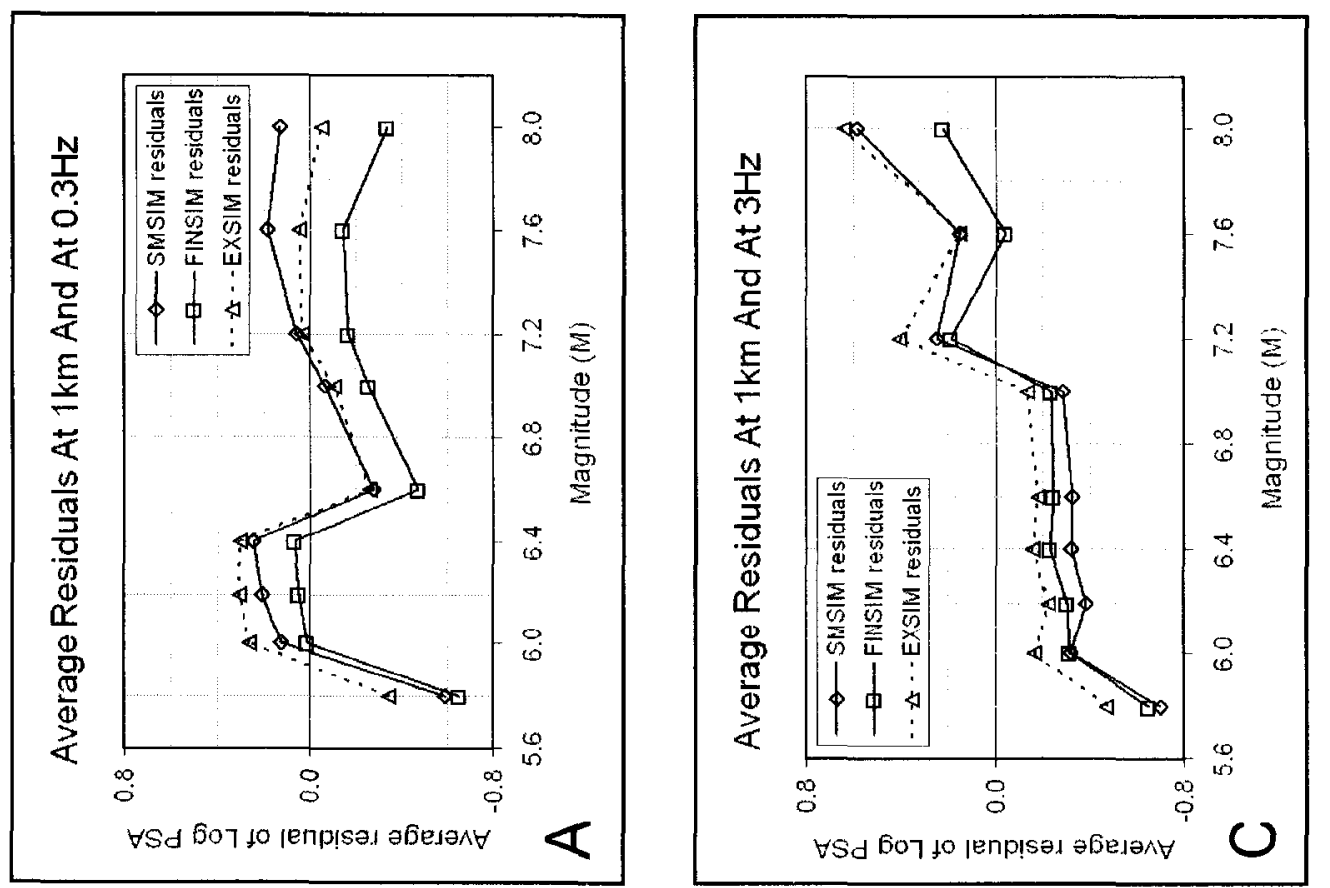

$\pi$

\%

$\Xi$

ส

望

$\infty$

$\stackrel{5}{4}$

0

疍

공

\pm I

䟺

3

\&

ङ

苛 
$\stackrel{\sim}{\sim}$
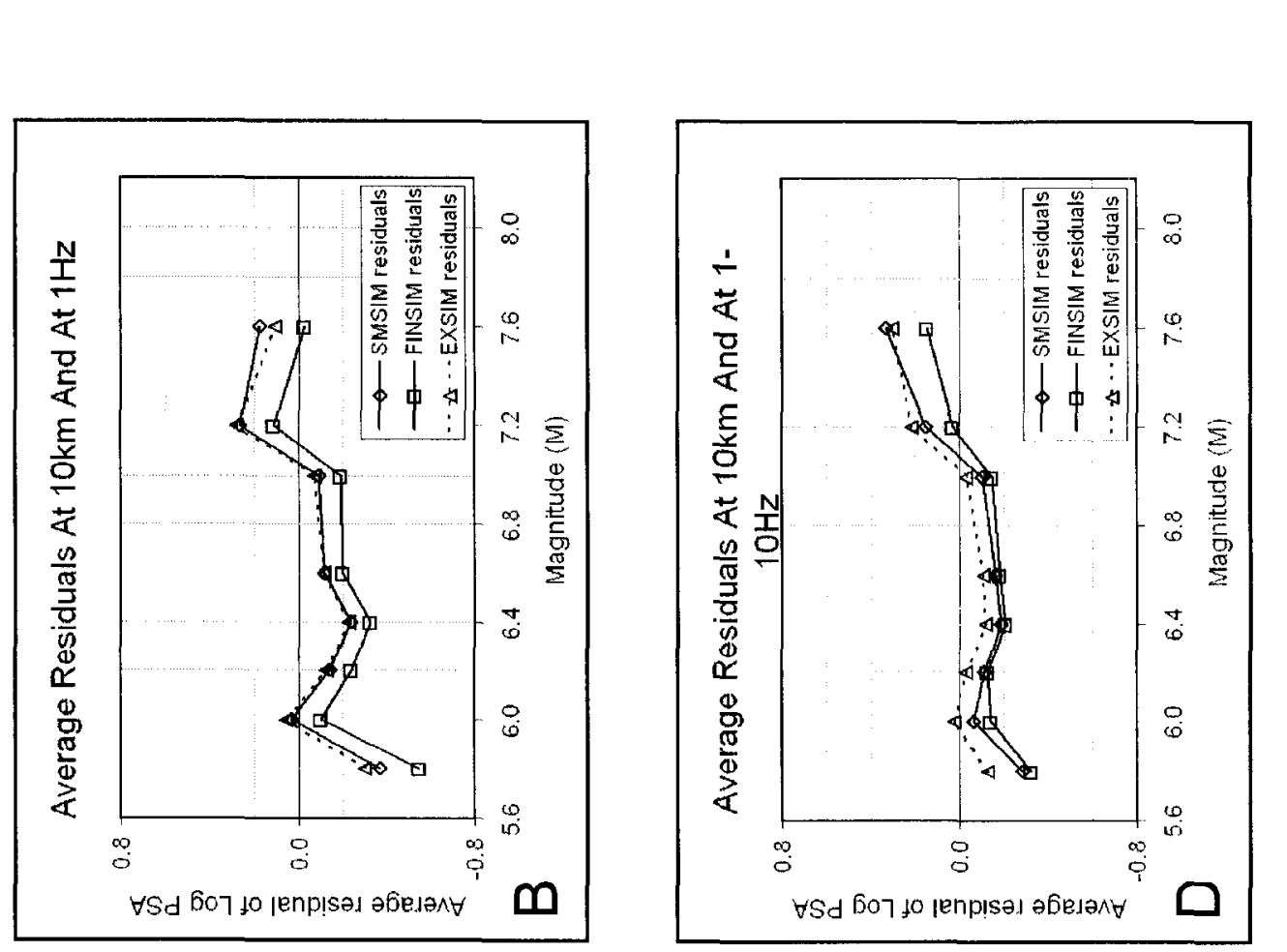

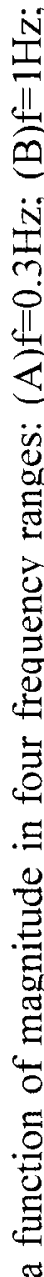
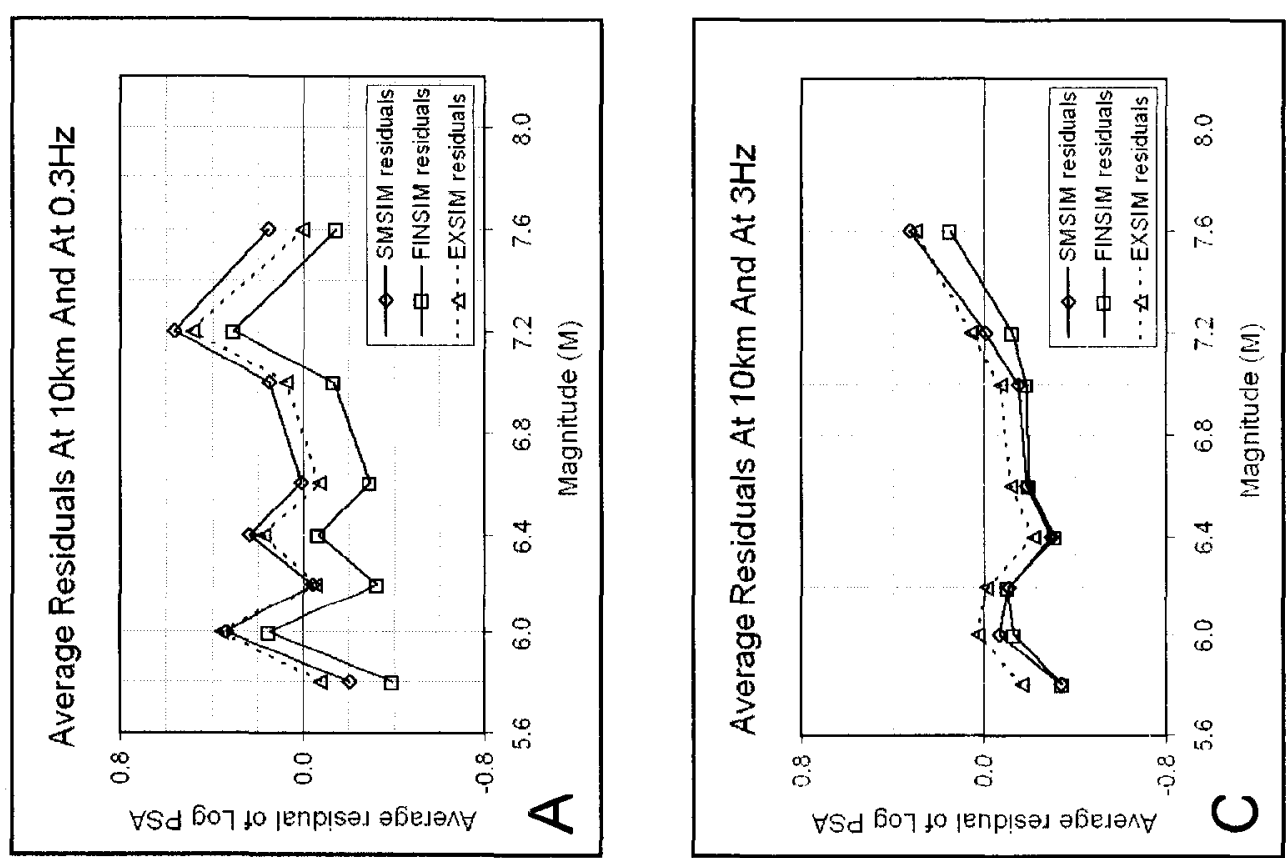

\&

픙

त

造

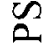

0
0
.

4

章

弯

ก

$\leq \frac{N}{9}$

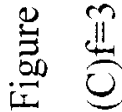


$\stackrel{\overbrace{}}{\sim}$
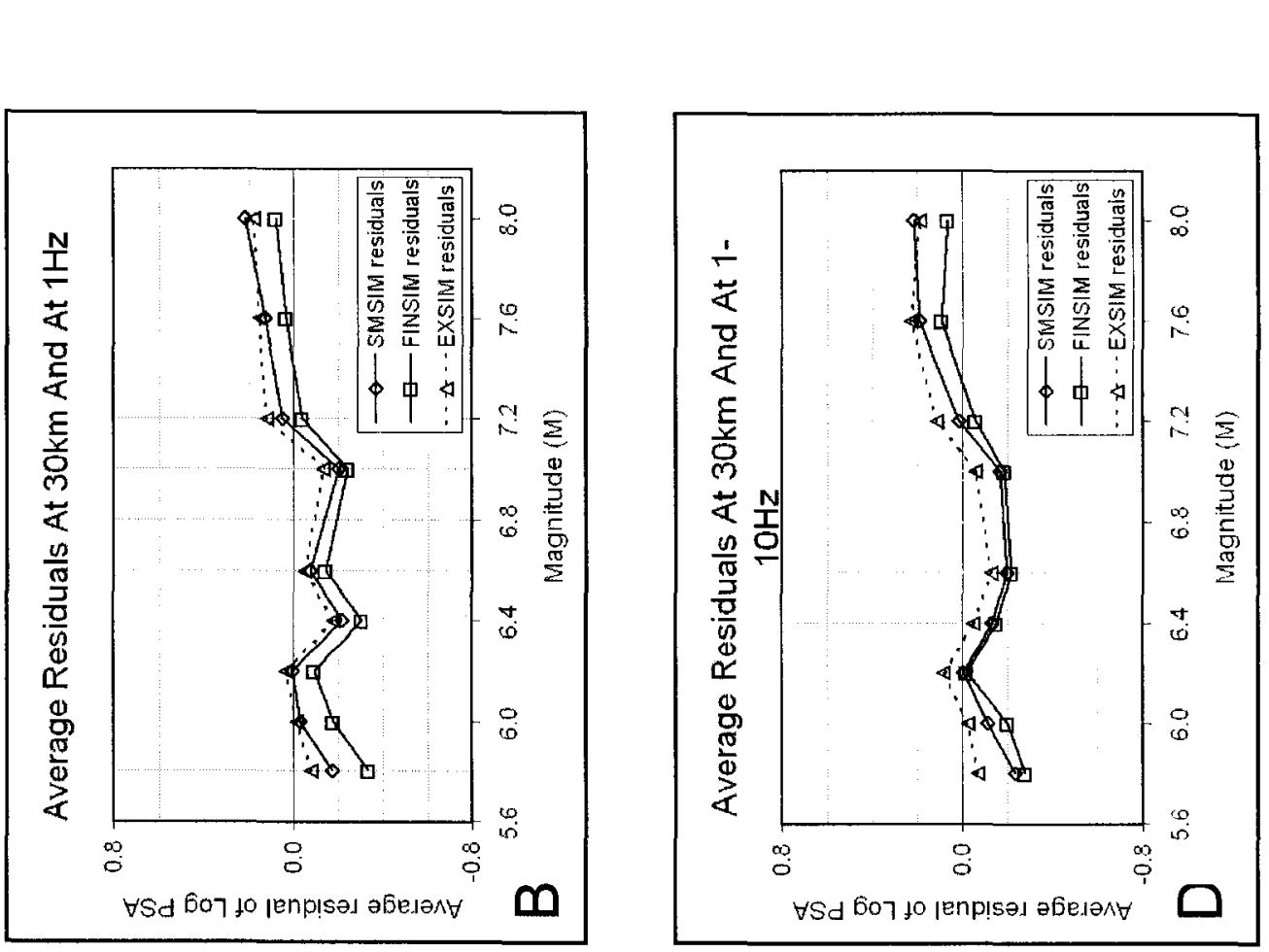

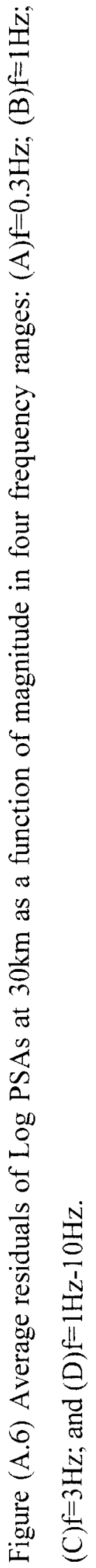
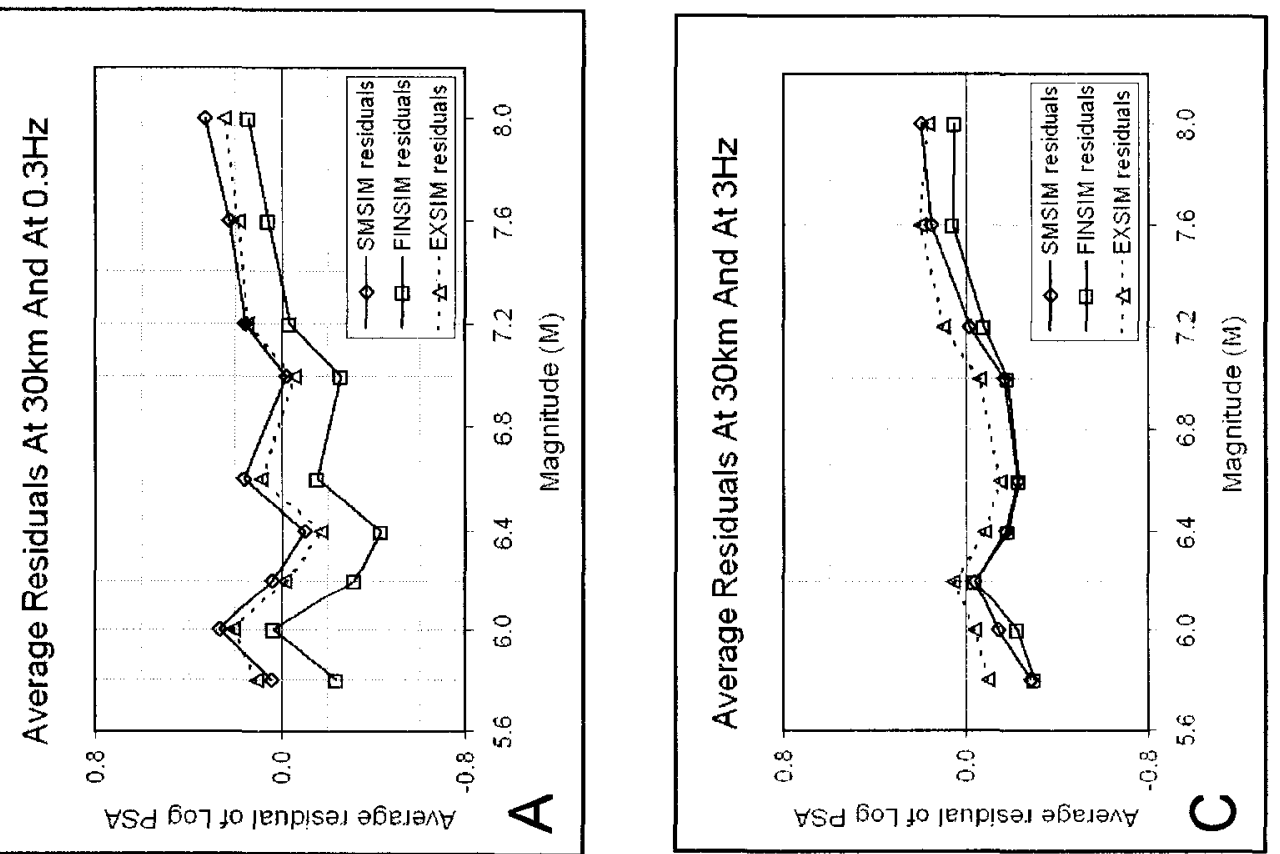
ষ
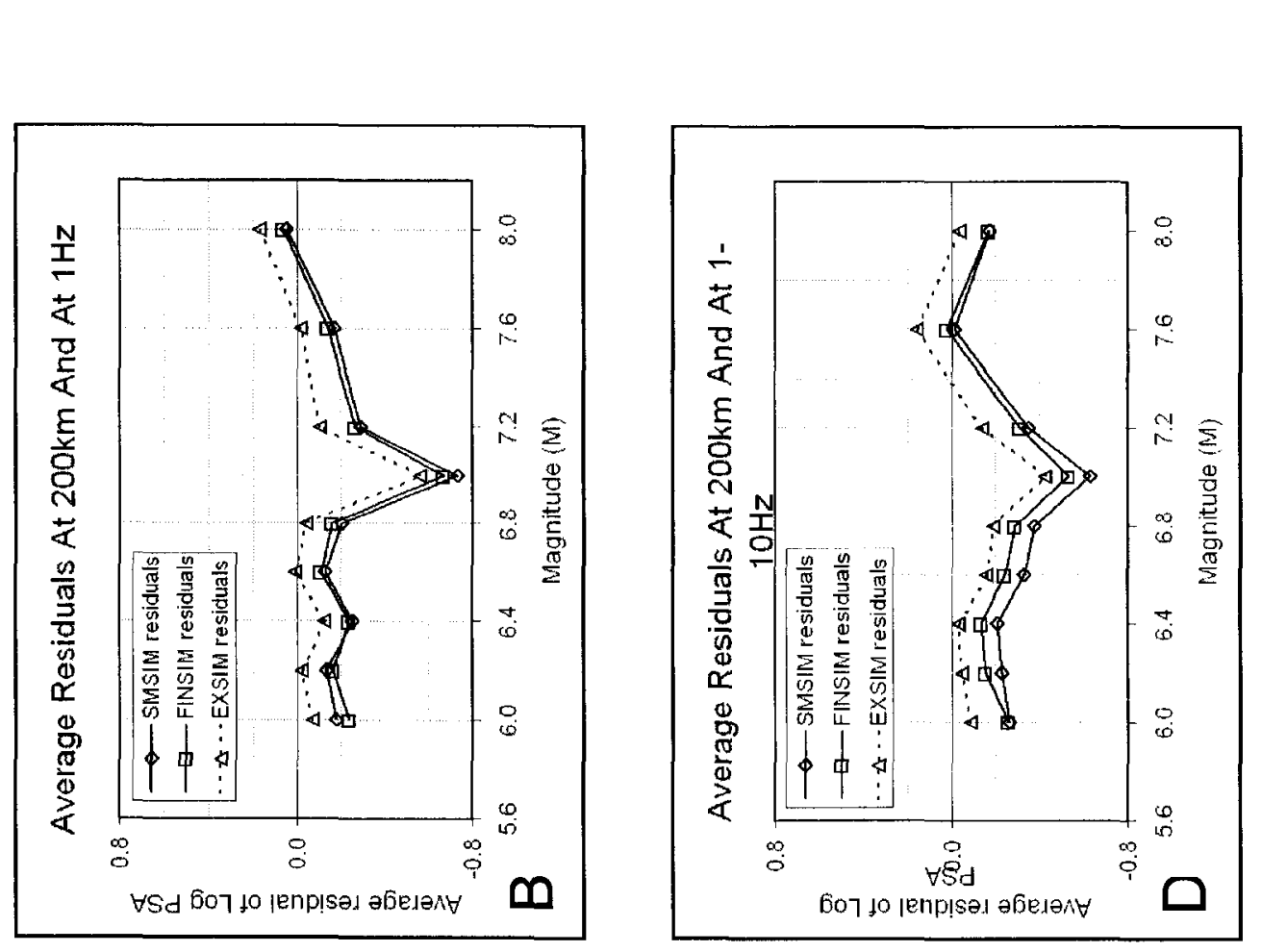

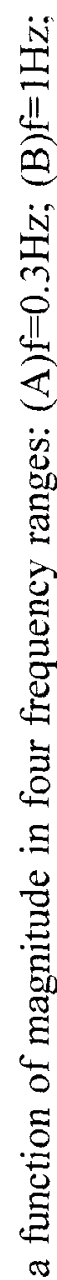
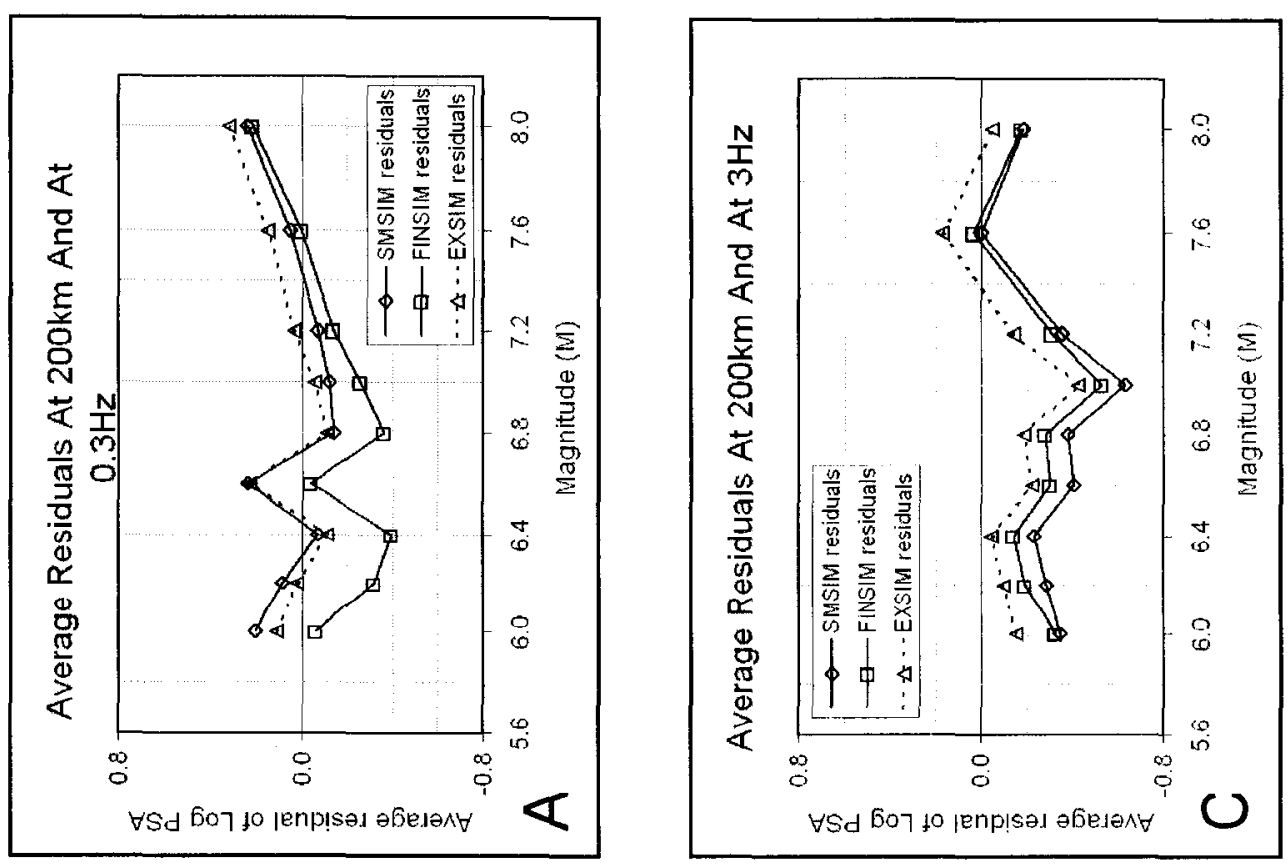

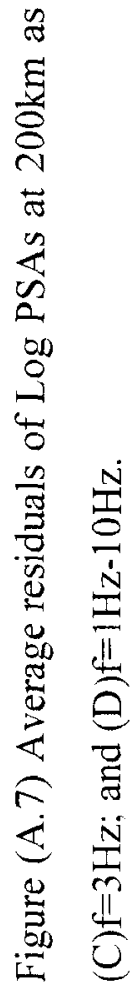




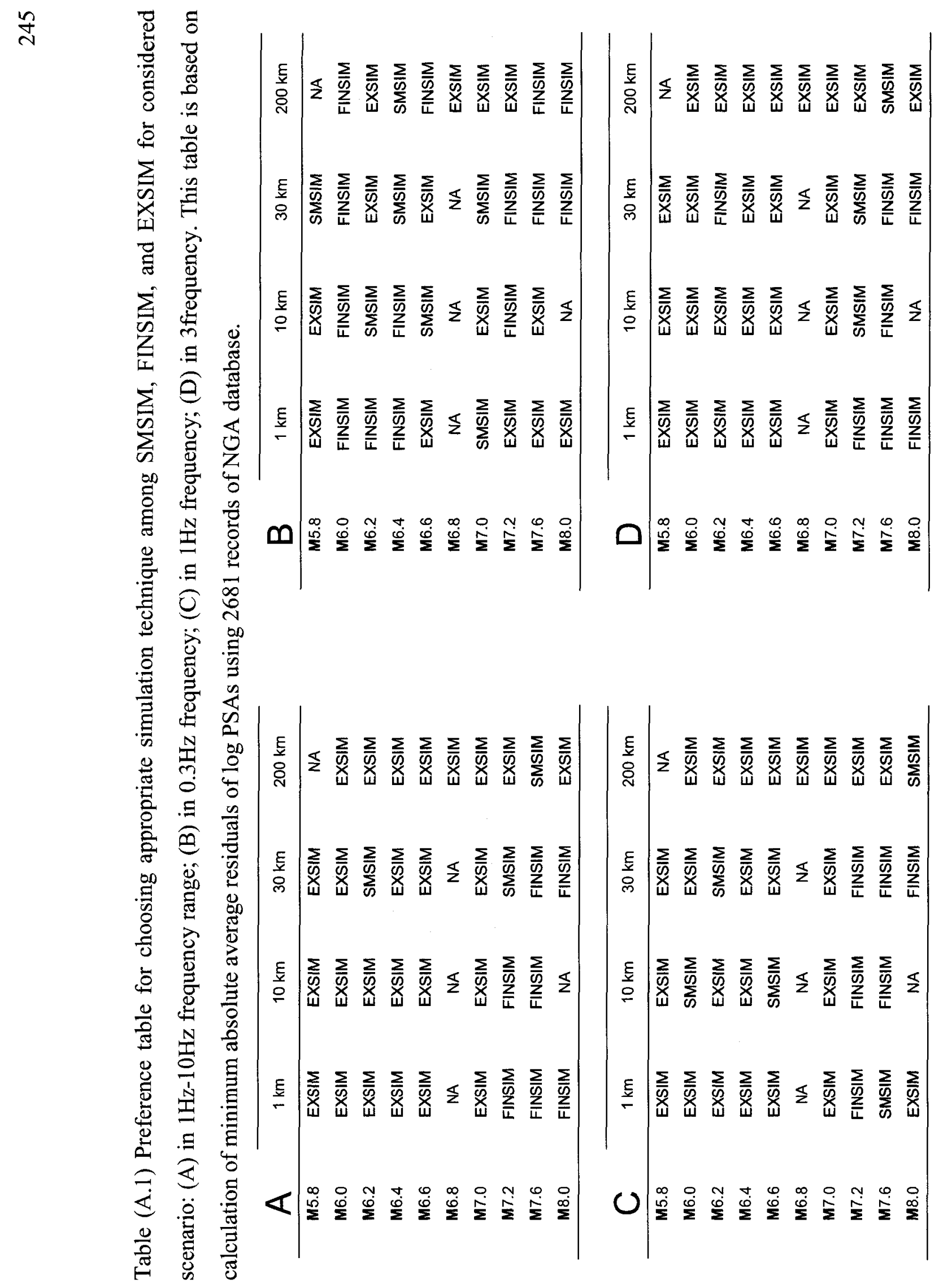




\section{References}

Abrahamson, N. A., and K. M. Shedlock (1997). Overview, Seismol.Res.Lett. 68, 9-23.

Aki, K. (1989). Deterministic and stochastic approaches in seismogram analysis; Observatory seismology; An anniversary symposium on the occasion of the centennial of the University of California at Berkeley seismographic stations, in Observatory seismology; An anniversary symposium on the occasion of the centennial of University of California at Berkeley seismographic stations, Berkeley, CA, United States, May 28-29, 1987. Univ. Calif. Press, Berkeley, CA, United States (USA), United States (USA), .

Aki, K. (1967). Scaling law of seismic spectrum, Journal of Geophysical Research 72, 1217-1231.

Anderson, J. G., and S. E. Hough (1984). A model for the shape of the Fourier amplitude spectrum of acceleration at high frequencies, Bulletin of the Seismological Society of America 74, 1969-1993.

Archuleta, R. J., J. H. Steidl, and M. Squibb (2003). COSMOS Virtual Data Center; a Web-based portal to the world's strong motion data; Abstracts of the 73rd annual meeting of the Seismological Society of America, Seismol.Res.Lett. 74, 218.

Armstrong, M. (1998). Basic linear geostatistics, Springer, Berlin, Federal Republic of Germany (DEU), Federal Republic of Germany (DEU).

Aster, R. C., C. H. Thurber, and B. Borchers (2005). Parameter estimation and inverse problems, Vol. ; v. 90, Elsevier Academic Press, Amsterdam ; Boston.

Atkinson, G. M., and D. M. Boore (1997). Stochastic point-source modeling of ground motions in the Cascadia region, Seismol.Res.Lett. 68, 74-85.

Atkinson, G. M., and D. M. Boore (1995). Ground motion relations for eastern North America, Bulletin of the Seismological Society of America 85, 17-30. 
Atkinson, G. M., and W. Silva (2000). Stochastic modeling of California ground motions. Bulletin of the Seismological Society of America 90, 255-274.

Atkinson, G. M., and W. Silva (1997). An empirical study of earthquake source spectra for California earthquakes, Bulletin of the Seismological Society of America 87, 97113.

Benioff, V. H. (1955). Mechanism and strain characteristics of the White Wolf fault as indicated by the aftershock sequence, [Article] 10; Pt. 2 of Oakeshott, G. B., ed., Earthquakes in Kern County, California, during 1952, 199-202.

Ben-Menahem, A. (1961). Radiation of seismic surface-waves from finite moving sources, Bulletin of the Seismological Society of America 51, 401-435.

Beresnev, I. A., and G. M. Atkinson (1998). FINSIM - a FORTRAN program for simulating stochastic acceleration time histories from finite faults, Seismol.Res.Lett. 69, 27-32.

Beresnev, I. A., and G. M. Atkinson (1997). Modeling finite-fault radiation from the $\omega^{\mathrm{n}}$ spectrum, Bulletin of the Seismological Society of America 87, 67-84.

Beresnev, I., and G. M. Atkinson (2001). Subevent structure of large earthquakes; a ground-motion perspective, Geophys.Res.Lett. 28, 53-56.

Bernard, P., and A. Herrero (1994). Slip heterogeneity, body-wave spectra, and directivity of earthquake ruptures; Earthquake source mechanics, Ann.Geofis. 37, 1679-1690.

Beroza, G. C., T. C. Hanks, and H. Krawinkler (1991). Near-source modeling of the Loma Prieta earthquake; evidence for heterogeneous slip and implications for earthquake hazard; The 1989 Loma Prieta, California, earthquake and its effects, Bulletin of the Seismological Society of America 81, 1603-1621.

Boatwright, J., and G. L. Choy (1992). Acceleration source spectra anticipated for large earthquakes in northeastern North America, Bulletin of the Seismological Society of America 82, 660-682. 
Boore, D. M., and G. M. Atkinson (2007). Boore-Atkinson NGA Ground Motion Relations for the Geometric Mean Horizontal Component of Peak and Spectral Ground Motion Parameters, PEER 2007/01,Pacific Earthquake Engineering Research Center, Berkeley, California, .

Boore, D. M., and G. M. Atkinson (2006). Boore-Atkinson provisional NGA empirical ground-motion model for the average horizontal component of PGA, PGV and SA at spectral periods of $0.05,0.1,0.2,0.3,0.5,1,2,3,4$, and 5 seconds, report to the PEER-Lifelines Next Generation Project [Revised 27 October 2006] [http://peer.berkeley.edu/lifelines/nga_docs/nov_13_06/Boore-Atkinson-NGA_1113-06.html]. .

Boore, D. M. (2003). Simulation of ground motion using the stochastic method; Seismic motion, lithospheric structures, earthquake and volcanic sources; the Keiiti Aki volume, Pure Appl.Geophys. 160, 635-676.

Boore, D. M. (2000). SMSIM; Fortran programs for simulating ground motions from earthquakes; version 2.0, OF 00-0509, 55.

Boore, D. M. (1983). Stochastic simulation of high-frequency ground motions based on seismological models of the radiated spectra, Bulletin of the Seismological Society of America 73, 1865-1894.

Boore, D. M., and G. M. Atkinson (1987). Stochastic prediction of ground motion and spectral response parameters at hard-rock sites in eastern North America, Bulletin of the Seismological Society of America 77, 440-467.

Boore, D. M., and J. Boatwright (1984). Average body-wave radiation coefficients, Bulletin of the Seismological Society of America 74, 1615-1621.

Boore, D. M., and W. B. Joyner (1997). Site amplifications for generic rock sites, Bulletin of the Seismological Society of America 87, 327-341.

Boore, D. M., W. B. Joyner, and L. G. Wennerberg (1992). Fitting the stochastic omega (super -2) source model to observed response spectra in western North America; 
trade-offs between Delta sigma and kappa, Bulletin of the Seismological Society of America 82, 1956-1963.

Borcherdt, R. D., and T. E. Fumal (2002). Shear-wave velocity compilation for Northridge strong-motion recording sites, OF 02-0107, 16.

Bour, M., and M. Cara (1997). Test of a simple empirical Green's function method on moderate-sized earthquakes, Bulletin of the Seismological Society of America 87, $668-683$.

Brune, J. N. (1971). Tectonic stress and the spectra of seismic shear waves from earthquakes; correction, Journal of Geophysical Research 76, 5002.

Brune, J. N. (1970). Tectonic stress and the spectra of seismic shear waves from earthquakes, Journal of Geophysical Research 75, 4997-5009.

Chiles, J., and P. Delfiner (1999). Geostatistics; modeling spatial uncertainty, John Wiley \& Sons, New York, NY, United States (USA), United States (USA).

Chin, B., K. Aki, T. C. Hanks, and H. Krawinkler (1991). Simultaneous study of the source, path, and site effects on strong ground motion during the 1989 Loma Prieta earthquake; a preliminary result on pervasive nonlinear site effects; The 1989 Loma Prieta, California, earthquake and its effects, Bulletin of the Seismological Society of America 81, 1859-1884.

Choi, Y., and J. P. Stewart (2005). Nonlinear site amplification as function of $30 \mathrm{~m}$ shear wave velocity, Earthquake Spectra 21, 1-30.

Chopra, A. K. (2007). Elastic response spectrum: A historical note, Earthquake Engineering and Structural Dynamics 36, 3-12.

Cohee, B. P., and G. C. Beroza (1994). Slip distribution of the 1992 Landers earthquake and its implications for earthquake source mechanics; The 1992 Landers. California, earthquake sequence, Bulletin of the Seismological Society of America 84, 692-712. 
Cotton, F., and M. Campillo (1995). Frequency domain inversion of strong motions; application to the 1992 Landers earthquake, Journal of Geophysical Research 100, 3961-3975.

D'Agostino, R. B., and M. A. Stephens (1986). Goodness-of-fit techniques, Vol. ol. 68, M. Dekker, New York.

Dong, G., and A. S. Papageorgiou (2002). Seismic radiation from a unidirectional asymmetrical circular crack model; Part II, Variable rupture velocity, Bulletin of the Seismological Society of America 92, 962-982.

Doornbos, D. J. (1984). On the determination of radiated seismic energy and related source parameters, Bulletin of the Seismological Society of America 74, 395-415.

Draper, N. R., and H. Smith (1998). Applied regression analysis, Wiley, New York.

Dreger, D. S. (1994). Empirical Green's function study of the January 17, 1994 Northridge, California earthquake, Geophys. Res.Lett. 21, 2633-2636.

Emolo, A., and A. Zollo (2005). Kinematic source parameters for the 1989 Loma Prieta earthquake from the nonlinear inversion of accelerograms, Bulletin of the Seismological Society of America 95, 981-994.

Frankel, A. D., C. S. Mueller, T. P. Barnhard, D. M. Perkins, E. V. Leyendecker, N. Dickman, S. L. Hanson, and M. G. Hopper (1996). National seismic-hazard maps; documentation June 1996, Open-File Report - U.S.Geological Survey OF 96-0532. 110.

Freymueller, J., N. E. King, and P. Segall (1994). The co-seismic slip distribution of the Landers earthquake; The 1992 Landers, California, earthquake sequence, Bulletin of the Seismological Society of America 84, 646-659.

Goldberg, D. E. (1988). Genetic algorithms in search, optimization and machine learning. Addison-Wesley., Reading, Mass. 
Gubbins, D. (2004). Time series analysis and inverse theory for geophysicists, Cambridge University Press, Cambridge, United Kingdom (GBR), United Kingdom (GBR).

Haddon, R. A. W. (1996). Earthquake source spectra in eastern North America, Bulletin of the Seismological Society of America 86, 1300-1313.

Hanks, T. C. (1979). b Values and $\omega^{-\gamma}$ Seismic Source Models; Implications for Tectonic Stress Variations Along Active Crustal Fault Zones and the Estimation of HighFrequency Strong Ground Motion; Fault mechanics, Journal of Geophysical Research 84, 2235-2242.

Hanks, T. C., and H. Kanamori (1979). A moment magnitude scale; Fault mechanics, Journal of Geophysical Research 84, 2348-2350.

Hanks, T. C., and J. Boatwright (1982). $f_{\max }$; Special papers on the dynamic characteristics of faulting inferred from recordings of strong ground motion, Bulletin of the Seismological Society of America 72, 1867-1879.

Hanks, T. C., and R. K. McGuire (1981). The character of high-frequency strong ground motion, Bulletin of the Seismological Society of America 71, 2071-2095.

Hartzell, S. H. (1978). Earthquake aftershocks as Green's functions, Geophys.Res.Lett. 5, $1-4$.

Hartzell, S. H., Liu Pengcheng, and C. Mendoza (1996). The 1994 Northridge. California, earthquake; investigation of rupture velocity, risetime, and highfrequency radiation, Journal of Geophysical Research 101, 20-20,108.

Hartzell, S., and C. Langer (1993). Importance of model parameterization in finite fault inversions; application to the $1974 \mathrm{M}$ (sub w) 8.0 Peru earthquake, Journal of Geophysical Research 98, 22-22,134.

Hauksson, E., L. M. Jones, L. K. Hutton, and D. Eberhart-Phillips (1993). The 1992 Landers earthquake sequence; seismological observations, Journal of Geophysical Research 98, 19-19,858. 
Hernandez, B., F. Cotton, and M. Campillo (1999). Contribution of radar interferometry to a two-step inversion of the kinematic process of the 1992 Landers earthquake, Journal of Geophysical Research 104, 13-13,100.

Hisada, Y. (2001). A theoretical omega-square model considering spatial variation in slip and rupture velocity; Part 2, Case for a two-dimensional source model, Bulletin of the Seismological Society of America 91, 651-666.

Hudnut, K. W., Z. Shen, M. Murray, S. McClusky, R. King, T. Herring, B. Hager, Y. Feng, P. Fang, A. Donnellan, and Y. Bock (1996). Co-seismic displacements of the 1994 Northridge, California, earthquake; The Northridge, California earthquake of January 17, 1994, Bulletin of the Seismological Society of America 86, 19-36.

Irikura, K. (1983). Semi-empirical estimation of strong ground motions during large earthquakes, Bulletin of the Disaster Prevention Research Institute 33, 63-104.

Irikura, K. (1992). The construction of large earthquake by a superposition of small events; Proceedings of the Tenth world conference on Earthquake engineering, Proceedings of the World Conference on Earthquake Engineering 10, 727-730.

Irikura, K., and K. Kamae (1994). Estimation of strong ground motion in broadfrequency band based on a seismic source scaling model and an empirical Green's function technique; Earthquake source mechanics, Ann.Geofis. 37, 1721-1743.

Isaaks, E. H., and R. M. Srivastava (1989). An introduction to applied geostatistics, Oxford Univ. Press, New York, NY, United States (USA), United States (USA).

Kolmogorov, A. N. (1950). Foundations of the theory of probability, Chelsea Pub. Co., New York.

Kolmogorov, A. N., and A. N. Shiriaev (1992). Probability theory and mathematical statistics, Vol. 26, Kluwer Academic Publishers, Dordrecht ; Boston.

Langbein, J., R. Borcherdt, D. S. Dreger, J. Fletcher, J. L. Hardebeck, J. R. Murray, R. Nadeau, M. J. Rymer, and J. A. Treiman (2005). Preliminary report on the 28 
September 2004, M 6.0 Parkfield, California earthquake, Seismol.Res.Lell. 76, 10. 26.

Lay, T., and T. C. Wallace (1995). Modern global seismology, Academic Press, San Diego, CA, United States (USA), United States (USA).

Lee, W. H. K., and And Stewart, S. W. (1981). Principles and Applications of Microearthquake Networks, Academic Press, New York.

Liu, P., S. Custódio, and R. J. Archuleta (2006). Kinematic Inversion of the 2004 M 6.0 Parkfield Earthquake Including an Approximation to Site Effects, Bull. Seism. Soc. Am. 96, S143-S158.

Mai, P. M., P. Spudich, and J. Boatwright (2003). Hypocenter locations in finite-source rupture models; Abstracts of the 73rd annual meeting of the Seismological Society of America, Seismol.Res.Lett. 74, 208.

Mai, P. M., P. Spudich, and J. Boatwright (2005). Hypocenter locations in finite-source rupture models, Bulletin of the Seismological Society of America 95, 965-980.

Marquardt, D. (1963). An algorithm for least-squares estimation of nonlinear parameters, J. Soc. Indust. Appl. Math. 11, 431.

McGuire, R. K., and T. C. Hanks (1980). RMS accelerations and spectral amplitudes of strong ground motion during the San Fernando, California earthquake, Bulletin of the Seismological Society of America 70, 1907-1919.

Menke, W. (1984). Geophysical data analysis : discrete inverse theory, Academic Press, Orlando, Fla.

Motazedian, D., and G. M. Atkinson (2005). Stochastic finite-fault modeling based on a dynamic corner frequency, Bulletin of the Seismological Society of America 95, 9951010.

Newland, D. E. (2005). An introduction to random vibrations, spectral \& wavelet analysis, Dover Publications, Mineola, N.Y. 
Ou, G., and R. B. Herrmann (1990). A statistical model for ground motion produced by earthquakes at local and regional distances, Bulletin of the Seismological Society of America 80, 1397-1417.

Papageorgiou, A. S., and K. Aki (1983). A specific barrier model for the quantitative description of inhomogeneous faulting and the prediction of strong ground motion; I, Description of the model, Bulletin of the Seismological Society of America 73, 693722.

Papageorgiou, A. S., and K. Aki (1983). A specific barrier model for the quantitative description of inhomogeneous faulting and the prediction of strong ground motion; Part II, Applications of the model, Bulletin of the Seismological Society of America 73, 953-978.

Parker, R. L. (1994). Geophysical inverse theory, Princeton University Press, Princeton, N.J.

Saragoni, G. R., and G. C. Hart (1974). Simulation of artificial earthquakes, Int.J.Earthquake Eng.Struct.Dyn 2, 249-267.

Sato, T. (1994). Seismic radiation from circular cracks growing at variable rupture velocity, Bulletin of the Seismological Society of America 84, 1199-1215.

Schneider, J. F., W. J. Silva, and C. Stark (1993). Ground motion model for the $1989 \mathrm{~m}$ 6.9 Loma Prieta earthquake including effects of source, path, and site, Earthquake Spectra 9, 251-287.

Shen, Z., B. X. Ge, D. D. Jackson, D. Potter, M. Cline, and L. Sung (1996). Northridge earthquake rupture models based on the Global Positioning System measurements; The Northridge, California earthquake of January 17, 1994, Bulletin of the Seismological Society of America 86, 37-48.

Silva, W. J., R. B. Darragh, I. G. Wong, and L. Huey (1989). Engineering characterization of strong ground motions with applications to the Pacific Northwest; 
Proceedings of Conference XLVIII; 3rd annual workshop on Earthquake hazards in the Puget Sound, Portland area, OF 89-0465, 40-41.

Somerville, P. G., M. K. Sen, and B. P. Cohee (1991). Simulation of strong ground motions recorded during the 1985 Michoacan, Mexico, and Valparaiso, Chile, earthquakes, Bulletin of the Seismological Society of America 81, 1-27.

Somerville, P. G., N. F. Smith, R. W. Graves, and N. A. Abrahamson (1997). Modification of empirical strong ground motion attenuation relations to include the amplitude and duration effects of rupture directivity, Seismol.Res.Letl. 68, 199-222.

Squibb, M. B., R. J. Archuleta, and J. Steidl (2004). COSMOS Virtual Data Center; SSA 2004; abstracts of the annual meeting, Seismol.Res.Lett. 75, 247.

Steidl, J. H., R. J. Archuleta, S. H. Hartzell, T. C. (1991). Rupture history of the 1989 Loma Prieta, California, earthquake; The 1989 Loma Prieta, California, earthquake and its effects, Bulletin of the Seismological Society of America 81, 1573-1602.

Stein, S., and M. Wysession (2003). An introduction to seismology, earthquakes, and Earth structure, Blackwell, Oxford, United Kingdom (GBR), United Kingdom (GBR).

Su, F., Y. Zeng, and J. G. Anderson (1994). Simulation of Landers earthquake strong ground motion using a composite source model; 89th annual meeting of the Seismological Society of America; abstracts, Seismol.Res. Lett. 65, 52.

Su, F., Y. Zeng, and J. G. Anderson (1994). Simulation of the Loma Prieta earthquake strong ground motion using a composite source model; AGU 1994 fall meeting, EOS Trans.Am.Geophys. Union 75, 448.

Iarantola, A. (2005). Inverse problem theory and methods for model paramenter estimation, Society for Industrial and Applied Mathematics, Philadelphia, PA.

Tarantola, A. (1987). Inverse problem theory : methods for data fitting and model parameter estimation, Elsevier; Distributors for the United States and Canada, Elsevier Science Pub. Co., Amsterdam ; New York; New York, NY, U.S.A. 
Toro, G. R., and R. K. McGuire (1987). An investigation into earthquake ground motion characteristics in eastern North America, Bulletin of the Seismological Society of America 77, 468-489.

Trifunac, M. D. (2006). Biot response spectrum, Soil Dyn.Earthquake Eng. 26, 491-500.

Wald, D. J., and T. H. Heaton (1994). Spatial and temporal distribution of slip for the 1992 Landers, California, earthquake; The 1992 Landers, California, earthquake sequence, Bulletin of the Seismological Society of America 84, 668-691.

Wald, D. J., T. H. Heaton, and K. W. Hudnut (1996). The slip history of the 1994 Northridge, California, earthquake determined from strong-motion, teleseismic, GPS, and leveling data; The Northridge, California earthquake of January 17, 1994, Bulletin of the Seismological Society of America $\mathbf{8 6}, 49-70$.

Wald, D. J., D. V. Helmberger, and T. H. Heaton (1991). Rupture model of the 1989 Loma Prieta earthquake from the inversion of strong-motion and broadband teleseismic data; The 1989 Loma Prieta, California, earthquake and its effects, Bulletin of the Seismological Society of America 81, 1540-1572.

Wells, D. L., and K. J. Coppersmith (1994). New empirical relationships among magnitude, rupture length, rupture width, rupture area, and surface displacement, Bulletin of the Seismological Society of America 84, 974-1002.

Wills, C. J., M. D. Petersen, W. A. Bryant, M. S. Reichle, G. J. Saucedo, S. S. Tan, G. C. Taylor, and J. A. Treiman (2000). A site conditions map for California based on geology and shear wave velocity; Seismological Society of America 2000; abstracts of the 95th annual meeting, Seismol.Res.Lett. 71, 248.

Wills, C. J., and W. Silva (1998). Shear-wave velocity characteristics of geologic units in California, Earthquake Spectra 14, 533-556.

Yu, G., K. N. Khattri, J. G. Anderson, J. N. Brune, and Y. Zeng (1995). Strong ground motion from the Uttarkashi, Himalaya, India earthquake; comparison of observations 
with synthetics using the composite source model, Bulletin of the Seismological Society of America 85, 31-50.

Zeng, Y., and J. G. Anderson (1996). A composite source model of the 1994 Northridge earthquake using genetic algorithms, Bulletin of the Seismological Society of America 86, 71-83.

Zeng, Y., and J. G. Anderson (1995). A method for direct computation of the differential seismogram with respect to the velocity change in a layered elastic solid, Bulletin of the Seismological Society of America 85, 300-307.

Zeng, Y., J. G. Anderson, and G. Yu (1994). A composite source model for computing realistic synthetic strong ground motions, Geophys.Res.Lett. 21, 725-728. 